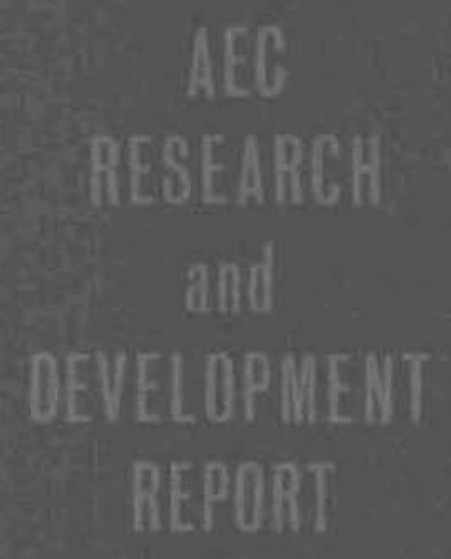

\title{
FAST FLUX TEST FACILITY DRIVER FUEL MEETING
}

\section{BNWL-268}

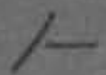

\author{
JUNE, 1966
}

\author{
RICHLAND, WASHINGTON \\ March 8, 9, and 10, 1966
}

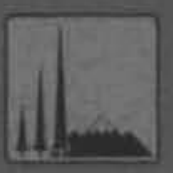

BATTELLEANDTHWEST BATTELIE MEMORIAL INSTITUTE/PACIFIC NORTHWEST LABORATORY 


\section{LEGAL NOTICE}

This report was prepared as an account of Government sponsored work. Neither the United States, nor the Commission, nor any persan acting on behalf of the Commission:

A. Makes any warranty or representation, expressed or implied, with respect to the occuracy, com. pleteness, or usefulness of the information contained in this report, or that the use of any information, apparatus, method, or process disclosed in this report moy not infringe privately owned rights; or

B. Assumes any liabilities with respect to the use of, of for domages resulting from the use of any information, apparatus, method, or process disclosed in this report.

As used in the obove, "person acting on behalf of the Commission includes any employee or contractor of the Commission, or employee of such contractor, to the extent that such employee or conHroctor of the Commission, or emplayee of such contractar prepores, disseminates, or provides access to, any information pursuant to his emplayment or controct with the Commission, or his employment with such contractor.

\section{PACIFIC NORTHWEST LABORATORY RICHLAND, WASHINGTON \\ operated by}

BATTELLE MEMORIAL INSTITUTE

for the

UNITED STATES ATOMIC ENERGY COMMISSION UNDER CONTRACT AT(45-1)-1830 
BNWL -268

UC-80, Reactor Technology

\section{FAST FLUX TEST FACILITY \\ DRIVER FUEL \\ MEET ING}

\section{Co-Sponsors:}

Pacific Northwest Laboratory and

United States Atomic Energy Commission

Richland, Washington, March 8, 9, and 10, 1966

$$
\text { June, } 1966
$$

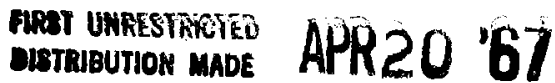

Compiled by

R. E. Peterson, Battelle-Northwest 
Printed in USA. Price \$7.00. Available from the

Clearinghouse for Federal Scientific and Technical Information National Bureau of Standards

U.S. Department of Commerce

Springfield, Virginia 


\section{FOREWORD}

This document is intended to provide a record of the proceedings of the FFTF Driver Fuels Meeting held in Richland, Washington, March 8-10, 1966 under the joint sponsorship of the Pacific Northwest Laboratory, operated by Battelle Memorial Institute, and of the Atomic Energy Commission.

Most of the presentations and discussions appearing in these proceedings were generated from tape recordings and shorthand trans criptions taken during the meeting. Thus, much of the informality and spontaneity of the meeting has been retained. Likewise, most of the illustrations with each paper were taken directly from the slides shown during the meeting. Although this less formal method for documenting the proceedings results in a somewhat less polished manuscript, it has the decided advantage of disseminating much worthwhile information before it is ancient history. During the course of transcription and editing, some remarks may have been lost or abbreviated. Hopefully, the essential information in all presentations and discussions has been faithfully reproduced without distortion.

R. E. Peterson

Publication Chairman 
ACKNOWLEDGEMENTS

Documentation of these proceedings in a relatively short time was made possible by the excellent cooperation of all of the authors in reviewing their papers for publication. The efforts of P. D. Cohn, who assisted with the technical editing, and L. R. Stevens, who performed the final edit, are especially appreciated. Preparation of the tape transcriptions by B. E. Leslie and J. K. Green is also gratefully acknowledged. 


$$
\operatorname{March} 8 \frac{\text { AGENDA }- \text { FFTF DRIVER FUEL MEETING }}{9, \text { and } 10,1966-\text { Richland, Washington }}
$$

Session Chairman:

Arrangements:

\section{SESSION I}

\begin{tabular}{|c|c|c|}
\hline $8: 00$ & $\mathrm{a} \cdot \mathrm{m}$. & $\mathrm{AEC} ; \mathrm{PNL}$ \\
\hline $8: 30$ & a.m. & PNL \\
\hline $9: 30$ & a.m. & PNL \\
\hline $10: 30$ & a.m. & PNL \\
\hline $11: 30$ & $a \cdot m$. & PNL \\
\hline $12: 00$ & p.m。 & PNL \\
\hline $1: 30$ & p.m. & ANL \\
\hline $2: 30$ & $p \cdot m$. & APDA \\
\hline $3: 30$ & $p, m$. & PP \\
\hline $4: 30$ & p.m. & $\mathrm{LA}$ \\
\hline
\end{tabular}

Tuesday, March 8, 1966

SESSION II

Wednesday, March 9, 1966

\begin{tabular}{rll}
\hline $8: 30 \mathrm{a} \cdot \mathrm{m}$. & BEW & L. R. Weissert \\
$9: 30 \mathrm{a} \cdot \mathrm{m}$. & NUMEC & K. H. Puechi \\
$10: 30 \mathrm{a} \cdot \mathrm{m}$. & WEST & F.M.Heck, W. E. Ray \\
$11: 30 \mathrm{a} \cdot \mathrm{m}$. & APDA & A. A. Shoudy, Jr. \\
$1: 30 \mathrm{p} \cdot \mathrm{m}$. & UNC & N. Fuhrman, A。H. Kazi \\
$2: 30 \mathrm{p} \cdot \mathrm{m}$. & ORNL & J.E.Cunningham, J.W. U11man \\
& & D. A. Douglas \\
$3: 30 \mathrm{p} \cdot \mathrm{m}$ & PNL & T. T. Claudson \\
$4: 30 \mathrm{p} \cdot \mathrm{m}$. & PNL & W. E. Roake
\end{tabular}

Thursday, March 10, 1966

\begin{tabular}{rll}
\hline $8: 30$ a.m. & GE & E. L. Zebroski, S. A, Rabin, \\
$9: 30 \mathrm{a} . \mathrm{m}$. & W. E. Bailey, . H. Field \\
$10: 30 \mathrm{a} \cdot \mathrm{m}$. & BMI & D. L. Keller \\
$11: 30 \mathrm{a} \cdot \mathrm{m}$. & ANL & J. H. Kittel, R. E. Macherey \\
$2: 00 \mathrm{p} . \mathrm{m}$. & C. E. Weber & \\
& PNL & E. R. Astiey-Chairman \\
& ANL & R. Avery \\
& APDA & A. Amorosi \\
& GE & E. L. Zebroski \\
& WEST & W. E. Ray \\
& CE & W. P. Chernock \\
& PNL & D. R. deHalas \\
& PNL & E. R. Astly-Final Remarks
\end{tabular}

J. J. Morabito, E. R. Astley

L. M. Finch

P. L. Hofmann, W. W. Little

P. D. Cohn

R. E. Peterson

D. R. deHalas

R. Avery, W. B. Loewenstein

J. B. Nims

D. R. de Boisblanc

W. H. Hannum, L. D。 Kirkbride

R. J. Hennig, PNL 

INTRODUCTION

J. J. Morabito and E. R. Astley... . 1.1 through 1.7

Design Concepts

L. M. Finch . . . . . . . . 2.1 through 2.26

Comparison of the Neutronics of Various

FTR Driver Fuels

P. L. Hofmann and W. W. Little.... 3.1 through 3.9

DISCUSSION D3.1 through D3.5

Capital Cost Parameter Survey

P. D. Cohn............ 4.1 through 4.14 DISCUSSION D4.1 through D4.1

FFTF Fuel Cycle Cost Analyses

R. E. Peterson........... 5.1 through 5.14 DISCUSSION D5.1 through D5.5

Cermet and Oxide Fue1s Review

D. R. deHalas.......... 6.1 through 6.4

Physics and Safety Considerations

R. Avery and W. B. Loewenstein. . . 7.1 through 7.6 DISCUSSION D7.1 through D7.2

Physics Comparisons in Fermi Size Cores

J. B. Nims........... 8.1 through 8.11 DISCUSSION D8.1 through D8.3

Applications of Thermal Test Reactor

Experience

D. R. de Boisblanc. . . . . . . 9.1 through 9.13 DISCUSSION D9.1 through D9.2

Liquid Plutonium Fuel Systems,

Their Status and Capabilities

W. H. Hannum and L. D. Kirkbride. . . 10.1 through 10.19 DISCUSSION DI0.1 through D10.8

Fabrication Processes-High Activity Fuels

L. R. Weissert......... . . 11.1 through 11.12 DISCUSSION D11.1 through D11.2

Plutonium Fuel Fabrication Experience at NUMEC

K. H. Puech1............. 12.1 through 12.11 DISCUSSION D12.1 through D12.4

Fuel Design and Oxide Development at Westinghouse

F. M. Heck and W. E. Ray. . . . . . 13.1 through 13.11 DISCUSSION D13.1 through D13.8 
vi i i

Irradiation Performance and Programs of Fermi Fuels

A. A. Shoudy, Jr. .. . . . . . 14.1 through 14.6

DISCUSSION D14.1 through D14.10

Carbide, Cermet and Clad Development at UNC

N. Fuhrman and A. H. Kazi .... 15.1 through 15.15 DISCUSSION D15.1 through D15.1

Cladding and Dispersion Fuel

Development at ORNL

$\mathrm{J}$. E. Cunningham and J.W. U11man . . 16.1 through 16.16

Cladding and Dispersion Fue1

Development at ORNL

D. A. Douglas......... 17.1 through 17.10

DISCUSSION D17.1 through D17.12

Materials Consideration

T. T. Claudson. ......... 18.1 through 18.12 DISCUSSION D18.1 through D18.3

Oxide and Cermet Fabrication at PNL

W. E. Roake............ 19.1 through 19.10

Oxide Fuel, Status and Stretch Capabilities

E. L. Zebroski, W. E. Baily, S. A. Rabin

and J.H. Field........ 20.1 through 20.30 DISCUSSION D20.1 through D20.3

Status of Stainless Stee1-UO $\mathrm{UO}_{2}$ Cermets

D. L. Keller.......2..... 21.1 through 21.11 DISCUSSION D21.1 through D21.5

Alloy Fuel and Carbide Development at ANL

J. H. Kittel and R. E. Macherey . . 22.1 through 22.25

Applications of Sodium Thermally Bonded

Carbide Elements in FFTF

C. E. Weber..... . . . 23.1 through 23.6 DISCUSंडION D23.1 through D23.3

SUMMARY DISCUSSION

INTRODUCTORY REMARKS

E. R. Astley。........... . SD24.1-SD24.2

R. Avery-Remarks。....... . SD25.1-SD25.3

A. Amorosi-Remarks。..... . . SD26.1-SD26.20

E. L. Zebroski-Remarks。...... SD27.1-SD27.4

W. E. Ray-Remarks ......... SD28.1-SD28.2

W. P. Chernock-Remarks....... . SD29.1-SD29:2

D. R. deHalas-Remarks . . . . . SD30.1-SD30.2

FINAL GENERAL REMARKS . . . . . . SD31.1-SD31.3 


\title{
FFTF DRIVER FUELS MEETING
}

\author{
J. J. Morabito and E. R. Astley \\ Pacific Northwest Laboratory \\ Richland, Washington \\ Operated by Battelle Memorial Institute
}

\section{INTRODUCTION}

The Pacific Northwest Laboratory has convened this meeting to enlist the best talents of our laboratories and industry in soliciting factual, technical information pertinent to the Pacific Northwest's Laboratory's evaluation of the potential fuel systems for the Fast Flux Test Facility.

The particular factors that should be emphasized for the se fuel systems are those associated with safety, ability to meet testing objectives, and economics. Specifically, the meeting should assist us in establishing for each potential fuel system:

- The development program and its associated costs including the research and development required for fuel and material fabrication and reprocessing

- The research and development required to establish the safety characteristics of the fuel system

- The research and development associated with the design of the fuel elements and subas semblies

- The effects of the fuel system on the plant capital costs and operating costs

- The effect of the choice on the driver fuel system of the plant's capability of carrying out its test mission with a high degree of availability.

A secondary though equally important aspect of this meeting is to keep our liquid metal fast breeder reactor contractors and laboratories fully informed on the current Pacific Northwest Laboratory efforts relative to driver fuel. This, I think, is within the general context of and consistent with the AEC's objectives of obtaining the maximum contribution to the overall liquid metal fast breeder reactor program.

I think I have now stated a rather general basis for this meeting. With that, I would like to turn it over to Gene Astley, the project manager on the FFTF. He will give the audience some background information and explain the current objectives.

Thank you, John and good morning. I think John covered the role of the meeting very well for me. I would like to talk briefly about how we got where we are; some of the things we look forward to; and where we are at this time.

The reactor concept of interest to us now has a history dating back to the late 1950's. The first actual documentation that we were able to find was published by Argonne (ANL-6194) called "The Fast Fuel Test Reactor," 
and authored by Bruebacker, Humme1, Kittel, McCarthy, and Smyrta. This document analyzes (in quite a bit of detail) the nuclear characteristics of the core, using $\mathrm{UO}_{2}$ flat plate assemblies in a square array. Argonne investigated cores in a range of about 238 to 400 liters, and they generally conclude that at that particular time, with what was known about fuels, a core of about 400 liters, with a thermal capacity of about $400 \mathrm{MW}$, seemed to be about the right size to provide stretch capability and immediate characteristics. They propose an initial operation at 300 liters; in other words, a core that could be varied in terms of its size, and an initial heat rating of around $200 \mathrm{IMW}$. This design would give a fiux of abcut $8 \times 10^{15}$ at the optimistic level of conditions, depending upon fuel performance.

The Argonne document included three important recommendations.

These were

- That the use of hexagonal rather than square grids be examined to enhance the irradiation of certain kinds of sample shapes

- That the use of skewed arrangements similar to the BR 2 Reactor should be investigated with the advantage, perhaps, of increasing accessibility to the core for test specimens and control rods

- That the use of $\mathrm{Pu}^{239}$ as a fuel instead of $\mathrm{U}^{235}$ should be investigated, with the hope that this might increase sample fission rates for the same power.

The next documentation, at least of general distribution (I am sure that the Ccmmission has had a lot of internal correspondence that is not available to us; was essentially the General Advisory Commitiee on Atomic Energy Report in July of 1963. This report requested the Commission to present a review of their fast breeder program. Their final report recommended the building of a fast test reactor even though they recognized that it called for a large investment.

In September, 1963, the office of the Commissioners asked Hanford Laboratories, then cperated by General Electric, to scope out a Fast Test Reactor that would meet the general requirements as outiined in the GAC Report and as outlined in generai by the field at that time. A small group in the Laboratory here, then, deveioped a concept based on the ANL recommendations. This work did result in a concept documented by ourselves, authored by Fred Albaugh of Hanford Laboratories (General Electric, at that time) and Paul Holsted of the local Commission, who is director of the USAEC Research and Development Division at Richland. This was the original concept that we had proposed and received quite widespread distribution. It has a. hexagonal, skewed core fueled with a plutonium cermet. After this report was issued the next year, between April 1964 and April 1965, there was very Iimited work performed in Pacific Northwest Laboratories. During the middie of this time, in January of 1955, the Laboratory was taken over by Battelle.

In April 1965, the AEC authorized Pacific Northwest Laboratories to undertake the conceptual design of the Fast Flux Test Facility. There were a large number of requirements given to us then, which it was hoped we could meet. Perhaps one of the most impcrtant ones was that we were asked to determine a concept that would be sufficiently detailed such that a reasonabie cost estimate could be made that would identify certain major features. The estimate would try to identify the difference between features 
that were a function of power level or flux, or others such as irradiation requirements and costs that were not a function of power or flux. For example, a fuel examination facility added to the complex might well be a functional requirement independent of power level or flux.

This work was finished by the end of August 1965 and documented in three comprehensive reports (BNWL-CC-175) by Battelle in a limited distribution. I would like to emphasize that the objective of this report was to create a conceptual design to identify costs and feasibility. This design was not necessarily developed for immediate initiation of construction. It included an investigative study to identify any parameter ranges, the costs, and some of the important problems that might arise in construction of such a facility. This work has continued at an accelerated pace at Pacific Northwest Laboratories in the last six months. A further progress report was issued in February (BNWL-CC-400).

I think the important point to consider and make clear at this time is what role Pacific Northwest Laboratory will play and what some of our objectives will be in the next few years. I think that it is most important to remember that the Fast Flux Test Facility will be a national testing irradiation facility which will be used by a large number of people throughout the United States in trying to satisfy programs that they have in support of the Fast Breeder Program. It is important that we not only include the requirements each of you feel are important, but at the same time, try to concentrate on this design the technical knowhow that your particular industrial outfits can submit in helping the design of this reactor. The next six months, in this regard, are reasonably critical because this is the period when wewill try to come up with a recommended concept. In order to assist in these studies and bring the talents of a large part of industry to bear on the FFTF, we have several subcontracts。

The first group of which you are aware is what we call the Alternate Study Contracts by Atomics International, General Electric, Westinghouse, and Phillips. Obviously one cannot hire the entire nation on subcontracts for information so it was necessary to make choices. I think the choices of these particular four are obvious. Atomics International has had as much experience, if not more, as anybody else in sodium components, engineering, as well as in the design and construction of large reactors. General Electric has had considerable experience in the general field and also in fast reactors and sodium. In the general reactor field Westinghouse has built a large number of plants; and, of course, Phillips has had extensive test reactor experience, which we thought was very important for this kind of reactor plant. As far as the general review of the concept, the se study contracts will essentially be finished this fiscal year.

Other work and assistance we have had has been subcontracted to Atomics International on a separate basis, direct design support. We have had a subcontract with Atomic Power Development Associates where we have received both design support, some alternate study work, as well as some review in certain areas that we requested. We have also had a small amount of assistance in the physics field from Argonne National Laboratory and are presently working with them in an attempt to outline a more extensive program of assistance. 
Besides these, we are looking forward to recommending to the Commission areas and development that are progressing in the fast breeder program that could have a secondary objective of supporting the FFTF even though they may have a primary objective of satisfying the fast breeder program. We have contacted a large number of you already and have made some site visits in trying to determine for ourselves what facilities might be applicable to support the FFTF program. With your help, I think we can get this done in the next few months.

So this meeting may we 11 turn out to be the first of a series of meetings from which we will plan on different subjects as the time becomes ripe. Today, we hope we can bring out the technical facts that are presently known about fuels and, as Mr. Morabito mentioned, the programs necessary to develop a Fast Driver fuel.

I want to talk briefly about some of the differences which we feel are important between fuel criteria for the FFTF and the Fast Breeder Reactor. The first one that is quite interesting and gives you adequate leeway is that there is no requirement to include fertile material in the FFTF fuel. In other words, it is not necessary to breed. That is not to say that it is not desirable. I am just saying that as a requirement, in contrast to a Fast Breeder Reactor, whether or not you include fertile material becomes a consideration of economics and overall balance of the system. Secondly, it is not required that the driver fuel coolant temperature be high because there is no power production involved. Therefore, there is no reason for optimizing steam temperatures. Again, I am not saying that it is not desirable for other reasons to have a high outiet coolant temperature. I am just saying that it is not a requirement per se. Number three, is the area of safety where indeed we have several additional factors for consideration.

I think the normal way of operating a fast breeder reactor is to develop a core, and sufficiently test it. Then when you operate the reactor, the core is running far enough suppressed, according to the data you have, that there is essentially no conceivable expected accident that would be catastrophic to the reactor in any way. This is obviously something that you do not print on the nameplate of these reactors--that such will not happen--but one does not need to consider such an accident an operational expected occurrence in the FFTF。 I think you have an opposite type situation, where you are considering operating with fuels in the core that have not been proven. In other words, you hope to extend limits by operating the fuel to its ultimate capabilities. An example might involve taking an oxide fuel rod and deliberately operating it with a molten fuel center. This is not what one would call a conservative approach to the fuel; therefore, one should expect that over a period of time of such typical tests that an accident, rather than being improbable, will be quite probable. We wish to have capabilities with this machine to extend fuel performance, and we have set a requirement that we do not destroy the reactor with a meltdown occurrence. This is not to say that retrieval is considered a routine operating problem. This adds, then, a considerable degree of safety requirements on the reactor safety considerations over what one might consider necessary for a fast breeder reactor.

Although the meeting today will certainly be focused on the fuel, one of the things equally important to us is what influence does the fuel have 
on the design of the concept itself. We have done quite a bit of parametric work on oxide and cermet driver cores, and this work will be the subject of the following morning sessions. We are not attempting to come to any conclusion in this meeting as to the proper choice for the driver fuel. We are, as I stated earlier, several months from having all of the necessary information for a considered judgment. I think it is obvious that if you build a large enough reactor with a large enough heat removal system, one can have whatever degree of versatility one can afford. The problem facing us then is to consider what investment you wish to make for versatility. The real problem is trying to set the range, or deciding exactly how large should this reactor be, what power level should it have, and what kind of flux ranges should it accomplish. In order to place some bounds on the problem, the Commission has set down a list of objectives, capabilities, and requirements. They are quite broad. Under the objectives and requirements portion of this list we have an adequately controlled instrument environment as one objective. Again, it doesn't say to what extent. They also say we should have capabilities to test fuel up to and including failure. But again, it does not specify as to what happens to the reactor in that extent so this is still to be decided. Another requirement calls for a reliable plant performance, but again to what extent needs to be determined in trade-off studies.

Now these are still very broad critera, and yet they still represent a couple of years of hard work by many different people; particularly, the AEC headquarters personnel. I think they do define pretty well a consistent set of objectives, and, perhaps most important, a realistic set.

Early in the study we set down a very broad gage set of bases that we though would be helpful in trying to evaluate the driver fuel. As you can see

FFTF DRIVER FUEL

Bases of Evaluation
Flux Intensity
Flux Spectrum
Flexibility
Safety
Reliability
R\&D Req'ts.
Schedule
Costs
R\&D
Testing
Capital
Fuel Cycle

Applicability To LMFBR Program

R\&D

Design

Engineering

Testing

Fabrication

Operation

SLIDE 1 here, on this slide we considered the flux

intensity the flux spectrum, safety of the reactor itself, safety of the environs, reliability and development requirements that might be involved in the selection of the driver fuel. Under costs, you must examine total costs. In other words, one needs to evaluate the various driver fuels in terms of how much it would cost for research and development to build the first core; how much it would cost for the testing program; what capital cost might be involved in either the building up of facilities within industry or, indeed, the effects of capital costs on the reactor plant itself as well as the operating costs brought about by the fuel cycle. And, of course, most important down at the bottom of the slide is the applicability of this particular machine to test fuel for the liquid metal fast breeder reactor program You should consider whether or not the research and development that you would do on the driver fuel is applicable to the liquid metal fast breeder program. In other words, if we can have as a secondary objective in the Fast Flux Test Reactor, 
the requirement that things that we do to get there also support the liquid metal fast breeder reactor program, we then have, perhaps, the most efficient program in terms of resource and dollars for the Commission.

More specifically, in trying to set some of the parameters, we have to worry about what environment we are going to provide in the reactor complex (Slide 2), and we have these particular kinds of items-the neutronic environment as to what kind of flux intensity level, what spectrum, what chemical properties in the coclant, what kind of impurity level control and what kind of wetted surface to maintain. We are interested in controlling these things not from the standpoint of protecting the driver fuel but controliing them in terms of trying to provide information to the tester.

Then, we have the thermal environment. What kind of inlet and outlet temperatures are optimum? Here you have several things to consider. One is the effect of inlet and outlet temperatures on the cost or operational cost of the driver fuel. Another is the auxiliary information of what temperatures can provide the most information to the Liquid Metal Fast Breeder Reactor Program. In the driver core, what temperatures do you have to have to make the driver core itself, perhaps, also be a fuel test bed. Important also is the time-integrated flux that one can provide in this facility.

In addition to the other requirements that one has, you also have to examine very closely what you are actually going to observe about these test specimens now that you have them irradiated. These have to be factored into the whole ccre design and we have a long list (Slides $3 a$ and $3 \mathrm{~b}$ ) of some of the kinds of things in which varous people are interested in examining fuel after it has been irradiated. These parameters, then, have gone into trying to describe the functional requirements and laying out what might be the cost of making a fully integrated facility that could stand on its own.

Finally, and perhaps the most important is consideration of what are the test cbjects that you are going to be dealing with; what are their composition: what are their chemical properties that you wish to test; what are their nuclear properties: what geometries might they have; and how many are you going to test ISlide 4\%. All of these things have been an important consideration in the work that's going to follow, and have been essentially our guidelines throughout all of our studies.

We will finish the se next three days with a brief summarization session. By means of recorders as well as the court reporter, we hope to issue a complete verbatim transcript of this meeting. We will hope to bring out in the summary session those facts which are germaine to the FFTF and are important in meeting those objectives that John Morabito outlined in his opening remarks. So with this introduction, I would like to go on to the next session by Battelle and get into the technical aspects of where we are. As a background we feel that it would help if you did have a brief review of the concept and some of the things at which we have looked. The presentation will not be complete in terms of trying to describe the complete concept. Instead it will be a digest of those important factors that we feel are helpful to explain some of the different kinds of things we have looked at and the influence on the design problem. 


\author{
ENVIRONMENTS \\ A. Neutronic \\ 1. Flux \\ 2. Spectrum
}

B. Chemical

1. Coolant

2. Impurities

3. Wetted Surfaces

C. Thermal

1. Inlet Temperature

2. Outlet Temperature

D. Hydraulic

1. Pressure

2. Pressure Drop

3. Flow Rate

E. Time

1. Cycle Time

2. Number of Cycles

SLIDE 2

\author{
OBSERVATIONS \\ A. In-Reactor \\ 1. Thermal
a. Coolant
b. Test Object

2. Hydraulic
a. Flow Rate
b. Pressure Drop

3. Chemical
a. Coolant Impurities
b. Test Object

4. Fission Gas
a. Pressure
b. Leakage

5. Dimensions
a. Spacing
b. Leng th
c. "Diameter"
SLIDE $3 \mathrm{a}$

FFTF FUNCTIONAL REQUIREMENTS

\section{TEST OBJECTS}

A. Composition

1. Chemical

2. Nuclear

B. Geometry

1. Shape

2. Length

3. "Diameter"

C. Number

11. Chemical

12. Nuclear

13. Metallurgical

* Note Chemical, Thermal, \& Time Limits 


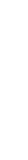




\title{
DESIGN CONCEPTS
}

\author{
L. M. Finch \\ Pacific Northwest Laboratory \\ Richland, Washington \\ Operated by Battelle Memorial Institute
}

\section{INTRODUCTION}

This morning's presentation by Pacific Northwest Laboratory is intended to provide a background (some basis for comparison if you wi11) for the Fast Flux Test Reactor driver fuel discussions of these next three days. To do justice to the driver fuel problems, they must be viewed from a number of vantage points. Some points of view, possibly because of proximity to other large problems, will not treat the driver fuel as the only large problem. So my pitch to you this morning is an appeal. Please consider that while the fuel is an important part of this reactor, it is not the only important part. It is important but not the only factor in reactor safety and capital cost. Again, it is important but not the only influence affecting the plant operability.

The other presentations this morning will depend rather heavily upon results of some preliminary parametric studies. In any parametric study, we seek to evaluate the advantages and penalties obtained by various tradeoffs among the parameters. In the discussion that follows, and presumably for the next three days, you will focus your attention upon ways in which driver fuel affects the reactor physics and the fuel cycle economics. It is important, however, to consider all the other ways the reactor facility might be affected by the driver fuel. While the parametric studies will show the relative affect of the parameters, they do not necessarily indicate that any particular parameter is a practical one throughout its range of interest. In fact, some of the apparent extreme conditions of some of these parameters are beyond the reach of our current technology.

The purpose of my presentation is not to sell this concept for the FFTF. Instead, it is to discuss, against this reference frame of a single reactor facility concept, some of the problems we have encountered in our efforts to match a reactor design to a given set of criteria. Although these criteria have not been carved in stone over these past few years, they have remained remarkably consistent. The most recent criteria (which you received this morning) as was issued as Attachment A to our most recent directive from the Commission. These consequently have some stone-like qualities. 
The directive did condition the criteria somewhat by means of another Attachment $B$ which served to explain and expand the criteria at some length. I will proceed, using Attachment $B$ as a guide for most of the remainder of this discussion, however, we will look first at the Attachment A criteria. These are the objectives, capabilities, and requirements, which Gene Astley touched upon a few minutes ago, that are listed as objectives for the Fast Flux Test Facility. OBJECTIVES, CAPABILITIES AND REQUIREMENTS

The first item under the Attachment A objectives is an adequately instrument-controlled environment in the fast flux. Presumably, this means testing stations; more specifically, it means closed loops. The second item listed under capabilities was: test fuel up to and including failure. This capability even more strongly suggests closed loops. Item three specified a high predictable and reliable plant performance factor. In the face of extensive testing requirements, this objective presents a significant challenge to obtain the plant performance factor.

Item four under the objectives, requires the FTR to test fuel for short time periods. This means that we must probably provide easy access and certainly immediate access to the core of the fast reactor. Item five, the interim nondestructive fuel examination objective is an in-plant requirement for fuel examination after irradiation. Presumably, this would also indicate examination of irradiated non-fuel material, samples, and specimens. Finally, the FFTF is to serve as a fast flux fest installation for users in the United States.

The requirements in Attachment $A$ listed instrumented closed loops about 6 in. OD with a core length of the order of 3 to 4 feet and a total of six to eight closed 1oops. This is a rather significant requirements statement, particularly if you consider that each loop of $6 \mathrm{in}$. diam and 3 to 4 feet long, will exceed 20 liters. Of course, six to eight loops in that size would constitute a very substantial part of the fast reactor core. The instrumented open loops, (about six in number) about the same size as the closed loops, sets the total space (total volume) allotted to testing positions in the core of the fast reactor at about $20 \%$ of the core volume.

Rabbit facilities, listed under item three in requirements, are the short time test installations. These are intergrated to be rapid-transit installations for immediate recovery of irradiated fuel samples and material samples.

The fourth item under requirements, cover provisions to meet programmatic needs of the LMFBR program. This is interpreted to mean that the reactor must be designed to be flexible, and must be arranged to take advantage of the LMFBR programs as they are developed. 
Item five of the requirements facilities for interim fuel examination, 1 ists a number of specific examination criteria. The word interior implies that the irradiated specimens will be returned to the reactor.

Under the first item of capabilities for the FFTF, the FTR should have an intergrated fast flux of the order of 1016 with a fast spectrum some $75 \%$ above 0.1 MeV. This capability requirement imposes a severe restriction upon the driver fuel and on many other parts of the reactor core.

Item two under capabilities requires a specific power range for the fissile fuel of 500 to $2000 \mathrm{~W} / \mathrm{g}$.

Item three specifies the high temperature capability $\left(1200^{\circ} \mathrm{F}\right)$ desired for the operating conditions in the FFTF at the outlet of the fuel channel.

Item four specifies the requirement for substantial sodium impurity controls in the FFTF. Impurity control certainly must be adequate to limit degradation of the structural strength or the integrity of the components within the reactor core and, potentially, of the test specimens that might be placed in open loops. Closed loops, of course, present impurity problems beyond this particular requirement.

The specific requirements of Attachment A call for certain details of the reactor or the facility that are deemed to be very important in meeting the objectives and capabilities that have been recited. The first requirement is for minimum of two reactor primary coolant systems to permit continued plant operation with one loop in the nonoperating status. This, quite obviously, is a capital expense item of a major magnitude.

Item two specifies that closed loops are to be accessible and removable externally to the reactor. In other words, we should not have to disassemble the reactor to make a change in closed loops. Item three specifies adequate provision for control of the closed loop conditions including instrumentation and possibly control applied to the specimen itself within the core.

Requirement Item four calls for adequate provisions for open loop instrumentation. Again, this is interpreted to mean that open loops must be capable of being fully instrumented within the reactor.

Item five specifies accessibility to make and break instrumentation connections from open loops. To the extent that open loops represent isolated or exclusive positions within the reactor core, this may mean that all core fue 1 positions should be accessible for instrumentation. 
Finally, the last item under specific requirements is for a plant availability factor of $75 \%$. This requirement is almost a contradiction to the requirement for large test loops. It represents the primary challenge in this reactor.

Using Attachment $B$, as a guide, I will elaborate upon these various requirements of Attachment A. The Attachment B items do not follow necessarily in the same sequence as Attachment $A$, but they do treat the same subjects.

In general, the principal objective of the FFTF is to provide a relatively large amount of relatively large closed loops. The second objective, and I believe this is quite secondary to the first, is to provide through the FFTF certain advantages to the fast breeder program in development of components, fuel fabrication, driver fuel experience, and general operation of sodium components, including the fuel handling equipment.

Under the general characteristics recited in Attachment $B$, plant availability again occupies a prominent place. It is stated that "the plant availability shall be the maximum practically obtainable." A listing of some of the problems implied would include an extensive design and development program for all components and all features that might affect operability, In this reactor virtually every function is interrelated so that we have few exceptions to the implied $R$ \& $D$ program. The use of proven components and technology is necessary to diminish the necessary design and development to practical levels. Use of proven components also assures we will take maximum advantage of past experience. Extensive proof and quality assurance tests are necessary to meet problems of operability and to assure that we can satisfy them. Redundant equipment and systems must be studied; having a second unit might double problems rather than halve them. However, in many cases redundant apparatus will serve to improve the reliability of the facility. We should, for certain cases where redundant apparatus is not the answer, provide a very highly developed maintenance philosophy. This might require a well developed technique for removing components and replacing them within a short time. It might require in-place repair techniques. It might require us to maintain a continuing development. program to upgrade the reliability of units demonstrated to be deficient in service.

Attachment B listed closed loop tests, which will be discussed here as the types of tests that should be performed in the FFTF.

Attachment $B$ lists the fuel types to be considered as plutoniumuranium fuels such as oxides and cermets, and also carbides, nitrides, carbo-nitrides, and various alloys. The attachment indicates that cladded materials should be metal, metal alloys, refractories, and refractory alloys. The types of fuel tests are specified as ranging from capsule irradiation (to obtain information on fue 1 behavior and characteristics after irradiation) to full scale single pin, clusters, small subassemblies, and finally, full-scale subassemblies. Burnup is expected to exceed $100,000 \mathrm{MWd} /$ ton of heavy atgms, and total exposures in neutrons of something in excess of $10^{24}$ nvt which, 
by implication, requires a large neutron flux. Closed loop tests themselves are treated in some detail in this attachment. We have been requested to design and cost this facility on the basis of $4,5,6,7$, and 8 tests.

Test fuel length depends on the selected core designed for the FFTF. However, the length must provide for specimens ranging from two to four feet of overall active core length, or active fuel section length. The diameter of the test space, that is, the closed loop diameter, has been a difficult problem. Significant controlling parameters apparenty are choice of coolant; the method of the coolant flow in the channel; coolant temperatures and temperature differentials; the size of the instrumented capsules; the size of the instrumentation package that is attached to the capsule (the provisions in the closed loop to limit the thermal or neutronic interaction with adjacent components in the core or other loops; any provisions that might be necessary to either control, to detect, or to limit the consequences of melt down; and finally, of course, the size of the test specimen (whether it be a subassembly, multiple pin, or single pin assembly).

Obviously, there are many other considerations: the number of loops, the amount of fissile material in the loop; and the amount of poison created affect the general safety requirements for the loop as well as the reactor.

Then, there is the question of where the loop should be located within the reactor core. The location, of course, determines the flux and the flux gradient that the particular fuel or the material test will see in the core. I think the first slide will give an indication of some problems and also some of the advantages of this core.

Slide 1 shows what we term the "initial core map for the FFTF. The initial case is a 600 liter core with a thermal capacity of 300 MW in this configuration. The center position in the core is occupied by a closed loop fuel test in this core map. There are also closed loop tests near the periphery and at two sector points on the periphery. These latter two positions are indicated here in 6 in. diam size. We have a number of open loop tests indicated (by the $\mathrm{F}$ in an open hexagon). They are rather heavily clustered about the reactor center position, although there are two peripheral locations shown for open loop tests.

The reactor driver fuel is zoned with increasing fissile content inventory to maintain essentially constant power per channel as we move from the center to the periphery of the core. Out beyond the driver fuel locations are additional 
tubes, which are essentially reflector tubes for the fast core. However, these do provide for material tests and for other auxiliary operations such as indicated on Slide 1 around the periphery. We have the oversize test positions indicated on the periphery in this core configuration.

Slide 2 shows a second core map representing the full test complement anticipated; perhaps this is even an excessive complement of fuel tests for the 600 liter core. Shown are four closed loops of 6 in. diam on the periphery; a materials test in the center; and two closed loops adjacent to the central position. There are other open and closed loops distributed about the core in greater quantity than on the previous core map. It might be interesting to point out that this core is controlled by three shim rods and by three safety rods. These are low in count number; the worth of the individual rods is very high and has been a source of concern for the physicists. Since the problem is probably one of economics rather than one of physics, there is a strong interest at present to provide shimming capability not in the core but at peripheral points about the core by using reflector tube positions numbering from 12 to perhaps the full complement of 30 to provide lower worth insertion values for the shim control. Presumably,
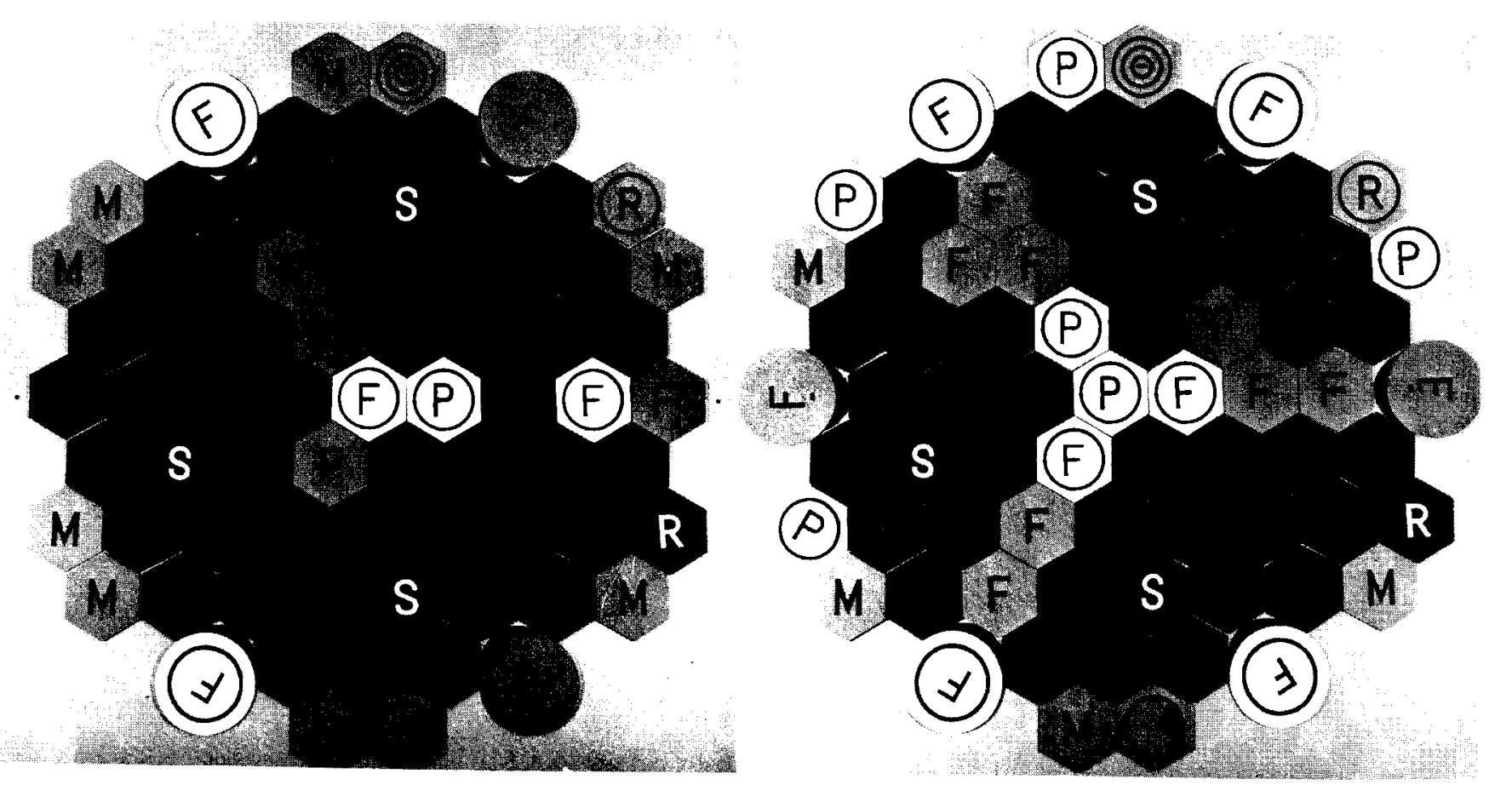

SLIDE 1 
the three or some similar low number of safety rods would be retained. Peter Hofmann will have more to discuss on core physics later on in these discussions.

Slide 3 shows the cross section of the reactor. The core map that we have just viewed was a horizontal cross section of the red spot in the center of this assembly view. The reactor concept at present is a tubed assembly with individual tubes occupying each of the lattice positions that we have seen on the core map. Each position is specialized either for closed loop, for open loop, for driver fue 1 , or the control requirements. The tubes extend through the cover of the reactor vessel, which is a pot type sodium filled container. Each tube is accessible at the upper end for refue1ing and for instrument connections to the instrumentation package on fuel and material specimens. The top of the tubes also are accessible for coolant connectors for closed loops (where closed loop coolant connections are made from the top of the reactor tube with extentions out through the containment vessel into an experimental cell containing the apparatus for the closed loops). The reactor tubes that constitute the main structural component of the reactor core will come in a variety of types.

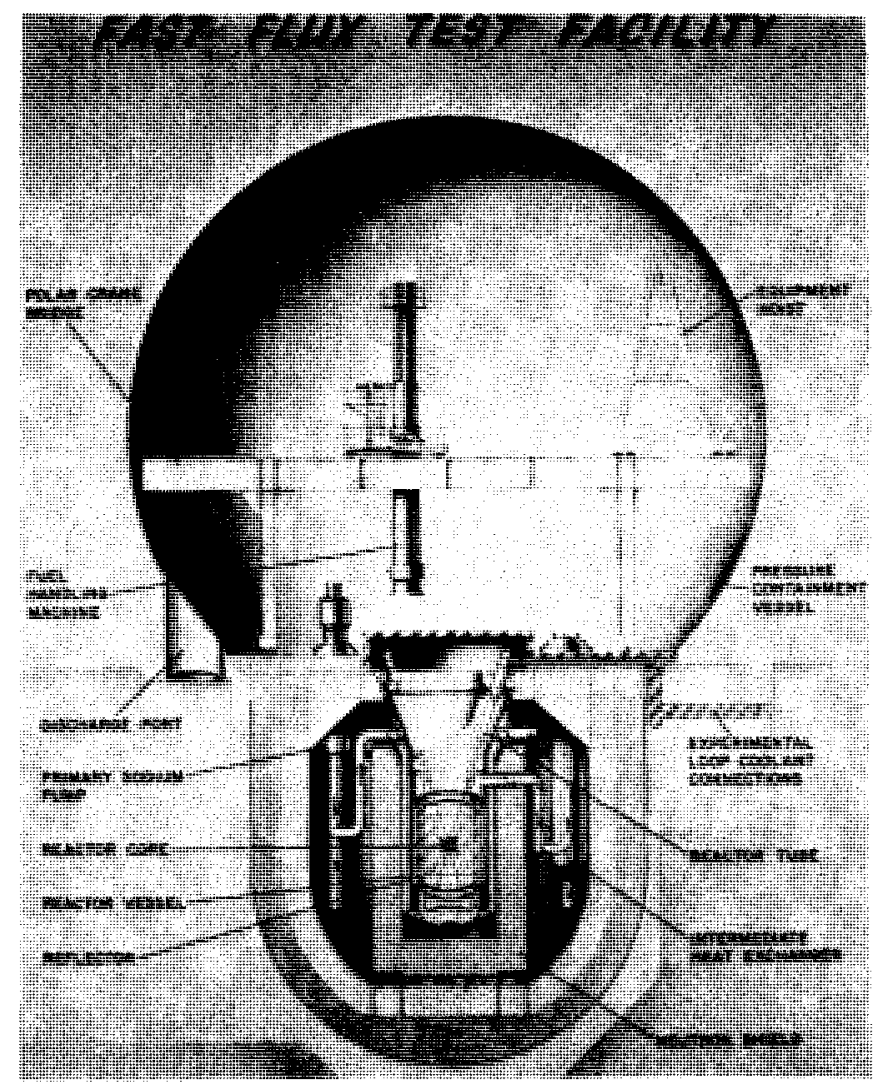


Slide 4 will show two types of reactor tube. The overall tube configuration is shown at the top. The tube shown in the center of the slide is a closed loop reactor tube with the fuel specimen carried in this core region. A bayonet type annular flow path within the general envelope of the reactor tube is supplied from the upper end of the reactor tube. A similar tube shown at the bottom of the view, is for the driver fuel. The driver fuel will be provided with a rod extending from the top of the fue 1 up to and through a shield plug at the top of the reactor tube.

Slide 5 is simply an elaboration upon the closed loop tube to show a tube concept for the 6 in. diam closed loop tube. It is substantially larger than the tubes shown on the preceding slides.

This schematic (SIide 6) shows a typical representation of the closed loop cell, pumps, and heat exchangers in position in the test cell for the primary circuit. The lower portion of the cell will contain storage tanks and emergency cooling circuits. The secondary coolant for the closed loop is piped to an isolated and separate heat sink.

Attachment $B$ has required that the outlet temperatures from the closed loops be maintained in the desing condition at $1400^{\circ} \mathrm{F}$. The coolant, as a general requirement, is sodium. Quite obviously other coolants could be used, especially, liquid metal coolants. It might be desirable to make provisions for at least one gas cooled loop. The particular concern with gas cooled loops is the need for extensive pressurization of the gas coolant itself. This in turn requires a high pressure component (reactor tube) within the core of the reactor. Because of the risk associated with gas cooled closed loops and to a lesser degree with the sodium and other liquid metal cooled loops, the failure of loop components, is a very significant issue from the standpoint of reactor safety and also from the standpoint of the plant operability.

Attachment $B$ also has made some requirements concerning the open loop test positions in the core. These requirements are much less specific than for the closed loops and, in general, reflect lesser concern. Presumably, if we can make closed loop positions accessable and available in the core, we also can do the same with open loops. The number of open loops, the test section length, and the diameter of test pins are roughly the same as closed loop requirements. The significant point here, perhaps, is that it is intended that open loops will receive full instrumentation of both the fuel specimen and the coolant channel. There is a notation that each test position should be in an essentially vertical position. This, of course, is of interest for this particular concept because, except for the central channel, each reactor tube has a vertical angle increasing with the radial position from the center of the core. Maximum inclination to the vertical is $15-1 / 2^{\circ}$ for the outer peripheral fuel position. 


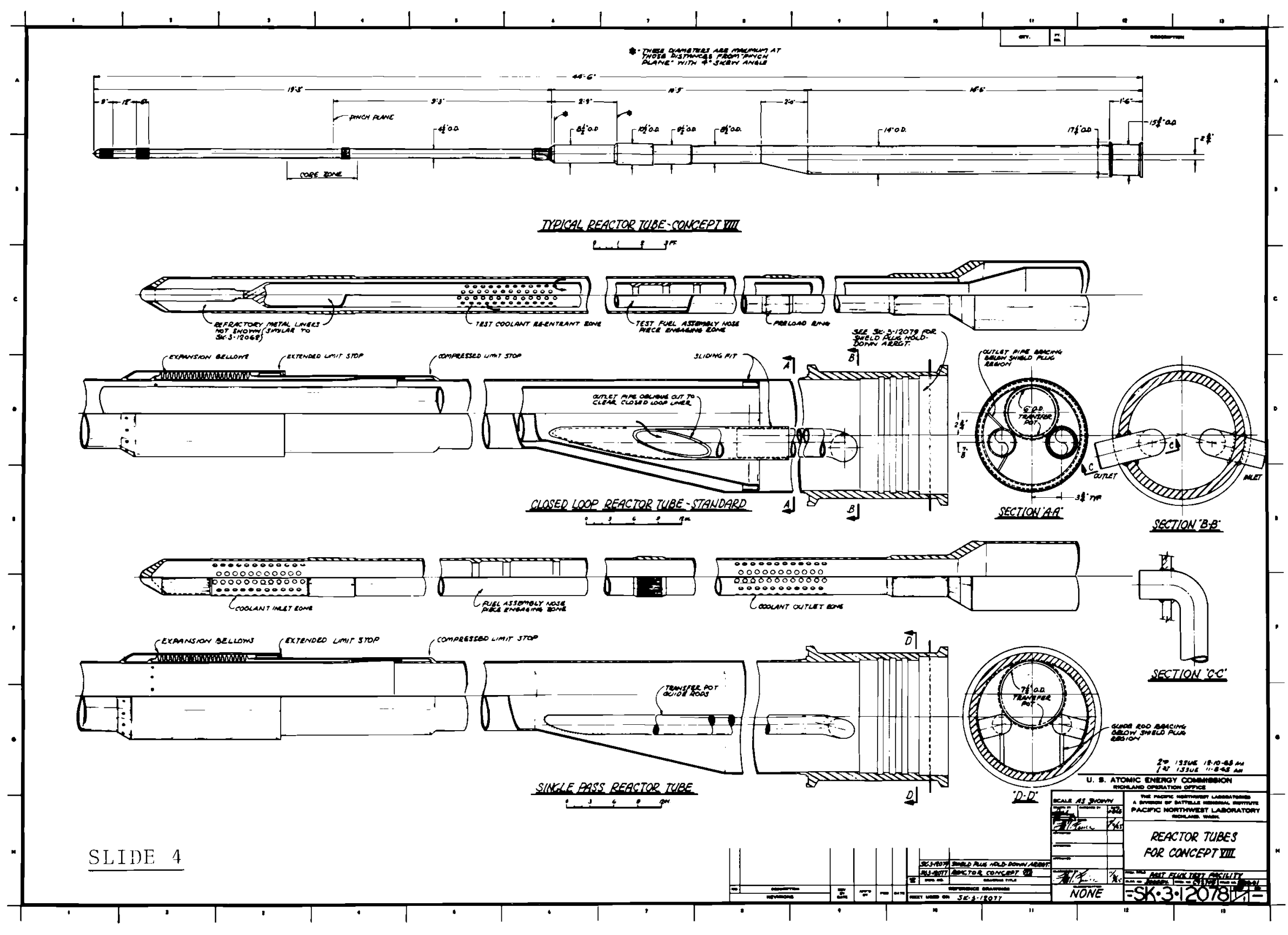




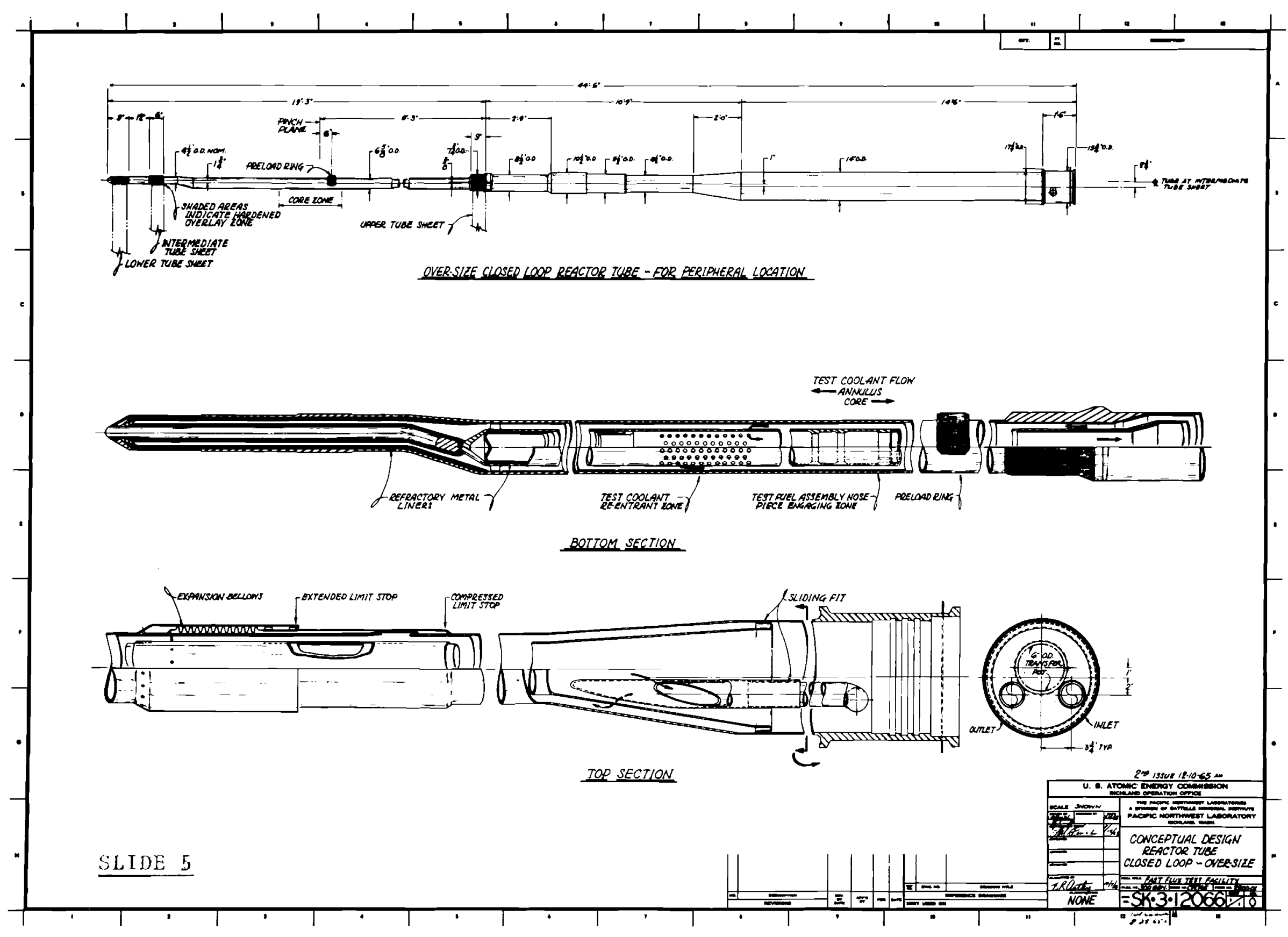




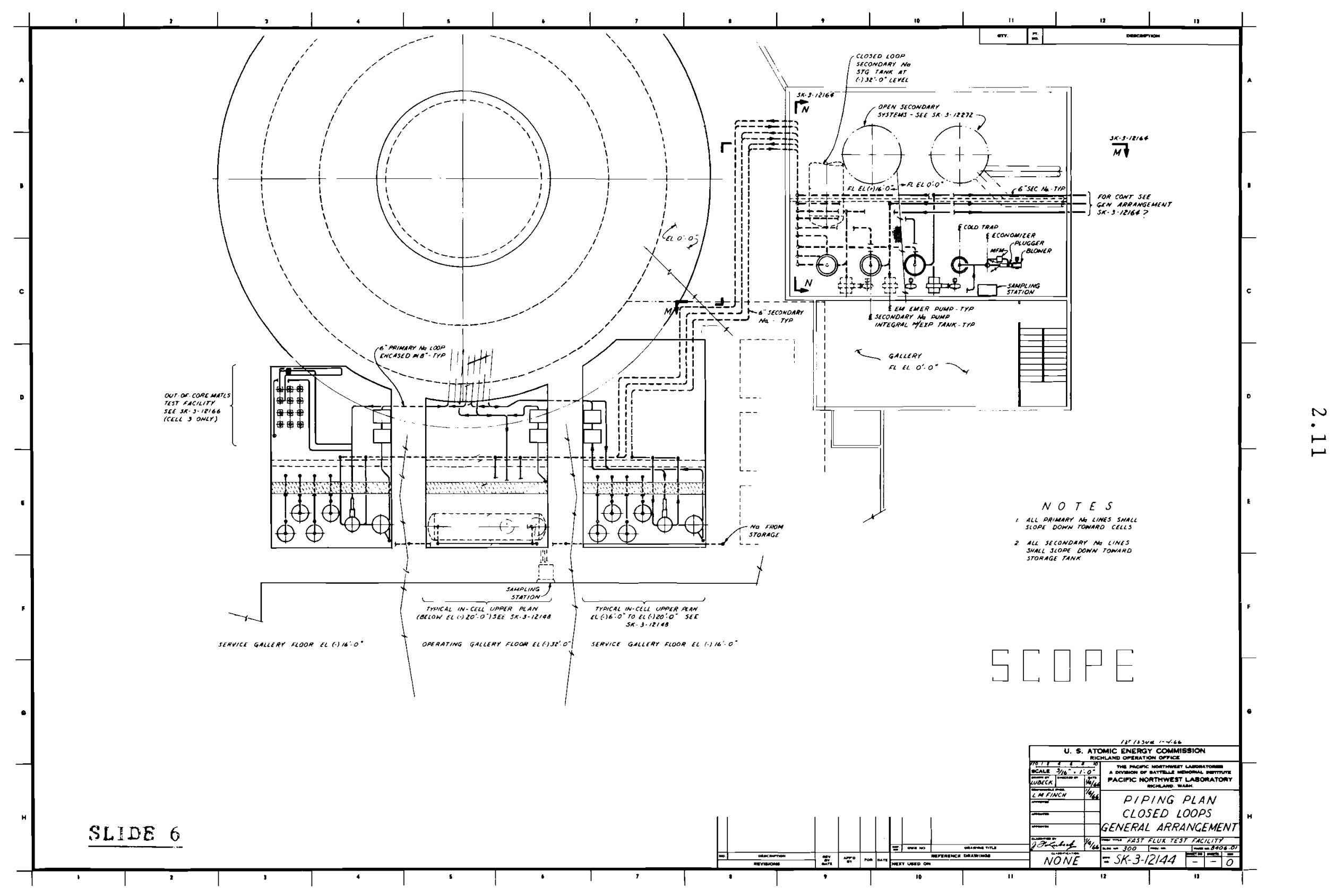


Attachment B specifies that each test position should be independent of other test positions in the core. This implies at least that there be a coolant separation for each test specimen.

There is particular mention made under these criteria (attachment B) for rabbit facilities. The rabbit facility is a reactor tube with a standard envelope which has a pneumatic transfer section within the upper end (large part) of the reactor tube and connections to this pneumatic transfer section extending outside of the reactor containment along the cover of the reactor in a manner similar to the closed loop coolant connections. The in-core section of the rabbit tube is isolated thermally by multiple gas barriers and annular stagnant zones.

Attachment $B$ treate I the core design as a specific topic. Maximum flux at the center of the core shall be evaluafed at three conditions of maximum flux. These levels are $0.7 \times 10^{16}, 1.0 \times 10^{16}$, and $1.35 \times 1016, \mathrm{n} / \mathrm{cm}^{2}$ sec. The referenced spectrum for each of these flux levels was $75 \%$ above $0.10 \mathrm{NeV}$. It was noted that means should be provided to permit the spectrum to be softened if desired to conform more exactly to expected fast breeder reactor in-core spectrum. The initial criteria conform to the $1000 \mathrm{MW}$ studies.

Driver fuels to be considered include a cermet or ceramic; and if other fuels show sufficient promise, they too should be considered.

We should have means to instrument the driver fuel positions and the open loop test positions. This places more generality upon the assumption we made earlier that open loops should be permitted in any position in the core and that they should be instrumented in those positions. This extends that requirement to include the driver fuel positions. Again, the temperature limitation appears in the requirement for the mixed-mean core outlet temperature evaluation over the range of 800 to $1200^{\circ} \mathrm{F}$. Bypass flow is permitted to reduce this temperature beyond the immediate outlet position on the reactor fuel section.

The next section of Attachment $B$ deals with the fuel handling system. We've already seen the reactor cross section earlier (Slide 3), but it will serve again as a reference for the discussion on fuel handling.

As we investigate this reactor concept further and further, the fuel handling machine assumes more and more importance. I think the most pertinent point of interest is the problem of maintaining a high degree of plant availability, and with that, the fuel handling machine apparently is the limiting system.

The machine has a large number of duties to perform. Obviously it must handle the driver fuel in and out of the reactor; but in addition, it must move the fuel samples and material specimens and it must complete their transit to and from the core. It must serve as a device to permit a wide variety of tubes to be removed from the reactor. Other maintenance operations in and about the core must be performed either by the fueling machine or by the gantry 


\subsection{3}

hoist. The fuel handling machine is located and positioned by the manipulator head on the main crane. In this concept the inadicted fuel is withdrawn from the reactor tube into the FHM cask, and placed in an irradiated fuel storage pot adjacent to the reactor vessel neutron shield for a decay period of the order of one fueling cycle. After the decay period, the fuel is then removed from the pot into the fueling machine and then it is transferred out to examination cells (in the case of fuel specimens) or to the fuel storage and shipping positions (in the case of the driver fue1). Of course, if a fast transit on specimens is desired, the use of the decay pots for an intermediate storage point will be omitted to permit the fuel to be transferred by the fueling machine directly into the examination areas. The examination facilities are quite closely associated with the fuel handling facilities. There is no clear way to separate them nor is there any particular purpose served by separation of these functions. The principle concern with the fuel handling system is that it must perform many crucial operations within this reactor containment zone.

Slide 7 shows the reactor core assembly. It is a cartridge assembly inserted within the reactor vessel. It provides the core region with its immediate reflector material and the high pressure plenum for coolant passage into the reactor tubes. The reactor tubes will discharge coolant into a plenum above the core for subsequent flow out into the coolant discharge region in the upper part of the reactor vessel.

Slide 8 will show the reactor vessel itself with phantom lines showing the reactor core assembly in place. Again, coolant comes in through the top ring header and flows down through six pipes to the inlet plenum. From the plenum, the flow is up through the reactor tubes for discharge from the reactor vessel to follow the recirculation path to the intermediate heat exchangers, pumps, and then back to the ring header.

At the top of the reactor vessel, the reactor cover has a diameter of 26 feet. Ninety-one nozzles for reactor tubes penetrate the cover. The reactor vessel is enclosed by a multiple element neutron shield. Slide 9 shows a typical coolant connection to a closed loop.

Slide 10 shows a view of the reactor tube nozzles at the top of the reactor. This is a much simplified version of the structure that will be used at the top of the reactor tubes. The tube itself lies in this general region, extending on down to the reactor core below. High pressure connections shown here are intended to constrain the components of the reactor tube and the shield plug against ejection in the event of a major pressurization of the core such as would occur in the extreme case of the hypothetical excursion. 


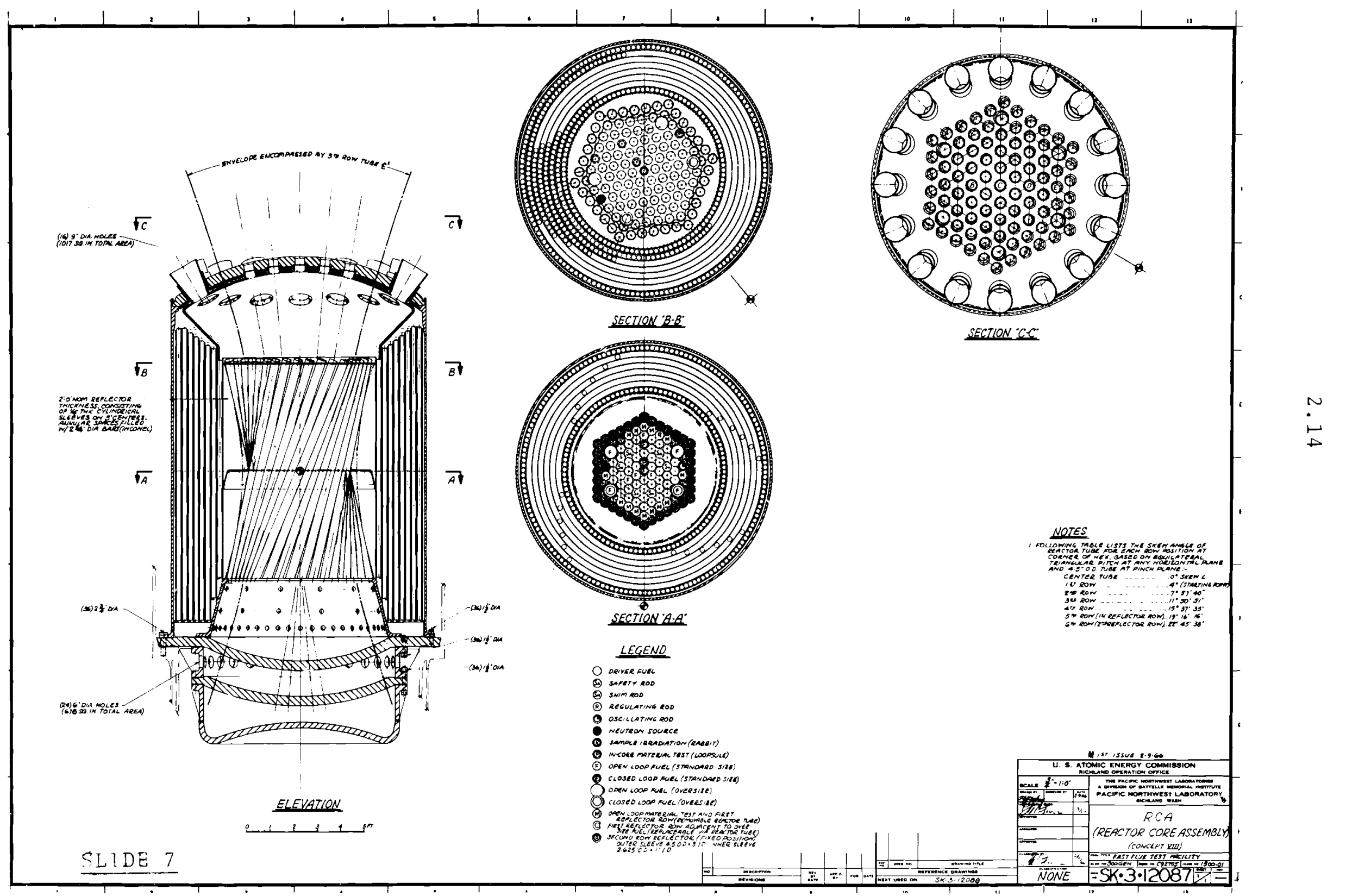




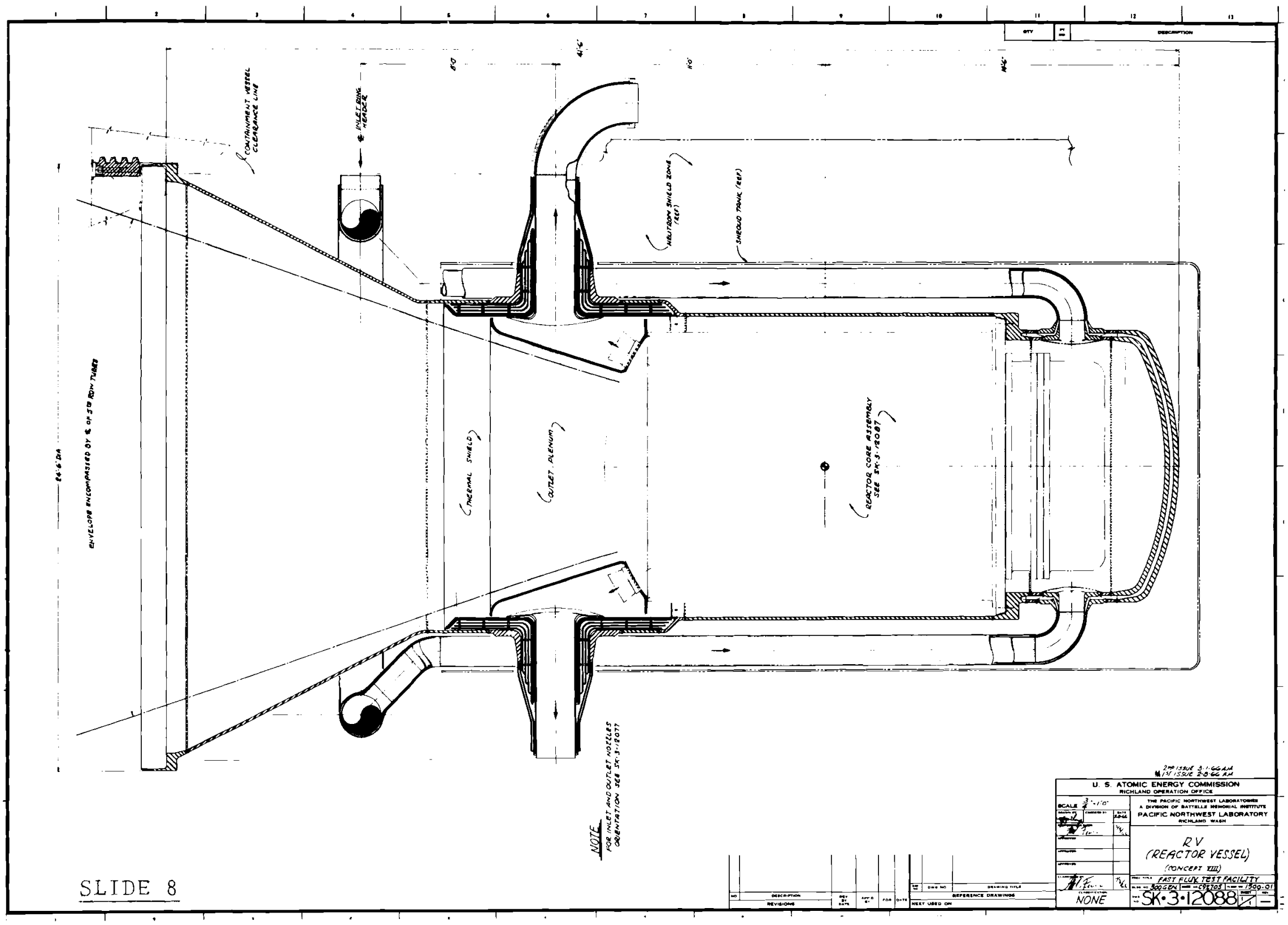

落 


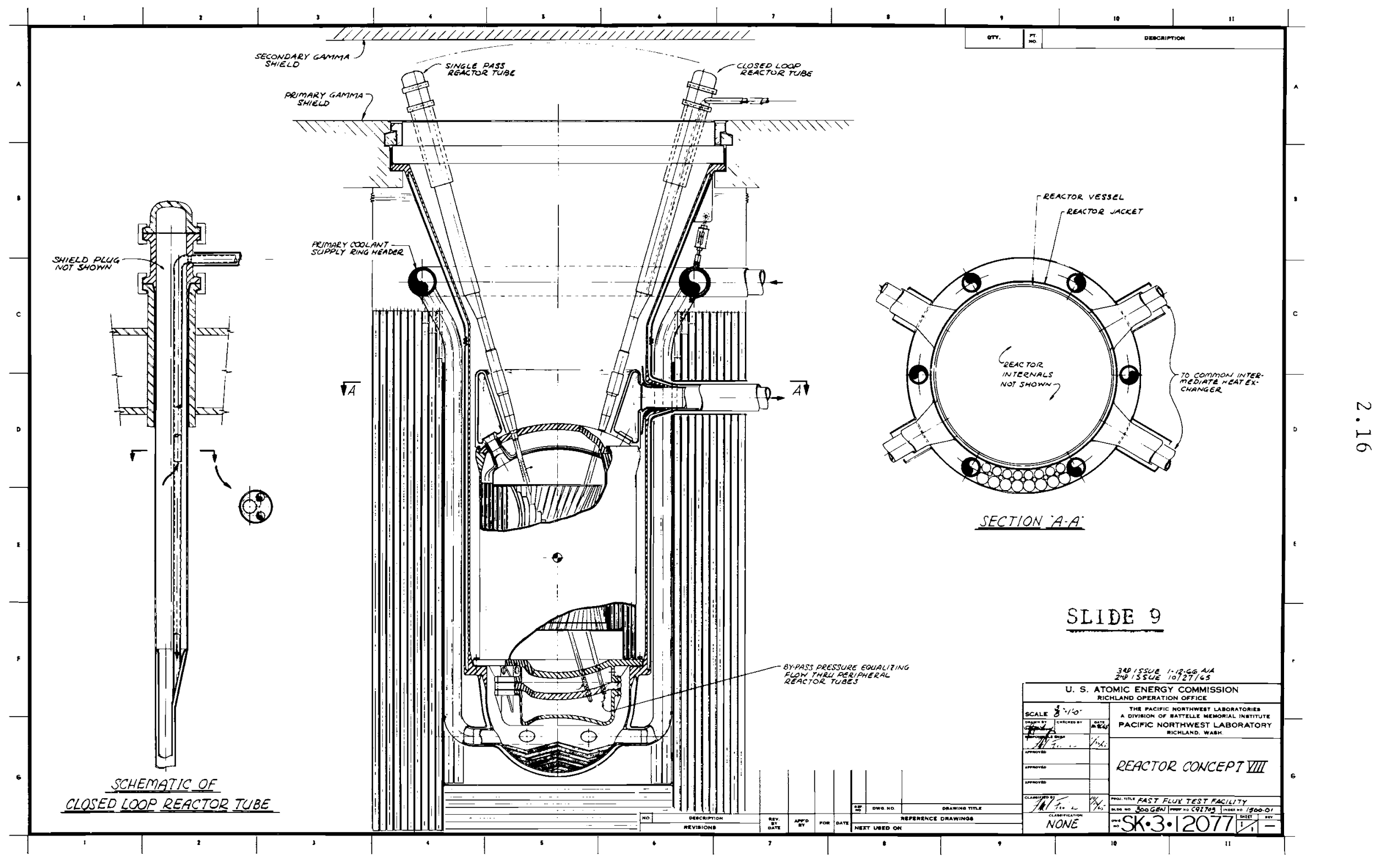




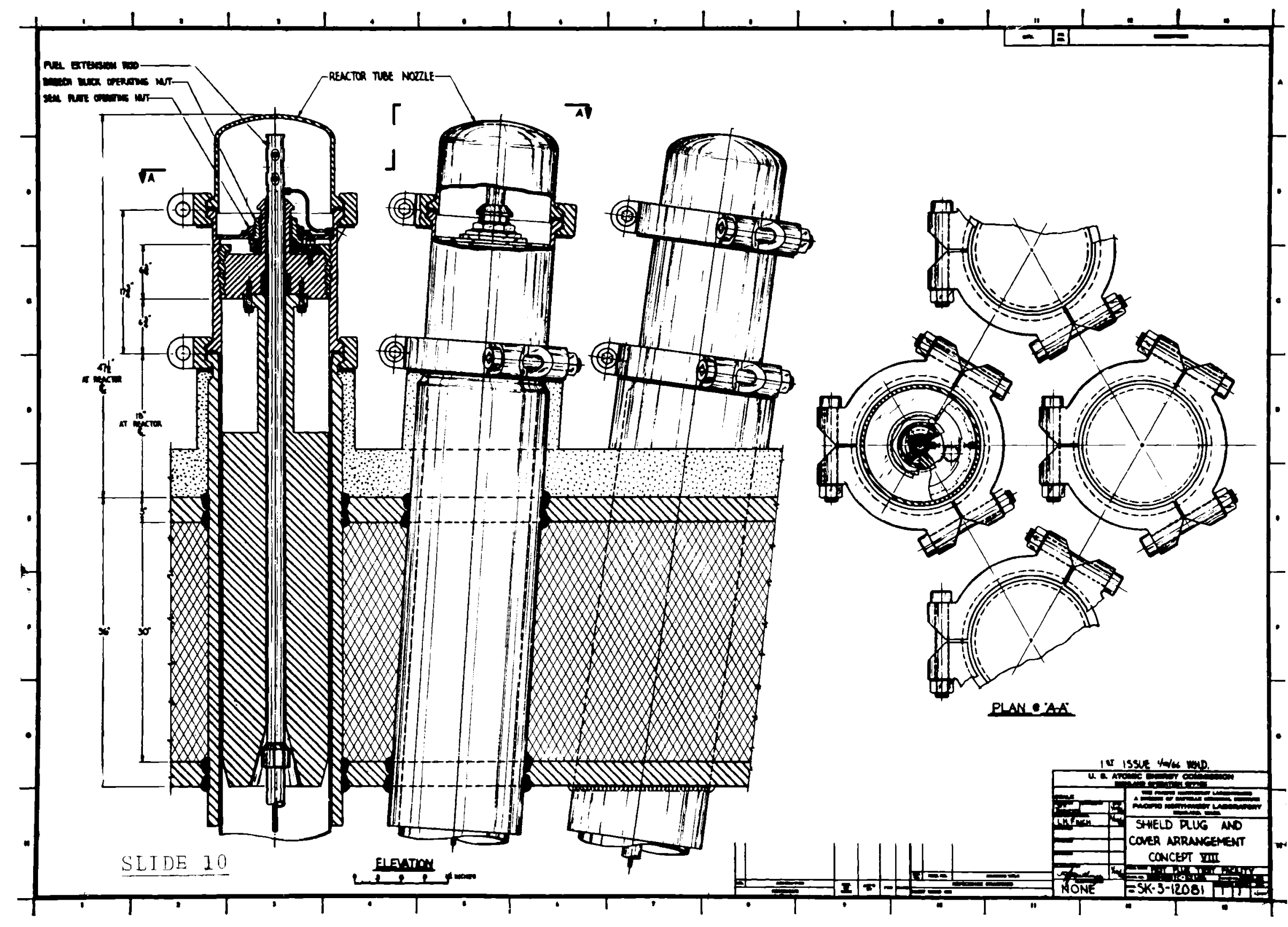


Slide 11 shows the floor plan of the reactor facility. In this area, the reactor vessel is shown with its annular array of primary loop components; the primary coolant pumps, the heat exchanges, and the decay storage pots for irradiated fuel. Note that there are six closed loop cell positions arranged in two groups of three on opposing sides of this reactor, each cell isolated from its neighbor and from the rest of the facility. Perhaps, more interesting is the rather extensive facility required for fuel examination. We have provided in this concept for two different examination techniques: an underwater examination of fuels that are not to be returned to the reactor (of course, this would include the driver fuel which ultimately goes to a storage position prior to shipment out of the reactor facility); an inert gas examination facility with a multiple cell complex for rather extensive gas environment examinations. There is a prevailing requirement for this facility to include a comprehensive radio-metallurgical facility in the vicinity.

On this same slide, secondary coolant from the main reactor circuits is transported by secondary coolant pumps for secondary to air blast heat exchangers which use the atmospheric air as a heat sink for the waste heat of the reactor. There are no provisions for power generation on this reactor. At the present time, the air blast heat exchanger is favored over sodium-water, sodium-water-steam, or sodium-steam waste heat techniques although these latter techniques are being investigated.

Slide 12 shows a lower level plan of the cells that are to be used for closed loops. The large cell is provided for maintenance on irradiated materials and contaminated components.

This slide 13 shows a cross section of the same building arrangement. The transverse cross section shows the loop cells in their position adjacent to the reactor containment; the spherical pressure containment above the reactor core; a very substantial concrete structure supporting the reactor vessel; and the general sodium system of the primary circuit.

This final architectural slide (Slide 14) shows a longitudinal. section emphasizing the large maintenance facility cell that was shown on the previous floor plan. Secondary pumps are shown in position for circulation of coolant from the reactor cavity to an outside air dump.

Slide 15 shows the types of components suspended within the reactor containment adjacent to the reactor vassel shield. These components are in the annular space about the reactor vessel. This first view is a heat exchanger similar to the Fermi heat exchanger; the next is a suspended centrifugal, mechanical pump in the primary circuit.

This next view is a line drawing of the irradiated fuel decay storage pot suspended within the annular area. Each pot holds approximately one-third of the core inventory in irradiated fuel subassemblies. Finally, in the view on the right is a unit serving as a gas circulator cooler to provide cooling for the neutron shield about the 


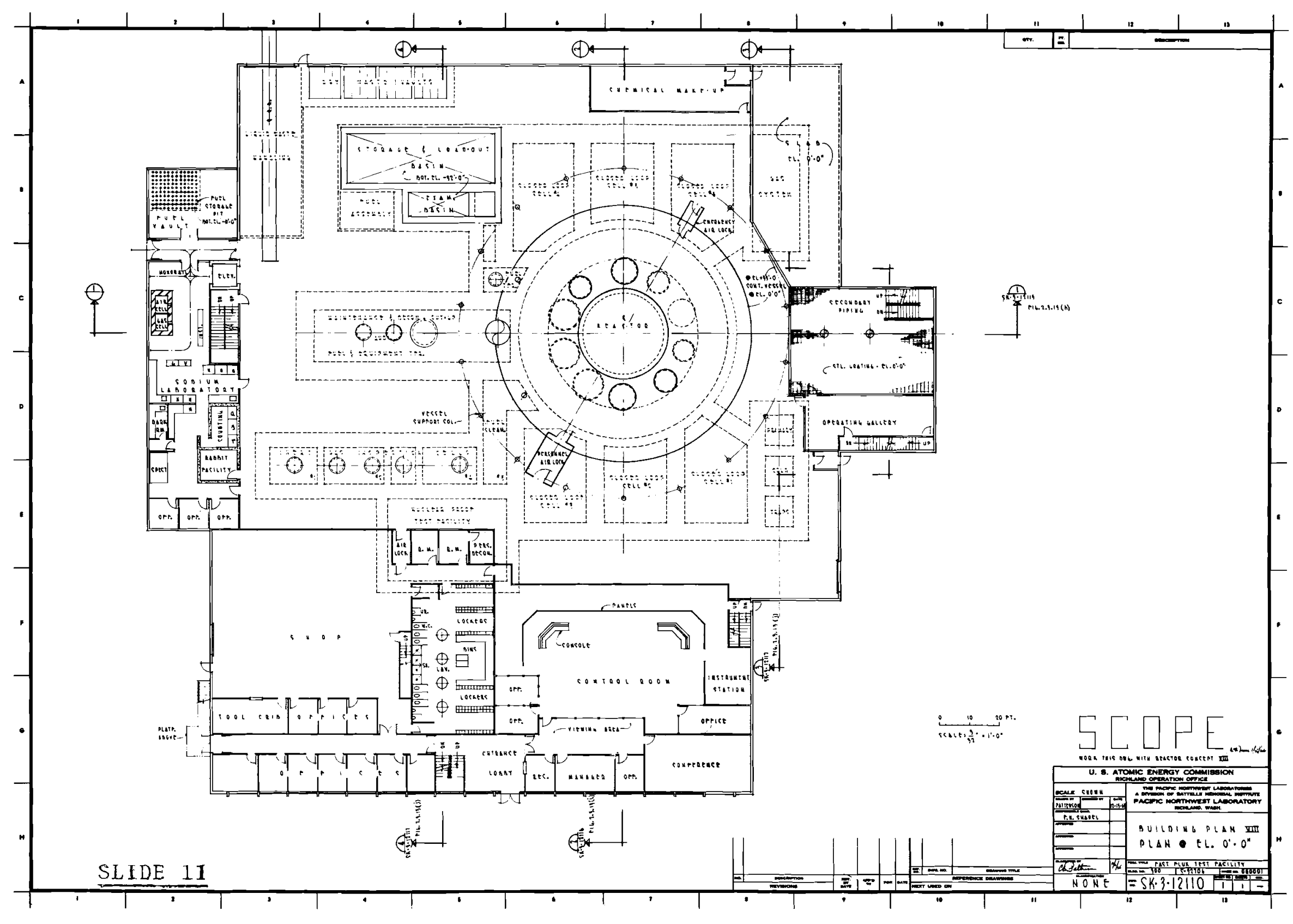




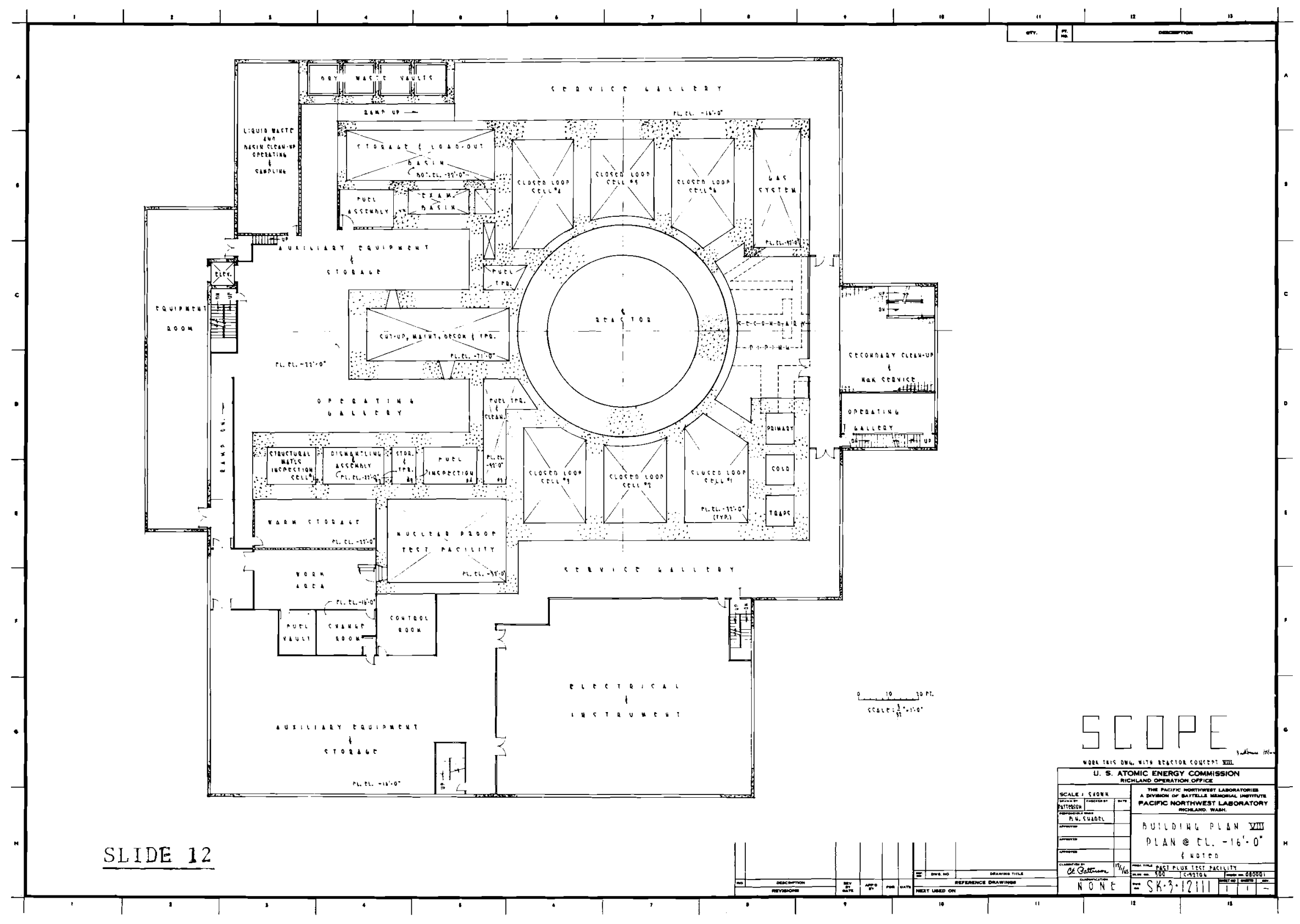




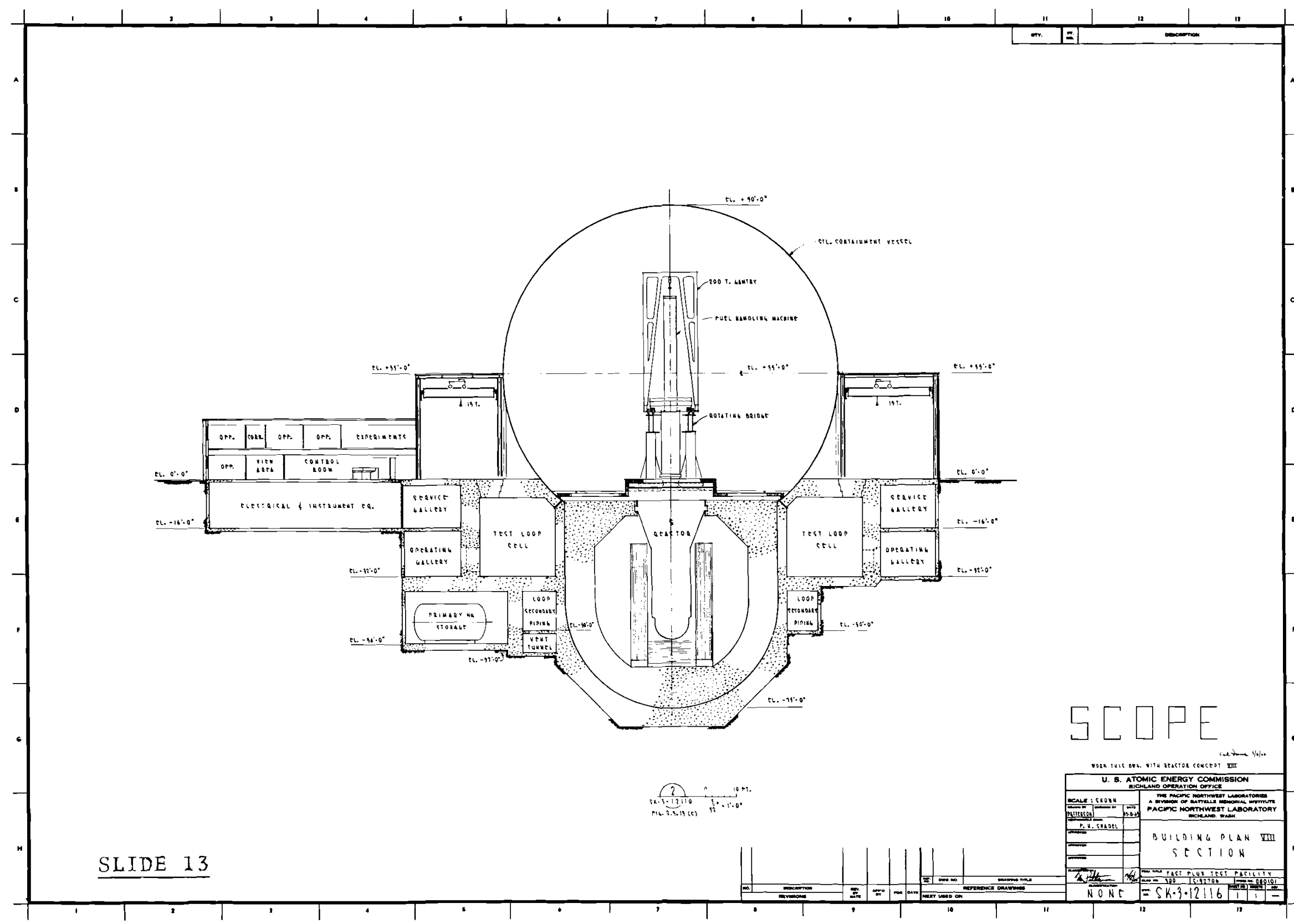




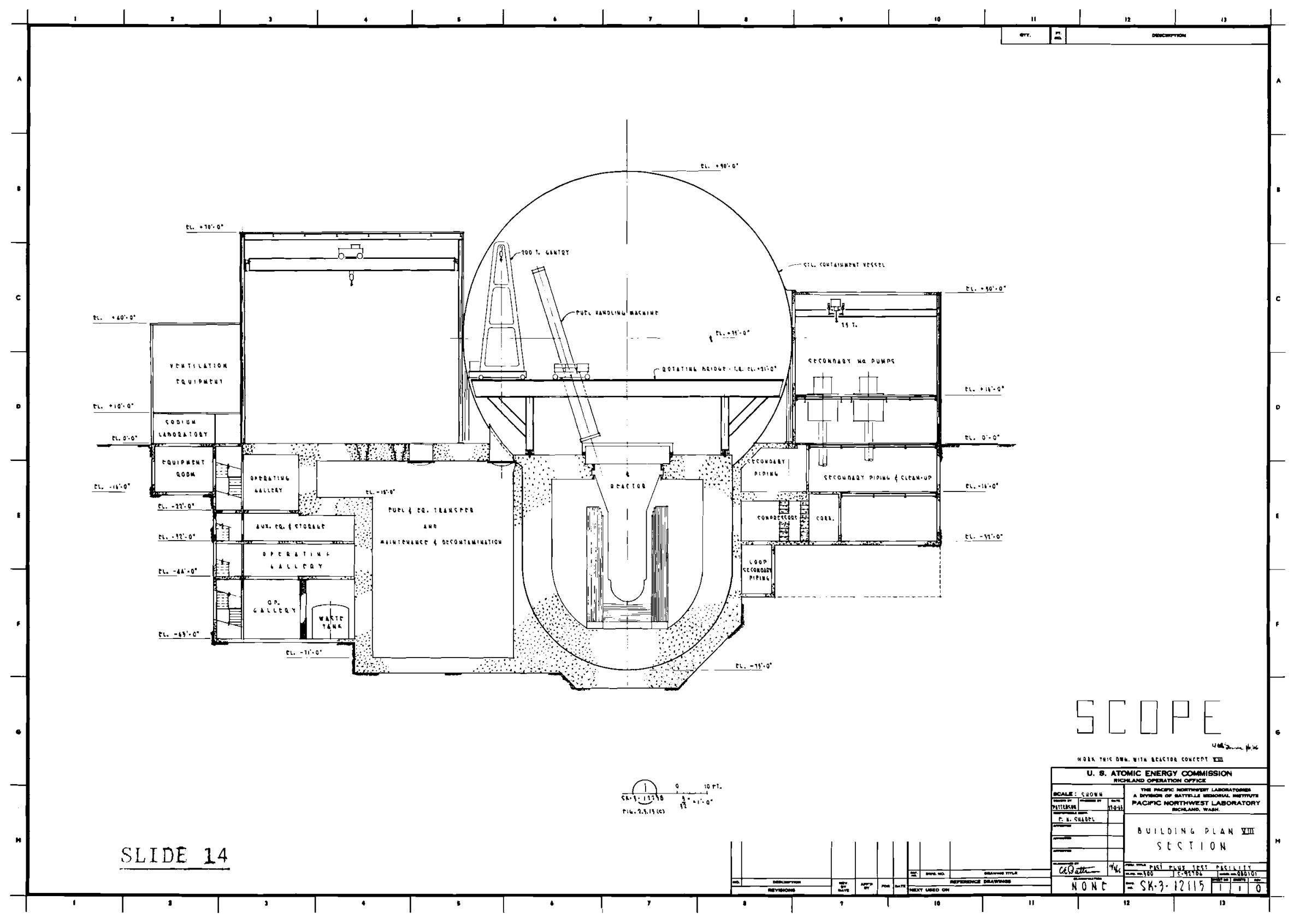




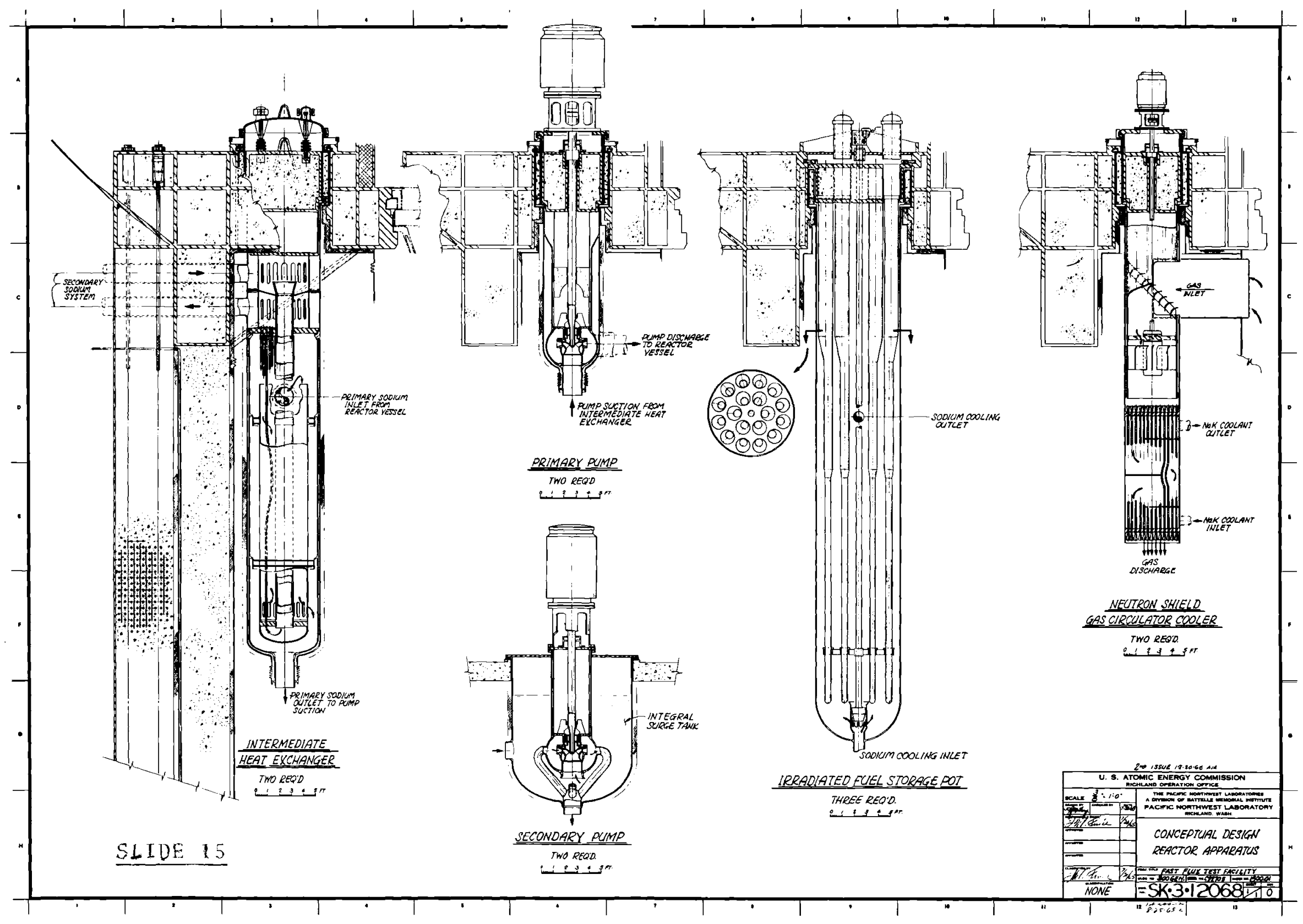


reactor vesse1. A11 of these components will be removed from the reactor containment zone by means of handling technique utilizing the gantry on the bridge of the fueling machine (S1ide 16).

Then on the subject of complexity, we've mentioned that closed loops do indicate a low reliability unless a great deal of attention is placed upon each part of a closed loop.

Show Slide 17 simply to indicate what schematic of a closed loop circuit would look like. In this case, the in-core section is indicated on the left of the view.

Finally, we have a mockup facility provided for, that will permit both reactor components and test components to be tested essentially in prototypic conditions. As a secondary issue, the mockup facility does provide a rather comprehensive simulation of the reactor core and its tubular components to permit special maintenance procedures to be developed in the event of a particular reactor emergency such as a stuck fuel or a melt down of an element within the core. 


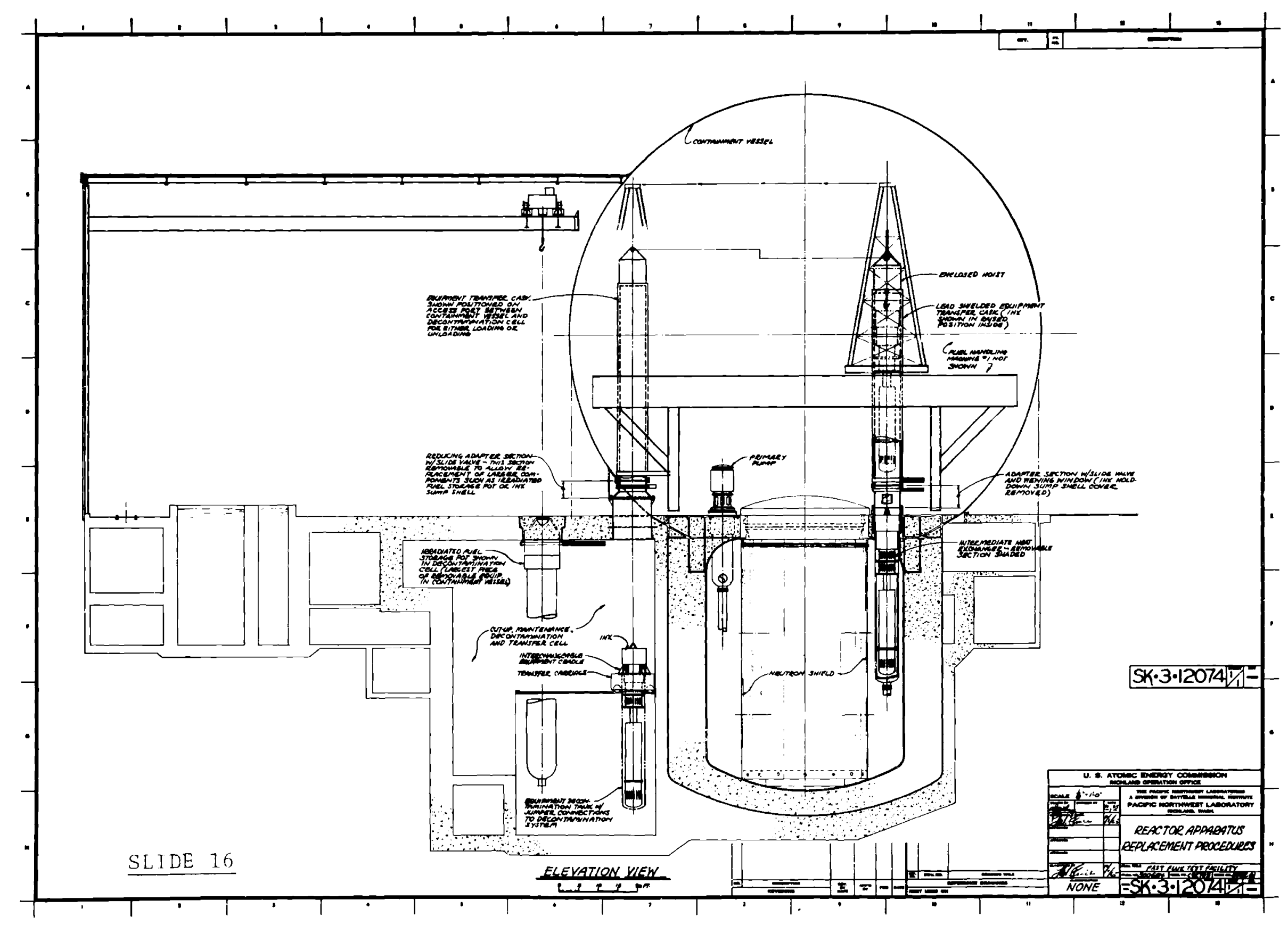




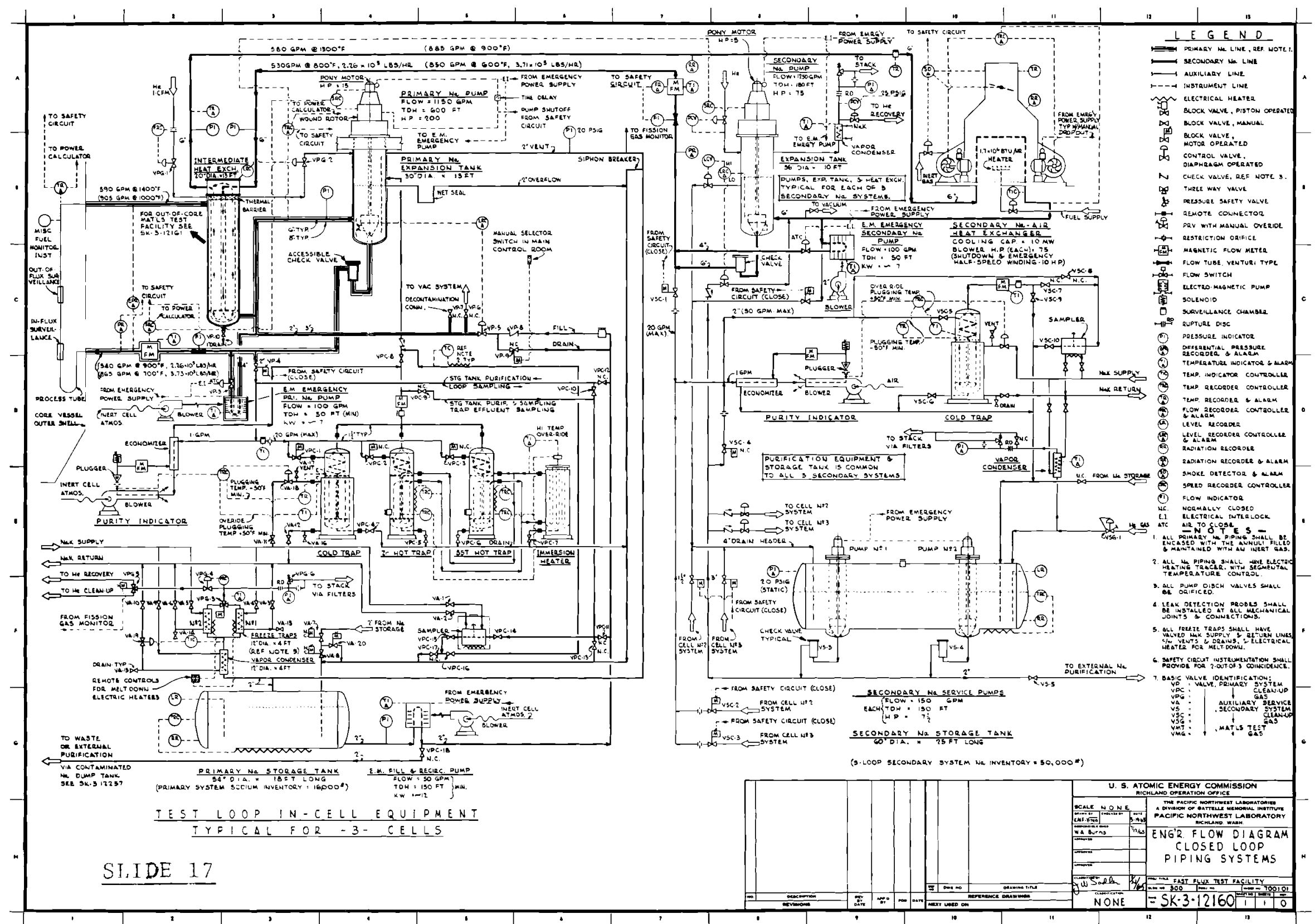




\section{DESIGN CONCEPTS}

By L. M. Finch

\section{DISCUSSION}

MR. HENNIG: Thank you very much. Gentlemen, do you have any questions or comments? If so, we will be happy to try to answer them. I would like to request that you not carry us of into a discussion of reactors or sodium components, or anything like that. Let's try to keep right on the line of the subject of the meeting, which is driver fuel.

CARL ANDERSON, JR.: (LASL) You are talking about a 75\% duty cycle, and I think someplace you mentioned a two weeks shutdown after six weeks run, for refueling. That means you have to have $100 \%$ duty cycling after refueling. Is that really what is meant, or are you compounding $75 \%$ by $75 \%$ ?

MR. FINCH: I think that is a good example of our problem. It is necessary to make definitions as to what these plant availability factors actually consist of and how they should be treated. I can't answer your question because I don't know the answer to these various factors. Obviously, if the plant availability of $75 \%$ includes the down time required for refueling or test installation, or any other, let us say, we can't talk about a $75 \%$ availability factor and still have any room left for failures.

J. H. WRIGHT: (Westinghouse) I try to understand the problem the way it is written: Try to design a reactor which meets the criteria. I think your interpretation is the fact that you want an overal 1 availability of $75 \%$ which is just not in the cards.

MR. HENNIG: I think we generally consider that the criteria are still open for adjustment.

D. R. deBOISBLANC: (Phillips) On the subject of plant availability, the scheduled on-stream time of MTR is roughly 75\%. The actual plant availability is a question of how often can a plant operate, when you want it operated, exclusive of the experiments. The EBR on an absolute basis has run, has fluctuated a little bit above $50 \%$ on full power and is actually edging up so the availability can be improved to around $62 \%$. This is a total fraction of time at full power. Now that represents around 95\% plant availability. A 95\% plant availability, in terms of which that question was addressed. When you want the plant to run, how often can it?

MR. ANDERSON: (LASL) My experience in using the MTR, they want a highly reliable fuel test, and if there is any likelihood of failure or anything else, the experiment is not allowed, and the first time that there is trouble in the loop, it is excluded from operation in the loop. This is the criteria I referred to when it says we are going to test failure. I don't believe failure is part of the testing.

MR. deBOISBLANC: It is in the ETR. 
MR. ANDERSON: It is in the MTR. I would say that is part of the operation, to handle a test and continue to operate. We have done this in the past on meteorological control, and of course you have a provision for avoiding the meteorological control. The continued operation of a plant is sometimes an experiment, in the case where reactors are doing just exactly what we want it to do. Therefore, it should be considered on-stream.

MR. deBOISBLANC: But if you count the competition for time with other experiments, they don't consider that on-stream time. You notice the cautious way this ground rule was set up. I think it is intended to say the plant should be available whenever you want it. The time it is not available is when you have to refuel it. That is not compatable.

MR. HENNIG: Do you accept this as a reasonable chart of the plant availability?

MR. deBOISBLANC: You will find the reactor is not going to be a problem.

MR. ANDERSON: I have another question. I understand you want to get fuel samples out in a hurry but I don't understand why you want to irradiate in a short period of time on the rabbit subject. Can you explain?

MR. FINCH: I think this is one I would just as soon throw to this meeting. There appears to be a high interest in very short term irradiation. I presume we will calibrate or assess damages over a short period. As I mentioned while I was talking, this has been a prevailing requirement.

MR. ANDERSON: Yes, what happens to it when you take it to 15 NPD?

MR. FINCH: I suspect... it swells.

MR. FIELD: (Argonne) The requirement here for rabbit is not only based on short NPD's but perhaps more so to operate experiments for a short period of time with unvarying thermal conditions, so if for example one wishes to determine what linear degrading processes take place in a particular type of fuel, this can be done very conveniently with rabbit experiments.

JOHN YEVICK, Headquarters: I would like to make a remark that Attachment $A$ is the goal you are seeking, is that correct? What does Attachment $B$ read?

MR. FINCH: $75 \%$ on $\mathrm{A}$, and it applies reasonably practical to $\mathrm{B}$.

MR. YEVICK: That is right. As you start designing the plant, and you have two weeks down time, and six weeks on, this indicates you cannot achieve $75 \%$, but the "highest available", I think is the wording you use. We are going to spend another seventy-five million dollars, plus or minus, for this facility, and another $15 \%$ of that for $\mathrm{R} \& \mathrm{D}$, we certainly should get a plant on line. If we can't we ought to quit. Closed loops will be operated $100 \%$ continuously. Your big problem will be your driver fuel, not the closed loop. Another thing, you only have six closed loops at the maximum. These are the center of everything. All the other loops you have, you have the ETR and the MTR and the ATR. In the ATR are nine loops and there are many more 
D2.3

in those reactors than this particular facility, so your real heart and soul of the plant are something in the order of six loops, plus or minus. I think $75 \%$ might be achievable, once we get good driver fuel. We are starting off with a driver fuel that requires you shut down two weeks but once you get to a good yield, to a ceramic, you will have a high rating.

MR. FINCH: I have one point, the $75 \%$ time set aside now for operation, $25 \%$ for down time, refueling, what have you, of course represents a very good place to work to improve this plant availability. If we are on a two-weeks, sixweeks cycle, anything done to reduce the two weeks can be quite helpful as Iong as the six weeks remains constant. So it isn't just the reliability of the componerts, but there is also a variety of opportunities for increasing plant availability.

MR. HENNIG: If we have the right kind of program, if the program turns out to be, for instance, all one year radiations of constant power, no transient testing, no further mention of system, we probably would push beyond $75 \%$.

MR. YEVICK: If in 90 experiments they are getting $60 \%$ of the availability on stream time, that is very good. That is a very complex reactor.

MR. HENNIG: I think of it this way: the Commission selects a testing program for the future, and this will probably have a substantial impact on the actual plant practice. 



\author{
3.1 \\ COMPARISON OF THE NEUTRONICS OF VARIOUS FTR DRIVER FUELS \\ P. L. Hofmann, W. W. Little, Jr. \\ Pacific Northwest Laboratory \\ Richland, Washington \\ Operated By Battelle Memorial Institute
}

\title{
INTRODUCTION
}

The purpose of this talk is to discuss the reactor physics aspects that enter into the selection of a suitable driver fuel for the Fast Test Reactor. As you we 11 know, the FTR is a rather complicated machine consisting of many interacting systems. The neutronic considerations, while important, represent only one aspect of this framework. My talk only concerns itself with the reactor physics. In subsequent talks, the FTR system will be considered in its entirety.

The topic of the comparative merits and drawbacks of various driver fuels has been uppermost in our minds during the 1 ast few months. I think we have a better understanding now of these questions than we did before, but considerable work still remains to be done.

\section{DRIVER FUELS}

The selection of the driver fuel has an important effect on three areas of reactor physics:

- It affects the core physics statics - fuel inventory, flux-topower ratios, spatial power distributions, and flux spectra.

- It affects the control statics - the control requirements and the control worth of the various control schemes and devices proposed.

- It affects the reactor kinetics - the transient core behavior in response to various postulated reactivity disturbances.

I'11 discuss each of these topics in turn.

Our investigations during the past few months have been concentrated on four kinds of driver fuels:

- $\mathrm{PuO}_{2}-\mathrm{SS}$ Cermets

- $\mathrm{UO}_{2}$-SS Cermets

- $\mathrm{PuO}_{2}-\mathrm{U}^{28} \mathrm{O}_{2}$ Ceramics

- $\mathrm{UO}_{2}-\mathrm{U}^{28} \mathrm{O}_{2}$ Ceramics. 
We also have made a brief investigation of metal fuels. Our results, however, are too incomplete to be reported here.

At the risk of saying the obvious, I am sure that all of you recognize that one of our main objectives in the design of the FTR is to achieve a high intensity, fast flux machine with considerable test volume available.

Since neutron flux is directly proportional to specific power (MW/kg fissile) and inversely proportional to the average fission cross section, plutonium offers obvious advantages as a driver fuel. The reduced plutonium inventories, compared to U-235, come about because of the high plutonium Eta value compared to that of U-235. For a typical FTR cermet core, $n-239$ is about 2.5, while $n-235$ is only about 2. The advantage in $n$ (or critical mass) is somewhat offset by the higher Pu-239 fission cross section $(\sim 15 \%)$. However, the net effect still favors plutonium considerably to give high flux cores.

In comparing the kinetic properties of plutonium-fueled versus U-235-fueled cores, the low delayed neutron fraction of plutonium is a disadvantage. It does make control of plutonium cores more difficult than control of U-235 cores, and it does make the plutonium core more susceptible to prompt excursions than the U-235 core. In addition, fast spectrum cores fueled entirely with plutonium have a positive Doppler coefficient. While this coefficient is quite small, it is nevertheless an undesirable feature.

Cermet fuels make it possible to employ plutonium without the presence of fertile fuel poisons, and, consequently, full advantage can be taken of the low plutonium inventories and resulting high flux-to-power ratios. Cermet fuels also can take advantage of the metal-matrix expansion coefficient which can be an important safety feature.

Ceramics, on the other hand, require higher plutonium inventories to offset the U-238 capture cross section. Consequent1y, they result in lower $f l u x-t o-p o w e r$ ratios, and larger core sizes might be required to achieve the desired high flux levels. The expansion co-efficients of ceramic fuels is less well established, . and, consequently, one must rely on the negative Doppler coefficient for shutdown. For FTR core sizes, this coefficient is rather small.

The core statistics data for the four major driver fuels have been calculated over a wide range of core sizes. Detailed descriptions of these calculations are contained in BNWL-197.(1)

(1) "Neutronic Considerations in the selection of the Driver Fuel for the Fast Test Reactor (FTR),"W. W. Littie, Jr. and R. W. Hardie, December, 1965 . 


\section{CORE STATICS DATA RESULTS}

The fissile fuel inventories of core volumes from 200 to 1600 liters are shown on Slide 1. These calculations were carried out with 1-D transport theory, employing a modified version of the 26 group Russian cross section set collapsed to 12 groups. Incidentally, we have also made comparisons with the ANL-224 set, and we obtain very good agreement. For small, hard spectrum cores, U-238 and steel have a comparable effect on critical mass. However for large, soft spectrum cores, U-238 becomes a significant poison. The behaviors of the U-235 and plutonium enriched cores are quite similar, except that the plutonium cores have, of course, lower fissile fuel inventories.

Slide 2 shows average core flux levels as a function of core size. In this slide a $2 / 3$ MW/1iter power density has been assumed throughout, i.e., $400 \mathrm{MW}$ for a 600 liter core. This normalization is somewhat arbitrary. However, it is a simple matter to obtain the appropriate flux levels for specified power levels. The low inventory plutonium cores show the highest flux levels over a wide range of core sizes.

Slide 3 shows the variation of the central peak flux with core volume. An axial peak/average flux factor 1.25 has been assumed for all cores; although strictly speaking, it has only been calculated for the 600 liter core. However, al1 cores do have the same length and the axial power factors, therefore, should not differ greatly. The central fluxes rise more steeply with core size than the average fluxes.

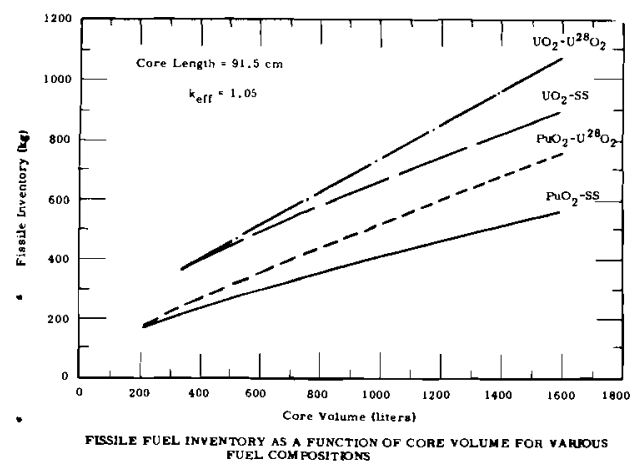

SLIDE 1

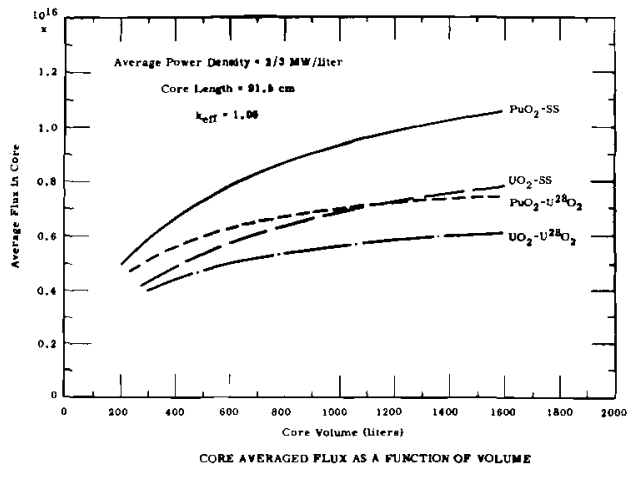

SLIDE 2

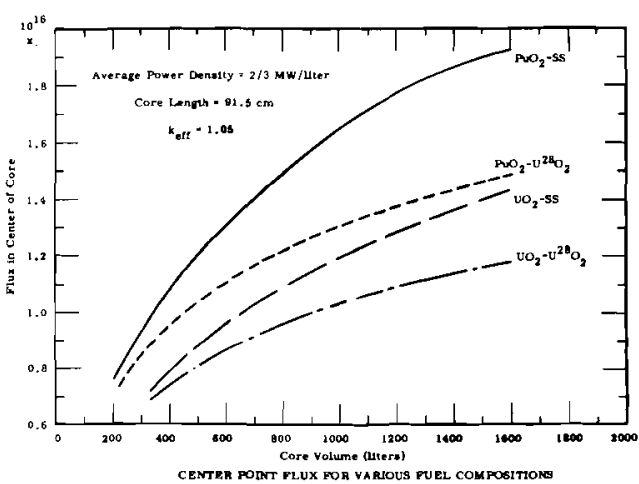

SLIDE 3

Since the 600 liter core has for some time been our reference core, we have summarized some of the more important static results on Siide 4. Note the marked differences in fuel inventories and the corresponding differences in flux levels. We have, of course, also carried out detailed flux spectrum calculations for the cores involved. The fraction of the flux above $0.1 \mathrm{MeV}$ is shown for the four cores. Comparison with typical breeder cores indicate that the 
$\mathrm{PuO}_{2}$-SS cermet FTR is somewhat softer than a metal reactor, harder than an oxide reactor, and reasonably close to a carbide reactor. We could, of course, soften the FTR spectrum using BeO.

\section{STATIC CHARACTERISTICS OF 600-\& REACTORS}

\section{SLIDE 4}

\begin{tabular}{|c|c|}
\hline Item & $\mathrm{PuO}_{2}-5 \mathrm{~S}$ \\
\hline$k_{\text {eff }}$ & 1.042 \\
\hline $\begin{array}{l}\text { wo fissile Fuel } \\
\left(\mathrm{PuO}_{2} \text { or } \mathrm{UO}_{2}\right)\end{array}$ & 5.5 \\
\hline $\begin{array}{l}\text { vo Fertile Fuel } \\
\quad\left(U^{28} \mathrm{O}_{2}\right)\end{array}$ & 0 \\
\hline Mass Pu239 (kg) & 300 \\
\hline Mass $\mathrm{Pu}^{240}$ & 33.5 \\
\hline Mass $U^{235}$ & 0 \\
\hline Mass $U^{238}$ & 0 \\
\hline $\bar{\Phi}(\text { core average })^{*}$ & $0.780 \times 10^{16}$ \\
\hline$\Phi_{0}$ (center point ${ }^{*}$ & $1.32 \times 10^{16}$ \\
\hline$\Phi 1>0.1 \mathrm{MeV} / \Phi^{\infty}$ & 0.710 \\
\hline
\end{tabular}

* Computed at $400 \mathrm{MWt}$

$\frac{P \cup O_{2}-\mathrm{UO}_{2}}{1.049}$
6.5
23.5
355
39.6
0
1362
$0.624 \times 10^{16}$
$1.10 \times 10^{16}$
0.679

$\frac{U 0_{2}-55}{1.044}$
9.1
0
0
0
489
37.3
$0.570 \times 10^{16}$
$0.96 \times 10^{16}$
0.760

\section{CONTROL STATICS CHARACTERISTICS}

For the 600 liter PuO-SS cermet core, the control requirements have been estimated to be about 10 to $12 \% \delta \mathrm{k}$. For 42 full power days of operation, the shim requirements are about $4 \% \delta \mathrm{k}$, the temperature defect is $1.5 \% \delta \mathrm{k}$, and the remainder is allocated to shutdown margin and other miscellaneous requirements.

The control requirements for ceramic cores are less. This is primarily due to the presence of fertile material and results in a. moderate initial conversion ratio. In addition, the sodium coefficient is less negative in the ceramic core; hence, the temperature defect is reduced.

On Slide 5 , the shim control requirements of the four 600 liter cores are summarized. The shim control requirements for the ceramic core are about one-half of those for the cermet core.

To meet the control requirements, a variety of control schemes have been proposed. In the early stages of our design we looked seriously at reflector control. We decided that for 600 liters FTR cermet cores, realistically achievable reflector control margins were probably only about 5 to $6 \% \delta \mathrm{k}$; hence, complete reflector control for the FTR did not seem practical. We then adopted complete 
in-core control and used six large in-core $\mathrm{B}_{4} \mathrm{C}$ rods for control. The small number of high worth rods was a direct result of trying to conserve core volume for test space and driver fuel. High worth rods are obviously unattractive for a variety of reasons; consequently, we are now seriously considering combinations of in-core and reflector control. Parenthetically, I might add we are also looking at moving fuel control.

If we retained three in-core safety rods, we would then only need about $6 \% \delta \mathrm{k}$ reflector control for the cermet core to cover shim and temperature defect requirements.

As shown in Slide 6 , the maximum number of reflector rods which one could physically accommodate in a 600 1iter, 61 tube core is 30. (This is the number of tubes in the next ring.) The results of some rather idealized reflector control calculations are compared in this slide. Reflector rod worth for the ceramic core is about half that for the cermet core, and the control requirements (you will recal1) are also less. Also note that the idealized rod worths are about twice of what is needed.

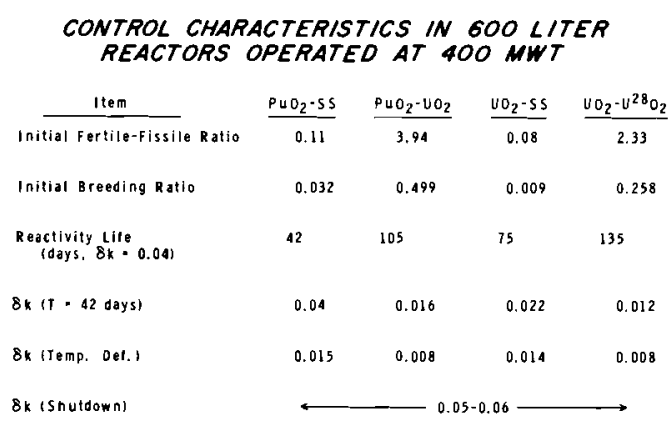

SLIDE 5
REFLECTOR CONTROL - 600 LITER FTR CORES, $30 \mathrm{~B}_{4}^{\mathrm{n}} \mathrm{C}$ RODS

\begin{tabular}{lc} 
Cermet core, Un-zoned & Control Swing.8P \\
\cline { 2 - 2 } Cermet Core, zoned & 0.126 \\
Ceramic Core, Un-zoned & 0.139 \\
\end{tabular}

It is fairly obvious that 30 control rods might not be tolerable from an engineering point of view; therefore, we have examined the decrease in reflector control worth as the number of rods are reduced. The results from $2-D(r-\theta)$ transport calculations are shown on Slide 7. If the number of rods were halved, reflector control would only drop by about $25 \%$. This is a fortunate, but not particularly unexpected result.

We recognize, of course, that reflector control calculations are subject to large uncertainties, since the determination of the low 
energy spectrum is uncertain. While the use of B-10 enrichment for in-core rods has an important effect on rod worth, it has little effect on the reactivity worth of reflector rods because it does not affect neutron leakage. This is illustrated on Slide 8. Most of the previous discussion has been confined to 600 liter cores. Slide 9 shows comparisons of reflector control over a wide range of core sizes. As expected, reflector control decreases with core size, but so do the control requirements.

The main conclusions we would like to draw from this discussion is that, if once a reactor configuration with a specific combination of in-core and reflector control elements is designed, a variety of driver fuels could probably be accommodated. This conclusion only pertains to the control statics aspects and not to the control dynamics of the system.

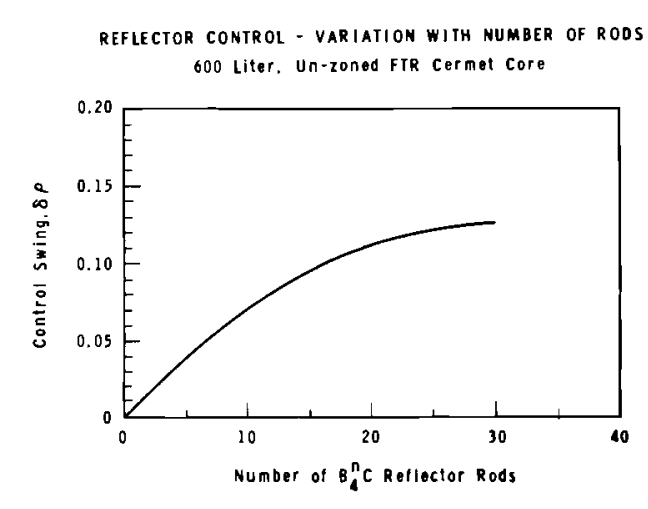

SLIDE 7

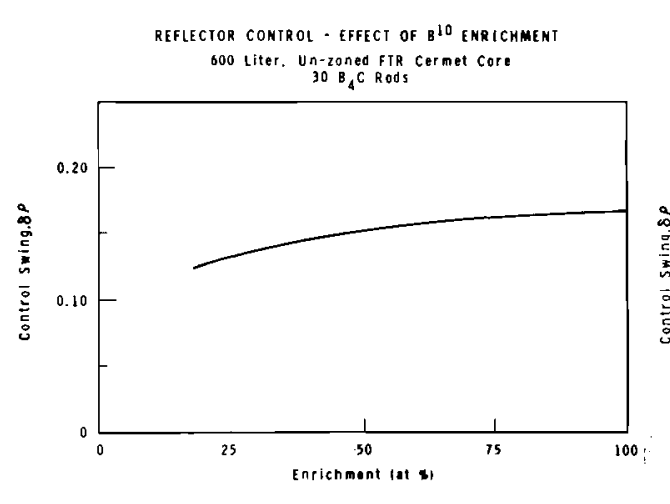

SLIDE 8

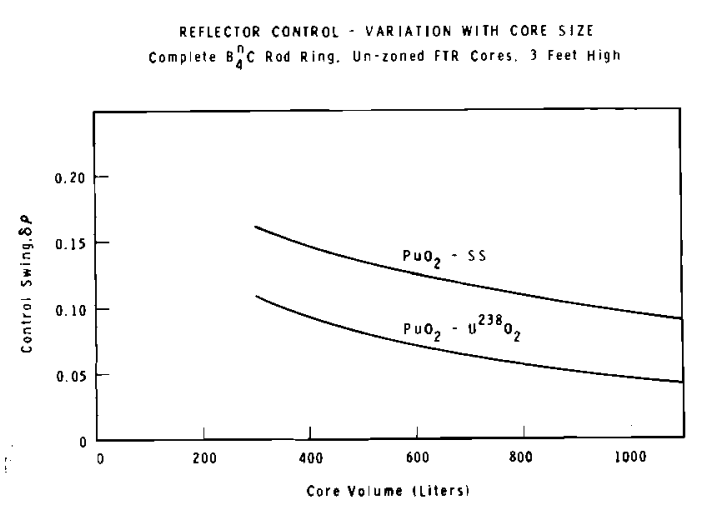

SLIDE 9

\section{KINETICS CHARACTERISTICS}

To make comparisons and carry out transient calculations requires a knowledge of the temperature coefficients, effective delay fractions and neutron lifetimes of the reactors involved.

Some of the kinetics characteristics of the four standard 600 liter cores are compared on Slide 10. The sodium coefficient for the cermet cores are quite negative, even for the central channel sodium, and the Doppler coefficient is small and positive. Fuel expansion coefficients for the ceramics have not been calculated; the Doppler coefficients are negative, but also not very large. The hard spectrum U-235 cores have even smaller Doppler coefficients. 
KINETICS CHARACTERISTICS OF 600-l REACTORS

\begin{tabular}{|c|c|c|c|c|c|}
\hline \multirow[b]{3}{*}{ SLIDE } & Item & $P \cup 0_{2}-55$ & $\mathrm{PuO}_{2}-\mathrm{UO}_{2}$ & $\mathrm{UO}_{2}-\mathrm{SS}$ & $\mathrm{UO}_{2}-\mathrm{U}^{28} \mathrm{O}_{2}$ \\
\hline & $\beta_{\mathrm{eff}}$ & 0.0022 & 0.0032 & 0.0070 & 0.0072 \\
\hline & $\Lambda_{0}(\sec )$ & $4.82 \times 10^{-7}$ & $3.55 \times 10^{-7}$ & $2.95 \times 10^{-7}$ & $2.59 \times 10^{-7}$ \\
\hline & Na worth (core) & 0.07339 & 0.02044 & 0.07492 & 0.03734 \\
\hline & Na Worth $\{r \leq 5 \mathrm{~cm}\}$ & 0.00114 & 0.00011 & 0.00120 & 0.00051 \\
\hline & Na Expansion Coef. $10^{-1}{ }^{-1}$ & $-19.6 \times 10^{-6}$ & $-5.46 \times 10^{-6}$ & $-20.0 \times 10^{-6}$ & $-9.97 \times 10^{-6}$ \\
\hline & Fuel Expansion Coet. & $-8.5 \times 10^{-6}$ & - & $\sim 7.0 \times 10^{-6}$ & - \\
\hline & Doppler Coet. $\left(\mathrm{T}=1000^{\circ} \mathrm{K}\right)$ & $+0.40 \times 10^{-6}$ & $-3.5 \times 10^{-6}$ & $+0.056 \times 10^{-6}$ & $-1.8 \times 10^{-6}$ \\
\hline & ooppler Coef. Ino Nal & $+0.27 \times 10^{-6}$ & $-1.6 \times 10^{-6}$ & $+0.023 \times 10^{-6}$ & $-0.94 \times 10^{-6}$ \\
\hline
\end{tabular}

As mentioned before, the addition of BeO might be used to soften the spectrum and to increase the Doppler coefficient. A $10 \%$ addition of BeO does indeed double the Doppler coefficient and reduce the fast flux component above $0.1 \mathrm{MeV}$. (Slide 11)

Actually, if a ceramic core were adopted, sizes in excess of 600 liters might be more interesting to recoup the loss in flux potential for this core type. Those core sizes might be in the neighborhood of 800 to 1000 liters. Perhaps the 800 liter core represents some sort of a dividing line, because for this core size, the central sodium coefficient seems to change sign. However, there is really nothing particularly magic about 800 liters.

The characteristics of an 800 liter ceramic core are summarized on Slide 12. The important thing to note is that the central channel sodium worth is about zero. The negative Doppler coefficient is somewhat larger than for the 600 1iter case, but considerably less than for the Be0 moderated 6001 iter core. At $2 / 3 \mathrm{MW} / 1$ iter power density, the central flux approaches that of the 600 liter cermet core.

With the kinetics parameters in hand we can now compare the transient responses of the cores. For the nuclear calculations, we have used a rather simple three node model, including feedback effects from the ceramic bead, the steel matrix, and the sodium 
coolant. We have assumed that relatively moderate accidents can be represented by simple ramp rates, and that power and temperature responses can then be calculated.

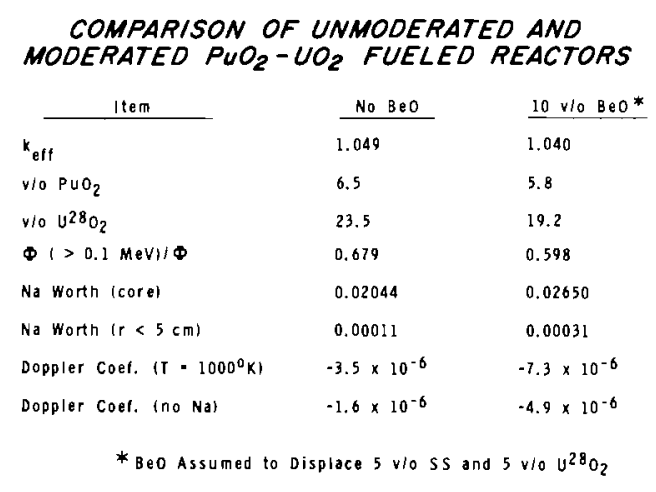

SLIDE 11

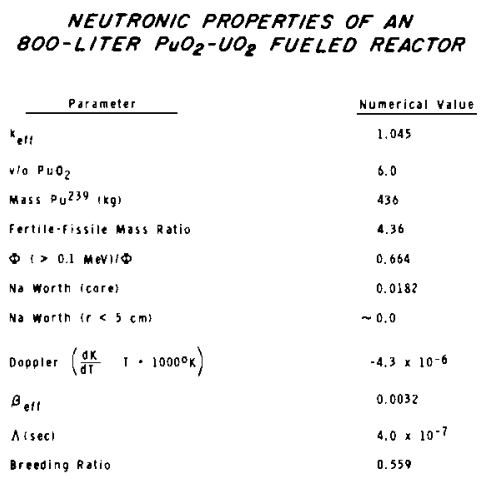

SLIDE 12

On Slide 13 we compare the power transients of cermet and ceramic cores for comparable reactivity ranges. For very low ramps, the power responses are quite similar. The ceramics lag the cermets because of the larger Beff value.

\section{SLIDE 13}

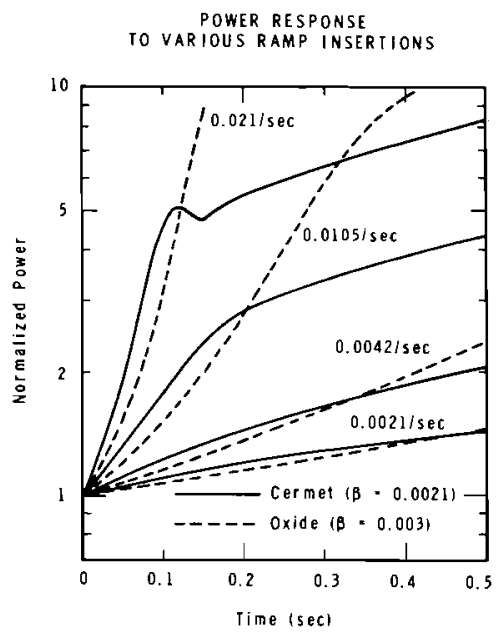

We have also carried out some analyses of very large accidents of the so-called Bethe-Tait type. Of course, in this type of accident one cannot take credit for the expansion coefficient of the cermet. In that case, the negative Doppler coefficient of the ceramic is a distinct asset, and the small positive Doppler coefficient of the plutonium cermet becomes a liability. However, the credibility of such accidents remains to be ascertained. 


\section{9}

\section{CONCLUSIONS}

The problem is quite complex, but perhaps one can draw two tentative conclusions.

- If a high intensity, fast spectrum flux is of prime importance, plutonium fuel without the admixture of poisonous fertile materials seems to be the answer.

- If doubts exist about $\mathrm{PuO}_{2}$-SS cermets, the core can probably be designed to accommodate a variety of fuels, though it might not be an optimized design for each fuel type. 


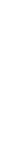


D-3. 1

PHYSICS CHARACTERISTICS

P. L. Hofmann and W. W. Little

\section{DISCUSSION}

MR. HENNIG: Are there any questions or comments. Jim?

MR. WRIGHT: Could you postulate what the effect of a more detailed analysis gives you on the ceramic? You started out by saying it wasn!'t good.

MR. HOFMANN: We calculated average temperature, so if you look at melting of the ceramic, bulk melting of the ceramic may occur later, but melting in selective spots will occur very soon. I don't know how much localized melting one can permit, but if melting at all is considered a failure, then this melting would occur sooner than one would conclude from average conditions.

MR. deBOISBLANC: I think one of the important kinetic considerations is the delayed neutron faction and its competitive position, shall we say, in this overall plant concept, and in order to fully appreciate what effect this would have on the experimental program, or the experimental utility of this, the parameters should be expressed in terms of mass change, for example...

MR. HOFMANN: That is an excellent point, of course...

Mr. deBOISBLANC: Because obviously there is a rather stringent limitation on the size of the central core facility, which is a part of the facility in the reactor and has been limited to $1 \%$ of the core mass, on that order of magnitude. If one were to examine this against the 235 core, it would be similar.

MR. HOFMANN: It is very hard. We have to postulate physical accidents really. I think this is the first time for this kind of : comparison, but in fact, suppose you look at an accident in the ceramic cores, if there are any ceramic cores worth less than the cermet core, the same thing in the ceramic would effect this more than the accident because it only draws out one rod. Therefore you might say that comparing it on the basis of the $\Delta k$ in terms of the ceramic, really penalizes the ceramic. It isn't fair because the same high broad accident in the ceramic, you have less $\Delta k$ per second. But since you know that in the ceramic core, rods are, in fact, worth less, it is conceivable that you might take advantage of this and couple rods. You might put in larger rods in your ceramic core. You can say, supposing I want to limit my accident, I want to limit my rod work to two dollars, I could then put a bigger ceramic rod in, or fewer cermet rods and might be back to $\Delta k$ or certain accidents. 
MR. deBOISBLANC: If you couple two rods together, for instance, you have two rod speeds. All the factors you have used up here have allowed a very reasonable withdrawal rate. There are two regimes, one is when you start the machine up, to begin with, which you can do extraordinary things about the start up time; and the other one is the requirements of broad motion during operation. And also there is the separation which you made in the concept between. Safety rods go only so far and then they are out: and the reactor is not critical when the safety rod is out and ali the other rods in, and the withdrawal accident on the safety rod is more hypothetical than a withdrawal accident in general.

MR HOFMANN: All these $\triangle \mathrm{k}$-per-seconds here don't say anything about how the acciden: comes about.

MR deBOISBLANC: The biggest thing is that the bigger the experiment, the worse the accident, is for a given system.

MR. HENNIG: For any system.

MR deBOISBLANC; For a given system. If you take advantage of delayed neutron fraction, you can have a larger physical mass to get the same $\Delta \mathrm{k} / \mathrm{k}$ system.

MR. HOFMANN: A larger inventory, because of the fraction, the best represents the smallest.

MR.AVERY (Argonne): I would like to make one comment on Peter's final conclusion, which, while I don't disagree with it, I think it sounds a little too simple in terms of stating the advantages of plutonium over uranium; or for that matter, the cermet over the oxide. Now one obviously has to look at the time average flux, including the reloading time, which is of the order of one-fourth, and if that number can be significantly changed by these other modifications, as apparently it can, a good part of this difference may disappear. I think even if one isn't limited by reactivity, and I don't know precisely what the limitations are, what numbers are used for permissible burnups, and we know that there are uncertainties in this for oxides versus cermets. I think they also imply a much longer cycle time for ceramic versus cermet. So it seems to me that this must be factored in very strongly in any overall future programming.

MR. deBOISBLANC: Did you make the $7 \%$ adjustment for comparing the uranium and plutonium? Actually, the fraction is .76 in the case of uranium oxide and cermet, and it is . 71 in the case of plutonium, and obviously if the interesting flux is above a tenth of a $\mathrm{MeV}$, then the obvious comparison should be above the $\mathrm{MeV}$, in which case, the $7 \%$, you have to take off the advantage that you spoke of.

MR. HOFMANN: Most of my comparisons have dealt with plutonium. While I did show the use of $\mathrm{UO}_{2}$ in the comparison, I certainly did not make any such adjustment. In fact I don't know exactly what value to attach to this $5 \%$ extra fast flux, but we have not made that test. 
CARL ANDERSON: You can design certain accidents out of existence, like the control rod withdrawal and so on. But there are some accidents by the nature of their facility; you have to consider a fast power accident does not have to consider such as someone inserting a fuel element which is designed to go to failure. When it fails, it slumps, and adds several dollars to reactivity in a fraction of a second. Your facility does have to accommodate that, doesn't it?

MR. HOFMANN: We have, of course, looked at the possibility of the live fuel slumping and test slumping. But I don't think we are designed for a fuel slump.

MR。ANDERSON: You mean the test packet must be designed-so they don't fail?

MR. HOFMANN: I don't know.

MR. ANDERSON: I think that is a restriction you shouldn't impose on the facility. Somebody is going to want to test this failure. Your driver fuel doesn't have to, certainly.

MR. HENNIG: The major point is, we are not designing core melting facilities. That is not part of the present objective. We are definitely considering the design and the analysis of facilities, and taking care of economic consequences of various degrees of accident. We recognize that this plays a major part in the decision of designing the subdividing core. How big the core should be would be covered in a major degree, but operational controls over testing, this kind of thing, will be covered largely by the Commission's desires on a testing program. If there is one big priority and it has a big risk associated with it, it may go, where another one may not.

MR. HOFMANN: We have looked, incidentally, at the test fuel slumping accident in the center. We think it is an incident which we can accommodate. In fact, we think we can accommodate it without damage to the driver fuel, but again this is not the extent of the proposed operation.

MR. HENNIG: Centainly one measure of the utility of driver fuel can be its ability to accommodate the more reactive tests.

MR. KATZI (United Nuclear Corp.): I was wondering in these kinetic traces of cermet versus ceramics, since in your opinion you would have a large fraction of test elements, whether they would effect your kinetic power? And this being so, maybe it would materially effect a comparison.

MR. HOFMANN: The test fuel obviously would effect the test parameters. Supposing it is a ceramic fuel, and assuming that ceramics are the fuel of the future, presumably many of the test fuels would be ceramic, so that the ceramic fuels would not be much changed. Suppose the core is a cermet core, and you have $15 \%$ or so occupied by test core, which might be (let us say) ceramics. Then in fact, the kinetics would be more on the side of the cermet, but I have not particularly looked at the combination. 
MR. KATZI: For example, from a smaller ceramic core to a larger core, it might conceivably be that if you have the smaller ceramic core, plus the experiments, that the associated softening of the spectrum would actually give you a smaller core.

MR. HOFMANN: The comparison here is a very preliminary comparison. There is no question that the presence of the test affects the kinetic parameters; how ever, the test occupies a relatively small portion of the core. From one point of view, I would imagine the change in the flux would not be large. But you are quite right, one would want to look at this.

MR. F. M. HECK (Westinghouse): It is certainly conservative in looking at the kinetics to neglect the expansion of ceramic fuel. How ever, in calculating the margins required for shutdown, it seems to me one should add in some reasonable number for fuel expansion.

MR. HOFMANN: We added in the sum of the expansion, it would not change the conclusions materially. If I assume the ceramic expands like a cermet.

MR. HECK: I have one further question. How did you treat the Doppler beyond the melting point?

MR. HOFMANN: I didn't. As far as the calculations are concerned, they are essentially all through the melting point, but the curves you have seen have been cut off pretty much at close-to-melting. The calculations assume that the Doppler co-efficient is present in molten fuel, but the curves that were shown have not been drawn all the way.

MR. ANDERSON: In your calculations, as you begin the transients, do you consider the positive Doppler feedback up to the time when you begin to get heat transfer, plus expansion of the stainless?

MR. HOFMANN: Yes, if we take a count of the positive Doppler, it would be in the expansion.

MR. LOEWENSTEIN: Does the peak flux change in a cermet during a duty cycle; and does the peak flux change in an oxide during the duty cycle?

MR. HOFMANN: The flux is about the same. Remember, the burnups are not that large. The burnup in one cycle, six kilos out of 300 , average. But if you are asking me what is the distribution with the burner, if that is what you are asking, I don't know. I imagine it isn't very much different than initially, because the fractional fuel burnup isn't so large.

MR. HENNIG: We have the zoned core. Really the zoned core is something to look at. Where the radial zoning varies by, roughly, increasing to the edge by a factor of $50 \%$, and the change of peak flux is on the order of $2 \%$, compared to unzoned. This is bigger effect than one we would expect to get for burnup. The uniformly loaded core is 6 tubes, with about five kegs in a tube, call it six per burnup, with a 
radial zoned core you have five in the seventh, six in the second, and seven in the eighth, and change in peak flux between the homogenously loaded core and the radially zoned core is only on the order of a couple of percent, but we aretalking about changes in loading, more on the order of 20 to $50 \%$ 。

MR。deBOISBLANC: The power density in the center determines the flux for a given plutonium loading. If you add more fuel outside you enhance the principal but you normalize the fact. When you talk about fractional changes, fractional change due to burnup, whatever it is, should be roughly the same for the uniform loaded core.

MR. HENNIG: The fluxes change very little in going from unzoned to radial zoned cores.

MR. deBOISBLANC: You don't change the core volume?

MR. HENNIG: No.

MR. deBOISBLANC: If the fluxes change at all, and you normalize it to the same power, what would expect the central power to do but go up at the end of the cycle, because the fission cross section is going down. I am thinking only if you deplete, by the same arguement that you use, that the power density determines the flux, given fissile material, and if you burn up 29\% of those atoms, you have burned up $29 \%$ of the fission cross section, unless there is gross change in the power distribution, which you have argued there isn't.

MR. HOFMANN: No, I have argued there is no gross change in the flux distribution. There are enormous changes in the power distribution, and in fact it is surprising how large a change in power distribution you can bring about by fuel zoning and how little effect this has on the flux distribution. The flux distribution seems to be remarkably insensitive to the fuel zoning. In fact, I have been trying to look for some scheme by which I could enhance fluxes locally by some fuel zoning, but I can't do that. I would like to have some sort of a fast flux reactor. So the flux distribution is quite insensitive to the fuel distribution, but the power distribution is very sensitive.

MR. HECK: I think pertinent to this, and also the other question about how much fuel can be put into an experimental region: We did some looking years ago when we were working on a reactor design at taking a test assembly and loading the outside of it with a ring of poison rods, for example. It turns out the flux depression is very, very small. So you can put a very high enrichment in the fuel and get very high power levels, if for instance you want a high level to deliberately melt the thing down. But the reactivity effect of this whole assembly can be held much lower than you can possibly get with the same number of pins and the same power density, so there are possible ways of enhancing the amount of fuel that you can put in an experimental hole so that the reactivity effect is low, even though the power effect is quite high. It is a little different than what you normally find in a water reactor system. 
.

•

- 


\section{CAPITAL COST PARAMETER SURVEY}

$$
\text { P.D. Cohn }
$$

Pacific Northwest Laboratory Richland, Washington

Operated by Battelle Memorial Institute

\section{INTRODUCTION}

The capital cost survey, which I am presenting today, examines the effects of FTR test volume, flux level, core power, and core volume on the capital cost; and the consequent effects from the use of mixed-oxide, or $\mathrm{PuO}_{2}-\mathrm{SS}$ cermet driver fuel. The slides, which I will show will attempt to demonstrate the differences between the capital cost for the FFTF facility and reactor with the reactor driven by an oxide or cermet core. The slides will portray the effect of individual variables as they affect the capital cost; which in turn are controlled by the fuel selection. The variables that will be examined include power level, core volume, flux, test volume, and number of closed loops. In each case, we have attempted to isolate the variable so that its individual affect may be seen.

Additionally, I will explore some of the bases for design selection including considerations of test volume growth and flux growth. I will also discuss the assumptions involved in the choice of the test variabled. These assumptions should be examined as part of this meeting's purpose. It should be pointed out that the capital cost survey is only one factor, albeit important, used in evaluation of FFTF. As would be expected, some aspects that are near-optimum from a capital cost standpoint may not be optimum from the standpoint of test capability, fuel useage and cycle cost, or safety. The capital cost bases to be presented have been jointly verified by two independent, offsite companies.

\section{COST ANALYSES}

Slide 1 is one of the major bases for the capital cost differential between an oxide and cermet fueled FTR. This slide, which is a modification of the physics data previously shown, presents core power versus core volume for various flux levels for oxide and cermet fuels. An understanding of these data is necessary to appreciate the differences between the oxide and cermet fueled cores. For instance, it may be seen that with a centerpoint flux of $135 \times 10^{16}$ (the top two 1 ines) and at a core volume of 800 1iters, the oxide core operates at a power approximately $20 \%$ higher than the cermet core in order to achieve the same flux level. That is, $475 \mathrm{MWt}$ for the cermet versus 585 MWt for the oxide. 


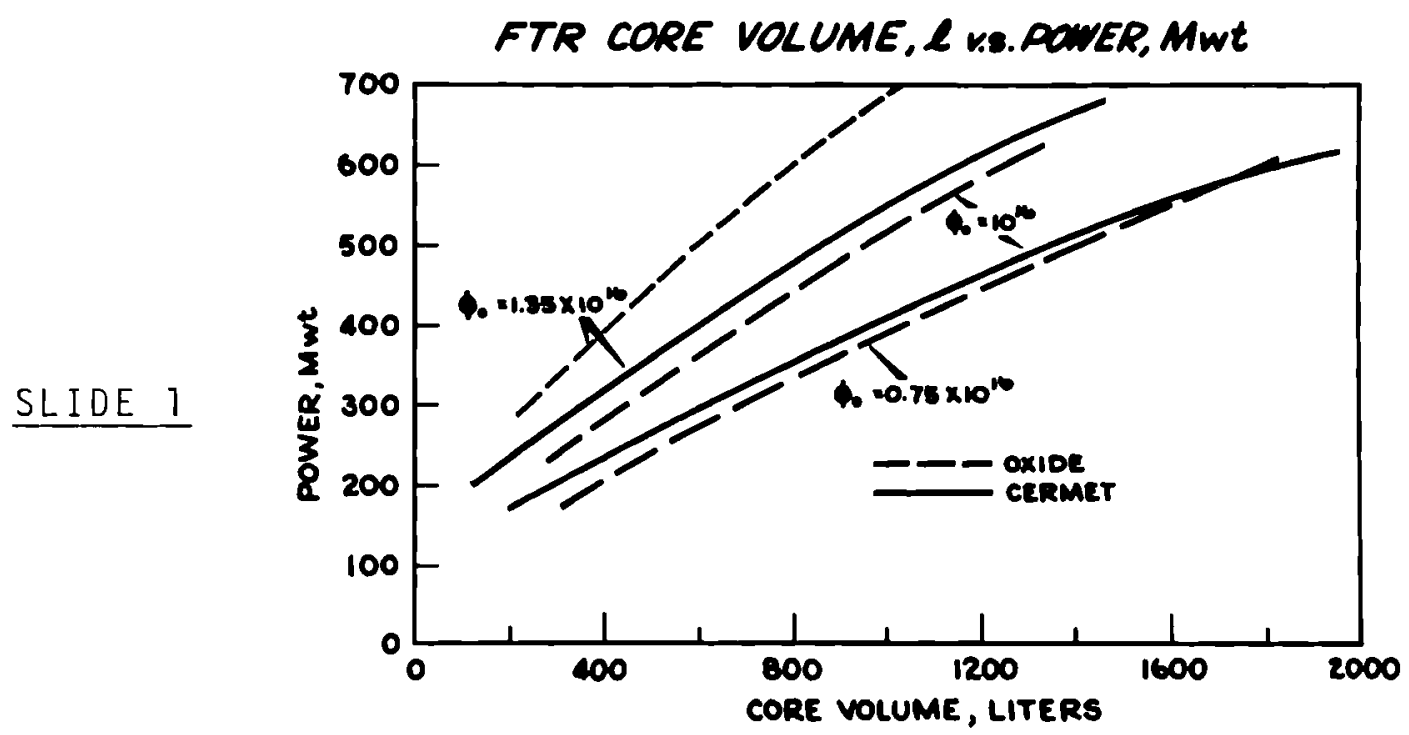

Similarly, at 600 iters, with a centerpoint flux of 1016 , the power of the cermet fueled core would be 300 MWt, and the oxide fueled core would be 365 MWt. Thus, the power level and the power density of the mixed-oxide fueled cores, in the range interest for FTR, operate at about $20 \%$ higher power at the same flux level. These curves are merely shifted downward but maintain the same spread if the power and flux level are reduced. As will be seen, this power differential at a fixed volume and fixed flux is the primary reason for the capital cost difference between an oxide and a cermet fueled facility. Since the primary product of the FFTF is flux and test volume, it is worthwhile to examine the allocation of test volume and its effect on the capital cost.

Slide 2 illustrates the allocation of test, driver fuel, and control spaces for the basic or initial test volume, 600 liter, 61 subassembly core array. These configurations are valid for either the oxide or cermet fueled cores.

Let me briefly describe the various symbols on the slide. The solid gray represents the driver fuel; the light hexagons are safety, shim rods, and the regulating rod; the uncircled hexagons are open test spaces; and "F" refers to fuel; "M" are materials tests; and the circled hexagons represent closedloop tests. Other nuclear tests are shown on the perimeter.

The allocation for the control spaces for the core is essentially fixed and is the same for either fuel. From our estimate of future testing requirements, the allocation of test space is based on criticality considerations and on the breakdown between materials and fuel test loops. It should 
be noted that for reactor volumes up to 400 liters, the cores were divided into 31 basic subassembly arrays. For cores between 400 and 700 1iters, the cores were segregated in 61 subassembly arrays; above 700 liters, 91 subassembly arrays were utilized. This choice of the basic hex pattern was based on the requirement for an adequate number of control spaces, plus control of the reactivity contribution on each driver fuel assembly. Additionally, the arrays were so fixed to maintain the driver fuel hex size at about 4.5 in. across the flat. This allows a near optimum open 4.5 in. in-core facility and the larger 6 to 8 in. test facilities on the core periphery. These sizes are based on our estimates of the testing needs as typified in the four $1000 \mathrm{MW}$ fast breeder reactor studies.

S1ide 3 presents an ultimate test volume configuration in a basic 61 element array. It may be seen that the number of packaged and material nonpower-producing test assemblies and the number of fuel testing assemblies assumed to produce about $3 / 4$ of the driver fuel power do increase, thus increasing the duty on the remaining driver fuel elements. While this increased test volume may appear attractive from a capital cost aspect, the increased fuel duty may be quite costly from the standpoint of fuel cycle costs.

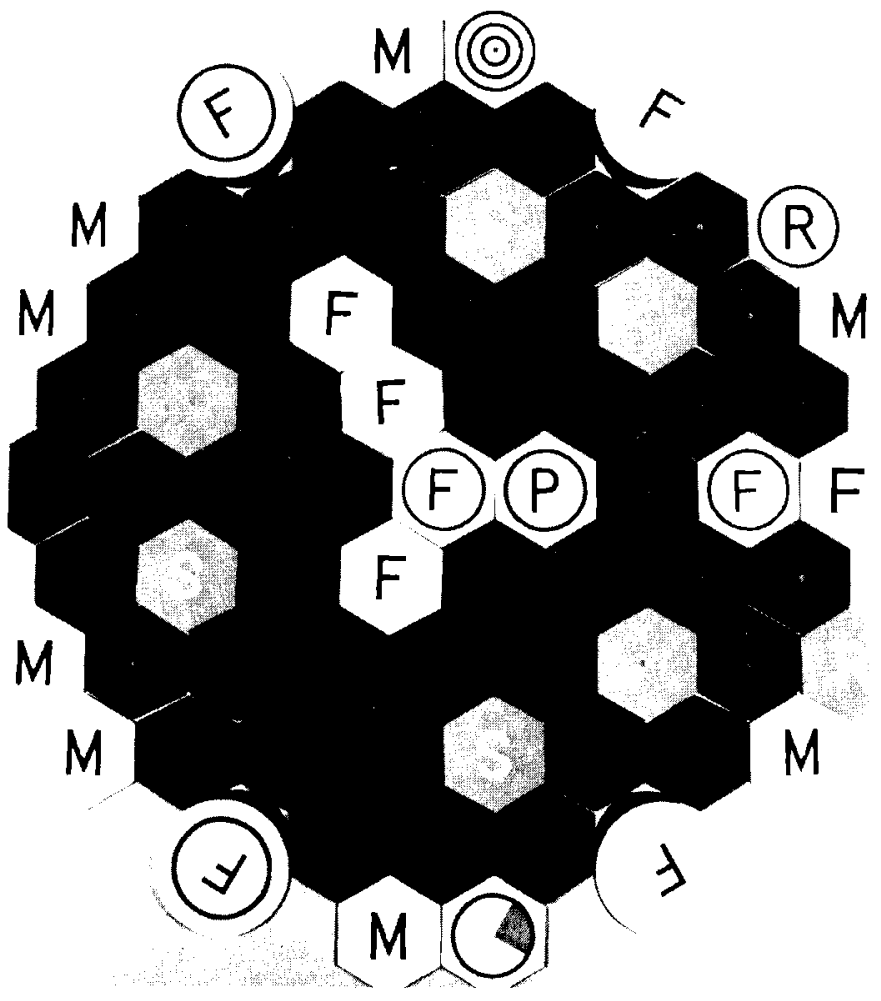

SLIDE 2. The basic or Initial Test volume, 600 Liter, 61 subassembly Core Array

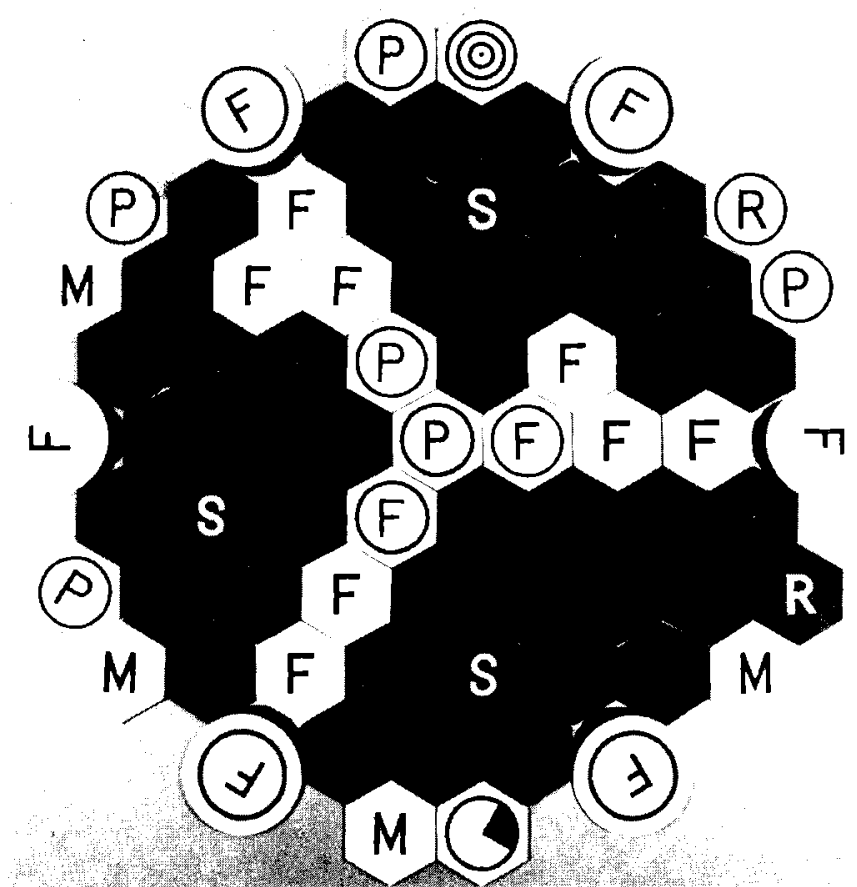

SLIDE 3. An Ultimate Test Volume Configuration In A Basic 62 Element Array 
Slide 4 presents the test volumes versus the reactor volume with the initial and ultimate test configurations shown on the previous slides. These data are valid for either the cermet or oxide fueled cores. The slope of the initial configuration line is 17 to $1 / 2 \%$ with a small amount of scatter, and the ultimate test configurations line slope is about $30 \%$ of the core volume. The spread between the ultimate and initial configurations represents an increase in test volume of about $70 \%$.

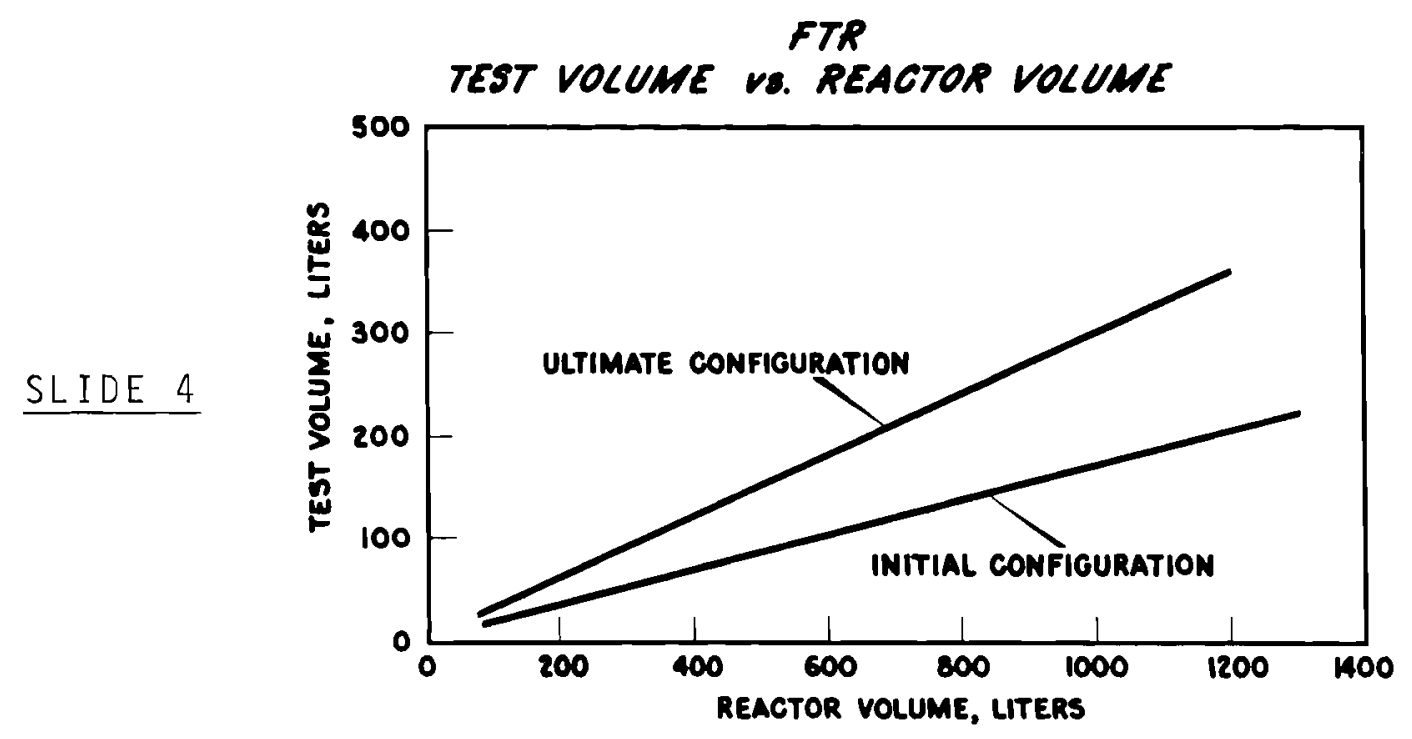

S1ide 1 has shown that at a fixed core volume and centerpoint flux, the oxide core operates at a power approximately $20 \%$ higher than the cermet fueled core. Based on the same curve, at a fixed power, the oxide core volume is about $30 \%$ to $35 \%$ below the cermet core volume. For instance, at a centerpoint flux of $1.35 \times 10^{16}$ (the top two 1 ines) at a fixed power of $400 \mathrm{MWt}$, the oxide volume is 440 1iters versus 600 iters for the cermet, or at a centerpoint flux of 1016 at 300 MWt, for instance, the oxide volume is 450 iters versus 600 liters for the cermet fueled core. In either case, the resultant reduction in core volume, and thus test volume, amounts to about 30 to $35 \%$ in the region of interest for FTR volumes.

It should be pointed out that technical feasibility has not been completely addressed as part of the capital cost analysis. For instance, it would not be practical to build a 200 iter oxide core with a centerpoint flux capability of $1.35 \times 10^{16}$ from the standpoints of fuel cycle cost and test volume. As have been and will be discussed, safety and operating characteristics are also important considerations. 
Slide 5 presents test volume versus core power, using the ultimate test volumes and a centerpoint flux of 1.35 as a basis for comparative purposes. This slide shows that at a fixed power, the oxide test volume is about $30 \%$ to $35 \%$ below the cermet test volume. Conversely, at a fixed test volume, a higher oxide power is required to achieve the same flux. Before we get into the cost effects, it is worthwhile to examine the allocation of construction costs to the various facility parts and the affects of power.

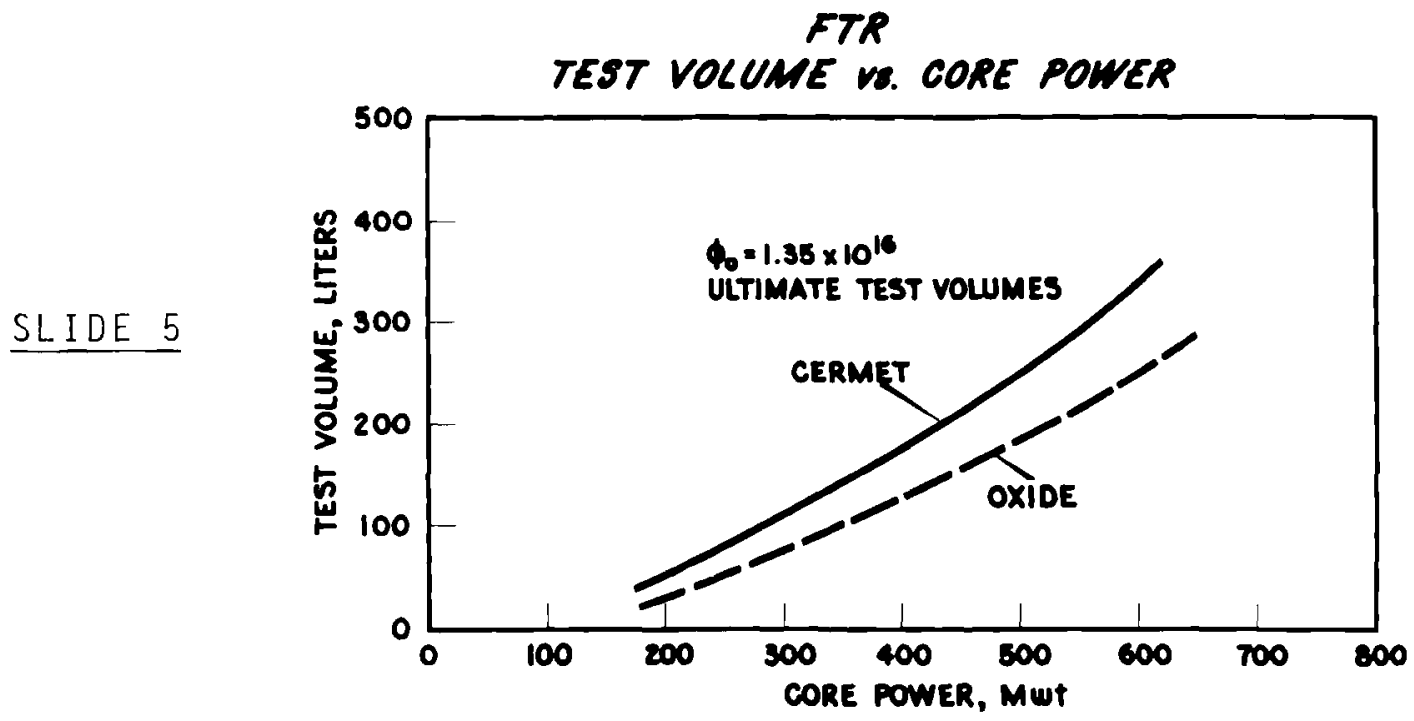

S1ide 6 shows the representative direct construction costs for the various FFTF items. The power level of the facility may be seen to effect the costs rather strongly as the heat removal costs represent a major fraction of the cost and are directly proportional to the reactor power. Similarily, the test loop costs, whose heat rejection requirements are directly proportional to reactor power, are also a factor. The reactor complex also has major affects on cost from the standpoint of thermal power change. Keeping in mind that the oxide operates at approximately $20 \%$ higher power than the cermet with the flux and volume fixed, the differences in cost between the two arises principally due to heat removal requirements, and we will be examining these requirements along with the capital cost effect of number of closed loops later on in this discussion.

The next series of slides that I will present are taken directly from BNWL-CC 503, a summary of our capital costs survey, written by Frances Shadel, and to be published shortly. As I mentioned previously, these capital costs have been verified jointly by two outside contractors. Since these slides are obtained directly from this document's graphs, please note that the scales vary from chart to chart. 
4.6

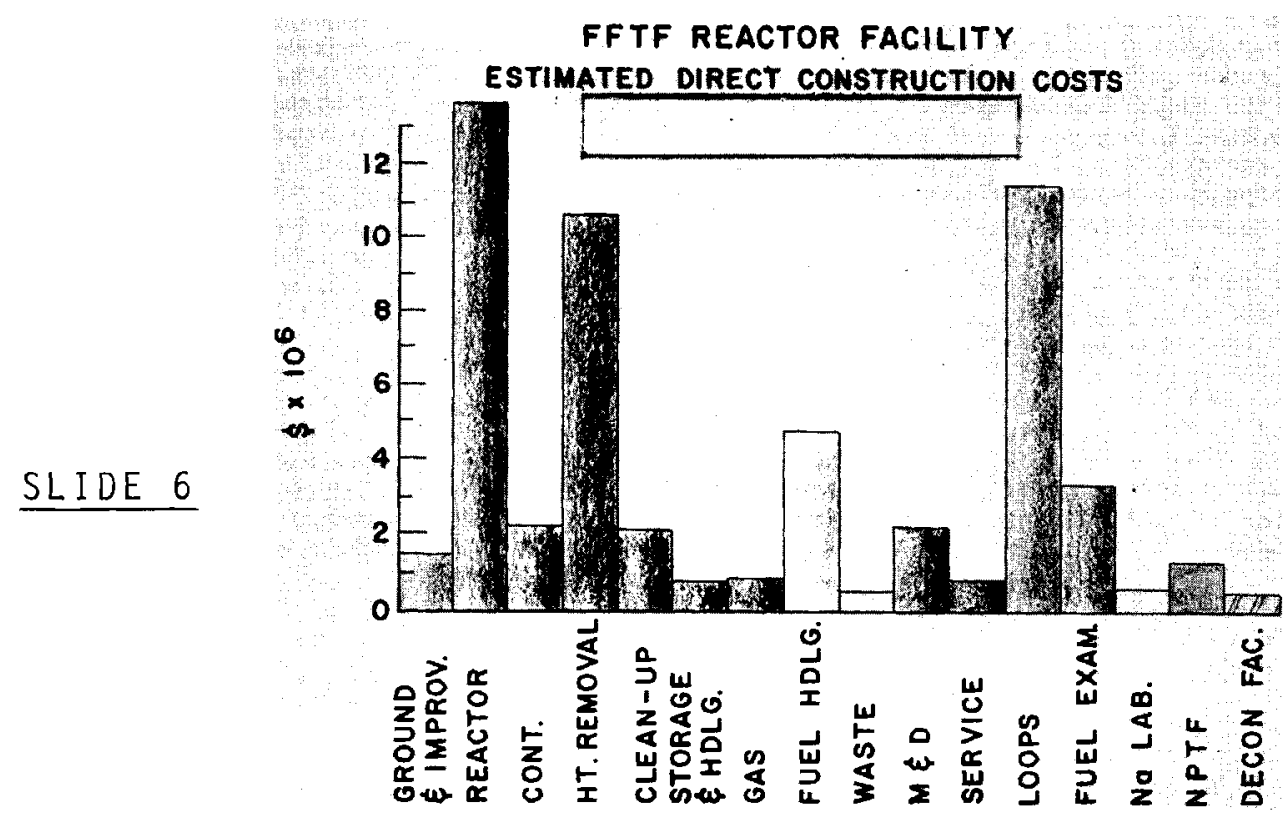

Slide 7 presents the direct cost in millions of dollars versus reactor power for the reactor complex, including those buildings and equipment associated directly with the reactor. The slope of this curve is about $\$ 1$ million per 100 MWt.

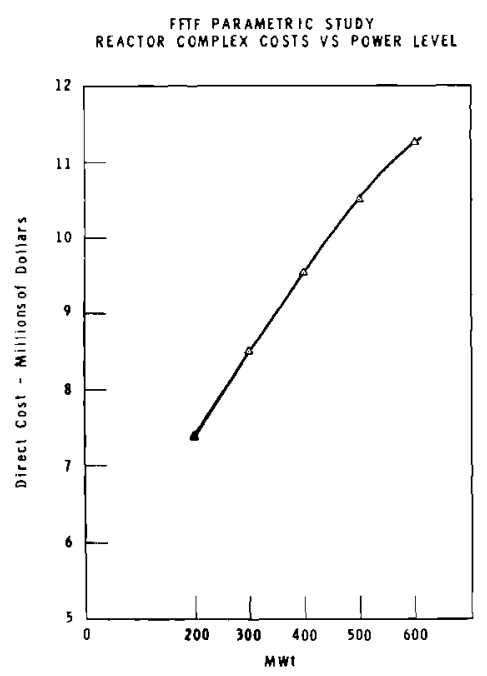

SLIDE 7
FFTF PARAMETRIC STUDY SODIUM SYSTEM BUILDING COST VS POWER LEVEL

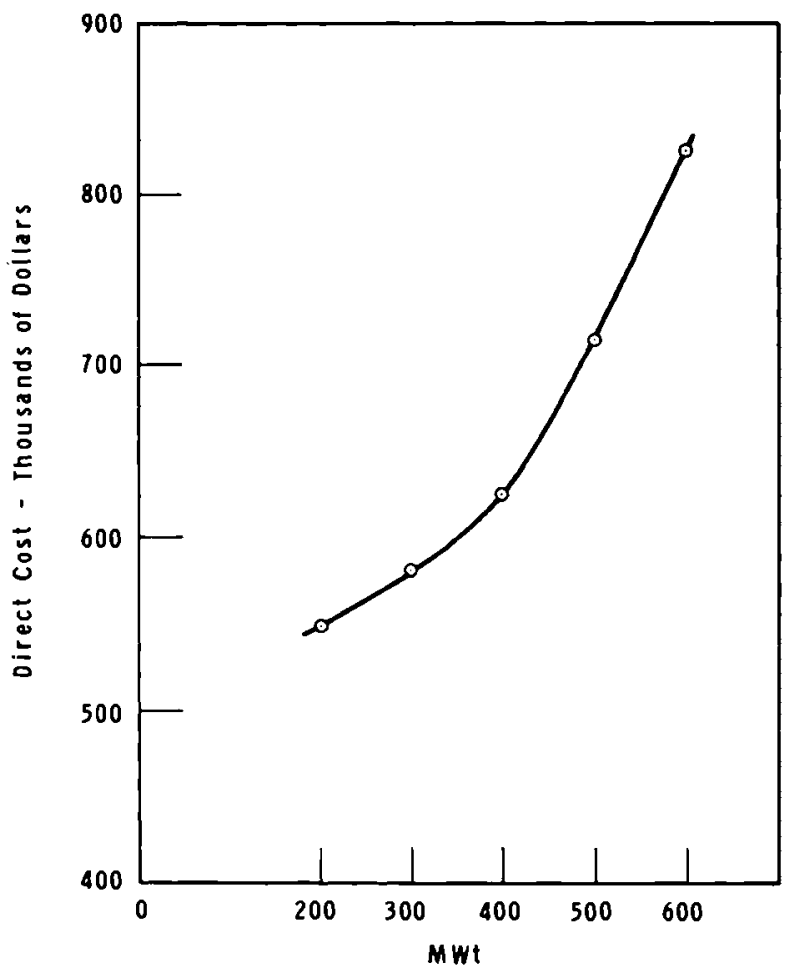


S1ide 8 shows cost versus reactor power for that portion of the building apportioned to the sodium systems.

Slide 9 presents two sets of data. The bottom figure represents fuel handling equipment costs versus power leve1, and as mentioned previously we see the effect of changing the number of tubes in the basic core array. Notice that the effect of changing the number of tubes and power level does not greatly change this cost. The upper curve presents the containment vessel costs versus reactor power level. This curve is quite flat with power because the containment vessel size is primarily determined by the refueling machine; and core height is a small portion of the total process tube height even though the core height does change as the power changes.

S1ide 10 presents data on the instrumentation costs versus power. The top curve presents the total instrumentation in the FFTF facility, and the bottom curve shows the reactor instrumentation on $1 y$. It may be seen that we are talking about a $30 \%$ cost change for a factor of three in power level. One of the purposes for having these costs data broken down in this fashion is that I wish to emphasize the large testing gain from the standpoint of testing volume with a relatively small investment increase. As we will see in the summary curves, the basic committment to building FFTF facilities is about $\$ 65 \mathrm{million}$. Then, to increase its testing usefulness many-fold including closed loops, the total investment increase above this basic figure amounts to less than $30 \%$. Thus it seems that there is high incentive for going to large reactors with large testing volume capabilities. Later in this talk, I will discuss the investment involved in flux level in a similar fashion.

CONTAINMENT VESSEL VS POWER LEVEL

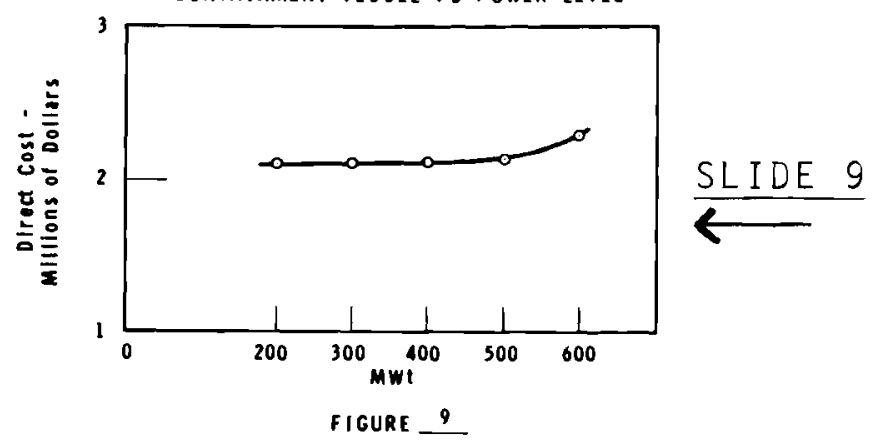

FFTF PARAMETRIC STUDY FUEL HANDLING SYSTEM

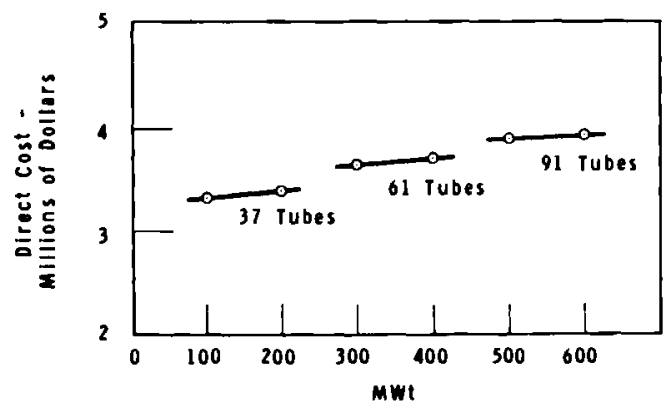

FFTF PARAMETRIC STUDY TOTAL INSTRUMENTATION VS POWER

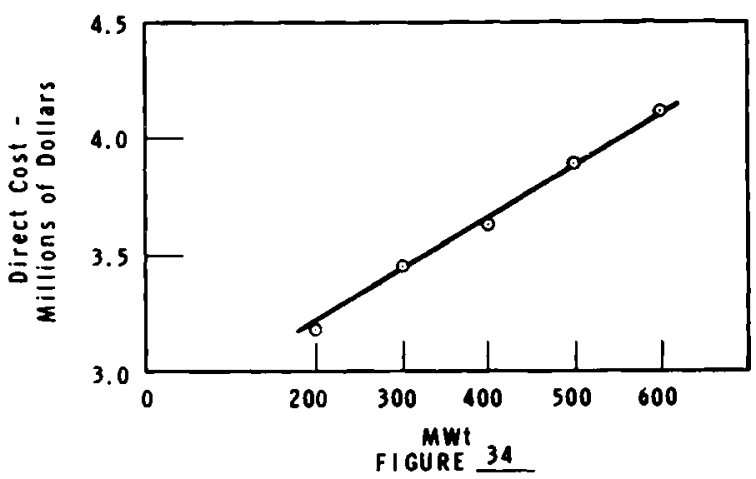

REACTOR INSTRUMENTATION VS POWER LEVEL

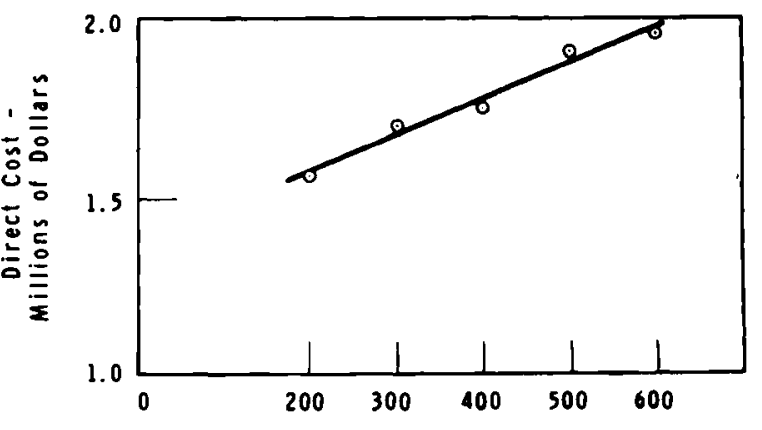


S1ide 11 shows the cost of loop construction versus the loop power in the closed testing loops. Since thermal duty is of major consideration in loop cost, a large cost change with loop power occurs. The closed test loops for the particular cores of interest, in the FTR volume range (that is above 500 liters), operate between 5 to 10 thermal megawatts.

S1ide 12 also presents two sets of data. The first shows the total coolant and gas system construction cost versus power leve1. This cost rises about $40 \%$ with a doubling of power. The bottom curve presents the primary pump cost versus power level. Since we are considering both fuels, it is worthwhile to remember that a $20 \%$ higher power is necessary for the oxide core to achieve the same flux level at the same core volume. Thus, in considering these curves and their variation with power, the oxide wil1 have an intercept $20 \%$ higher on the power scale.

Slide 13 also presents two sets of data. The top curve is for the secondary pump costs, and these vary in a manner similar to the primary pump costs. The bottom curve shows the secondary piping cost versus power level for the two loop or three loop systems. There are several considerations involved in loop number selection, including the pump capacity, safety, and other operating considerations.

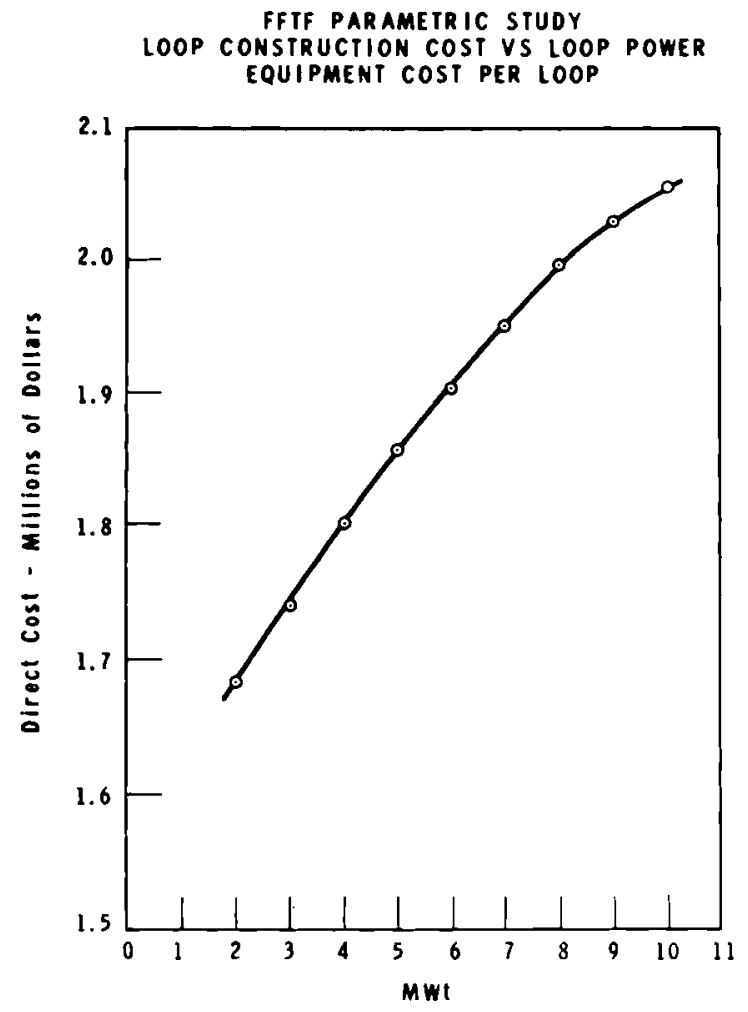

SLIDE 11

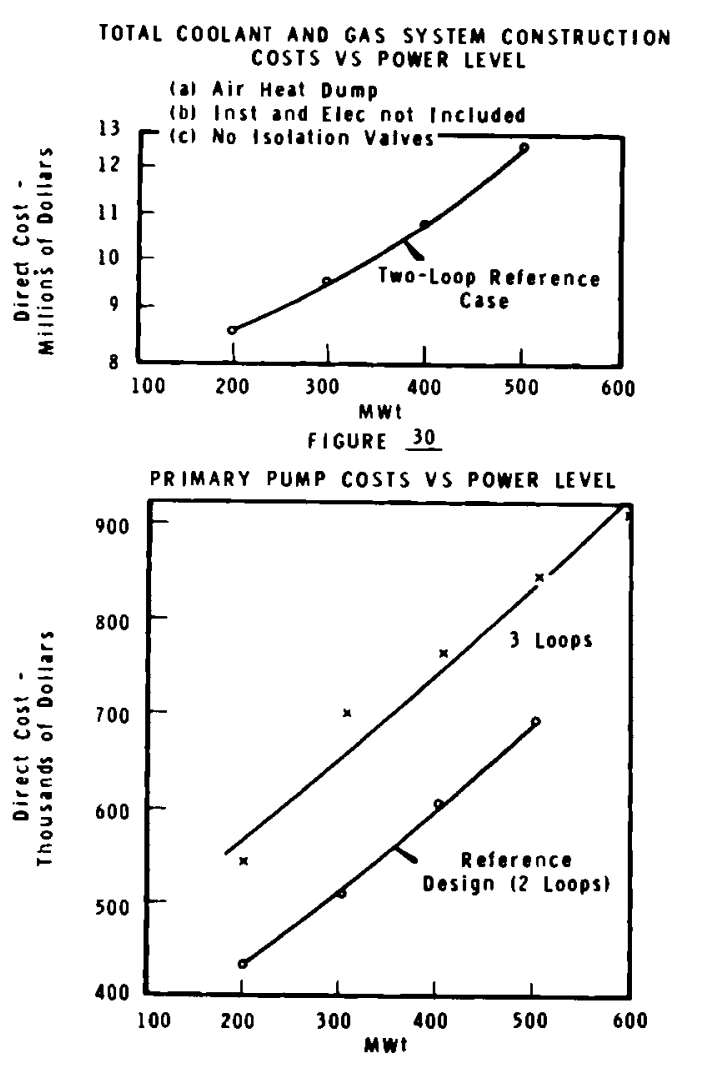

SLIDE 12 
FFTF PARAMETRIC STUDY SECONDARY PUMP COSTS VS POWER LEVEL

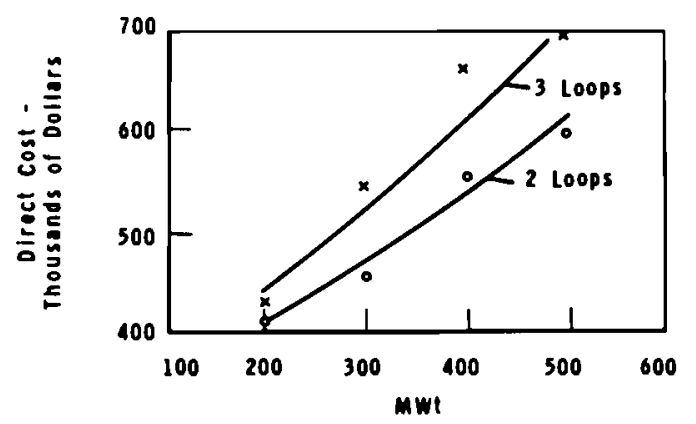

FIGURE 25

SECONDARY PIPING COSTS VS POWER LEVEL

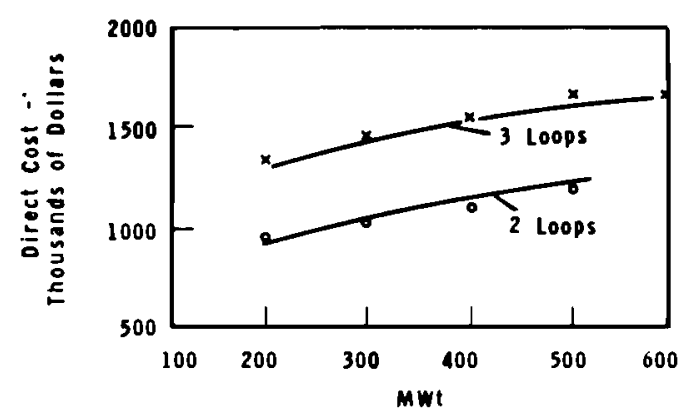

SLIDE 13

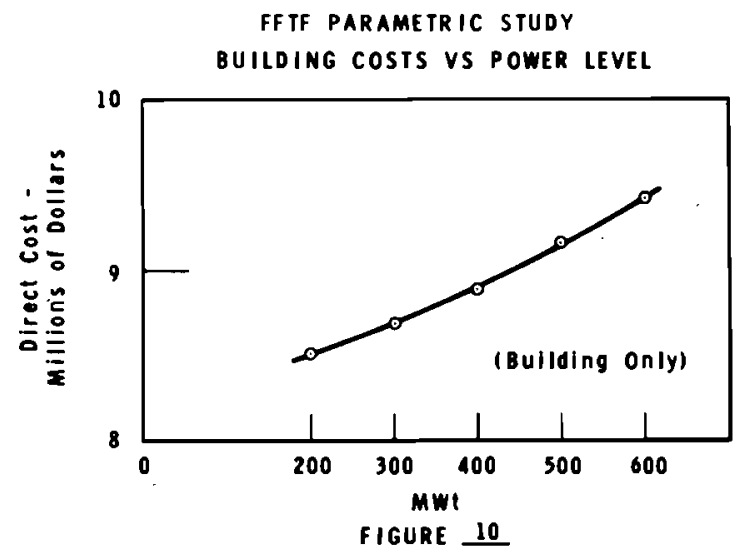

heAt REMOVAL SYSTEM BUILOING COST VS POWER LEVEL

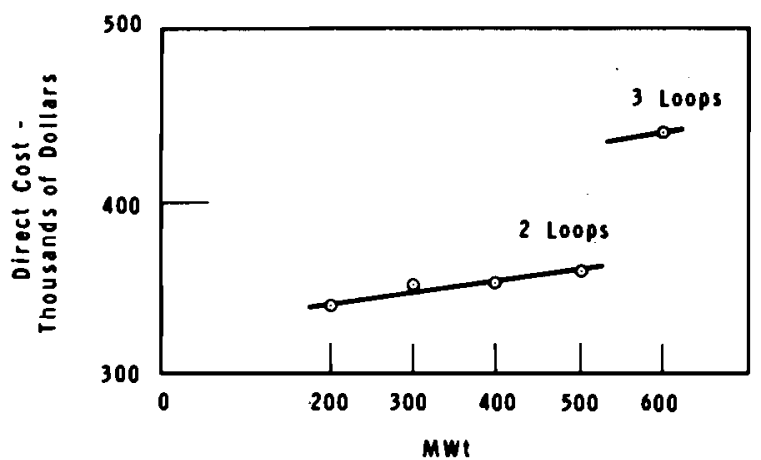

$\underline{\text { SLIDE } 14}$

Slide 14 presents two more sets of data, showing the building costs versus power level. The upper curve is for the total building cost, and note that this slope is less than $20 \%$ for a factor of 3 change in power level. The lower curve shows the building costs apportioned to the heat removal system and shows the cost difference between the two and three loop systems.

Heat removal costs represent one of the major differences between the oxide and cermet fueled cores; the FFTF heat removal direct construction costs versus reactor power is shown in Slide 15. In the range of interest at $300 \mathrm{MW}$ and $\mathrm{up}$, the increase in reactor power amounts to a direct construction cost increase of about $17 \%$ per $100 \mathrm{MW}$ or about $1.9 \mathrm{million}$ dollars/100 MW.

Slide 16 presents the cost of the FFTF facility versus core volume in liters. The centerpoint flux is constrained at $1.35 \times 10^{16}$ for comparative purposes. This slide and the following slides are summary curves, and the costs given are the direct construction costs times the contingencies which gives the total cost. It may be seen that the spread between the 
4.10

oxide and cermet costs is between 7 and $10 \%$ in the range of interest from 500 liters up, or about 5 to 8 million dollars. At this point, it is worthwhile to note those items which contribute to this 5 to 8 million dollars difference between the oxide and cermet capital costs. Heat rejection due to the higher heat load in the oxide represents $59 \%$, or about $\$ 3.8$ miliion of the cost differential. The reactor facility represents $25 \%$, or about 1.6 million dollars, of the cost difference. These later costs for the reactor are due primarily to larger nozzles, plenums, and larger reactor diameter (smaller H/D ratio) in the case of the oxide core to facilitate near-optimum testing conditions, pressure drop, and fuel cycle costs. The other items are relatively minor. The closed loops contribute $9 \%$, or about $\$ 600,000$, to the cost difference because of their higher thermal duty in the oxide core. Other items not specifically called out contribute $7 \%$, or about $\$ 450,000$, to the cost difference.

\section{FFTF HEAT REMOVAL DIRECT CONSTRUCTION COSTS \\ vS. REACTOR POWER}
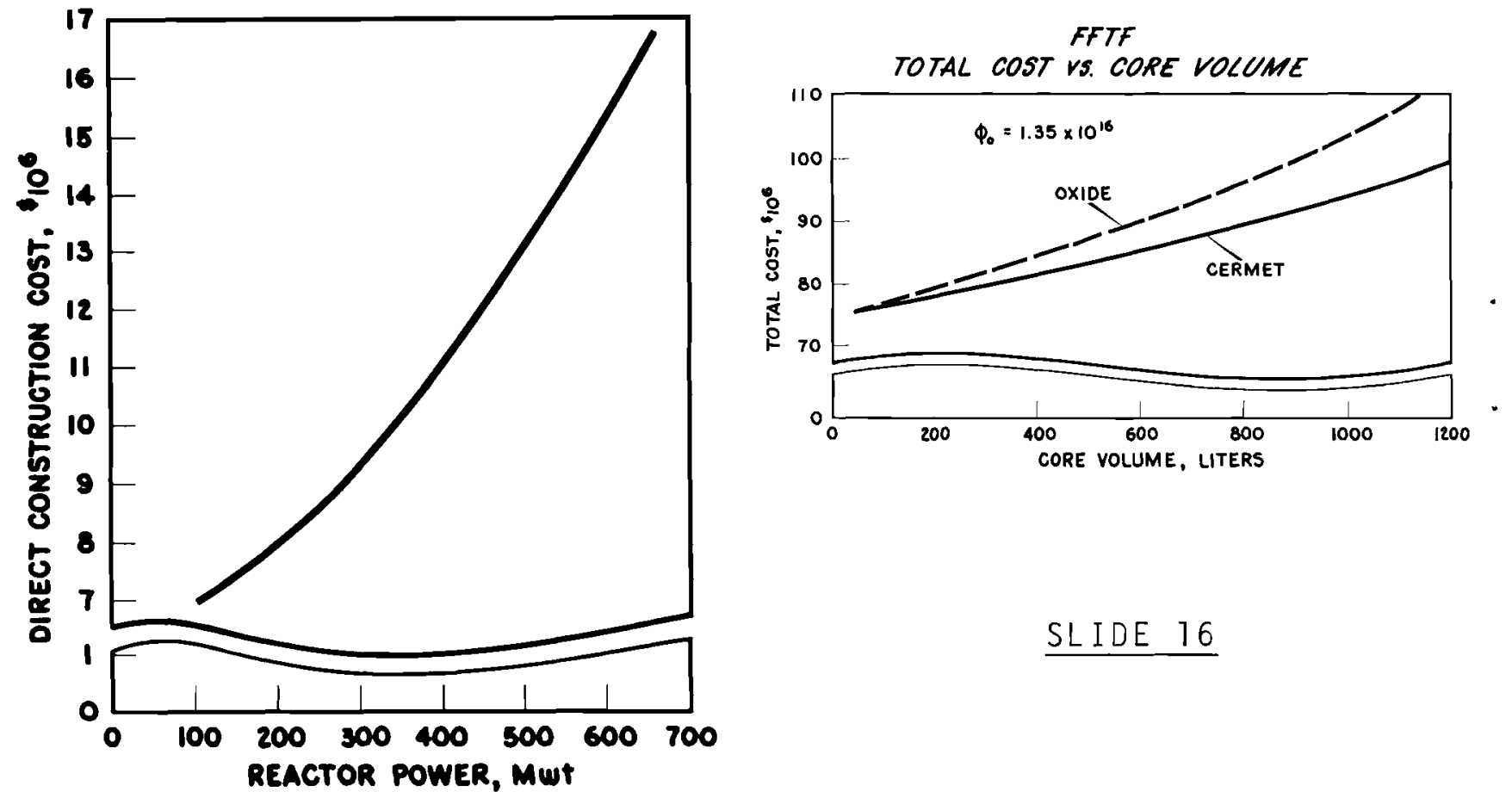

SLIDE 15 
Slide 17 has the same ordinate as the previous slide, and it presents the cost versus test volume for the oxide and cermet 6 fueled cores. The centerpoint flux is constrained at $1.35 \times 10^{16}$, and the ultimate test volumes are used for comparative purposes. These test volumes are based on the approximate $30 \%$ of core volume (shown previously). It is interesting to note that the direct costs are just as flat with increasing test volume as they were with core volume. For instance, the cermet fueled core increases in capital cost approximately $10 \%$, from 100 to 250 liters of test volume. Thus, there is a large gain in testing facility, with a relatively small increase in the cost. Again, the spread of about $7 \%$ in the total costs is evident between the oxide and cermet fueled cores.

S1ide 18 presents more dramatically the benefit from the increased investment on test volume. On it we have plotted cost divided, now, by liters of test volume versus core volume in liters. It may be seen that core volumes above about 500 liters yield the more optimum investment from the standpoint of dollars per test 1iter.

As in the previous slide, the centerpoint flux is constrained at $1.35 \times 10^{16}$ and ultimate test volumes are used for the comparison. If the initial test volumes were used, both the oxide and cermet curves would be displaced upwards by about a factor of 1.7 .

\section{FFTF}

TOTAL COSTS PER LITER OF TEST VOLUME

vs.

CORE VOLUME

FFTF

TOTAL COST VS. TEST VOLUME

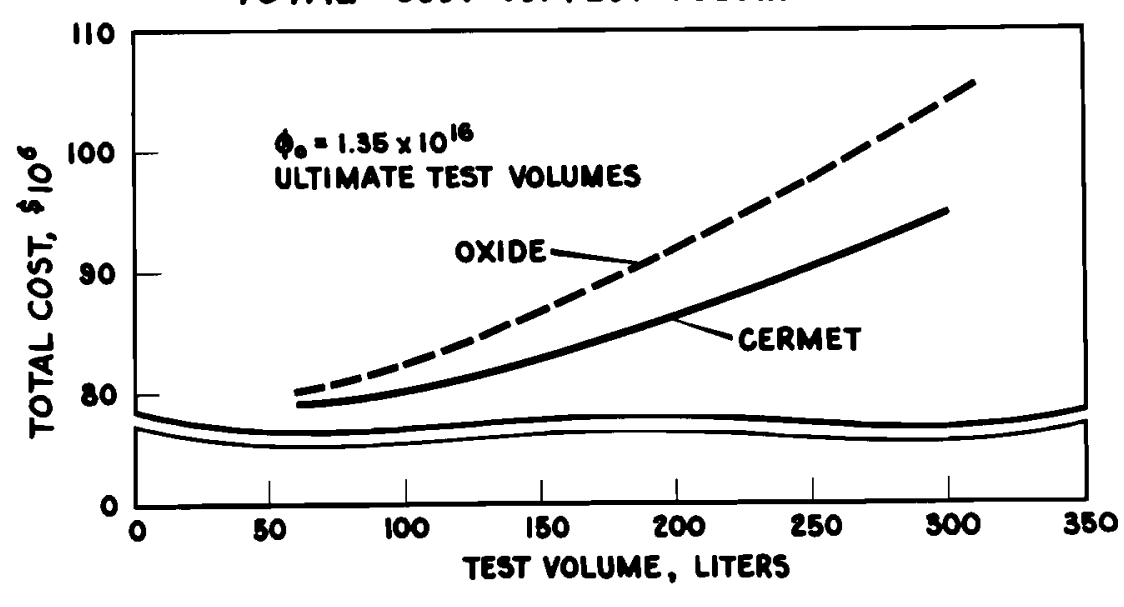

SLIDE 17

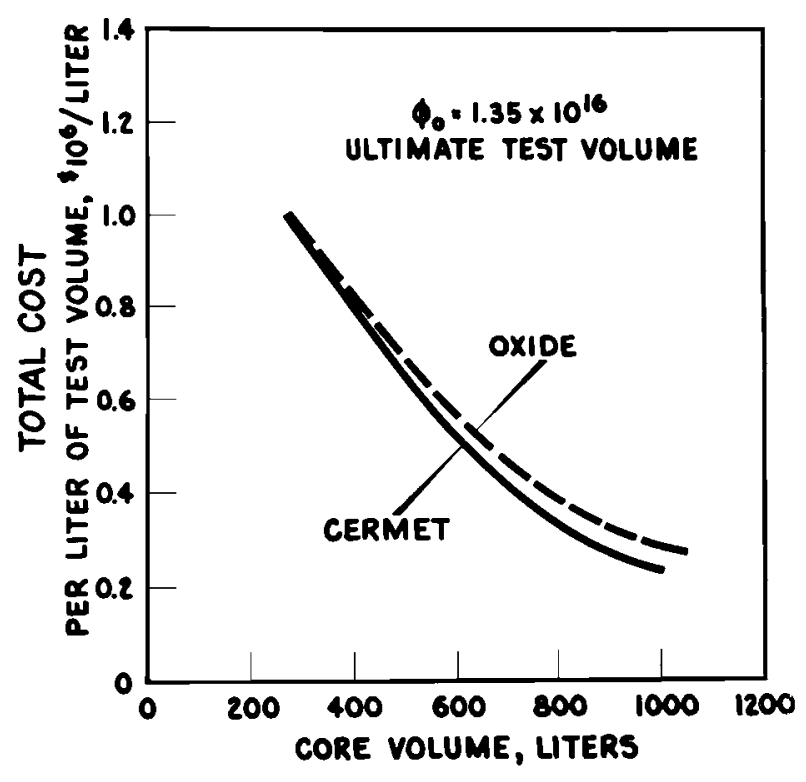

SLIDE 18 


\subsection{2}

In either case, the 7 to $10 \%$ cost spread between oxide and cermet fuel cores is maintained in the core volume range of interest. Again, this curve emphasizes the large investment benefit from the standpoint of test volume by going to larger volume cores.

Slide 19 presents cost versus core centerpoint flux. The particular core utilized for comparative purposes is a 600 1iter core. As in the case of increased test value, increased flux costs vary little when compared to the testing benefit gained. It should be kept in mind, however, that the capital cost is only one part of the consideration for increased flux level, and fuel duty must be taken into account (as shown in Russ Peterson's presentation). The increasing cost with increased centerpoint flux (as shown in Slide 19) is due primarily to the increased heat rejection requirement as the power grows to achieve the higher flux. Notice that in either the oxide or cermet case, the capital cost difference, in going from a centerpoint flux point of .7 to 1.35 , costs 1 ess than $10 \%$ in total cost. This slide points up the advisablility of designing a system that has the capability of operating at the higher flux level even though initial operation may not be at the peak flux potential.

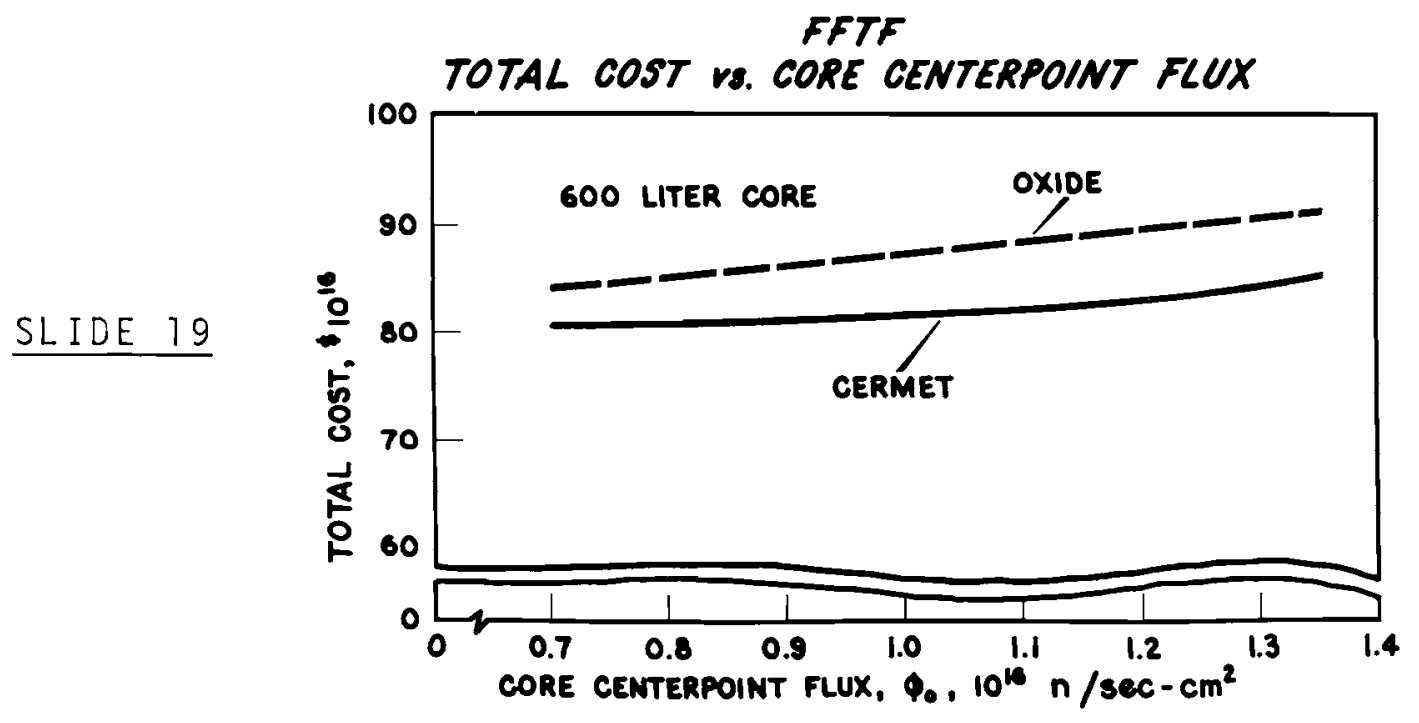


The question arises how to allocate test volume between open and closed loops. The closed loops increase the cost due to their equipment requirements to maintain their testing purpose. This includes test cells, heat rejection equipment, materials and coolant handling, pumps, and reactor penetrations to accommodate the loops. The effect of the number of closed loops on the facility cost is shown in slide 20. For comparative purposes, a 600 liter core was chosen, and the centerpoint flux was constrained at 1.35. The incremental total cost for closed loops is approximately 3.5 million dollars per closed loop for either the oxide or cermet fueled cores. Again, the spread between the oxide and cermet fueled cores is evident because of the $20 \%$ higher power density required in the oxide fueled core to achieve the same centerpoint flux.

In either case, based on the enhanced testing versatility available from the closed loops the additional capital cost is probably justified. For instance, the capital cost increase is about $\$ 18$ million, or about $24 \%$, when going from one to six closed loops (Slide 20).

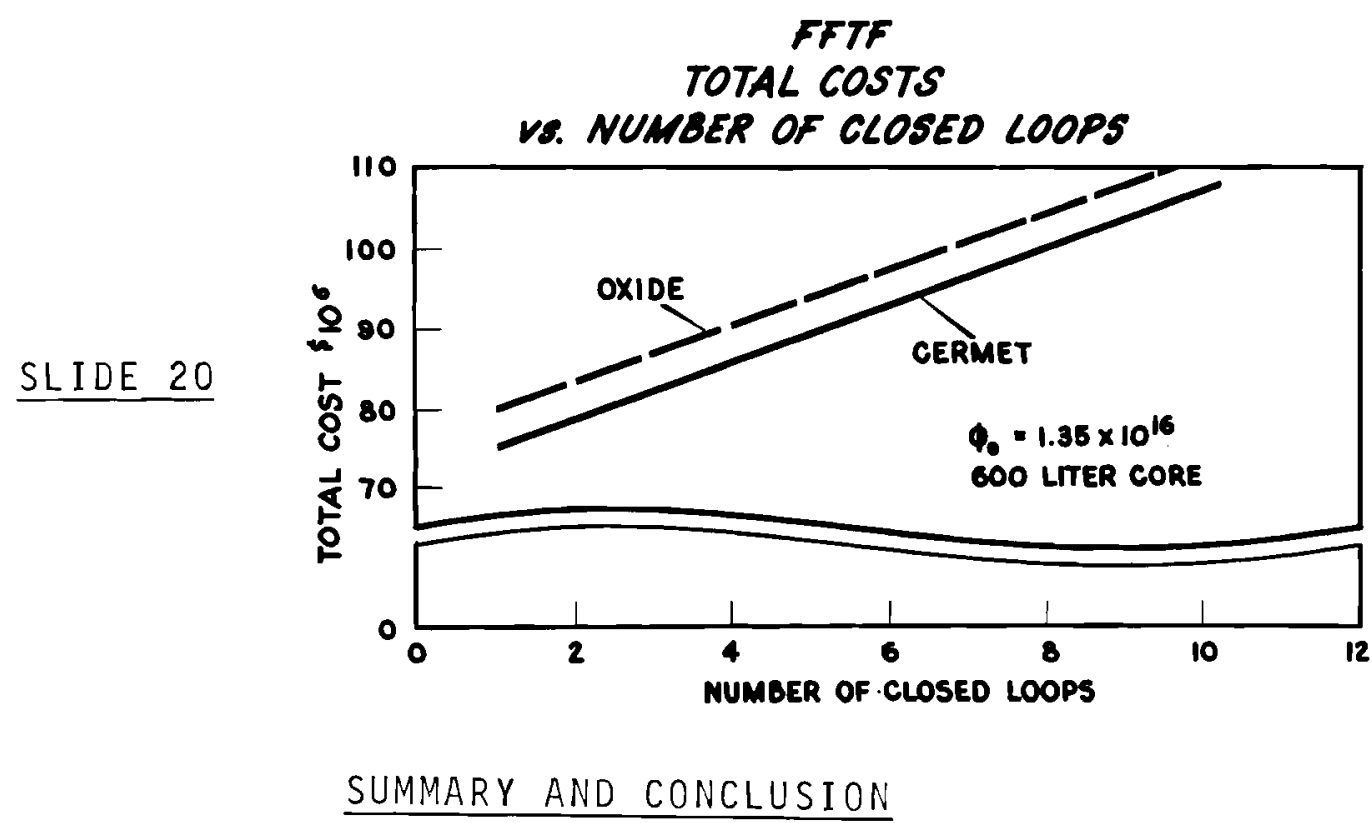

In the summary, we have attempted to examine the capital costs and the effects on these capital costs of flux level, test volume, reactor power, reactor volume, and test configuration. One conclusion, I believe, that may be drawn from the data is that the large cores above 500 liters, coupled with their ultimate flux potentials, yield significant increases in test volume and test capability at a relatively minor increase in total cost. 
A1so I have attempted to demonstrate that the basic FFTF commitment amounts to approximately $\$ 65$ million. Therefore to make the facility capable of handling testing for a considerable period into the future, a relatively small increase in investment is necessary to insure the capability of future testing usefulness. By this I refer to a total increase in cost of about $30 \%$ over the base cost to yield the ultimate flux level of $1.35 \times 10^{16}$, the higher test volumes, and the $1 \mathrm{ar}-$ ger number of closed loops. Additionally, the facility is designed for high availability, turnaround, the necessary fuel examination facilities, and all of these attendant facilities required to enable complete and efficient usage of the total FFTF complex. In other words, the increased cost over the fixed functional requirements is small compared to the testing benefit gained from the standpoints of flux, power level, and test volume. Generally, the cermet fueled cores yield the lower unit cost per test volume and also the lower total cost at fixed flux and volume because of the higher thermal duty of the oxide cores required to achieve these fixed variables. However, the cost spread, due to fuel choice represents less than $10 \%$ of the total cost within the range of interest.

In conclusion, it should also be pointed out that the capital cost picture is only part of the total FFTF cost study, and the fuel cycle costs, which will be presented in the next presentation, help complete the picture for comparison of oxide and cermet fueled FFTF facilities. 
D. 4.1

CAPITAL COST PARAMETER SURVEY

P. D. Cohn

\section{DISCUSSION}

JAMES WRIGHT (Westinghouse): I had two or three interesting thoughts here as you went down the pike. One is, you equated oxides to ceramic many times during your story, drawing conclusions about ceramic versus cermet, where oxide was the only constituent of your ceramic studies. I would submit he would find higher performance in ceramic fuels giving quite a different picture here as compared to oxides.

MR. COHN: By ceramics, I mean mixed oxides. We are only talking about mixed oxides for the analysis.

MR. WRIGHT: Well, I don't think it should be limited to that, obviously, which is why I mentioned it. A second point which strikes me as interesting: As long as we are dealing with test reactors running only $60 \%$ of the time, and then down for refueling, it would seem to be of interest to look at the ceramic, even your oxide fuel requirements downtime for refueling, interval, and time versus the cermet.

MR. COHN: These are going to be examined in the next talk.

MR. WRIGHT: Going back to your capital cost. Your talk has been based primarily on the volume of fuels available at certain maximum numbers. Of course, as everybody in the room recognizes, the test volume is a key problem. (the specific volume and the specific flux); but how much flux is in a specific volume at a given period of time? Now again remembering the ground rules as we said earlier in the day about the availability of these things, if you found a principal difference was in availability for the total cycle time as compared to refueling time, I would suggest that the ceramic, in this case the oxide, would have an improvement of something like $20 \%$ of the available downtime, which would be available for test operations, and if so, your maximum flux would be weighted by this number. If you do that, your difference is going to cost a dollar a day, and your case falls apart. effects.

MR. COHN: In a piecemeal fashion we are looking at the individual

MR. WRIGHT: I am saying your capital cost differences are exaggerated by the factor of two or three because the time of availability is not included.

MR. COHN: But availability doesn't enter into the capital cost per se, but it will be included in a figure-of-merit evaluation.

MR. WRIGHT: Of course. 


\section{.}

. 
5.1

FFTR FUEL CYCLE COST ANALYSES

R. E. Peterson

Pacific Northwest Laboratory

Richland, Washington

Operated by Battelle Memorial Institute

INTRODUCTION

In addition to safety considerations, test capability, and capital costs, the selection of a driver fuel should also be based on an evaluation of fuel cycle costs. Consistent with the present conceptual objectives to provide a facility of maximum versatility, fuel cycle analyses are not being limited to any single fuel type. Rather, a variety of potential fuels are being investigated in some depth. In this way, not only can present cycle costs be contrasted, but incentives for future development can be identified and a more versatile reactor design will evolve.

Presently, cermet and mixed oxide fuels have been investigated to about the same extent. Efforts are now being made to evaluate metaliic a1loys. Eventually, data will be developed for most conceivable fuel types. The value of early definition of cycle costs is, perhaps, further emphasized by the fact that the product of the reactor is fast neutrons. Since there is no off-setting revenue from the large amount of heat energy produced, potential customers will exert strong pressures to reduce operating costs. Related parametric studies are also attempting to identify an optimum reactor design from the standpoint of the customer (i.e., provide a facility of maximum value to the customer at the lowest cost).

\section{CONSIDERATIONS AFFECTING COSTS}

The evaluation of driver fuels cycle costs is substantially clarified by considering the parameters that affect fuel design. These will be discussed separately to illustrate the basis for the analytical model used in these studies.

Fuel Pin Diameter

Diameter of the fuel pin is a factor in fuel fabrication cost because the difficulty of fabrication increases with decreasing diameter. If the core fuel density is maintained, the smaller pin diameter increases the number of pins and tends to increase the cladding fraction. On the other hand, the smaller diameter permits a lower operating temperature. Thus, the tendency for fuel with low conductivity is towards smaller pin diameters when high flux is a requirement. 
5.2

Fue1 Temperature

The fuel temperature is a limitation from the standpoint of attainable exposure as well as reactor safety. Fuel temperatures above given limits (depending on the fuel) tend to reduce fuel life drastically; hence, increase fuel throughput. Thus, an increase in specific power may be traded off against fuel life which is an important consideration in attaining high neutron flux.

Fuel Exposure

Fuel and clad damage in the driver fuel can be a more serious 1 imitation than reactivity 1 ifetime, and is a situation common also to fast breeder reactors. Fission gas pressures and other damage mechanisms become significant at high burnups. Fue 1 throughput again is affected adversely where exposure is limiting.

Fuel subassemb1y

The size of the fuel subassembly and the pins per subassembly are factors in the fabrication costs, increasing with finer core subdivision. The number of subassemblies and the pins per subassembly are constrained to discrete quantities in the practical case to fit geometrically regular patterms. This has the effect of quantifying fuel cycle costs.

Fue1 Length

Fuel length, important in a test reactor to accommodate a wide range of test fuel length within the active zone, affects the coolant pressure drop across the core. Aside from pumping costs, if the pressure drop is fixed by an upper 1 imit on pump head, or mechanical constraint, then the fuel pin diameter must decrease to allow a larger hydraulic diameter to counteract an increase in height.

Cycle Costs

of course, driver fuel characteristics that influence reprocessing, handling during fabrication, and conversion costs must be accounted for in any fuel cycle comparisons. Materials that do not dissolve readily or require special head end treatment and remote handing are examples.

\section{ANALYTICAL MODEL}

Identification of minimum fuel cycle costs requires the investigation of a large number of reactor designs, each differing in one or more fundamental parameters. Since this is such a morumental task, one usually limits the scope of any parametric survey to a reasonably narrow range. Battelle-Northwest studies have been carried out while attempting to limit constraints to those that are physically realistic and principally dictated by either economic or safety considerations. This task has been considerably enhanced by an analytical model developed by R. J. Hennig, D. T. Aase and R. D. Benham of Battel] e-Northwest. 


$$
5.3
$$

The model that performs a complete reactor core design is constructed from the functional relations existing between the design variables. Calculations are carried out by using both analog and digital computer techniques. Instantaneous and simultaneous observation of major design variables and economic factors are achievable as a selected parameter is varied on the analog computer. Increased calculational precision is afforded by using digital equipment to investigate specific points.

The calculational flow diagram portraying the model used for the cermet design is shown in slide 1. From a set of eight major parameters (H/D, core pressure drop, core volume, outlet temperature, number of subassemblies, number of pins/ subassembly, $\Delta \mathrm{T}$ and neutron flux), the core, fuel, and subassembly dimensions are calculated. The large number of interconnections in the flow diagram indicate the high degree of interdependence between the design variables. A feedback control technique is used to iterate on a design variable automatically. For example, the fuel pin diameter is calculated by feeding back the core pressure drop to match that specified. Several features are worth noting:

- The critical mass is determined from a functional relationship between it and the core volume, previously determined by core physics calculations. This avoids the problem of performing these complex calculations within the model.

- This approximate treatment does not represent a serious compromise because the critical mass does not vary strongly with height/diameter ratio in the range we are considering.

SLIDE 1.

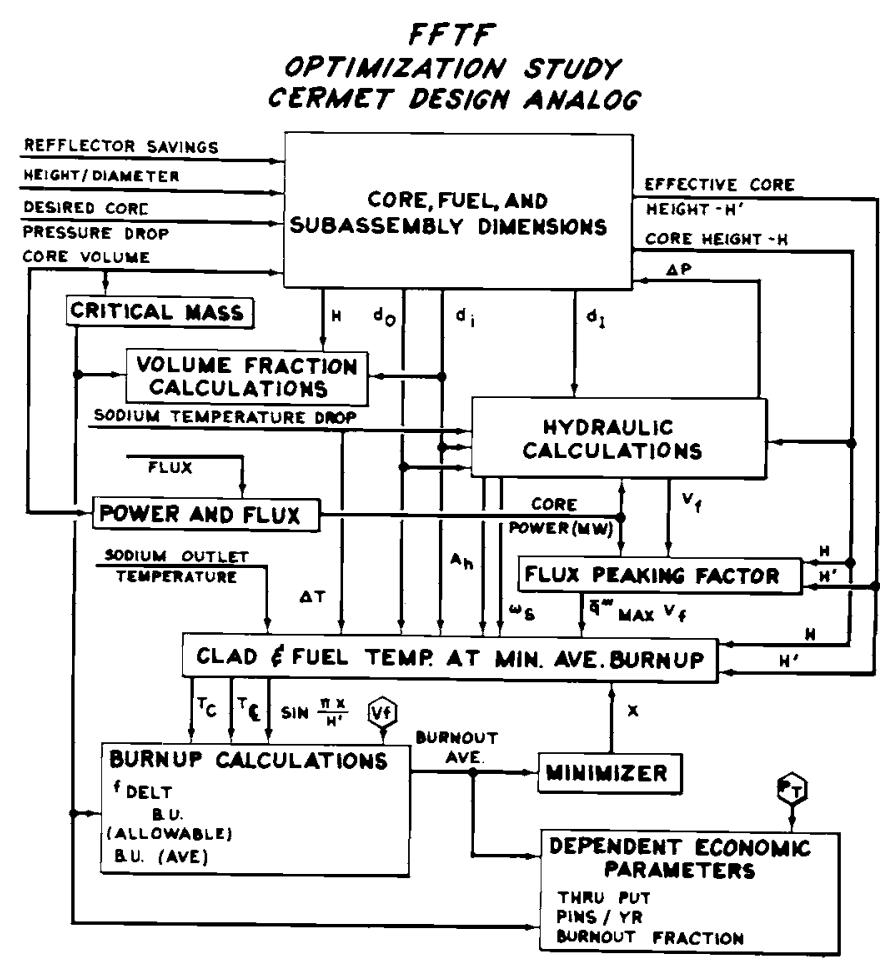




$$
5.4
$$

- As indicated, feedback techniques are employed to iterate on pin diameter and on reactor power to achieve a set pressure drop and a set neutron flux, respectively. The allowable burnup is calculated from an empirically determined correlation between centerline temperature and attainable exposure. (Discussed later in greater detail.)

The model utilized for the oxide calculation shown in Slide 2 is essentially similar with respect to the core, fuel, and subassembly dimensional calculations. Pressure drop calculations take account of added length to accommodate a fission gas reservoir and a different fuel subassembly spacer design. A significant difference is the specification of the maximum fuel centerline temperature as opposed to the cermet case where this is a calculated quantity limited by attainable burnup. The maximum centerline temperature is set at the melting point of the oxide, with an overpower factor incorporated to assure steady-state operation at some centerline temperature below this point at full power. The average burnup is specified independently as well; and, of course, all factors that tend to reduce this quantity, including high temperature operation, must be taken into account.

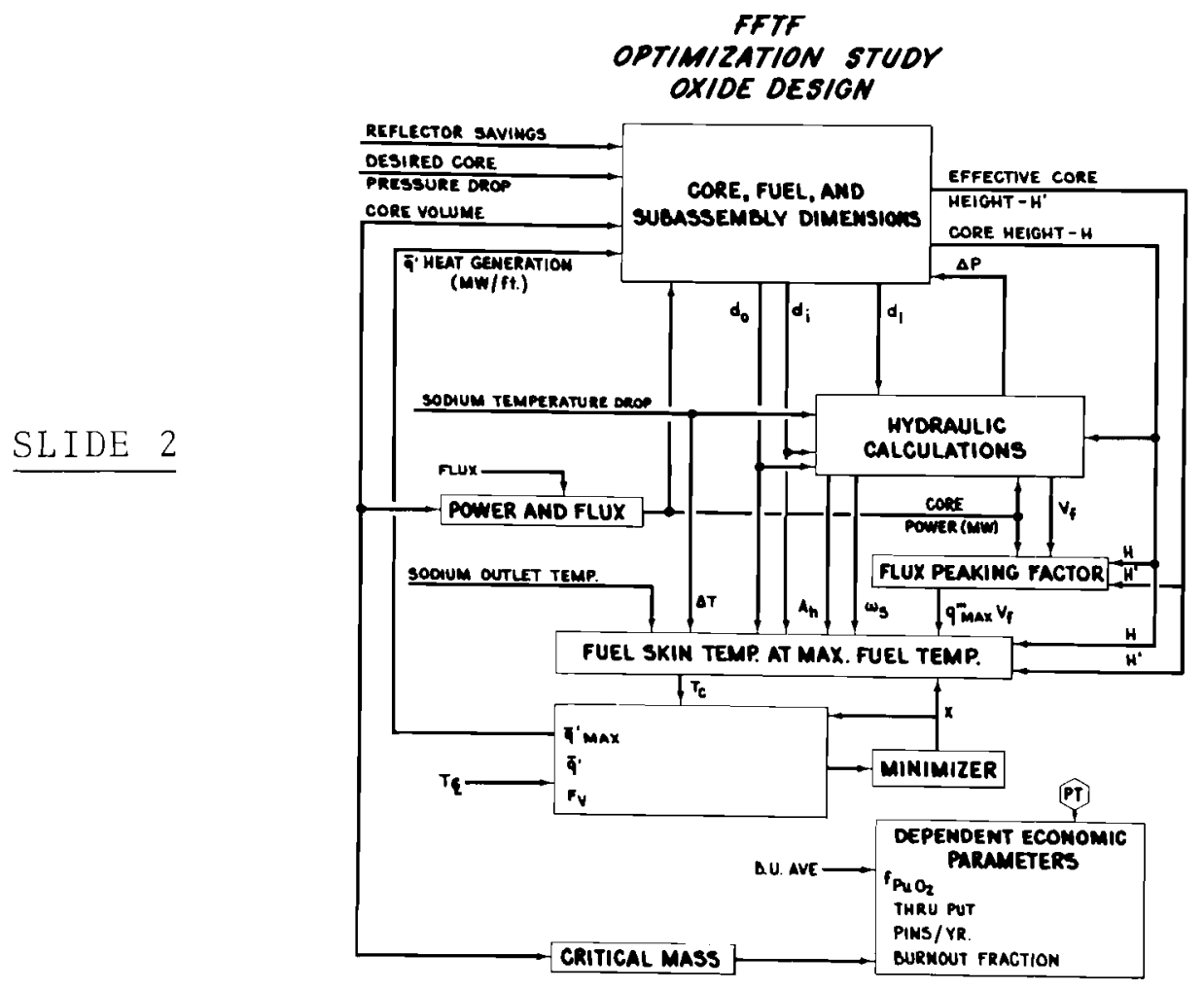

Economic parameters are calculated as shown in slide 3 . The total fuel cycle cost consists of the sum of conversion, fabrication, prereactor shipping, postreactor shipping, scrap recovery, reprocessing 
and burnup and loss costs. The calculations are for both fuel types with differences in the factors employed to reflect the different cost penalties. The value ascribed to plutonium is $\$ 10 / g$ fissile in the present studies.

\section{FFTF OPTIMIZATION STUDY ECONOMIC ANALYSIS}

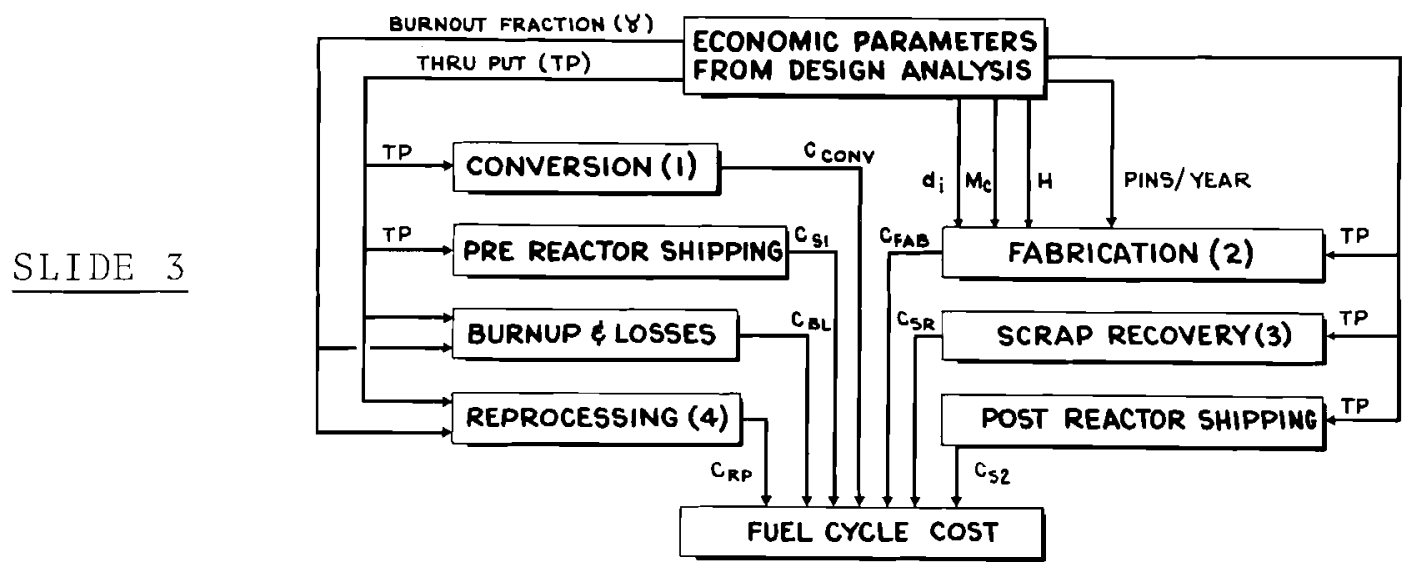

An example of the computer output is shown on Slide 4 . It is a particularly mixed oxide case and is typical of calculations directly off the analog. The large number of variables that one is able to observe at one time is quite impressive. In this example, the number of pins per subassembly is varied independently, and the resulting design and fuel cycle costs are shown in the

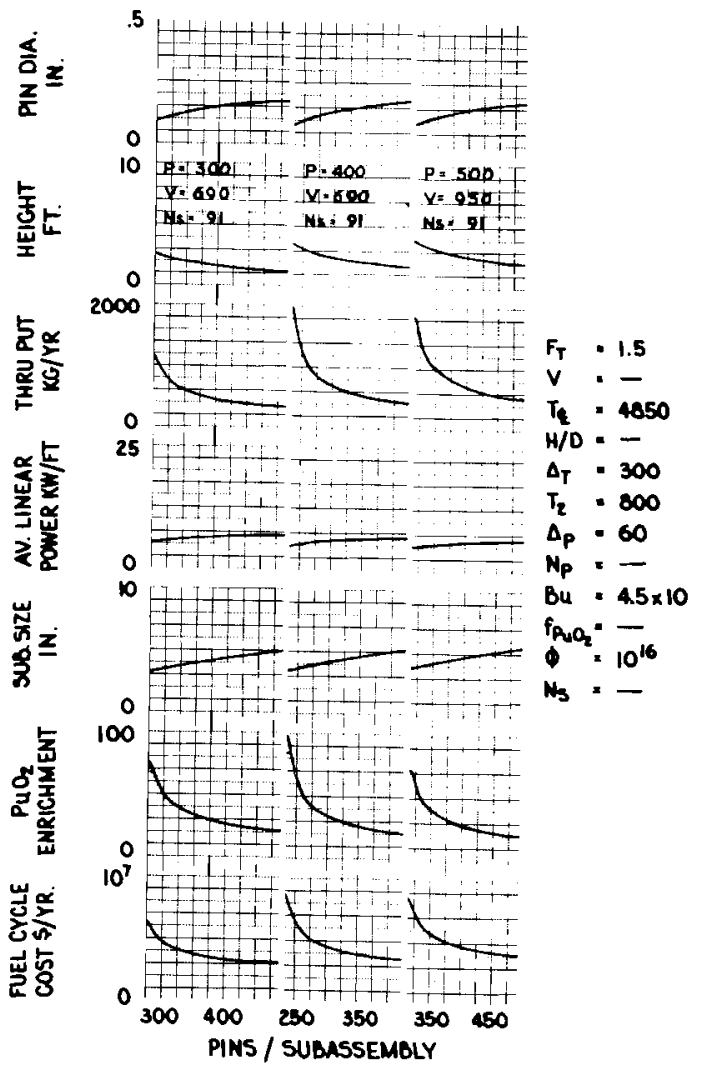




\section{6}

seven output channels. Additional output channels may be employed if desired for other design parameters. The great potential of this model to the reactor designer for initial surveys is evident, since the effect of variation of any of the design parameters on cycle costs or on any other parameters in the design may be observed instantly.

\section{OXIDE FUEL RESULTS}

We will now take up some of the results of these analyses, first as related to $\mathrm{PuO}_{2}-\mathrm{UO}_{2}$ oxide fuels.

Because of the low thermal conductivity and slump potential of the oxide fuel type, the parametric calculations are constrained by a safety consideration; that is, the centerline fuel temperature is not permitted to reach the melting point within a factor of safety referred to as an overpower factor. In other words, the reactor power, if increased by the overpower factor, would just raise the centerline temperature to melting. It is important that this factor be established and that it not be overly conservative so as to bias the fuel cycle costs on the high side. However, the very nature of the FFTR may require more restrictive fuel criteria than a conventional oxide fast breeder. We will not attempt a recommended value at this time, but we have investigated a range from 1.0 to 1.5 .

The calculation of fuel temperatures, of course, depends strongly on the thermal conductivity data employed. The data employed in these calculations for oxide fuel are shown on slide 5. It represents data which has been utilized locally within Battelle-Northwest for oxide fuel irradiation in PRTR analyses, and is characteristic of vibrationally compacted fuel material. The $/ \mathrm{k}-\mathrm{dt}$ to the melting point is near $75 \mathrm{~W} / \mathrm{cm}$. Account is taken of in-core sintering by reducing the radial conduction path and by the discontinuity in the thermal conductivity curve showing the transition from 85 to $100 \%$ theoretical density. That uncertainties exist in the conductivity data is well known and, perhaps, there will be further discussion on this point during the meeting.

As in the cermet case, a further consideration is the attainable exposure achieved with oxide fuel. Presumably, one can trade off overpower factor against burnup since the lower fission gas pressure and clad damage at lower exposures may permit containment of the fuel under higher temperature conditions. We have not attempted to utilize a correlation between the two for oxide fuels, however.

Next, let us consider pin diameter. As previously indicated, the penalty for going to small pin diameters to decrease the centerline temperature is an increase in fabrication costs. On the other hand, with increasing pin diameter, cycle costs tend to a constant minimum because the attainable exposure is considered to be independent. However, a decrease in core height results because an increase in the number of pins per subassembly accompanies the increased pin diameter. This happens because the linear heat rate is also maintained for a given centerline temperature so the pin 


\section{7}

length decreases. These considerations are illustrated on the next siide.

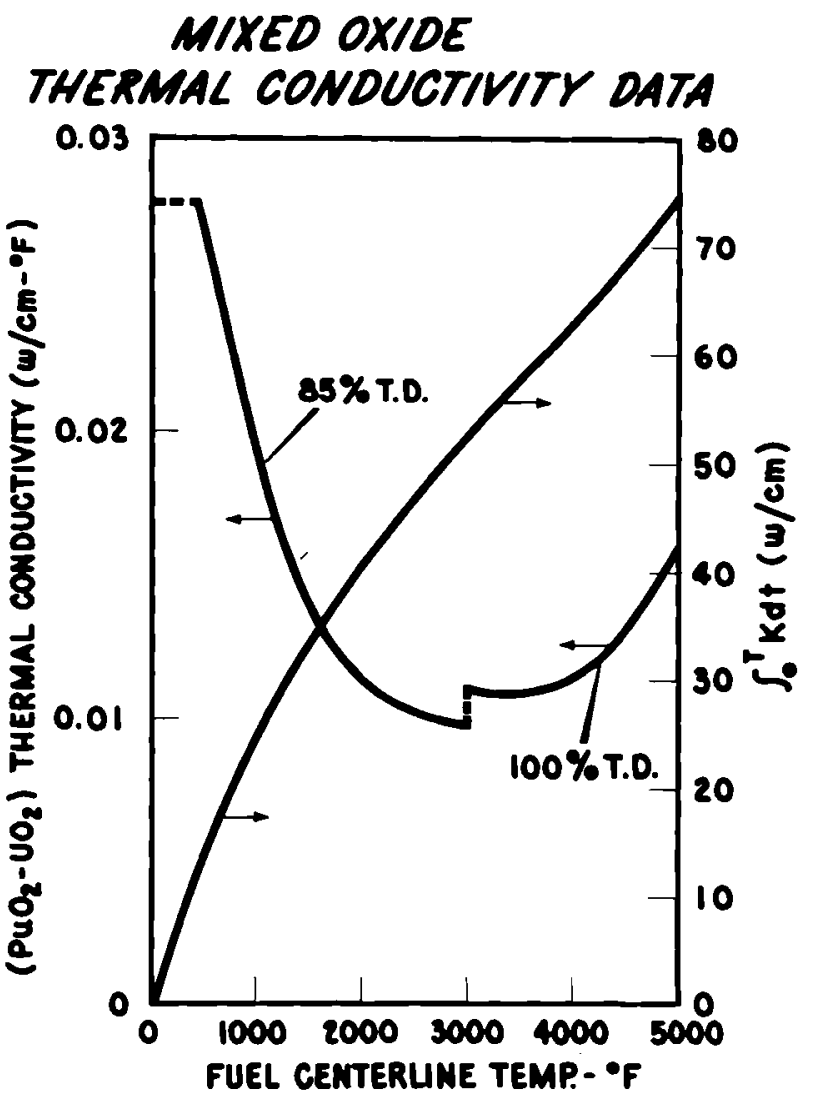

On Slide 6 , the fuel cycle cost results as a function of fuel pin diameter for an oxide fueled core of 690 liters, operating at $400 \mathrm{MW}$, yielding a peak flux of $1016 \mathrm{n} / \mathrm{cm}^{2}-\mathrm{sec}$. Two cases are shown here: one for an overpower factor of 1.0 , and the other for 1.5. Also two values of exposure are shown: $100,000 \mathrm{MWd} /$ ton and 45,000 MWd/ton. The number of subassemblies has been held constant at 91 , but the height varies from $1.3 \mathrm{ft}$ at a pin diameter of 0.26 in. to nearly 3 ft at a diameter of $0.12 \mathrm{in.}$. Again, the rise in fuel cycle costs at the smaller pin diameter is caused by increased fabrication costs. In fact, the difficulty in fabricating an oxide pin of the order of 0.1 in. diam may well result in a cost penalty even higher than that shown.

The differences in costs due to changing the required exposure from 100,000 to $45,000 \mathrm{MWd} /$ ton are perhaps most significant. Although it appears that the penalty for increasing the overpower factor is not large, it must be pointed out that the H/D ratio for the dotted curve and the solid curve at the same pin diameter is not the same. Therefore, a tradeoff in core height is incurred that affects core test requirements and, perhaps, control requirements, although fuel cycle costs remain nearly the same. 
5.8

The cycle costs decrease in the direction of increasing fuel pin size. Unfortunately, the penalty in core height becomes rather severe at these larger pin diameters. For a conservative oxide design, it would appear that cycle costs are in the range of $\$ 3$ to $\$ 4 \times 10 \% / y r$ for a core of this size with an II/D near unity.

\section{FFTF \\ TOTAL FUEL CYCLE COSTS, \$106 v. FUEL PW OIAMETER, INCHES}

\section{SLIDE 6}

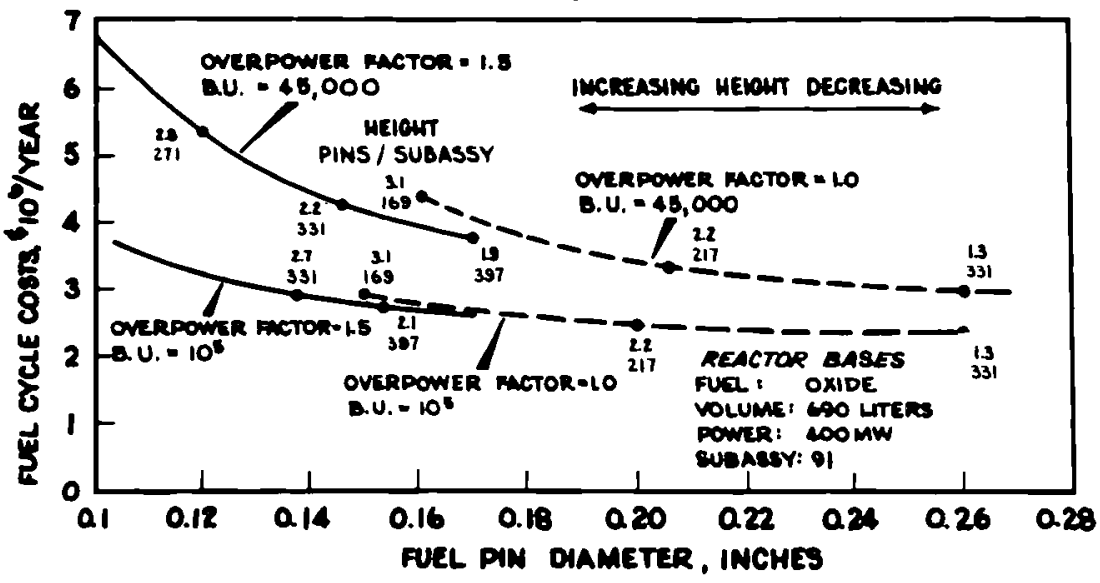

Perhaps, the effect of overpower factor on cycle costs is better illustrated by considering reactors of different size. An increase in the overpower factor at fixed burnup and reactor size causes a decrease in pin size and increased pin throughput; hence, increased fabrication cost. In the smaller reactor sizes, the pin diameter is already comparatively small, so that the cycle costs climb much more rapidly with overpower factor. These variations are illustrated on Slide 7 and show cycle costs for a range in overpower factor of from 1.0 to 1.5 and reactors with power levels of 300,400 , and 500 Whit.

FTR OXIDE FUEL CYCLE COST V.S. OVERPOWER FACTOR

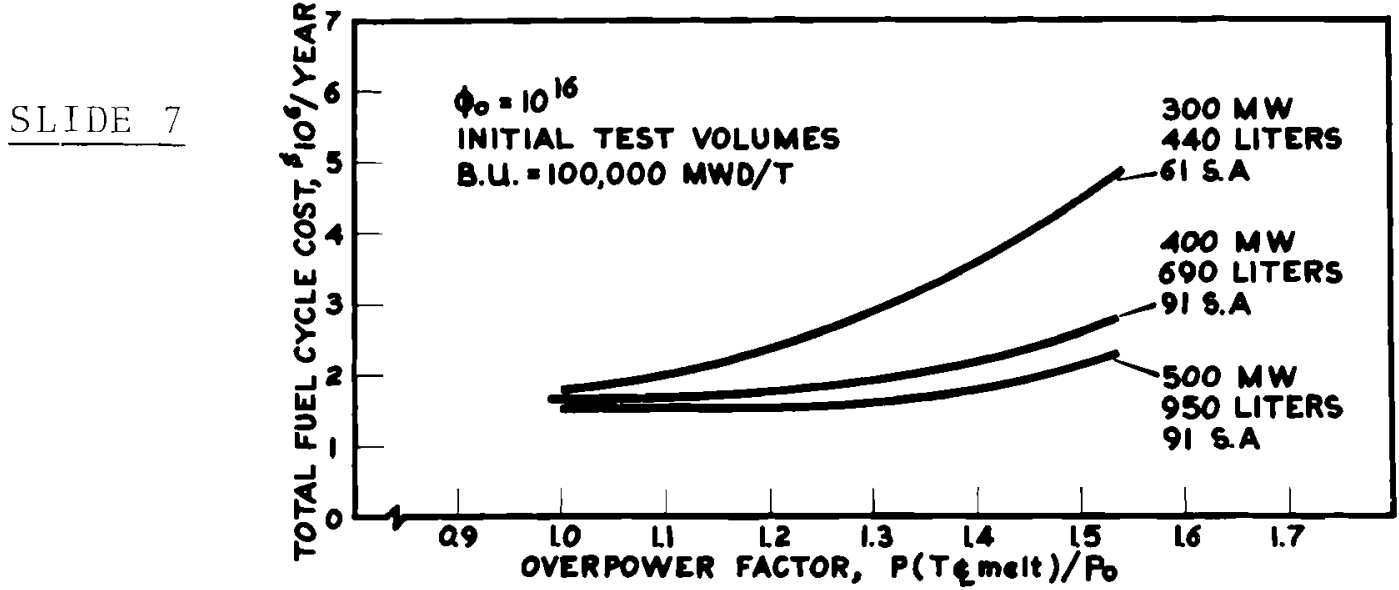


5.9

For the small cores, the increase in cycle costs are of the order of a factor of two. Over this range, the cost penalty for the larger cores is more on the order of $30 \%$. Thus, the importance of setting fuel safety criteria realistically is important to minimizing operating costs, and this is more important in small cores than in large.

\section{CERMET RESULTS}

In evaluating the cermet core, the irradiation stability of the fuel is considered as shown in Slide 8. The relationship between attainable exposure and surface temperature of the cermet is shown. The centerline temperature is of the order of $100^{\circ} \mathrm{F}$ higher than this. The tradeoff between fuel temperature and fuel exposure is seen to be very significant above $800^{\circ} \mathrm{F}$. The solid line is the correlation used in the calculations. The concept of overpower factor does not strictly apply in the cermet as it did in the oxide because attainable exposure limits the operating temperature far under the melting point. Again, these data are subject to uncertainty. Perhaps more discussion will be generated during the meeting regarding considerations such as this.

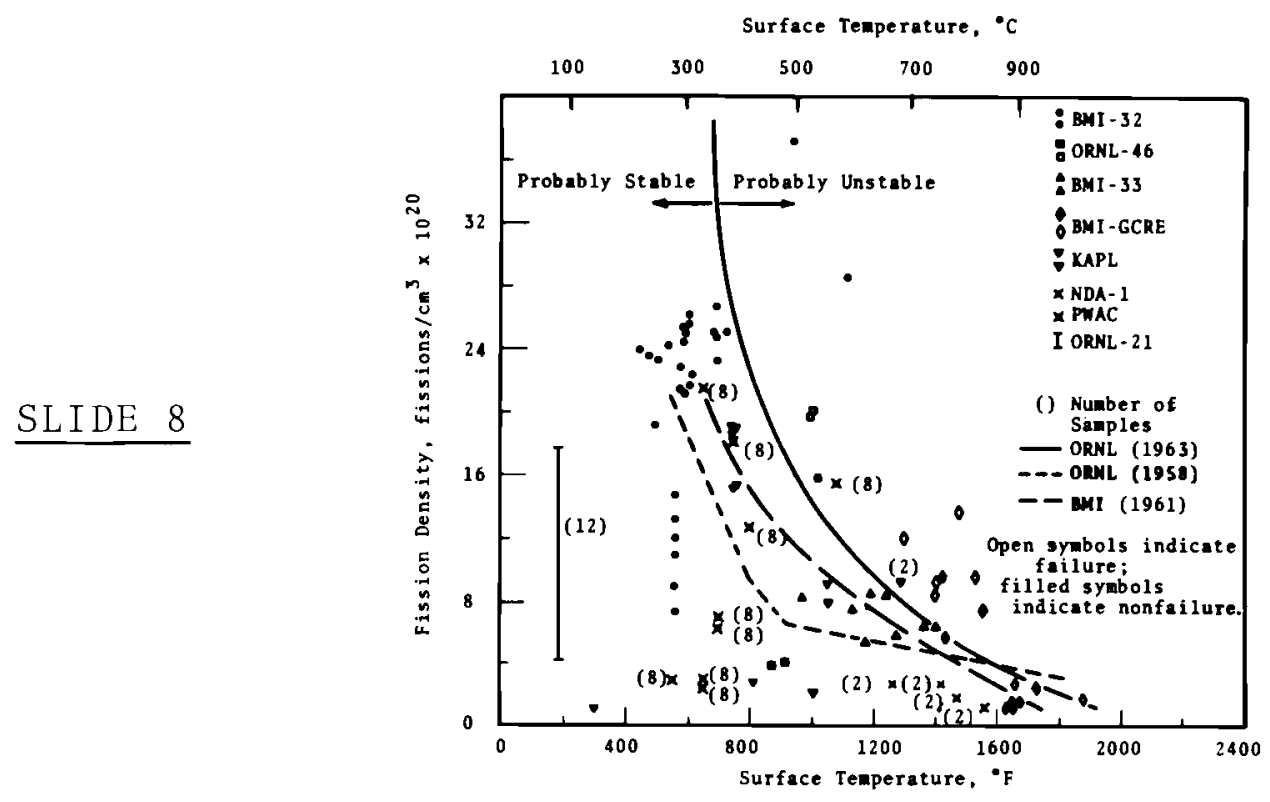

Irradiation Stability of $\mathrm{UO}_{2}-\mathrm{SS}$ Dispersions

The effect of pin diameter in the case of cermet fuel is the same as that of oxide fuel for small pin diameters; that is, the fabrication costs begin to dominate. However, in this case, since the fuel temperature is tied directly to the attainable exposure, the penalty for increased throughput at higher temperatures dominates the larger pin diameters. The net result is a minimum in cycle costs as a function of pin diameter.

In Slide 9 we have shown the total fuel cycle costs as a function of fuel pin diameter for a core height of $2.8 \mathrm{ft}, 61-$ subassembly core, operating at $300 \mathrm{MW}_{t}$ to yield a peak flux of 


\subsection{0}

$10^{16} \mathrm{n} / \mathrm{cm}^{2}-\mathrm{sec}$. In this case, the minimum fuel cycle costs of about $\$ 2.8 \times 106 / \mathrm{yr}$ occur for a pin diameter of 0.16 to 0.20 in. diam, and 271 to 397 pins per subassembly. It may be noted that the number of pins per subassembly increases discretely as the pin diameter falls. As the pin diameter decreases below 0.16 pin, the fabrication costs begin to rise. However, as the pin size increases above 0.18 in., the fuel centerline temperature rises, decreasing the attainable exposure as shown along the right-hand scale. The increased throughput then results in a rising fuel cycle cost. It is interesting to note the relatively high dependence of burnup (and centerline temperature) on pin diameter.

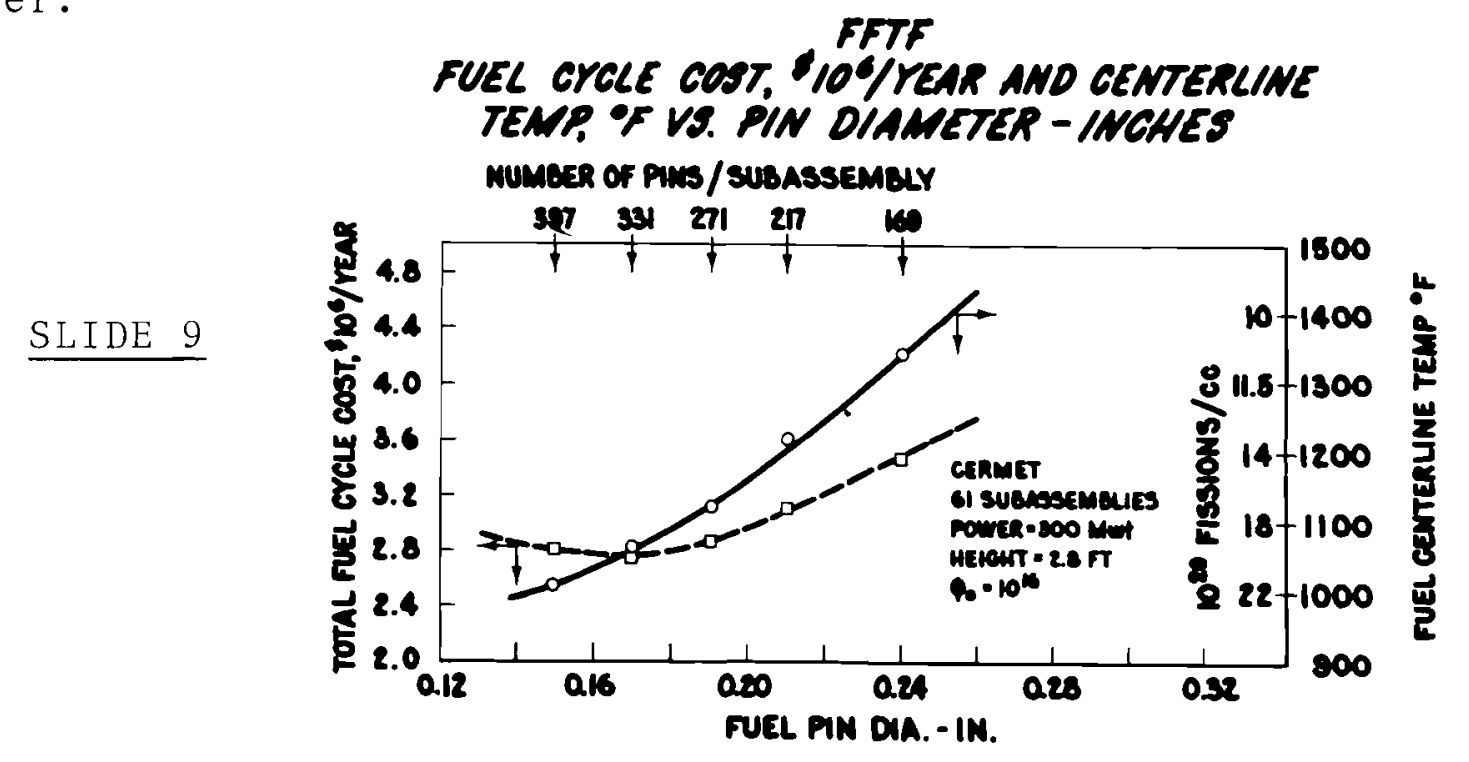

S1ide 10 shows the same relationships but with an increase in height to $3.1 \mathrm{ft}$ and an increase in power to $370 \mathrm{MW}$. The minimum cycle costs are relatively unchanged, but the minimum occurs at a larger pin diameter. Thus, the dependence of cycle cost on reactor power does not appear to be a strong function of power level. This is demonstrated in Slide 11 where the fue 1 cycle costs as a function of pin diameter have been summarized for a range of reactor power levels from 150 to $600 \mathrm{MW}_{t}$. A plot of the locus of minima is shown to result in a rather broad minimum from about 250 to $350 \mathrm{MWt}$. The $\mathrm{H} / \mathrm{D}$ is constant at 0.9 , and the peak neutron $f 1 u x$ is held at $1016 \mathrm{n} / \mathrm{cm}^{2}-\mathrm{sec}$. The minimum fuel cycle costs are seen to be near $\$ 2.7 \times 10^{6} / \mathrm{yr}$. It may be further noted that the core subdivision varies discretely with reactor power. 

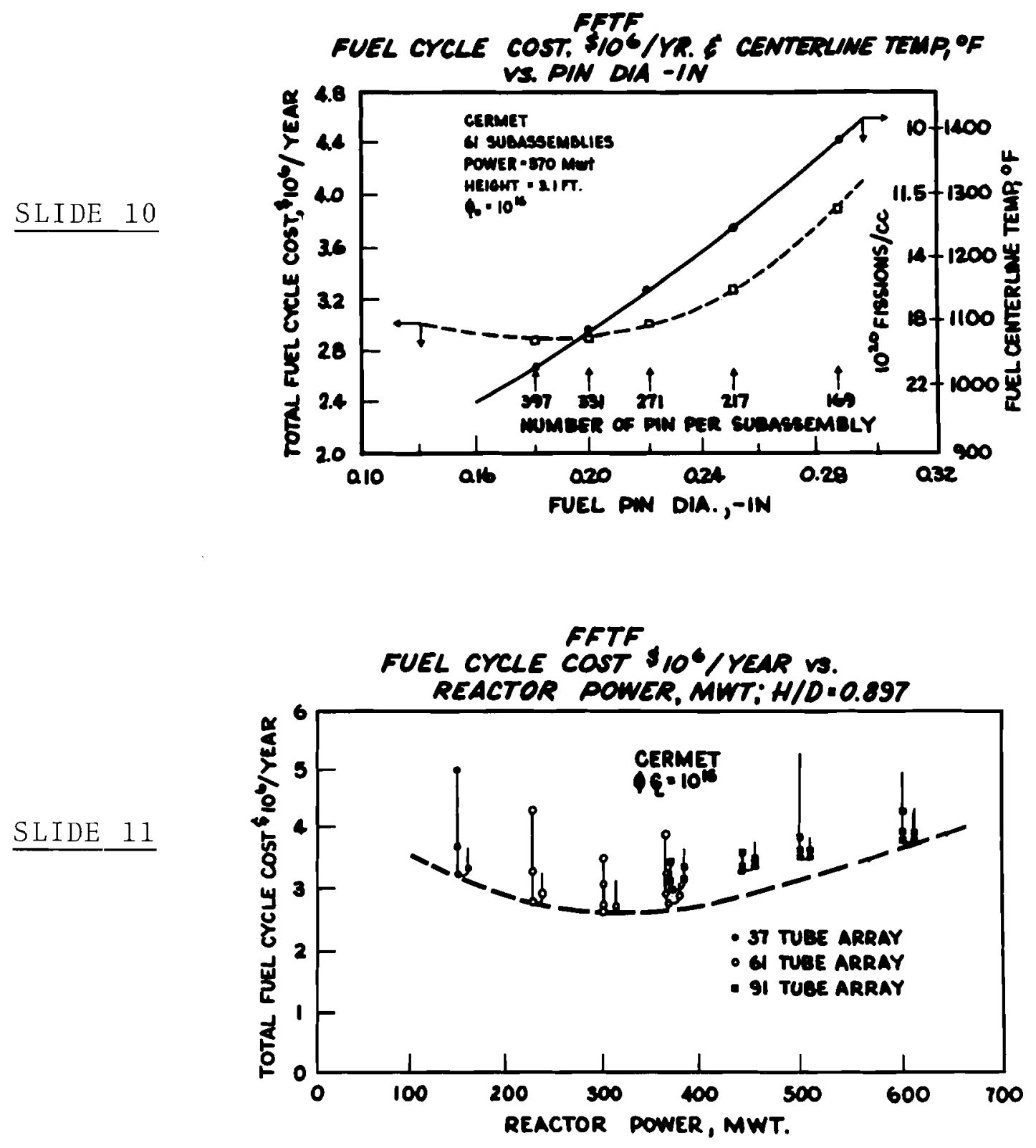

COMPARATIVE RESULTS

Similar data have been developed for the oxide reactor designs, and it is of interest to compare cycle costs for the two fuel types. Results of the present slide are compared with the locus of minimum fuel cycle costs for the oxide in Slide 12 . The oxide design shown is one of conservative rating. That is, an attainable exposure of 45,000 $\mathrm{MWd} /$ ton and an overpower factor of 1.5 has been assumed rather arbitrarily. In all cases, the peak neutron flux is held constant at $1016 \mathrm{n} / \mathrm{cm}^{2}-\mathrm{sec}$. The minimum fuel cycle costs are roughly a $\$ 1$ million/yr higher for 
5.12

the oxide, being near $\$ 3.6 \times 10^{6} / \mathrm{yr}$ as opposed to $\$ 2.6 \times 10^{6}$ for the cermet. These minima appear to fall in the 300 to $400 \mathrm{MW}_{t}$ power range and are about the same for both the oxide and cermet cases.

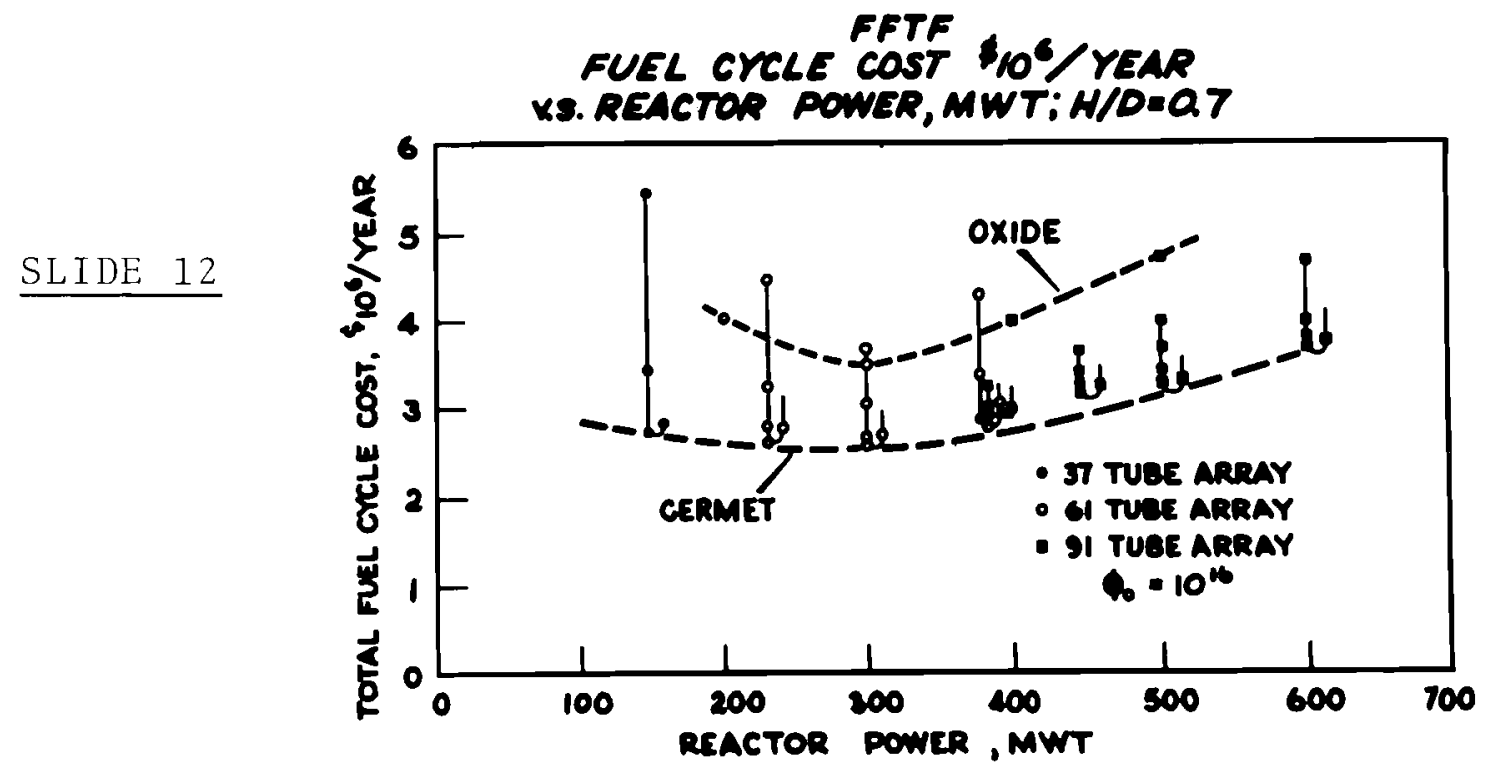

Finally, the stretch capabilities of the two fuels is of interest. The fuels from the present slide have been reevaluated with proposed performance improvements to allow higher exposures and operating temperatures. These are shown in Slide 13.

FFTF

FUEL CVCLE COST VE REACTOR POWER

$\underline{\text { SLIDE } 13}$

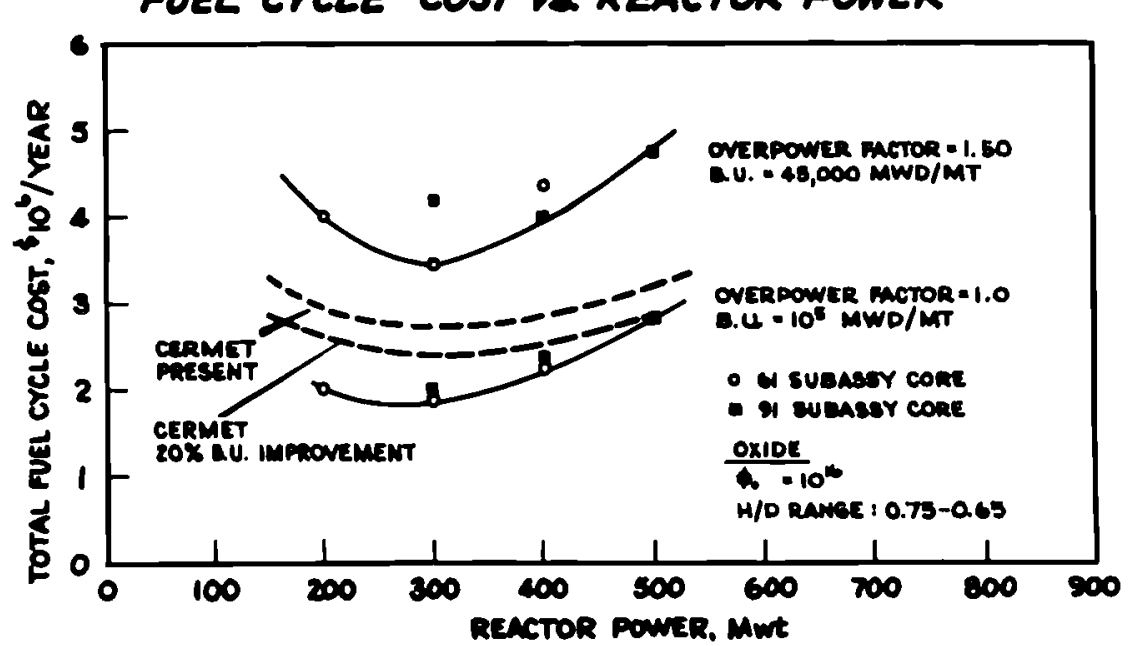

The improvement in the oxide case is assumed to permit an attainable exposure of $100,000 \mathrm{MWd} / \mathrm{ton}$ with the centerline fue 1 temperature just approaching melting. The improvement in stainless steel cermet capability, on the other hand, is assumed to increase the burnup by $20 \%$ for a given fuel temperature. The 


\subsection{3}

smaller improvement for the cermet is due to the selection of less conservative initial conditions. Although the selection of these particular increases in performance is again somewhat arbitrary, it seems reasonable that the cycle costs of cermet and oxide fuels are ultimately quite close and in the range of $\$ 2 \times 10^{6} / \mathrm{yr}$.

The data shown previously have been represented at only one neutron flux, namely $1016 \mathrm{n} / \mathrm{cm}^{2}-\mathrm{sec}$, to avoid showing too many parameters at one time. We will now consider the sensitivity of the cycle cost to neutron flux. The comparison is shown in Slide 14 where conservative and advanced fuel conditions are again illustrated for a 600 liter core. Since the increase in flux is brought about by increasing specific power, the penalties associated with increased fuel temperature are responsible for the rising cost. It appears that on the order of a $\$ 1 \mathrm{million} / \mathrm{yr}$ is required to increase the flux by $20 \%$. It may well be, however, that the value of an increase of this magnitude is greater than the increased cost. Not shown here, is the fact that the oxide core has some $30 \%$ less test volume. If a weighting of test volume were applied, the results would appear to favor the cermet.

SLIDE 14

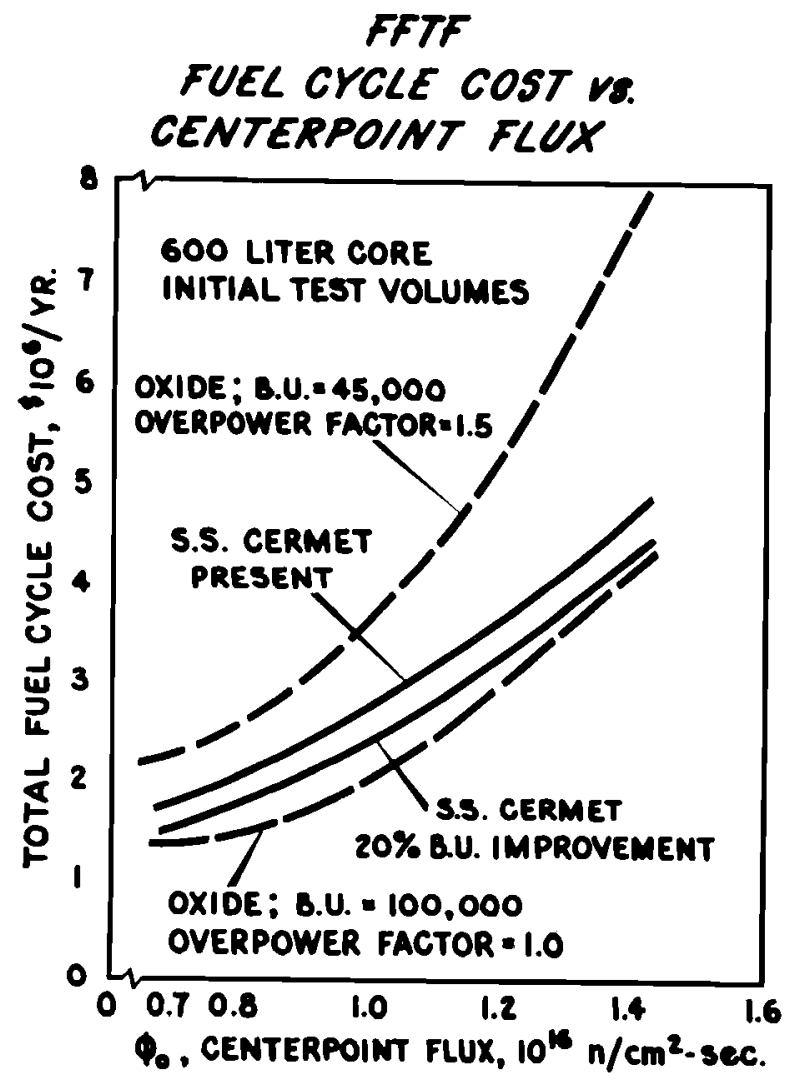




\subsection{4}

\section{CONCLUSIONS}

A number of conclusions are indicated from the foregoing ana1ysis:

1. On a conservative basis, cermet fuel cycle costs appear to be less than those of oxide for a neutron flux close to $10^{16} \mathrm{n} / \mathrm{cm}^{2}-\mathrm{sec}$.

2. The ultimate cycle costs of both fuels appear reasonably close. The stretch capability of each fuel type and the fuel safety criteria become extremely important in this regard.

3. From a standpoint of fuel cycle costs alone, the optimum cores for both oxides and cermet driver fuels are in a range of 300 to $400 \mathrm{MW}_{\mathrm{t}}$.

4. Minimum cycle costs at a neutron flux of $10^{16} \mathrm{n} / \mathrm{sec}$ for reasonable $\mathrm{H} / \mathrm{D}$ ratios $(>0.7)$ are about $\$ 2.5 \times 10^{6} / \mathrm{yr}$ and up to $\$ 3.5 \times 10^{6} / y r$ for initial cermet and oxide, respectively. Both fuels appear capable of costs near $\$ 2 \times 10^{6 / y r}$ in the ultimate.

5. Increasing flux by $20 \%$ near $1016 \mathrm{n} / \mathrm{cm}^{2}-\mathrm{sec}$ represents approximately a factor of 2 increase in fuel cycle costs.

Finally, it should be emphasized that constraints due to achievable test volume, reactor safety, and capital costs for each fuel system must be given weight in addition to the cycle costs to properly evaluate the driver fuel in total. 
D5。1

FUEL CYCLE COST PARAMETER

$R$. E。 Peterson

\section{DISCUSSION}

MR. deHALAS: I am quite confused on one important point here. Looking at Hofmann's slides, I got the feeling the cermet was reactively limited in burnup, and I am not sure about the oxide. I didn't eyeball that one. The curves we are using here and essentially burnup, limited on a material basis. How was this compatible?

MR. PETERSON: Well, this was taken into account on the critical mass calculations where an allowance was made for access reactivity to accommodate the burnup.

MR. deHALAS: The point is this. Even if the cermet did take the burnup you would get from the reactivity consideration, which looks maybe a reasonable number, it looks like you have very limited possibilities for fuel shuffling because of the degraded core. Now, just as an example, suppose that the curve which you showed; burnup versus temperature, gave twice the exposure that Hofmann says you are allowed, how do you account for this in your fuel cycle cost?

MR. PETERSON: The critical mass has a constant allowance in it already above a ${ }^{1 "} \mathrm{k}$ " of one.

MR. deHALAS: It is probably a simple answer such as that.

MR. HENNIG: Any fuel burned up in any reactor would experience a reactive change. If you make the initial assumption you are going to use partial refueling, then the question of reactivity change per unit type at full power comes on as interest only in terms of how much control rod you want to put in to handle it.

MR. HECK: Not if it means you have to shut down every week-end, even for a week.

MR. HENNIG: We didn't say we were necessarily going to limit ourselves to less than one dollar in control rods worth for burnup. In fact, we never suggested anything even near that. Taking any other number at this time would seem to be very premature. We can just say that the longer you burn it, the more reactivity change takes place. Built into the basic analysis is a technique, as you burn off more fuel, burn off $20 \%$, you have to put that in to begin with. We have correction factors for penalizing the cermet burnup as a function of the amount of ceramic phase in the cermet. This is in fact a feedback that is used in the work done. The higher the burnup, the higher the initial ceramic load, which means the greater penalty. It is a negative feedback. 
MR. PUECHL: Have you looked at all the higher 240 content fuel? Since on your economics you don't pay for $240--$

MR. PETERSON: This is not taken account of.

MR. PUECHL: And aiso because of the conversion, it should be sort of a compromise between a reactivity--

MR. PETERSON: Yes, the conversion to 240 does not take account of it at the moment. It is a small fraction, however.

MR. PUECHL: You have been losing $10 \%$.

MR. PETERSON: Yes.

MR. HENNIG: Peter, did you have a comment?

MR. HOFMANN: Most of our calculations we have been using $10 \%$. We have, however, also looked at fuels containing 20 to $25 \%$ of 240 , clearly, from the point of view of what the critical inventory would be and what the flux level would be. We can certainly accommodate use of the higher 240 fuels, the increases in the critical mass for reasonable composition is not very large. The decreases in flux level are very minor.

MR. AASE (BNW): What was the basis of your fuel fabrication costs for the fuel system?

MR. PETERSON: These were supplied to us by our fuel people.

MR. deHALAS: Weli, the bases, we hope, were reasonable. We went to a brief analysis of a cermet scheme and an oxide scheme. I am sure you can get more details on these if you want it.

MR. PETERSON: We have all the detail on these calculations, if you care to see me after the talk.

MR. deWITT MOSS (AEC): When you had two graphs up there, one for the cermet core, and the other for the ceramic core, the figures I put down were on 4 million fuel cycle costs per year for the oxides, 2.7 million for the cermet. People who have worked with cermets tell me you couldn't have picked a more expensive fuel system to work with; yet your numbers say there is a $50 \%$ fuel cost difference.

MR. PETERSON: I don't think we are in a position to make any statement of that kind. I think it is quite evident. The point I was trying to illustrate here was the dependence of these costs on the parameters that you select for fuel performance.

MR. MOSS: I guess $\mathbb{I}$ am questioning the 62 or $63 \%$, whatever it is, increase fuel cycle cost of cermet versus the oxides.

MR. PETERSON: Welì, this is a function of the particular choice of parameters I chose here. Now take the overpower factor as an example. One could argue certainly whether or not to approach unity for this figure or 1.5. You saw the striking difference in obtainabie exposure in the oxide case. Obtainable exposure in the oxide certainly is a factor, too, in fuel cycle costs here. 
D5.3

MR. MOSS: I question that also because General Electric and Oak Ridge run fuel cycle costs, but G.E. says about 1.7 mills--this is on a mills per kilowatt hour difference between 60 and 100,000. Oak Ridge says 0.25 . It doesn't bear that $50 \%$ difference.

MR. HENNIG: You are arguing not with the pieces of paper we furnished you last spring showing you all the constants and all the formulas, you are arguing with the results which these formulas and constants produced. I suggest that you should start from this basis and build up.

MR. SHOUDY (ADPA): I would like to know your fabrication costs on a cermet, were they ever compared with what some of the Army costs were? It seems to me that, on just fuel costs alone, it turned out to be 12 mills or 14 mills per hour, which sounds like a big fraction of that two million dollars a year costs you are quoting. In a paper of this type I don't quite understand why there is not $50 \%$ of it dealing with the input that has gone into your fuel cycle costs, but I would like to have a feeling of your relative reprocessing costs of cermet versus oxide. I know the British were dealing for quite a long time with a cermet fuel, and they've had quite a difficult time in finding an acceptable reprocessing scheme. I think, implying at least to me, that the cermet fuel on the reprocessing is quite a bit more expensive.

MR. PETERSON: Well, part of the reason for this was the matter of time, trying to get across the essential results of our study without running into too much detail, but I have prepared a list of the constants that went into both calculations, which I will be glad to provide you with.

MR. deHALAS: To answer part of your question, the cermet calculation costs are indeed higher in our studies than the ceramic costs. I hope we can make them cheaper than the Army reactor cermet. I think this is a reasonable goal. This depended on pin size. One number that we came up with here, the cermet, was about $25 \%$ more expensive than the oxide.

MR. SHOUDY: Mr Cunningham might want to comment on that, but can you really make 200 pins cheaper than 14 plates, or whatever a comparable number might be, of oxide material?

MR. J.E. CUNNINGHAM (ORNL): All our studies and almost all analyses show that cermet is more expensive particularly on the fabrication costs. It soars way up in favor of the bulk ceramic.

MR, deBOISBLANC (Phillips): I think it should be recognized, for example, that the head-in process for chemical processing, using perhaps an electrolytic dissolution step is principally the main factor involved. But it doesn't really add a great deal.

MR。CUNNINGHAM: I want to come back and to the point, if the dispersion fuels are burnup limited, relative to oxides, and they cost more on the basis of existing technology today, what, in the analytical analys is that was made--how come they are cheaper? 
D5. 4

MR. PETERSON: The point I am trying to make here is that these are not absolute data, necessarily. They depend upon the performance assumed for these two fuel.s, and there are sweeping differences in fuel cycles costs made here by the differences in assumptions made.

MR. HENNIG: I have asked Wayne Hoskins to ship down here for use this afternoon the input data that were used both in the core design analys is section and the fuel section, cost analys is section; and I think th is is a fact of some significant interest, we can look at how we compute the fabrication costs.

MR.COHN: For the center point flux of $10^{16}$ and the 600 liter reactor, the pin fabrication cost of cermet is about $\$ 109$., and the oxide is about $\$ 75$., so it is cheaper. But the throughput for the oxide at an overpower factor of 1.5 and 45,000 burnup is higher because you can't burn it up. We are allowing a higher cost of burnup, so even though the cost per pin is lower, you are putting through more pins, and you are really processing more fuel and plutonium. burner.

MR. CARL ANDERSON: So the hook is, you catch the assumed spread.

MR. deHALAS: That is right. That is why there is this smaller in there?

MR. ANDERSON: There is a factor of four of uncertainty thrown

MR. deHALAS: No, solid uncertainty.

MR. COHN: No, it is not an uncertainty. We don't know what the achievable burnup is, so we are looking at 45,000 and 100,000 $\mathrm{MWd} /$ ton.

VOICE: And 100,000 was cheaper.

MR. HENNIG: Bennett?

MR.BENNETT: I think Carl answered it pretty well. You really have to go into all the parameters to determine why the difference in ceramic and oxide fuel. It is conceivable if you raise the sodium temperature high enough, you have a high enough flux on the oxide fuel of any reasonable diameter, it just isn't useable. The cermet has a much better conductivity. You can't keep the temperature down and therefore the burnups are better. So you can't look at any simple reactor, like one of these water reactors, and compare the cost of cermet fuel versus an oxide fuel under those conditions, because they are different, entirely different things.

MR. A。H。WILLIS (Atomic International): It seems to me that the cermet is like the oxide to build, if you assume $45 \%$ burnup of oxides, you would have to conclude you are running your dispersion up to 60 to $70 \%$ due to your correlations, is that correct?

MR. HENNIG: That is a very small amount of the cermet. 
D5. 5

MR. deBOISBLANC: We have been talking about ceramic for one thing, we also should remember that cermet is for one thing. This is for stainless steel cermet. There are other cermets, there are other pos sible materials which have a higher allowable centerline temperature. As I understand it, this temperature is limited, and if you would allow higher centerline temperatures you would have a gross change in the performance of the fuel. You could make some gross changes in the costs, too. So there may be other systems.

MR. PETERSON: These may be traded off on increased processing costs, too. If you go to nickel cermet or something like that.

MR. GORDON: The speaker on capital costs did us a favor by finally dividing the capital costs out by the test volume available and showed us what the cost per unit of test volume was. I wonder if a fuel cycle can be figured out on--an annual fuel cycle cost has been figured out?

MR. PETERSON: I hinted at that on the last slide on the cost of obtainable flux, because of the lower test volume capability of the oxide, or actually the lower flux capability of the oxide that it would probably tend to run the costs up something in the order of $20 \%$, if that factor were included, here, for the oxide case. The weighting depends on the oxide. 
. 
CERMET AND OXIDE FUELS REVIEW

D. R. deHalas

Pacific Northwest Laboratory

Richland, Washington

Operated by Battelle Memorial Institute

\section{INTRODUCTION}

I'm going to speak on the problem of driver fuel based on material considerations. Initially, this wasn't on the program; because it's fairly controversial. However we've been thinking about materials for some time, and it isn't really fair to indicate that we haven't any opinions, because we do.

There are many ways to approach a problem, and this creates the basis for much of the controversy on fuel selection. You can't really cast out any fuel that's been seriously proposed because you can probably make just about anything work if you work on it hard enough. So what we have to look at is our "druthers". For material selection this is the fuel we think will work best with the minimum amount of effort. I will, therefore, give our reasoning for why we favor a cermet from the materials standpoint now. This will give everybody a chance to take shots at our reasoning as they make presentations on their own fuel choices.

I think that some of you, who have been following our progress for a little while, realize that this is somewhat of a reversal of our initial position on fuels from a materials viewpoint. The design people showed that there was incentive for using a cermet, and we thought it would probably be easier to develop an oxide; but it wasn't really clear one way or another. We now support the cermet from a materials standpoint.

\section{FUEL TECHNOLOGY}

Now at the risk of making a bunch of people mad by indulging in oversimplifications, I'll go into some of our reasoning.

In terms of developed technology, I would rate the oxides first and cermets second; then carbides; then alloys; then sulfides, carbo-nitrides, liquid metals. This last group, sulfides, liquid metals, etc., is promising. I think there is no doubt about it, it is promising from the long term development standpoint. We felt, from our own choice of materials, 
6,2

that these required too much development to count on at this time as a driver fuel for the reactor. The carbides, on the other hand, for the FFTF conditions do not seem to be too far out. I wish to point out that we have not looked hard at the carbides from a design standpoint, nor have we looked at them hard from the material standpoint. I'll only indicate that one of the major questions in our minds is the unknown factor in the large scale fabrication of a mixed carbide fuel, and this is something that has yet to be overcome

The alloy fuels-we initially threw these out of our reasoning because of swelling and somewhat because of compatibility problems. The recent advances at Argonne make us look at the alloy fuels with a bit more interest, and I would only say that the alloy fuels seem very attractive because of ease of fabrication. If the Argonne progress continues, I think there is the chance that there might be a late switch made to an alloy fuel for the reactor. But that's from a materials viewpoint, and I'm not trying to reflect all the design considerations involved.

Getting down to oxides and cermets, which are the only two categories of things I left in the running, there is no doubt that most of the experience is on the oxides. On the other hand, the cermets have also been widely investigated in the US, and Britain. I think that more work has been done in the U.S. than in Britain, although a lot of people have the opposite idea.

The choice between oxides and cermets on a materials basis is not simple, and we do not feel that it can be made with any $100 \%$ certainty. This is one reason that, a11 along, we've been talking about a backup fuel as well as a primary fuel, and we still feel this way. We could choose oxide as a primary fuel with a cermet as a backup or vice versa. The amount of effort placed on a backup from an RED basis would simply be inversely proportional to the amount of success you had on the primary fuel.

\section{POROSITY}

Now I'll give some simplified reasoning-so simplified. indeed, that it is a little risky-for our choice of a cermet over an oxide. At the burnups and temperatures for this reactor, it is clear to us that you will have to introduce porosity into the oxide phase, whether it be a cermet or a straight oxide ceramic fuel. Exactly how much porosity that you must introduce is one of the questions. With the oxide fuel at the burnups of 45,000 megawatt days per ton, and at the temperatures in this reactor there'11 be between 10 and $20 \%$ porosity required to allow for the swelling due to burnup and the thermal expansion. Now $10 \%$ might not be too hard, but when you get into the 15 to $20 \%$ porosity range, you're entering a relatively 


$$
6.3
$$

unknown region as far as performance of fuels is concerned. With the cermet you'll need even more porosity in the ceramic phase. You'll need probably 20 to $30 \%$ to hit the burnups that we want.

Now the main problems involved in both of these fuels stem from the porosity. With the cermet, I believe that the biggest problem stemming from the porosity is fabrication; that is, how can you fabricate these things and keep a porous ceramic base in the metal? We don't claim to have a ready answer to that, although the small amount of work that's been done makes it appear that it is feasible.

With the oxide, on the other hand, the major question is whether the porosity will remain distributed where you want it. Now there're several ways of introducing porosity into the oxide fuel. You can use pellets and put it as a gap on the outside, you can use an annular pellet, or you can use porous pellets. I think that at the present time I personally would favor use of a low density or porous oxide. Also, you could use a vi-pack rod to get the lower density.

If the porosity moves about, though, which it will do in thermal gradients for example, you create problems for which we don't have ready answers. One of the normal type problems that you would create is that you would get into clad strain problems because of fuel relocation giving you more dense oxide than you want in some regions and less dense in others. This is also related to the change in thermal conductivity that would occur. I think that probably the biggest problem that you're going to get if you have to go to too low a density oxide is the safety problem during transient behavior. Now, $I^{\prime} m$ not saying that this is insolvable, and I'm sure that it is going to be solved before anybody makes a fast oxide breeder. However, it is very hard to say now that we could go to the ACRS and claim that we have a reactor that's safe, and it doesn't matter if we go into a transient and all the oxide runs to the bottom of the rod. It is going to take a lot of work to prove just what happens during transients whether or not an unsafe condition could result.

Another factor, unrelated to the porosity but related to the use of an oxide fuel, is that the can is really the only pressure vessel. All of the fission gas is contained by the can itself, and this puts some relatively strict requirements on the can. It also gets you again into some hypothetical accidents that are more severe than with the cermet core.

With the cermet fuels, however, the void volume is where you want it. You put the porosity in the little particles of oxide, and that's where it stays. Also, you don't have to rely on the clad to take up the strain due to the swelling and the fission gas. 


\section{4}

The latest look that people have taken at cermet performance tends to lead to a somewhat more complicated correlation than the curve that was shown on the board of burnup-versustemperature. The simple way of stating this is that the cermet won't start swelling until the void volume equals the swelling volume in the oxide. This means that if you have $20 \%$ swelling (and this includes the fission gas swelling) and you leave $20 \%$ void volume, it looks like you're in pretty good shape.

FUEL LOADING

Another point I would like to bring out, because it hasn't been brought out earlier, is important. We're talking of cermet loadings in 20 to 30 vol\%. These are lower than the 50 to 60 vol\% loadings for some of the water reactor concepts that people have had experience with. This lower ceramic content will give us à lot more leeway in fabrication, and it will do a lot to reduce our fabrication costs. It will also give us a lot better chance of getting the higher burnups in the cermet. To summarize, what I'm saying is that based particularly on the one consideration of void redistribution, we favor the cermet fuel over the oxide at the present time. 
PHYSICS AND SAFETY CONSIDERATIONS IN THE FFTF

R. Avery \& W. B. Loewenstein

Argonne National Laboratory

Argonne, Illinois

\section{INTRODUCTION}

It might be appropriate to begin with a disclaimer. We have not been involved, except rather incidentally last summer, in any FFTF studies; therefore, the remarks that Walt Loewenstein and $I$ have prepared represent rather qualitative considerations. We have performed in the last week some relatively simple calculations. I will give some of the results along with other comments related to them.

To scope the problem, we looked at various driver fuels, mainly the cermet and the oxide. We also looked at a metal alloy, a uranium-plutonium mixture with 25 at\% zirconium : id with a fair amount of void within it to allow for swelling.

Some of our other general considerations relate to a few other design parameters and alternates that have been considered for FFTF. One obvious parameter is size. We looked at 500 liter and 700 liter core systems which bracket the current reference 600 1iter system. We also looked at the effect of $\mathrm{U} 235$ as the fuel, rather than plutonium; the use of beryllium oxide as a moderator for either or both of several purposes, either to lower the flux and thus enhance reaction rates or as a means of enhancing the Doppler effect in the systems containing $U^{238}$ and thus having a negative Doppler effect. We also took a very brief $100 k$ at what the effect of a higher amount of $\mathrm{Pu}^{240}$ would be. The results were rather uninteresting; there were no particularly significant changes. The reason for looking at the higher 240 case would be largely motivated simply by considerations that may be what's available rather than any other specific consideration. It ifs as a means of reducing reactivity loss during the cycle, but we made no such studies.

The areas that I will comment on will, to a large extent, parallel those that Peter Hofmann considered. One group of parameters relates to the flux, the critical mass, the power density, and the effect of size and power on these variables. Another area relates to the effect of these various alternates 
on the spectrum in the system and whether any significant effects exist. Another group of questions relate to the burnup, and the associated reactivity and control requirements. These involve questions such as the core conversion ration and possible means of shimming and the reactivity by the multi-zone or multi-enrichment configurations.

The next group of questions relate to safety parameters. We had no opportunity to do any kinetic studies but we looked at the various reactivity coefficients and these include the sodium coefficient, the Doppler effect, the expansion coefficient, and the delayed neutron fraction. I might say at this point that it is difficult to say much about the safety of the driver fuel without getting involved in questions related to the loops. They are so closely tied, in terms of criteria, that the loops may specify what is required for safety critgria of the driver. The final area relates to the effect of $\mathrm{U}^{23}$ and beryllium oxide.

\section{REACTOR PHYSICS}

Generally the critical masses seem to be roughly the same for the oxide and the metal in the particular cases under our study. For the 700 liter core systems the critical mass was around $450 \mathrm{Kg}$. These numbers are not to be taken seriously because we didn't allow anything for excess reactivity and a number of other details. We were primarily interested in comparative values. The critical mass for the cermet was about $350 \mathrm{Kg}$. The ratio is about 10:8; which may be a useful number to keep in mind for the critical mass. The fluxes for the cermet, oxide, and metal systems are in the ratios of 10:8:7, respectively. These numbers are based on the assumption of equal size and power density in all of these systems, which are very questionable assumptions. Obviously, before one translates this to actual fluxes, one would have to consider the maximum permissible power densities in the different systems. I won't make any comments on that except that it might be a reasonable assumption to expect the oxide power density to be somewhat lower than that of metal and cermet.

Flux

There are relatively straightforward conclusions with respect to the effect of size on flux. For example, if one goes from a 500 liter to 700 liter core system with equal power density in two cores, one finds that the flux goes up in the ratio of 10:12. If one increases the size but retains the same total power, letting the power density go down by the same factor as the size increases, one finds that, in going from 500 iters to 700 liters, the flux decreases in the ratio of $10: 8$. 
With respect to the spectrum of the flux that one gets in these different systems, I will discuss some of the questions we considered. The first question is whether among the various alternatives there is a significant difference in the spectrum. With one exception, which I will mention in a moment, the answer is no. The spectra are sufficiently alike, particularly when one considers that the spectra of the power breeder systems one is interested in vary considerably among themselves insofar as the spectrum is concerned. The exception is if one considers moderating with beryllium oxide and thereby lowering the spectrum considerably. In our results we find that lowering the spectrum with beryllium oxide the reaction rate in plutonium would increase by about $15 \%$ which is certainly an important factor. This result is based on the assumption that the power density remains the same as the beryllium oxide is added. Since this is probably an incorrect assumption the proper normalization would have to be made.

There is another area where reaction rate, as opposed to just the flux level, may be quite important; that is, when one considers the very high energy end of the flux, which may be important for certain studies relating to damage on clad or structural materials. One does find substantial differences, and here the only comment I would want to make is that this is an area that one should look at very carefully. Another factor which may have been ignored, at least from what I have seen, is that the effect of the structure associated with the loops may be a very large perturbing factor, particularly on the high energy flux. Any phenomena that one wishes to study that relate to very high energy neutrons probably deserve a very thorough and close look in this connection. Apart from that question, I don't think questions of spectra as they differ among the various alternates are particularly relevant except for the beryllium oxide case.

Burnup

I will now discuss some of the various questions related to burnup. I was confused earlier in the day on some of the questions related to this. As I understand it now, the core cycle time of about 6 weeks has nothing whatever to do, per se, with either a reactivity limit or a radiation damage limit on the fuel itself. Taking very rough numbers, a power of $400 \mathrm{MW}$ roughly corresponds to burning half a kilogram of fuel in the reactor of plutonium per day. Assuming that our critical mass is of the order of $400 \mathrm{Kg}$ and that in the case of a cermet we will be burning $15 \%$ to $20 \%$ of the fuel, we then burn about $75 \mathrm{Kg}$ corresponding to rough1y a 150 day residence time. In the oxide case, if we have a burnup of $45,000 \mathrm{MWd} / \mathrm{ton}$ or rough1y $5 \%$ atom burnup this a1so corresponds to burning about $20 \%$ of the fissile isotope because of the about 4 to 1 dilution. We again have roughly the same core residence time. One finds that the reactivity loss for equal burnups is less in the case of the oxide than it is in the case of the cermet. This is primarily 
because one has a core conversion ratio of about half, in the oxid case, so therefore one is building in about a half of the burned fuel.

The metal case is approximately like that of the oxide. One has to decide how much burnup can be reasonably assumed. Numbers in the range $41 / 2 \%$ to $5 \%$ burnup would lead to results similar to that of the oxide. The metal case would also have a core conversion ratio of about a half.

On the question, which I think came up earlier in the day, on what the flux variation might be during the 6 week cycle period, I'm not sure I agree with some of the comments which implied that it might be completely negligible although the effect is certainly not large. Certainly the flux value per unit power density changes as the enrichment varies. There may have been rather complex factors taking place in the zoned arrangement discussed earlier in the day that seemed to show that the effect was negligible. Generally, the flux should vary during a 6 week period, perhaps the same fraction as the fraction of fuel that is being burned. This is not very large, of the order of $5 \%$. In fact this would only be one of the factors one would be concerned with in maintaining some sort of constant conditions for the test samples. The test sample itself is also burning up fuel so one would have to look at that factor also.

Another factor, which I suppose is not, strictly speaking, within the limits of this meeting, but which I think does relate to driver fuel indirectly, is the question of loop perturbations. on the flux. We certainly don't have any detailed studies of this, but these may not be negligible factors, particularly, in the high energy end of the spectrum.

One might also be concerned with the interaction of loops, but I expect these are rather small effects.

The multi-zone approach, on which studies are being made, certainly seems a proper way to shim. The only question I wish to raise is that there seem to be a large number of different enrichments, four, if I read the figures correctly. I wonder if that may not be too much. Some of the older studies we made indicated that two zones can do a fairly good job of flattening. From there on out as one increases the number of zones the gains become rather marginal.

In the area of safety, I made the comment before, which I would like to repeat, that it is difficult to separate the criteria that you will ask from the driver fuel without saying what the requirements are on the test loops. To follow up on what was touched upon several times during the morning, the question of beta, the delayed neutron fraction is an extremely important one. It is important because it might very well be that one will wind up with safety criteria that relate to a meltdown in the loop. I am fully aware that no one is planning to do this, 
but nevertheless, if one is going to test samples to possible failure, one may have to accept in the safety analyses the possibility of such an event. A possible criterion is that the amount of reactivity added in the loop meltdown is less than a dollar. One might then find that the small value of the plutonium dollar might become extremely restrictive. Perhaps the advantages of $U^{235}$ have not received full consideration in such studies, even though I certainly accept the basic factor that the fluxes are lower with the $U^{235}$ fuel than they are with plutonium. But certainly the setting of specific safety criteria with respect to the dollar may become an extremely important question.

With respect to the sodium coefficient, I think I would agree with what has been said, that it is almost certainly not going to be a problem, but it might be for the larger system that as one gets towards the 800 liter system for the metal and oxide. This ${ }_{4}$ problem would be slightly worsened if one used the higher $\mathrm{Pu} 240$ fuel.

The Doppler effect, as shown in the calculations, the results of which were given this morning, is less than the expansion coefficient even in the oxide system. If one moderates with beryllium oxide, it then becomes of equal size. If one, for example, did decide to moderate with beryllium oxide, and used the cermet, I think one might start running into considerable worry about the positive nature of the Doppler effect in the cermet since it only has fissile isotopes. As some of you know who have been following the Doppler effect, it is not at all certain that the sign is positive even for a pure fissile isotope. There is some evidence that it may be negative. There is even more evidence that it is rather small. For all other cases it will either be negligible, or it might be an important safety criteria if one has it both large and negative. It will certainly be negative in all of the loadings that have U238. It will also be large if one has a degraded spectrum. With respect to the Doppler effect and the expansion coefficient, a rather important question comes up, which people designing the reactor ultimately must resolve in their own minds, and that is: Is it absolutely necessary that there be a negative feed back coefficient? If so, there are really only two possibilities, and they are the expansion coefficient and the Doppler coefficient.

There are certainly a number of questions that would have to be resolved rather early in the game on what the loop criteria would be insofar as such questions are concerned; how much reactivity can be added, and under what circumstances. Otherwise, if one ignores the loop criteria, then one is essentially asking the same questions as might pertain to, say, the prototype, as to whether any of these fuels are inherently unsafe? My only view would be that all of them are in themselves sufficiently safe. If one adds specific criteria that they must override other effects associated with the test loops, then one fuel might be favored over the other. 
We have not made any kinetic studies so that I cannot comment about some of the results that Peter Hofmann showed on kinetic studies. I would have to admit to a certain amount of skepticism until I see all the details on the studies that seemed to imply that for the relatively high ramp rates the cermet fuel seemed to be doing much better than the ceramic fuel. I should say at this point, that there is another important variable in such ramp rates studies which involves the expansion coefficient in the cermet and/or the Doppler effect. One would have to look at the question of how much reactivity is available before failure. Obviously, if one is at a certain point, the expansion coefficient is no longer there after melting. If this amount of reactivity is relatively small, it will do you no good if you were involved in an accident that is adding in more reactivity. The same consideration, of course, holds for the Doppler effect. There is a certain amount of reactivity that is available. Of course the Doppler coefficient does have the advantage that it is still operable as you go to higher temperatures, though the value becomes progressively smaller.

Design Alternates

The final comments I wish to make relate to the design alternates. In the higher Pu 240 case, we did not observe anything significant; the spectra still seemed to be the same, as well as the flux per unit power density. For all of the systems the flux is reduced by a ratio of about $10: 7$ as one goes to $U^{235}$ fuel, assuming the same power density.

Beta, the delayed neutron fraction which is around 0.002 for pure plutonium or around 0.003 for the mixed plutonium $U^{238}$ increases to the order of around 0.007 with $\mathrm{U}^{23}$ fue 1 . This may be a significant factor as one goes to beryllium oxide but the flux is relatively unchanged; the reaction rate, which is the parameter that would determine what you are getting out of the sample, increases by about 15 to $20 \%$. In conclusion, it seems that there are a number of factors that change in the range of 15,20 , or $30 \%$ as one changes driver fuels. One would want to be very careful to include them all before one reaches any final conclusions on their comparison. 
D7. 1

PHYSICS AND SAFETY CONSIDERATIONS

R. Avery

$\underline{\text { DISCUSSION }}$

MR. YEVICK (AEC): With respect to the capture rate, would you care to comment on that?

MR. AVERY: I would make a guess based only on the fact that the cermet is a less black system. The absorption rate is less. I think the movement of the specific other thing, whether it be a control rod or a bunch of molten fuel, tends to be a little bit more, but that is a guess. In fact, I think that any calculations of meltdown reactivity are very tricky things based on detailed geometric factors or factors which tend to be negative. It is a very tricky subject, and I think there is a slight tendency to attach a little bit more cermet for this one factor, seeming it a less absorbing material throughout. The question, as I understood it, was, if there is a meltdown of some sort in a test group, is there any factor which one might guess would be worse or better than cermet or ceramic insofar as the amount of reactivity. Was that the question?

MR. YEVICK: Let's take another one, the fact that you have got, in the case of expansion, your stainless steel has been heated up. This is an instantaneous effect. I am talking about the time lag association.

MR. AVERY: We11, of course, the Doppler effect, I think, (for all practical purposes) is instantaneous. I think in something like a mixed oxide or separate particles there still has to be heat conduction from the plutonium to the uranium, but I think studies have been made of this which show that this is essentially negligible. I seem to recall some study made at Hanford, in relation to this question in connection with a thermal reactor where the enrichment was much less, and the time delay was sufficiently small that it was negligible. I think in a fast reactor where the enrichment is much higher, the time for each plutonium oxide particle to transfer heat to the uranium particle would be less. I think that part of it is true. As far as the time element associated with expansion of the cermet, I would be guessing, but it is rather sma11. It is not important. This is just a guess, purely on the basis of a good conductor. 


$$
\text { D } 7.2
$$

PETER HOFMANN: In discussing the question of contact resistance between the ceramic bead and the steel, I might just briefly refer to my talk again. It is a question that I think is not completely resolved. In other words, if there is a heating factor, it is a very complex factor between the ceramic bead and the steel matrix. Now the question of what this heat transfer coefficient here is, is a complicated question, and I have no answer to that.

MR。CARL ANDERSON: On the same subject, if you have some history of irradiation, and the porous oxide allows a space between the oxide and the metal, this should affect it adversely also.

MR. COHN: After about 20 hours of operation and power, the heat transfer coefficient, due to the fission gases is high enough between the bead surface and the stainless steel matrix to give you a reasonable response. And it gets better as time goes on, presuming you have a gap.

MR. YEVICK: Do you have any numbers on this?

MR. COHN: Yes. They are quite preliminary in nature now. I got the impression that you implied it might be conceivable to build a reactor without an inherent natural shutdown. Would you elaborate on that for us?

MR. AVERY: Well, I don't think it has any particularly profound meaning. I think it would depend on how much you decided that for maximum credible accidents you would rely on your control system. It might very we11 be that you will convince yourself that this is not necessary. If you do not put any reliance on the control system, I think you generally would wind up with a much larger maximum credible accident, where you will get very little benefit from the expansion for example, so this is the type of consideration that it would have. 
8. 1

\title{
PHYSICS COMPARISONS IN FERMI SIZE CORES
}

\author{
J. B. Nims
}

Atomic Power Development Associates, Inc.

Detroit, Michigan

\section{INTRODUCTION}

This presentation describes some of the physics and safety studies carried out at APDA in connection with Fermi programs which have some bearing on the FFTF program. The analyses described here, of course, do not relate directly to our FFTF concept but, nevertheless, should provide some useful insight and background information for the choices which have to be made. This information was developed in two separate programs; the first was made for the purpose of selecting an advanced core design suitable for installation in the Fermi reactor; the second is concerned with the use of Fermi Core A for fast fuel irradiation purposes. The first study considered both $\mathrm{PuO}_{2} / \mathrm{SS}$ cermet fuel and mixed oxide core loading. The latter considered both a uniformly enriched core and also a three-zoned enriched system. The study included the calculation of temperature and power coefficients, and the core conditions that occurred under certain accident situations. The core sizes were in the intermediate range $(\sim 800$ liters) which is somewhat larger than the contemplated FFTF sizes. Since safety is a major consideration in the FFTF fuel selection, these studies should be useful in assessing the relative safety characteristics of the two fuel systems under consideration.

The second Fermi program mentioned above investigates the problems associated with fast fuel irradiation testing in a metallic fueled driverFermi Core A. It is planned to irradiate up to 15 test subassemblies in a core lattice of approximately 100 core subassemblies. There are many aspects to this program but, for the purpose of this meeting, the material discussed will be concerned principally with the accident studies and the protection required to prevent the occurrence of a severe accident such as primary flow failure or test subassembly collapse from causing multiple test subassembly failure. This is, of course, one of the more serious aspects of the FFTF program. The Core A test bed results cannot be related directly to FFTF but they do present evidence that a test bed type operation which utilizes moderate sized pin bundles and tests under elevated temperature and power density conditions can be done quite safely and without the possiblity of a single test bundle failure propagating to other test bundles.

\section{COMPARISON OF CERMET AND OXIDE CORE DESIGNS}

The core designs considered were the maximum size that can be accommodated in the high pressure plenum. This consists of 130 core subassemblies, 10 safety and control rods, a neutron source, and an additional 9 core positions for flexibility. 
8. 2

\section{CORE DESCRIPTIONS}

Slide 1.

The core designs are summarized and compared with Core A in

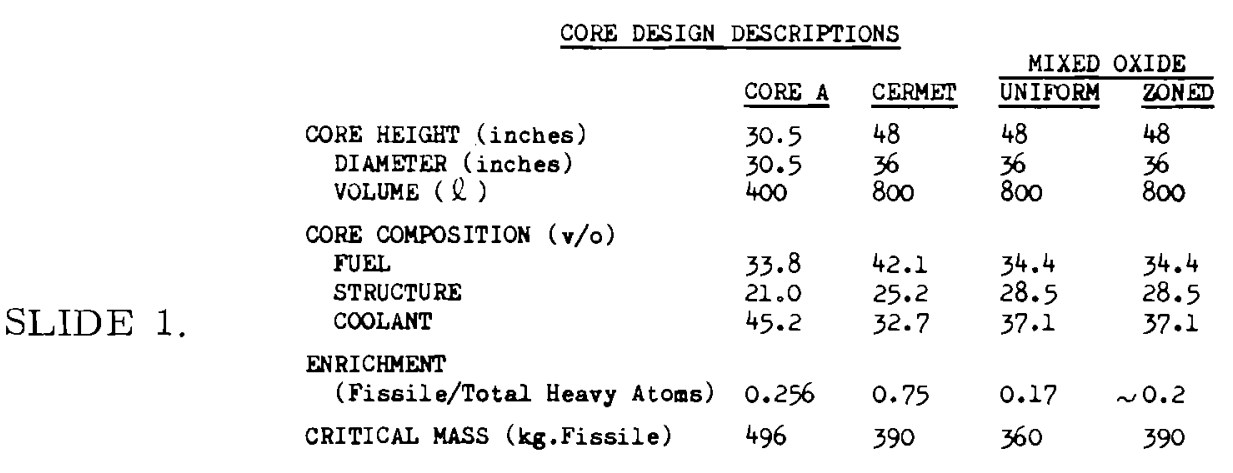

\section{TEMPERATURE AND POWER COEFFICIENTS}

The individual components of the temperature coefficients are given in Slide 2. In addition, the total power coefficient as determined from kinetics calculations are shown for both the zero power and full power conditions.

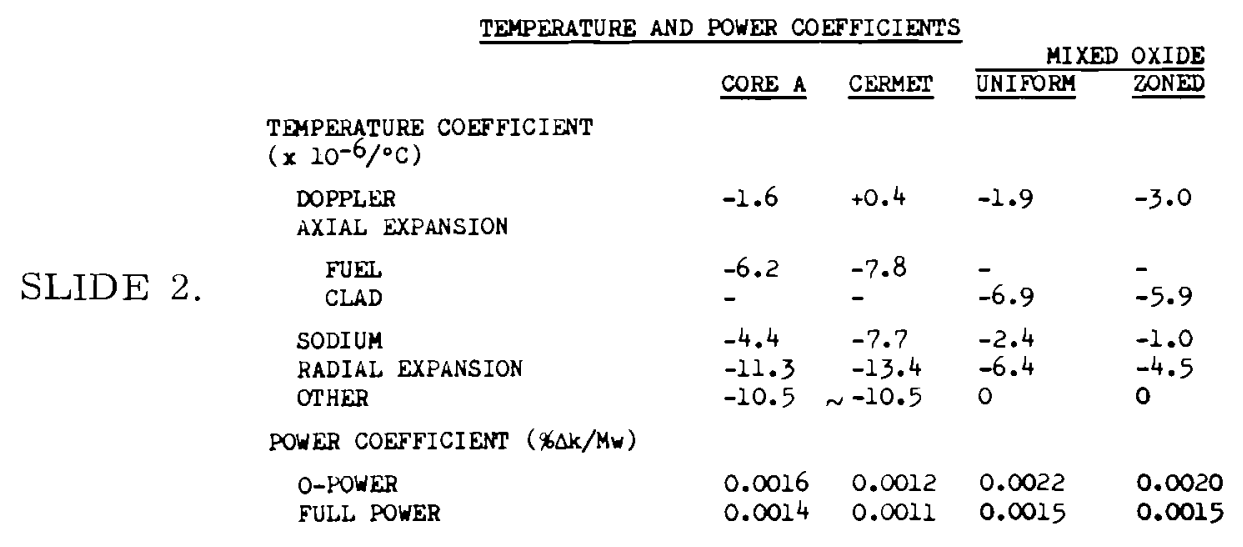

When the temperature and power coefficients of the cermet core are compared with those of Core $A$, it is seen that the values for the cermet design compare very closely with Core A. It has an axial fuel expansion coefficient slightly larger than Core A and a Doppler coefficient which, although positive, is small in comparison to the former. The prompt feedback is then essentially as strong as in the Core A design. The sodium 
coefficient is calculated to be nearly a factor of 2 larger than that of Core A. The radial expansion coefficient is shown to be larger than Core $A$, but this value is believed to be too large. A value approximately one-half this size is perhaps more reasonable, particularly if a radial nickel reflector is used in preference to the breeder blanket present in the calculated design.

In the oxide cores, the fuel elements were designed to minimize differential expansion of oxide and clad, and with a mechanical bond between fuel and clad in order to assure that the fuel moves with the clad material. Therefore, no feedback due to oxide expansion is assumed, but fuel displacement by clad expansion will occur and produce feedback. The prompt temperature coefficient due to the Doppler effect (both uniform and zone loaded cores have an enrichment in the $20 \%$ range) is considerably smaller in magnitude than the fuel expansion coefficients of the metal and cermet cores. However, the power coefficient is a more significant quantity as far as inherent safety is concerned; the larger temperature rise in the oxide material per megawatt of power as compared to the other two fuel materials, weights the Doppler effect heavily so that it is more effective than the magnitude of the Doppler temperature coefficient would indicate. Therefore, at low power the feedback is actually stronger in the oxide cores. Because of the dependence of the Doppler on temperature, this component decreases in magnitude with increasing power. At full power conditions, the total power coefficients of all systems are similar in magnitude. The coefficient for the oxides would continue to decrease significantly in overpower transients.

Comparing the two oxide cores, it is noted that the zoned enrichment approach benefits the Doppler effect because of the lower enrichment in the central core region where the reactivity effect is greater. The positive sodium void problem and the sodium temperature coefficient become more positive for the same reason. For cores of the size considered here, the void coefficient is not large and the problem is not serious. The reduction in the sodium portion of the power coefficient is small and more than compensated by the increase in the Doppler component.

In Core $A$ there are feedback effects, other than the four principal core effects specifically evaluated, that make a substantial contribution to the feedback. These total $10^{-5} \Delta \mathrm{k} / \mathrm{k}^{\circ} \mathrm{C}$, of which sodium density effects in the blankets and blanket material expansion are major contributors. Similar effects are expected in the other designs, although they are probably smaller in magnitude than in Core A because of the larger core size.

\section{ACCIDENT CONSIDERATIONS}

Kinetics studies were carried out to investigate two hypothetical accident situations; the first study determined what reactivity insertions at moderate rates can be tolerated at full power without exceeding the coolant boiling temperature; and the second investigated the effects of a primary system flow failure accompanied by failure to scram. These results and other pertinent information are shown in Slide 3.

As can be seen from the table, the oxide cores can tolerate a substantially smaller nonstep reactivity insertion than can the other designs. Whereas the metal and cermet systems can withstand a reactivity addition of 0.6 to $0.7 \%$, the ceramic designs can only tolerate 0.24 to $0.3 \%$. 
8.4

Actually the oxide core results are somewhat more conservative than those obtained for Core A and the cermet because the blanket feedback effects were not included. Including a nominal effect would increase the tolerable $\Delta \mathrm{k}$ to the 0.3 to 0.4 range. In addition, the design basis for the oxide cores is more severe in that the core inlet temperature is higher by $50^{\circ} \mathrm{F}$. How ever, the coolant temperature rise is the same for all of the designs. If for the oxide cores the inlet temperature is dropped $50{ }^{\circ} \mathrm{F}$, then the $\Delta \mathrm{k}$ to boiling conditions is in the 0.35 to 0.45 range, which is still somewhat less than that of either Core $\mathrm{A}$ or the cermet core.

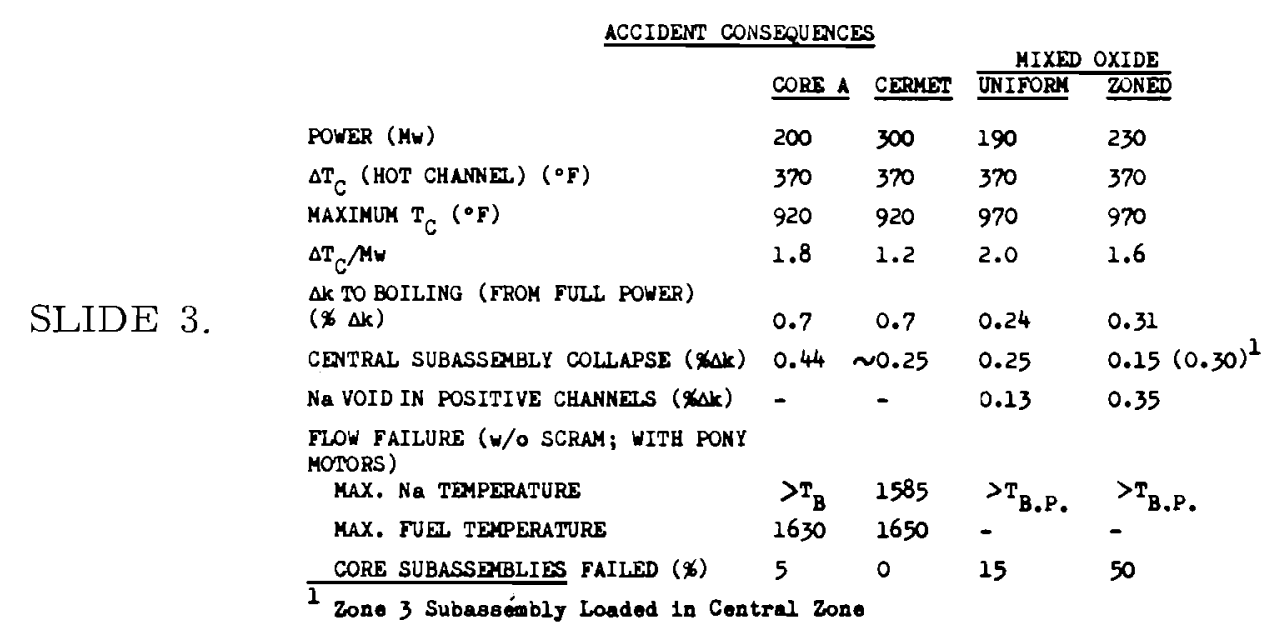

One possible reactivity insertion mechanism is a single subassembly meltdown. This has been evaluated on a very conservative basis--complete compaction of a central subassembly around the central plane. For the metal and cermet cores an insertion equivalent to the complete collapse of a single subassembly could be withstood without causing gross boiling and meltdown. For the cermet core the maximum amount available exceeds $1 \$$ by about $20 \xi$. Therefore, it is apparent that the rate must be slow enough to permit enough negative feedback to prevent $\mathrm{k}$ ff from exceeding $1 \$$. For the oxide systems on which the original analysis was performed, the amounts inserted are less than $1 \$$, but are approximately large enough to cause gross boiling. When one includes some reasonable values for other feedback effects, the $\Delta \mathrm{k}$ required to reach the boiling point would be larger than the hypothesized insertions, but with only a small margin for error.

The flow failure accident calculations clearly show an advantage for the cermet design over the oxide and Core $\mathrm{A}$ as well. The zoned oxide is the most seriously affected in such an accident, as might be expected, since the design intention was to make all channels operate closer to maximum temperatures. Obviously, when something happens that affects the reactor power level, a larger fraction of the core is affected. However, the calculations do not include all feedback effects. A supplementary analysis showed that additional feedback equivalent to the radial expansion effects would reduce the number of channels affected but substantial boiling would still occur. 
8.5

The results in general indicate an advantage to the cermet design as far as inherent safety is concerned. The problems of safety in oxide systems is one which has to be faced and resolved for power reactor use. It is APDA's opinion that the oxide core systems described can be operated safely in normal power reactor use. In this application, the sources of extraneous reactivity insertions are more easily controlled by design and management and therefore accidents involving such sources are much more improbable.

\section{EXTRAPOLATION TO FFTF}

\section{EFFECT OF TEST BUNDLE MELTDOWN}

A feeling for potential reactivity insertions that can be anticipated from a meltdown in a test loop can be had from studies made in connection with some proposed Core A oxide pin irradiations (Slide 4). The original numbers were calculated for pins $3 \mathrm{ft}$ long and having an OD of 0.25 in. The ratio of $\mathrm{PuO}_{2}$ to $\mathrm{UO}_{2}$ is $4: 1$. These numbers have been approximately adjusted to apply to FFTF and scaled to correspond to various test bundle dimensions. The loop sizes considered are 6, 5, and 3.5 in. in diameter and would contain 271,169 , and 19 pins respectively.

$\triangle k$ DUE TO COLLAPSE OF UPPER HALF OF TEST BUNDLE

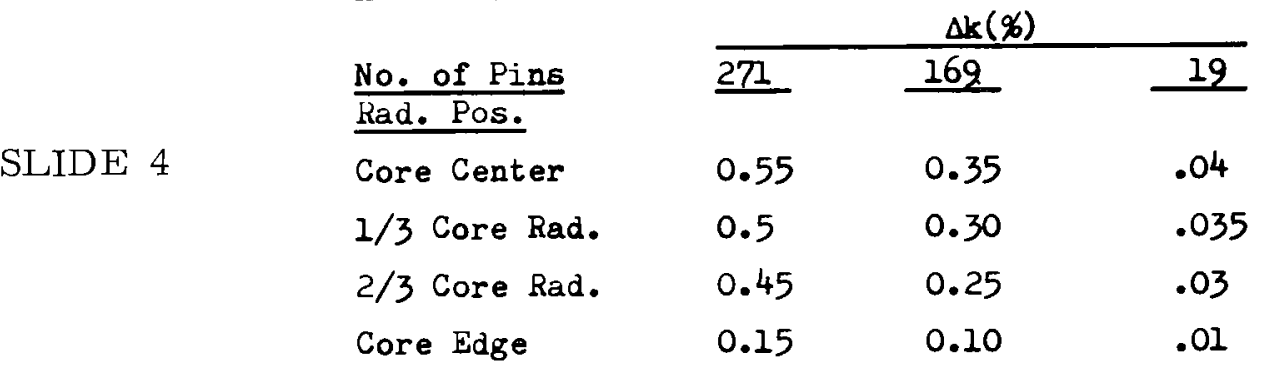

The $\Delta \mathrm{k}$ increase for collapse to half height of the material above the core midplane is shown below for different radial positions of the bundle.

The numbers represent reasonable, not conservative, estimates of the upper limits as far as the magnitude of collapse effects for a single bundle are concerned. To refine them on any justifiable basis (other than to make similar estimates directly for FFTF) is beyond presently available technology. The information needed to specify a realistic reactivity addition and rate is essentially the same as that required to improve the realism of the maximum hypothetical accident--namely, the velocity profile of the fuel mass in the channel in the presence of liquid sodium and high velocity sodium vapor. The need for this information has been recognized for many years but no real progress has been made toward 
this goal. The "best" information available is from the APDA $\mathrm{Pb}-\mathrm{H}_{2} \mathrm{O}$ experiments used to simulate metallic uranium pin meltdown in sodium. These tests indicated in general that the fuel in the lower half of the core did not redistribute, which is some justification for assuming the type of collapse that the data in the table is based on. The fuel tended to move away from the core center initially and subsequently toward the center but at rates less than those produced by gravitational acceleration. However, since displacements of about $1 \mathrm{ft}$ are pertinent, the observation does indicate that the fuel motion may we $\geqq 1$ be completed before the scram system can shut the reactor down. Therefore it is necessary to depend on the magnitude of the effect being within the limits of the natural response of the core for protection or a combination of inherent feedback and safety system action before the heat irput exceeds that required to produce boiling, or fuel element failure in other channels.

The relationship between the metallic meltdown experiments and oxide fuel meltdown is obscure but there are several important differences. With the oxide, failure is more likely to cccur near the core midplane where the fuei temperatures are highest. In metal fuel elements, because the fuel $\Delta \mathrm{T}$ is smaller, failure is more likely to occur near the top of the fuel element. Therefore with the metal it is probably easier for the molten fuel to be expelled cut the top of the core. Also, the oxide may have a greater tendency to freeze and stick because of its higher freezing point. Self-obstruction by the oxide may therefore tend to prevent dispersal. On the other hand, the fission product gases contained in the fuel, if significant burnup has been reached, should have the effect of reducing fuel derisity and reactivity, These remarks are highly speculative and the effect of meltdown should be explored experimenta ly.

However if we accept two points from the $\mathrm{Pb}-\mathrm{H}_{2} \mathrm{O}$ tests, namely that the fuel redistribution is limited to the top half of the test bundie, and secondly that the movement can take place at rates similar to those produced by gravity, the corclusion would be as fallows: The reactivity insertion would be completed in a time shorter than the system time constants and the magnitude of the addition shculd not exceed a value of approximately 1\$. For this slide we then see that the bundle size should be limited to approximately 100 pins in the inner ccre positions, which is probably adequate. Larger bundles could be tested in outer parts of the core.

In the case of the oxide cores the Doppler feedback as given in Slide 2 is optimistic if the oxide system is limited to the same size as the cermet. The actual cermet design (as studied by PNL, by the way) has stronger feedback than the Fermi system. This is partly explained by the reduced $P^{240}$ isotopic content in the fuel which detracts from the sodium component. If a smaller oxide ccre is used, the $\mathrm{U}^{238}$ concentration will be smaller. For a 600 liter oxide core, the fertile-to-fissile isotopic ratio would probably be 2 to 3 rather than 4 to 1 in the Fermi design. This would make ar appreciable rediction in the power coefficient. Since the Doppler effect accounts for approximately $70 \%$ of the total feedback, the reduction in the power coefficient wculd be about $30 \%$. The $\Delta \mathrm{k}$ from full power to boiling conditions would then be about 75 and $1,0 \$$ for the uniform and zoned cores respectively. Although the test bundles could be restricted in size so as not to exceed these limits, the rate of addition becomes important because most of the feedback is now delayed. 
It appears that the cermet design has an advantage over the oxide core. However, it is clear that a very thorough analysis of the test bundle failure accident is necessary with particular attention to insertion rates and test bundle size.

\section{TEST BED STUDIES}

As an example of the safety analysis that is necessary in test bed type operation the studies, performed for the Core A program are described briefly.

The program calls for irradiating simultaneously up to 15 test subassemblies. Each subassembly can contain up to 24 test pins. The outlet temperature in the test thimbles is not allowed to exceed $1200^{\circ} \mathrm{F}$. The maximum Core A outlet temperature is $617^{\circ} \mathrm{F}$. The studies have considered several typical test subassemblies. The most reactive of these is shown in Slide 5.

TYPICAL TEST SUBASSEMBLY DESCRIPTION

$\begin{array}{ll}\text { NUMBER OF PINS } & 28 \\ \text { FUEL DIAMETER } & .22 \text { INCHES } \\ \text { CLAD THICKNESS } & .015 \text { INCHES } \\ \text { FUEL LENGTH } & 30.5 \text { INCHES } \\ \text { FUEL COMPOSITION } & \\ \mathrm{UO}_{2} & 80 \mathrm{w} / 0 \\ \mathrm{PUO}_{2} & 20 \mathrm{w} / 0 \\ \text { ENRICHMENT } & \\ \mathrm{U}_{-235} & 93 \mathrm{w} / 0 \\ \text { U-238 } & 7 \mathrm{w} / 0 \\ \text { PU-239 } & 93 \mathrm{w} / 0 \\ \text { PU-240 } & 7 \mathrm{w} / 0 \\ \text { FUEL DENSITY } & 90 \% \text { OF THEO- } \\ & \text { RETICAL }\end{array}$

One question of interest is the effect of 15 test subassemblies on the reactor power coefficient. This was the subject of a $2 \mathrm{D}$ analysis in $\mathrm{x}, \mathrm{y}$ geometry with the test subassemblies distributed in a reasonable pattern throughout the lattice. The temperature coefficients for the base loading and test bed loading are compared in Slide 6. As the table indicates, the changes are small and change the total power coefficient by about $2 \%$. Some of the effects are actually more pronounced than the table indicates. In the case of the Doppler coefficient, for example, the test subassemblies decrease the Doppler coefficient, but the reduction in driver fuel temperatures at 110 MW gives a net increase in magnitude.

In the credible accident studies, the major factor is the large $\Delta \mathrm{T}$ $\left(750^{\circ} \mathrm{F}\right)$ and high outlet temperature $\left(1200^{\circ} \mathrm{F}\right)$ in the test subassemblies. The two most serious accidents are primary flow failure and reactivity insertion from test subassembly collapse. The criteria followed is that two independent scram signals must be activated and safety system response must be rapid enough to prevent hot channel boiling anywhere in the core. As an example, the scram signals which are effective in the flow failure accident are shown in Slide 7. 
TEMPERATURE COEFFICIENTS FOR 15

SUBASSEMBLY TYPICAL LOADING

$\left(10^{-6} \Delta \mathrm{k} / \mathrm{k} / \mathrm{c}\right)$

SLIDE 6.

DOPPLER

FUEL PIN AXIAL EXPANSION

CORE SUBASSEMBLY WRAPPER TUBE RADIAL EXPANSION

NA EXPANSION

NA EXPANSION IN UPPER AXIAL

BLANKET

OTHERS

THESE COEFFICIENTS RESULT IN a TWO PERCENT DECREASE IN THE POWER COEFFICINNT RELATIVE TO CORE A
SLIDE 7.
TYPICAL LOADING * CORE A $110 \mathrm{MH}$ $200 \mathrm{MW}$

$-1.85$

$-1.68$

$-5.85-6.96$

$-16.9 \quad-17.96$

$-5.86 \quad-4.16$

$-2.61 \quad-2.61$

$\begin{array}{ll}-2.89 & -2.89\end{array}$

FLOW FAILURE SCRAM SIGNALS

NEGATIVE

POWER RATE

TRIP LEVEL

TIME ACHIEVED, SEC.

DELAY, SEC .

AUTOMATIC CONTROL

ELECTRONIC

DEJATCH

LAG

\begin{tabular}{cc}
$-1.8 \mathrm{Mw} / \mathrm{sec}$ & $85 \%$ \\
2.1 & 2.2 \\
& \\
.100 & 0 \\
.700 & .100 \\
.150 & .150 \\
.238 & .238 \\
\hline 1.188 & .488
\end{tabular}

EXIT

TEMPERATURE

$120 \% \Delta T$

3.1

238

$488 \quad>2$

This accident for the case of test subassembly operation is more important due to the relatively small increases in power to flow ratio than can be tolerated before coolant boiling in the test subassembly begins. For example, hot channel boiling in test subassemblies would begin approximately 5 sec after flow failure and bulk boiling at about $8 \mathrm{sec}$ if there were no scram. These short times remove the core outlet temperature as a means of initiating scram due to the relatively long time constants involved. This omission leaves the flow indication itself and the - $d n / d t$ signal as possible means of scram initiation.

The scram trip points for those two signals (Slide 7) were chosen to assure a scram with sufficient speed to prevent hot channel boiling in test subassemblies with a steady-state hot channel sodium outlet temperature of $1200^{\circ} \mathrm{F}$. With the settings listed in Slide 7 for flow and $-\mathrm{dn} / \mathrm{dt}$, the $-\mathrm{dn} / \mathrm{dt}$ 
signal will be the slower acting of the two. Therefore, the flow failure accident was analyzed, assuming scram to be initiated by the $-\mathrm{dn} / \mathrm{dt}$ signal with the given trip point value of $-1.8 \mathrm{MW} / \mathrm{sec}$. The resulting maximum hot channel clad and coolant temperatures for the Type 1 test subassembly are shown in Slide 8. Note that the maximum hot channel temperature is about $200^{\circ} \mathrm{F}$ below boiling; the maximum nominal temperature is some $400^{\circ} \mathrm{F}$ below boiling. The maximum test fuel temperature is essentially the steady-state value since there is an immediate drop in power following flow failure due to the negative power coefficient. The maximum Core A driver sodium temperatures are shown in Slide 9.

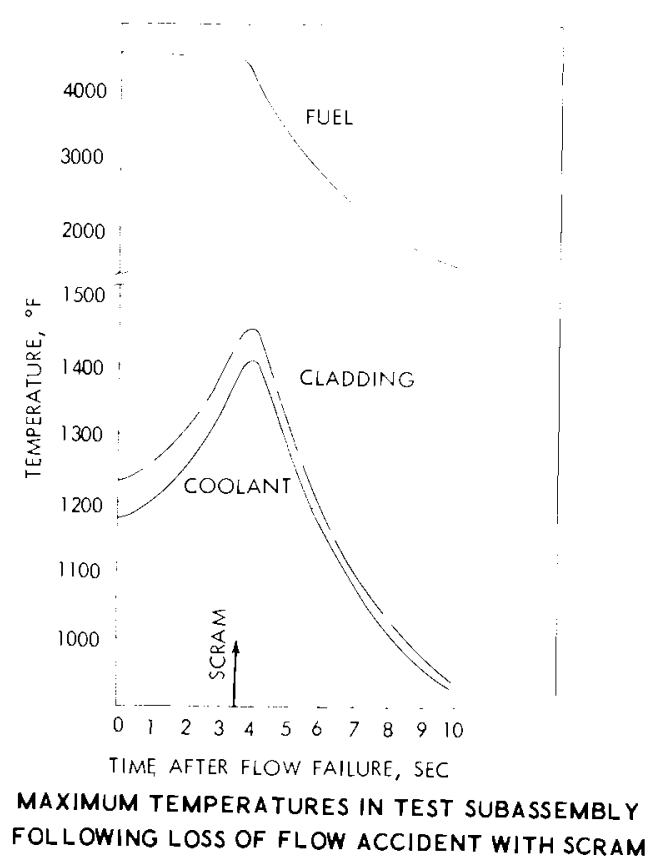

SLIDE 8.

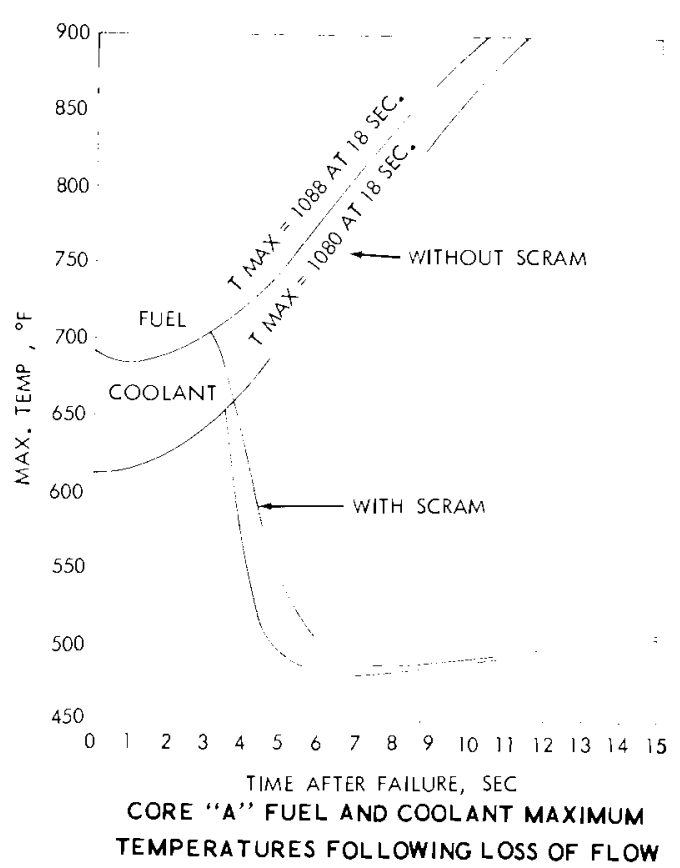

SLIDE 9.

The maximum credible accident for Core $\mathrm{A}$ is the melting of some or all of the fuel in one core subassembly. For the case of test subassembly operation, the maximum credible accident remains the same but includes the possibility of meltdown of a test subassembly. On a per subassembly basis, the meltdown of a test subassembly is more likely, due to its higher operating temperatures and small orifices, and the somewhat greater unknowns concerning its in-pile performance. Moreover, it should also be stated that the more likely form of meltdown for the four-thimble reference test subassembly is for the fuel in only one thimble to melt. Each thimble is insulated and separated from the others by high velocity, low temperature by-pass sodium. Simultaneous fuel failure in all four thimbles due to hot spots or metallurgical behavior is highly improbable even if the fuel is of the same type. Partial or complete blockage of the total subassembly or undetected design error are the only likely causes of total test subassembly meltdown. 
Nevertheless, it will be assumed that the total meltdown of a high worth test subassembly represents the maximum credible accident since its consequences are slightly more severe than for Core A subassembly meltdown. If all the fuel in a Type 1 test subassembly at core center were to melt, violate the thimble containment, and collect at core midplane, there would be a resulting reactivity increase of $50 c$. The consequences of such a reactivity insertion are analyzed based on the additional conservative assumption that it is inserted as a step rather than taking finite amount of time. Reactor power would overshoot to a very high value, partially recover, and then start its asymptotic rise from about $200 \mathrm{MW}$. Both the power level and intermediate range scram trip level settings would be almost instantaneously exceeded. The period circuit would respond somewhat slower than the power level circuit. Assuming a scram is initiated by the former, the maximum hot channel fuel and sodium temperatures (Slide 10) in the undamaged test subassemblies are calculated to be $1330^{\circ} \mathrm{F}$. Core A driver sodium temperatures are considerably lower. Thus, the power perturbation resulting from complete meltdown of the high worth fuel test subassembly followed by scram is not sufficient to induce coolant boiling in other test subassemblies or in a driver core.

SLIDE 10.

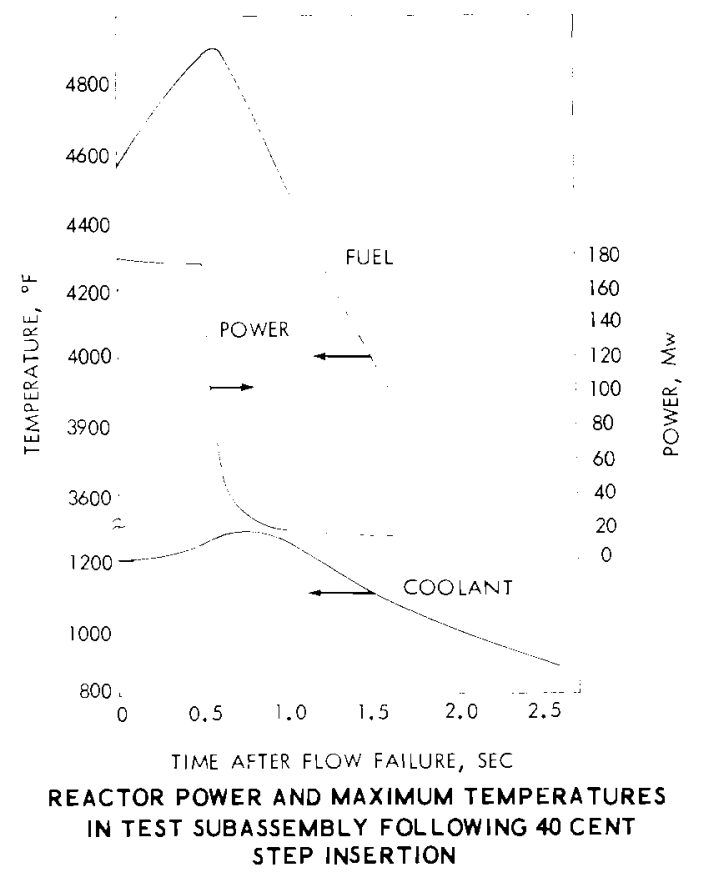

These results simply serve as an example of the type of analysis required for FFTF and provide some basis for optimism for being able to provide essential protection.

$\underline{\text { SUMMARY }}$

MAJOR POINTS

1. Based on the analysis of Formi sized cermet and oxide cores, the cermet has the stronger inherent feedback. The feedback of the FFTF cermet is apparently stronger than that of the Fermi design. If the oxide cores are reduced in size to approximately 600 liters, 
the concentration of fertile material will be reduced and the Doppler component of the feedback reduced proportionately. Therefore, for the FFTF application the cermet fuel is preferable for safety reasons.

2. Based on the $\mathrm{Pb}-\mathrm{H}_{2} \mathrm{O}$ tests, which simulate meltdown of metal pins in the presence of coolant, it appears that a reasonable model to estimate the effect of collapse of the top half of the bundle rather than total compaction. Furthermore, the tests do indicate that the fuel rearrangements can take place in a time interval which is short compared to the time constants for heat transfer in the system. Therefore it is reasonable to limit the test bundle size so that the amount of reactivity insertion is under $1 \$$. Refinement of this number requires knowledge of the insertion rate and detailed analysis of the system response for this insertion rate.

3. Based on the rather crudely scaled numbers for the collapse of a Core A test subassembly to FFTF, the amount of excess reactivity on the basis of the model described above can be limited to less than $1 \$$ if the test bundle is limited to approximately 100 pins in a central position.

4. Based on the kinetics studies for Core A test bed operation, a safety system can be designed to prevent a flow failure accident from causing failure and collapse of one test subassembly which would cause multiple test loop failures. 
. 
D8. 1

PHYSICS COMPARISONS IN FERMI SIZE CORES

J. B. Nims

\section{DISCUSSION}

MR. HOFMANN: What is the blanket?

MR. NIMS: The blanket in this case is a metal, uranium..

MR. HOFMANN: I was wondering what it was because your sodium coefficient is about half the value we calculate. So the composition is slightly different.

MR. NIMS: That is very possible. It also depends, I think, on the sodium volume fraction.

MR. HOFMANN: They are quite close, because ours was around $40 \%$. The differences don't seem to be large enough to account for it.

MR. NIMS: The $\mathrm{Pu}^{240}$ contents could also be important. We used a dirty grade of $\mathrm{Pu}$. I would expect this could make a difference of $25 \%$.

MR. HENNIG: Are there other questions or comments?

MR. YEVICK: Let me ask Pete Hofmann this one. I notice that John Nims indicated that for 28 pins he would get $40 \zeta$. Have you done anything comparable with respect to your closed loops? For instance, Avery mentioned that you may have to look at the dollar insertion, and how many pins, let's say, of unenriched plutonium do you have to have to obtain a dollar?

MR. HOFMANN: The only thing we have done comparable to this type of calculation is the early calculations on the collapse of a central test assembly. Now what we assume is a very reactive test assembly, in fact one that is equal to $100 \%$ enrichment. And if you recall, we talked about this half collapse.. Now we assumed only that the upper portion of the fuel slid down, and the reactivity rate was about $4 \$$ a second. But the whole element, I think a central driver element, was worth about $6 \$$ total. As I recall, the upper quarter being about a dollar, the middle and third quarter being two dollars, and the bottom quarter a dollar. So again you slide the top quarter into the second quarter, and you gain essentially a dollar. So we are saying this extreme case would introduce a maximum of a dollar.

MR. YEVICK: What is the total number of test pins?

MR. HENNIG: Seventy-five pins.

MR. YEVICK: What is the maximum number of pins you could get in a closed loop right now?

MR. HENNIG: One percent of the critical mass is about $3 \mathrm{~kg}^{\prime} \mathrm{s}$. Add $12 \mathrm{~kg}$ 's of 28 along with it; bring it down to $20 \%$ enrichment and add 
D8. 2

$200 \mathrm{~g} / \mathrm{pin}$, which is a good round number. You should be able to get 75 pins in the center loop before we hit our model meltdown accident.

A VOICE: Physically you can't get that in there.

MR. HENNIG: In the center loop?

MR. deBOISBLANC: In the upper half of the element, the upper quarter he has calculated.

MR. HOFMANN: The total fuel element contains about $5 \mathrm{~kg}$.

MR. deHALAS: It is way too big, Bob. It is $90 \mathrm{~g} /$ driver pin.

MR. HENNIG: I have got a mixed oxide pin.

MR. deBOISBLANC: That would be 75 pins added to the center.

MR. HENNIG: No, based on our model, it says here is the 3 foot long piece, and the top half collapses in position 4 and position 3 . It is a half compaction accident that Nims just described. We said you could put in about $1 \%$ of the critical mass in the center loop. That is about $3 \mathrm{~kg}^{\prime} \mathrm{s}$ of plutonium. It doesn't make much difference how you distribute it. Dilute it with stainless steel or dilute it with 238 oxide, or with plutonium. I don't suppose it makes much difference. You could put in about $3 \mathrm{~kg}^{\prime} \mathrm{s}$ and fail it that way without destroying the core. But for Yevick's question, it still works out to about 75 pins.

MR. YEVICK: Seventy-five pins was correct. What is the maximum number of pins in a closed loop at the present time, assuming a 6 in. OD?

MR. HENNIG: Put it where?

MR. YEVICK: It doesn't matter where you put it. Let's say you had a $41 / 2$ in. loop, as you have now, (your cross section shown this morning by $F$ inch) how many test pins can you get in that center closed loop?

MR. YEVICK: The question has been raised essentially about the amount of reactivity having a closed loop. That is the one I want answered now.

MR. HOF MANN: I am saying in the central closed loop, the kind of accident you would get is one the order of $4 \$$ a sec if you put in this central line test loop; presumably, you would test some sort of ceramic, so the amount of plutonium in a typical test will be considerably less than a driver element so it is less by a factor of four.

MR. HENNIG: Between 61 and 91 pin bundles (0.3 in. pins).

Mr. deBOISBLANC: What would you be allowed for a dollar of seven-tenths of a percent? 


\section{D8. 3}

MR. NIMS: If it is a large sized oxide system, $0.42 \% \Delta \mathrm{k}$ will get you up to the boiling point; so if a reactivity insertion approaching $0.7 \%$ occurs, you would fail the fuel.

MR. deBOISBLANC: So your boiling point is the failure point?

MR. NIMS: At least for the cermet, and we take it to be so for the ceramic. 


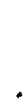




\section{1}

APPLICATIONS OF THERMAL TEST REACTOR EXPERIENCE

D. R. deBoisblanc

Phillips Petroleum Company

Idaho Fal1s, Idaho

\section{INTRODUCTION}

"What possible correspondence is there between the problems of the thermal reactor with a water cooled, aluminum-based, plate-type fuel element to a fast reactor using a fuel material with ceramic particles in a stainless steel matrix, or some variant thereof? I searched through the documentation and attempted to derive some generic principles that have evolved in the design and execution of experimental programs at the NRTS, principles which could be applicable to any high power density test reactor. There is, in fact, quite a bit of correspondence in certain key areas that are important to the future of this project or, for that matter, any other such project. There are those things, of course, that affect the continuing feasible operation of the machine at high power densities. There are those factors that affect the fuel cycle cost, about which we learned something this morning. There are those factors that relate to the relationship between the fuel and intended experiments, and which to some extent determine the geometry allowable in a test reactor. As a result, it is almost impossible to divorce oneself in a design effort from a consideration of what it is that the reactor is supposed to do. I, being no different from anyone else, said, "We11, what kind of experiments do you wish to do?" In this respect, I would say, and this is not intended to be criticism of Battelle-Northwest's approach to this thing, that I feel a little as I felt back in 1952 in looking at the beginning of the experimental program in the MTR.

The MTR was not designed to do loop experiments. It was intended to be a beam reactor. The core leakage considerations, power densities and all of these things were really ordained on the basis of critical experiments, of consideration of neutronic properties of relatively clean core materials, and of almost ideal geometry for the core and reflector. Dr. Wigner, I think, even now would be surprised to know how far the original MTR has actually departed from the original concept. The beam tubes, of course, did serve their purpose. In the early days they were made to serve in a rather awkward manner the needs of an experimental loop program. There are people I can see in this audience who were involved in the tedious and very difficult engineering problems of designing high temperature loops to operate in a horizontal position. We talked about fuel slumping and how you get a fuel element in-to and out-of a facility that is horizontal in view of distances on the order of $20 \mathrm{ft}$ of length. Somehow these problems were solved; somehow these problems were contended with; and a great deal of information, without which this country's atomic energy program would be in a pretty sad shape, was obtained. 
When the decision was made for an engineering test facility to do a specific job for a class of reactors, then something like the proper design of an Engineering Test Reactor took place. As a participant in the design of the ETR, I was almost saying the same things then that I am saying today, "What kind of experiments do you want to do?" After hammering on the subject long enough, we were able to get at least a tentative complement of experiments which were intended to serve the needs of the ANP project. As you know, the ETR was designed primarily to serve the purposes of the ANP project, hence, the $9 \times 9$ hole and certain of the other parameters about the geometry of the core that maybe you today find a little mystifying. It was true that the ETR evolved around a particular class of experiments, but it is also true that before the design was consummated people like John Yevick were asking the questions, "We11, suppose you want to do something else in that reactor besides gas cooled experiments using up to $30 \mathrm{lb} / \mathrm{sec}$ of air at 1100 to $1400^{\circ} \mathrm{F}$; what do you do?" To add versatility to it, the core size was increased beyond the immediate needs of the ANP program and was led in the direction of providing some $3 \times 3,5 \times 6$, and $9 \times 9$ facilities, etc. It would be difficult for me to contend that the choice to make the reactor versatile was not accompanied by an increase in the cost of what might be called the minimum facility for the ANP program, because it is true that this increased the cost if for no other reason than to delay decisions on the design.

After the demise of the ANP program, we became very conscious of the need to make the ETR function as a versatile test facility and we were very glad indeed for the design decisions that our Mr. Yevick had

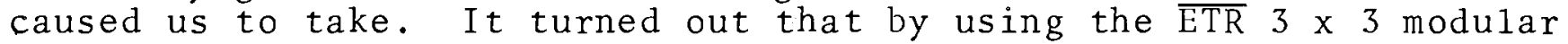
dimension, it was possible for us to accommodate a large number of loops of the type that proved so useful in the Naval Reactor's program and in other high pressure water studies. This led to the establishment of much of what might be called the fundamental technology of loop operation. Today these loops run on a routine day-in and day-out basis, with a minimum but not negligible amount of interference with the operating duty cycle of the reactor.

ATR CONCEPT

After the ATR concept was completed, I wrote a report on the reexamination of the capabilities of the ETR as a high flux facility, following the exodus of the ANP program. The aim was to provide an optimum loading of experiments into the ETR to give the highest combination of power density, flux, etc. That report was written really to pose the question, "Should we build an ATR when we have an ETR with no ANP program in it?" The answer was that there is a man in this country who wanted an ATR, and he got it. It is really a little unfair to say that, because certain criteria were set down that could not be met in the ETR in its upgraded state. We could have operated the ETR at $240 \mathrm{MW}$; we could have had a complement of four $3 \times 3$ facilities and five 6 x 6 facilities; and into these latter facilities we could have loaded essentially an array of flux traps very similar to the configuration, that in an optimum sense, was achieved in the ATR. But, 
it is just that difference between the optimization and the square array which allows us to accomplish the ATR objectives at $250 \mathrm{MW}$. It would really require around $480 \mathrm{MW}$ in ETR geometry to do the same thing we do with $250 \mathrm{MW}$ in the ATR.

The lessons that we learned from the design of the ETR were that a little versatility is a good thing, but too much can be a bad thing. This affected the design of the ATR. We first were given to believe that the ATR would be designed to serve the needs of a certain program and that the needs of this program would dominate the design of the reactor. Indeed, they did dominate the design. The modulus size which is established in the Naval Reactor's loops became a point of departure for the design of the ATR. As much as I like to point to the creative aspects of the ATR core configuration, it is little more than what any one of you would arrive at if you were to try to optimize the design for a set of cylindrical tubular loops of a specified diameter with a foot greater length than the ETR could accommodate. The annular fuel surround is an attempt to make use of the principle that if one locates the fission sources equidistant from an experiment, then the probability of a neutron being captured in an experiment is independent of where it originates in the fuel. This allows you to do wild things with the power distribution in the fuel with a second order effect upon the experimental conditions. Hence, the concept of allowing you to operate the reactor in multiple modes of unbalanced power was possible, so that you could simultaneously satisfy the requirements of a number of experiments without having to move them around in the core. While the ATR has been designed and has not yet been operated, this is not because it is not a good design--it is just because it is having construction problems. I don't have to defend the design until it isn't able to achieve full power.

I have drawn this analogy between the design of the ATR and the FTR because Battelle is precisely in the same position now, with this one exception, they do not have as a point of departure a well defined set of experiments. Hence, I think they are being led a little bit down the road of too much versatility. I will raise my voice against this a little bit for I believe that it is possible to make a rubber band too flexible. (On the other hand, when you are spending 75 megabucks \pm 15 , you are entitled to dream of having everything.) I, myself, would do about the same thing--especially if it were my money. You have to end up at some time, however, as we are today, discussing the importance of defining a class of experiments. How can one dispose fuel around these experiments, and what conditions must prevail in that fuel in order to fulfill the requirements? I would like to say that the requirements seem much more crystal clear because they don't necessarily exist. That is a paradox, because we say that--we want a spectrum $75 \%>0.1 \mathrm{MeV}$; we talk about 1 x $10^{16}$; we can talk about these objectives as if they were really necessary objectives, but we would be much harder pressed to defend those objectives as being vital to the LMFBR program. I don't propose to do it, and I don't think anybody in this room really proposes to defend any objective other 
than to speak of it in a philosophical sense. In a philosophical sense, one can say if we are at 5 or $6 \times 10^{15}$ in some reactors now, certainly, you would allow me, for 75 megabucks \pm 15 , a factor of 2 just to give me a little growth in the future. I might say that this is not an unreasonable request, but it may be unrealizable. What it does lead to is a $2 / 3$ MW/1iter power density, which places us in the class of what we might call a high power density machine. It is a little on the low side, but it is still a high power density machine in which burnup effects begin to play a major role on the life expectancy of the fuel. Hence, the nicely evolved figure-of-merit of so many fissions per cubic centimeter deposited in the matrix containing the fuel becomes a way of expressing it.

Now, don't forget we are building this reactor because we want to know how fast reactor fuels perform. It is an odd thing, however, that virtually all of the information about the fuel which we are going to use in this fast reactor comes from thermal irradiations. Very little high burnup data exist for fast reactors. This is another paradox to which I wish to address myself because it turns out not to be so much of a paradox. When one attempts to operate a fuel sample in a thermal neutron environment, we all know that one is led to severe self-shielding problems. Hence, it is almost impossible within practical metallurgical schemes to get the kind of power distribution in the sample and fission distribution across the sample which you would like to have. It is not quite impossible because, of course, we are trying to approximate this in one of the ATR loops. It is very difficult to do, however, and it doesn't take much of a metallurgist to argue that if you don't have the proper temperature distribution, if you don't have the proper nvt on the cladding, if you don't have the proper fission density in the sample and the total fission rate, you do not have a representative test. All you have is fissions per cubic centimeters and temperature and not very much more. What I would like to show you here is some work that we have been doing on a system so far removed from FFTF that if you laugh, I wll not be offended. I am going to talk about the behavior of ceramic fuels in what, for your purpose, would be a fluid-like water, actually in aluminum almost at its melting point.

Cost Analysis

In the cost analysis for the ATR, we were led to one of two choices of fuel: some fuel element that could have an extremely long lifetime, such as was proposed for the $\mathrm{A}^{2} \mathrm{R}^{2}$, or some fuel element that was extremely cheap in construction and could thereby be changed out more frequently without prohibited fuel cost. Because of the optimization problem, which I mentioned before, trying to keep the total power within bounds, the total volume of the fuel elements is 262 liters in the ATR and it 
surrounds, and is in close communion with, a large number of experimental facilities (nine to be exact). A consequence of this is that, if one tries, it is possible to load a stainless steel or a Zircaloy core into the ATR and to get a cycle that is roughly 12 weeks in length, but when one costs this out against the aluminum systems, he comes to only one conclusion. If one had a much more favorable geometry, one could have a critical mass that is sufficiently low; then it would be possible to load a very long lived core of perhaps 90 to 120 days where one could pay four times as much for the fuel, and thereby get an acceptable fuel cost. With the aluminum systems as our goal then, it wasn't a question of desire, it was a question of need.

The MTR fuel element, which was one that could be accomplished within the easy range of 18 wt: Al-U alloy, and which could be rolled very neat1y, was succeeded by the ETR element to which boron was added as a distributed poison to lower the fuel cost and increase the operating lifetime. The distribution problems of the boron in the fuel, as everybody knows, was a monumental problem that was never satisfactorily solved in alloy technology. Powder metallurgy was costed out in the early days against alloy technology in each of the test reactors. It was concluded then, as Jack Cunningham said this morning that, "It's just too damn expensive." I would like to te 11 you that today the fuel elements for the ETR and the MTR are made by powder metallurgical methods as the preferred way. I am not sure that this vendor will be in the business after this contract, but at least he was optimistic enough to enter into the contract and to offer the low bid. In searching for some other form in which the uranium might be contained, the possibility of using the oxides in powder metallurgy was examined and this, of course, is now the reference basis for the ATR fuel element. This prescription was a multiple joint venture, to which we have all become accustomed in the AEC laboratories, between Oak Ridge National Laboratories, Babcock and Wilcox Company, and Phillips Petroleum Company, with some assistance from others. This led, starting from the technology of the HFIR fuel element, to the present prescription of the ATR. Of course, this prescription per se was made because the fabrication problems were contended to be the prime problems to be solved. They were difficult problems, but they were solved much more readily than some of the other problems.

Irradiation Studies

An irradiation program was instituted to find out how this material would perform under the high fission density conditions that would prevail in the ATR and, of course, also in the HFIR. The results of the initial tests were pretty dismal because the first samples which were irradiated in the ETR failed miserably to pass their test. This failure as it turned out, occurred because the matrix of the material exceeded the temperature at which it must operate in either of these reactors, but it led 
us into an interesting discovery of how much you could learn about particulate systems by a study of individual particles. I would like to depart a minute now to sort of tick off the logical possibilities for a solid powder fuel system. If you have a particle (now I am talking for the first moment about system in which the particle can be identified and in which the matrix can be identified and which they do not interact with each other chemically) which retains the fission gases to the desired degree with reasonable volume change, thereby exercising little or no hydrostatic force upon the matrix, this places the minimum requirements upon the matrix. The fuel element only has to hang together and hold its geometry at the requisite temperature. Secondly, you could have a particle completely unable to hold anything but its fissionable material, whereby the gaseous fission products would discharge entirely to its matrix. Then you would have the leaky particle and would require a strong matrix. At the operating temperature the properties of this fuel element would be dependent upon the way that the matrix would accommodate itself to the fission gases. Other possibilities exist in which you could have a combination of the two. of course, the worst combination is the combination of the leaky particle and the weak matrix. This is the one we chose. It turns out that aluminum at the temperature of operation is really not very strong. When I talk about yield strength, I am I am talking about a few thousand pounds per square inch. In the proposed FFTF cermet we are talking about magnitudes at least an order higher than that. It is possible to consider this thing in an abstract sense and trying to do some sealingwax and string-type of research and to examine how a particle in complete isolation would behave; to study this and see if you could arrive at a point of understanding about when you would expect a given particle to retain its fission gases and when you could expect it not to retain its fission gases.

We were fortunate in the sense that we found three apparently usable materials, two of them fairly familiar to you ( $\mathrm{UO}_{2}$ and $\mathrm{U}_{3} \mathrm{O}_{8}$ ), one we $11-$ known and probably about which there is more irradiation data than any other fuel material in existence, but yet which is still the subject of some mystery. This latter is one of intermetallic compounds of aluminum in uranium.

I am going to show you now a picture of an ETR 1oop in which ATR irradiation studies have been carried out.

The loop shown in Slide 1 is installed in one of the $6 \times 6$ holes in the ETR and has no special distinction, because it is a very modest loop in terms of its pressure and temperature capabilities. It is only capable of operating samples in the neighborhood of $250{ }^{\circ} \mathrm{C}$, operates at $900 \mathrm{lb} / \mathrm{sq}$ in., is a feed-andbleed type of loop and is what we like to refer to as our "zerocost" facility. That is precisely why we have it--because it costs us virtually nothing as loops go--about half a million dollars. In this position, however, it does have the benefit 


\section{7}

of a respectably high thermal flux (in the neighborhood of $5 \mathrm{x}$ 1014). Hence, one can achieve fission rates in the sample and heat fluxes in the neighborhood of 2 and $2.4 \times 106 \mathrm{Btu} / \mathrm{hr} / \mathrm{ft}^{2}$ and fission densities, in a few cycles, in the neighborhood of $2 \times 10^{21}$ fissions/cc. So you see that it is capable of studying materials at least to the degree of burnup that is required in the ATR and incidentally the FFTF.

$\underline{\text { SLIDE } 1}$

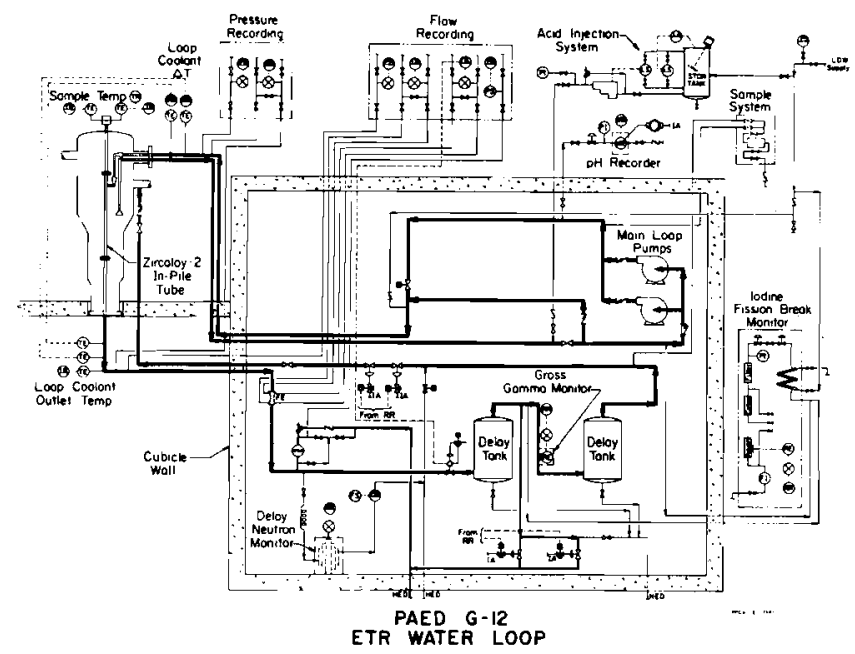

This is a sample train (S1ide 2) which shows how we overcame the problem, which I mentioned about self-shielding (e.g., the use of thin plates). These are loaded into a nolder which simply positions them in the vertical direction. These are essentially vertical experiments because they wouldn't work well in the horizontal position or even, I believe, at an $18^{\circ}$ angle. When we get failure in one of these experiments, we get a simple failure. I feel that we would be in some difficulty if the samples were in a horizontal position. That is one of the reasons that we chose this particular facility, instead of $\mathrm{HB}-2$ horizontal loop already installed in the MTR.

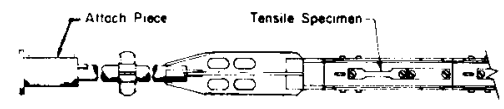

$\underline{\text { SLIDE } 2}$

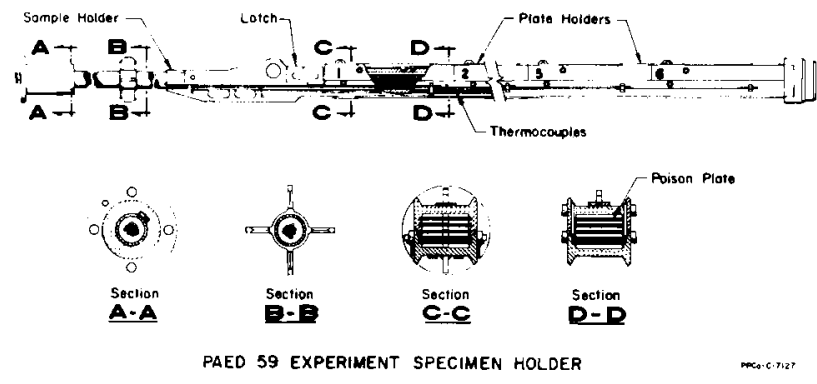


Slide 3 shows a series of cross sections of materials that have been irradiated to $1.6 \times 1021$ fissions/cc and just shows essentially a gross cut across the plate, as polished. On the left you see an intermetallic particle of uranium; on the right you see niobium coated $\mathrm{UO}_{2}$ particles; and in the center is the $\mathrm{U}_{3} \mathrm{O}_{8}$ reference fuel for the ATR. I would like to say that all of these fuel elements, at the temperature of the ATR, operated successfully.

Success is a relative thing, and I would now like to call your attention to the behavior of the separate particles in these systems. On the left in Slide 4, you see the U-A 3 particles. On the right, you see the niobium coated $\mathrm{UO}_{2}$ particles which have become like so much Swiss cheese and which have an interesting behavior. The question this morning was raised as to what the temperature internal to these particles would be. Because these holes are spherical in shape, I would ask the question in another way, "What is the temperature at which the uranium oxide is essentially plastic?" I would not say, and I do not intend to say that the particle is at its melting point, but it is up at a respectable temperature. The central one shows again the agglomeration of the fission products which become identified as thermodynamic gases exerting a partial pressure. On your left are visible the single particles of $\mathrm{U}-\mathrm{Al}_{3}$; actually it can be shown by other information that the particles can either be $\mathrm{U}-\mathrm{Al}_{3}$, can completely react to $\mathrm{U}-\mathrm{Al}_{4}$, or can be surrounded by a little shell of U-A1 4 going into U-A1 3 . In either one of these particles you see essentially the same behavior. You see no evidence of a local accumulation of fission gases. You might immediately say, "Well that's because they get out of the particles." There is strong evidence against this hypothesis.

SLIDE 3

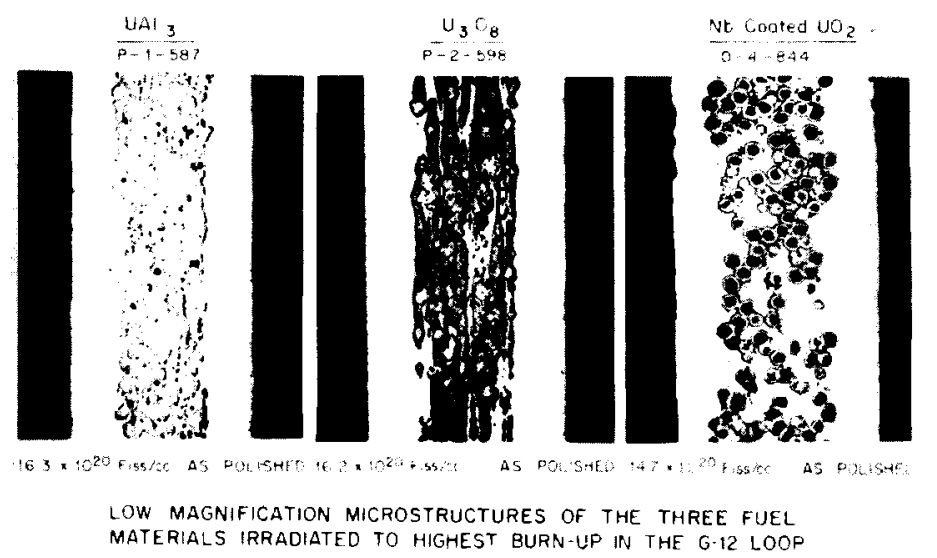


SLIDE 4

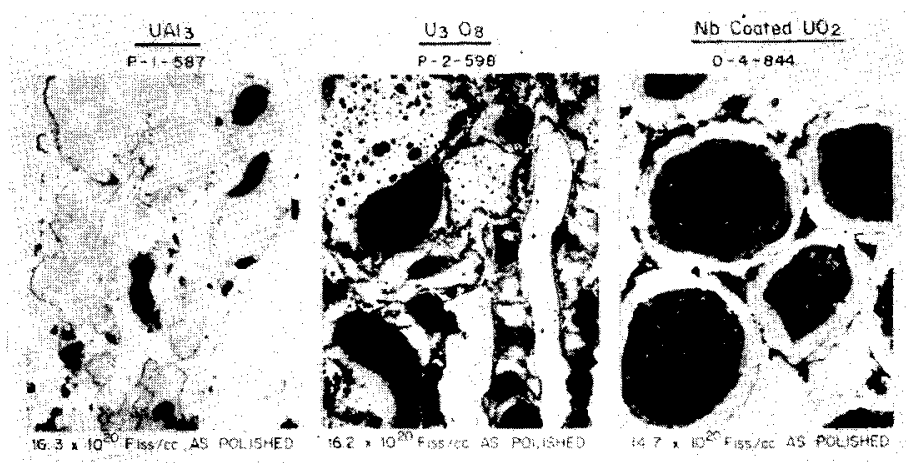

HIGH MAGNIFHCATION MICROSTRLCTURES OF THE THREE FUEL MATERHALS HRFADIATED TO HIGHEST BURN - LPP IN THE G.12 LOOP

I will go back now to fill in the information on the $\mathrm{UO}_{2}$. The aluminum system shows a niobium coated particle and below it a particle of $\mathrm{UO}_{2}$ which is just simply there in the matrix (Slide 5). Look at the coated particle itself which can be identified there. There is no reaction zone which would make it necessary to talk about the heat transfer characteristics. There is some evidence, however, that there might be a thermal barrier in the niobium coated particle because the gases diffuse to the wall and seem to collect there. One would not have to stretch his imagination too much to say that there is a film of fission gases separating these two. Nonetheless, these particles all show that at least at the temperature at which they were irradiated, they were able to retain the gases; hence, this plastic matrix did not blister and did not fail.

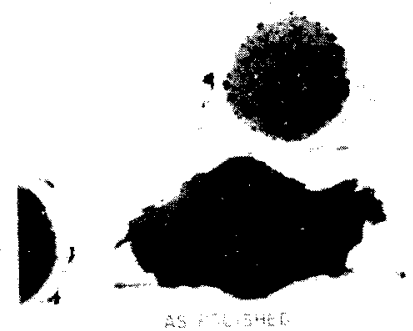


However, as you raise the temperature, as in the irradiation that I mentioned before that had failed in the process water system of the ETR, the examination of the particles indicated that most of the particles had fractured and released the fission gases. Slide 6 shows you what the failure looked like. It was not a cladding failure but actually a splitting of the matrix in two, which is the classical expected behavior of an agglomerated gas in the middle of the matrix where the matrix hasn't got enough strength to accommodate the forces involved; hence, it bulges. This one happened in the reactor, not in the hot cell.

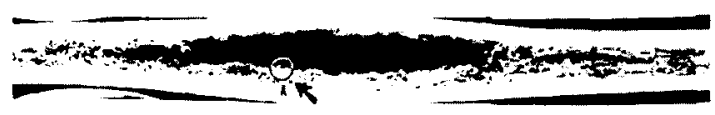

SLIDE 6

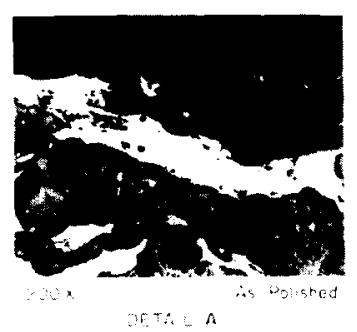

WORGTRUCTURAL DETAL OF UOz PLATE CORE N THE BL:STERED AREA

I am not attempting to scoop Argonne on their own plates, I only want to show S1ide 7 for the continuity of the message I have here. This is a particle of $\mathrm{UO}_{2}$ in an $\mathrm{A}^{2} \mathrm{R}^{2}$ stainless steel cermet plate.

SLIDE 7

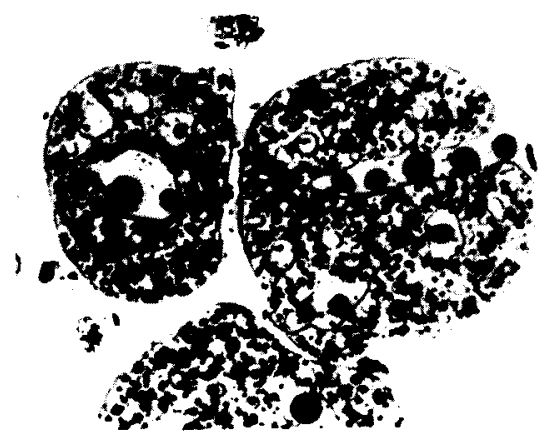




\subsection{1}

Slide 8 shows that plate when it was taken into the hot cell and given a postirradiation anneal to $1060^{\circ} \mathrm{F}$. You see not a cladding failure, but again you see a failure in this cermet as in the previous slide of the matrix. If one examines these particles in detail he sees a similar indication of that release of the fission products from at least a good fraction of the particles. If one examines the density as a function of the irradiation of a low temperature irradiated plate, he sees something like this. This is the high exposure aspect of a low temperature irradiation. You see that the $\mathrm{UO}_{2}$ is distinctly capable of swelling even at the lower temperatures in order to accommodate all the gases produced in the particle. On the other hand, it is odd that $\mathrm{U}_{3} \mathrm{O}_{8}$ is able to exhibit a much smaller amount of swelling, and the intermetallics are correspondingly lower. None of these plates, of course, have reached the point where they would form a blister.

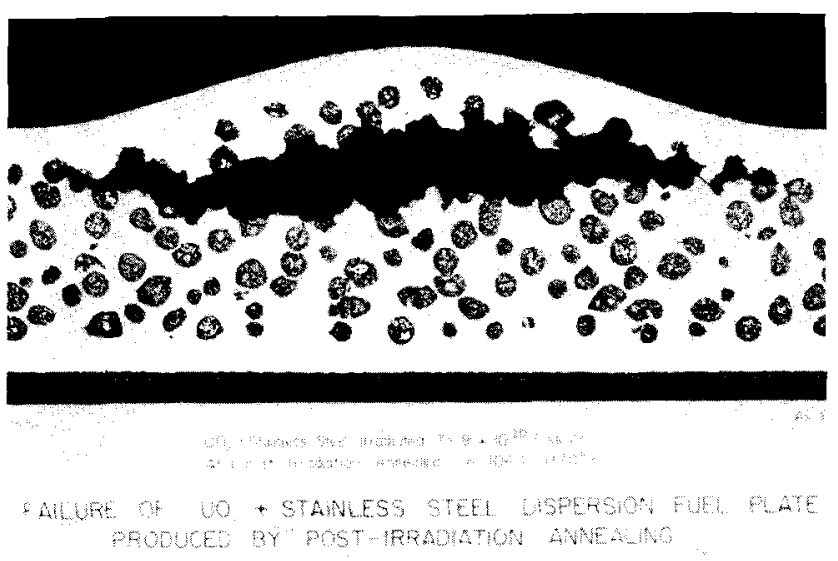

SLIDE 8

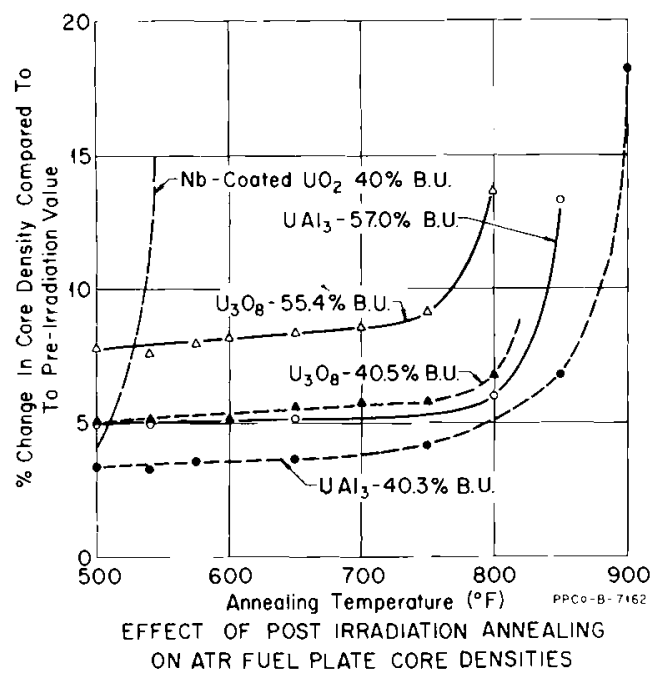

SLIDE 9

If one examines the density versus postirradiated annealing temperature (S1ide 9), he again observes the transition in core density from the point where the particle dimensions are essentially fixed to the point where the particle releases its gases and the matrix begins to exhibit large deformations. This, of course, leads to the formation of blisters; hence, this inflection point is a point beyond which you would not expect to operate those plates at the corresponding temperature. You will immediately say, "But you are talking about operation at a low temperature and annealing at high temperature, and this is not characteristic either in diffusion times, migration, agglomeration, etc., of the operating conditions." 


\section{CONCLUSION}

Let me show, in Slide 10, however, some remarkable features of this simple experiment. If one takes that band shown in the slide (this is essentially the information that is known about the performance of $\mathrm{UO}_{2}$ stainless steel up to a certain point of time, and it hasn' $t$ differed much since then) as a function of irradiating temperatures. These samples were mostly all irradiated at the temperatures indicated, and the band of uncertainty is essentially the uncertainty in the point of failure of a given material at a given fission density. First we look at $\mathrm{UO}_{2}$ alone. When these aluminum plates are blister tested, they fail at $470^{\circ} \mathrm{F}$ when irradiated to $10^{21}$ fissions/cc. The blisters form immediately. $\mathrm{U}_{3} \mathrm{O}_{8}$ in aluminum is able to go just a few hundred degrees higher. Notice, however, the $A^{2} R^{2}$ plates with a stainless matrix. They fall right in the place where one would expect them to fall had those irradiations been performed at the temperatures at which swelling is indicated. These plates were irradiated to the two levels of burnup indicated at low temperatures and postirradiation annealed to the temperatures at which they formed blisters. Just for kicks, the $\mathrm{U}^{-\mathrm{Al}_{3}}$ aluminum plots on a line that, up in the range of high burnups, exceed the irradiation capability of the stainless steel matrix. This is not meant to say that they would be acceptable as a fuel element at those temperatures because we all know that the aluminum is like so much putty at this temperature. But, it does suggest that if it were possible to take the particles indicated and to surround them by a strong matrix, perhaps, you would have the basis of an acceptable fuel.

SLIDE 10

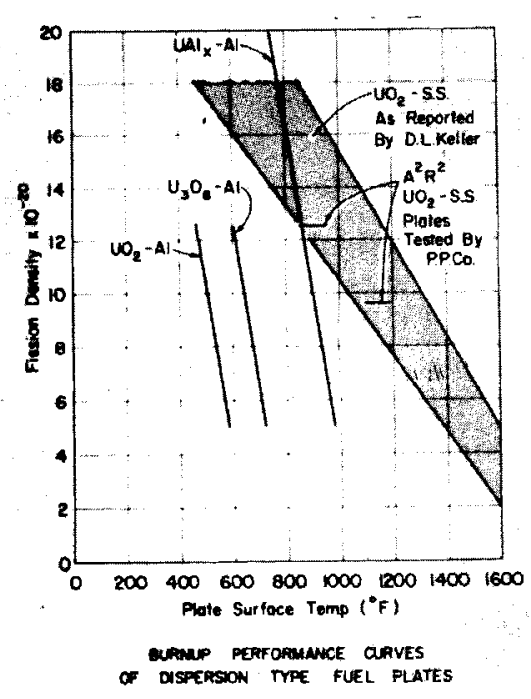


Anyone who has tried to do this will immediately know there exists reactions that occur with the uranium-aluminum intermetallic and with stainless steel. These reactions occur at fairly elevated temperatures, but we have found it possible to build fuel elements which are essentially cermet fue 1 elements with $\mathrm{U}-\mathrm{Al}_{3}$. This is without going to any coating of the particle which, of course, is still a possibility. On the other hand, if one looks at the uranium-aluminum phase diagram, he would say that the intermetallics are thermodynamically unstable compounds and should dissociate into other compounds and essentially move along the horizontal isotherm. However, this is true only at infinite times. What is important is to derive the rate constants exhibited by such a dissociation. In order to address ourselves to this problem we have done some experiments where the U-AlX particles were heated to elevated temperatures and held in an X-ray diffraction system at elevated temperatures of $600,800,1000{ }^{\circ} \mathrm{C}$, and, from the analysis, the rate constants for the decomposition can be determined. It becomes reasonably clear that it is possible to relate the melting point of a intermetallic particle phase and the departure from its melting point on a thermodynamic scale to the usable lifetime of that material. It appears from our preliminary work, and this is by no means a convincing proof, that even this mild-mannered material does have a future at considerably elevated temperatures in a properly strong, nonreacting matrix. The second thing that a11 of this suggests is that you can't really go much further with cermets no matter what you do, unless you either change the particle or go to a matrix stronger than stainless stee 1 . I don't think that this is a surprising conclusion. 

D9.1

APPLICATIONS OF THERMAL TEST

REACTOR EXPERIENCE

D. R. deBoisblanc

\section{DISCUSSION}

MR. MOSS: How much scale formed on those cermet radiations?

MR. deBOISBLANC: On the plates?

MR. MOSS: Yes, externally on the plates.

MR. deBOISBLANC: That, of course, was a prime variable which we were studying in the G-12 10op. Specifically, the formation can be predicted by a correlation developed at Oak Ridge, and it amounts in the ATR to about $1-1 / 2$ to $2 \mathrm{mils}$ of oxide. It stands up well and it also holds the plate temperature up.

MR. WILLIS: You imply a plasticity on the $\mathrm{UO}_{2}$ during radiation. You say it is not a sintering phenomena. Several years back PL was reporting that their materials were cracking during irradiation. There was no indication of plasticity. Do you have any information on this?

MR. deBOISBLANC: It is difficult to convince yourself that the temperature departure from the matrix is much in excess of 500 of so I would say that the most these particles are running is $1,000^{\circ} \mathrm{F}$, but plasticity is a relative thing and the energy of deformation is also an important parameter. The energy of deformation is considerable, therefore this could be a fission rate induced phenomenon. In any case the $\mathrm{U}_{3} \mathrm{O}_{8}$ and $\mathrm{UO}_{2}$ both show spherical gas bubbles, indicating plasticity.

MR. WILLIS: Is it fission rate or parameter level?

MR. deBOISBLANC: We11, our work hasn't answered that. Does Jack Cunningham happen to be here? I think Oak Ridge has some opinions on this subject.

MR。CUNNINGHAM (ORNL): I think there is plenty of evidence to show that a $U_{2}$ is plastic at a remarkably low temperature, below melting.

MR. deHALAS: Everybody observes this same phenomena. It is probably related to burnup because the bubbles don't get that big.

MR。deBOISBLANC: There is a lot of pressure involved. Let me add just one note to what I have said here. I didn't mean to propose a new fuel material. What I meant to propose 
was a new way of looking at an old fuel material. A new way of looking at even uranium oxide. There is a way, it seems to me, of doing this. One has to take account of the separate properties of the matrix and the properties of the particles, the average temperatures at which they are operated, and the departure from that temperature. It is clear that the behavior of the gas within the particle is much less determined by temperature than has been previously expected, and the atoms diffuse around and accumulate at the low temperature. It is not until they receive high temperatures or large burnup that they get out of the particle. But if it is true that a $U_{2}$ particle does give off fission gas at relatively low temperatures, then one should not talk about retention of gases in that particle. One must talk about retention of gases in the matrix. Encapsulate it with a strong matrix that allows it to be molded or whatever you wish and still retain the fission gas. Your are dealing with the properties of the matrix. There is a chance in the intermetaliics that with stainless steel, one could go at the temperatures you propose io operate, into the range of $2 \times 1022$ fissions per $c c$ while yet retaining fission gases in the particle.

MR BURNHAM: Do you propose that all you need is a barrier between the fuel particle for $\mathrm{UO}_{2}$ steel?

MR。 deBOISBLANC: If you didn't know all the things wrong with that simple idea, I think there is much to be learned about intermetallics and a good fraction of my technical 1 ife has been spent in worrying about the technology of the intermetallics in just one system. There are many other intermetallic compounds--one should look at the available properties of them. You see they have indeed some remarkable properties. But believe it or not--believe it, I should say, these are indeed stoichiometric compounds and can be separately retained for appreciable periods of time, identifying themselves as UAL 2 and UAL ${ }_{3}$. The equilibrium phase diagram does not provide the information to determine how long such a compound will persist when isolated. This must be approached by reaction kinetics. You can get in reasonable amounts of uranium, certainly far in excess of the FFTF loading requirements. Therefore, it is a class of materials. that should be included and I propose to consider it. 
LIQUID PLUTONIUM FUEL SYSTEMS - THEIR STATUS AND CAPABILITIES*

W. H. Hannum and L. D. Kirkbride

University of California

Los Alamos Scientific Laboratory

Los Alamos, New Mexico

\section{INTRODUCTION}

This morning Gene Astley indicated that a wide variety of fuels would be considered for FFTF driver fuels. We would like to suggest the possibility of using a liquid plutonium driver fuel for FFTF. We do not consider liquid plutonium to be in the class which Don deHalas so pointedly described as "God knows what else." Perhaps the reason liquid metal fuels are sometimes put in this class is not so much a lack of technology, as the fact that you do have to change your point of view somewhat when thinking about them. One fact is clear: liquid plutonium does not fit into the same category and classification with most solid fuels.

What we propose to describe is not a design of FFTF. Even if a design of FFTF based on liquid plutonium were to be done, it is not at all clear that 1 iquid plutonium would be the best driver fuel for FFTF. There are, however, certain very attractive features of such a fuel system which, we feel, warrant its being included in our considerations. We will describe first an illustrative survey calculation of FFTF, using liquid plutonium, to show that it is possible to obtain satisfactory performance. We will also comment on the flexibility of such a system-the safety and stability, which is the area where this fuel offers major advantages for this application. We will touch very briefly on what further developments might be required to make such a system realizable. Then we will discuss the material studies that have been done which illustrate the capability and performance characteristics of such a fuel. For that last item, I will turn the microphone over to Dale Kirkbride. Certainly, in the limited time available, we cannot hope to discuss fully all of these areas. But should there be any interest, we will be more than happy to go into details with anyone.

\section{DESIGN AND PERFORMANCE CONSIDERATIONS}

The material we will be discussing is based largely on two sources: first, is the design and operation of LAMPRE I, a 1 MW reactor experiment, which has been completed; second, the major source of information, other than the few survey

* Work done under the auspices of the United States Atomic Energy Commission 
calculations for FFTF, is the detailed design (which is well under way) of a $20 \mathrm{MW}$ experiment called MPBE. I might comment at this point that during the last six to nine months of LAMPRE I operation, an actual 69\% load factor was achieved. This might be of interest relative to the discussion this morning on plant availability.

The illustrative case (we have considered) is based on information received from BNW, particularly from Winston Little. The design from which we started was what Les Finch and Peter Hoffman described this morning as the "More-or-Less Reference Case," a 600-1iter, 61-module core. It is not of interest to do a direct substitution of liquid plutonium fuel elements into the design, because of the different limitations and performance that one faces with a liquid fuel as compared with a solid fuel. In particular, much of the discussion this morning on the design optimization for the cermet and ceramic fuels was based on the centerline temperature (i.e., How hot can you operate the centerline before you run into difficulties associated with fuel melting?) Obviously, you don't worry about fuel melting, if the fuel is already molten. For liquid fuels, centerline temperature is not a serious concern. We do not know with any certainty what the centerline temperatures are, and we do not care. They are far from any phase change. The limiting factor considered is the temperature of the interface between the liquid fuel and the container. The basis for selection of the design temperature will be described by Dale Kirkbride in the second half of this presentation. So, if you are not worried about centerline temperature, the first thing you do is to go to a much larger capsule than Peter Hofmann was talking about this morning. The LAMPRE I experiment operated with capsules approximately 0.42 in. OD. This capsule dimension has been used for all the survey calculations which will be discussed today. This is certainly not the only capsule diameter that could be used, and if it happened to be optimum in any respect, that would be a coincidence.

For the illustrative cases, a power level of $400 \mathrm{MW}$ was selected. The initial parameters chosen are shown in slide 1. The core height is reduced somewhat from that used in the Battelle study. This reduction is not absolutely necessary, but it is convenient for our calculations. The process tube size for the distance across flats was specified as that used by Battelle, or larger; that is, 4.388 in. or greater across flats. The number of subassemblies used in the core is 61; the subassembly 1 iners are separated by about 0.35 in. Sodium coolant velocity was 1 imited to $30 \mathrm{ft} / \mathrm{sec}$. A sodium inlet temperature of $460 \mathrm{C}$. selected, which is substantially higher than that presently used with solid fuels. This temperature is selected because it is safely above the melting point of this fuel alloy. Thus, the entire system will operate at modern sodium temperatures.

The first problem is to find a convenient parameter that will give some illustration of the sort of performance you can achieve in this type of system. One convenient parameter was 
the subassembly dimension across flats. The 4.05 in. 1 iner size is the size used by Battelle in their calculations. If some of the 61 subassemblies do not contain fuels that contribute to the power production, the interface temperatures get somewhat higher than we would like, as shown in Slide 2 . We, therefore, choose to go to a somewhat larger subassembly size. We more or less arbitrarily picked a 500 liter core. That allows, among other things, a 4-1/2 in. diam subassembly for the test holes. The peak flux for any of these cases is essentially 1016 . The cases selected were all (by definition) limited to $400 \mathrm{MW}$. At this power level, there is considerable margin in the fuel-clad interface temperature. If the power were raised by $10 \%$ (to $440 \mathrm{MW}$ )

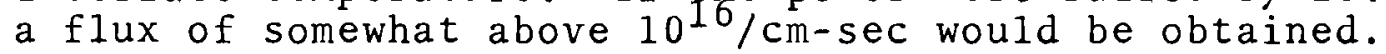

These fluxes are not dependent on a prior selection of fuel composition. Essentially, the flux is determined by the available heat transfer surface. The interface temperature is a slowly varying function of the number of inert subassemblies that are inserted into 61 available spaces. This means that it is quite reasonable, without violating any $1 \mathrm{imit}$, to put in perhaps as many as 16 out of 61 subassemblies as nonreactivitycontributing tests. These might be low-enrichment fuels; they might be fuels with multiple containment, thereby having a reactivity penalty associated with them; or they might even be materials tests. These can be operated in the peak flux region.

\begin{tabular}{|c|c|}
\hline \multicolumn{2}{|c|}{$\frac{\text { BASIS OF STUDY OF LIQUID PLUTONIUM }}{\text { AS FFTF DRIVER FUEL }}$} \\
\hline POWER & $400 \mathrm{MN}$ \\
\hline CORE HEIGHT & $24 \mathrm{IN}$. \\
\hline $\begin{array}{l}\text { PROCESS TUBE SIZE } \\
\text { (ACROSS FLATS, OUTSIDE) }\end{array}$ & $\geq 4.388 \mathrm{IN}$ \\
\hline NUMBER OF SUBASSEMBLY SITES & 61 \\
\hline $\begin{array}{l}\text { THICKNESS (STRUCTURE, ETC.) } \\
\text { BETWEEN SUBASSEMBLY LINERS }\end{array}$ & $0.338 \mathrm{IN}$. \\
\hline NA COOLANT VELOCITY & $30 \mathrm{FT} / \mathrm{SEC}$ \\
\hline NA INLET TEMPERATURE & $860^{\circ} \mathrm{F}\left(460^{\circ} \mathrm{C}\right)$ \\
\hline $\begin{array}{l}\text { FUEL CAPSULES (TA-5W) } \\
\text { OUTSIDE DIAMETER } \\
\text { WALL THICKMESS }\end{array}$ & $\begin{array}{l}0.420 \mathrm{IN} . \\
0.020 \mathrm{IN} .\end{array}$ \\
\hline $\begin{array}{l}\text { PINS PER SUBASSEMBLY: } \\
\text { FUELED CAPSULES } \\
\text { GUIDE RODS }\end{array}$ & $\begin{array}{r}58 \\
3\end{array}$ \\
\hline
\end{tabular}

SLIDE 1

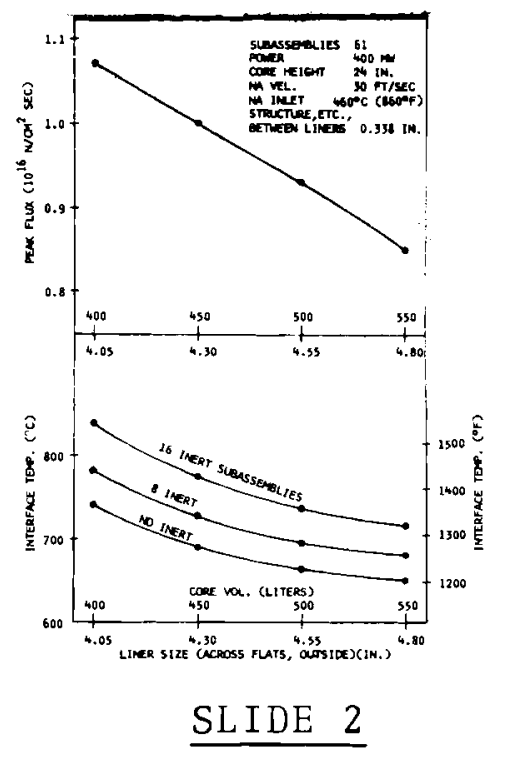

Going on to the selection of a fuel material, we obviously have to get this thing critical. Slide 3 shows a summary of the parameters for the reference case. This case uses a liner size sightly in excess of 4-1/2 in. The core volume is 500 liters. The flux, based on two-dimensional calculations, is $1 \times 1016$." 
The spectrum is slightly harder than that which has been spoken of by the Battelle people; it is about $80 \%$ above $0.1 \mathrm{MeV}$. The median fission energy is about $410 \mathrm{keV}$. It is assumed that there are 8 inert subassemblies and 53 fuel subassemblies. The sodium temperatures are comparable to those quoted in design studies of power reactors.

The loadings for a variety of cases are shown in Slide 4. This figure illustrates that the loading is basically a function of the average fuel density. The amount of diluent can be compensated for quite directly by increasing the loading density in other fuel pins. If the average fuel density is about $4 \mathrm{~g}$ $\mathrm{Pu} / \mathrm{Cc}$ of fuel and we want to put in $30 \%$ of the core as inert test, we simply increase the loading in the remaining pins by $30 \%$ (this is still an acceptable alloy), and the core is operable again.

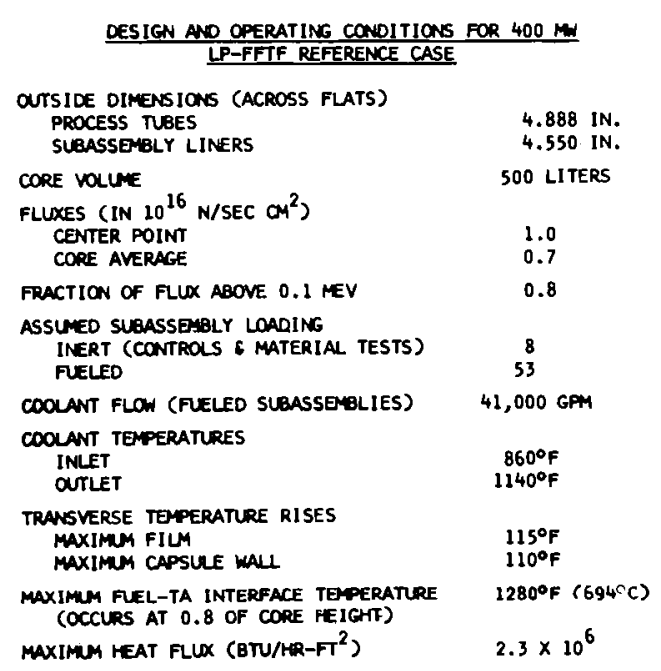

SLIDE 3

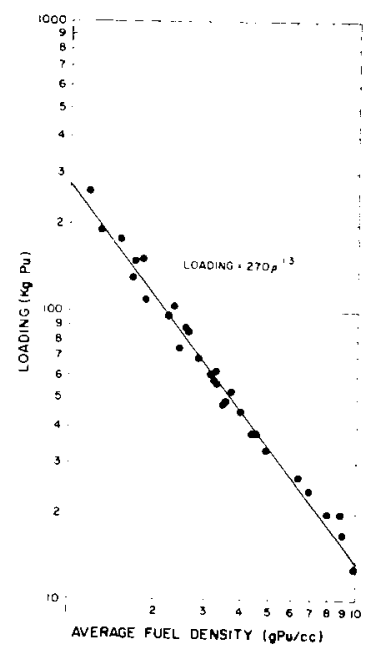

SLIDE 4

The fuel properties of the reference case are described in Slide 5. Following the lead of Battelle, a zone loading is used. Zone sizes and relative loadings are the same as the Battelle design. The plutonium density in the fuel varies from slightly under 3 to almost $4 \mathrm{~g}$ Pu/cc. The power in watts per cubic centimeter of fuel is essentially flattened by this zoning. The burnup varies from 3 to almost $5 \%$ of the plutonium, or a constant $3.3 \times 10^{20}$ fissions $/ \mathrm{cm}^{3}$ average in each zone, per six weeks cycle. This is certainly not felt to correspond to the limit of burnup for the fuel, but as we will mention again later, we do not know what parameter or physical property will determine the burnup limitation of this fuel. Presumably, the fuel can be left in for several cycles, and a partial reloading (as Battelle has mentioned) would be used with this. 


\section{5}

The data shown in Slide 6 illustrate the control swing that can be designed into such a reactor as this. (These particular numbers happen to be for the MPBE.) As Peter Hofmann has suggested, two types of control are used. We use moving fuel for gross control and inert reflector poison control elements on the periphery for the fine control. The total worth for this experiment is comparable with that suggested to be appropriate for the design of FFTF.

FUEL CHARACTERISTICS FOR $400 \mathrm{MW}$ LP-FFTF REFERENCE CASE

\section{A. CORE REGIONAL DATA \\ 6-WEEK CYCLEX}

CONTROL WORTH CALCULATIONS

\begin{tabular}{cccccc}
$\begin{array}{c}\text { CORE } \\
\text { REGION }\end{array}$ & $\frac{G P U}{\text { CC FUEL }}$ & $\frac{W}{C C \text { FUEL }}$ & $\frac{1}{\text { BUPNUP }}$ & $\frac{10^{20} \text { FISS. }}{\text { CC FUEL }}$ \\
\hline 1 & 2.67 & & 2920 & 4.60 & 3.3 \\
2 & 2.85 & & 2920 & 4.30 & 3.3 \\
3 & 3.17 & & 2920 & 3.87 & 3.3 \\
4 & 3.81 & & 2920 & 3.22 & 3.3
\end{tabular}

$\begin{gathered}\text { Fuel } \\ (\mathrm{g} \text { Pu/cc })\end{gathered}$
6.2
6.2
8.0

Temp.
$\frac{\left({ }^{\circ} \mathrm{C}\right)}{530}$
160
530

\begin{tabular}{|c|c|}
\hline Control & $\begin{array}{l}\text { Worth } \\
(\bar{q} \Delta \mathrm{k})\end{array}$ \\
\hline $\begin{array}{l}\text { Total } \\
\text { Fueled Only } \\
\text { Inert Only }\end{array}$ & $\begin{array}{r}11.3 \\
7.3 \\
4.6\end{array}$ \\
\hline Total & 10.7 \\
\hline $\begin{array}{c}\text { Total } \\
\text { Inert Only }\end{array}$ & $\begin{array}{l}9.3 \\
3.3\end{array}$ \\
\hline
\end{tabular}

PU-239 $407 \mathrm{KG}$

PU-240 $45 \mathrm{KG}$

MWUTIPLY BY NUMBER OF CYCLES SELECTED FOR FUEL LIFETIME.

SLIDE 5

SLIDE 6

The spectrum of a system containing no moderating components is hard. The dashed curve of Slide 7 is a bar graph for the spectrum of a typical molten plutonium-fueled fast reactor. This does not happen to be for this particular design but is for a similar case. The solid lines typify the spectra obtained in the ceramic-fueled $1000 \mathrm{MWe}$ studies. You can see that in the very high energy range (namely, that energy range in which $n-\alpha$ cross sections are significant) the spectrum is representative of that for the large power reactors. In the range around $1 \mathrm{MeV}$, the $f l u x$ is higher. There are no resonance neutrons in this type of reactor.

The major areas of advantage for this type of system are stability and safety. The stability of the system is based, in principle, on the large negative feedback coefficient from fuel expansion which overrides all other coefficients. Our confidence is based on our experience in operating LAMPRE I, a very stable system. 


\section{6}

The basic safety of the system is eatablished by the following three factors:

- All feedback coefficients are favorable; there is no significant positive coefficient.

- The over-all tolerance of the system to overpower, overtemperature, and overreactivity is great.

- We have eliminated a major autocatalytic mechanism, namely, fuel slump.

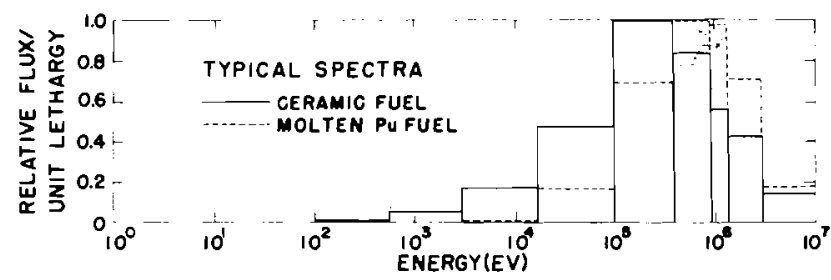

SLIDE 7

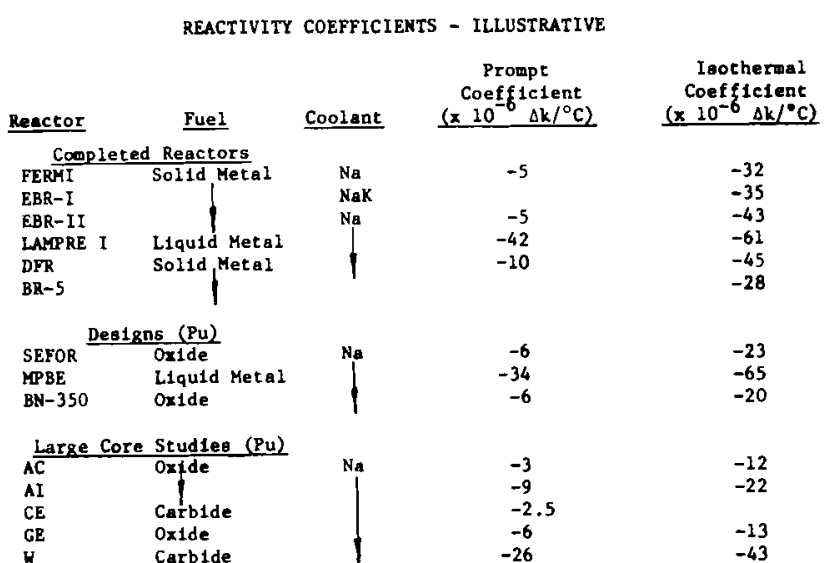

SLIDE 8

In Slide 8 we have listed the prompt coefficients and isothermal coefficients of a number of reactors: those that have operated, those that have been designed in detail, and a few large core studies. As Mr. Shaw indicated in the JCAE hearings last week, all fast reactors operated to date have been stable. He failed to mention that all fast reactors that have operated in this country to date have used metal fuels. There may be a correlation between those two statements.

The prompt coefficient that stands out as being tremendously larger than any of the others is that associated with LAMPRE I. This is based exclusively on the fuel expansion coefficient. The container has a very low expansion coefficient; therefore, the entire volumetric expansion of the fuel is effective in removing fuel from the core. This yields a very large coefficient, three times that available with a solid metal fuel. Of those cores that have been designed in detail, the liquid metal fuel is outstanding in its prompt coefficient, and the total isothermal coefficient remains substantially in excess of that available in other fuels. 


\section{7}

As Gene Astley suggested this morning, a very large reactivity coefficient is advisable, in that it may be desirable to test large samples of fuels (such as oxides) to the point that they can be expected to slump and add reactivity. It would be very convenient in the design of a test reactor if there were a coefficient adequate to override such reactivity insertions.

As for the transient performance of such a system, we must again turn to the design of the MPBE to obtain numbers. While these would not necessarily apply directly to FFTF, they are certainly illustrative of the sort of response such a system could give. If you have a traditional control rod withdrawal accident (withdraw one control rod at its maximum speed starting at a power of $1 \mathrm{~kW}$ ), after $30 \mathrm{sec}$ you will find that the reactor power of this $20 \mathrm{MW}$ core has gone up to $4 \mathrm{MW}$. Therefore, it would seem that removing one control rod at its maximum rate would be a very reasonable way to start this core up, and would not constitute an accident at all. If you increase the reactivity ramp to 20 cents/sec, again you will find that this is a very reasonable way to start the reactor up. If you withdraw one control rod at maximum speed at full power, the feedback coefficient is so strong that nothing significant happens. At full power, if al1 control rods are withdrawn from core mid-height (which is their maximum worth position) and at their maximum rate, we find that after $15 \mathrm{sec}$ the rated core power has doubled, and none of the temperatures are at all seriously out of 1 ine. Again, this is a very modest transient, and certainly, no damage occurs. If we insert $1 \$ / \mathrm{sec}$ we find that after about $10 \mathrm{sec}$ when we have inserted ten dollars worth of reactivity, the power has climbed to roughly three times nominal power, and the temperatures are a bit high. We would not want to have the system in this condition for more than a few hours. Again, that is not a very serious accident. The first trouble you will get into with this sort of system is sodium boiling. This occurs, for example, on a $1 \$ / \mathrm{sec}$ ramp, after 13 dollars of reactivity has been inserted; that is $13 \mathrm{sec}$. It seems unlikely that you could not have done something by that time.

There are some other factors you can talk about with regard to the stability of the system, and one of these is transfer functions. Transfer functions, as measured in LAMPRE I, are shown in Slide 9. About the only thing you can learn from this slide is that transfer functions are really very dul1 in this sort of system; nothing happens. One other situation you can get into is a sudden power demand. Slide 10 shows the experimental results obtained with LAMPRE I while operating at $51 \mathrm{~kW}$ when $11 / 2$ times that power was demanded. The power simply adjusts itself to account for the new demand. In fact, this was the way the reactor was operated. When we wanted to 
raise the power, we just increased the power demand, and the power went up with no overshoot. The second curve of this figure shows the case of an initial power of $45 \mathrm{~kW}$, with a demand of $100 \mathrm{~kW}$ (4.5\% to $14.5 \%$ of full power). Again, the power comes up without event.

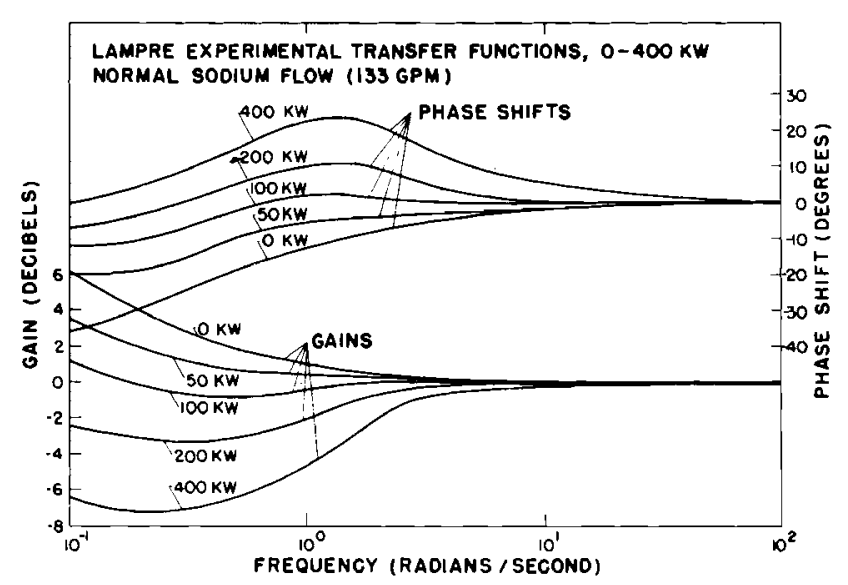

SLIDE 9

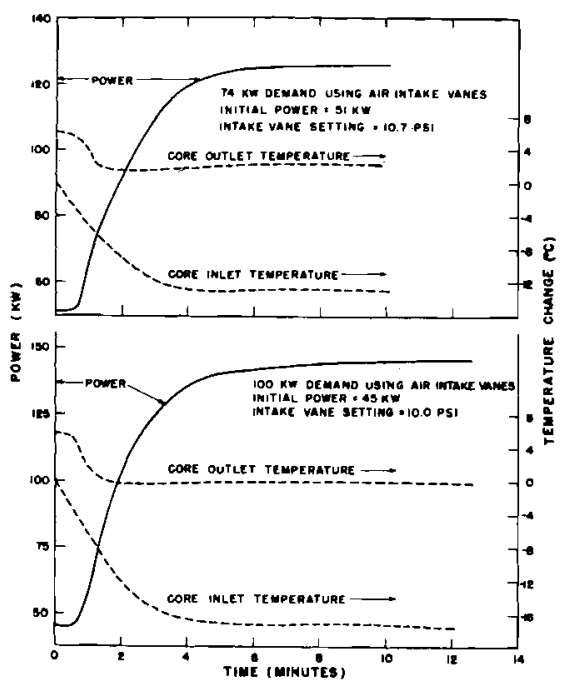

SLIDE 10

In the range of the more serious accidents, which impose very large reactivity insertions, you again have to assure yourself that the system will suffer no damage. A series of calculations has been done for cases in which the system starts from prompt critical. Reactivity is. inserted at 10 cents, 1 dollar, 10 dollars, and up to 100 dollars per second. Slide 11 shows the temperature rises for various cases; all are quite nominal. At extreme ramp rates, the internal pressure in the capsule can get so high as to damage the capsule. In this realm, the presence of the fission gas bubbles actually may decrease the severity of given ramp rates. It may be noted that for credible ramps, the pressure rises are quite nominal. A few tens of pounds per square inch are obtained for a reactivity ramp insertion of $10 \$ / \mathrm{sec}$; that is not going to be damaging.

If you continue to insert reactivity beyond the first pulse so that a cumulative effect of these temperature and pressure rises occurs, you will eventually get into trouble. Peter Hofmann erased the data of his curve above $10 \$ / \mathrm{sec}$; mainly for laughs, we left $500 \$ / \mathrm{sec}$ on Slide 12. Of course, that is an utterly ridiculous number. The peak power does get rather large as you get these astronomical insertion rates, and the time to the first power maximum becomes shorter. Therefore, the more frequent will be repetition of criticalities. The maximum pressure again builds up, and at some point is going to be damaging; however, the 
maximum temperature $\mathrm{rise}$ is not severe. If you go to this fantastic $500 \$ / \mathrm{sec}$ reactivity ramp, you will find that the equivalent burst energy is about $41 \mathrm{~b}$ of $\mathrm{HE}$.

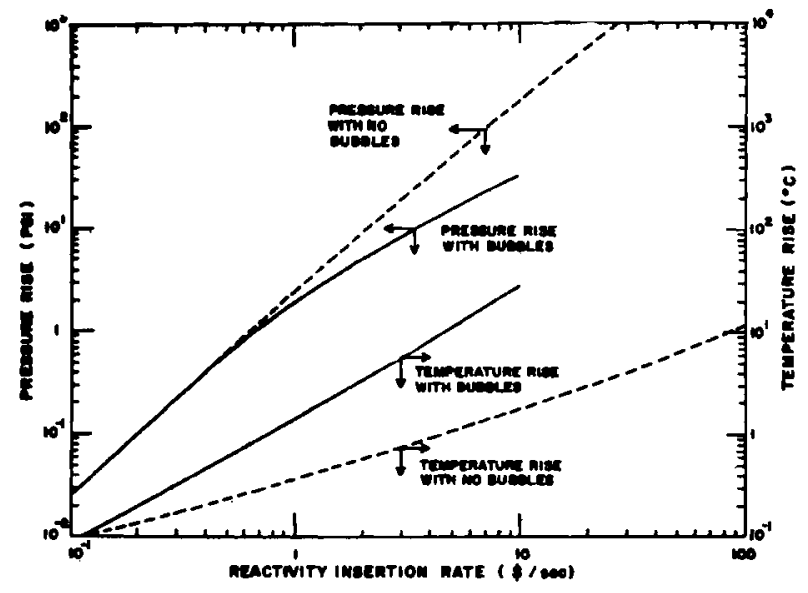

SLIDE 11

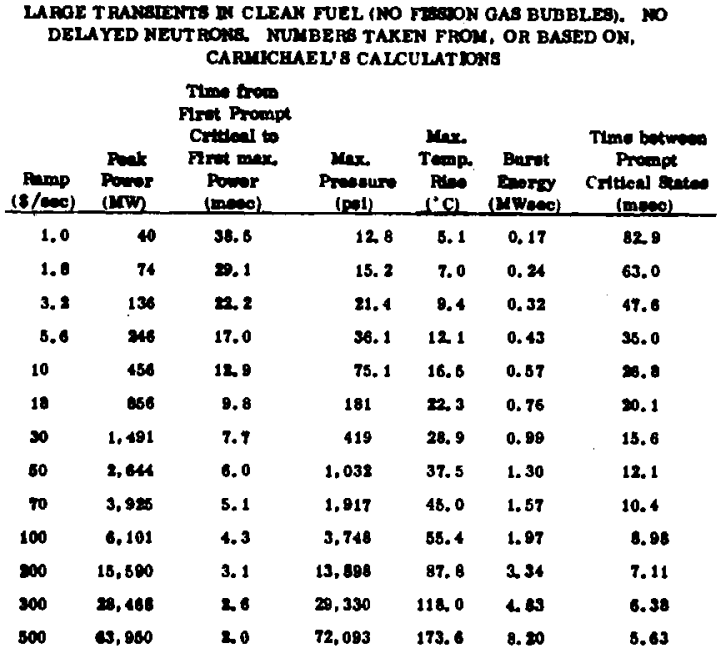

SLIDE 12

Slide 13 shows where we may possibly get into trouble with successive pulses of very large ramp rates. The time prior to obtaining excessive stresses is quite long (many seconds) for these lower ramp rates, and the reactivity coefficient associated with the sodium will be effective. In the very short range, where a very small fraction of a second elapses prior to damage, the sodium expansion coefficient will not help. By inserting reactivity at $10 \$ / \mathrm{sec}$, you can insert a total of $\$ 40$ reactivity, and the system will not fail. At $40 \$ / \mathrm{sec}$, you can insert approximately $\$ 20$ worth of reactivity, and the system will not fail. These safety calculations were conservative, in that delayed neutrons were not included; these will help to taper the later pulses so that the buildup will not be as rapid.

SLIDE 13

CAPSULE FAILURE TEM PERATURE VERSUS RAMP RATE

\begin{tabular}{|c|c|c|c|c|c|c|}
\hline \multirow[b]{2}{*}{ 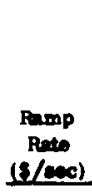 } & \multirow[b]{2}{*}{$\begin{array}{c}\text { Max. } \\
\text { Ceppule } \\
\text { Woll } \\
\text { straed } \\
\text { (pol) }\end{array}$} & \multirow[b]{2}{*}{$\begin{array}{c}\text { Capoule } \\
\text { Yiold } \\
\text { Temp. } \\
(\mathrm{C})\end{array}$} & \multicolumn{2}{|c|}{$\begin{array}{l}\text { Buened on Fuel } \\
\text { Cooffictent Culy }\end{array}$} & \multicolumn{2}{|c|}{$\begin{array}{l}\text { Ineludiag Badium } \\
\text { Conffletere }\end{array}$} \\
\hline & & & $\begin{array}{c}\text { Reactivity } \\
\text { Inserted } \\
\text { (\$) }\end{array}$ & $\begin{array}{l}\text { Timo } \\
\text { To } \\
\text { Ytold } \\
\text { (uec) }\end{array}$ & $\begin{array}{c}\text { Reactivity } \\
\text { Inserted } \\
\text { (\$) }\end{array}$ & $\begin{array}{l}\text { Timo } \\
\text { To } \\
\text { Yiold } \\
\text { (cec) } \\
\end{array}$ \\
\hline 1.0 & 1,250 & $>2750$ & $>37.50$ & $>37$ & $>64$ & 264 \\
\hline 1.8 & 1.270 & $>2750$ & $>37.50$ & $>20$ & & \\
\hline 3.2 & 1,330 & $>2750$ & $>37.50$ & $>11$ & & \\
\hline 5.6 & 1.480 & $>2750$ & $>37.50$ & $>6.7$ & & \\
\hline 10 & 1.870 & 2650 & 35.90 & 3.6 & & \\
\hline 28 & 2.930 & 2500 & 33.40 & 1.85 & $\geq_{40}$ & $\geq 22$ \\
\hline 30 & 5,170 & 2150 & 27.70 & 0.92 & & \\
\hline 50 & 11,100 & 1650 & 19.60 & 0.39 & & \\
\hline 70 & 19,840 & 1380 & 15.25 & 0.20 & & \\
\hline 100 & 37,860 & 650 & 3.43 & 0.034 & 3.43 & 0.09 \\
\hline
\end{tabular}


In comparison with the cermet, there are two major advantages in the expansion coefficient: first, there is no chance of a substantial time delay for heat transfer; second, when the cermet does expand, only a linear expansion coefficient is effective while for the liquid fuel, the entire volumetric expansion is effective.

The safety features of a liquid-fueled system may be summarized as follows: The large negative feedback coefficient allows this system to operate under conditions that would be extremely damaging to any other type of system. If there were an accident where very large amounts of temperature, pressure, or reactivity were involved, the materials in the core are such that they will not undergo any substantial change in properties. With regard to the autocatalytic mechanisms, the sodium void coefficient for such systems should not be substantially different from any of the other systems we have spoken of today. (It will be somewhat less negative because of the tantalum captures.) We have eliminated fuel slump-the fuel obviously cannot melt and slump and add reactivity.

In terms of the status of liquid fuels (to illustrate what can be expected from them), we have listed in Slide 14 some data from the LAMPRE I operation, now completed. We have 1 isted in the slide the $20 \mathrm{MW}$ experiment under construction at the moment, and we have listed an illustrative case of what might be done with this type of fuel system in the FFTF. The first two columns of this figure are for the operation of LAMPRE I. This experiment ran at $1 / 2 \mathrm{MW}$ for most of the operation; there were 13 days of operation at $1 \mathrm{MW}$. At $1 / 2 \mathrm{MW},{ }_{3}$ the central pins in LAMPRE I operated at approximately $450 \mathrm{~W} / \mathrm{cm}^{3}$; at $1 \mathrm{MW}$, of course, it was $900 \mathrm{~W} / \mathrm{cm}^{3}$. The average number of grams of plutonium fissioned per cubic centimeter for the operation was slightly over 0.1 total.

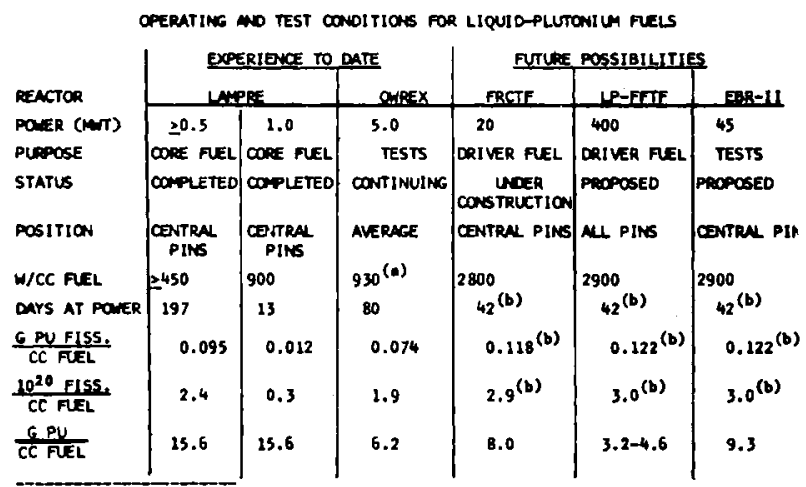

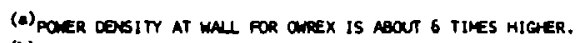

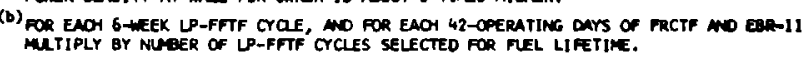


There are two tests running in a thermal reactor now (the Omega West Reactor at Los Alamos). In these tests, the power density within the fuel is about the same as that obtained in LAMPRE I at MW. One of the experiments, which is presently in core, has operated for 80 days. The burnup level is presently about 0.075 grams plutonium fissioned per cubic centimeter of fuel. In the illustrative case for FFTF, the power density within the fuel is three times that of these tests. In the FFTF column, the grams plutonium fissioned per cubic centimeter per cycle is about what has already been observed on massive samples in LAMPRE I. It is our expectation that the burnup capability of this fuel would be many cycles, but that has not yet been demonstrated. The $20 \mathrm{MW}$ experiment, which is under construction now, will have approximately the same power density (W/CC fuel), and again will have about the same grams plutonium fissioned per cubic centimeter for each cycle of operation as FFTF.

If there is concern over this factor of 3 extrapolation in power density, this particular item can be checked by utilizing the variable density of plutonium in the fuel. In testing in a fast reactor, specific power remains approximately constant, being proportional to the flux. By making the fuel density three times that of the reference case, we obtain power densities in the capsule about three times that which would be obtained for the normal fuels. That would allow a demonstration of the capability of operating at this specific power.

$$
\underline{\text { PART II (L. D. Kirkbride) }}
$$

\section{MATERIALS CONSIDERATIONS}

In this section of the liquid plutonium fuel presentation, we will discuss the materials properties and data appropriate to this fuel system. Slide 1 shows the phase diagram of the present reference fuel - - the Pu-Co-Ce ternary alloy. Before discussing this fuel in any detail, it may be appropriate to point out that we will be talking about three distinct liquid plutonium fuels during the course of this discussion: this (Pu-Co-Ce) ternary alloy (melting point $435^{\circ} \mathrm{C}$ ); the binary Pu-Fe alloy, which was used in LAMPRE I (eutectic melting point $410^{\circ} \mathrm{C}$ ); and plutonium itself (melting point $640^{\circ} \mathrm{C}$ ). The system that we are proposing for the Fast Reactor Core Test Facility first experiment (MPBE), for this (FFTF) application, and for large power reactors would use a Pu-Co-Ce fuel. The comparison of $\mathrm{Pu}-\mathrm{Fe}$ results with those for $\mathrm{Pu}-\mathrm{Co}-\mathrm{Ce}$ is analogous to the comparison of sulfides with nitrides with carbides with oxides; the two alloys are different beasts, even though they are both used in the liquid state. 


\subsection{2}

The area of interest in the ternary phase diagram is the low melting valley that runs at almost constant cobalt concentration. The compositions of interest are in the vicinity of 40 wt: plutonium. The valley, however, extends from zero grams plutonium per cubic centimeter up to 12 grams plutonium per cubic centimeter. All the compositions in this range have melting points below $443^{\circ} \mathrm{C}$. This allows large flexibility in loading.

The next point which may be worthy of note is that, with this fuel system, we do not observe gross failures such as those seen in oxide, carbide, or other solid fuel elements. With solid fuel, failure normally arises when the cladding is strained by the swelling of the fuel. While there was one gross failure in LAMPRE I, j.t was not caused by fuel swelling. Since our emphasis has shifted to the Pu-Co-Ce system, we have not seen gross failure, and we do not expect to see it. We have to pick some criterion, however, to use in our testing program. We have picked as a criterion that no detectable plutonium shall reach the surface of the cladding material. The limit of detection for surface contamination is about $1 / 100$ of a microgram. A quantity as small as this obviously has no operational significance in a reactor. However, there is a possibility (the situation was observed in LAMPRE I) that, if enough diffusion is allowed to occur through the grain boundaries in the material, a quantity of plutonium (sufficient to constitute a thermodynamic entity) could be transported through the capsule wall. Thus, the complete stoppage of this diffusion is the objective for our materials development program. If there isn't any diffusion, then a transport mechanism can't be developed by this process.

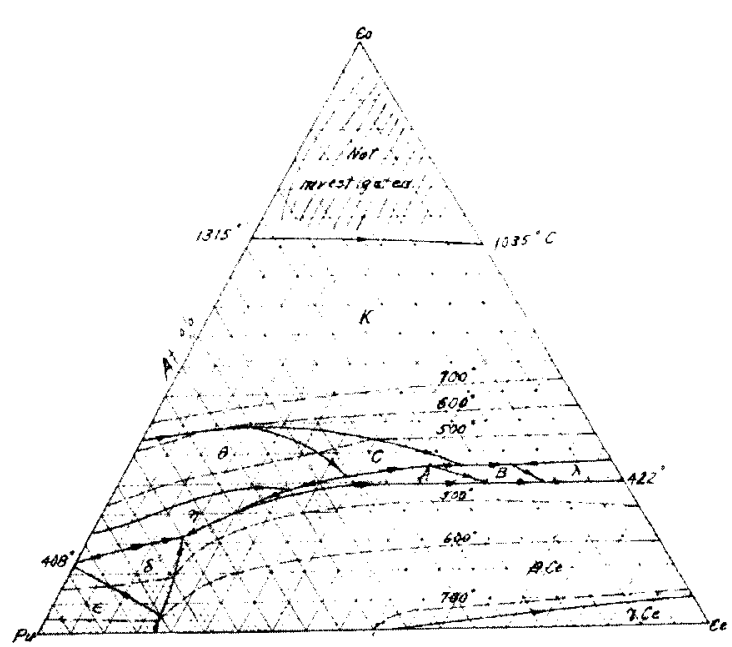

SLIDE 1
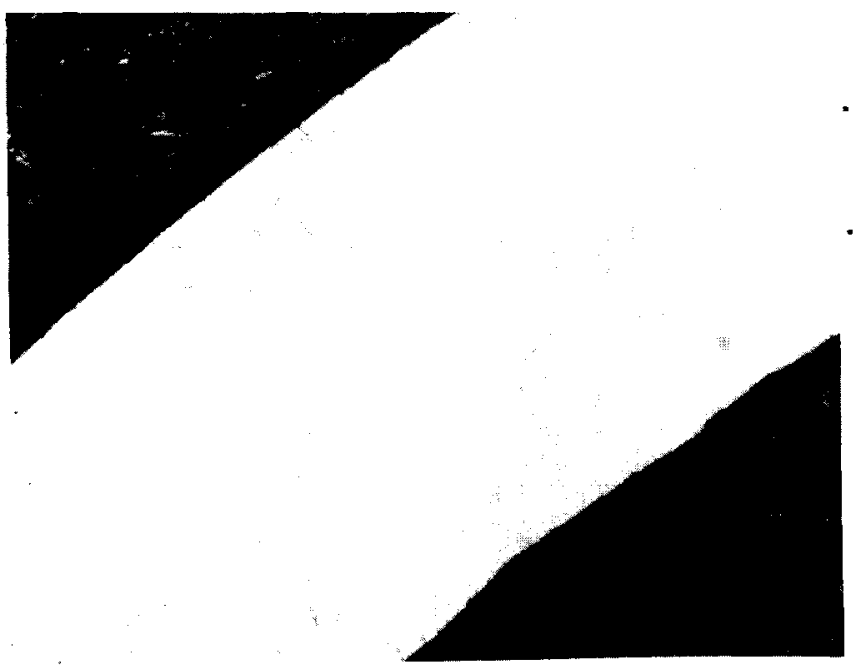
Slide 2 illustrates the type of diffusion we are discussing. This is an 0.02 in. wall tantalum capsule that is completely penetrated by plutonium in the grain boundaries. It has about $10,000 \mathrm{cpm}$ of plutonium on the external surface. As you can see, it hasn't fallen apart; it is still relatively strong; it is still quite ductile; and it certainly maintains its geometry.

Another point is that, in our particular case, where we were talking about diffusion, thermal history and not irradiation time is important. It is thermal history that will determine diffusion. We do not get lattice diffusion in tantalum, because the plutonium is absolutely insoluble in tantalum and because of too large a mismatch in atomic diameters. However, we do get grain boundary diffusion.

S1ide 3 summarizes a study of out-of-pile characteristics of various plutonium alloys. The data plotted is what we will call lifetime -- the time for diffusion of detectable $\left(10^{-8} \mathrm{~g}\right)$ plutonium through a constant wall thickness of 0.02 in. tantalum vs $1 / T$. Line $B$ is for the Pu-Fe fuel, the fuel used in LAMPRE I. We notice that the data obtained for sodium and the data obtained for a vacuum of $10^{-8}$ torr, fall on the same line and indicates no effect (to first order) of oxygen concentration in the external environment of the capsule. Line $\mathrm{C}$ is for pure plutonium. You can see that the time involved here is very short ( 10 hours or so) at temperatures of 900 to $1000^{\circ} \mathrm{C}$. The maximum temperatures we are interested in for almost any of our reactor applications would be about $750^{\circ} \mathrm{C}$. You can see that there is considerable tolerance here for overtemperature for considerable periods of time. Line $A$ is the line for pure plutonium in capsules having a carburized layer of $3 \mu$ thickness on the internal surface of the tantalum container and a $200 \mathrm{ppm}$ carbon top cap. As you can see, it is difficult to get data in a reasonable amount of time. Since we are interested in capsule integrity over a year or so, we would certainly not expect to have very much trouble, even if we were trying to contain pure plutonium.

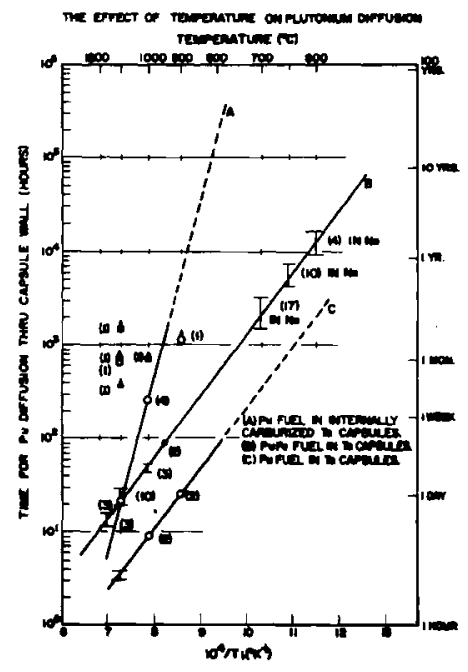


As we go from pure plutonium to $\mathrm{Pu}-\mathrm{Fe}$ to $\mathrm{Pu}-\mathrm{Co}-\mathrm{Ce}$, the time for this diffusion gets even longer. We are having trouble trying to get capsules containing Pu-Co-Ce to show any diffusion whatsoever. In fact, we have four capsules right now that have been in test at $1100^{\circ} \mathrm{C}$ for 1500 hours. All four of them are still operating, and they are expected to continue in operation for a considerable period of time. The slope of the lines in this figure are the activation energy for grain boundary diffusion of plutonium in tantalum. Consequently, since we are looking at a single mechanism here, we can extrapolate from the available data to the temperature range of interest with some confidence. You can see that at $700^{\circ} \mathrm{C}$, even without carburization, the diffusion through the clad is going to be apparent only after many years. We definitely will get an improvement with Pu-Co-Ce fuel by carburizing the capsules. Data for carburized capsules will fall to the left of Line A. Consequently, there appears to be no containment problem whatsoever, as long as somebody re members to put the bottom in the capsule.

One thing that does happen with these fuels is that all the transition elements form intermetallic compounds with tantalum. The cobalt in the $\mathrm{Pu}-\mathrm{Co}-\mathrm{Ce}$ fuel forms intermetallic compounds of $\mathrm{Ta}$-Co of varying composition. The particular compositions depend upon the fuel used. Because of wetting action, the fuel creeps up the wall. It then forms the intermetallic compound with the tantalum, becoming enriched in plutonium by the depletion of cobalt. Then, various temperature fluctuations move the concentration up to almost 90 at.\% plutonium. We are now talking about corrosion, not by Pu-Co-Ce but more nearly by pure plutonium again. We do start to obtain diffusion through the walls. We take care of this very easily by putting a $3 \mu \mathrm{Ta}=\mathrm{C}$ layer on the wall. This carbide layer does not have to be continuous; its only function is to keep the Ta-Co intermetallic compound from forming, and that it does quite well. Analyses to as high as one foot above the fuel level indicate no enrichment of the fuel whatsoever. The fuel film is maintained at the composition of the bulk fuel; consequently, we are again looking at behavior typical of the $\mathrm{Pu}-\mathrm{Co}-\mathrm{Ce}$ system.

We have not seen anything, nor do we anticipate that anything will happen to the $\mathrm{Ta}=\mathrm{C}$ layer during irradiation. of course, the only place that we really care about this layer is above the fuel; that is, in the gas plenum above the fuel level. In addition, the carbon solubility in $\mathrm{Pu}$-Co-Ce fuel is quite small. Slide 4 shows a carbide layer that was in contact with pure plutonium. There, the solubility is several thousand parts per million. You can see that this layer was quite stable at quite high temperatures. 


\subsection{5}

There are a few irradiation questions that may come to people's minds. One is the disengagement of fission gas bubbles. The first question that comes to mind is, "Does this fuel froth up like shaving cream"? The answer is "No". Slide 5 shows a radiograph of a capsule irradiated to about $2 \times 1020$ fissions $/ \mathrm{cm}^{3}$, which corresponds to about 1 at. $\%$ burnup of a $6 \mathrm{~g}$ plutonium per cubic centimeter fuel. Note the very clean meniscus. A shorter term irradiation $(\simeq 1 / 8$ as long) performed, where the capsule was loaded with exactly the same amount of fuel, came out within 0.003 in. of the same height as the higher burnup case after irradiation, indicating that we are effectively releasing $100 \%$ of the volatile fission products. However, there is an initial period where one builds up fission product bubbles along the wall. You can't see them in this photograph, but I will show them to you in a later slide. In this particular case, we have wall-to-average fission density of a factor of 6 , so that we actually have an integrated fission density corresponding to about 6 at. $\%$ burnup at the wall with respect to fission recoil damage. The volume percentage of fission gas bubbles in this case was about 6 vol.\%. In LAMPRE I, the corresponding value was measured as about 4 vol. $\%$. We would expect in most fast reactor systems that the retained fission gas content will be in the vicinity of 4 to 5 vol.o fission product bubbles. Slide 6 is a macrograph of the same capsule showing the distribution of $\mathrm{fis}$ sion gas bubbles. The two bubbles seen in this figure are the largest ones we have observed. I use a very poor analogy, but I like to look at this as either a teakettle or a glass of beer. We saturate the nucleation sites along the wall; then the capsule sits there and boils; and the gas comes off and is freely disengaged at the gas-liquid surface.

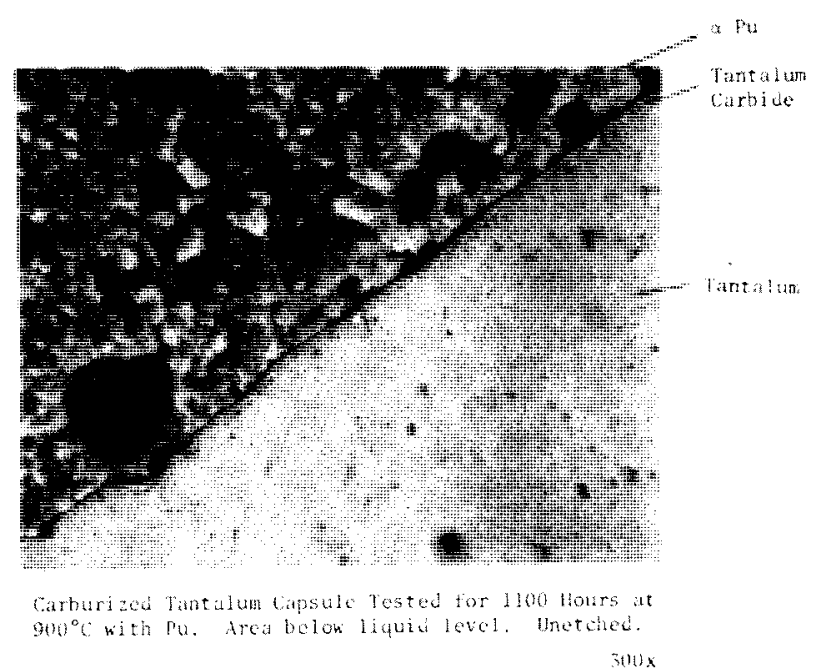

SLIDE 4

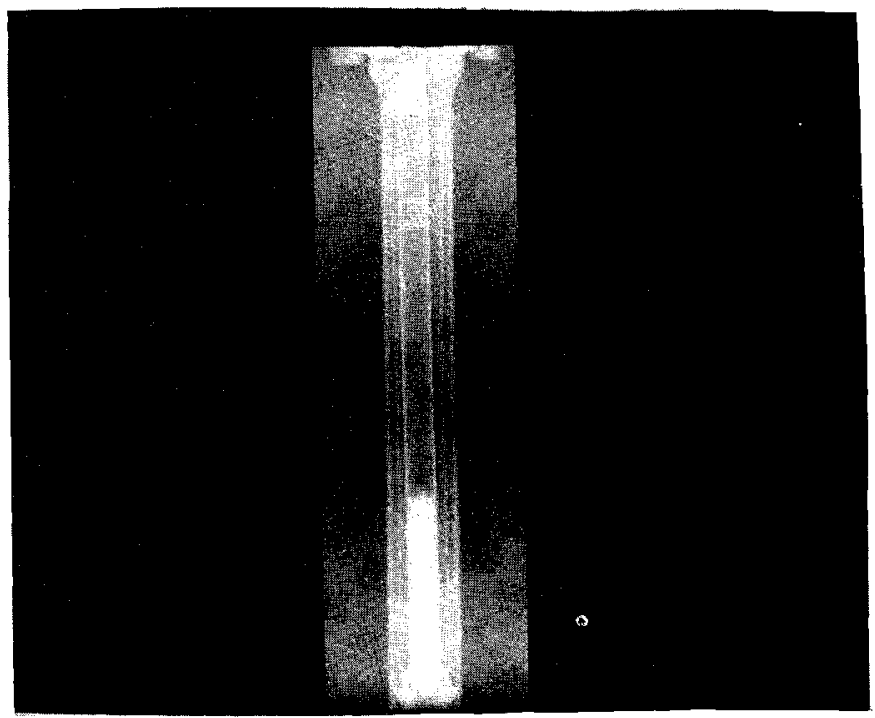

SLIDE 5 
In the solid state, these fuels are extremely brittle, and they expand rather than contract when they freeze. In the particular case (Slide 6), the fuel was quenched from power; that is, the reactor was scrammed with no compensation in the heat dump system. The capsule cooled very rapidly, and yet there was absolutely no deformation of the capsule. The fuel has many cracks in between the bubbles which indicates that the $\simeq 3$ vol. $\%$ expansion occurring during the phase change is being accommodated by the brittle fue 1 ; in this case, by the 6 vol.o of bubbles that occur along the wall.

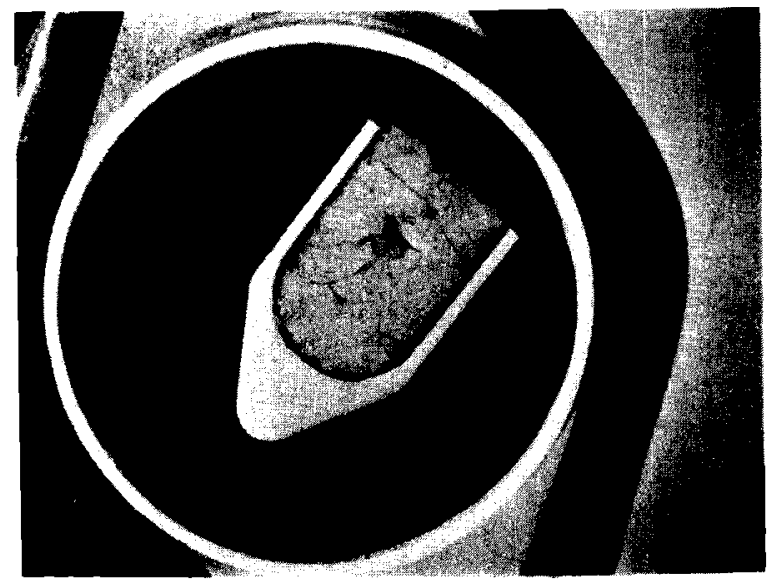

We have observed one effect of recoil damage on the cladding. Slide 7 illustrates that effect. This is a section of tantalum wall from the capsule shown in the previous slide. In the unetched condition, we see a $4 \mu$ band, a bright-appearing band, along the edge; here the structure is about 0.002 in. thick. It is a dendritic type of structure, and it looks like something growing from a melt. We think this structure is caused by certain fission products (not yet identified) diffusing in certain crystallographically preferred directions in the tantalum crystals. Slide 8 shows the same structure at a higher magnification, and you can again see the dendritic type of structure. 


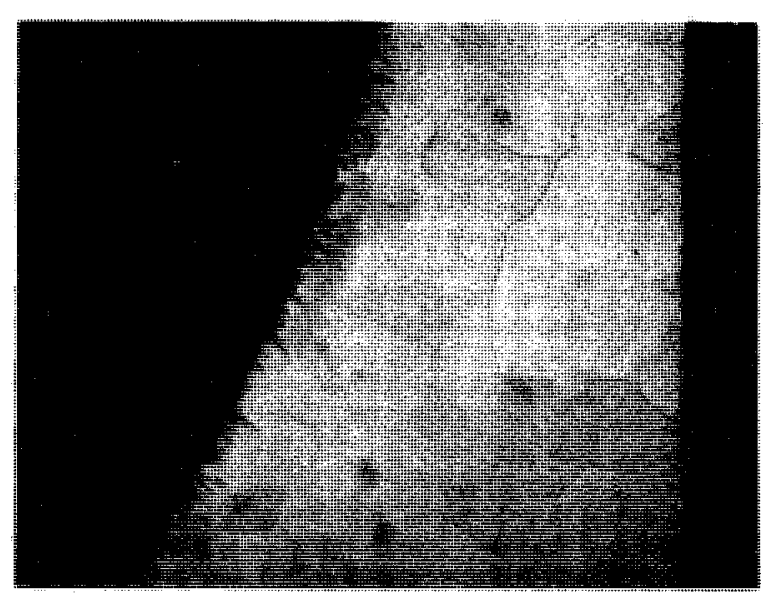

SLIDE 7

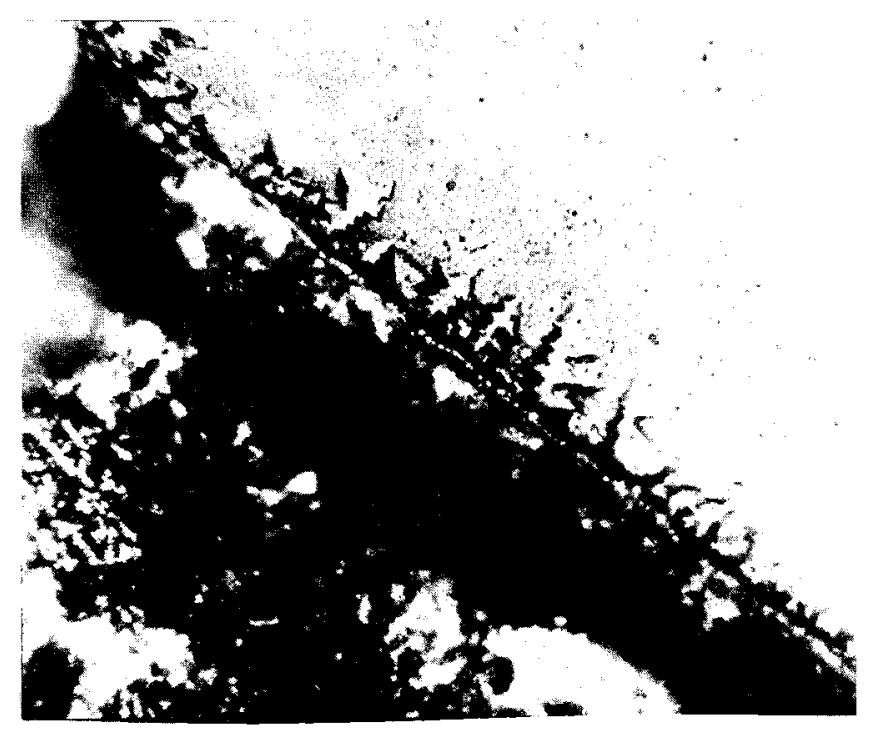

$\underline{\text { SLIDE } 8}$

While it is certainly not a dendrite, it is clear from its appearance that a cubic-type phenomenon is occurring. It is far too deep to be the result of direct recoil of fission products.

In an effort to determine what will actually limit the burnup capability of the molten plutonium fuels, we have made analyses of the chemical behavior of the various fission products. We anticipate that about $45 \%$ of them will either precipitate or be volatile and escape into the gas space; the remainder of them will be soluble in the fuel. One expects that $\mathrm{Zr}-\mathrm{Nb}$ couple, being refractory metal and thus having a very low solubility in these fuels, will precipitate along the wall. Indeed, by using a pinhole gamma scanning technique (as illustrated in slide 9), you can look at the activities of the various isotopes. Tantalum is used as a base to get the geometry effect. Indeed, you can see that the $\mathrm{Zr}-\mathrm{Nb}$ is precipitating along the wall. The Lal40 would be expected to show the same type of behavior; it is insoluble in the fuel, and it appears to be doing the same thing. On the other hand, ruthenium should be soluble in the Pu-Co-Ce fuel; indeed, the data indicate that it is soluble and remains in the fue 1 .

We have estimated the change in the melting point that might occur in going to $20 \%$ burnup of a $6 \mathrm{~g}$ plutonium per cubic centimeter fuel. If the melting point of the fuel increased too much, it would be difficult to keep the fuel liquid. If we start at the melting point of about $443^{\circ} \mathrm{C}$ (SIide 10), it will be about $455^{\circ} \mathrm{C}$ (to the best of our ability in analyzing this type of system after $20 \%$ burnup). It doesn't look like the change of melting point will be a limiting factor. 
DIAMETRICAL GAMMA SCAN OF CAPSULE 2285 WITH PIN HOLE COLLIMATOR (SEPT. 22, 1965)

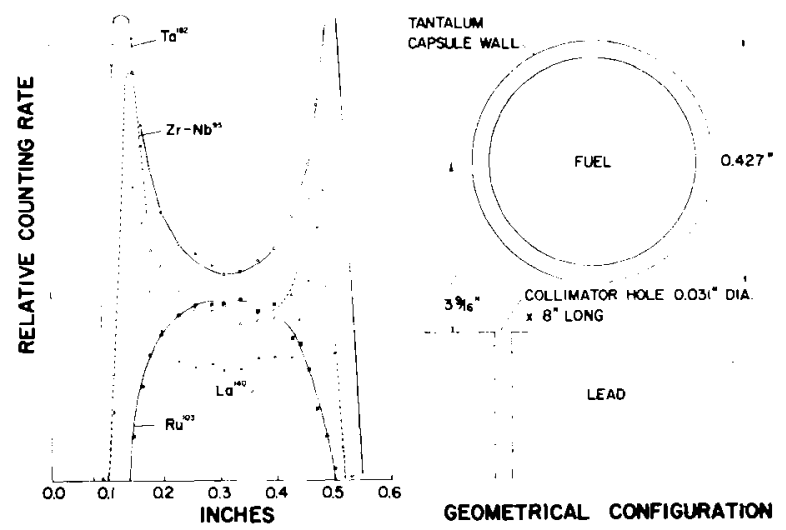

SLIDE 9

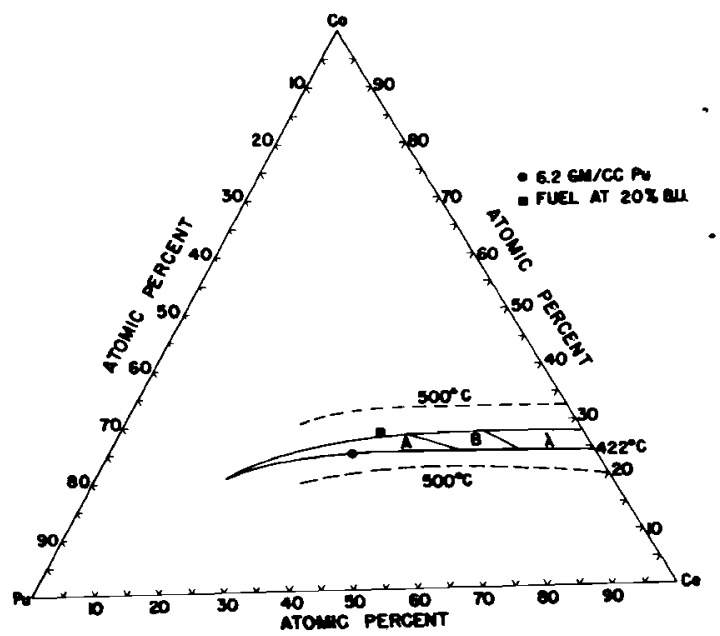

SLIDE 10

We can't see that irradiation damage to the clad is going to have any particular effect. Present designs utilize fission gas plenums having a gas-to-fuel ratio of about 0.8. At 20\% burnup, combining the pressure stress with the thermal stress in the capsule, we come up with about $1 / 2$ to $2 / 3$ of the yield stress of the unirradiated material. We are using $\mathrm{Ta}-5 \% \mathrm{~W}$ alloy because it is much stronger than pure tantalum.

Consequently, it does not appear that we are going to have mechanical problems, such as creep. It does not appear that melting point is going to limit burnup. One thing that could limit burnup is the chemical behavior of the fission products as they burn into the fuel. This, of course, can only be demonstrated by burning up. the fuel, which has not yet been done. We do not anticipate that the presence of chemically significant quantities of fission products would produce dissolution effects on the capsule wall material.. All of our mass transfer studies to date show rates in the range of $1 / 10$ of a mil per year (and down) under temperature gradients far higher than we would expect in any real system. The only thing we can't really simulate in our mass transfer and solubility tests is the convective currents which do, of course, exist in a capsule that is generating power. Perhaps, the factor that will limit the lifetime will be excess reactivity. 


\section{SUMMARY}

In conclusion, I would like to say that we have presented a case here that we think merits consideration. Certainly, some extrapolations and a test program need to be carried out, but that is also true for any of the fuel systems which have been and will be discussed at this meeting. The outstanding feature of this system, beyond the shadow of a doubt, is safety. You can do practically anything to this system and nothing will happen. We think that the materials situation is determined by thermal history and not by irradiation. We are looking at the effect of irradiation on the wall by utilizing the factor of 6 to 1 peak-to-average fission density in our OWR tests. We expect to have two tests coming out at $20 \%$ equivalent burnup (that is as far as the fission density at the wall) by about July. These tests are using capsules with the carbide layer throughout the whole capsule. We will see then the effect of fission recoil on that carburized layer. I think that that is probably the biggest problem; if there isn't a problem with fission recoil in that layer, there probably won't be a recoil problem. We have described a test here that could possibly be carried in EBR II to demonstrate the power density requirements for the FFTF. 
D10.1

\section{LIQUID PLUTONIUM FUEL SYSTEMS, THEIR STATUS AND CAPABILITIES}

W. H, Hannum and L. D. Kirkbride

\section{DISCUSSTON} isn't it?

MR. HENNIG: Trank you very much. At a minimum it is intriguing,

MR. deHALAS: I get the feeling you skipped lightiy over the fission gas problem. I noticed that capsule had a large plug and you just mentioned the plenum as long as eight-tenths of the fuel length. The actual analyses that $\mathbb{I}$ saw, at least, the way $\mathbb{I}$ read up there, there was no fission gas pressure in the capsule. What happens, for example, if you get sodium boiling with a good head of fission gas behind it? Does your capsule bulge cut?

MR. HANNUM: No. . The size depends on how much fission gas you intend to release. For a $4 \mathrm{~g}$ fuel with a $20 \%$ burnout, which I think is reasonable, about eight-tenths of the fuel should be allowed to stay safe below the fuel pressure.

MR, derALAS: What happens if you get a hole in the cladding?

MR. KIRKBRIDE: Where?

MR. deHALAS: Below the metal level. I assume you biow the plutonium out?

MR. KIRKBRIDE: It wouldn't stop. Not strictiy. In Lampre, I can't possibly go into the detailed analys is of it in this short time, but in Lampre we actually did have a hole that developed 2 in. below the level of the fuel. Two inches worth of fuel blew out. There was an immediate loss of reactivity, and people tried to keep up with it and didn't extract any more reactivity. However, we lost about $60 \mathrm{~g}-$ or $70 \mathrm{~g}$, in that case. That fuel stayed in the reactor for the rest of the time. It turns out this fuel will wet stainless very nicely. What it does, it hits the surface of the stainless and wets it into a very thin film and then the ... compounds form that we have heard about, and that is the $\mathrm{Pu}_{6} \mathrm{Fe}$ that melts at very high temperatures, $3,000^{\circ} \mathrm{C}$.

VOICE: $\mathrm{PuFe}_{2}$ compound.

MR. KIRKBRIDE: The pcint is, simply the film freezes. It immediately forms this protective layer and then it picks up more iron by diffusion from the stainless and then the whole thing freezes. Now we have looked at the hot traps of Lampre, operated through the entire history of Lampre, and less than 10,000 counts/min of plutonium in contrast, none whatsoever on the zirconium. And only after we cleaned the sodium film off the stainless steel did we pick up this 10,000 count $/ \mathrm{min}$, 
so effectively there are several ways of getting rid of that fuel. Of course, the more that comes out, the bigger the problem.

MR. KITTEL (Argonne): I was also struck by the large plenum you showed on your fuel there. I wondered if you have completed any experiments for plenums of more reasonable size of eight-tenths of the fuel length?

MR. KIRKBRIDE: We contend that eight tenths is a reasonable size. In Lampre there was $6 \mathrm{in.}$ of fuel and 2 in. of gas plenum.

MR. KITTEL: Are these the capsules that went to $3 \times 10^{20}$ ?

MR. KIRKBRIDE: No, these were up here. Fission gas pressure was $20 \mathrm{psi}$, which is sort of trivial. The only reason they are designed that way is because we happened to have the capsules on hand. These capsules were for Lampre, which we did not actually put into the core. We had them on hand and used them. The reason there is only 2 in. of fuel in there is because our circulating sodium system taking the heat from this capsule, can't handle too much more. It could handle 4 in. quite easily. There is no reason not to, but we haven't put it in. The fission gas pressure doesn't bother us at all.

MR. KITTEL: I guess what I was wondering, how the can behaved when it was under stress? Apparently there is no experiment completed yet where there has been paramount pressure stress on the can, is that right?

MR.ANDERSON: Maybe it would clarify it a little. You mentioned the tensile strength of tantulum is $34,000 \mathrm{psi}$. Is that the tensile strength on plutonium grain boundaries?

MR.KIRKBRIDE: We are not getting plutonium in grain boundaries.

MR. ANDERSON: I know, not on the outside, but is there any penetration?

MR. KIRKBRIDE: There is a very interesting situation and I neglected this, I am sorry. It is a very important point. The only location where plutonium in grain boundaries has ever been seen is in a weld. There is never plutonium in the raw material grain boundaries, and if you are sufficiently interested in this we can go into the theory on why. But only the welds. Consequently, there was no plutonium-in any grain boundaries. We don't expect any in grain boundaries. Plutonium iron, you do get plutonium. in the raw material boundaries, and in plutonium you definitely get it in the plutonium raw material boundaries but in the cobalt series it has never been observed, and I doubt that it ever will be. I neglected to sav that of all the data we have, the plutonium has come through the wall and has been through the weld material. That is a very important point and one that I should drive rome. Not through the raw mater:al.

MR. KELMAN (Argonne): I would like a little more clarification about this penetration through welds and about your thin carbide layer, how you put it on. Ho: do you, for exampie, cover the weld area to protect it? 
D 10.3

MR. KIRKBRIDE: It turns out you don't really have to have the carbide layer. It is the carbon that is fusing and into the boundary that is important.

MR. KELMAN: I thought I saw a tantalum carbide layer.

MR. KIRKBRIDE: Yes, you did. I need a lot of blackboard space and a lot of time to develop this in a logical fashion. However, the carbide layer, even though it is not going to stay,... diffuses down these boundaries. Now it is, that carbon is important in stopping plutonium. Apparently what has happened, if you noticed on the slide showing the plutonium data and the carbon data, there was a difference in slope. The self diffusion coefficient of tantalum is about 110 . You would expect $30 \%$ of that not to be too bad for grain boundary diffusion. The plutonium is somewhat bigger than tantalum and you would expect the 30 kilocalories not to be too bad. It is 70 kilocalories in the case of carbonized material, and it appears (and this is very difficult to try to substantiate quantitatively, ) that there is a carbon interaction, either chemical or another type of mechanism. Remember, there is no last diffusion of plutonium, and this is very important. It has to go in the boundary, and so not only do we have that process but we have to either break a bond with carbon or pull it effectively in the direction of plutonjum. It is quite obvious that it is a more energetic process. In effect, what we are doing is exchanging activation energy. Now the carbide layer is important to keep the cobalt in, and that layer is laid down by using amorphous carbon granules that have been outgassed and then backfilled with argon. They are then poured into the tantalum tube in a quartz induction heating system, pumped out, fused at $1700^{\circ} \mathrm{C}$ for 5 min. hydrogen pumped out and then the granules are poured out and one gets a very uniform content.

MR. KELMAN: My experience with layers is that quite frequently you can't rely on them being continuous. How much effort do you put into looking for continuity?

MR. KIRKBRIDE: We have never seen discontinuity. As I mentioned, we don't particularly care if it is or isn't continuous. And that is because of the reaction of the cobalt in the zone. Now you are talking films. I must say, we are worrying about something that may be hypothetical, because this film, as it gets 6 in. above the fuel level in 1 in. of $0.420 \mathrm{in.}$ OD capsules contains about $11 \mathrm{mg}$ of fuel. On the carbide layer if contains about $100 \mathrm{mg}$ of fuel. Consequently, we are not pumping much fuel at those high levels. When we worry about this pumping mechanism, we are talking about a hole below the fuel level or a hole just at or just above the fuel level. It appears that the limiting height may be 1 in. Any hole above 1 in. will not pump any substantial quantity of fuel, and it may be $\mathrm{mg}$, and things you can see by count but nothing substantial that a reactor would ever see at all, as far as its operation goes. I will be glad to go into more detail on this item, and I undoubtedly will. 
D 10.4

MR. deBOISBLANC: I just have a little accident haunting me in this infinitely safe system. Suppose the reactor is cold and you put in $100 \$$ per second step. Then what happens?

MR. HANNUM: The first comment I would make on that is that we don't intend to have the reactor cold.

cold?

MR. deBOISBLANC: Suppose you don't intend to have it cold but it is

MR. HANNUM: A serious accident can occur in this type of system when you have a solid metal fuel and your feedback coefficient is down by a factor of three.

MR. deBOSIBLANC: Well, the inside melts first, doesn't it, and doesn't the restraint then develop some nice pressure?

MR. HANNUM: Oh no, the fuel contracts on melting.

MR. deBOISBLANC: But it doesn't keep on melting, it has a very high expansion rate.

MR. HANNUM: Yes, very high expansion.

MR. deBOISBLANC: I would postulate that the ends are solid and the middle is melted and because of the power distribution the middle always leads and then finaliy a reversal in sign occurs, and then everything breaks loose.

MR. HANNUM: I suppose you could postulate such a thing. I don't know what the time scale would be. It would have to be pretty fast. Yes, very fast so as not to aliow any heat from the center to the outside.

MR. deBOISBLANC: The second question. You mentioned that because of this retrograde expansion upon freezing that the gas bubbles took up--accommodated itself, so you didn't rupture the container. Suppose this happens the first hour, that you just get a scram and cool the system off. Then what happens?

MR. KIRKBRIDE: We have thermally cycled elements, certainly not of three or four feet of fuel, only of half a foot of fuel, and this was without the radiation. It seems to indicate, and this is between 10 and 20 cycles, we get a linear increase in deformation with the capsule, the higher the cooling rate. Now we have looked at a large system, in particular our 20 megawatt system, trying to determine the most rapid cooling rate we can have. That cooling rate we anticipate will be one of operating at 20 megawatts, scramming... and it turns out the actual cooling rate we get is 11 degrees per minute. And the 11 degrees per minute indicates that we will get, in about 15 cycles, about $1 \%$. . The clad will take it quite easily.

MR. HANNUM: Relative to the spirit of the thing you talked about here today, I might also add that the amount of expansion you get on 
D10. 5

freezing is shown as a function of plutonium in the fuel. The $4 \mathrm{~g}$ fuel is what we are talking about here and this is about $1 \%$, and this expansion is very easy to accommodate.

MR. KIRKBRIDE: The thermal cycling experiments have been $6 \mathrm{~g}$ fuel. That is 6.2.

MR. deBOISBLANC: You wouldn't want to keep doing this, would you?

MR. KIRKBRIDE: We try to avoid it.

MR. deBOISBLANC: You get 1\% each time?

MR. KIRKBRIDE: No, no. The $1 \%$ is after the 15 cycle buildup. One thing that would be very interesting to determine in a reactor is how often one can freeze, and under what conditions with the presence of the bubble. On the other hand, I think one would not want to operate a reactor like this.

MR. deBOISBLANC: You mentioned the bubbles. One of the first things that occurred to me, do they go away?

MR. KIRKBRIDE: No, they are like a glass of beer.

MR. deBOISBLANC: But if you leave the glass of beer long enough, they will go away.

MR. KIRKBRIDE: I suppose if you leave the fuel long enough they will go away also, but as long as you fission--the bubbles will keep growing at the nucleation centers. We actually have a photograph. I have a slide of it.

MR. deBOISBLANC: A better analogy would be a glass of beer that freezes when you don't drink it.

MR. KIRKBRIDE: Well, that is just what we did. When we quenched this fuel, we actually caught this nucleation site with a string of bubbles coming up from it.

VOICE: Have you looked into what might happen if the bubbles dislodged from the surface?

MR. HANNUM: If you have shaken a glass of good beer, you can't get all the bubbles to come off at the same time.

MR. KIRKBRIDE: The bubbles rise at under $4 \%$ 。

MR. HANNUM: It doesn't go away that fast.

MR. ANDERSON (LASL): Let me correct that. If you have any bubbles and they all rise at once, if they go up as buoyancy dictates, it would be a couple of dollars a second, but as you say, it is trivial.

MR. HANTHORN (BNW): What is the reactivity worth in one capsule somewhere near the middle of the reactor? about.

MR. HANNUM: That depends on what type of reactor you are talking 
D10.6

MR. HANTHORN: I am talking about this reactor.

MR. HANNUM: The particular FFTF?

MR. HANTHORN: Your reference reactor.

MR. HANNUM: That is right, that has about 3600 capsules--something less than 60 in fuel.

MR. KIRKBRIDE: Fifty-three.

MR. HANNUM: Fifty-three fuel capsules, so if you assume it is an average worth position it would be $0.03 \%$ of the reactivity; and if you assume it is the center capsule, it might conceivably be worth three times that, or 1. $1 \%$.

MR. HANTHORN: It is in the range of 1 to 3 dollars then ?

MR. HANNUM: No. It is in the range of up to a half a dollar.

MR. HANTHORN: Oh. Anything that happens quickly in one capsule is negligible.

MR. deHALAS: I realize this is sort of an unfair question, but you have probably thought about it? What sort of fuel cycle cost do you get as compared to oxide? You have to go through some sort of chemical processing, I know but what?

MR. HANNUM: For the FFTF type application, we have not looked at what it would cost to operate. We have compared with what you would get in the large breeder reactor, using the ceramic type, and the cost comes out quite comparable, depending on whether you want to cheat or not. You can save a few cents a dollar or come out the same. They are quite comparable.

MR. KELMAN (ANL): I am concerned about anything that might embrittle the container, such as impurities in the sodium, the fission products that might do this, or irradiation itself. I wonder how much experience there is in being sure that you retain some ductility. I keep thinking of my own experience with liquid metal in pipe, and what happens when for some reason, you have large expansion changes with temperature. You have a tremendous pressure that builds up, and that cladding better have some ductility. Do you have experience, for example, with engineering type facilities, such as the sodium you might expect to see in an engineering system?

MR. KIRKBRIDE: To take your points at random, we consider the sodium we used for these tests to be of high quality. The loops that we run are zirconium gettered .. We are presently building a loop to do sodium testing at much higher levels. Those we think are totally outlandish, we are running into them. So to answer your question, sodium impurities on oxygen, you only look in the 1 to 2 million part range, say up to 5. Hydrogen, we don't think there is any problem. We have a linear buildup of nitrogen in cover gas. It is apparently coming through the stainless steel, which may be of concern in any fast reactor system... As far as fission products embrittling cladding, that is a possibility. We don't know. That is another 
D10.7

thing on which we will have to get the irradiation data. As far as plutonium brittling the cladding, it doesn't appear to. Even in the cases where we have run pure plutonium, to quite a large amount of plutonium as used in the fuel material, still the clad appears to be quite ductile. We even performed one test in which we tried to remelt pure plutonium . . . blew wide open because of the changes in the solid. That was a completely ductile failure. Let's see, what were some of your other points?

MR. KELMAN: To go back to your sodium impurities, I don't know that we know how to measure oxygen in 1 or 2 parts per million.

MR. KIRKBRIDE: I was just thinking, it is hard to get a part per million on sodium, as you are well aware. When I say 1 or 2 parts per million, the way we determined this was, we have quite an amount of data on the zirconium oxidation at the levels where we can measure them quite accurately. If you take and extrapolate the weight gains from zirconium down (and all of these loops run on zirconium traps) and look at the weight gain, the extrapolation comes out in $0.1 \mathrm{ppm}$ for $10 \% \mathrm{ppm}$. We run these zirconium traps to exhaustion and they don't change too often. Sometimes we are actually taking capsules out of tests that are coated on the surface with a very loosely adhering film. One which you can wipe off with a pen knife. It doesn't appear to make any difference whether that is carbide, which it could be, or whether it is oxide, which it also could be, we do not know.

MR. YEVICK: Do you have time for any tests in TREAT?

MR. ANDERSON: I think the time scale in TREAT is much too long to get into action. As you saw, until we get to the level of $100 \$$ insertion rate, we don't think you have any trouble but that insertion rate . . . is extremely short and I don't think a model in TREAT would do. We have considered, however, the use of Super Kukla.

MR. YEVICK: What volume percentage of the cobalt-cerium fuel is plutonium?

MR. KIRKBRIDE: I had that number, but I know you don't talk about volume percent. I have it.

MR YEVICK: Weight percent then.

MR. KIRKBRIDE: The weight percent for which, for this case?

MR, YEVICK: The FFTF case.

MR. KIRKBRIDE: About 20.

MR. HANNUM: The weight percentage would be sornewhat higher, about $4 \mathrm{~g} / \mathrm{crn}^{3}$ on plutoniurn and the density of fuel about 10 , so about 40 .

MR. YEVICK: The next questions is, the high nvt involved here for high burnup, what effects are you going to get out of the cobalt and ceriurn? 
D 10.8

MR. KIRKBRIDE: Cobalt and cerium are neutronically inert...

MR. HANNUM: Neutronically this is a very slow process.

MR. deBOISBLANC: What do you consider your maximum technical credible uncertainty?

MR. KIRKBRIDE: Maximum credible technical uncertainty?

MR. HANNUM: We talk very glibly about a $20 \%$ burnup for the simple reason we can't think of anything that would stop short of this.

MR. deBOISBLANC: I would like to ask, what is the stability of carbonization layer at $10^{22}$ nvt.

MR. KIRKBRIDE: This again is irradiation data and we don't have a large number of tests on it. However, I do want to mention one thing that might put us in perspective and that is the $20 \%$ burnout. When we are talking about fissions per cc to burn out the oxides or carbides and other things, you are talking about $25 \times 10^{20}$ fission per cubic centimeter. So if you put it on the fission per centimeter basis, which of course we think is the way to talk about liquid (because you are looking at the chemistry of fission products), this is how much cubic centimeter per concentration. We are actually at a lower fission per cubic centimeter at these numbers than the ceramic.

MR. deBOISBLANC: However, the other factor is that irradiation damage in a constant flux is essentially a matter of time, so you have more equal number days of operation rather than fission per cubic centimeter.

MR. KIRKBRIDE: The irradiation data.

MR. HOF MANN: . . . if I can heat the core to $4 / 5 \mathrm{MW} /$ liter, the peak flux process we get would be on the order of the 1.4 , but the one you employed was $1 \times 10^{16}$ ?

MR. HANNUM: Something like 1, I suspect.

MR. KELMAN (Argonne): I feel kind of betrayed by our physicist. I wonder why it is, in our consideration of metal fuels, over the years tantalum has been avoided like the plague for a cladding material. From a physicist's point of view, what happened?

MR. HANNUM: Tantalum is a very bad poison. The thing that allows us to live with it is the very hard spectrum that we use. If we get into any system with a large scale softening in the spectrum, then we would be in trouble. If for a particular test, you want to locally soften the spectrum, I don't see any reason you couldn't do that. But to try to run the entire reactor this way would be out of the question.

would?

MR. KELMAN: You say in consideration for a large reactor, you still

MR. HANNUM: For a large reactor, you have to be careful of the spectrum, and so far we have only talked of going to the totally external breeder for that very reason. I might also throw out sort of a miscellaneous comment on both of these. We are instituting a program to look at other containers than tantalum, but we don't have any data on that. 
11. 1

\title{
FABRICATION PROCESSES-HIGH ACTIVITY FUELS
}

\author{
L. R. Weissert
}

Babcock and Wilcox

Lynchburg, Virginia

\section{INTRODUCTION}

It seems appropriate to address outselves to a new direction in the FFTF driver fuel question and probably, a better title for the first talk this morning might be commercial Aspects of Fabricating High Activity Fuels. Certainly, judging by the remainder of the speakers this morning it seems to be the main subject for this morning's session. In the discussion portion of this presentation, I would like to hear some comment on where the source of plutonium would be for fabricating these FF'TF fuels. If my intelligence is right from last week's Plutonium Symposium in Washington, most of it will come from the stockpile. In any event, this has a rather distinct bearing upon the activity considerations in fabricating the fuel. Moreover, the ground rules that have been given for the fabrication of FFTF fuel are very strict in that they must have high reliability and low cost. These, combined with the fact that the fuel is going to be plutonium, impose rather strict requirements.

In talking about the generai considerations concerning this subject, we might discuss for a moment the aspects of early production of high activity fuels, specifically plutonium. Again, judging from last week's discussion in Washington, one can certainly say from a commercial standpoint that there will be a rather low volume to process for quite a while. There just isn't enough plutonium to go around. Of course, by the same token, that would indicate that early fabrication costs will be rather high. A lot of the costs which we hear being quoted for fabricating plutonium fuels are generally based on somewhat of an equilibrium situation assuming a certain throughput of plutonium. The early aspects of production will also be featured by nonstandard designs. When one considers a commercial fabricating plant for high activity fuels, labor influences must be considered. With the kind of semiskilled labor that is used in a production plant, we have to consider what the impact of union activity will be for those that are in the sphere of union infuence. Right now, of course, we are able to consider working up to certain maximum permissible limits as set by the state and federal authorities. As we all know, there are distinct pressures from the labor front to work on the linear theory which means that there is no exposure limit. You have to consider their total dose and work as low as possible. Also from a commercial standpoint, we have to consider licensing requirements. This turns out to be a continuing, formidable task to convince them that you can work in a safe fashion both from the nuclear safety and radiological standpoint. 
Let us now discuss various aspects of early production of high activity fuels. In thinking back to the general considerations I mentioned earlier, a low volume situation on the near term will require low plant capital cost to achieve low fabrication costs. Additionally, simple processes and equipment are indicated. Of course, ease of equipment mainterance is a must. This is particularly important with high activity fuels because if glove box entry is required to maintain a piece of equipment then the least amount of time should be spent fixing it. to minimize personnel exposure. High yield is needed, or conversely, low scrap generation. It costs money to process the scrap that is building up in radioactivity. Low material holdup is highly desirable. Both plutonium and $\mathrm{U}^{233}$ are characterized by increasing gamma activity with time so that the more material you have left in glove boxes or in your equipment, the more background activity is generated. These are the major aspects. Other factors are important also. One is the adaptability to automation. To help keep personnel doses down, automation can minimize direct handling in boxes as much as possible. Rapid processing can accomplish a part of the same result.

These features characterize the ideal objectives in considering a near term processing of high activity fuel. This situation changes, of course, when large volumes are encountered. One can then justify higher capital costs for shielding, for instance, to attain high production rates at minimum radiation hazard.

By way of illustration, I wanted to take a few moments this morning to describe a project which Babcock and Wilcox just completed. This is a million dollar, company sponsored project which started in 1961 in an attempt to come to grips with the commercial problems that might be encountered with handling high activity fuels. The first phase of this work involved handling $\mathrm{U}^{233}$. One would, of course, ask, "Well, that 's fine. But what is the relationship with the subject for this morning?" As I will show later on, there is not a one-to-one relationship but there are other similarities--close similarities--which I think make some of our experiences and those of others on handling $\mathrm{U}^{233}$ applicable to the particular subject at hand. This particular project was started because we felt that one could never really come to real grips with the commercial problems with handling high activity fuel unless we actually did the work in a production oriented manner using the kinds of people that you would have to use in a production plant. The project had as an objective the design, construction, and operation of a pilot fabrication plant which could be easily scaled up to full production.

In looking at the periodic tables in the $\mathrm{Th}-\mathrm{U}^{233}$ system (Slide 1), $\mathrm{U}^{232}$ is generated in the process of forming $\mathrm{U}^{23}$ from thorium. In a water reactor system, this can be formed by two routes, $(n, 2 n)$ reaction on $\mathrm{U}^{233}$ and $(n, 2 \mathrm{n})$ on $\mathrm{Th}^{232}$, going through the protactinium route to $\mathrm{U}^{232}$. This latter route contributes about $90 \%$ of the yield. The other contributes about $10 \%$. The $\mathrm{U}^{232}$ itself is a rather high activity alpha emitter. Through a series of alpha decays, it ends up with two daughters-- $\mathrm{Bi}^{212}$ and $\mathrm{Th}^{208}-\mathrm{C}_{-}$ both of which are highly energetic gamma emitters. It is from these sources that one encounters the problems of handling $\mathrm{U}^{233}$. The specific alpha activity of $U^{233}$ and $U^{232}$ is not as high as plutonium but it is high erough to require glove box handling. 
11. 3

SLIDE 1.

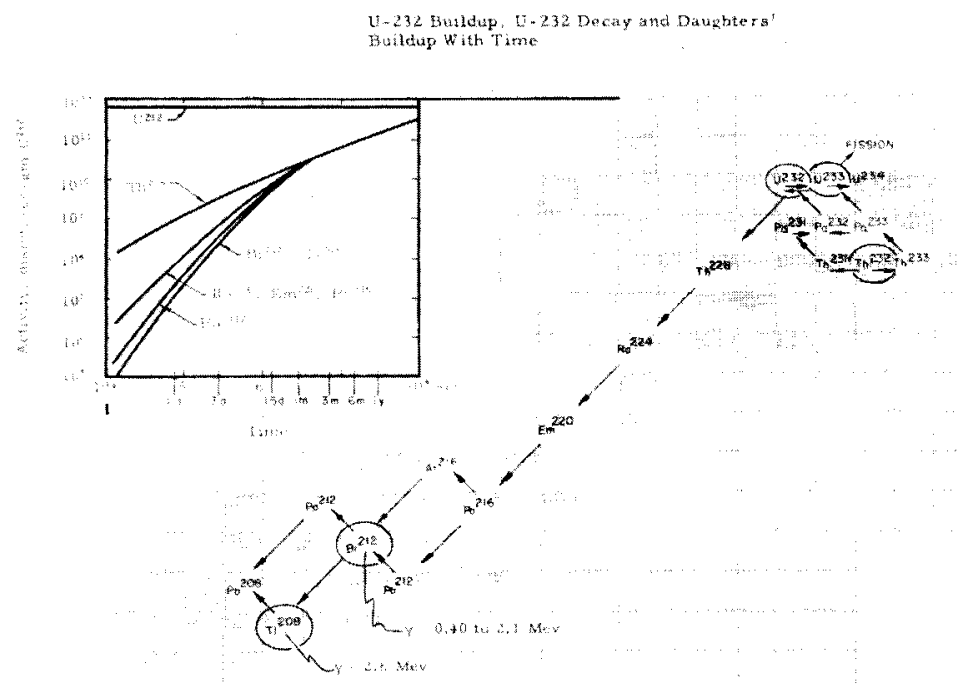

In the engineering sense, one would say, "Well, what does this mean in terms of activity from fuels?" The activity involved is a function of the time from purification or partition of the uranium from the throium in the solvent extraction plant. Slide 2 shows the dose rate at $1 \mathrm{ft}$ as a function of the $\mathrm{U}^{232}$ content in total uranium. One can see that it rises with time beginning with one day, six days, and then on into weeks and then on into weeks and months. As an example, the $\mathrm{U}^{232}$ content in the Indian Point Core A fuel will be about $125 \mathrm{ppm}$ of $\mathrm{U}^{232}$ in uranium. For a water reactor optimized on the $\mathrm{U}^{23}{ }^{3}$ cycle, such as the Spectral Shift Control Reactor, equilibrium $\mathrm{U}^{232}$ contents of up to $4000 \mathrm{ppm}$ are encountered. A large and definite increase in the dose rate with time is shown. At the same time, fast neutron activity must be considered because of an $(\alpha, n)$ reaction on light elements such as beryllium and fluorine. However, within the specification of light elements in the solvent extraction plant product, we found that the neutron activity was very low and essentially insignificant as compared to the gamma activity.

SLIDE 2.

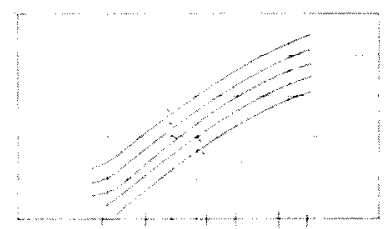


11. 4

In considering the process flow (Slide 3 ) and using the guidelines for what one would build as an ideal near term plant for handling high activity fuel, we ended up with three major parts:

1. The Sol-Gel method for making high density thoria-urania particles using the early work of Oak Ridge National Laboratories adding some modifications of our own

2. Vibratory compaction of the particles in the fuel tubes and

3. Underwater assembly of fuel elements.

By means of these three separate processes, we were able to quite nearly approach the goals that we sought for this near term processing. I will detail the processes.

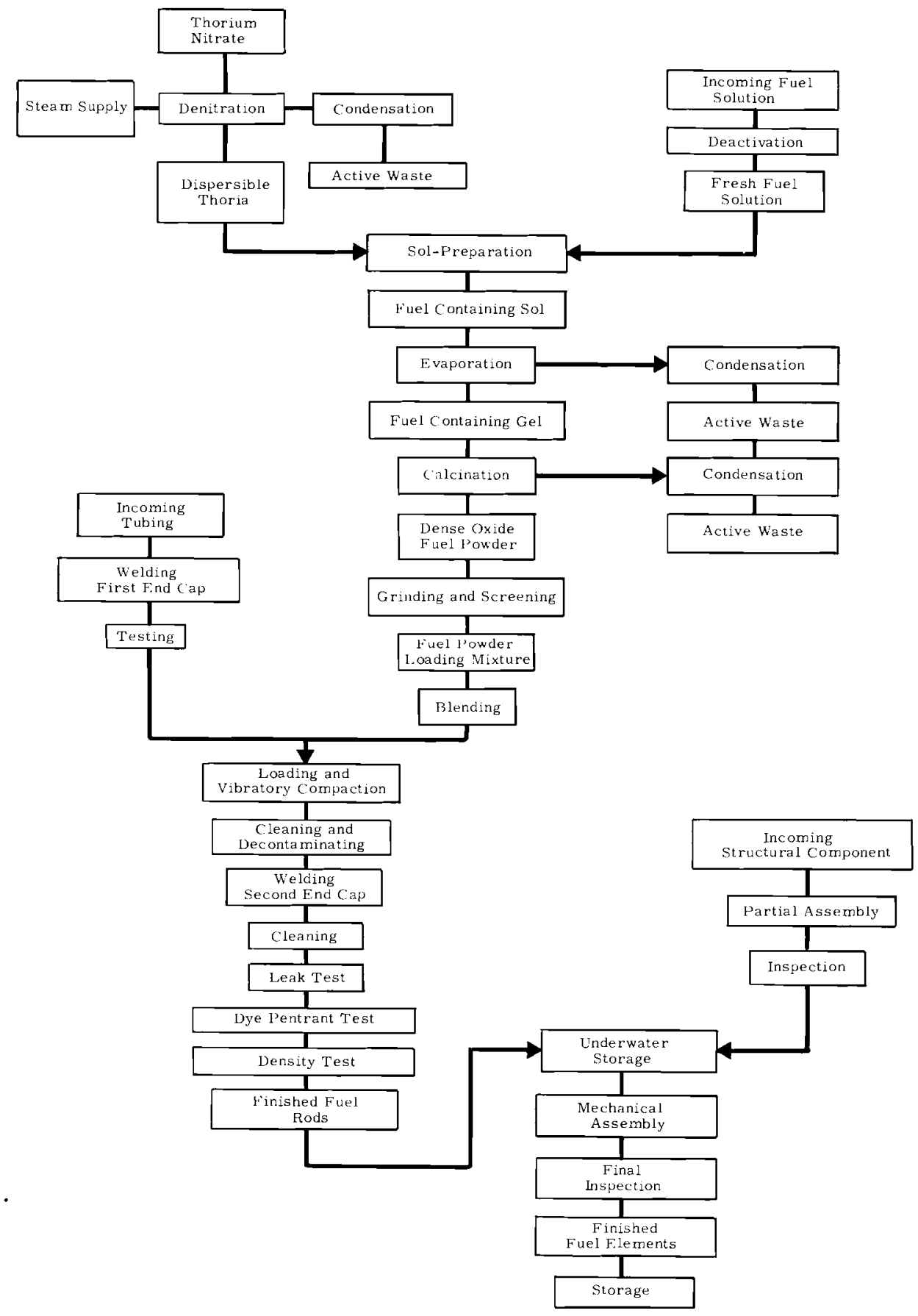


A very important aspect of design and operation is criticality control. This, of course, is established by the size of boxes and equipment and how much material can be put through it. The thoria is quite useful here because it helps to self-shield the gamma rays and increases criticality limits. For example, Slide 4 shows the allowance factor as a function of the amount of fissile material in the total fuel oxide.

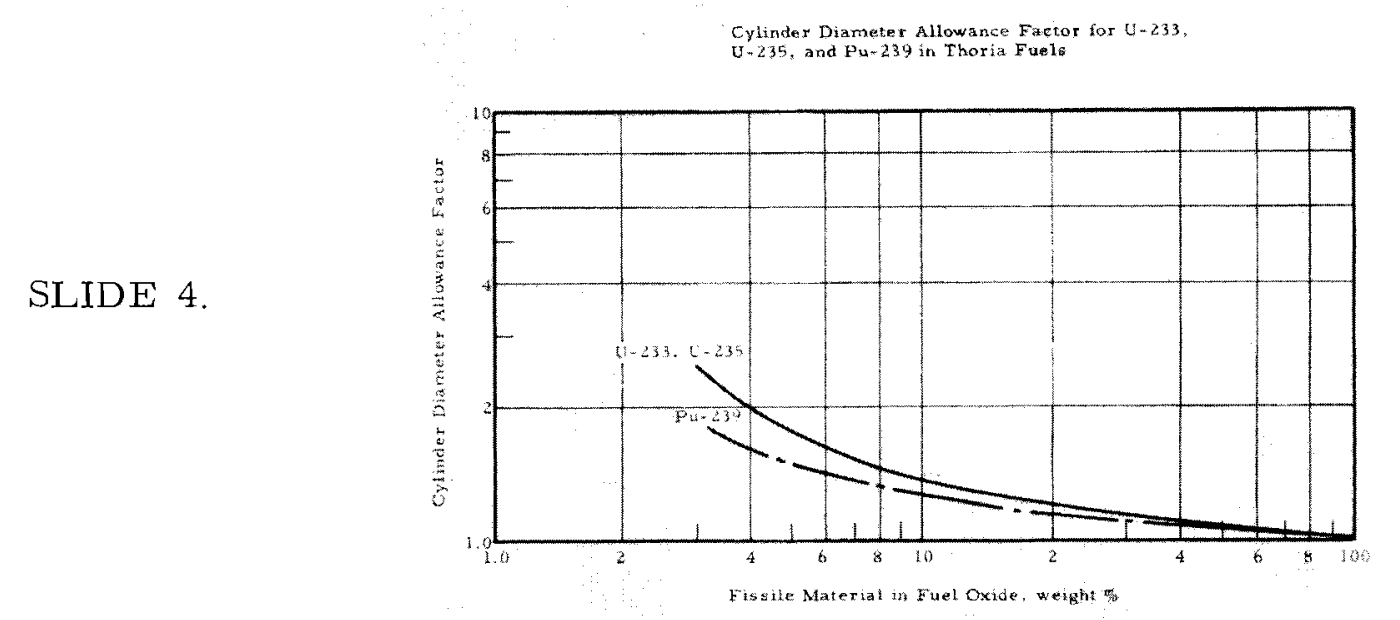

Slide 5 shows geometry allowance factors. In the thorium system a lower allowance factor is applied for $\mathrm{Pu}^{239}$ and then for uranium isotopes.

\section{SLIDE 5.}

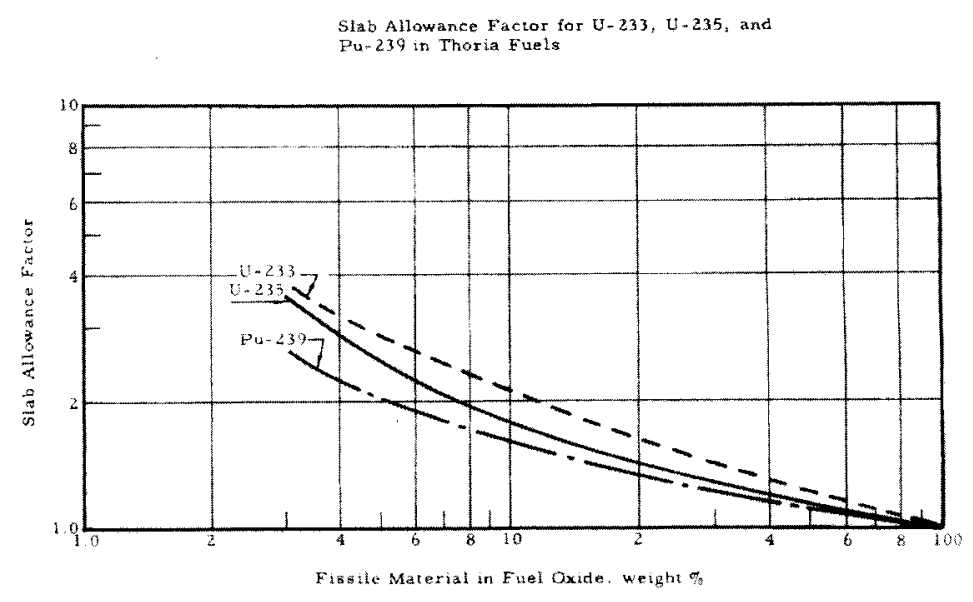

Slide 6 shows that for the case of mass control there is quite a penalty for going up into the higher percentage ranges. The range that we worked in was about $3 \%$ which happens to be about optimum for a water reactor. If one were to go up into higher ranges there is a large difference 
in the factor. The following series of slides is a pictorial tour of the line itself. Briefly, the Sol-Gel process is simply a low temperature process for making high density oxides. Thorium nitrate-tetrahydrate crystals are hydrothermally denitrated (Slide 7) in a superheated steam atmosphere, resulting in a very finely divided thoria which has a controlled quantity of residual nitrate ions.

SLIDE 6.

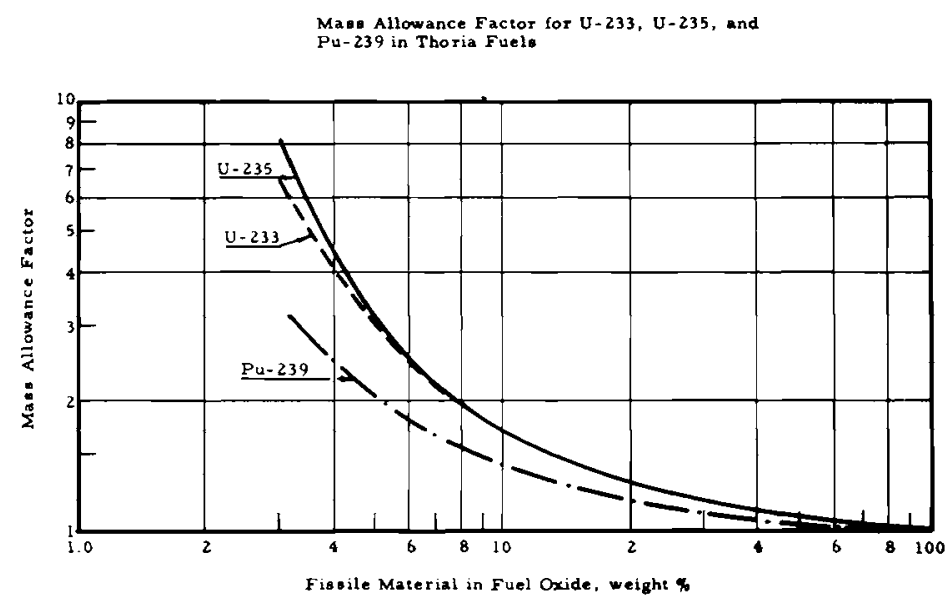

SLIDE 7.

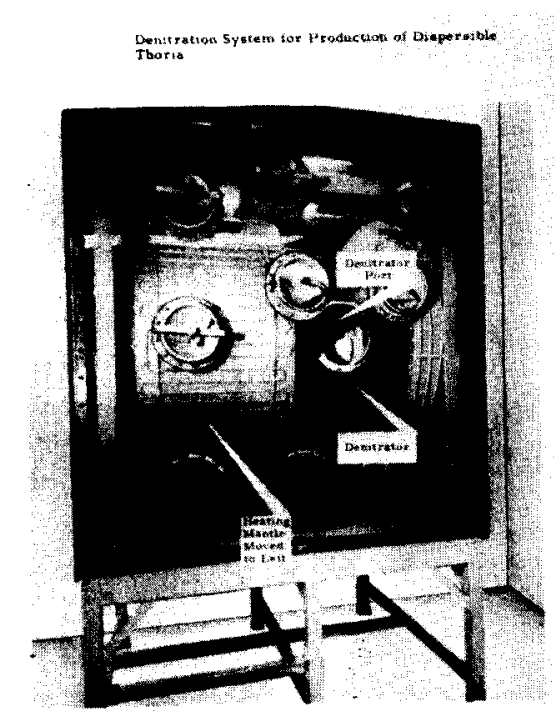

Slide 8 shows the glove box for ion exchange. As mentioned earlier, one can gain some time in handling this material by partitioning the $\mathrm{Th}^{228}$, which is the daughter of $\mathrm{U}^{232}$, just before processing the material, essentially starting from ground zero again. We use ion exchange methods for 
separating the uranium from the thorium at the beginning of the process. It is a rather simple process--the passing of uranyl nitrate through

Dowex 50 resin. For a production plant, solvent extraction would be used for this purpose since the additional benefit of scrap recovery would be obtained.

SLIDE 8.

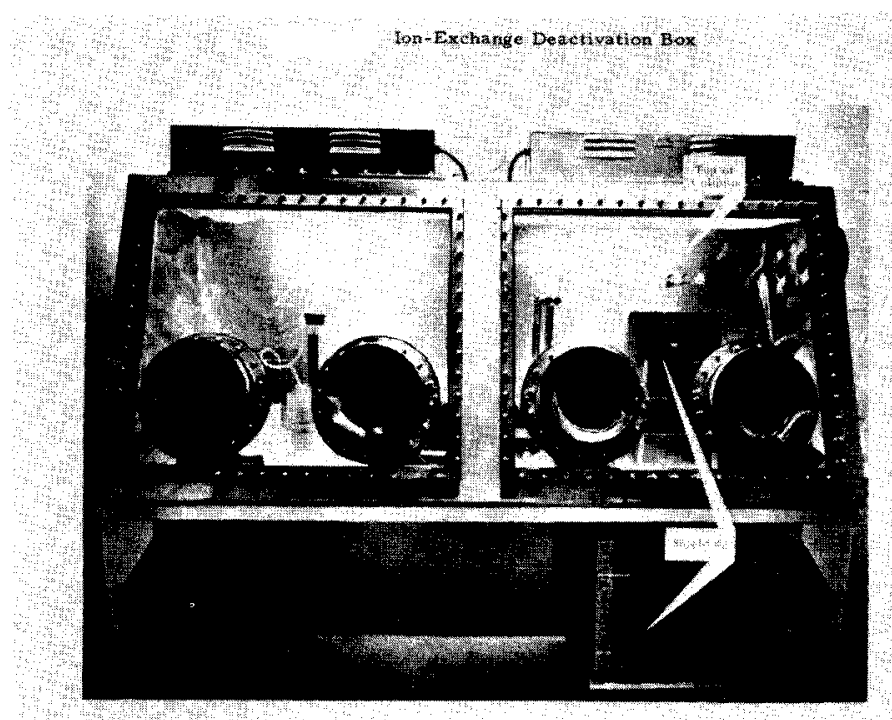

Slide 9 shows a long end view of the Sol-Gel line. The thorium oxide particles are dispersed in an aqueous acidic medium in this slab tank (for geometry criticality control) and the uranyl nitrate is added. The uranyl nitrate attaches itself by ionic processes to the surface of the thoria particles. This forms a true colloid, or sol, of a very homogeneous mixture. After circulation for a short while in the tank, the sol is pumped to pans in a drying oven at about $200^{\circ} \mathrm{F}$. The resulting gel looks like the bottom of a dried up creek bed. These particles are then moved into the calciner box where they are heated to 1100 to $1150^{\circ} \mathrm{C}$ in a reducing atmosphere to produce particles of $99 \%$ plus density. . The particles are then moved into the powder preparation box where they are classified for vibrational compaction.

SLIDE 9.

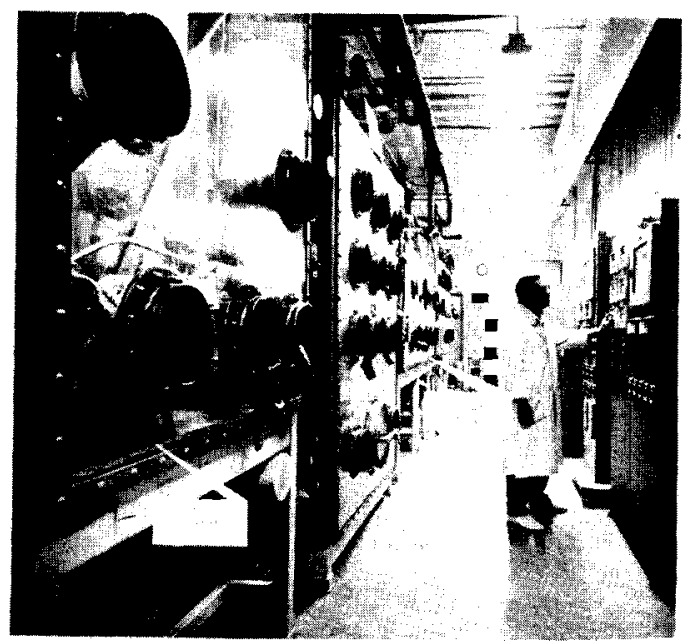


Slide 10 shows the vibrational compaction line which was optimized for the highest production possible consistent with this particular method of fabrication. The proper size fractions are introduced into the blending box at the top where they are weighed and blended. The blended mixture is fed into the cladding while vibrating. The rods were $0.420 \mathrm{in}$. OD by $10 \mathrm{ft}$ long Zircaloy-4. The fabrication was all performed in conformance with power reactor specifications. The end caps are inserted and welded. The rods were then moved to the quality control boxes for checks on weld quality, helium leak test, and also gamma absorption for density variation. After ultrasonic cleaning, the surface is smeared for activity, then taken out the end of the box. At this point, the rods are complete.

View of the Rod Fabrication Clove-Box Line

SLIDE 10.

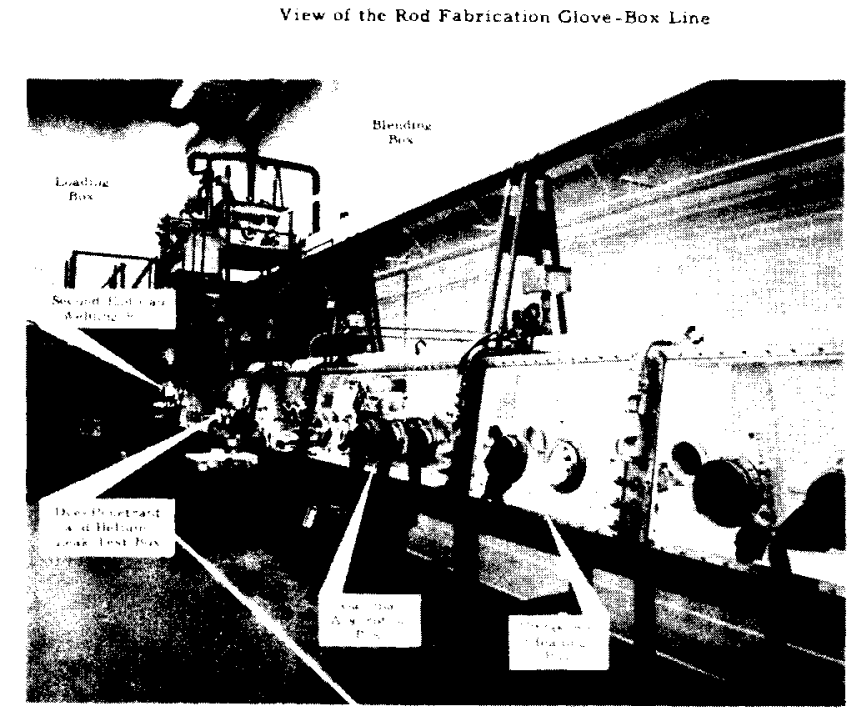

Slide 11 shows a machine which we built for assembling fuel elements under water. When enough fuel rods are stored to make a fuel element, the activity begins to build up to a considerable extent. This building is not only due to time but also due to the large number of rods involved. Underwater assembly allows two advantages: ready, visual access to the rod storage and ease of mechanical assembly. This machine is automatically controlled so that it will pick up the rods from a designated storage spot and insert them in the right places in the fuel element grid.

Slide 12 shows the finished fuel element that we pulled up out of the canal for a short while to get a surface reading. It is roughly a 170 rod design, about $10 \mathrm{ft}$ long. This was all assembled under water including the end fittings. This completed the job.

Slide 13 shows the analysis of the U 233 shipped to us by the Commission from Oak Ridge. Note that it had about $42 \mathrm{ppm}$ of $\mathrm{U}^{232}$ and $\mathrm{U}^{233}$. The high fluoride level caused difficulty in the ion exchange. In "cold" tests, we obtained 15 -fold reduction in gamma activity. With the $\mathrm{U}^{233}$, it was only 2 -fold because of the high fluoride level and a uranium concentration twice as high as specified. After one run we abandoned ion exchange, resulting in our handling 60 day old rather than 3 day old material. It turned out to be an interesting experience. 
11.9

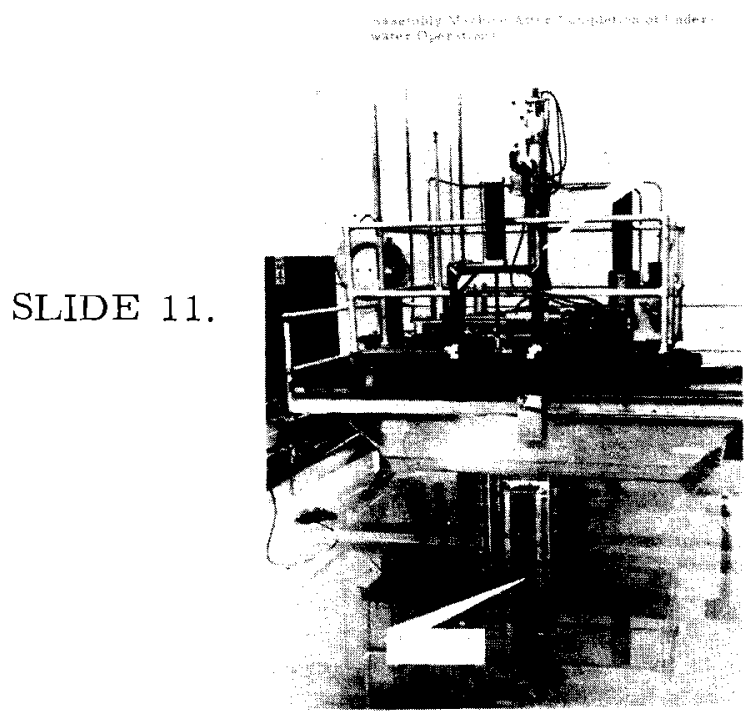

SLIDE 12.
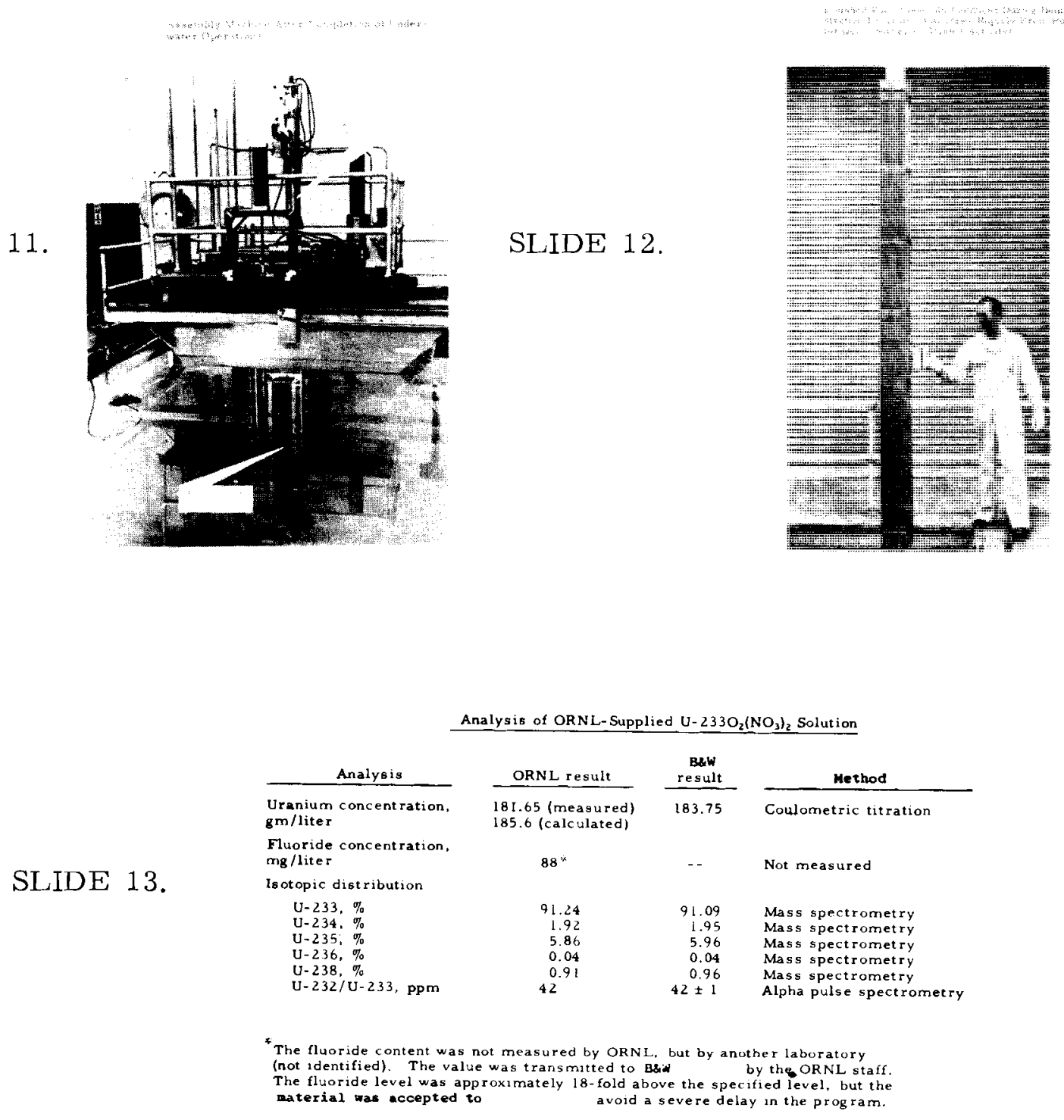

Slide 14 is a chart showing the growth of the gamma activity in the $\mathrm{U}^{233}$ as actually measured. The first drop in activity after partition is due primarily to the decay of the $10.6 \mathrm{hr} \mathrm{Pb}^{212}$. Then, the buildup of $\mathrm{Th}^{228}$ begins to take over causing a rise in the curve. The material we worked with was 50 to 70 days old.

With pulse height spectrometry, alpha spectrum measurements were made as shown in Slide 15.

Slide 16 shows the gamma spectrum. The energy of the gamma activity from $\mathrm{U}^{233}$ is quite a bit more than one sees in current plutonium. 
11. 10

SLIDE 14.

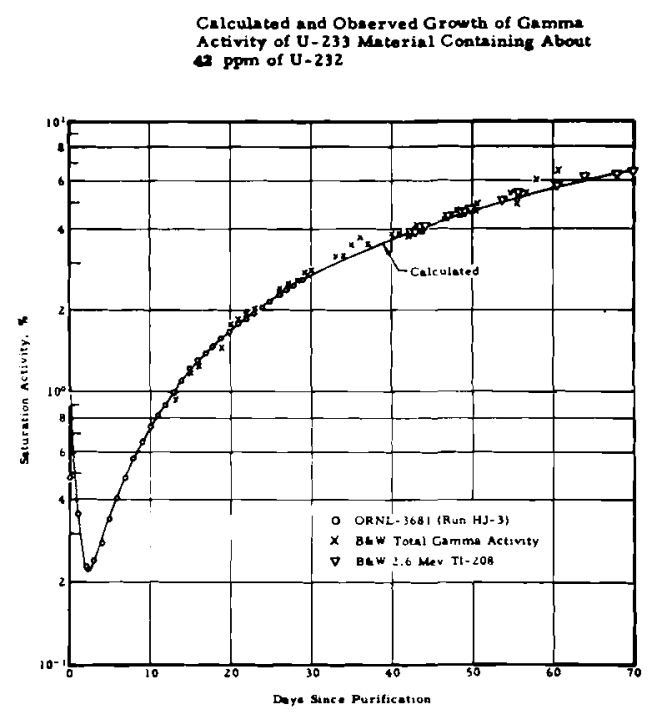

Alphe Spectrum of the Uned U-233 Material

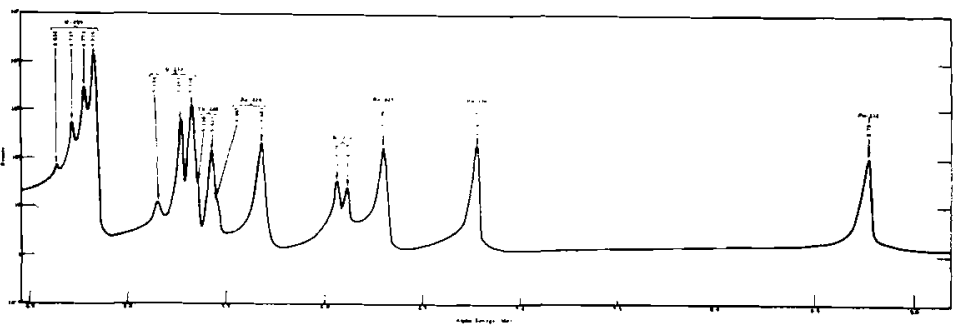

SLIDE 16.

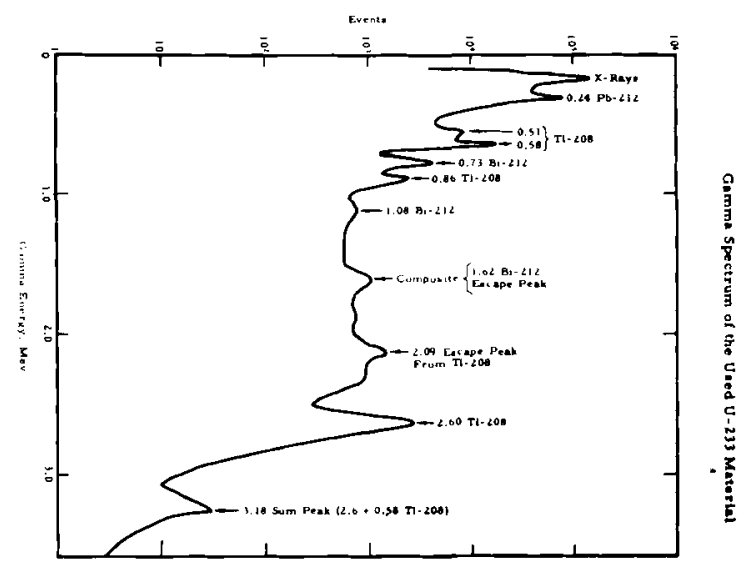


The table in Slide 17 summarizes the gamma doses that we have experienced in handling this material. We have found that we were able to process material from the nitrate to the completed fuel rods in $48 \mathrm{hr}$. The total dose for all people involved in the project as determined by film badges and confirmed by ion-chamber dosemeters was $880 \mathrm{mR}$. Factoring in the number of people and the amount of material involved, results in 54 millirem per man per week. for average 60 day old $\mathrm{U}^{233}$ containing $92 \mathrm{ppm} \mathrm{U} \mathrm{U}^{232}$. The present permissible dose on a weekly basis is 100 . The quarterly dose is $1.25 \mathrm{rem}$ per quarter per man. The principal dose contributor was in the powder preparation box (which is what one would expect). It is a very dirty operation and there is a lot of material that ends up hanging in the screens.

For measuring dosage, the men wore pocket dosimeters and film badges all the time they were in the plant.thus picking up activity from all sources. Also, pocket dosimeters and film badges were kept in lead containers at each work station. As each operation was performed, each man removed this particular film pack and dosimeter and put it on his person. When he was through, he put it back recording the time involved. The result is "station dose" or the exposure he received doing that particular operation. By comparing the total and station doses one can see a significant amount of radiation received from other sources--primarily, scatter from other parts of the line. This does indicate that it is desirable from an administrative standpoint to operate a plant like this in a manner that reduces scatter from other radiation sources.

To conclude, these data indicate that $\mathrm{U}^{233}$ containing up to a thousand parts per million of $\mathrm{U}^{232}$ in uranium can be processed by direct means.

The implication to the FFTF driver fuel situation cannot be drawn directly. For instance, in this process we use a Sol-Gel process for making the thoria-urania. At this time that process cannot be used for making high density plutonia-urania economically. However, there are lessons to be learned in the assembling of the fuel elements and vibrational compaction of the rods. With plutonium, there is a problem of gamma activity buildup with time due to the buildup of the daughters of U237 and Am ${ }^{241}$. Certainly, the people here at Hanford have had a lot more experience with this particular situation. In addition to the neutron activity, the spontaneous fission from $\mathrm{Pu}^{24} 40$ is something that has to be contended with. In general, the same principles apply in handling any high activity fuel. These have to be recognized early in the program.

If there is one message that I would leave, it would be to urge those who are involved with designing the FFTF driver fuel give early recognition to the implications of commercial fabrication. I would like to hear some comment on this, particularly with respect to the tentative plans as far as the purchase of the cores. I can see where the first core or the second core might be custom built in a laboratory, but I would think that it should quickly be relegated to the commercial domain. Thus, it will be very important to feed the technical and economical implications of commercial manufacture back into core design. 
11. 12

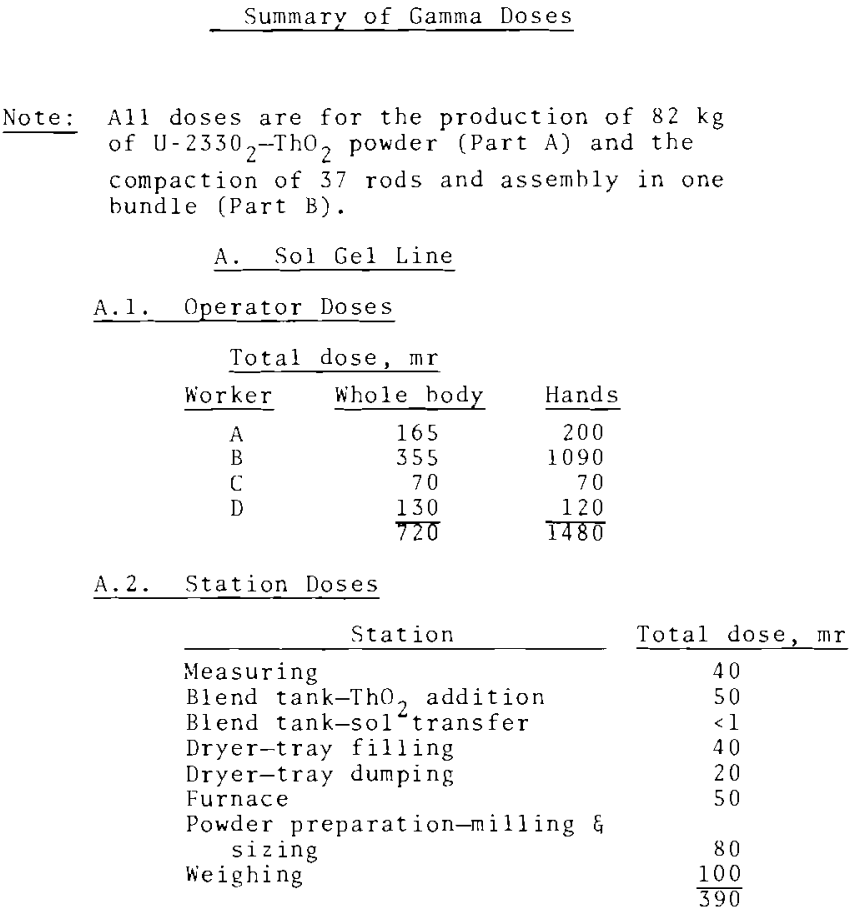

SLIDE 17.

B. Rod Fabrication Line and Mechanical Assembly

B.1. Operator Doses

Total dose, $\mathrm{mr}$

Worker Whole body Hands

A $60 \quad 170$

B $\quad 50 \quad 130$

\begin{tabular}{rrr}
$\mathrm{C}$ & 40 & 170 \\
$\mathrm{D}$ & 10 & 30 \\
\hline
\end{tabular}

B.2. Station Doses

Station

Total dose, $m \mathbf{r}$

Blending

Loading

Cleaning

he ighing

helding

Leak test

Gamma absorption

Final decontamination

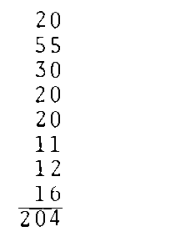

C. Totals

C.1. Operator Doses

Total dose, mr

Operator location Whole body Hands

Sol-gel line $720 \quad 1480$

Rest of plant $\quad \frac{100}{880} \quad \frac{500}{1980}$

C.2. Station Doses

Station _. Total dose, mr

Sol-gel line

Rest of plant

190

$\frac{204}{594}$ 


\title{
FABRICATION PROCESSES-HIGH ACTIV_TY FUELS
}

\author{
L. R. Weissert \\ Babcock and Wilcox \\ Lynchburg, Virginia
}

\section{DISCUSSION}

MR. HENNIG: Are there any questions or comments?

MR. BURNHAM (Battelle-Northwest): When will high activity fuels be commercialiy feasible?

MR. WEISSERT: I can't comment on when the commercial people will be producing this fuel. I trust it will be rather soon. But I think a couple of comments are in order on the plutorium versus U233 problem. You are stuck with some high m. e.v. gammas, which are a problem. And then you have the neutrons on top of this. In the plutonium we have looked at very recently, Roberts \& Van Tile have very carefuliy analyzed some $31,000 \mathrm{~m}$. w. v. yankee fuel, and this gets up to about $1 \%$, weight percent $\mathrm{Pu} 238$, and a lit:le less than a-part-per-million of 236. (In fact, considerabiy less.) It works out that actually for the alpha $-\eta$, the $\mathrm{Pu}^{238}$ is really the big problem. The gammas are not a problem. We have looked at this rather carefully. It works out that a combined $1.2 \mathrm{~cm}$ of the 3.2 density lead glass are more than adequate to handle the gammas. The neutron problem with this fuel now is potentially a problem but it is something that you are working away from. We envision a potential problem when you have a large bundle, and you ask a man to work right on top of it, such as final assembly, and calculations show that $4 \mathrm{in}$ of borated paraffin is more than adequate to handle anything there.

Again, you are going to have to handle it in body doses. You are going to have to think about it, as you say. How are you going to make some of these final inspections? Now maybe som ebody else has better ideas on what the plutonium isotopics will be for the FTR. I would be surprised if it were this high burnup material.

MR. HENNIG: I believe that we happen to be one of the few applicants coming up soon where there is a genuine motive to use non-prototypic plutonium. In that particular area we go to the cermet fuel。 It is a little less clear for other ceramics. For experimental work, your applications in, say, Fermi, or the next prototype, one would want high burnup plutorium fuel to run all the tests, including the first core. It doesn't seem to be true for us. We always have some benefit, even maybe very marginal, in using a very low burnup.

MR. WEISSERT: The point is well made, and I think with a proper plant, and a given design, a given fabrication process, a great deal can be done to administer and control the process and keep within limits. But a lot of inquiry has to be put into the original design of the fuel on the particular fabricating process. In some fabricating processes it is just an inherently built-in difficulty, when we are talking about handling highly active fuel. I thirk that is certainly my principal message at least. 


\section{Dll. 2}

MR, SHOUDY (APDA): What is the design capacity of your line and did you ever reach that capacity?

MR. WEISSERT: With the amount of material available to us, we did not reach the design capacity of the line.

MR. SHOUDY: And what was it?

MR. WEISSERT: As far as the design capacity of the line, it was about $300 \mathrm{k}$ per day, but with rather low cost modification that could be increased considerably. 
PLUTONIUM FUEL FABRICATION EXPERIENCE AT NUMEC

K. H. Puech 1

Nuclear Materials and Equipment Corporation

Apollo, Pennsylvania

\begin{abstract}
INTRODUCTION
I had planned on concentrating on our particle forming and coated particle technology and assorted dispersion technology, but after yesterday, I don't think there is anything we have done in the way of plutonium fabrication that might not be applicable to FFTF. So, I would rather generalize my talk, and in fact, I will use some slides I used in Washington last week. I hope that the few of you who attended the washington meeting won't be too bored.

We have been in the plutonium business now for six years, and in that time, we have been continually striving towards complete horizontal integration. We are trying to make all plutonium compounds, alloys, and any plutonium-bearing fuel material. This across the board capability should allow us to produce anything that the industry wants. To a large degree, we are already there (as far as the R\&D facilities go), and we are fast putting in the production capability. For example, we can supply in tonnage quantities, encapsulated fuel elements that contain $\mathrm{PuO}_{2}, \mathrm{UO}_{2}-\mathrm{PuO}_{2}, \mathrm{Pu}-\mathrm{Al}$, or $\mathrm{Pu}-\mathrm{U}$ alloys. Orders for these fuels that require processing of contained plutonium at a rate of $1,000 \mathrm{~kg} / \mathrm{year}$ can be satisfied routinely.
\end{abstract}

\title{
FACILITIES AND PROJECTS
}

Slide 1 shows the magnitude of our facilities. The building is about 380 feet long. On the one end is a hot cell facility for post-irradiation examination and also for encapsulation of high activity material. The next area is the research and development area, which has many glove boxes with specialized and versatile small equipment such as precipitation equipment, calciners, sintering furnaces, casting furnaces; also analytical chemistry equipment and physical property measurement equipment for determination of such properties as melting point, thermal conductivity, etc. The next area is the metal working facility where we have been doing primarily, plutonium-uranium alloy work; precision casting, machining, and welding of ZPR-type elements. The next area is our mixed-oxide production line. We have facilities for both precipitation of the plutonium to the oxalate, or coprecipitation with uranium to ADU-hydroxide and then reduction to oxide, and a 
pelletizing line to go along with this. The next small room is the neutron source manufacturing area where we manufacture neutron sources containing plutonium, plutonium-238, americium, or polonium. In the far end is our scrap recovery facility to allow recovery of all the cold scrap we generate, as well as for performing outside jobs. Along the front wall of the fabrication area are many associated laboratories, such as analytical chemistry, particle coating and particle forming laboratory, electron microscopy laboratory, a small metals fabrication area where we have a small rolling mill (for making foils), arc melting furnaces, and a hot press.

SLIDE 1

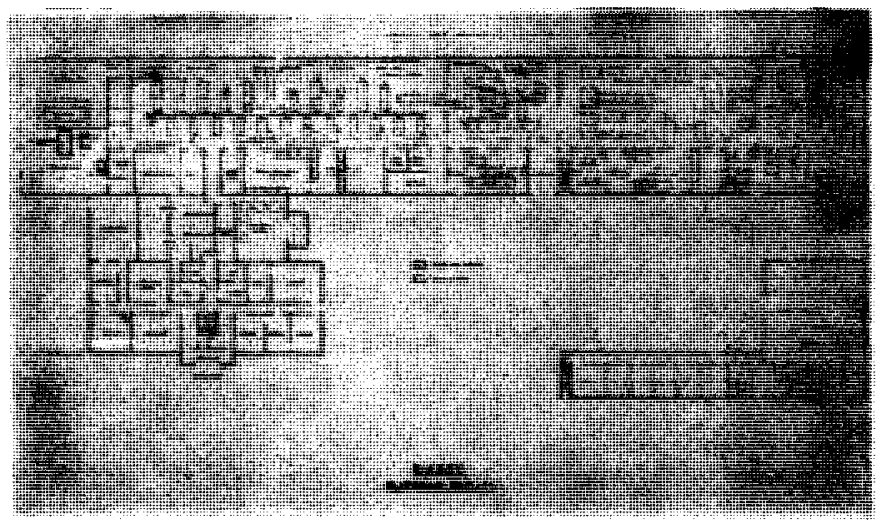

S1ide 2 gives you an idea of the magnitude of the facilities inside. This is the research and development area where the smaller equipment is located and where we have quite a bit of flexibility. All the glove boxes in this area are connected to the central transfer tunnel, to minimize generation of plastic bag scrap.

SLIDE 2

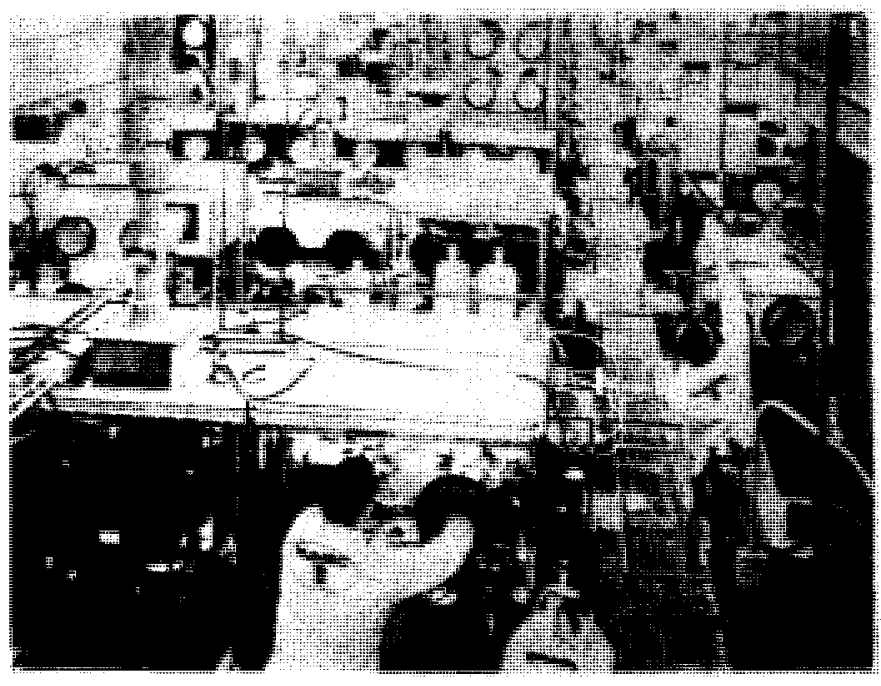


Slide 3 is a view of the ceramics production area. You see the larger glove boxes for the wet chemistry operations, and the more continuous 1 ine for the pelletizing and powder handling operations.

\section{SLIDE 3}

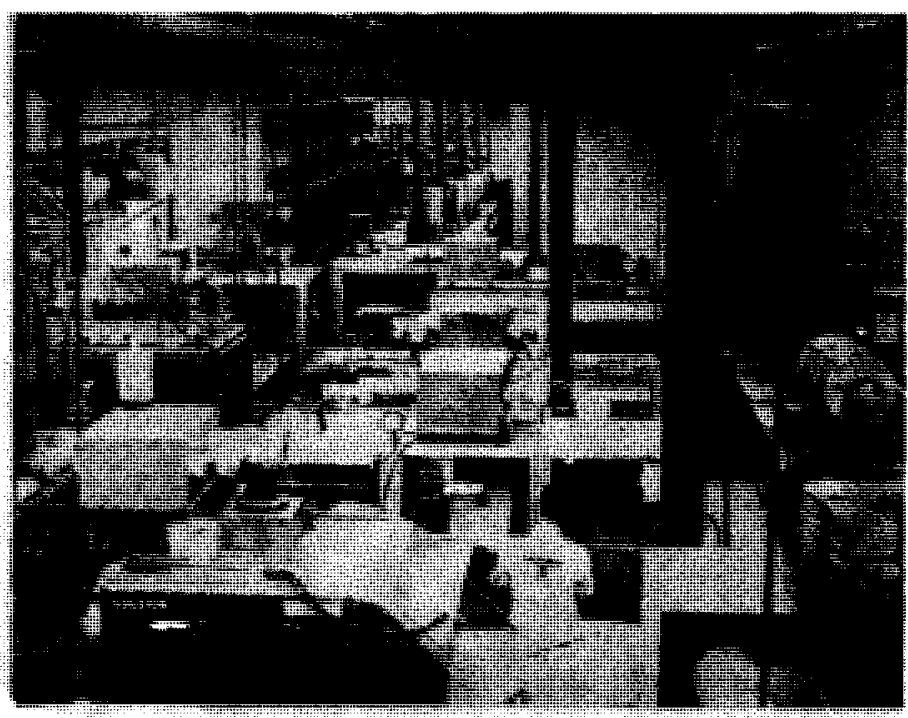

Since Mr. Weissert brought it up, we've had no particular problem with licensing. It takes time to educate licensing or to get liaison going with the people, but once this is accomplished, there is no difficulty in obtaining approval to handle material to the same 1 imits as you do here at Battelle-Northwest. For mixed oxides, (for example) we can take credit for dilution with the uranium; in dry form we can go up to a 30 to $50 \mathrm{~kg}$ limit of plutonium in one glove box. For the magnitude of the fabrication jobs we have now, this is perfectly adequate. In fact, production is limited, by the size of the equipment rather than by criticality considerations.

Some of our research work could be related to FFTF. I would like to describe a few of these projects. We have a contract for trying to grow single crystals of both alpha and delta-stabilized plutonium metal. Here we are using strain annealing techniques as well as high pressure transformation techniques. This is the most basic program we have; so far, I am sorry that we cannot report amazing success. We are trying hard and we are getting some grain growth, but as yet, we are far away from single crystals.

On mixed oxides, we have an irradiation program where we have made the powder, fabricated pellets and fabricated capsules. The capsules were radiated at GETR, and two of them are now back at our hot cells being examined. In these capsules, we prepared $\mathrm{UO}_{2}-5 \% \mathrm{PuO}_{2}$ material and $\mathrm{UO}_{2}-20 \% \mathrm{PuO}_{2}$ material; so we are looking 
at plutonium recycle as well as fast reactor application. We prepared the material both by coprecipatation and by mechanical blending, and we sintered under conditions to get both hyper-stoichiometric and hypo-stoichiometric pellets. We also prepared some pellets that had built-in inhomogeniety. We prepared $\mathrm{PuO}_{2}$ spheres, we sintered some, we left some unsintered, and then packed these into a $\mathrm{UO}_{2}$ pellet to see what kind of diffusion effects we would get with time in pile. This is a way of evaluating how sloppy one could be in blending procedures for mixing $\mathrm{UO}_{2}$ and $\mathrm{PuO}_{2}$, and still not get into trouble. As I said, two of these capsules are in our hot cell, and these are the thermal fuels. They were irradiated to 10,000 and $20,000 \mathrm{MWd} / \mathrm{ton}$. In general, there were no surprises; fission gas release seems to be comparable for the various materials, and it shows, essentially, total release for the fuel that operated above $1800{ }^{\circ} \mathrm{C}$. The effect we did notice was that the sub-stoichiometric fuel seems to have a somewhat higher thermal conductivity than the stoichiometric fuel. The other three fast fuel capsules are still in pile and are being irradiated to $25,000,67,000$ and $100,000 \mathrm{MWd} / \mathrm{ton}$.

We have another irradiation-type contract which covers fast fue 1 development. Here we are going into EBR-II. We are now making up 42 EBR-II pins for this irradiation program. We are looking at many variables: stoichiometry, coprecipatated versus mechanically mixed, low density versus high density pellets, different pellet to clad gaps, different cladding materials for cladding restraint, dished pellets, and undished pellets. From the comparison of performance of materials, we will be able to provide firm specifications for mixed oxide fast breeder fuels. In this program we are placing more emphasis on low density fuels than originally planned. We are going down to average densities (over a fuel rod section) of between 80 and $85 \%$. If you try to sinter pellets to this low density without doing anything but changing time and temperature generally you find that the pellets don't have enough strength and that disintegration occurs during subsequent operations such as grinding. One can use additives; at present we are using. methoce 1 and burning this out, and this seems to work quite we 11. The density is apparantly quite sensitive to the quantity of methocel added.

Another R\&D program we have that may be of interest is with the Joint EURATOM Board. This is for recycle fuel. What is interesting for the FFTF program is that the fuel contains a large quantity of inert diluent. You could look at this as a cermet, as far as behavior goes, but it is really a ceramic. What we are looking at is development and irradiation testing of a $\mathrm{PuO}_{2}-\mathrm{UO}_{2}-\mathrm{ZrO}_{2}$ fue 1 , with $\mathrm{ZrO}_{2}$ contents ranging from 25 to $75 \%$. We did not propose this fuel concept primarily for irradiation stability; we proposed it to allow recycle in present day thermal reactors without changing the lattice. By way of explanation, plutonium has a higher cross section than $\mathrm{U}^{235}$, therefore a looser lattice (wetter lattice) is required to achieve optimum economic fuel cycle costs with plutonium. Since it is rather difficult to change lattice spacing in an existing reactor, and also since you can't get the same amount of heat out of a given sized pot if you loosen up the lattice, putting $\mathrm{ZrO}_{2}$ into the fuel, allows optimization of the plutonium-to-water ratio with- 
out changing flow or heat transfer characteristics. We have tried to make this fuel by a number of routes, coprecipitation as well as mechanical mixing of the various components. A good single-phased material has resulted in all cases. Some out-of-pile testing has been performed to determine phase stability, and this also looks good. We are now fabricating test samples, capsules to go into GETR, and this irradiation program. should start next month. Next year we will be doing phase diagram studies and more physical property measurements out-of-pile to support the irradiation program.

Now, I would like to discuss the fabrication projects, the fixed price jobs we have performed in-house to other customers specifications. This will give you an idea of the variety of things we can do, the quantities of materials that we have handled, and generally will show that it hasn't been a problem handling relatively large quantities of plutonium.

Slide 4 is a tabulation of the significant production jobs that we have performed over the past two years. The first job listed was making plutonium-alumium alloy extrusion billets for Savannah River for their curium program. This was casting the alloy, canning it in aluminum, leak checking it, and so on. We handled $80 \mathrm{~kg}$ of plutonium for this order, and I might say, it took us only a month to perform this work. About 200 billets were cast.

In another similar project with $\mathrm{Pu}-\mathrm{Al}$, but for the Australian AEC surprisingly enough, we fabricated MTR-type fuel plates containing $\mathrm{A} 1-33 \% \mathrm{Pu}$. This was a real tough alloy to work with, and we eventually had to roll the filler close to the melting temperature. We made 420 plates of this material.

For the ZPR-III, we made a fue 1 which is a uranium, $18 \%$ plutonium, 2\% molly alloy. We fabricated fuel plates, which are quite thin and are of different lengths, to fit into the ZPR drawers. Over 2000 plates were processed containing about $180 \mathrm{~kg}$ of plutonium. These plates were precision cast, machined, and canned in stainless steel.

Another plutonium-aluminum alloy job was for Brookhaven, for critical experiments. This was a $10 \%$ al1oy, and fuel was injection cast into pyrex glass and subsequently canned in Zircaloy-2. The problem here was the control of porosity, and we found that satisfactory control could be achieved because of low conductivity of the pyrex.

On mixed oxide work, we made some interesting material for Argonne about a year and a half ago; it was $\mathrm{UO}_{2}-5 \% \mathrm{PuO}_{2}$ and contained simulated fission products. We put these in, saw how many burned out during sintering, and then adjusted to give about the amounts desired for the simulated irradiations. It wasn't surprising, but the pellets were mighty hard to sinter to density because of gas evolution of the fission. 


\begin{tabular}{|c|c|c|c|c|c|}
\hline \multirow{2}{*}{ Production Type } & \multirow{2}{*}{ Fuel Composition } & \multirow{2}{*}{ Plutonium Content, $\mathrm{kg}$} & \multicolumn{2}{|c|}{ Number of Units } & \multirow{2}{*}{ Clad Material } \\
\hline & & & Elements & Pellets & \\
\hline Extrusion Billets & A1-10\% Pu A110y & 80 & 200 & - & A1 \\
\hline MTR-Type Fue1 Plates & Al-33\% Pu A1loy & 5 & 420 & - & A1 \\
\hline 2PR-Type Fuel Plates & U-18\% Pu-2\% Mo A1loy & 180 & 2,082 & - & Stainless Steel \\
\hline Fue1 Rods & A1-10\% Pu A110y & 65 & 800 & - & $2 r-2$ \\
\hline Oxide Pellets & $\mathrm{UO}_{2}-5 \% \mathrm{PuO}_{2}+\mathrm{Fissia}$ & 1.5 & - & 8,000 & - \\
\hline Fue1 Rods & $\mathrm{UO}_{2}-6.6 \% \mathrm{PuO}_{2}$ & 26 & 530 & 70,000 & $2 r-2$ \\
\hline Fuel Rods & $\mathrm{UO}_{2}-1.5 \% \mathrm{PuO}_{2}$ & 7.5 & 545 & 30,000 & $2 r-2$ \\
\hline Carbide Pe1lets & UC $-20 \%$ PuC & 0.3 & - & 500 & - \\
\hline
\end{tabular}

SLIDE 4. Significant NUMEC Plutonium Fabrication Projects 
In a more standard mixed oxide job, for the Saxton reactor, we supplied the pellet fuel elements. A total of seven fuel elements (530 fuel rods) composed of $\mathrm{UO}_{2} 6.6 \% \mathrm{PuO}_{2}$ and containing $26 \mathrm{~kg}$ of plutonium were fabricated.

A similar job was done for the AEC for use by GE in their recycle critical experiment program; this fuel was $\mathrm{UO}_{2}-1.5 \% \mathrm{PuO}_{2}$. Here again over 500 rods were fabricated, I would like to point out here that this material had $17 \% \mathrm{Pu}^{240}$ content, while most of the other projects used the standard 8\% plutonium. We had absolutely no trouble with the high Pu240. One of the reasons, obviously is the low percentage of plutonium, getting a tremendous amount of self-shielding from the uranium. On another project, which isn't listed here, we've handled, at one time, up to 600 grams of $\mathrm{Pu}_{2} 38$ in a series of three glove boxes. While here we had to watch the dose rates of the people, and we had to use lead-glass and leaded gloves, we handled this material without personnel over-exposures. High exposure plutonium will not be a serious problem for some years.

In addition to oxide and alloy work, we have recently gotten into the carbide business. We now have orders in house to fabricate approximately $8 \mathrm{~kg}$ of mixed carbide. We're doing this by the reaction of carbon with the oxides in a vacuum furnace.

In addition to these major plutonium jobs, we have also done much uranium work that bears on the FFTF program; pelletizing rod welding, brazing, etc. We have made fuel elements for some small reactor facilities and critical experiment programs.

Now I would like to get into the particle forming and coating work where we again do much more with $\mathrm{UO}_{2}$ than with $\mathrm{PuO}_{2}$, although we have made appreciable quantities of $\mathrm{PuO}_{2}$ spheres, $\mathrm{PuO}_{2}-\mathrm{PO}_{2}, \mathrm{PuC}$, and plutonium metal, both alpha and delta stabilized. The standard or the easiest way we have found to make spheres out of the oxide powders is to snowball these, that is to gently mill powders to get pick up until spheres are formed. These materials after they are formed are sintered. We have sintered to densities as low as $50 \%$ and as high as what you would normally expect from pellets, above $90 \%$ This is a typical snowball product (S1ide 5). They are not perfectly spherical, but for most dispersion applications, there is nothing wrong with them. They stand up very well with essentially no stringering. We have also tried to coat the $50 \%$ material for a particular application. One thing we tried was nickel-carbonyl. We found that when you use a carbonyl, the gas goes into all the open porosity, and you end up with a solid metaliic sphere rather than the low density one with which you started. If you try another coating technique on a low density substrate, for example, electron beam vaporization of the metal, you end up with a solid shell around a low density particle. Something like this may be good for fission gas retention and accommodation of local swelling.

Slide 6 is a picture of $\mathrm{PuO}_{2}$ plasma torched spheres. Here the sphericity is quite good. The one problem we have had with the plasma torch, which.I am sure can be solved, is the lack of control 
on the formation of central voids in the spheres. Under some conditions we have been able to have just a thin shell of oxide with a large central void, essentially, a bubble. Under other conditions, we have achieved nearly theoretical density. We have not been able to control this sphere forming technique to the degree that we would like but good sphericity is generally achieved. One can also run the torch in a manner such that nothing is done to the interior part of the feed particle; i.e., only the outer surface is scorched to give a smooth surface, and the inside stays at low density.

SLIDE 5. Snowballed

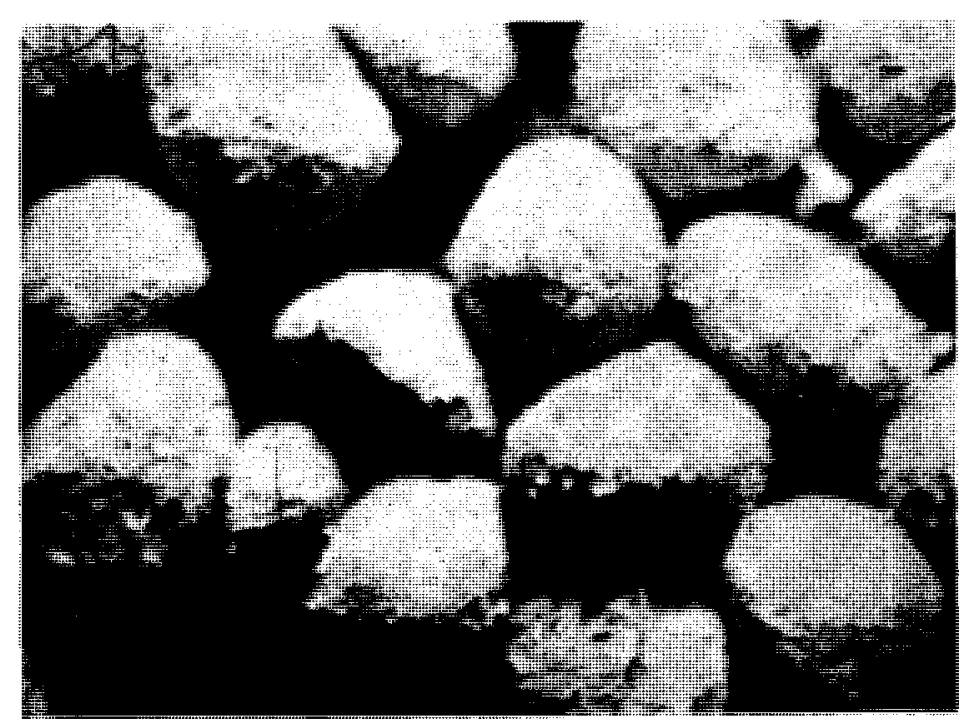

SLIDE 6. $\mathrm{PuO}_{2} \mathrm{Plasma}$ Torched Spheres

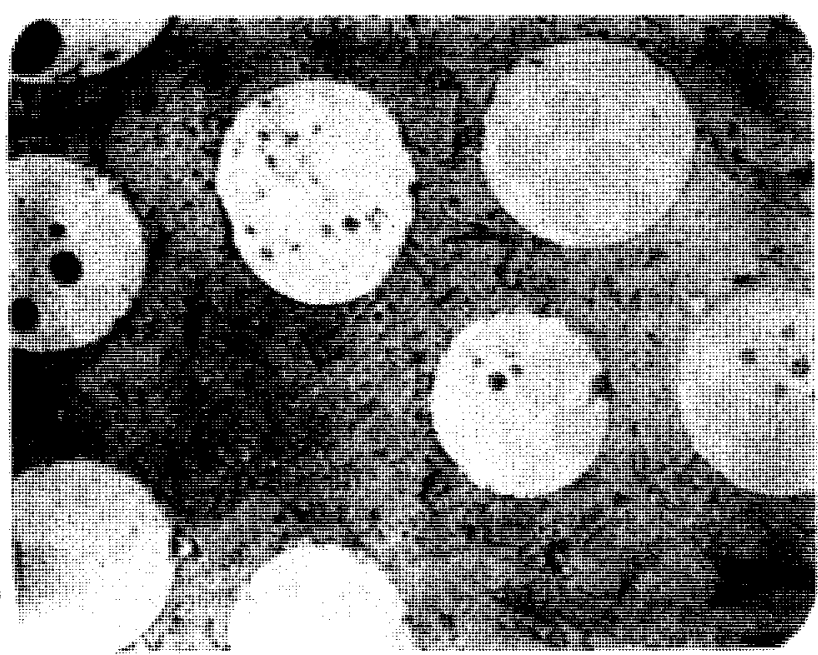


I have a few slides here to show different coatings and the character of them. Slide 7 shows niobium on $\mathrm{UO}_{2}$. You can see it is a good integral coating, and that there is adhesion with the substrate. The coating about $6 \mu$ of niobium. Slide 8 is the same, but magnified. Slide 9 is molybdenum again on $\mathrm{UO}_{2}$. I might say a little about how we make these coatings. We use a variety of techniques: Carbonyls, where you can get a carbonyl compound; nickel, and we have also tried tungsten, and molly. The problem with some of the carbonyls is you tend to get carbon inclusions, so you may end up with a carbide rather than with a metal. Halide reduction is another coating technique. This generally gives very pure coatings, especially of tungsten, molly, and other refractory metals.

SLIDE 7. Niobium Coated $\mathrm{UO}_{2}$

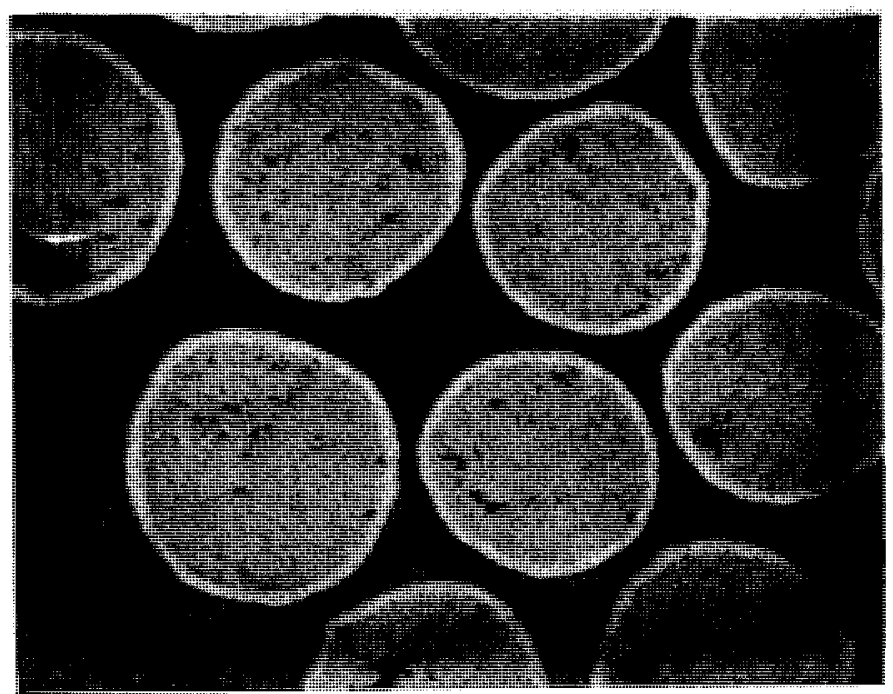

SLIDE 8. Niobium Coated $\mathrm{UO}_{2}$, Magnified

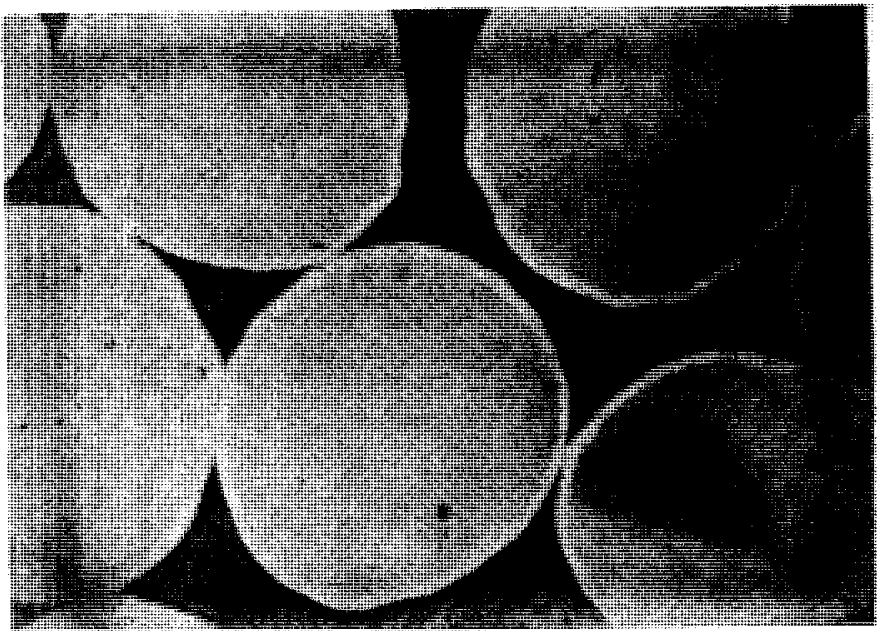


Lately we have been going more and more to electron beam vaporization. With this technique you focus an electron beam on a solid target and locally vaporize it to produce the coating gas. Almost any material can be vaporized and coated this way. With appropriate geometry and agitation of particles, one can get very good coatings. Slide 10 is $\mathrm{UO}_{2}$ with an $\mathrm{EB}$ coating on it.

SLIDE 9. Molybdenum Coated $\mathrm{UO}_{2}$

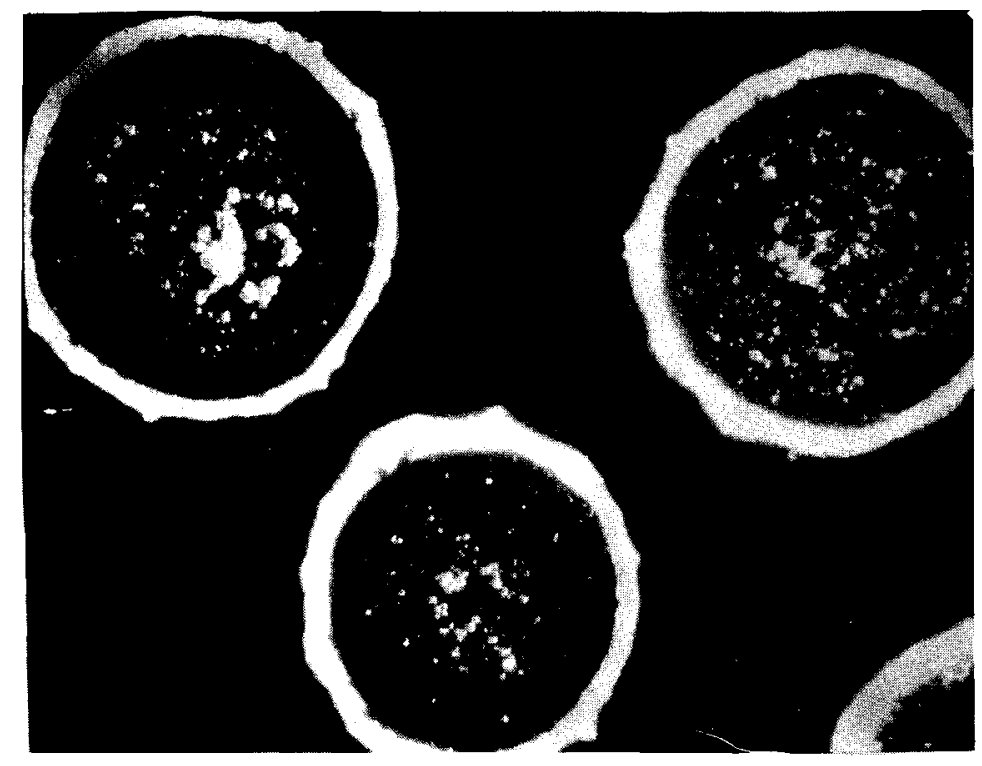

SLIDE 10. EB Coated $\mathrm{UO}_{2}$

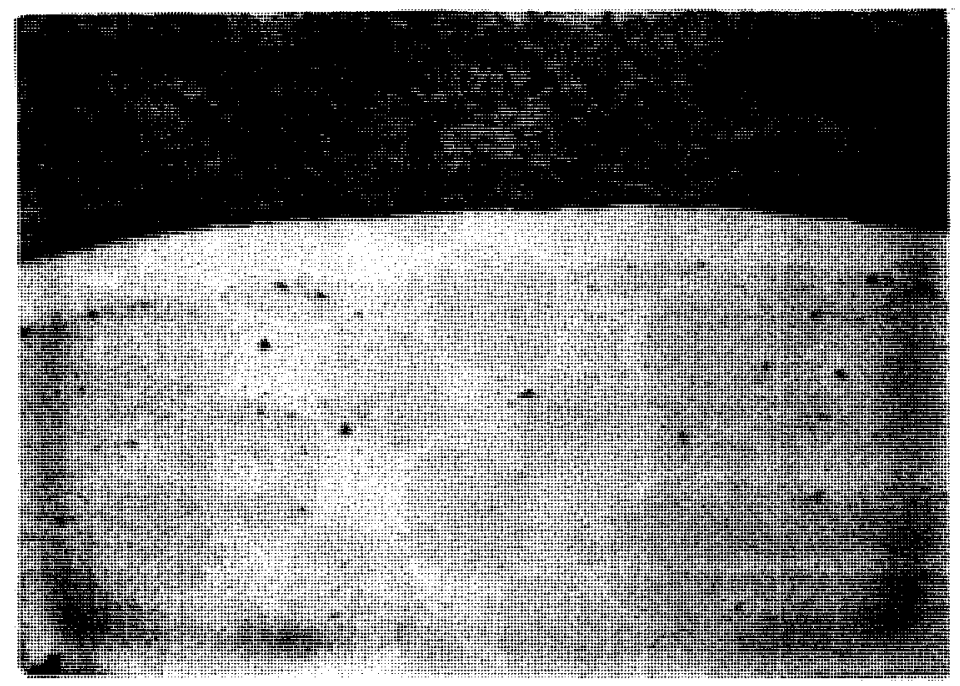


We have also taken these spheres either coated or uncoated, and fabricated dense bodies by hotpressing and by ceramic extrusion. We have two hot presses in the glove box line. One has a vacuum chamber, resistance heated die and is capable of pressing at $2000{ }^{\circ} \mathrm{C}$, and 50 tons pressure. The second press is induction heated and it has a 15 ton capacity. We also have a 75 ton ceramics extrusion press in the glove box, another 25 ton vertical ceramics press, and a 100 ton metal press that we have been using for extruding plutonium metal wire. All these boxes are glove box enclosed. With our glove box line we can use air or inert gas and therefore we can handle a variety of materials. Besides plutonium capability, next to the Plutonium Laboratory is our Metals Plant wherein one of the most versatile pieces of equipment is a 750 ton extrusion press. We have done both coextrusion and extrusion on this press. Such items as $\mathrm{UO}_{2}$ in zircaloy rods, $\mathrm{B}_{4} \mathrm{C}$ poison strips, zircaloy boron alloys, copper, nickel, manganese, zircaloy beryllium alloys and so on, have been fabricated. This facility is also licensed to handle plutonium, provided that the plutonium is first encapsulated. We will take our chances regarding a break-through when working with plutonium in the Metals Plant. The facility is rigged up with ventilation, so if we should break through the cladding, no plutonium will be forced out of the Plant.

Our Uranium Plant is also licensed to handle encapsulated plutonium. There we have facilities for corrosion testing of long rods.

In conclusion I think we can do a variety of things with regard to FFTF. I don't see any particular problems from the fabricator's viewpoint at this stage of the game. For economy though, and this, goes for all plutonium work, it is desirable to freeze upon a standard product, and to stay there once you do freeze. I suggest that you do not keep changing specifications, and changing the fabrication process, which changes could result in the need for additional equipment and therefore higher costs. Take time before you freeze a design, but when you have frozen, stay there. This gives the commercial people time to get the proper equipment. Before investing hundreds of thousands of dollars, they must be certain that it will continue to be the proper equipment. We are willing to invest funds but only after there is reasonable assurance of a continuing market. 

D12. 1

\title{
PLUTONIUM FUEL FABRICATION
}

\author{
K. H. Puechl
}

\section{DISCUSSION}

Mr. CARL ANDERSON: I think there has been some oversimplification, and I would like to try to look into it a little bit. First you mentioned you had 40 fuel pins going to EBR-II, and I think you commented that the objective here was to establish specifications for a test reactor core.

MR. PUECHL: No, the test reactor fuel, not the over-all reactor.

MR. ANDERSON: With 40 fuel pins?

MR. PUECHL: Yes.

MR. ANDERSON: Yesterday I heard Mr. deHalas say that he was concerned about oxide fuel and fast reactors, and I am kind of planning on talking about that, too. I am not sure you can do it with 40 pins.

MR. PUECHL: Obviously, I am sure we won't have enough statistical bases for doing this. But you can give future program direction. Does the mechanically mixed behave as well as the coprecipitated? If it does, and we have every reason to believe it does, why bother with coprecipitation from here on? You can decide whether you need low density pellets to allow for expansion. There will have to be a final test of many elements to obtain statistical data.

MR. ANDERSON: This many pins are required somewhere. I would like to inquire what you think $1 / 4$ in. pin, $20 \%$ plutonium, $\mathrm{PuO}_{2} /$ $\mathrm{UO}_{2}$ would cost today? What do you think it might cost about five years from today; and further, what is the effect if you did get more $\mathrm{Pu}-240$ ? I am really asking this because we see big advantages of plutonium in the fast test reactor. I think it is about 20 to $30 \%$ Flux. I would like to find out what are the disadvantages. Is there a disadvantage in a plutonium core from a fabrication standpoint, as against, say, making a $\mathrm{UO}_{2}$ core with a $\mathrm{UO}_{2}-235$. Is it big, $10 \%, 20 \%$, $100 \%$ ?

MR. PUECHL: I didn't bring my estimator slide rule down, so I am going to answer this generally. I did touch on this subject at the Washington meeting. For all types of plutonium fabrication estimated on a relatively large scale on today's market (handling say a $1000 \mathrm{~kg}$ of plutonium), we have always come out with numbers between $\$ 3,000$ and $\$ 5,000$ a kilogram of contained plutonium. Now before you write that number down, this number includes many nonfabrication items which apply 
only to today's situation when you use plutonium at $\$ 43$ a gram of fission isotopes. For example, if you look at that number for your plutonium value and compute use charges, you will find that about $20 \%$ of this 3000 to 5000 dollar number is use charges. You find that another significant fraction is insurance, needed insurance in case you have fire that gets rid of this material. The cost of plutonium losses again can be $20 \%$ of this total number. When you are considering commercial plutonium at some price lower than the $\$ 43$ a gram, I think you can take that 3000 to 5000 dollar number and cut it approximately in half.

The other thing you talked of is cost differential. Today the differential is quite high because we are comparing fabrication in a small-sized plant with fabrication (in the case of uranium) in a largesized plant. Eventually the difference (the extra cost you will have to put into plutonium when you do talk large plants), will be rather insignificant. Certainly, you will need glove boxes and you have to maintain equipment in glove boxes. These are the two major additional costs. However, when I am talking about a large plant I am considering a plant automated to the hilt. Under such automation, it doesn't really make any difference whether I am operating outside of glove boxes or in glove boxes. But with the present plutonium requirements, which are like no more than $3000 \mathrm{~kg}$ (per year) of plutonium to be fabricated between now and probably 1975, you cannot afford the capital cost to go with an automated large scale plant. You can't afford to put this plant in. After 1975, yes, but then it will be mostly for plutonium recycling and still not for the fast reactor market.

MR. ANDERSON: Okay, one final question. You were talking about your particle work, and I think you made some comments about the porosity in the cermet fuel. I think deHalas was looking for a particle about $80 \%$ of theoretical density. Would you care to comment on a commercial ability to make that type of particle?

MR. PUECHL: We can make that type of particle.

MR. ANDERSON: Commercially?

MR. PUECHL: Yes. You have to look at the overall fabrication process, how you want to make this, and what the characteristics should be.

MR. SHAPIRO (NUMEC): There are two comments I would like to make: one about the previous question and one about the present question. There are two ways that you can look at the fabrication of material having lower density in the $80 \%$ range. One is to have the generally uniform porous material; the other is to have the material where you have a high density matrix (with maybe 96 or $98 \%$ theoretical matrix with built-in porosity): If you look at the material, say, with a high density matrix, which gives you presumably the maximum fission gas retention for the density but also aliows for internal accommodation for growth, etc., you have a material essentially mechanically stable and easily worked with. The latter would be the material that I 
think should be recommended for general application in core construction. (Where you have to take mechanical forces without crushing, without stringering, etc.) The other way that you can do it, as Karl had pointed out, is to use a material with a fairly low density. This you can achieve in two ways: one by having a dead material which will not sinter and the other is having a material that you sinter up to $80 \%$ theoretical density. The probability is, if you get rather hot, you are going to be changing the structure during operation; if you get a fairly low density material, simply because you deaden the material, you probably will not have decent mechanical strength in the material. However, you could (if you wanted to) coat it, and hold things together. But generally speaking, I feel that you would want to go to a high density matrix with built-in porosity, and I think you would accomplish the objectives all the way around.

Getting back to the other question that you asked, regarding the difference between production of $\mathrm{PuO}_{2}$ today and tomorrow, and the comparisons of the production of $\mathrm{PuO}_{2}$ with $\mathrm{UO}_{2}$, I think there is a tendency today to be hyper-careful or critical of material in certain respects. Apparently we don't apply uniform specifications with regard to the nature of material. For example, if you take $\mathrm{PuO}_{2}$ material and you look at the vibratory packed material versus pellet-type material, there is a tendency on the part of the person, who is making the specifications, to take into account what he can do with the material. That is, if you look at Vipac material, you know that you can get maybe $88 \%$ or $90 \%$ theoretical density with a variability of plus or minus a couple of percent; so it is written into the specifications. But with regard to pellets they will say, what we want is something having such and such clearances, and very high tolerance, plus or minus a quarter of a per cent, etc. Actually, it is going into the same application, but the guy who is designing the core and writing his specifications doesn't acknowledge that it is going to be in the same thing. What I am saying is that, if the people are reasonable in setting specifications and doing it on a uniform basis (regardless of the methods of fabrication) I think you can do these things fairly inexpensively, within reason. However, if you are going to be hypercritical about these things and you are going to be even more so in the case of plutonium than you are in uranium obviously it is going to cost you a lot more. You are going to effect it, and you'li have to pay for it. So my appeal to you here is for goodness sakes, be reasonable, consider what the applications are and what the risks are, and I think you will find the cost to be reasonable.

MR. PUECHL: To elaborate on one more point. You talk of automation. It is relatively easy to automate what you call a processing line and fabrication line, but when you come to analytical chemistry and quality control you have problems. These are very difficult to automate; they are more or less limitea to hand operations, and plutonium hand operations are costly because you are inglove boxes. No matter how much you automate a line, if you pile such specifications on your product that the guys are in glove boxes all the time in analytical chemistry, it, is going to be much more costiy. 
$\mathrm{D} 12.4$

MR. KITTEL: I wonder if you could say a little more about your capsule irradiation program? In particular, I was wondering what body materials you are using, what thicknesses and what fuel density you are looking at.

MR. PUECHL: The cladding is 316 stainless. The pellets, I think, are around a 0.25 to $0.3 \mathrm{in.}$ The densities in the first five capsules, I believe, are 92 plus or minus 1 . density?

MR. KITTEL: That is the pellet density. What is the smear

MR. PUECHL: The smear density, I recall there was a 2 mil gap. I don't think I have calculated any smear on that.

MR. KITTEL: And what cladding thickness, do you recall?

MR. PUECHL: Do you recall that?

VOICE: I think it is $20 \mathrm{mils}$.

MR. PUECHL: That is about the same as I recall.

MR. BURNHAM (Battelle): I think we have here a cost problem for you, Karl. Given $500 \mathrm{~kg}$ a year for $\mathrm{PuO}_{2}$ particles, say $80 \%$ density (any way you want to figure the density) what would be the relative cost of this quantity per year in the coated versus uncoated?

MR. PUECHL: You have got to be sort of specific about the coating. It is a question of specifications to apply to the material. When the amount or percentage of rejection starts running up, the relative costs will build up. I would say if you apply a reasonable specification on the coating (again depending upon the nature of the material that you are coating with) you might add possibly no more than $20 \%$ on to the total cost of the material.

MR. SHAPIRO: Do you want to say anything about the thickness?

MR. BURNHAM: I think in this case, now you are talking about a cermet, you are not interested in tight control in thickness.

MR. SHAPIRO: I think you are interested in the loading.

MR. BURNHAM: That is right, and the chemistry.

MR. SHAPIRO: It depends on the nature of the coating you put on, the particular metal you put on, and the method of application. If, for example, you are looking at certain types of coatings where you are interested in assuring (after all, you are dealing with fuel particles, you literally have millions upon millions of individual fuel elements) that these are going to be completely tight, and you have to throw them in acid, for example, and boil them to determine whether or not you have any activity in the liquid afterwards, then obviously it is a tight specification. On the other hand, if you are simply interested in taking coated particles and maybe allowing a pinhole or so, it doesn't matter because you are going to compact them into a cermet type fuel, this is cheaper. 
FUEL DESIGN AND OXIDE DEVELOPMENT AT WESTINGHOUSE

\author{
F. M. Heck and W. E. Ray \\ Pittsburg, Pennsylvania
}

\title{
INTRODUCTION
}

The fuel design problems for the FFTF, as for any reactor, start with the criteria and objectives established for the performance of the system. The commission has established a set of objectives, capabilities, and requirements called "Appendix A" and "Appendix B". These describe the desirable characteristics of a versatile Fast Flux Test Facility capable of providing closed loop testing.

\begin{tabular}{|c|c|}
\hline $\begin{array}{l}\text { Skimming } \\
\text { B2.a.(1) }\end{array}$ & $\begin{array}{l}\text { through the two documents, we find the following: } \\
\text { "The primary objective ... is ... reliable }\end{array}$ \\
\hline B2.a. (3) & closed loop testing." \\
\hline $\begin{array}{l}\text { B3.i. } \\
\text { B3.1. }\end{array}$ & $\begin{array}{l}\text { "Each loop should be ... essentially vertical .. } \\
\text { "The closed loops must be accessible and }\end{array}$ \\
\hline B3.h. & $\begin{array}{l}\text { "Loops wili have service equipment (Pumps, heat } \\
\text { exchanger) located external to the reactor ..." }\end{array}$ \\
\hline B3.g. & $\begin{array}{l}\text { "Instrumentation should include ... monitoring } \\
\text { of ... the test fuels performance. Accessi- } \\
\text { bility sha11 be provided to disconnect (make } \\
\text { and breed) instrumentation connections external } \\
\text { to the reactor." }\end{array}$ \\
\hline
\end{tabular}

These requirements virtually define a vertical bayonet (re-entry) type process tube for the closed loops, with the tubes extending through the top of the reactor vesse1 to provide coolant and instrumentation connections outside the reactor vessel.

Bottom entry with through tubes is essentially 1imited by plant availability and $100 \mathrm{p}$ removal, since $100 \mathrm{p}$ removal would require draining the core sodium, which in turn would require removing the driver fuel (unless all fuels were in closed process tubes). Bottom bayonet entry has the same difficulties plus extremely complex handling problem for test specimens.

El shaped closed loops entering from the side of the vessel would require making and breaking of coolant and instrumentation lines inside the reactor vessel. Even if the objectives permitted, this would be undesirable from the reliability and versatility standpoint. 


\section{2}

B3.a. "Number of loops ... should be ..." (in the range)" $\ldots 4,5,6,7$, and 8 closed 1oops".

At this point then, we have (say) six vertical re-entry bayonet process tubes extending through the top of the reactor vessel in undefined positions.

B3.f. " ... loops should be located ... after careful consideration ... to ... flux, reliability, safety, reactivity effects, cost, and maintenance. A center loop should be given prime consideration."

Consideration of reliability, safety, reactivity effects, cost, and maintenance would all point to the preferred location for all loops on the periphery. This would permit nearly any driver core design and driver fuel handling system if closed loop tubes were slightly canted so that they cross the external faces of the core at a small angle. This would permit bringing the closed loop process tubes out of the top of the vessel on a sufficiently large diameter circle, and would permit a separate small closure or plug over the driver fuel.

Attainment of high flux on the other hand, along with the preference for a central loop, would indicate placing the closed loop tubes within the core region of the reactor. Placement of the closed loop tubes within the core region essentially establishes the splayed process tube core structure of the FTR reference design. Otherwise, either the fuel handling system would be an exceedingly complex piece of gadgetry operating under sodium or sodium vapor, or the closed loop tubes would have to be removable for refueling. Neither system is as likely to meet the objective of high plant availability as the reference design.

\section{DESIGN AND DEVELOPMENT}

\section{B.4 (Open Test Positions in Case)}

"The definition of an open test position in any position in the reactor core used for test purposes ...

... Instrumentation will include ... monitoring of

... the test fuels performance."

This reinforces the need for the process tube design for the driver fuel because test fuel performance monitoring requires thermocouples attached to the test fuel. Electrical leads from multiple fuel assemblies to the outside of the reactor vessel create nearly insurmountable fuel handling problems in a sodium cooled reactor unless it is of the process tube type.

Thus, the experimental requirements for open and closed loop testing essentially force the core structure. The degree of tilt of the structure is a compromise which must be worked out with the fuel handing machine design. 
Because of the sensitivity of small fast cores to fuel movement, it is necessary to constrain the process tubes in the vicinity of the core. Since there are tolerances on tube structure dimensions, clearance, and straightness of the process tubes, constraining then essentially means bowing them slightly.

At this point the fuel element designer recognizes a potentially difficult problem. The driver fuel subassemblies must be located within the process tube with sufficient firmness to obviate the possibility of subassembly vibration. Some resilient members must be provided between the process tube and the fuel subassembly; and repetitively inserting and removing a presumably straight subassembly (with a firm fit) into a slightly bent process tube strongly suggests that galling or sticking may occur. This would seem to require a development program with full scale testing.

Now the flux, for uniformly loaded core, bears a rather simple relationship to certain other important core properties. The peak flux is directly proportional to the total reactor power and the peak-to-average flux ratio; and inversely proportional to the fissile mass and the fission cross section.

$$
\phi_{\mathrm{m}} \simeq\left(\frac{1.23 \times 10^{16}}{\bar{\sigma}}\right)\left(\frac{\mathrm{p}}{\mathrm{f}}\right)\left(\frac{\phi_{\mathrm{m}}}{\overline{\mathrm{\phi}}}\right)
$$

where $\mathrm{P}=$ total power in $\mathrm{MW}_{\text {th }}$

$$
\begin{aligned}
& M_{f}=f i s s i l e \text { mass, } K g \\
& \bar{\sigma}=\text { av. fission cross section, barns } \\
& f
\end{aligned}
$$

$$
\left(\phi_{m} / \bar{\phi}\right)=\text { peak to average flux }
$$

Now the value of $\bar{\sigma}$ typically varies only slightly with the fuel form (oxide, carbide, or cermet). The peak-toaverage flux ratio depends primarily on core geometry and reflector materials. Hence, specifying the peak flux very nearly specifies the specific power, MW/kg fissile, for a given fissile material. For the reference FFTF at $400 \mathrm{MW}$ and $300 \mathrm{~kg}$ fissile, the specific power must be about $1.3 \mathrm{MW} / \mathrm{kg}$ fissile.

There is little that the designer can do to reduce this specific power requirement except go to a larger core with more total power, in which case the fissle mass per unit volume is reduced, and the peak-to-average flux increases, 
permitting a lower specific power. Economicilly, of course, one desires the least total power which will achieve the objectives.

But now the designer has a very difficult problem. The experimental requirements along with capital and operating cost considerations have set a thermal-hydraulic performance level for the driver fuel in the test reactor which is equal to or higher than that of postulated future large commercial breeder reactors. The other experimental requirements, seeming to necessitate the skewed process tube core structure, result in a reduction in the useful core volume of perhaps $15 \%$; since the average fissile mass per unit volume of core region is higher in the small test reactor than the large commercial core, the designer must shove more fuel into a smaller available volume and take out more heat per unit volume than is postulated for future large commercial reactors. This leads to the use of small fuel pins and still pressing the temperature capabilities of the materials.

This is in direct oppositjon to the type fuel design, which would be preferred for a reactor intended to permit testing fuel to destruction, since one would prefer all the driver fuel materials to have large margins of safety (so that it could ride through significant excursions without damage).

The apparent need for high specific powers and high power density per unit volume of useful core space magnifies already difficult problems. Small rods with close clearances have higher mechanical hot channel factors than large rods with generous spacing. This results from the fact that a given numerical variation in rod diameter, location, or bowing represents a larger fractional variation in crolant flow. The fuel rod support structure (grids for instance) must not only fit into a smaller clearance, but must position the rods more exactly, and the possibilities for structure are more limited because of the pressure drop they represent in the small coolant channels. Local maldistribution of fissile material within the fuel rod will produce larger hot channel factors because of the smaller rod cross section.

In addition, test fuel assemblies must have a moderator incorporated into them if the experimentor wishes to have a close simulation of large power operating cinditions. This is desirable to match damage and annealing rates to avoid being trapped by phenomena that are both fission rate, neutron energy, and temperature dependent. This may :ause some power peaking in the adjacent driver fuel assemblies. 
Since the average specific power and power per unit volume must be held up to obtain the flux levels, all the "little" hot channel effects (mentioned above) have the effect of raising the possible temperature levels to be experienced by the materials.

Thus, the present preferred experimental requirements seem to require a tightly packed fuel assembly which exceeds postulated future commercial cores in its thermal performance. I do not imply that this cannot be achieved. Now that PNL has done sufficient work on the concept to begin identification of the extent to which the present requirements are stretching the technology in the design, data, and fabrication process areas, I do suggest that a careful reevaluation of the importance of the experimental requirements be performed.

Now let me return for a moment to the flux-specific power relationship. It can be rewritten as

$$
\phi_{\mathrm{m}}=\left(\frac{1.23 \times 10^{16}}{\overline{\mathrm{\sigma}}}\right) \frac{(\mathrm{P} / \mathrm{V})}{\left(\mathrm{M}_{\mathrm{f}} / \mathrm{V}\right)}\left(\frac{\phi_{\mathrm{m}}}{\overline{\bar{\phi}}}\right)
$$

where $V$ is the gross core volume.

Now the specific fissile mass $\left(M_{f} / V\right)$ is a function of core volume. For H/D equals one and for a core volume of 200 to 500 liters, its reciprocal $\left(\mathrm{V} / \mathrm{M}_{\mathrm{f}}\right)$ varies approximately as the volume-to-power values between 0.4 and 0.5 . The peakto-average flux varies less rapidly than the reciprocal of the specific mass, but it also rises with increasing core volume.

If $P / V$ is taken to be the heat removal capability of the system, it decreases slowly with core volume; especially if the H/D is maintained. The product then of $(P / V) \times\left(V / M_{f}\right) \times(\phi m / \Phi)$ tends to be a monotonic increasing function of core volume. Hence, as we have seen in the PNL calculational results (for a given core geometry and fuel form), there is a minimum volume required to achieve a specified neutron flux; hence there is also a minimum power level.

Now suppose we change the form of the fuel (say) from cermet to ceramic. The average fission cross section is very little changed, even though the neutron spectrum shifts slight $\frac{1}{2} y_{9}$, because it is shifting over an energy region where the Pu 39 cross section is nearly constant. The peak-toaverage flux may rise a little because of the lower leakage. If we neglect these two usually opposite minor effects, the maximum flux depends essentially on the ratio of allowable heat removal capacity $(\mathrm{P} / \mathrm{V})$ to the required specific mass. 
The specific mass for oxide is on the order of $15 \%$ higher than for the cermet. If we assume the same $\Delta \mathrm{T}, \Delta \mathrm{P}$, and core size, we can estimate the relative heat removal capacity if we know the required volume of fuel rods in each case. In order to get into the 20 to $25 \%$ enrichment level for Doppler effect, it is a good first approximation to set the total rod volume the same. Then, if we take (say) $8 \mathrm{~kW} / \mathrm{ft}$ for the average oxide rod versus about $12 \mathrm{~kW} / \mathrm{ft}$ for the cermet rods, $\mathrm{P} / \mathrm{V}$ for the oxide is only about $76 \%$ of that for the cermet. Then peak flux is about $2 / 3$ of that for the cermet. Raising the enrichment to reduce the total rod volume can be used to raise this value somewhat, but this has limits if the ceramic advantages are to be retained.

If the ceramic were carbide, the specific mass would be perhaps $10 \%$ higher than that of the cermet. If the carbide is sodium bonded, then the heat removal capability can be greater than that of the cermet design. A linear power level of $16 \mathrm{~kW} / \mathrm{ft}$ is quite conservative with respect to hot spot temperature, which will be less than $2000^{\circ} \mathrm{F}$, with a bulk fuel melting temperature of $ح 4200^{\circ} \mathrm{F}$. Again using the same $\Delta \mathrm{T}, \Delta \mathrm{P}$, and core size, along with the same volume of fuel pins, the heat removal capacity is higher than the cermet by about $20 \%$. Hence, a carbide core might obtain a higher peak flux in a given core volume than the cermet.

The heat sink requirements for the carbide core would probably lie between those of the oxide and the cermet for a given core volume and neutron flux. The burnup reactivity requirements should be slightly lower than that of the oxide.

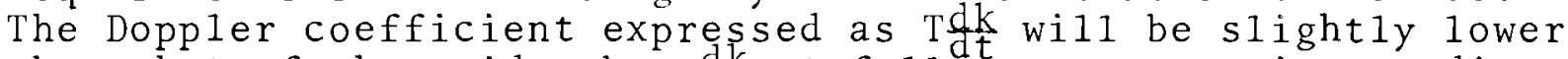
than that of the oxide, but $\frac{\mathrm{dk}}{\mathrm{d}}$ at full power operating conditions should be about the same; also, the integrated Doppler from full power to melting about the same as the oxide. The neutron spectrum will be intermediate in hardness between oxide and cermet. The slightly harder spectrum should yield a lesser reduction in Doppler on loss of sodium.

In the course of evaluating controlled expansion type fuels, we have performed a few preliminary calculations on ceramic fuel rods compartmented to permit clad expansion to move fuel and provide an additional negative temperature coefficient. This mechanism is particularly effective with sodium bonded carbide fuel rods. The preliminary results indicate that, for physically realizable reactivity insertions, this mechanism provides an effective negative feedback control.

Thus, a sodium bonded carbide fuel offers significant technical advantages as a fast fuel test driver fuel. Sufficient time remains in the FFTF schedule to develop the necessary fabrication and processing procedures, and attain essential performance data on stabilized mixed carbide. An initial burnup 
performance of 30 to $40 \mathrm{MWd} / \mathrm{kg}$ would compare favorably with the reference fuel at a burnup of $16 \%$. As additional data occurred, the burnup could have been extended to beyond $100 \mathrm{MWd} / \mathrm{kg}$ with a core design that was relatively conservative thermal-hydraulically, economical to operate, and directly transferable to large commercial breeder power plants.

Stabilized sodium bonded carbide fuel should, therefore, be given serious consideration as the driver fuel for FFTF.

\section{PART I I W. E. RAY (WESTINGHOUSE)}

\section{INTRODUCTION}

Most of the people present at this meeting have previously heard formal presentations describing Westinghouse development work on mixed uranium-plutonium monocarbide fast breeder reactor fuels. Therefore, it seems unnecessary on this occasion to cover details of the theoretical and experimental work. Briefly, the stabilization technique identified is to effect an addition to the carbide, which combines with the excess carbon hyperstoichiometric carbide or with the excess uranium-plutonium metallic phase in hypostoichiometric carbide. Iron is used as the additive to "hypo-" material, and the structure produced is (UPu)C $+(\mathrm{UPu}) \mathrm{Fe}_{2}+\mathrm{Fe} . \mathrm{A}$ chromium addition is used with "hyper-" material which yields a three-phase [(UPu)C $\left.+\mathrm{Cr}_{23} \mathrm{C}_{6}+\mathrm{Cr}\right]$ equilibrium structure. Ordinarily, the total volume percentage of nonfissile phases in chromium-stabilized material is about 1.3 , and the percentage of $\left[(\mathrm{UPu}) \mathrm{Fe}_{2}+\mathrm{Fe}\right]$ in iron-stabilized material is about 3.8 .

The program has progressed enough that the experimental demonstration of the originally postulated thermodynamic equilibria can be considered achieved. We have conducted out-of-flux compatibility test, showing that stabilized carbide fuels are inert when sodium bonded with stainless steel claddings. Temperatures as high as $1500 \mathrm{O}$; times as long as 1500 hours have been investigated.

Work in Westinghouse facilities has been restricted to experimentation, with UC (both "hypo-" and "hyper-") used as a stand-in for the mixed carbide. We have, within the last year, initiated work in cooperation with Argonne National Laboratory (and using ANL facilities) on the mixed carbides. Presently, there is no reason to suspect that differences exist between the behaviors of UC and (UPu)C insofar as stabilization is concerned. However, there are definite differences in process response (for example, in sintering 


\section{8}

parameters required to achieve a given density) which affect final product attributes (such as phase distributions).

We intend to continue our work to include an irradiation in EBR-II. The initial experiment (as now conceived) consists of 20 pins. Half of the samples will contain stabilized hyperstoichiometric material; whereas, the others will contain stabilized hypostoichiometric fuel. Preparation of these specimens is now in progress. Objectives of this initial test are as follows:

- Confirmation of fuel-cladding compatibility in sodium-bonded elements.

- Measurement of unrestrained stabilized fuel swelling rates as a function of density.

- Measurement of fission gas release rates as a function of density.

Obviously, the two candidate stabilization methods will be directly compared from a performance viewpoint. Data will be taken at several burnup levels, and emphasis will be placed upon engineering rather than fundamental information.

With regard to the FFTF, our review has progressed through initial evaluation of the reference cermet driver fuel element. We are not satisfied that the currently postulated fabrication process can provide an element containing low-density fuel particles dispersed in a high density matrix. Also, in addition to the technological problems, there are certain business considerations involved. The type of fabrication facility necessary to produce this type of element requires a rather substantial capital investment. It is unclear that sufficient market justification exists at this time for installation of such a capacity. However, business considerations of this variety are, perhaps, not within the scope of this meeting. Let us then restrict our considerations to the technical aspects of FFTF driver fuel design and production.

\section{QUALITY CONTROL TECHNIQUES}

The first task, of course, is production of appropriate ceramic grade $\mathrm{PuO}_{2}$ powder. There are known procedures for accomplishing this objective (as NUMEC and others have shown in earlier talks), but it does seem important to us that we are considering a rather high volume product in this instance. Further, FFTF driver fuel will be produced on a recurring basis, with stringent requirements on lot-to-lot fuel element variability. Consequently, there is need for consistent, reproducible input material. It seems probable that $\mathrm{PuO}_{2}$ variations from batch to batch will require full, complete characterization of every shipment of ceramic grade powder. This can result in drastic increases in fuel element costs.

The second task to be accomplished is production of appropriately spherical low density particles. We concur with the NUMEC position that the best approach to this problem is the 
"snowballing" technique. The current "rounding" method, cited in FFTF documentation, is economically inappropriate on a commercial scale. We also recommend that the spheroidized particles be provided with a high-strength coating (for reasons cited by earlier speakers). First, such coatings can provide sufficient strength to avoid fuel particle crushing, stringering, and densification during fabrication. The second incentive for high strength coatings is assurance of an effective heat transfer bond between fuel particle and matrix phases so that fuel element axial temperatures can be minimized during operation. The heat transfer bond may also be effective in avoiding excessive fuel particle temperatures during reactor transients. If we postulate that the high-strength coating is used in usual technique ranges, we can hope for fission product retention within the dispersed phase. We concur with the views on this question treated yeaterday morning by Phillips Petroleum.

Particle coatings also offer the advantages of a secondary corrosion protection barrier, which might be quite useful in the event of a cladding failure. The final advantage we wish to cite, for coated fuel particles in the FFTF reference cermet element, is that it is frequently easier to assure uniform fuel distribution with coated particles than with uncoated particles of the same size, shape and density. However, this fuel uniformity advantage is somewhat specific to the fabrication process used.

With regard to fabrication of cermet fuels suitable for the FFTF application, we feel that appreciable development work is required to assure acceptability from a manufacturing standpoint. It appears definite that alternate fabrication process to the presently defined extrusion should be carefully evaluated. There are several such candidate alternates, one of which is a swage bonding technique developed some 12 years ago. We believe swage bonding offers a superior potential of providing the desired high density matrix and low density dis persed phase fuel structure without unacceptable modification of fuel particle morphology.

Another consideration deserving equal emphasis with the fabrication question is the area of quality control and product reliability. We share the concern expressed by NUMEC and others that inspection operations cannot be easily automated. Two possible approaches seem evident. The first is use of appropriate statistical sampling methods which minimize the number of samples taken. This method of reducing the number of man-hours devoted to quality control measurements and imposes the requirement of assuming a known fuel perform. ance risk. An alternate approach is development of more sophis ticated nondestructive testing techniques which would then be applied to $100 \%$ of the fuel elements produced. We will 
13. 10

cite, then, the following specific requirements for refinement of quality control techniques:

- A uniquely acceptable method for quantitative measurement of fuel particle integrity is required.

- An improved nondestructive technique for economic and routine determination of fuel districution must be identified and applied to pin type of fuel elements.

- Appropriate methods are needed for assessment of fuel particle clumping and segregation on a local (microscopic) basis.

- A method for routine evaluation of heat transfer bond continuity is required regardless of whether or not the bonding material involved is liquid sodium.

- A high-speed procedure for assuring that cladding thicknesses in final fuel elements are acceptably uniform is not presently available for cermet type elements.

We feel that until questions of this type are resolved (and again it is emphasized that considerable technical work is involved), it cannot be assumed that acceptable product reproducibility and reliability has been realized with any given process. Without such assurances, the safe and reliable operation of FFTF is open to question.

Proceding to the presently identified alternate fuel element consisting of a ceramic oxide gas bonded with stainless steel cladding, it again appears that appreciable development work must be done. The WAPD preference is for a pellet type fuel. The reason for this preference is that certain operating questions might arise with vibratory compacted ceramic fuels; questions that cannot be characterized at this point. For example, one such uncertainty is that contact conductance between vibratory compacted fuel and cladding cannot be quantitatively deleted at the present time. Also, we feel that pellet fuels might be less susceptible to slumping and ratcheting problems during test reactor operation; however, this requires experimental verification. We are certain that local fuel homogeneity can be more readily assured with pelletized fuels than with vibratory compacted fuels.

There are some problems that will be encountered in synthesis of FFTF fuel powder for ceramic elements, and these are similar to the synthesis problems briefly mentioned in connection with cermet fuels. Despite this similarity, the solutions to the two problems will likely be different. The attributes of ceramic-grade powder needed for preparation of particles to be dispersed in cermet elements are, of course, different from the attributes required for powder to be pelletized.

\section{CONCLUSION}

There is, then, considerable materials and process development work required before the FFTF reference fuel element can be defined in detail. It appears significant that the two candidate elements (the dispersion type and the alternate ceramic type) require entirely different commercial capabilities. To 
assure that appropriate commercial facilities are available on a timely basis, it seems crucial that the designer establish (at the earliest possible date) which type of element is preferred for FFTF. This will assist in definition of capital cost requirements necessary for support of the FFTF program. It seems prudent to initiate parallel development efforts on the two types of fuel elements. Only in this way can the designer achieve a direct comparison of performance and manufacturing characteristics on which to base a timely selection. 


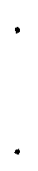


$\mathrm{D} 13.1$

FUEL DESIGN AND OXIDE DEVELOPMENT

F. M. Heck and W. E. Ray

\section{DISCUSSION}

MR. deHALAS: You seem to have a distorted picture of the cermet processes which we propose. First of a11, there is no doubt that fabrication is one of the major problems of a cermet fue1. We have not settled on extrusion as the fabrication process. This is one of the processes we have looked at. We do not agree that snowbal1ing is the best process. We on $1 y$ used rounded particles in radiation testing, where we had to get our hands on something and I would like to strongly disagree with the need for coating. First of a 11 , if we have to coat, there is an economic penalty for this. In these low loadings we believe there is no need for coating to get a good distribution. If you are going to $15 \%$, yes, you have to coat. If you go to $20 \%$ you can blend and get it.

The other thing you mentioned was a bond between the particle and the cermet. If you use a low density particle, the first thing it does when it heats up is to shrink away from the stainless anyhow. So it doesn't really make a lot of sense to talk about bond there. At best, it swells back up against the stainless again. The matter of an oxide bead is not one that is particular to cermets alone. There are a lot of people who have interest in mechanical blending as a means of producing mixed oxide fuels.

In your comments on the oxide, I am not sure what we said about Vipac versus pellet, although I assure you we have no strong feeling either way. Presently, I don't think there is a lot to choose between Vipac and pellet. As I mentioned yesterday, one of the major problems with an oxide fuel is porosity where you want it, and I am not sure whether it is better to use annular pellets. Regarding thermal conditions in the element, I am not sure whether you are any worse off calculating for Vipac fuel over pellet fuel. In particular, I think probably the gap conductance is better known with Vipac than it is with pellets, although the data seems to indicate that you don't have to assign the gap conductance to Vipac that is chargeable in the pellet fuel.

MR. RAY: Let me see if I can recall your points in some sort of order. It seems to me, if one conducts his irradiation experiments with a particular type of fuel pellet, that pellet immediately becomes reference. Take a rounded particle of high density, or low density, (it doesn't make any difference). I believe yours were rather high density, were they not? 
D13.2

MR. deHALAS: Yes.

MR. RAY: I think that this does lend some weight to that process simply for the reason that it constitutes a demonstrated constituent at that point. If you obtain data on the other fuel particles produced by the different processes, I think the tendency would be to specify this design.

Mr. deHALAS: I strongly disagree with you, but our basic criteria right now, which is preliminary, calls for a low density particle in the first place: this has no more bearing on what we have put in the preliminary irradiation tests, which have to be done cheaply and rather rapidly (as somebody at Argonne would irradiate sulphide). It gives some information on compatability.

MR. RAY: I would like also to talk to you about the matter of the particles slipping away from the matrix itself. Is that your point about coating?

MR. deHALAS: Yes.

MR. RAY: I am not familiar with the data showing this occurrence. Unless I am mistaken, all the irradiation samples I have seen seem to indicate that the fuel particles and the coating are in relatively close contact.

MR. deHALAS: I don't think you will find that this is the case at zero burnup.

MR. RAY: No, no.

MR. deHALAS: They heat up the first time.

MR. RAY: That is correct. I was thinking of the later situation. If your coating is bonded with your matrix and then you proceed, your fuel material contact enters into the inner surface; you now have no heat transfer barrier at all. I think it is somewhat of an advantage.

MR. MERCKX (BNW): Regarding this particle in the matrix, I think we are having a bunch of heat transfer arguments. Actually, if it were bonded, it would have to give your particle quite a bit of tension because the plutonium doesn't expand as much as the stainless steel. It actually comes out that with this bond you conduct way too much heat away, and the stainless steel pulls away from the plutonium oxide. If you look at the type of bond conductance you need to follow there, only a portion of what you would need is in a contact conductance type of situation. So it looks like if you don't even bother bonding, you are not at temperatures where you are going to get sintering. You have a lot of pressure. You are just going to have a situation where the particle follows the matrix and just gets hot enough to go right along with the matrix, exerting no more than a minimal type, as far as the yield stress, minimal type stress on the material. You just have an effective particle following the matrix. If it pulls away, the gas conductance will be enough, and before you get any sort of incidence, it will heat up enough to touch the materials. I think we are looking at a false problem when we look at all of 
the things that might happen. You almost can't imagine that situation where you don't heat up. If there ever was an incident where you have all this gas in the form of a barrier, then your material would get enough pressure and you would be in a bad situation anyway. The stuff would swe11 up like dough, and you would shut down the reactor. I think we are being real unfair to the cermet. You can imagine a lot of things, and if you real1y get deeper into the mechanism, many of these ghosts are not ghosts at all.

MR. RAY: I don't mean to leave a false impression here. It is not our position that the cermet fuel is in any way deficient. It is simply our position that there are probably as many questions which can be raised in ceramic fuel as with the other.

MR. W. P. CHERNOCK (Combustion Engineering): I would like to raise a few points concerning the carbide. Maybe I haven't followed this as closely as other people have, but the term of stabilizing carbide (as you explained it) implies to me that you have essentially (in the case of hypo-fuel) licked the problem of plutonium and uranium by tieing it up, which could conceivably solve two problems: the reaction of the free metal with the cladding, and (which I don't think it solves) the free metal phase in the grain boundary containing some fissile material. Your other argument, of course, is the fact that you have solved the carbon transport problem by the addition of chromium. I think this is a momentous solution because if one had his "druthers," I think he would pick a hyper-fuel in a sodium bonded situation. I wondered why you mentioned both in the same vein, because if the hyper-fuel problem is solved, is there any incentive really to talk in terms of hypo-fuel? What I am merely asking is, what is the evidence that you have that the carbon transport problem is essentially solved? Secondly, I don't think in carbide we would try to turn up a margin of melting like we do in oxide. I think again (going back to your reference situation), if you are talking hypo-carbide, your margin is not margin-o -melting, it is a margin to $1800^{\circ} \mathrm{F}$. If you are talking about hyper-carbide, I think your margin again is not margin-to-melting, it is margin to a conceivable high swe11ing temperature which might very we11 be 2500 or $2600^{\circ} \mathrm{F}$.

MR. RAY: First of all our primary interest at this point is in connection with the chrome addition to hyper-fuel, for the very reason that you say. The reason for mentioning both is that the bulk of the early work was done on hypo, simply because we attacked that problem first. It so happened at that stage of the game, the material available on the market was a hypo materia1. I think the situation has changed somewhat. I think hyper material is now more common. What was your other question?

MR. CHERNOCK: I was wondering what your evidence is in support of the fact that you have no carbon transport in the hyper-fue 1 . 


\section{D13.4}

MR. RAY: Well, we have been running compatability capsules now for approximately a year. There is a rather large number of tests involving times up to $1500 \mathrm{hr}$ and temperatures up to $1550^{\circ} \mathrm{F}$. The capsules are the normal compatability type. We examined the stainless steel member both metallographically and chemically. After the completion of the tests, we have (as I indicated) no indication regarding the situation under irradiation. It is this lag that we hope to fill with the proposed EBR-II tests.

MR. CHERNOCK: What percent of carbon?

MR. RAY: Most of the material is 5.1 .

MR. SHAPIRO: How long are these capsules?

MR. RAY: The compatability capsules?

MR. SHAPIRO: Yes.

MR. RAY: Three and a half inches; they are alternating disks of carbide and flat material.

MR. SHAPIRO: May I make some comments here on that?

MR. RAY: Yes.

MR. SHAPIRO: In connection with the carbides, it seems to me that one can be very seriously misled about the compatability problem when one is doing tests on small capsules of uniform temperature. I think the problems really arise when you have larger capsules that are deliberately at nonuniform temperatures, which you might experience under reactor operated conditions. It is under those conditions that you cause materials transfer and that you get solution and redeposition of the material. Consequently, it seems to me that the tests, in order to really be valid from the standpoint of operation, must do this. And I think from the standpoint of the drawing (at least at this particular point), conclusions regarding the possible use of carbide on the basis of tests with uranium versus mixed carbides are not necessarily valid. It is here, I think I would like to make the following comment. With the FFTF generally, it seems that what we are looking for is a device which can be designed and which can be built and operated in the shortest possible time. The idea is to use this device for testing other materials so that we can tool for the design of experimental things, fuels or what have you, it would seem to me that we are going to find ourselves in a great deal of difficulty unless we are extremely lucky. I think, therefore, insofar as the design is concerned, that the design should be based on currently known techniques with currently known compatability and methods, even though there may be a small penalty paid from the standpoint of physics or what have you. Then, in this way, a selection can be made of the materials and a reactor can be designed. I doubt very much whether there would be many surprises occurring under these conditions that would necessitate redesign and alterations in the schedule. If for example, a sodium bonded carbide turns out to be a good thing, it can be used for the future: but I think for now we are treading into unknown territory, and I for one would advise against it. 


\section{D13.5}

With regard to some of the other comments that were made on the irradiation testing, I think that it has certainly been shown on many occasions that irradiation testing at best is a pretty "hairy" kind of thing. You don't know your conditions very well, and they are very difficult to define. Therefore, in view of the law of perversity of science (which I am a firm believer in), it would seem that one should not perform irradiation tests just for hasty results because I think that these hasty results will, in fact, result in a lot of surprises later on. It seems more important to design the experiments very, very carefully, and also to select the materials carefully to be representative of the kind of things you intend to do. For example, the utilization of high density material for a compatability test could be misleading when in fact you are thinking in terms of using a low density material. It could be misleading because you are talking about the different contact conditions and, consequently, different reactivity would occur. Certainly, in the case of zirconium and $\mathrm{UO}_{2}$, if one looks back at the history of that and at the compatability testing work, you will find a lot depends upon the nature of the contact that one gets, insofar as the nature of the action that one gets. And I would express some alarm at this approach. I feel that if you want to determine what happens under a particular set of conditions, then you don't use something else to do it.

With regard to the distribution of material and the matrix (say, $80 \%$ versus $50 \%$ ), it seems to me that if one is worried about the clumping or about the possibility of having areas in a cermet fuel which may not have any matrix material distributed around, when you get to a higher volume percentage of oxide, the probability of these things occurring is, in fact, a great deal greater. Therefore, more precautions must be taken to assure uniformity of distribution. I think it is precisely under those conditions that one has to really worry. I agree with Bill in favoring the coated particle over the simple mixtures. There are ways of doing the coating. It doesn't have to be done by a chemical technique. The British (for example) have used the technique of sticking the material on the surface by rolling it, instead of extruding. I simply contact the material and use a regular ceramic technique that can be employed.

I think there was a study done back in 1951 or 1952 on this very point. It was done on the distribution and the probability of clumping occurring, and I think it was shown that the limit was about $20 \%$ to assure no particle to particle contact. But I would still suggest that the probability is a lot higher than by using some simple technique.

Regarding the availability of pellets versus the Vipac and its use of low density particles, the point that I made previously regarding the utilization of high density matrix material (where you build in the porosity), I think I would, in fact, 


\section{D13.6}

eliminate the movement of the porosity. This ( I think) has already been shown in other applications; the LSBR is a good example of that. The Shippingport PWR fuel is another example of that, which has been proven where you can use a high density matrix with built in porosity: get a total low density ceramic and yet have high dimensional stability. It seems to me that one could use the same techniques here.

MR. RAY: As I recall, at the outset you indicated that our position was that we had a developed fuel here. I think our position is that the work is still in progress. We simply have nothing negative at this point; everything we see looks good. We see no reason to stop the work because it may be atypical.

MR. SHAPIRO: I wasn't suggesting that, Bill. What I was suggesting was simply that if one is going to build this reactor, one ought to work in view of the schedule involved and the desirability of having this reactor. One should work with things currently well known as against working with something which is still under a high degree of development and which may cause the continued alteration of the design based on new data (which are coming out).

MR. RAY: We11, the only reply or comment that I would make on that remark is that $I$ am not so certain there are that many things well enough known to assure continuing design change won't occur. An example is the cladding and the bonding problem. There will be a multitude of such phenomena as time goes on.

MR. SHAPIRO: Let's not add to it.

MR. HECK: He asked about the melting. You are quite right. There is a swelling problem in the high 2000 degree reading. But if we are concerned about safety, I don't think we really care if it happens to swe11 a 1ittle extra due to heating up for a few minutes. We are concerned whether the fuel is going to be mobile.

MR. HANNUM: I think that depends on what your other margins are with respect to gas.

MR. HECK: And swelling.

MR. HANNUM: As far as the 2000 , by the way, I think that is probably a little high for hypo and a little low for hyper.

MR. HECK: We11, the 2000 is too high for the FFTF. It wouldn't be that high.

MR. HANNUM: I was going to comment a little further on Mr. Shapiro's observation. I think we all agree that the FFTF should be filled with the material that is understood and demonstrated (which is not a major extrapolation), but one of Milt's main points was what are necessarily basic criteria you can set down. (Talking about operating, at 1.3 and $1.4 \mathrm{MW} / \mathrm{kg}$ of a cermet or the mixed oxide.) Now I raise this question: if we followed your observation that tests should be done to precise materials in question, how many such fuel arms have been irradiated at 
higher densities than $1.3 \mathrm{MW} / \mathrm{kg}$ and how many oxides, ceramic plutonium bearing elements, have been irradiated in a fast flux? How many test reactors have run either with ceramic or cermet fuel elements? I think you would come to the rather embarrassing conclusion (if you follow this thin reasoning), that the only material that has been in the fast reactor is the metallic element, either solid or liquid.

MR. RAY: Admittedly this element is in an early stage of development; I simply don't know one that isn't.

MR. SHAPIRO: I think that particular remark was taken out of context. What I said was (insofar as the specific test is concerned), if you want to find something out such as the compatability of $80 \%$ dense material with a particular matrix, then that is the kind of material that you should try to irradiate, not a material having $100 \%$ density with the same matrix. Otherwise, you may come up with some very serious surprises. I think you seem to be agreeing with that point--

MR. RAY: That is exactly what we are doing. Did I leave the impression we were not?

MR. SHAPIRO: No, my comment was directed to Mr. deHalas' statement about irradiation testing where he used a high density to demonstrate compatability, when in fact he was using a $8 \%$ density material.

MR. RAY: That is the point, but I was much more clumsy.

Mr. deHALAS: I agree fully that one would like to test as close as possible to the condition that you are actually after. We are going to have to pull ourselves up by our boot straps: this means a lot of thermal reaction testing. Likewise, our thinking on what is required in the cermet has changed, but more than that, the materials are still not available. We have been trying to make them, with a fair amount of success. So you can't do everything sequentially, as much as you would like to. About the other point discussed here, like carbides versus oxides, etc., it is true that none of these fuels is a developed fuel. Even the oxide, which is the best known, is not a developed fue1. However, in designing a reactor $I$ think that you want to begin from as far down the road as you can. If you start from scratch (so to speak), as on a stabilized carbide, it is a long way to go to get as much data as on oxides for cermets. So this reactor does have to be built in a relatively short order: you start down the road as far as you can and add to that.

MR. RAY: Don, I heartily concur. I would like to throw out one thought for consideration. It looks like there is a definite possibility that something 1 ike $80 \%$ of the neutrons generated in this country for the next few years are going to be devoted to fast fuel testing, and possibly directly to the FFTF fuels. Wouldn't it be nice if we got some data that might be used for eventual power reactor application? A11 I am saying is that I think that it is virtually necessary that we establish an FFTF fuel, but why don't we pick a system that might do double duty? 
MR. deHALAS: I think this point was covered yesterday by Les Finch. One of the primary goals is to get a workable machine; and the secondary goal (and how far secondary it is I don't know), is to develop information useful for the fast breeder program. Perhaps my own opinion, the primary goal of getting a workable machine, far overshadows the secondary one.

MR. RAY: Yes, I simply suggested making a workable compromise.

MR. deBOISBLANC: I suppose we already recognize. the logical inconsistency of deciding on a limited or, perhaps, one material for a reactor like the FFTF, but when we have launched the program, we will have to use two-thirds of all of the neutrons to demonstrate that we were right. I would say that most of those who are promoting in one way or another an interest in a particular fuel system will be able to make a case of gathering some experience to demonstrate the passiveness or lack of same for his system for the FFTF application by using some of that two-thirds of all the neutrons. I think the Commission would not be in a responsive mood if they neglected any reasonably promising system totally, and tried to zero in on "the promising" system at this time. I think this is substantially the simultaneous conclusion ofthe set of opinions that seem to exist here. 
14.1

\title{
IRRADIATION PERFORMANCE AND PROGRAMS \\ OF FERMI FUELS
}

\author{
A. A. Shoudy \\ Atomic Power Development Associates, Inc. \\ Detroit, Michigan
}

\begin{abstract}
INTRODUCTION
Most everything has been said that I intended to say, so I think I'll review Don deHalas's discussion yesterday, and try to re-emphasize some of the points he made. They are quite consistent with our feelings in early 1965. For the advanced core for Fermi, we considered oxide fuel, cermet fuel, and also a $\mathrm{PuO}_{2}$ stainless steel cermet. We also considered carbide, but (after the Los Alamos discussion last night) I'm not even going to mention "also rans" or downgrade them at this point. Essentially, they are the three that we considered in detail. We also looked at a thouium-uranium alloy; however, since the data was so meager, it was soon dropped from consideration. We had certain ground rules, which we followed in our advanced fuel evaluation, and these are shown in Slide 1. Looking back at them now, I'm not so sure that they weren't right.
\end{abstract}

\section{SLIDE 1}

FERMI FUEL SURTEY GROUND RULES

1. SELECTED FUEL SYSTEM MUST UTILIZE PLUTONIUY (PERHAPS U-233)
2. TECHNOLOGY OF SELECTED FUFL ADVANCED TO POINT WHERE FULI-CORE DEMONSTRATION IS TIMELY

3. SELECTED FUEL SYSTEM MUST OFFER FROMISE OF LONG-RANGE ECONOMIC POTENTIAL 


\section{FUEL SYSTEM}

First, the selected fuel system must have plutonium or $U^{233}$ as the fissile component. This ground rule was set by management. They felt that in the long range, we must have breeding; if we have breeding, we have to have plutonium; if we have to have plutonium, we have to use it. The objective here then, is to see if we can find a way to use plutonium in the fast reactor.

Skipping to item 3 we say we had to have a fuel system with a long range economic potential in a fast reactor. Perhaps, this is the point of departure from the discussion that we had here today and yesterday, and from Don deHalas's comments about the fuel for the Fast Test Reactor. You don't have to feel that this must be a fuel with a long range economic potential in a power reactor.

We felt we must select a fuel that is sufficiently advanced technologically so that it would not take 10 years to be ready to put a core loading in Fermi. It might take us 10 years anyway, but let's try to pick out one that might go a little bit faster. I think Don mentioned that they had not considered carbide to any great extent, primarily because there is little known on large scale fabrication of mixed carbide fuels. I was going to add to that, but the point was brought out ten or fifteen minutes ago with a rather detailed discussion of what a carbide fuel element looks like. We did not know a year ago, and I don't think we know today whether it is a sodium bonded hypo-carbide element, a gasbonded hype rcarbide element or a sodium bonded ihypercarbide with chromium in it. It is going to take some time to find out. I do not think that Fermi should be shut down for the entire period of time that we're waiting to determine what a carbide element should really look like. I had one more comment relative to Don's talk. Don felt that to get the kind of burnup you need for an oxide element, you have to introduce a rather high percentage of porosity. When you run the oxide fuel element at high power levels, the porosity doesn't stay where you put it; and we're not so sure that it doesn't go where we don't want it. I think we feel exactly the same as Don does, and for this reason we have an oxide program that, perhaps, does not really emphasize the future and total economic capabilities of the oxide fuel; but a least, we feel it will permit us to license an oxide core for operation in the reactor (we hope) within the next three or four years.

In Slide 2, I mentioned that we looked at carbide and a thoriumuranium alloy. I think it is clear that metals are good fuel for a fast reactor if they will stand up under irradiation. The thorium-uranium alloys appear to do that. We did not give it much consideration, primarily because no significant effort has gone into its development. We would have had to support the total effort ourselves, and we felt we could not do this. 
14.3

FUEL SYSTEMS CONSIDERED

SLIDE 2

1. MIXED CARBIDES OF PLUTONIUM AND URANIUM

2. THORIUM-URANTUM-233 ALIOY

3. MIXED OXIDFS OF PLUTONIUM AND URANIUM

4. PUO2-STANLESS STEEL CERMET

FUEL SELECTION

On Slide 3, I've tried to list the basis for selecting the oxide fuel. Relative to the current technology, there is a large amount of work going on oxide fuel. We felt with this large effort, our own effort could be relatively small and since last year, this has been further emphasized. The British effort includes about $60 \%$ on oxide fuel, $30 \%$ on carbides. Euratom countries are planning to irradiate fuel in the Fermi reactor; they are going to irradiate four subassemplies, each subassembly having 24 fuel pins, and all of these will be oxide. The total Euratom effort now on carbide fuel is limited to fabrication development and to radiation testing in thermal reactors.

SLIDE 3

BASIS FOR SELECTION OF FUEL

CURRENT TECHNOLOGY

1. EXTENSIVE EFFORT UNDERWAY THROUGHOUT THE WORLD ON OXIDE

2. UO 2 -SS CERMET TECHNOLOGY CONSIDERED APPLICABLE TO PuOO2-SS CERRET

3. MINIYAL WORK UNDERWAY ON MIXED PLUTONIUM-URANIUM CARBDE

4. NO CURRENT WORK ON THORTUY-URANIUM ALLOT

ECONOMIC POTENTIAL

1. MIXED OXIDE OR CARBIDE SHOW CONSIDERABLE PROMISE FOR LONG-RANCE ECONOMIC FAST REACTOR FUEL

2. CERMET FUEL SUFFERS IN LONO-RANGE POTENTIAL DUE TO POOR BREEDTNG RATIO IN LAROE FAST REACTORS 
I meant to check DeWitt Mosses' list on what they would like to irradiate in Fermi, but again, I think it is on the order of $30 \%$ carbide, and $60 \%$ oxide. I don't know what they are currently testing in EBR-II, but the oxide is getting major effort today, and we do need that kind of effort to support our program and to help us go in the right direction. With respect to the $\mathrm{PuO}_{2}$-cermet, we felt UOZ-cermet technology was applicable. There has been a lot of discussion here today on whether it is or isn't I think at this point, I am inclined to agree with those that feel we have demonstrated the burnup capability of this cermet fuel. I think we may improve things by a few percent but with a great deal of effort. I mentioned that we did not consider thorium-uranium very long because there is just no work going on with this fuel system. As an economic potential, certainly a carbide shows considerable promise.

We ran a study two or three years ago and tried to compare a plutonium oxide cermet with a plutonium-uranium oxide fuel in the power reactor. Economically, we attempted to compare these, and I think the prime thing we were able to show is that from the economic and long range point of view, the cermet clearly suffers from its lack of breeding in the core. It looks like no matter what you attempt to do with fabrication costs, you just can't make up the loss in plutonium breeding.

I would say that this is primarily the reason we did not have the difficulty in making our choice that obviously will be encountered in making the choice for the test reactor.

\section{DESIGN CHARACTERISTICS}

Slide 4, shows what our preliminary fuel pin designs for Fermi look like. Not too surprisingly, they're not too different from a fast test reactor design. The core volume, as was mentioned yesterday, is 800 liters; for the test reactor you are thinking of 600 . We have a cermet pin at $0.20 \mathrm{in}$. in diameter, and we have an oxide pin at 0.25 . We have a temperature gradient in the cermet pin of $475^{\circ}$. I think short of the long range application standpoint, this probably was the one thing that gave us some concern about operation of the cermet pins in Fermi.

\section{$\underline{\text { SLIDE } 4}$}

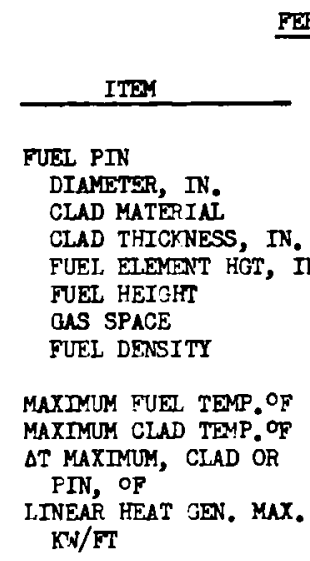

FERYI FUEL PIN CHARACTERISTICS

\begin{tabular}{|c|c|c|c|}
\hline \multirow[t]{2}{*}{ CORE A } & \multirow[t]{2}{*}{$\mathrm{PuO}_{2}$-SS } & \multicolumn{2}{|c|}{$\mathrm{PuO}_{2}-\mathrm{UO}_{2}$} \\
\hline & & $230 \mathrm{M}$ & 4307 \\
\hline \multirow[t]{4}{*}{$\begin{array}{l}0.158 \\
2 R \\
0.005 \\
30.5 \\
30.5\end{array}$} & $\begin{array}{c}0.206 \\
5 S \\
0.007 \\
4.8 \\
48\end{array}$ & \multicolumn{2}{|c|}{$\begin{array}{c}0.250 \\
58 \\
0.25 \\
67 \\
48 \\
19 \\
87.56 \text { T.D. }\end{array}$} \\
\hline & $\begin{array}{r}1270 \\
930\end{array}$ & $\begin{array}{r}<3300 \\
1100\end{array}$ & $\begin{array}{r}4800 \\
1150\end{array}$ \\
\hline & 475 & 60 & 110 \\
\hline & & 12 & 22.5 \\
\hline
\end{tabular}


We show two power levels under the oxide design, one at 230 , (and I would like to put an "about" sign there), and one at 430 , which is the maximum capability of the reactor. The 230 design essentially is one in which the peak generation rate is about 1 kilowatt per inch. We think and we hope that this is a point at which we do not move the porosity in oxide fuel. We think that somewhere in that range we will not form a central hole, and that we will not be concerned about having to prove that a central hole is a safety problem. We think that this is not a safety concern, but we do not know how we can improve it. Since we would like to license this core for use in the reactor within the next couple of years, we intend, initially, to avoid that problem. We do not want to have to face up to it, in so many words. I don't know whether it's going to take a long time to convince the licensing people that this is not a problem, or whether it will be easy to do. We think it clearly is not a problem, but we don't know.

Subsequently, we would like to operate this core under the power rating conditions that the $1000 \mathrm{MW}$ design-studies came out with, a peak heat rating of approximately $24 \mathrm{~kW} / \mathrm{ft}$.

Under these conditions, then, we would reach the $430 \mathrm{MW}$ power level, and finally demonstrate real breeder fuel in the Fermi reactor. I show stainless steel as the cladding material. A little while ago, Carl Puechl told us that the specimens in his program are 316. Most everybody talks about 316 , but I haven't been able to find out exactly why. I think 347 has certain mechanical properties that are superior to 316 , and I have been trying to find out why nobody looks at 347 . We already know that in the test reactor (or any reactor) that ductility is not something that you can count on, so I'm not sure why we should count on it in the initial design. Ductility appears to be the prime advantage of 316 over 347 in the unirradiated condition. We, have as most people, relatively low density fuel. I've listed $87-1 / 2 \%$, we've talked 85 to $90 \%$. Our final selection will depend on what we find we are able to make (from a standpoint of just making pellets). In Slide 5, we see 600 to 900 sodium inlet-outlet temperature. We have felt that the cermet fuel was such that

FERQI FUEL DFSIGN CHARACTERISTICS

SLIDE 5

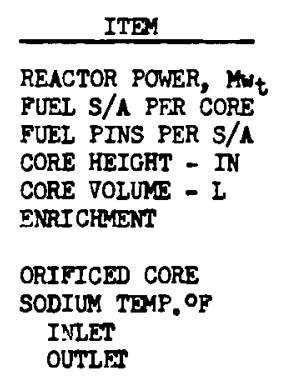

$\underline{\text { CORE A }}$

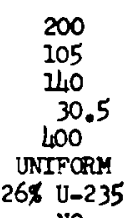

No

550

800
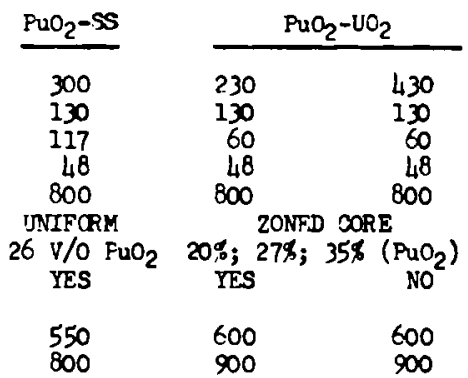
14.6

if we attempted 600 to 900 , we would be operating the fuel up in the $1350{ }^{\circ} \mathrm{F}$ range; one where the burnup capability did not appear to be what we would need to have anything approaching an operation that we could economically accept. 
D 14.1

\section{IRRADIATION PERFORMANCE AND PROGRAMS \\ OF FERMI FUELS}

A. A. Shoudy

Atomic Power Development Associates, Inc.

Detroit, Michigan

\section{DISCUSSION}

MR. SHOUDY: I will try to answer any questions.

MR. HENNIG: I will start it myself. Do you actually segment the

pin?

MR. SHOUDY: No. We felt that the actual segmentation of the pin, if we asked how many segments does he want, his answer would be, how many can you give me. If we can give him 100, he would take 100 . If we could give him something approaching a cermet fuel, one every pellet, he would take one every pellet.

MR. SHOUDY: I think that one or the prime reasons that we did not select, or elect, to go to segmenting pellets is that when you look at the big reactor (and I guess perhaps Mr. Zebroski, or somebody, can talk better about this in their design) they do not worry about fuel movement to the extent that we must. in a smaller core. So we felt ther, in the future, a segmented element will not be the thing to do. Collapsing the clad was, perhaps, not departing too much from what happens with a fuel pin, so that maybe it is coming closer to demonstrating something 10 or 15 yr away than would we have, had we used the segmented element, so we did not choose this one.

MR. HENNIG: I neglected to mention that Belgium Nuclear is participating in this development program. I would say the L/D at this point is like one. I don't know what it is. They have not gotten to the point where they are ready to specify this, but this will be in the Belgium Nuclear province.

MR. CHERNOCK (Combustion Engineer): I would like to discuss a few of the comments made about the existing state of technology with respect to mixed oxides and carbides. I think you inferred that there were so many selections on which carbides were used and we didn't know which one to go to, and I look at the particular mixed oxides situation, and we talk about dish versus nondish, and we talk about pellets versus vibrational compaction, and we talk about high density versus low density. You take the specific design that is being used in APDA, or the one that is being proposed, and I don't see very much in existence in technology that is being fallen back on so that I would have to question as to whether the present selection is based really on an existing technology. I would like to carry this one point further and point out that if we look at the general comparison of oxides and carbides, I will agree that there is a preponderance of 


\section{14. 2}

planned work for the mixed oxide versus the mixed carbide, some of which gets into this previous discussion of differences or variables. Some of this is associated with fuel recycle with significantly lower plutonium contents than you are considering in the fast reactors. I would like to separate the fuel recycling preponderance of that from the other planned efforts for fast reactors. I would like to raise one very important question. That is, if it is true that lower doubling times are in effect obtained with carbides, and if we are not today, in this country, planning an extensive power reactor program with fast reactors, couldn't we honestly state a year from now (if we change our plans today), that instead of $70 \%$ of effort on radiation being in the oxide area and $30 \%$ in the carbide, that $70 \%$ in the carbide and $30 \%$ in the oxide wculd lead us into a better future situation for the fast reactor concepts.

MR. SHOUDY: I will attempt to comment on the first comment relative to the fuel technology standpoint. I think, when we look at what we have done with the fuel pin as being a relatively minor type of thing, like a comparison with, shall I sodium bond the fuel or shall I gas bond it? Do I have to use hyperstoichiometric material or do I have to use hypostoichiometric material? I think these are major unresolved things.

I think my only comment to ycur latter question of shifting the emphasis on an oxide fuel-carbide fuel problem might better be answered by-I was just going to say it without Mr. Amorosi's pointing it out $-\infty$ but, might better be answered by the people in the metal fuel area. I think if you acknowledge that breeding ratio is the important--or doubling time is the important-thing, that then you can not reduce your development effort on a solid metal fuel. You cannot consider eiminating your effort on molten metal fuel. I think metal fuels clearly give you the doubling times that are quite large by even a comparison to carbide fuel.

MR。L.E。 GLASGOW (Atomics International): I would like to ask a question with respect to reactive change due to fuel movement.

MR. SHOUDY: John, do ycu want to comment?

MR. JOHN NIMS: It seems to me we figured you could have a result of differential exchanges of gaps of something like half an inch or so. If you make a very conservative assumption that this is all concentrated at the plate, that is about as small as I can recall the numbers. Incidentally, if anybody can show these cracks are distributed, that they are distributed uniformly over the length of the --

MR. SHOUDY: You can get them to collapse, then we could save probably two miilion dollars on our program. Don mentioned yesterday that they were concerned about the melting of the low density oxide eiements. When you get to the point of melting, I think the oxide is probably not worse than any other kind of fuel. What we are concerned about is the likelihood or the probability of getting to melting, which leads us into this actual fuel movement problem. We are not really saying that we think this is our problem. We are just saying we have never seer, anybody attempt to show it is not, and that is the only way that we feel we can license an operation in Fermi. Howard?

MR. KITTEL: I would like to ask another question. What is the boiler code allowance that we have always had? 
MR. SHOUDY: I am just a poor old metallurgist. I don't know. I did look--we did look recently. Tom Claudson sent us some data on the most recent boiler code allowances for these particular materials and I know we looked at them and now I don't remember what we saw.

MR. ZEBROSKI: If I could offer a couple of comments on your last question first and then a question after that. On your $230 \mathrm{MW}$ operation of the oxide fuel with the centerline temperature of about $1800{ }^{\circ} \mathrm{C}$ you will surely make central voids even at that temperature. You don it get holes.

MR SHOUDY: I think it said less than 1800。

MR. ZEBROSKI: Less than 1800, you would have to go to one-half of the $\Delta \mathrm{T}$ or to about $115 \mathrm{MW}$...

On the question of 347 , the most recent boiler code data does not really show significant advantage for the 347 over the 304 or 316 . How ever, the question of strength does not come into play. Very little of the fuel cladding is used in way of boilers without neutrons. The change in carbon during radiation surely must be the chief criteria of comparison and therefore it is really quite relevant.

Coming now to the question, and I think this should go both to you, $\mathrm{Al}$, and to deHalas. The British carried on the cermet fuel to a fine degree of development. They got the burnups quite a long way up. Do you have a good feeling why they felt they should abandon this line of development, even though they also needed dry runs?

MR. SHOUDY: I guess I would say I don t have a good feeling for why. There clearly are still some real proponents of cermet fuel in England. I think it was probably for the same reason that we did not carry it too much further than we did. If you are really talking of fast reactors from the standpoint of doubling time, then a cermet fuel doesn't make sense. The ceramic itself is bad enough from a 238 density standpoint, but then if you go ahead and cut it down (I think the British ended up in the range of 50 or 60 vol\% material), you now practically eliminate a doubling time limit. So I think that may be the reason, but I don't think arybody can really say. Do you know, Don? Did you ever get a feeling?

MR. DON deHALAS: The only comment I was going to make is the same thing; that as far as I know, it is simply a question of degree of stainless steel, and there, of course, I think everybody realizes they are continuing efforts on cermet. I didn't know if there were any technological problems they had with cermet itself. It should be reaized that the initial British cermet was much worse than those turned out by the United States at the same time.

MR. KITTEL: I want to ccmment on one of the comments, too, which had to do with the selection of stainless steel. The data we have seen--and maybe Larry Kelman could comment more authoritatively than I--show that 304,316 , and 347 are all so close together there is very little basis for choosing among them on these accounts, so I wondered if you had looked at all the $304, \mathrm{Al}$, as a possible candidate for your fuel studies, because it certainily welds better than 347 , at least in many applications. The other thing I wanted to ask concerns ancther design parameter which has come to Iight in the last year or so. This relates 
to the location of the gas lines in the outside oxide fuel element. What is your feeling on this? Are you looking at both the upper and lower plenum, or do you figure one over the other?

MR SHOUDY: I will take the first question first. As I recall, looking at the boiler code, it seems to me and perhaps Larry can qualify that. Will you, Larry? Is it 316?

MR. KELMAN: You talked about yield. It is a short time yield?

MR。KITTEL: Yes.

MR. KELMAN: Where these various stainless steels tend to come together is a long term property. In the short time it is true, 347 and 316 have advantages over 304 in about that order. But I think the comment Mr. Zebroski made was much more pertinent here. What is the effect of irradiation on these things? Frankly, I am not sure why you prefer yield over a long time...

MR SHOUDY: I guess I am not sure at this point, either. Let's say we more or less backed into it. In testing fuel in Fermi, we acknow1edge to ourselves this was not going to be a simple thing to obtain licensing from the testing fuel. We have had enough trouble just getting a license to operate. We felt that we must be quite conservative in the kind of tests we do rur, so we looked at what does being conservative mean. We considered the British approach to the design of an oxide fuel line and theirs is essentially the type of thing we have adopted. It says that the internal pressure stress with one quarter of the thermal stress shall not exceed the yield--and then there are a couple of other variations of the same thing. Now I think that we fuel people will acknowledge that it is really not--probably not--the fission gas release that ruptures your friel tank, but it is the oxide pressing against the clad and straining it to the point to which it just won't strain any more. In any event, though, if we go back now and apply a criterion that we developed for testing in Fermi to the various failed oxide fuel pins, we find in almost every case that we have examined so far, we would not have permitted that burnup, or the burnup a at which point it fell. I think we have not gone far enough as yet in this area to know whether we are too conservative or whether we are just mildly conservative. Other than that, it is not too bad an approach, although it is really not what we think happens. But it is, I think, emphasizing the wrong thing, as you point out. It is probably the long term thermal property that we should be concerned with. I think that in the long run we will settle down to that, but at this point, we don't have the basis.

MR. KELMAN: Let me just add in this area of slang from the family of steels, do consider the British work where they are considering strongly what they call an FB548 alloy. The type 318 stainless steel, the 316--we have it verified; but, they certainly claim much better strength properties than others.

MR. SHOUDY: Howard, you had one other question I didn't answer, but I wanted to get into it.

MR. KITTEL: Where are you putting the gas plenum?

MR SHOUDY: Oh yes, yes. That is another thing we just backed into with this fuel test assembly design. The Core A design is right in the 
middle of the fuel test subassembly; so obviously then, that is where you want to locate your fuel--right where the Core A fuel is. Then it just follows from that, that now you also have some free space down at the bottom below your fuel and you also have some up at the top. So we ended up our design--or somebody ended up drawing a fuel pin. Here is what your fuel pin look like, and it was drawn in, and there is your gas space at the top and at the bottom. But I think a lot of the fuel pins are going to have to be tested in Fermi, and have gas spaces at the top and the bottom. Now if there is an advantage to having it at one place or the other, I might say that we have both advantages; but some of you smart characters will say there are also some disadvantages having it one place of the other, and we have all the disadvantages. I don't know what the core will be, Howard. We want about a $4 \mathrm{ft}$ high core to get something on this order of 200 to $400 \mathrm{MW}$ of power. This just does not leave any room to put an axial blanket in the subassembly and it increases the flux on the support plate and hold down fingers. I don't think we like it, We will attempt to incorporate some degree of shielding within the fuel pin. How much we are willing to sacrifice for an achievable power level just because fuel metallurgists are concerned about nvt's, I don't know; but, it sure increases the incidence of flux by about a factor of 20 or so.

MR. KITTEL: What I was interested in was the core design for the outside reactor rather than the Fermi test itself. We also have the upper and lower plenums.

MR. SHOUDY: I think what I really went back to was the core design. We are in the process of looking at it. Like most people, we feel we would like to have it at the bottom because it is colder. We become somewhat concerned about what happens if it ruptures at the bottom. But at the same time if we put the gas plenum at the bottom, we are moving that fuel about 12 or 9 in. closer to the hold-down, and we increase again by a factor of 30 or $40 \%$ incidence of flux on the hold-down finger, so we haven't made this decision, I think it is going to be really a difficult one to combine.

MR. KIRKBRIDE: I would like to make a couple of comments, if I may, on the stainless steel business, and in particular the ductility in a fast flux. We had Iour stainless steel reflector pins in the LAMPRE experiment, and we are doing a program at Oak Ridge at this time, doing the tensile properties on these four stainless steels. Most of you who were here yesterday will remember the flux is quite hard in this molten fuel reactor. Consequently we are looking almost purely at fast neutron data. The preliminary data that we have are only on elevated temperature, short time yield strength; and these four stainless steels that we had were 316 , 316-L, 304, and 1748. In all cases the three normal stainless steels started to lose their strength, or their ductility, at $600{ }^{\circ} \mathrm{C}$ and became progressively worse. However, the 1748 remained ductile. In fact, the 1748 material looks exactly as though it had not been irradiated. It just matched. The ductility data matched the material. In all cases, since the irradiation was done at $550{ }^{\circ} \mathrm{C}$, there was no strengthening of the stainless steel as far as yield was concerned. The irradiation was high enough that we lost all the plastic damage. So I might suggest, in a further point, that we will deplete. This is in process now by Bill Martin at Oak Ridge, so I might suggest that one might look at the 
D 14,6

precipitation hardening alloys .... and forget--or at least start to phase out-your thinking on the ordinary run-of-the-mill stainless steel.

VOICE: What heat treatment condition was that in? Was it in the hardened condition?

MR. SHOUDY: Which?

VOICE: The 1748.

MR. SHOUDY: I believe the 800 to 1100 condition.

MR. HECK: Al, in your formal presentation you mentioned that the collapsed cladding was a good thing to do. Later, answering one of the questions, you mentioned that you thought it was quite well agreed that fuel swelling and so on was going to fail the clad. How do you reconcile these two?

MR. SHOUDY: Yes, good question. I think that fuel swelling--I think that we hope to incorpcrate enough porosity in the cladding to accommodate a given amount of swelling. We are not, I don't believe, anticipating that we have to go about $100,000 \mathrm{MWd} /$ ton to convince somebody we should buy it for the reactor. I think that our principal feeling is that the smear density is the same as that, which you might have had, or if you had a high density pellet of $90 \%$ or so. I don't really see how you can avoid the fuel movement problem short of shelving. If you go to shelves, this may be the way to do it. I don't know. We felt that we had to take one path and go down it; and we thought this would still be relatively consistent with what you might like to do 10 or 15 yr from now

MR. SHAPIRO: Warren made a comment very early after your talk regarding the selection of the fixed oxides versus the mixed carbides. He made the point that we know little about the oxides and that we don't know whether it will be pellets or with or without dishes, etc., versus the carbides. The difference between the two that has to be pointed out is that in the case of the carbides, you have some basic problems to contend with (stability problems and so forth); whereas, in the case of the oxides, you are not so much worried about that. Your thinking here is the specific physical form in which they should be incorporated. I think these are problems of entirely different orders of magnitude.

MR. WILLIS (AI): I would like to know what stability problems we have in carbides.

MR. SHOUDY: I don't know, Art. Why are we still looking at three or four basically different types of carbide elements? I think if somebody can answer that, then we have the answer to your question.

VOICE: What was the question? carbide?"

MR. SHOUDY: He said," "What is the basic stability problem with

MR. WILLIS: No, the question just raised was, what is the difference between $\mathrm{UO}_{2}$ and carbide. There was a question of whether there was a basic difference instability with carbides. I got the problem raised, and since we are working with carbides in various compositions, I was just curious as to what problems we have in carbides that we don't have in oxides. 
MR. MOSS: I don't think you have to look too far to open up the door on the big question of carbides and the compatability and swelling. I think fuel swelling is a serious problem to be considered with carbides. Now Al Strasser radiated to about $114,000 \mathrm{MWd} / \mathrm{ton}$, but he did this in about 15 or $25 \mathrm{mil} \mathrm{clad,} \mathrm{and} \mathrm{the} \mathrm{true} \mathrm{performance} \mathrm{of} \mathrm{this} \mathrm{material} \mathrm{did}$ not evaporate. Now the compatability situation is again open to question. I believe also Westinghouse would say that they got some results even with their modified material.

MR. CHERNOK: Regarding your last comment about the porosity results with a thick clad, I would like to point out that there has been a magical number going around on mixed oxides of 98,000 , and if one examines what that magical number is, one finds that that is a thick clad. If I am not mistaken it is a rod at reasonably low density. I think the density runs $85 \%$ of theoretical.

MR. SHOUDY: You are referring to the G-E work, and I think, indeed, they were also gunbarrels. Now, we do have a number of oxide fuel pins that have gone up to 60,000 or 70,000 without having gunbarrels on them. Al Strasser pointed this out to me when I gave him the same deal about a gunbarrel, and he replied that your own oxide tests were gunbarrel (and they were).

MR. CHERNOK: I am not trying to give anybody the needle. I am trying to scope in on the thing from the light of where do we want to go in fast reactors, and where are we today. But where do we want to go? If we all agree that ( $\mathrm{I}$ am not so sure we do) doubling time is a very important parameter, and if we all agree that we are not going to build a power utility reactor today, I still raise the very important question: what, then, drives us today to expend a major portion of cur efforts on mixed oxides when it appears that the mixed carbide is the future road to go?

MR. SHOUDY: I am not sure your are going to get a lot of people agreeing with you. We are talking about power reactors now. I didn't point it out, but I hope most people know that we would like to order core in the next 2 to $3 \mathrm{yr}$. We did not think we were ready to pick out the best way to use them.

MR. PUECHL: I think the big difference between the carbides and the oxides program is that today we know that the oxides, we are putting in, are producing; they can be characterized. We also know what the results will be. On carbides, this isn't the case. If people were asked to irradiate carbide samples again in the same equipment, they could not possibly do it. In different equipment, you have different seal over pressure, or slight temperature differences, the effects of oxygen, the effects of nitrogen, etc., we just don't know (it is meaningless). I think until we fully understand the carbides (uranium-plutonium-oxygen system), the radiation results are going to be rather meaningless.

MR。 RAY: I would like to characterize these two flukes that deWitt Moss mentioned. The first fluke we encountered consisted of a pretty conventional sort; namely a pin hole in the weld. We picked up some oxygen in the flexibility capsule, and the sample just deteriorated (for cbvious reasons). The second fluke we encountered in the compatability series was with the chromium modified material. The first one was with 
iron, as I recall, and the second was with hyperstoichiometric carbide. The difficulty we encountered was that some of the feed material had $\mathrm{UC}_{2}$ in quite large amounts. I am still trying to find out how it got there. I don't quibble for one moment with Mr. Puechl's remark that there are problems in the processing of carbide that reproducability is difficult to achieve. I do not agree, however, that you cannot.

MR. SHAPIRO: I don't think the contention was that it can't be. I think the contention was there is a lot more that needs to be learned at this particular point. You are much farther ahead if you are thinking in the terms of the near term versus the long term.

MR. RAY: I guess the position I am trying to indicate here is that it really doesn't make much difference what the problem is; but if it takes an irradiation to resolve, the time lapse is about the same. If you are talking about putting an addition in the pellet or changing where the pellet has been, it still requires an irradiation proof test.

MR. SHAPIRO: We agree with that, but I think Mr. deHalas' remark previously is applicable--that you are farther ahead today; you are farther down the road with oxides than you are with carbides. You have a lot more to learn about the carbide than you apparently have to learn about the oxide.

MR. SHOUDY: I would like to add a little bit to that. In speaking about testing fuel in Fermi, we clearly (like I mentioned before) would like to test them under conditions that we are fairly confident we are not going to fail with fuel pins, that plutonium will not be going all through our system, or anything of this sort. I think we can justify putting an oxide fuel pin with something like 10, 15, or $20 \mathrm{mil}$ clad into Fermi. We can pick out numerous tests that have gone to the kind of burnouts that we can reach in Fermi core operations, 50,000 to 60,000 . If we put an oxide pin in like deWitt wants to do (running at a couple of kilowatts per inch) and they want to go to 90,000 MWd/ton--I don't know how we can put that pin into Fermi.. I don't know how we can justify or give a feeling that it is not going to fail. Can you prove that it can go to 25,000? I don't think we have a real fast reactor carbide fuel pin that has gone to 25,000 . So I think it is that order of magnitude difference between the material. The oxide has gone 60,000. The carbide hasn't gone there, yet. I am not saying it won't, but it hasn't.

MR. HECK: Except for the gunbarrel clad problem, it has gone more than the oxide has.

MR. SHOUDY: If that is right, the cermets have gone to 50 or 60 or $70 \%$ burnup, too.

MR. HECK: The point I want to make is that we tend to agree with you. You are talking about a core on a different schedule than we are talking about. I think that neither Bill nor I said that carbide should be the fuel for the FFTF, but we said it should it be given serious consideration as an alternate fuel development during the time we are getting ready to produce fuel for the FFTF.

MR. MOSS: I think the first priority is on the oxide and its demonstrated performance. Breeding will not be so important to the 
utility, when in fact, the large plant is sold, whether it is a prototype or a $1000 \mathrm{MW}$ job. Because what are we going to do, for example, if we get part way down the pike and we find out stainless steel will not perform? We obviously will look at another cladding material. But I don't think we will let it stop us. What I am saying is that economics, industrial acceptance, and performance are going to dictate to the first one or two generations. I think it is something potentially to keep in the back of our minds, and to realize generally what the program is for. Al questioned also, earlier in the day, what our relative limits of efforts are. On fuel materials and fast breeders, we have about ten million dollars this year. This is just a good crosscut; about $30 \%$ is going to oxides, $20 \%$ to carbides, $10 \%$ to metal, $10 \%$ to clad, and $10 \%$ to others, and a small percentage of the "others" is driver fuel--FFTF driver fuel.

MR. HENNIG: A third of the generation capacity power in the Uinited States is at PRDC and APDA.

One of the reasons that this group is represented here by Shoudy is that it has made the decision not to use $\mathrm{PuO}_{2}$ stainless steel cermet, because it won't breed. That is a major difference between it and the oxide. To this utility group that is the thinking right now. It really does make a difference.

MR. SHOUDY: Yes, I think you made some assumptions there, Bob.

MR. deBOISBLANC: I think he was referring to using it as a figureof-merit. That is, how high does it have to be when it is no longer a figureof-merit? If a system can breed (and indications are that oxides can be made to breed), the question is: What is the minimum doubling time before it ceases to be an economic penalty to the utility?

MR. MOSS: This is right. I think basically, if breeding is the criterion that justifies AEC's expenditures, you would take another serious look at metals, and metal plutonium, and you have doubling time down into 6 or $7 \mathrm{yr}$. Change clad materials and accept niobium, and I think even by a wrong factor of 2 or 3 , it is better than anything else. So I think economicaily reliable performance and industrial acceptance is going to be an early decision. I don't know what $\mathrm{Al}$ 's decision is up here--other than there is a relatively large program going. I might ask (looking to the past), do you really feel that you could decide on a mixed oxide pin for the future? I think whatever the answer is, it is going to be better than cermet.

MR. SHOUDY: You mean from the burnup standpoint and heat rating?

MR. ZEBROSKI: I think it is a very fair point. I think maybe we are tied up in a bit of semantics here in that we are trying to make an ultimate. solution to any of these problems. If that were a valid hope, we would see only Chevs or only Fords or only Plymouths on the roads and not a mixed economy of several different kinds. I think the question of the relationship of carbides to oxides is one of, "Can you, in fact, keep design flexibility in a plant as part of your hoped-for stretch capability on oxides? " The solution might be the employment of an eventual replacement carbide core that meets all of the requirements (not yet assured!. The complication there has to do with safety characteristics of the core, which obviously is the main issue. Next to the fuel feasibility, you may have a feasible 
fuel, but if you can't license the thing--the safety issue is really paramount to an industrial use of large reactors. I would say in this area, the carbide also suffers by a less conservative tax in the safety province than oxides have so far, and that you can equate the years of delay as two or three years back. However, it certainly doesn't rule it out as an eventual candidate. I would still say, if we commit (as we hope to) a prototype in 1969, we will do our best to keep open the option for a second or third core of carbide.

MR. MOSS: Ed, what would you permit yourself to work on in terms of exposure, because this is the problem facing FFTF today? Would it be on fuel cycle economy or in terms of plant availability (which is a factor or reprocessing onstream time and the like)? With G. E. 's primary extensive experiments in our country on mixed oxides, what would you say regarding mixed oxides today versus cermet? This is where the decision is going to have to be made on the FFTF.

MR. ZEBROSKY: Well, I would say that any as sumption much lower than 60,000 is faced with a problem. I am going to steal my thunder from the concluding statement, and maybe put out the challenge of the problem that I see is objective here. It seems to me, the problem of objectivity and the credibility of objective judgment as to the correct fuel selection can or will be made. It centers on this: Is there any way you can demonstrate that if by choosing a ceramic fuel for an FFTF, we haven't violated the basic premise of need for the FFTF?

MR. SHOUDY: Does anyone care to answer that one? It looks like that did it. 
CARBIDE, CERMET, AND CLAD DEVELOPMENT
AT UNC

N. Fuhrman and A. H. Kazi

United Nuclear Corporation

White Plains, New York

\section{INTRODUCTION}

My presentation will be devoted to recent carbide, cermet and clad development work at UNC pertinent to the FFTF Driver Fuel selection problem. Hal Kazi will follow with some remarks on the application of a particular carbide fuel concept in large fast reactors such as the FFTF. For a detailed discussion of the potential of carbide fuel for fast breeder reactors, I refer you to Uranium-Plutonium Carbide Fuels for Fast Breeder Reactors, ANS-100.

\section{CLAD DEVELOPMENT}

To provide an economic driver core for the FFTF, it is desirable that the fuel selected be capable of achieving high burnup. Solid solution (UPu)C has been irradiated to high burnup, $38 \times 1020 \mathrm{fissions} / \mathrm{cm}^{3}$, or $113,000 \mathrm{MWd} / \mathrm{ton}$. The fuel was in the form of pellets fabricated by a compacting and sintering process, that used a carbide powder prepared by carbothermic reduction of the mixed dioxides. Pellets made with nickel sintering aid were sintered at $1550{ }^{\circ} \mathrm{C}$, while those made without sintering aid were sintered $1925^{\circ} \mathrm{C}$. Both types of materials exhibited a hyperstoichiometric microstructure, i.e. contained a higher carbide phase. The former contained 15 vol\% sesquicarbide, and the latter traces of dicarbide.

The densities were $96 \%$ theoretical density (T.D.) for the pellets produced with nickel sintering aid, and $91 \%$ T.D. for those without nickel.

Thirteen to sixteen pellets of each type (approximately 3 in. of fueled length) were loaded into columbium and 316 stainless tubes $(0.030$ in. wall thickness), and assembled under helium with a cold diametral gap of 0.0005 and 0.0025 in. The two columbium clad specimens and the 316 stainless clad specimens were encapsulated in separate capsules and irradiated in the GETR (that is, in a thermal flux.) 
- The irradiation conditions are shown in Slide 1. Note:

- Average fuel center temperature ranged from 1750 to $2255^{\circ} \mathrm{F}$.

- Average clad OD temperature ranged from 850 to $1070{ }^{\circ} \mathrm{F}$.

- Specimen heat generation rate was $15.0 \mathrm{~kW} / \mathrm{ft}$ to $17.4 \mathrm{~kW} / \mathrm{ft}$. Fuel power density was 1195 to $1325 \mathrm{~W} / \mathrm{g} \mathrm{Pu}$.

Note that the higher power density is consistent with the lower heat generation rate for the bottom specimens because of their lower density. (Four other capsules were also irradiated under the same conditions except for lower burnups: 2 contained $\mathrm{Cb}-$ clad specimens and 2 contained 316 SS-clad specimens.)

Upon completion of the test and capsule disassembly, the two columbium clad specimens were found in excellent condition and appeared as they did prior to irradiation. The two stainless steel clad specimens each had one large and several smal1 longitudinal brittle fractures in the cladding, as shown in Slide 2. These ruptures were attributed to a drastic decrease in the ductility of the steel (integrated flux at specimen surface $1.9 \times 10^{21} \mathrm{nvt}$ ) to a level below that required to xcommodate strains from fuel expansion and thermal stresses. Similar clad failure was found in the $316 \mathrm{clad}$ specimen taken to 40,000 to $50,000 \mathrm{MWd} /$ ton, but not in the specimen taken to $23,000 \mathrm{MWd} /$ ton.

The results of the post irradiation examination are summarized in Slide 3.

- Note the difference in clad OD increase, and that the measurement indicates columbium clad offered some restraint to swelling. (In fact, measurement of whole pellets removed from columbium clad indicated a possible "relaxation" occurred after decladding).

- Fission gas release was about $30 \%$.

- The immersion densities indicate a maximum volumetric swe 11 ing of $18 \%$.

- Autoradiography indicated no plutonium redistribution.

- Microstructures were essentially unchanged by irradiation except for disappearance of dicarbide phase.

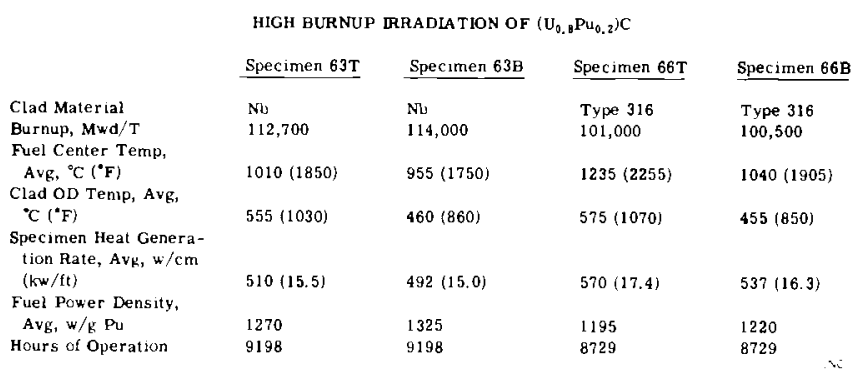

SLIDE 1

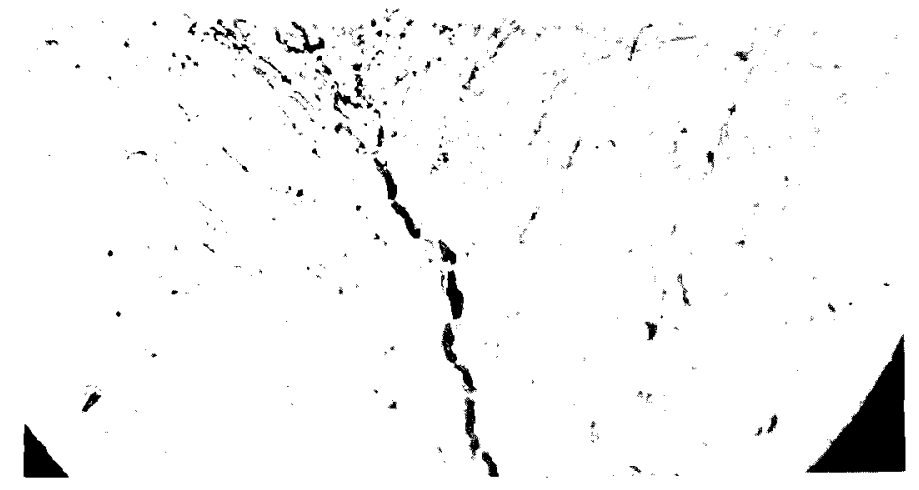

SLIDE 2. Longitudinal Fractures In 316SS CIad Specimen 


\section{3}

The microstructure of the four specimens are shown in the next series of slides. (S1ide 4) Note the sesquicarbide phase as well as the intact appearance of the columbium clad.

POST-IRRADIATION EXAMINATION OF HIGH BURNUP $\left(\mathrm{U}_{0.8} \mathrm{Pu}_{0.2}\right) \mathrm{C}$
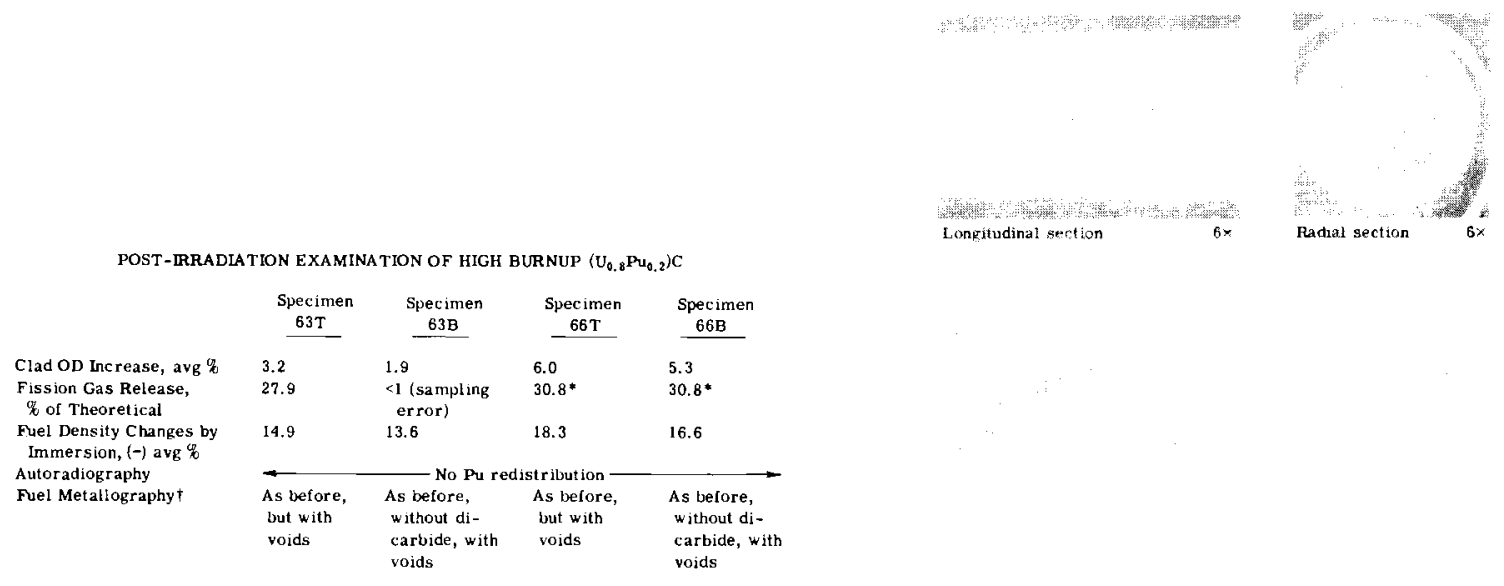

*Based on gas in capsule.

$\nmid N o$ significant change in grain size in any specimen.

Slide 5 shows the columbium clad carbide fabricated without nickel sintering aid. The irradiated structure is essentially single phase, similar to the as-fabricated structure except that dicarbide is no longer present.

Type 316 clad carbide prepared with nickel sintering aid is shown after irradiation. (Slide 6). Again, the sesquicarbide phase can be seen.
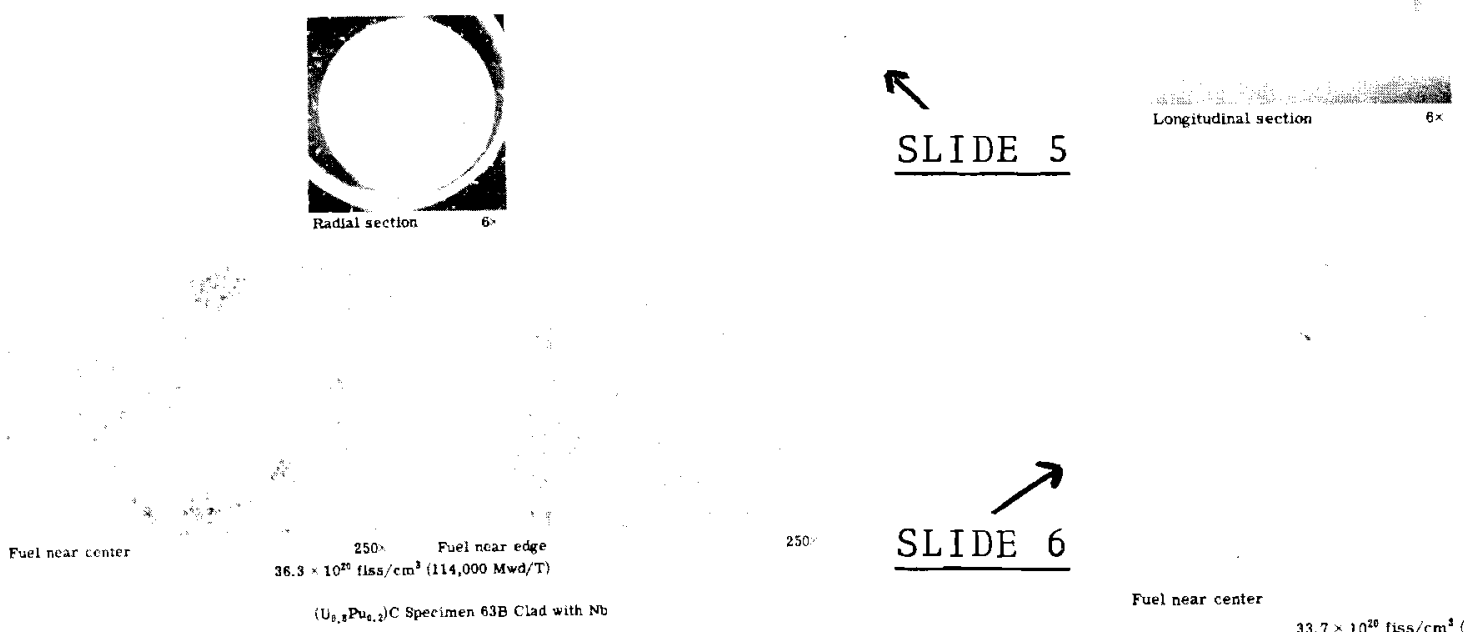
Slide 7 shows Type 316 clad carbide prepared without nicke1 sintering aid. Here one can see a significant amount of intergranular porosity at the fuel center, as compared with fuel near edge. This is believed to represent fission gas agglomeration.

No interactions occurred between the fuel and the columbium clad in either specimen. The clad was ductile and could be bent $180^{\circ}$ over $1 / 2$ in. radius without cracking. Slide 8 shows the columbium-fuel interface. Note the deformation of the clad.

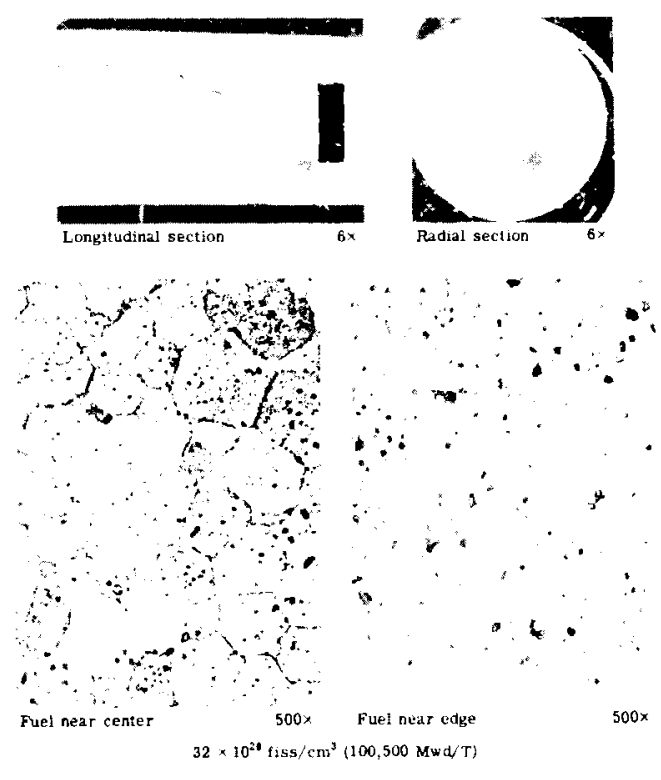

$\left(\mathrm{U}_{8,8} \mathrm{P}_{9,8}\right) \mathrm{C}$, Specimen 66B Clad wilh Type 316

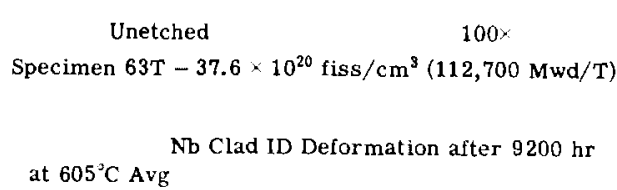

SLIDE 8

\section{SLIDE 7}

Similarly, no interactions were found between the fuel and the Type 316 stainless steel where the materials were in tight contact, as shown in Slide 9.

At this point, it is worth reviewing helium contact compatibility data developed by UNC prior to this irradiation program. Slide 10 tabulates the results with the various carbide-clad couples tested. No interfacial reaction was found after $4000 \mathrm{hr}$ between hyperstoichiometric (UPu)C and 316 stainless, columbium, vanadium or $21 / 4 \mathrm{Cr}-1$ Mo alloy steel. (Note that the equivalent carbon content $(\mathrm{C}+\mathrm{O}+\mathrm{N})$ of the carbides place them in hyper condition.) The high nickel Inconel alloy is not compatible.

Currently, helium-contact compatibility tests are in progress with other nickel base alloys that offer strength advantages over stainless steel and have better corrosion resistance to sodium than vanadium, which is being considered as a substitute for stainless steel. Vanadium as well as V-20Ti, another 
candidate clad, have the disadvantage of higher cost than that of stainless steel. Two medium nickel alloys, Incone1 625 (approximately 60\% $\mathrm{Ni}$ ) and Incoloy 800 (approximately $30-35 \% \mathrm{Ni}$ ) are being tested. Preliminary results after $1000 \mathrm{hr}$ at $800^{\circ} \mathrm{C}$ are favorable.

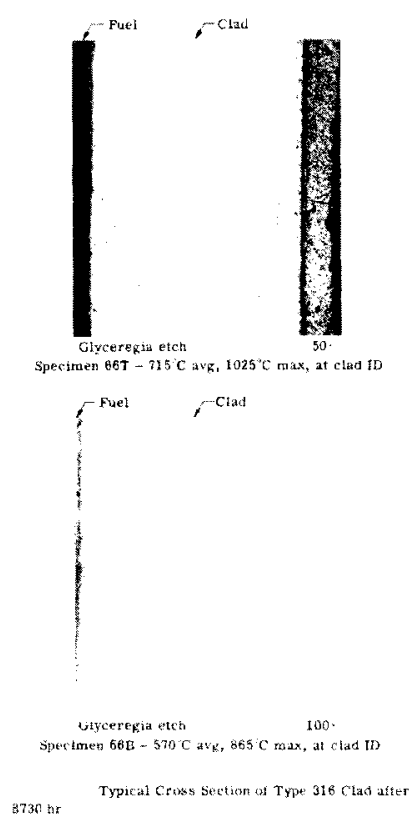

SLIDE 9

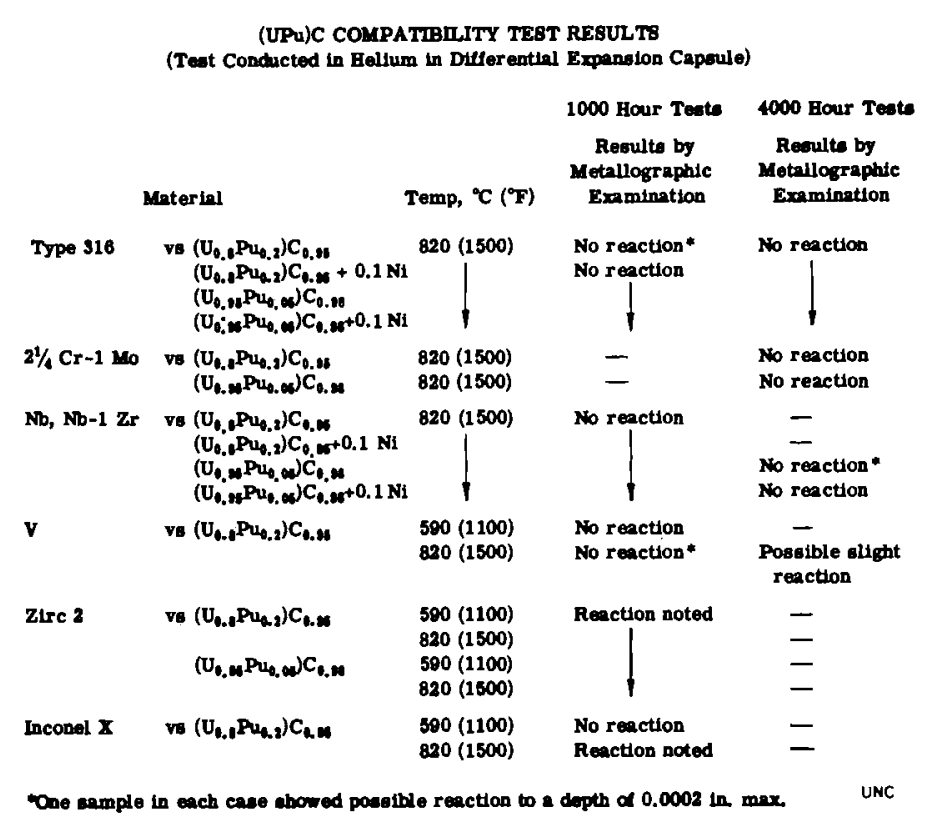

SLIDE 10

Clearly, the basic problem in developing carbide fuel (as well as any solid fuel clad in stainless steel) is to maintain clad integrity to high burnup. The current UNC carbide fuels program seeks to accomplish this by employing combinations of clad restraint with some form of fuel swe1ling accomodation within the fuel. Alternate cladding materjals are also being investigated. Along these 1 ines, 3 EBRI rods, helium bonded with hyperstoichiometric fuel, are about to be irradiated with the objective of comparing the performance of vanadium and vanadium-20Ti with that of 316 stainless. Power levels of 20 to $30 \mathrm{~kW} / \mathrm{ft}$ and burnups of $20,000 \mathrm{MWd} / \mathrm{ton}$ are planned and results are expected late this year.

Ten additional helium-bonded rods containing hyperstoichiometric carbide are planned for EBR-II irradiation. Diametral gap will be a major fuel parameter to be examined (also oxygen content). Cladding will be 316 stainless and V-20Ti, and

power levels will be 20 to $30 \mathrm{~kW} / \mathrm{ft}$. Burnups to $100,000 \mathrm{MWd} / \mathrm{ton}$ are planned; post irradiation data are expected to be deve1oped from late 1967 to early 1969.

Still in the planning stage is a full length fuel rod irradiation program to be conducted in EBR-II and Fermi, which will investigate additional fuel parameters as shown in slide 11 . 


\section{6}

1. Under structure, the three types are

- $\mathrm{MC}+$ Metal - hypostoichiometric

- $\mathrm{MC}$ - single phase monocarbide

- $\mathrm{MC}+\mathrm{M}_{2} \mathrm{C}_{3}$ - hyperstoichiometric.

2. Under chemical composition, we are interested in varying the oxygen content primarily.

3. To accommodate fuel swelling, methods of porosity distribution such as, low density solid pellets or annular high density pellets will be investigated.

4. The sodium-bonded fuel studies will involve only the $M C$ and $M C+M$ fuel structures.

5. These irradiations will involve power levels of 20 $30 \mathrm{~kW} / \mathrm{ft}$, and burnups to $100,000 \mathrm{MWd} / \mathrm{ton}$.

In addition, thermal flux capsule irradiations are planned to study unrestrained swelling of the various fuels as a function of fuel temperature. Also, fission gas release and in-pile thermal conductivity.

Results on fast fuel rod tests are expected from early 1968 to late 1968 , and those from the capsule irradiations from early 1967 thru 1968. As back up for the sodium-bonded fuel effort, sodium compatibility tests are planned as indicated in S1ide 12 .

CURRENT UNC CARBIDE PROGRAM

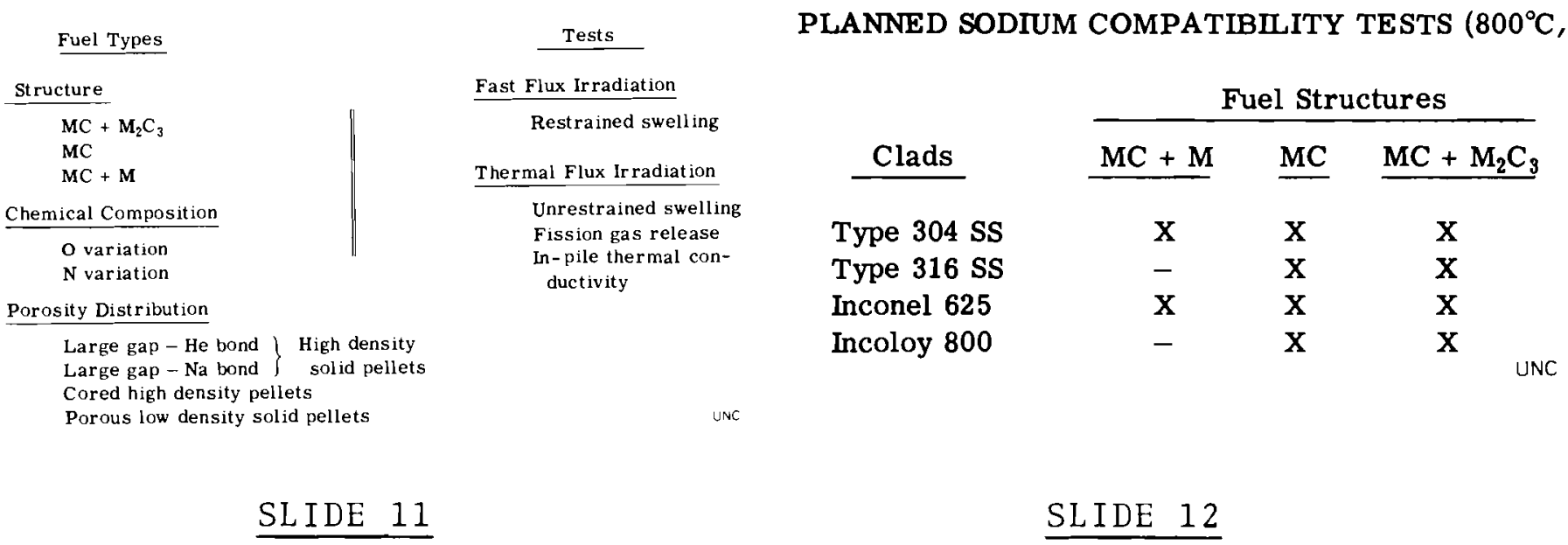

In the area of cermet fuel development, UNC has conducted a thermal irradiation test of high loaded (that is, 70 vol\% and 80 vol\% ceramic phase) $\mathrm{Cb}-\mathrm{UO}_{2}$ cermets. The fuels tested were metallurgically-bonded to Cb-1Zr cladding and represented three fabrication processes:

- Hot isostatic pressing (with coated shot as the starting material)

- Hot pressing (also with coated shot starting material)

- Compacting and sintering (starting with spherical porous oxide agglomerates blended with $\mathrm{Cb}$ power). 


\section{7}

The results of the irradiation are presented in Slide 13 . Note that diametral swelling at higher burnups was about $1 \%$. The 80 vol\% agglomerate ran hotter and exhibited more fission gas release, as well as slightly greater swelling. In every case, the clad was intact.

The fuel microstructures after irradiation are shown in the next series of slides.

Slide 14 shows 83 vol\% UO hot isostatically pressed fuel. Note the crack, otherwise the microstructure is the same as the fabrication condition. Whether such cracks would affect the thermal expansion feedback mechanism postulated for safety is not clear. Also note the absence of particle contact.

Cb-UO, CERMET IRRADIATION RESULTS

\begin{tabular}{|c|c|c|c|c|}
\hline \multicolumn{5}{|c|}{$70 \mathrm{v} / \mathrm{O} \mathrm{HIP}$ and HP } \\
\hline $\begin{array}{l}\text { Burnup, } \\
\text { Mwd/T }\end{array}$ & $\begin{array}{c}\text { Calculated } \\
\text { Max. Center } \\
\text { Temp, }{ }^{\circ} \mathbf{F}\end{array}$ & $\begin{array}{c}\text { Heat } \\
\text { Generation } \\
\text { Rate, kw/ft } \\
\end{array}$ & $\begin{array}{c}\text { Diametral } \\
\text { Swelling }\end{array}$ & $\begin{array}{l}\text { Fission Gas } \\
\text { Release, \% }\end{array}$ \\
\hline 16,000 & 2165 & 15.3 & $<1.0 \%$ & $<0.2$ \\
\hline 28,850 & 2425 & 16.6 & $<1.0 \%$ & 3.3 \\
\hline 32,000 & 2060 & 13.0 & Nil & 5.2 \\
\hline 38,500 & 2450 & 17.9 & $<1.0 \%$ & 7.6 \\
\hline \multicolumn{5}{|c|}{$80 \mathrm{v} / \mathrm{O} \mathrm{HIP}$ and HP } \\
\hline 16,000 & 2350 & 12.7 & $<1.0 \%$ & $<0.2$ \\
\hline 22,400 & 2595 & 20.0 & Nil & 1.1 \\
\hline 29,000 & 2180 & 11.3 & $<1.0 \%$ & 1.0 \\
\hline 34,100 & 2745 & 16.5 & $1.0 \%$ & 7.9 \\
\hline \multicolumn{5}{|c|}{$80 \mathrm{v} / \mathrm{o}$ Agglomerate } \\
\hline 37,200 & 3035 & 23.8 & $1.5 \%$ & 21.0 \\
\hline
\end{tabular}

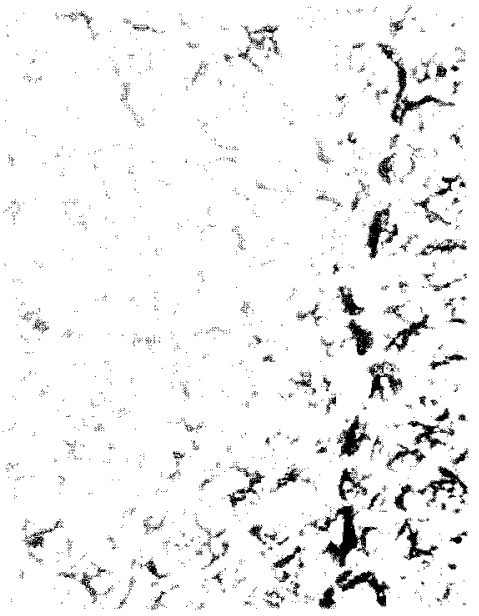

$100 \times$

$83 v / 0 \mathrm{UO}_{2}-\mathrm{Cb}$, Hot Pressed Type $-34,100 \mathrm{Mwd} / \mathrm{T}$

SLIDE 13

SLIDE 14

As seen in Slide 15 , the structure of 73 vol\% $\mathrm{UO}_{2}$ hot pressed fuel indicates very little (if any) cracking. Indications are that lower loadings would probably eliminate this effect.

SLIDE 15

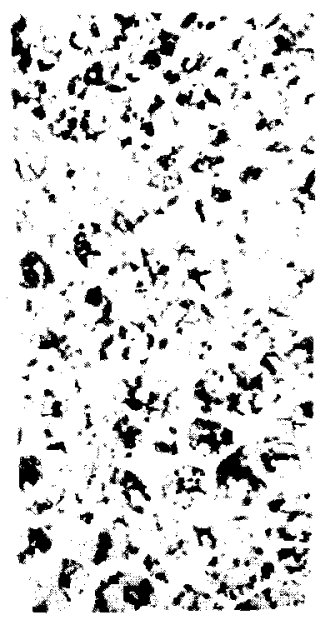


The 80 vol\% UO agglomerate type fuel structure is illustrated in Slide 16. ${ }^{2}$ In contrast with previous structures, we see particles contacting each other. It is not clear, however, whether the performance of this fuel was adversely affected by such contact.

It should be noted that a process was developed to make fairly long metallurgically-bonded pins (16 to 17 in.) by the hot isostatic pressing route.

In another area of fuel fabrication development, I should mention that $\mathrm{Cb}-\mathrm{UO}_{2}$ plate type specimens containing 25 vol\% ceramic phase were prepared. An attempt was made to retain porosity in the fuel particles of the dispersion during the fabrication steps.

What is of greater interest from the FFTF standpoint, perhaps, is the United Nuclear fuel development for the Settied Bed Reactor Concept of Brookhaven National Laboratory during which UNC fabricated 60 vol\% UO,-stainless steel cermet pellets by a compacting and sintering tèchnique. The intended fue 1 particle density was to be in the 85 to $90 \%$ T.D. range, and the matrix density was to be maximized. Fuel particle size was in the 350 to $400 \mu$ range. Evaluation of the pellets indicated an overal1 cermet density of about $85 \% \mathrm{~T} . \mathrm{D}$. with the fuel particle density somewhat higher (perhaps, $88 \%$ T.D.). The microstructure of a typical pellet is shown in slide 17.

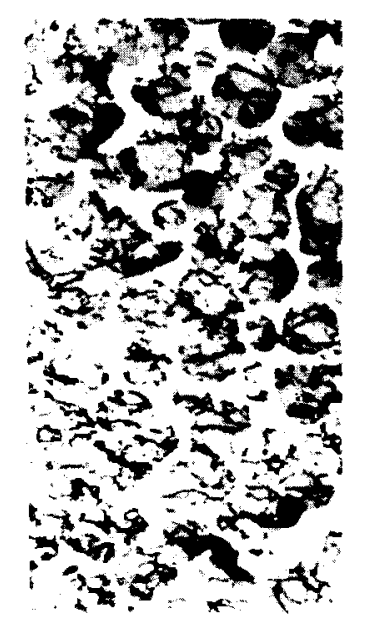

100 .

$80 \times 0$ LO $-\mathrm{Ct}$, Agnlomerate Type $-37.200 \mathrm{Mud}$ T

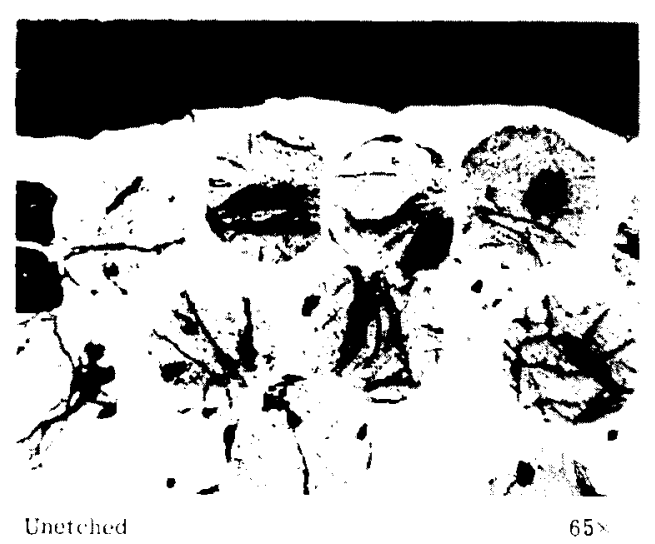

$60 \mathrm{r} 0 \mathrm{LO}_{2}$-Stannless Steel Pellet Cuated with Stanless Steel. Fuel Partirle Size 350400 r: 
Brookhaven irradiated six capsules containing these pellets in the ETR; two were removed after $1 \%$ burnup. Brookhaven no longer has funds to examine the fuel. I understand, however, that these two capsules are to be sent to Battelle-Northwest for the post irradiation examination. It appears, therefore, that there is interest here in the behavior of this particular fuel material.

This completes my remarks on recent fuel and clad developments at United Nuclear. Hal Kazi will now present an analysis showing how a porous carbide pellet fuel concept leads to acceptable characteristics for FFTR driver fuel.

\section{PART II DESIGN CHARACTERISTICS OF CARBIDE FUEL CONCEPT}

$$
\text { A. H. Kazi }
$$

\section{INTRODUCTION}

While no specific design analyses have been performed at UNC on a (UPu)C FFTF driver fuel element, a number of specific optimization studies have been performed on a (UPu)C fuel rod for fast breeder application. The characteristics of this fuel rod are well within the range of FFTF requirements.

The design requirements for a FFTF driver fuel rod include a specific power of 500 to $2000 \mathrm{~W} / \mathrm{g}$ of plutonium, burnun objective of 100,000 to $200,000 \mathrm{MWd} / \mathrm{ton}$, and reactor core outlet sodium temperature capable of operating from 800 to $1200{ }^{\circ} \mathrm{F}$. The flux objective is an integrated fast flux $\geq 1016,75 \%$ of which should be above $100 \mathrm{KeV}$.

Basically, we have looked at a fuel rod design (apart from the overall reactor design) that will use existing physical property and irradiation test data, keep the maximum fuel temperature below $3500^{\circ} \mathrm{F}$, and keep the maximum fuel rating below $30 \mathrm{~kW} / \mathrm{ft}$.

S1ide 1 summarizes the principal conceptual design characteristics of especial of interest to FFTF driver fuel application are the average specific power of $1200 \mathrm{~kW} / \mathrm{kg}$ and the fairly high core conversion ratio of 0.70 . The latter, coupled with the reasonable enrichment of $14.6 \%$ and $100,000 \mathrm{MWd} / \mathrm{ton}$ burnup, makes for attractive economics. I should point out that the present design consists of three core modules of about 1400 liters each. This is, thus, about twice the core volume considered for FFTF. Using similar fuel in a 635-1iter core, results in an increase of enrichment to $18.2 \%$. Coolant temperatures and core composition are compatible with FFTF requirements. Notice the maximum fuel temperature of $3200^{\circ} \mathrm{F}$, which is reasonable for (UPu)C. The flux objective of FFTF 
is met with (UPu)C at a power level slightly higher than a metal core and slightly less than with a mixed oxide core.

\section{SLIDE 1}

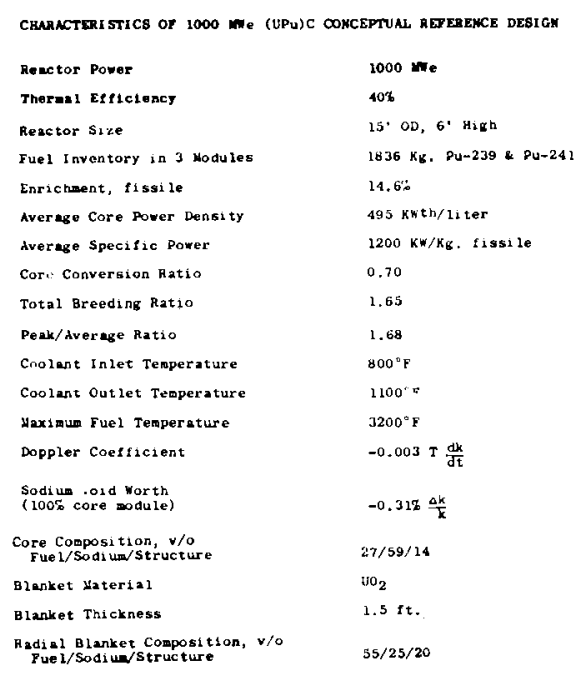

Helium Bond

In the present design, helium is preferred to sodium as a thermal bond material for the following reasons:

- Clad carburization is facilitated by the presence of sodium.

- Fabrication costs are higher with sodium.

- Uncontrolled presence of bubbles in the sodium can lead to excessive fuel temperatures.

- Contact of sodium with fuel at the center of the pellet may cause excessive internal pressures.

Fission gas venting to the coolant is not taken as a nearterm design objective.

Accomodation of Swelling

The (UPu)C fuel rod is designed to allow for currently expected fuel swelling at $100,000 \mathrm{MWd} /$ ton and to keep the maximum fuel temperature below $3500{ }^{\circ} \mathrm{F}$ at a fuel rating of $30 \mathrm{~kW} / \mathrm{ft}$. Two principal methods can be considered to accommodate swelling: in the first, fuel-clad interference is permitted and the cladding is allowed to deform plastically; in the second, lower effective fuel density is assumed to absorb swelling. Both approaches to solve the fuel swelling problem are being investigated in current irradiation tests in EBR-II and FERMI (as discussed by Dr. Fuhrman in the preceding paper). Fuel with high and low "effective" densities will be irradiated in Type 316 SS clad to determine the best compromise between clad strain, fuel temperature, and effective fuel density. Only the results of such experimental data can guide the further design philosophy for ceramic fuel rods for high burnups. 
If the fuel center temperature is to be kept below $3500^{\circ} \mathrm{F}$ at the beginning of 1 ife, a clad strain of $2.8 \%$ must be accomodated at the end of the $100,000 \mathrm{MWd} / \mathrm{ton}$ life for a $95 \%$ density fue 1 .

At the present time, data on the affects of fast flux on the ductility of clad materials are lacking. The alternate approach of lower effective density of fuel to prevent excessive clad strain at end of life was, therefore, considered for the present conceptual design.

Material properties and irradiation effects for the (UPu)C fuel were taken from data recently obtained by United Nuclear Corporation. The specific values of the properties used, together with properties of the cladding, the filler gas, and the sodium coolant, are shown in Slide 2. The gap conductance (hg) across the helium filler gas between the fuel and the clad is calculated, based on the actual gap thickness between pellet and cladding, by taking into account thermal expansion and swelling.

When the fuel and ${ }_{2} c_{0}$ ad are in contact, a maximum value of $\mathrm{hg}=2000 \mathrm{Btu} / \mathrm{hr}-\mathrm{ft} \mathrm{t}^{2} \mathrm{~F}$ is used.

A maximum volume increase of $18 \%$ at a burnup of 100,000 MWd/ton is used and assumed to occur isotropically for fuel of $95 \%$ theroretical density, based on irradiation swelling data recently determined. For fuel of less than $95 \%$ theoretical density, the internal voids are assumed to be effective in reducing the external dimensional increases of the fuel pellet. For a pellet with a density of $p / p T$, the external swelling is calculated as $0.18-(0.95-\rho / \rho T)$.

\begin{tabular}{|c|c|c|c|c|}
\hline & & TERIALS PROPERTIES & & \\
\hline \multirow{5}{*}{ SLIDE } & Material & $(U P u) C$ & $316 \mathrm{SS}$ & $\mathbf{N a}$ \\
\hline & Density, $\mathrm{g} / \mathrm{cm}^{3}$ & (100\% theoretical) & 7.8 & .83 \\
\hline & $\begin{array}{l}\text { Thermal conductivity, } \\
\text { BTU } / \text { hr }_{-} \mathrm{ft}_{-}{ }^{\circ} \mathbf{F}\end{array}$ & 11.6 & 12.0 & 40 \\
\hline & Specific heat, BTU $/ 1 b-{ }^{\circ} F$ & .062 & .134 & .3 \\
\hline & $\begin{array}{l}\text { Coefficient of thermal } \\
\text { expansion, of }{ }_{F}^{-1}\end{array}$ & $6.1 \times 10^{-6}$ & $10.0 \times 10^{-6}$ & \\
\hline
\end{tabular}


The fuel conductivity is assumed to very directly with the fuel density over the range investigated.

The determination of the fuel density and assembled radial gap width for the reference fuel rod design was conducted by means of a parametric survey with maximum fuel temperatures and end-of-life pellet-clad interference calculated as a function of effective density and assembled cold gap thickness. Effective density is defined as the mass of fuel per unit volume inside the fuel rod, which includes the fuel pellet and the radial gap. Effective density is lower than the fuel pellet density by the ratio of (pellet diameter/clad inside diameter).

All calculations were made with the reference fuel design of 0.265 in. diam with a clad thickness of 0.015 in.

The effects of the assembled radial gap and effective density on maximum fuel temperature are shown in slide 3 . The maximum fuel temperature, which occurs at the beginning of Iife, is strongly dependent upon the gap width because of the inverse relationship of gap conductance with gap width.

Since the thermal conductivity of the fuel is quite high, the maximum fuel temperature is not strongly dependent on fuel density, although fuel conductivity is assumed to be directly proportional to fuel density.

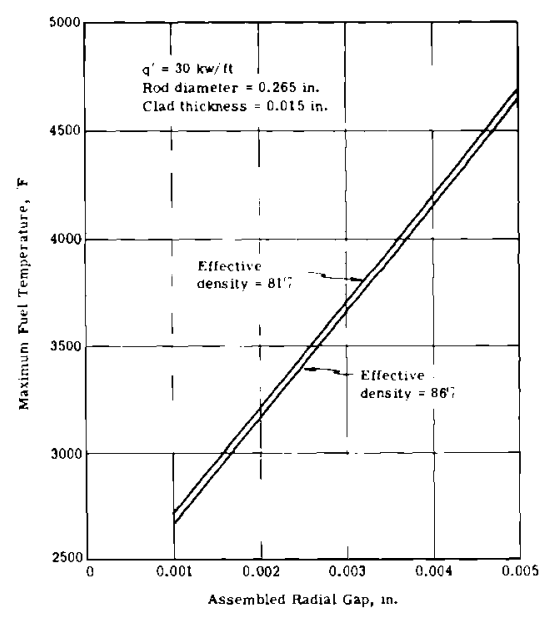

Effect of Assembled Radial Gap on Maximum Fuel

SLIDE 3

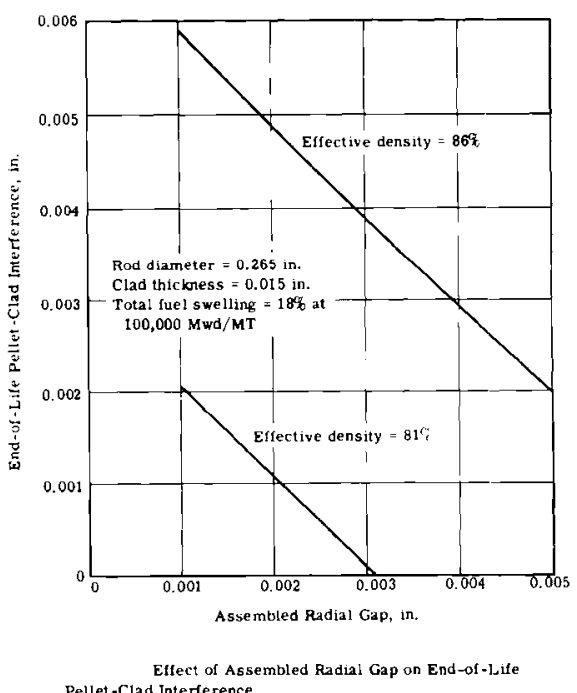

SLIDE 4 


\subsection{3}

Examination of Slide 3 reveals that an assembled gap width less than $\sim 0.0025$ in. is required to keep the maximum fuel temerature below $3500 \mathrm{~F}$. Either of the densities shown would be acceptable.

However, as the assembled radial gap thickness is reduced, there is less room available to accomodate irradiation swelling of the fuel pellet, and the end-of-life pellet-clad interference increases (S1ide 4). This interference is strongly dependent on effective density because of the swelling model discussed above. An assembled radial gap of greater than $0.003 \mathrm{in}$. is required to insure no pellet-clad interference at the end-of1 ife with the lower effective density (81\%). An effective density of $81 \%$ shown on the figures and an assembled radical gap of $0.002 \mathrm{in.}$ are chosen at present to preserve acceptable nuclear characteristics. This corresponds to a pellet density of $83.7 \%$ theoretical.

The selected values of the assembled radial gap width and effective density for the present reference fuel rod represents a compromise among the mutually conflicting requirements of breeding ratio, maximum fuel temperature, and pelletclad interference. In the case of FFTF, the question of breeding ratio is not as significant. The nominal gap thickness of 0.002 in. and the effective density of $81 \%$, chosen for the reference fuel rod, result in a maximum fuel temperature of $3200 \mathrm{~F}$ and an end-of-life cold pellet-clad interference of 0.001 in. The corresponding hot pellet-clad interference at the endof- 1 ife is 0.0005 in.

The characteristics of the reference fuel rod are shown in Slide 5. From an FFTF point of view, perhaps the most interesting thing here is that the characteristics of this fuel rod do not differ very much from designs that have not taken swelling into account, except for the lower fuel density. The penalty of going to a lower fuel density is mainly felt in breeding ratio; hence, fuel cycle costs. This penalty is less severe in the case of FFTF driver fuel application than in large fast breeder design. It should be noted that a similar problem of swelling at high burnup exists with mixed oxide fuel. Lower effective fuel density offers a possible solution here also. However, the $\mathrm{UO}_{2}-\mathrm{PuO}_{2}$ is at an inherent disadvantage because of its lower $100 \%$ dense fuel density.

REFERENCE FUEL ROD CHARACTERISTICS

$\begin{array}{lc}\text { Rod outside diameter, in. } & 0.265 \\ \text { Clad thickness, in. } & 0.015 \\ \text { Rod inside diameter, in. } & 0.235 \\ \text { Radial gap thickness, in. } & 0.002 \\ \text { Fuel pellet diameter, in. } & 0.231 \\ \text { Effective density, } q \text { theoretical } & 80.9 \\ \text { Actual fuel density, } \% \text { theoretical } & 83.7 \\ \text { Active core length, ft. } & 3.0\end{array}$


Composition - Configuration Survey Calculations

Slide 6 shows examples of composition-configuration survey calculations performed in connection with the present (UPu)C design. The data is self-explanatory. The seven-coremodule configuration involves 635-liter core regions which are very similar to the FFTF.

A calculation was performed to assess the effect of increasing the stainless steel clad thickness from 15 to 30 mils. The stainless steel volume fractions corresponding to these two clad thicknesses are 6 vol\% and 13 vol\%, respectively. Other structure accounts for 8 vol\%. It is seen from Side 6 (Case 7 ) that the resultant penalty is small: an increase of $50 \mathrm{~kg}$ in fissile inventory, and a reduction in breeding ratio of $\sim 0.02$.

SLIDE 6

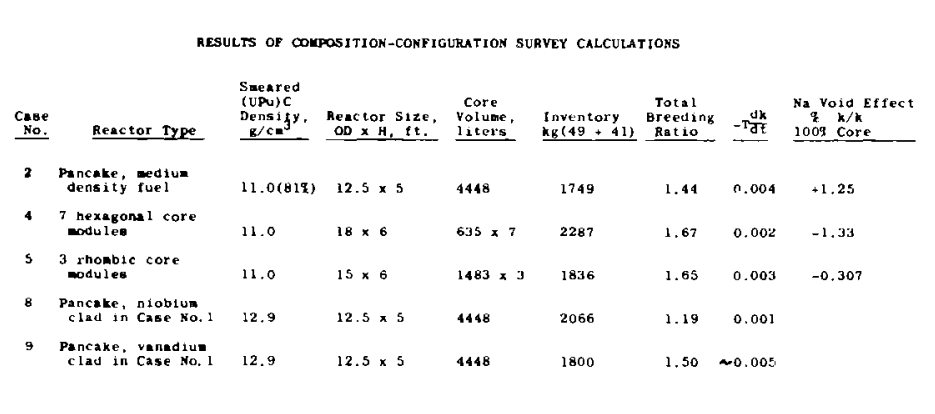

Effect of Noibium and Vanadium Clad

Due to the present lack of adequate fast flux irradiation data, it is not clear at this time whether 316 SS will prove to be a satisfactory clad material. A number of calculations have been performed substituting niobium or vanadium clad for stainless steel. In agreement with earlier studies reported by Argonne, it is found that (from a physics point of view) vanadium is acceptable while niobium is not. There is an increase in Doppler coefficient with the use of vanadium. Niobium results in poor physics characteristics all around compared to stainless steel. One problem in doing such comparison calculations on a generalized basis is that they only indicate trend. In a specific design, the differences, say, between stainless steel and niobium clad, may well be great. Niobium is of interest because of compatability with (UPu)C, and greater strength and resistance to embrittlement than stainless steel. In the analyses reported to date, self-shielding of the niobium resonances, and possible resonance overlap, has not been taken into account. Since the niobium resonances overlap the important $U^{238}$ resonances, it is expected that proper treatment of the niobium resonances could appreciably alter the predicted effect of niobium on fast breeder performance. Therefore, calculations have been performed to assess the importance of the niobium resonances to fast reactor performance. The data are summarized in slide 7 . 
Three calculational models are considered for niobium:

$\mathrm{Nb}-1$. The $\mathrm{Nb} \mathrm{RI}$ is 11.3 barns as given by the LASL HansenRoach cross section 1ibrary.

$\mathrm{Nb}-2$. The $\mathrm{Nb} \mathrm{RI}$ is 7.5 barns consistent with the data of Jackson.

$\mathrm{Nb}-3$. The $\mathrm{Nb}$ resonance absorption peaks are neglected, and a smooth $\sigma_{a}$ of 0.4 barns is used in the range 0.1 to $17 \mathrm{keV}$.

From S1ide 7 , complete self-shielding of the Nb resonances versus a RI of 11.3 barns is seen to reduce fissile inventory by $5 \%$, increase core conversion ratio by $10 \%$, increase tota 1 breeding ratio be $3.5 \%$, increase the magnitude of the negative Doppler coefficient by $76 \%$, and make the reactivity effect of $100 \%$ sodium loss from the core $62 \%$ less positive. It is seen, at least from the present example, that niobium cannot yield as satisfactory a set of physics parameters as does stainless steel; however, the actual performance of a niobiumcontaining fast-spectrum core is likely to be better than currently predicted. This could be of importance to the FFTF design, not only in terms of cladding, but of structure such as irradiation loops.

SLIDE 7

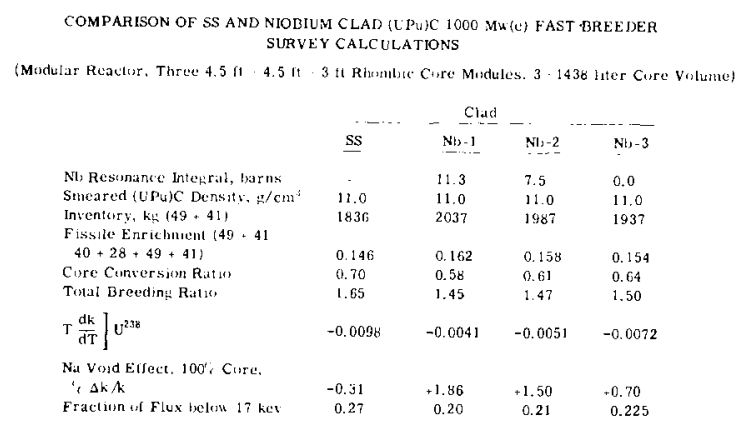

CONCLUSION

In the preceding discussion, I have tried to summarize some of the considerations which have gone into the conceptual design of a (UPu)C fuel rod for fast breeder application. There appears to be no reason why one could not design a satisfactory (UPu)C fuel rod to serve as FFTF driver fuel. This is especially true if one considers the fast flux irradiation data and fabrication facilities which will be available by 1969 , and if one retreats somewhat in the beginning from the design goals that appear necessary for economic breeder design, such as 100,000 MWd/Mton burnup. What is required evidently is a detailed study which evaluates the use of (UPu)C in FFTF. This is something we have, as yet, not had a chance to do. 
CARBIDE, CERMET \& CLAD DEVELOPMENT AT UNITED NUCLEAR CORPORATION N. Fuhrman and A. H. Kazi

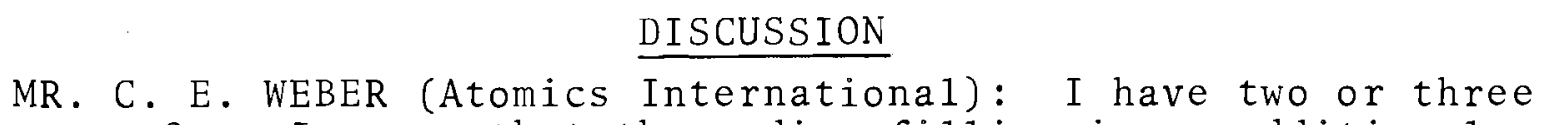
comments. One, I agree that the sodium filling is an additional cost. Two, sodium logging and high density carbides is not the system for our problems. Three, I don't think we need to go into it, but it would be interesting to discuss your analytical model wherein you have small gas bubbles of sodium. We find that with sodium wetting inside the surface, it should have small gas bubbles. In fact, if it does, it would be quite excellent, and this would not be a problem. I don't quarrel with your general presentation but it would be interesting sometime later to see your theory or your experimental data with reference to sodium logging as a problem or small bubbles as a problem.

MR. KAZI: I would make only one comment on this, and that is that I feel that the kind of analytical models especially in pellets and tubes during a radiation is always subject to many approximations. That is exactly one of the reasons why we have irradiation tests. 
16.1

\title{
CLADDING AND DISPERSION FUEL DEVELOPMENT AT ORNL
}

\author{
J. E. Cunningham and J. W. Ullman \\ Oak Ridge National Laboratory \\ Oak Ridge, Tennessee
}

\section{INTRODUCTION}

The presentation from Oak Ridge National Laboratory will be given in three parts. The subject and speaker for each part is as follows:

1) Dispersion Fuel Technology by $J$. E. Cunningham, 2) Fuel Reprocessing by J. W. Ullmann, and 3) Fuel Cycle Economics and Radiation Effects in Cladding Materials by D. A. Douglas.

UO2-STAINLESS STEEL CERMET FUEL EXPERIENCE AT ORNL

In reviewing the technology of the $\mathrm{UO}_{2}$-stainless steel dispersion fuel, I shall confine my remarks mainly to irradiation experience accumulated over the past $15 \mathrm{yr}$ and attempt to point up shortcomings of this fuel type for application in FFTF.

As most of you know, the stainless steel-uranium dioxide dispersion fuel technology for the Army Water Reactor Program was developed in the early 1950's. The product that evolved for startup of the SM-1 reactor at Fort Belvoir, Virginia, was a brazed assembly of thin plates. A representative cross section of such a composite fuel plate is shown in Figure 1 (LS-4278, Y-25961) for reference. It consists mainly of a 20-mil-thick fuel-bearing section with 5-mil cladding of type 304L stainless steel on each face to give an overall plate thickness of $30 \mathrm{mils}$. The fuel is incorporated into the fuel-bearing section in the form of $26 \mathrm{wt} \%$ or $19 \mathrm{vol} \% \mathrm{UO}_{2}$. The matrix material is type $302 \mathrm{~B}$ stainless steel powder, which contains $2.5 \mathrm{wt} \%$ silicon.

These composite fuel plates were prepared by the conventional roll-bonding technique. Most of the fragmentation and stringering of the oxide that is apparent in Figure 1 occurred during cold rolling (25\% reduction) to final thickness. These composite fuel plates of 1950 design performed quite well in the 10-MW thermal reactor at Fort Belvoir; in fact, they accumulated a total power output of over $60,000 \mathrm{MWd}$ (10\% greater than the expected design lifetime of $15 \mathrm{MW}$-yr) before discharge at burnout. The operating conditions were not very severe. At a power level of $10 \mathrm{MW}$, for instance, the heat flux was only 55, 000 Btu $\mathrm{hr}^{-1} \mathrm{ft}-2$. The average surface temperature was $450^{\circ} \mathrm{F}$, while the maximum centerline temperature of the fuel was $650^{\circ} \mathrm{F}$. The elements were subjected to over 3000 thermal cycles during startup and shutdown of the reactor, because the reactor was used primarily as an operatortraining facility. The initial core loading accumulated a total reactivity 
lifetime of 16. $4 \mathrm{MW}$-yr before discharge. The average fuel burnup in terms of depletion of ${ }^{235} \mathrm{U}$ atoms was $26 \%$ or $1 \times 10^{21}$ fissions/cc.

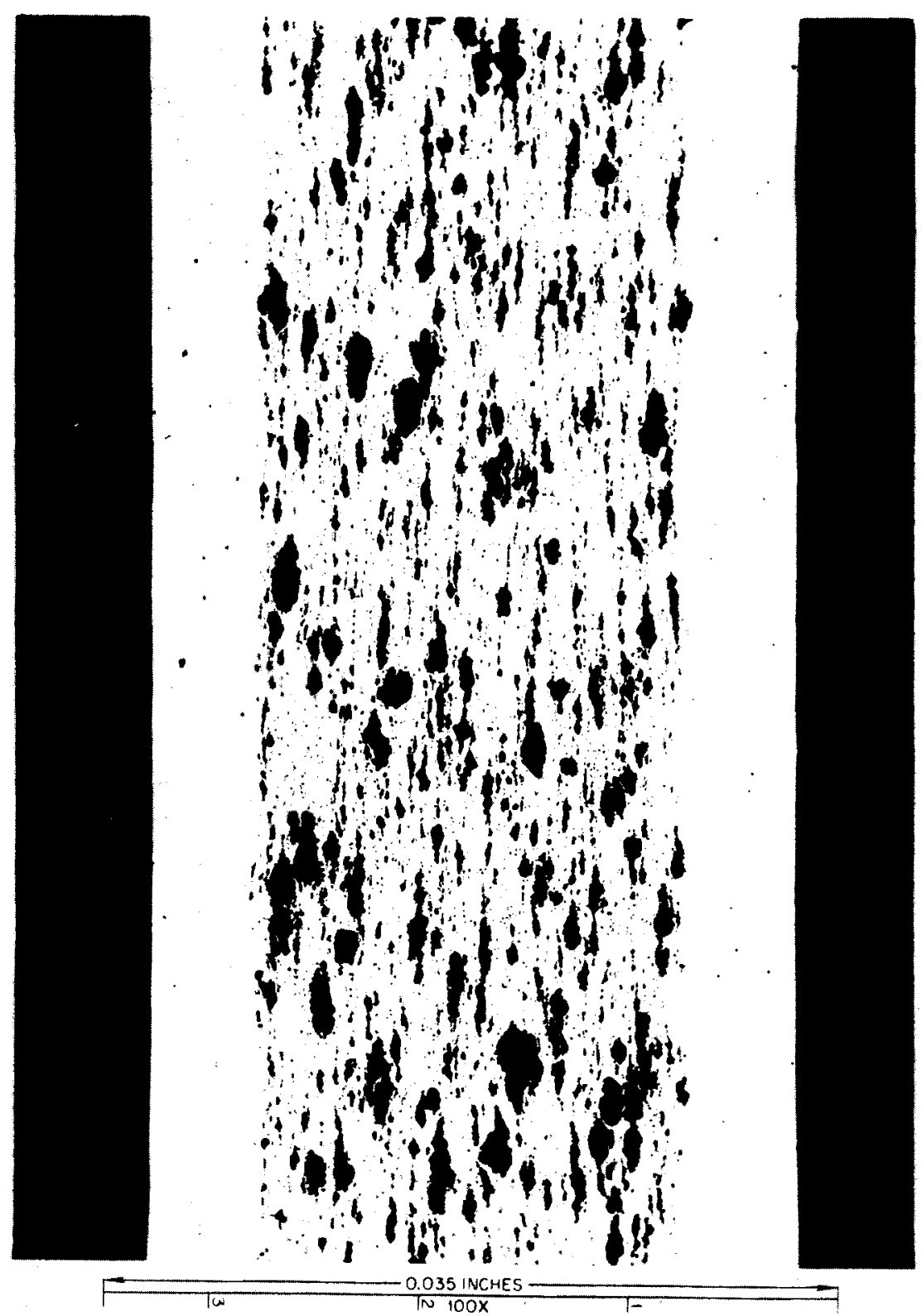

Figure 1. A Composite Fuel Plate with Cladding of Type 304L SS 
The effect of irradiation on dimensional stability and structural integrity was checked by hot-cell examination of stationary fuel elements that had been removed after various levels of exposure in the reactor. The first fuel component was removed after 10.5 MW-yr life, the second at the end of the core life or 16.5 MW-yr, and the third after 19.5 MW-yr (high exposure was achieved by reinsertion in Core II). In-cell examination revealed the following:

- Progressively greater tendency toward plate rippling with increasing exposure. Maximum ripple amplitudes of 23, 33, and 45 mils were observed, respectively, for $10.5,16.5$, and 19.5 MW-yr exposures.

- A maximum plate increase in thickness of 3 mils or $10 \%$ occurred at the axial peak burnup position in the fuel element with the greatest neutron exposure. The burnup at this location was $62 \%$ depletion of $235 \mathrm{U}$ atoms or $2.2 \times 10^{21}$ fissions/cc.

- The frequency of intergranular cracks in the cladding varied directly with burnup. In a few instances, cracking was so severe that whole grains were removed at the cladding surface. Some transgranular cracking was noted in the grain structure of the low-cobalt low-carbon stainless steel cladding in the high-burnup fuel element.

No evidence was found of matrix cracking nor sign of gross deterioration in these dispersion fuel plates. Yet, swelling of the order of $10 \%$ did occur under the modest operating conditions prevailing in the SM-1 reactor, and such behavior must be factored into the design of the FFTF if the dispersion is used for the driver fuel.

In 1962 , we examined 34 dispersion fuel specimens contained in $6 \mathrm{NaK}$-filled capsules that had been irradiated to relatively high burnup in the ETR. These irradiation tests were initiated by BMI to evaluate various parameters in connection with their program to develop an improved dispersion fuel for service in the Army SM-2 reactor. The cermet specimens were clad with type $347 \mathrm{SS}$ and contained either $\mathrm{UO}_{2}$ or UN dispersed in type 347 SS along with small but varying amounts of burnable poison, in the form of $\mathrm{B}_{4} \mathrm{C}, \mathrm{NbB}_{2}$, and $\mathrm{ZrB} \mathrm{B}_{2}$. In addition, the behavior of fused and hydrothermal oxides was compared. The highly enriched fuel was incorporated in the fuel-bearing section to a nominal composition of 26 and $38 \mathrm{wt} \% \mathrm{UO}_{2}$ or $34 \mathrm{wt} \%$ UN.

Of the 34 specimens examined, 11 showed failure. These specimens varied in degree of failure from surface blistering to complete disintegration, as shown in Figure 2 (LS-13896, R-9043, R-9045). The 6 specimens with the high fuel loading operated at a surface temperature of $950^{\circ} \mathrm{F}$, and all 6 failed. Four of these were badly swollen and the other two compietely disintegrated. Of the remaining 5 failed specimens, which contained 24 or 25 wt $\% \mathrm{UO}_{2}, 3$ were severely damaged and 1 developed a large blister over the fuel region. 
16. 4

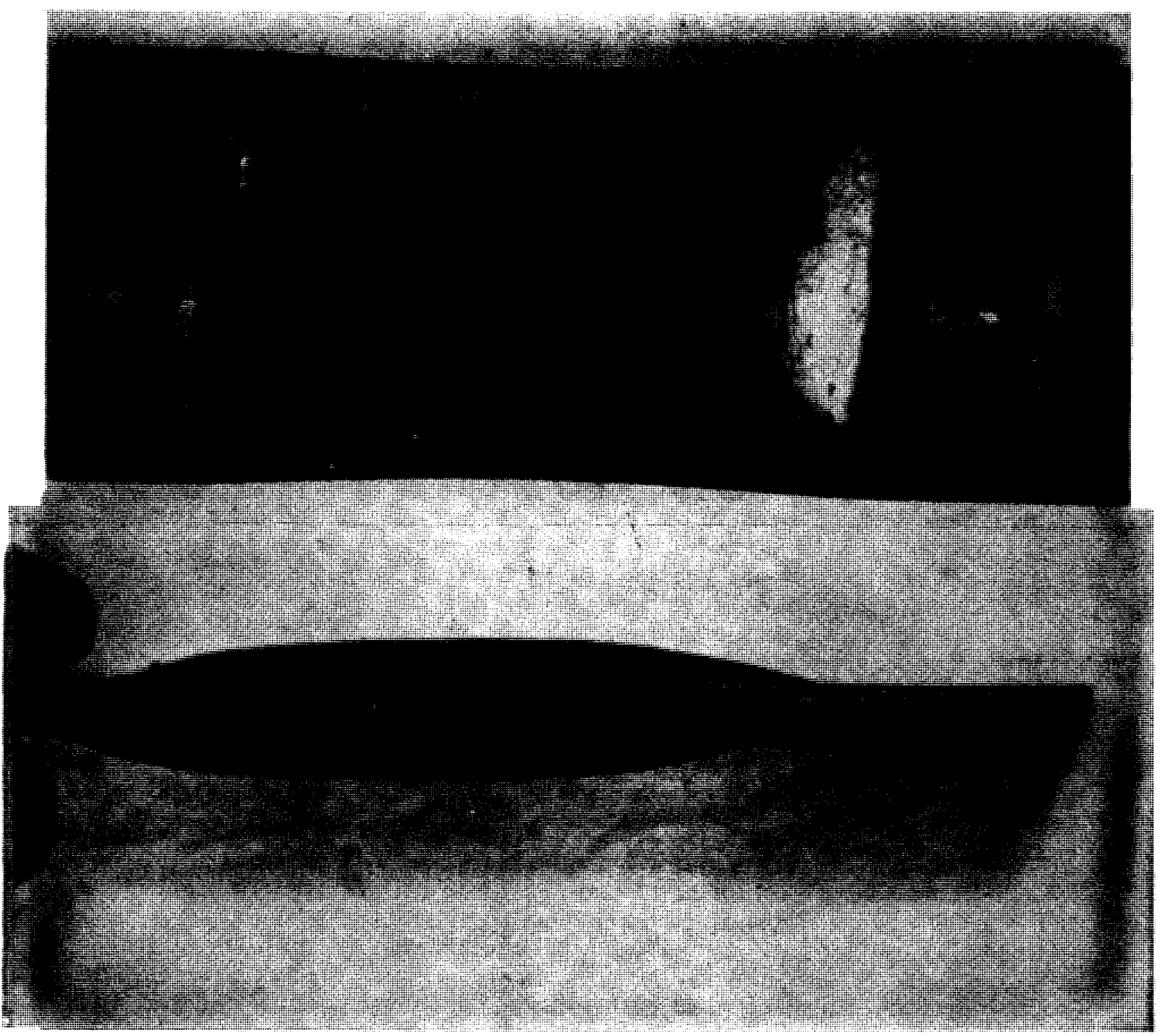

Figure 2. Typical Swelling of a Specimen with a High Fuel Loading

The micrograph in Figure 3 (LS-8870, R-11235) shows the degree of swelling noted at the edge of a specimen loaded with $26 \mathrm{wt} \% \mathrm{UO}_{2}$ and exposed to a fuel depletion of $74 \%$ of the ${ }^{235} \mathrm{U}$ atoms $\left(2.9 \times 10^{21}\right.$ fissions/cc) at $1000^{\circ} \mathrm{F}$. Matrix cracking was observed toward the center of this specimen. A typical picture of severe cracking is shown in Figure 4 (LS-13897, R-11229). This particular specimen contained $34 \mathrm{wt} \% \mathrm{UN}$, operated at a peak temperature of $950^{\circ} \mathrm{F}$, and achieved 63 at. \% burnup. 
16.5

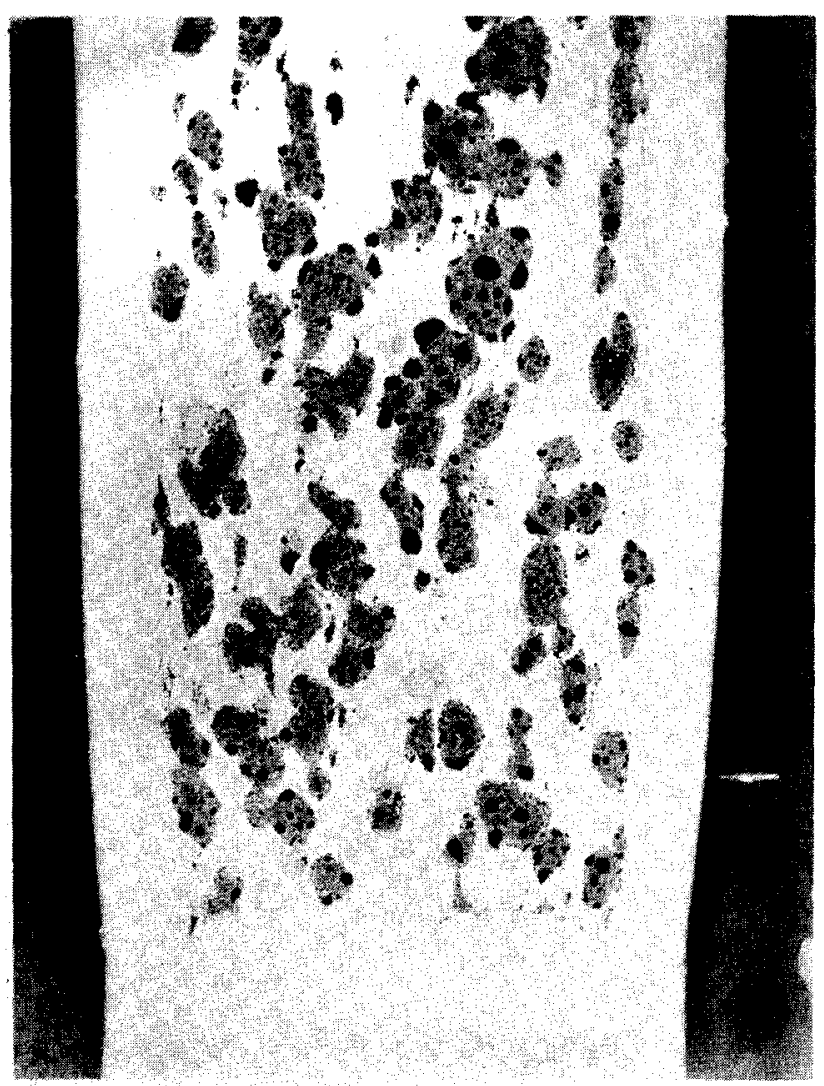

Figure 3. Swelling Associated with $74 \%$ U ${ }^{235}$ Depletion

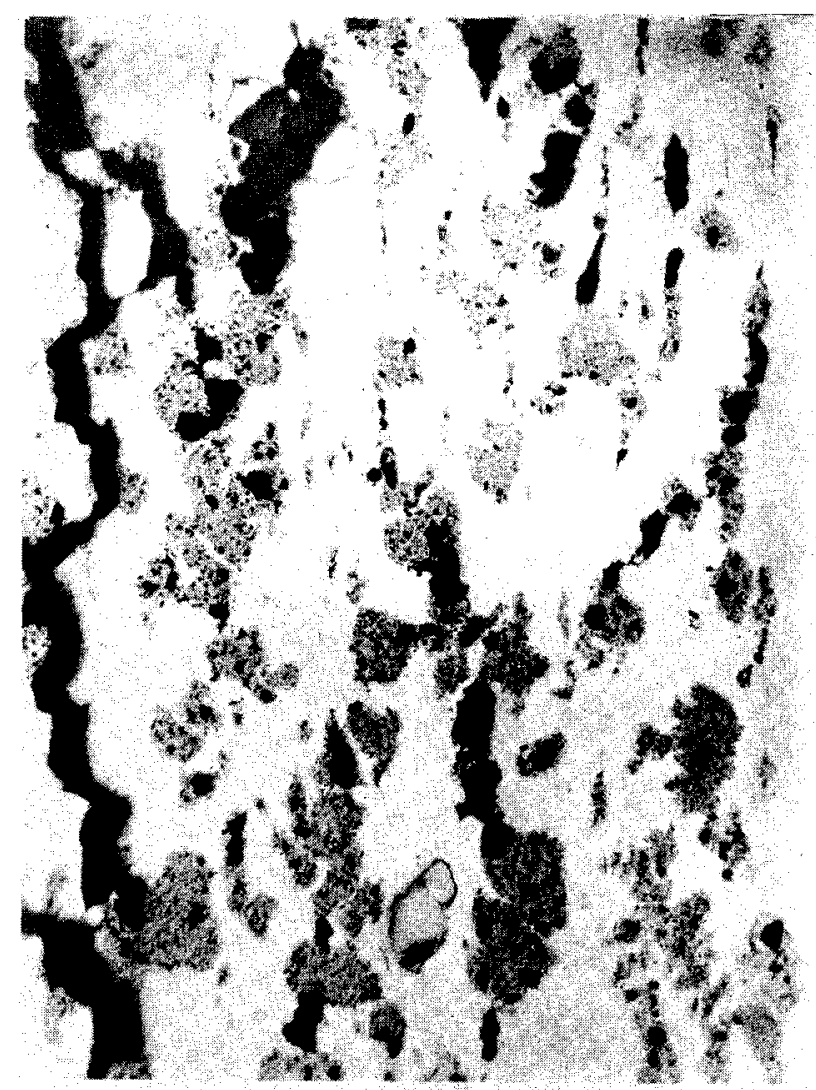

Figure 4. Specimen at $63 \% \mathrm{U}^{235}$ Depletion

Metallographic examination of the 23 undamaged specimens revealed no evidence of matrix cracking or blistering. These specimens all contained 24.2 or $26 \mathrm{wt} \% \mathrm{UO}_{2}$, operated in the temperature region of 420 to $600{ }^{\circ} \mathrm{F}$, and achieved burnups of 52 to $67 \%$ of the $235 \mathrm{U}$ atoms (1. 9 to $2.6 \times 10^{21}$ fissions/cc). No significant difference was noted between specimens prepared with spherical (fused) or irregular-shaped (hydrothermal) $\mathrm{UO}_{2}$ particles not between specimens containing burnable poison of different chemical form. The microstructure illustrated in Figure 5 (LS-9601, R-11306) is typical for these specimens and is quite similar in appearance to that found in the high burnup region of the SM-1 fuel elements.

From mid-1959 to 1962 , we worked on a cooperative program with APDA to develop an improved $\mathrm{UO}_{2}-\mathrm{SS}$ fuel dispersion for use in Core B of the Fermi Reactor. The scope of the program embodied 1) characterization of spheroidal $\mathrm{UO}_{2}$ particles, 2) fabrication of composite plates, 3) feasibility studies on nondestructive testing, 4) establishment of suitable assembly procedures, 5) measurement of fuel material mechanical properties, and 6) irradiation testing. 
16. 6

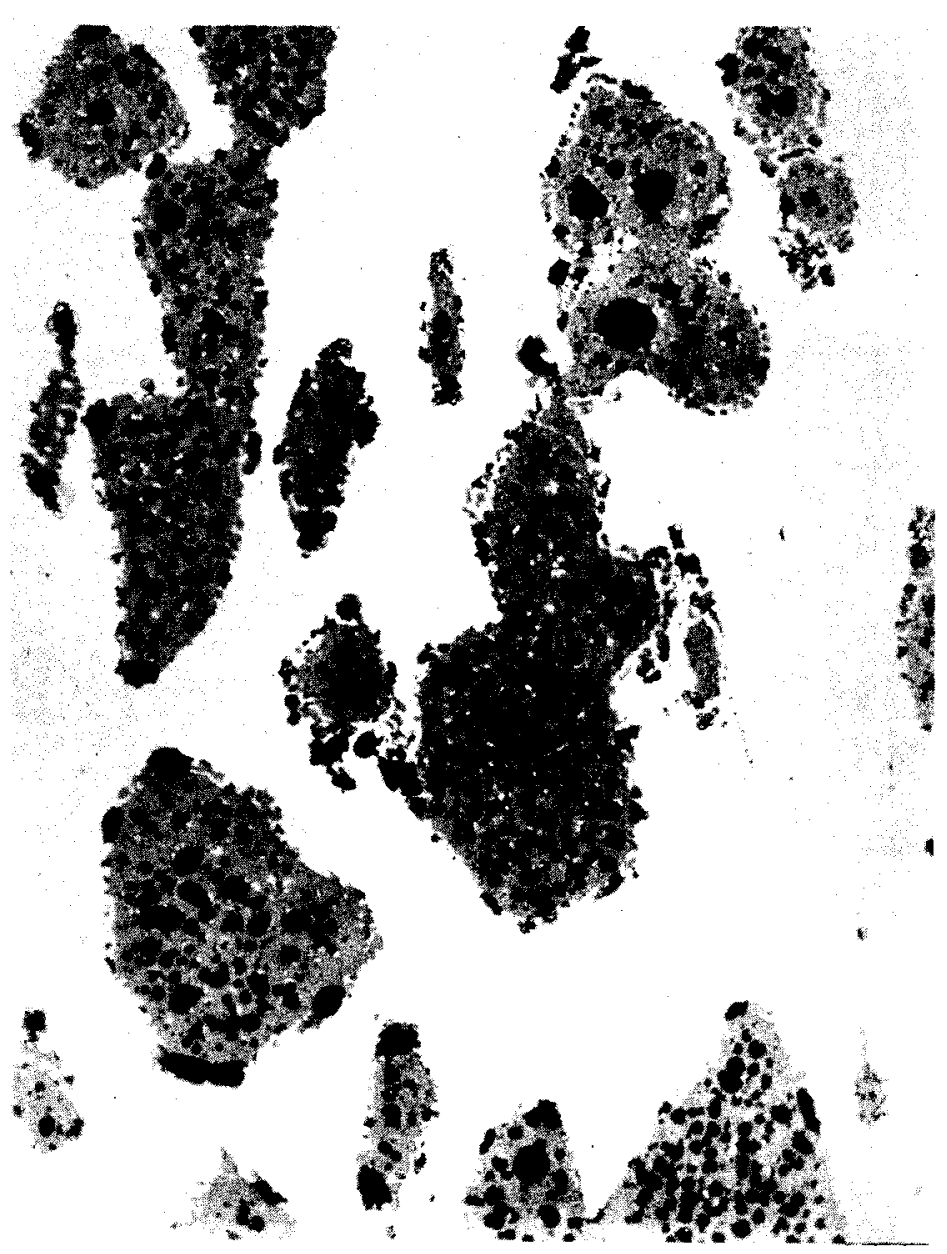

Figure 5. Microstructure of Undamaged Specimen

Early in the development program, the concentration of fuel was 1 imited to 27.5 vol\% (34. $5 \mathrm{wt} \%$ ) $\mathrm{UO}_{2}$ in order to enhance radiation performance. Spheroidal fuel particles in the 105 to $149-\mu$ size range were chosen, because we felt that this shape would minimize stress concentration and reduce radiation damage in the fuel matrix. Type 347 SS was selected as the structural material because of its superior strength compared to other conventional grades of austenitic steels in the 800 to $1000^{\circ} \mathrm{F}$ temperature range.

The effect of cold deformation on the integrity and shape of the oxide in the finished plate was investigated and the results are summarized in Figure 6 (LS-7260, Photo 52670). Cracking of the oxide was noted at all reductions investigated and stringering became pronounced at 20 to $30 \%$ reduction in thickness; hence, we decided to hot roll the fuel plates to finish size to minimize oxide fragmentation and stringering. During plate 
16.7

development, we also noticed a wide variation in the behavior during hot working of spheroidal $\mathrm{UO}_{2}$ particles procured from different vendors, as illustrated in Figure 7 (LS-7021, Y-38004, Y-38989). Our characterization studies showed that we needed some means to separate good from bad quality starting powder; hence, a new technique based on solid embedment and density was developed to serve this purpose. A specification was also written that allowed us to procure good quality spheroidal particles with a high degree of confidence.

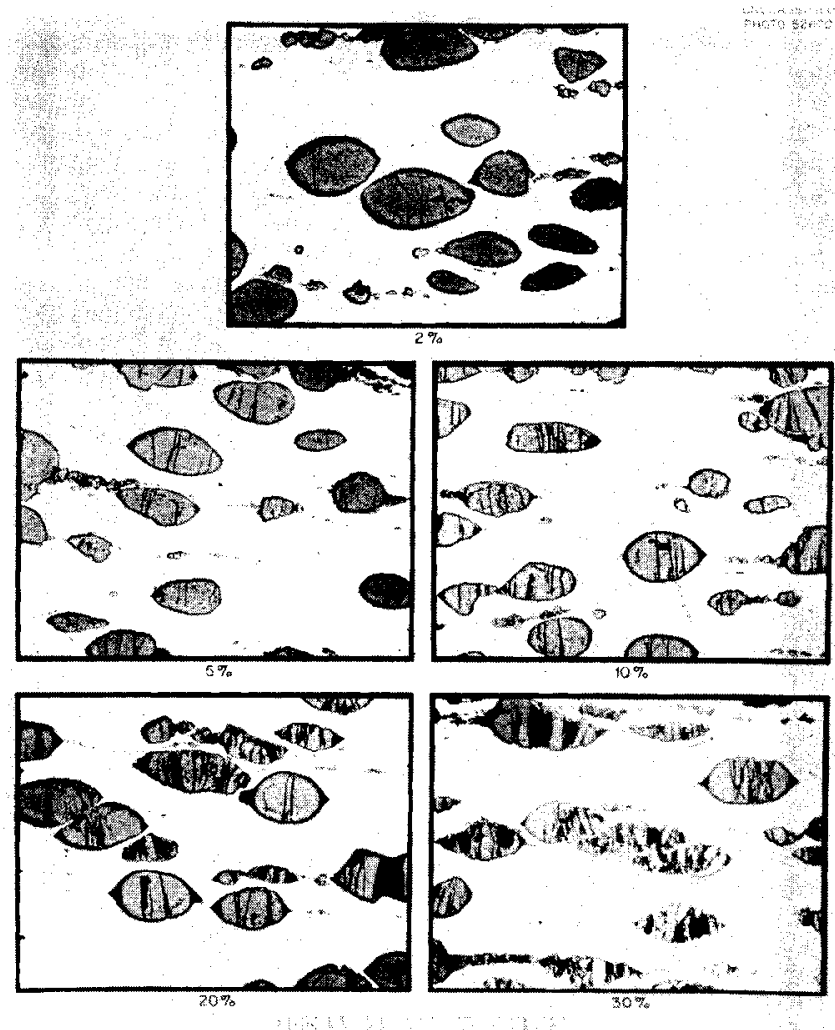

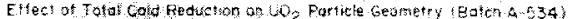

Figure 6. Cross Sectional View of Rolled Plates

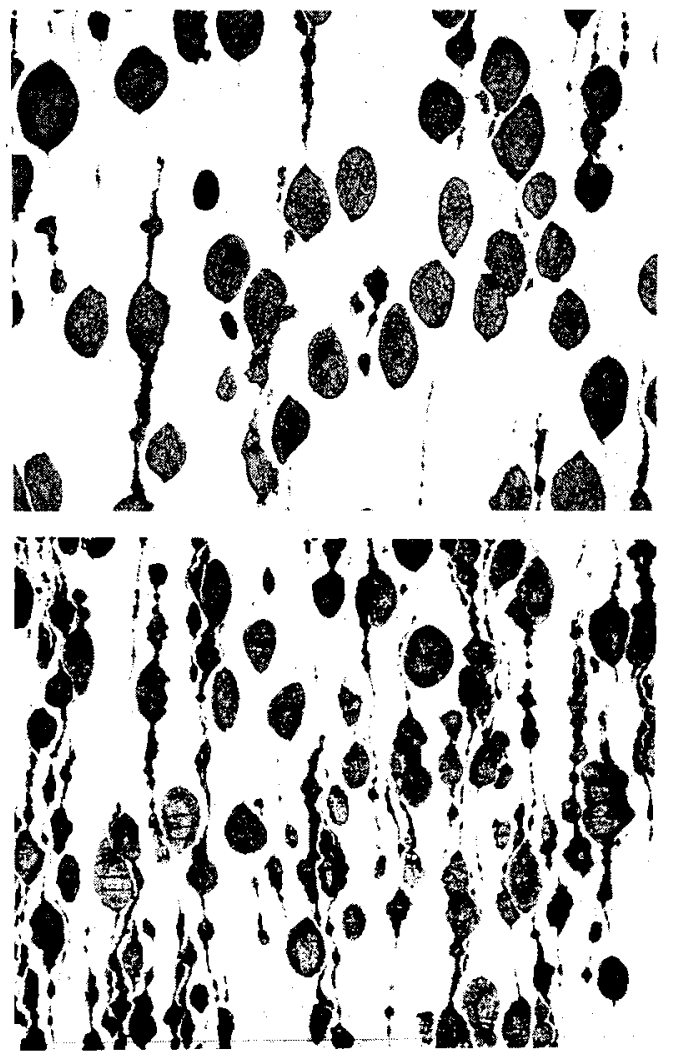

Figure 7. Difference in Spherical $\mathrm{UO}_{2}$ After Hot Working

Three instrumented capsules were irradiated in the MTR to assess the in-reactor performance of the Fermi Core B fuel plate. Each capsule contained two miniature plates, $2 \times 0.5 \times 0.116$ in. The fuel-bearing section contained $33 \mathrm{wt} \%$ spheroidal $\mathrm{UO}_{2}$ of $105-$ to $149-\mu$ particle size, homogeneously dispersed in type 347 SS as shown in Figure 8 (LS-6986, Y-35413). Two thermocouples were attached to each specimen. 
16.8

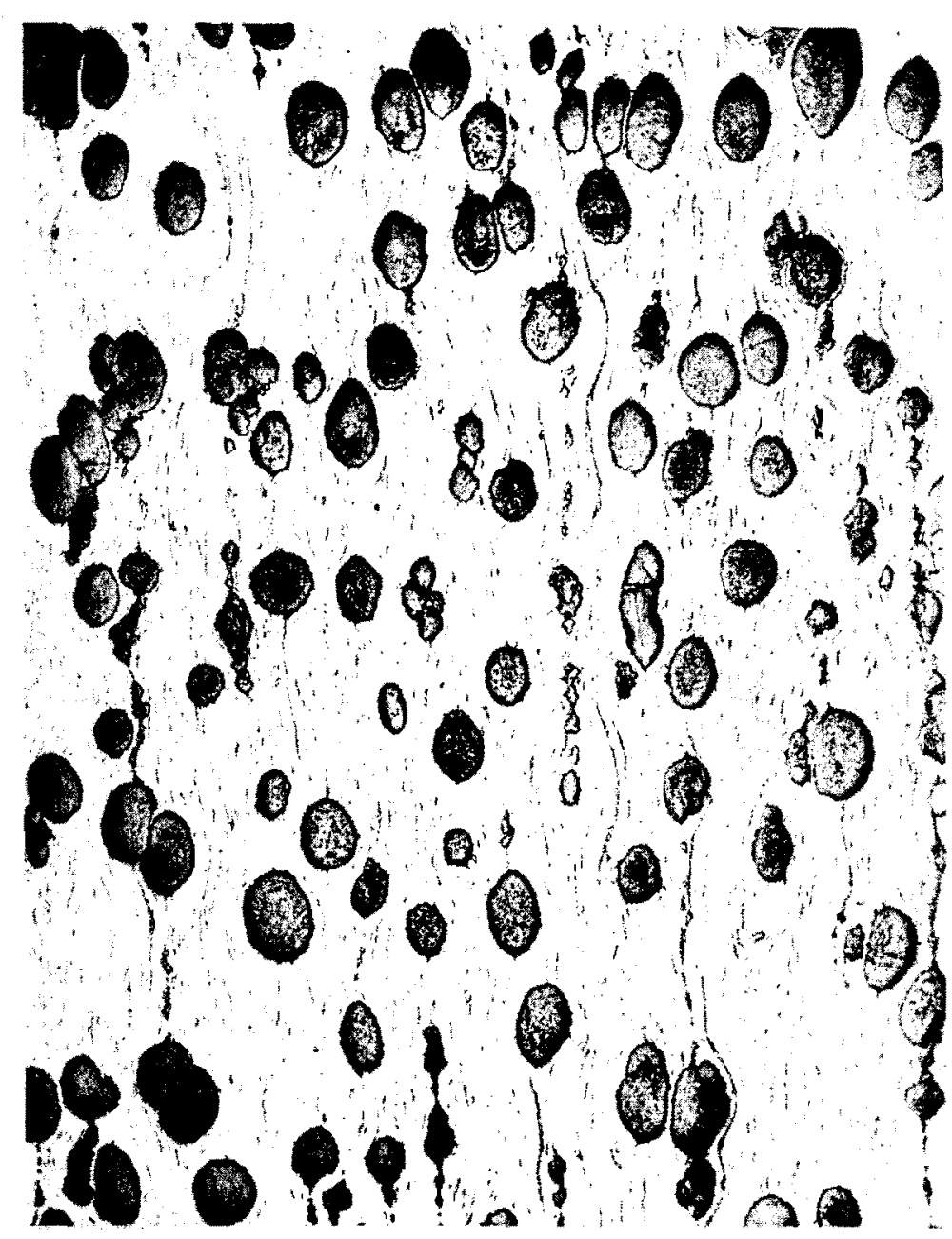

Figure 8. Cross Section of MTR Test Plates

The nominal irradiation condition selected for in-reactor testing were three different burnup levels- $-10,20$, and $25 \%$ of the $235 \mathrm{U}$ atoms-and a surface temperature of 925 to $950^{\circ} \mathrm{F}$. These conditions were chosen on the basis that Core B would be used to operate the Fermi reactor at a maximum core thermal power of $270 \mathrm{MW}$. At this level, the calculated throughput burnup would be $20.7 \%$ of the initial $235 \mathrm{U}$ atoms; hence, our burnup targets bracketed this level. Nominal fuel temperatures at the reactor center would be $750^{\circ} \mathrm{F}$ at the plate surface and $970^{\circ} \mathrm{F}$ at the fuel centerline. Hot spot and hot channel factors increased the centerline temperature to a maximum of about $1040{ }^{\circ} \mathrm{F}$.

The first capsule was discharged after an estimated burnup of $9.5 \%$ of the $235 \mathrm{U}$ atoms. Subsequent measurement revealed, however, that the actual fission burnup was only $6.3 \%$ (average of two samples). Consequently, an experience factor of $6.3 / 9.5$ or 0.66 was applied in fixing the discharge dates for the remaining capsules. On this basis, 
the other two capsules were dissharged after an estimated fuel depletion of 16 and $26 \%$ of the ${ }^{235} \mathrm{U}$ atoms.

Specimens 1 and 10, which were contained in cpasule ORNL-MTR64-4 were unaffected by the irradiation. The burnup of $235 \mathrm{U}$ atoms was $6.3 \%$. The general appearance of specimen 10 is shown in Figure 9 (LS-8898, R-4549). All four thermocouples remained operational throughout the test and indicated that the surface temperature on these specimens ranged from 800 to $950^{\circ} \mathrm{F}$. The appearance of the microstructure was as expected.

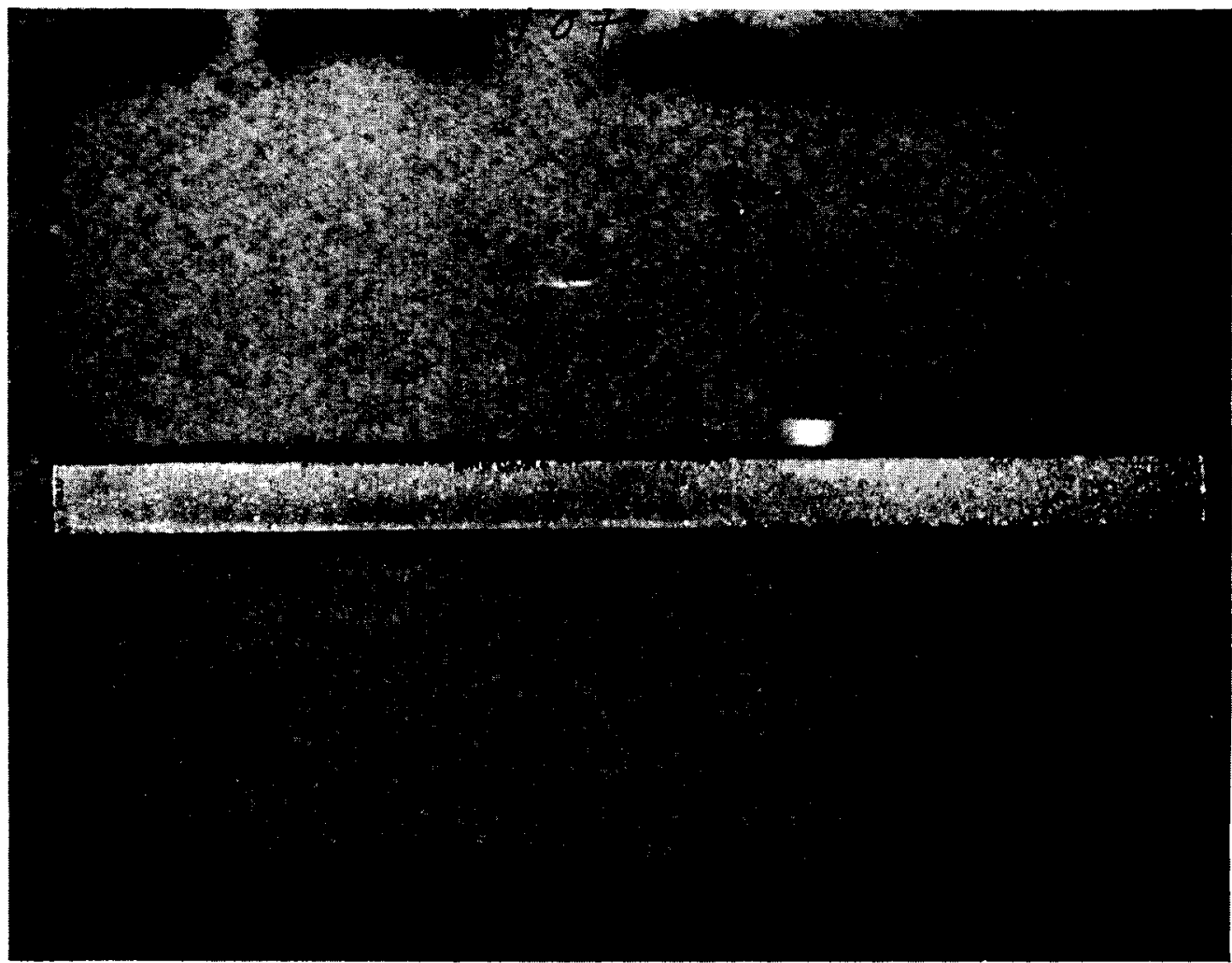

Figure 9. Survived MTR Test Specimen

Specimen 6 from ORNL-MTR-46-2 showed severe damage, as illustrated in Figure 10 (LS-8901, R-11567). Two full-penetration holes were noted in the test plate. A major crack also developed at one edge of the specimen, as shown in Figure 11 (LS-13898, R-11569). Marked swelling was noted in specimen 5 located at the bottom of the capsule. The appearance of specimen 5 in transverse section is depicted in Figure 12 (LS-13899, R-13091). Gross swelling $(\Delta \ell=-13 \%)$ and matrix cracking are apparent. In this specimen the surface temperature ranged between 850 and $1150^{\circ} \mathrm{F}$ throughout the life of test and the measured burnup was $29 \%$ of the $235 \mathrm{U}$ atoms. 


\subsection{0}

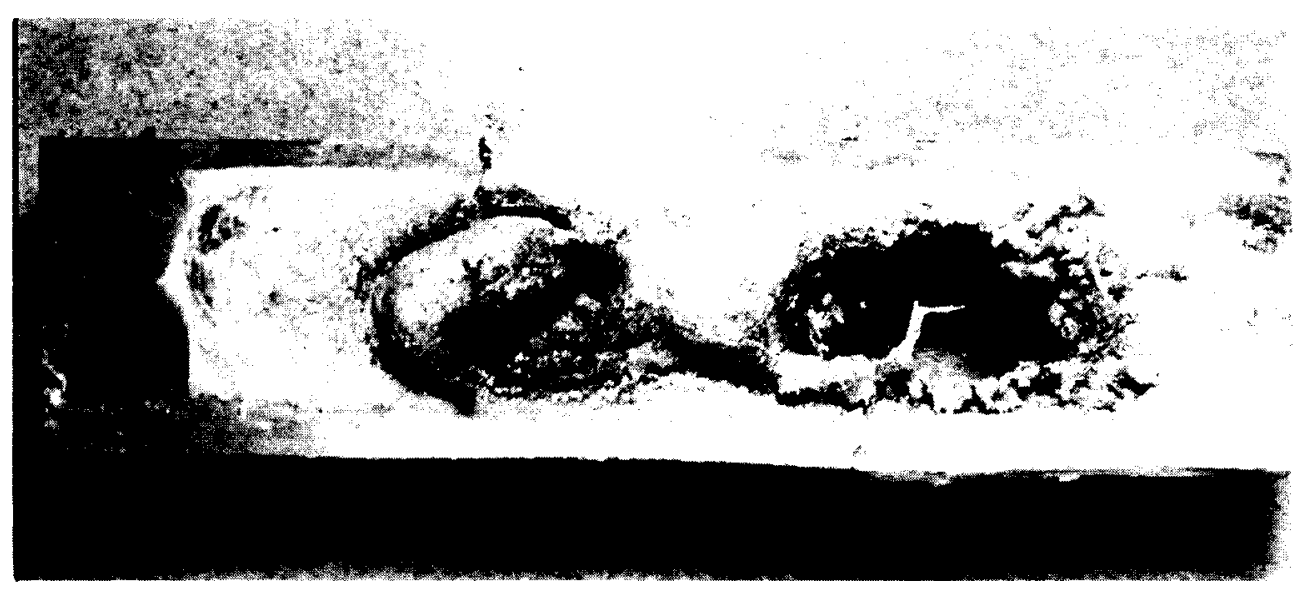

Figure 10. Damaged Test Specimen

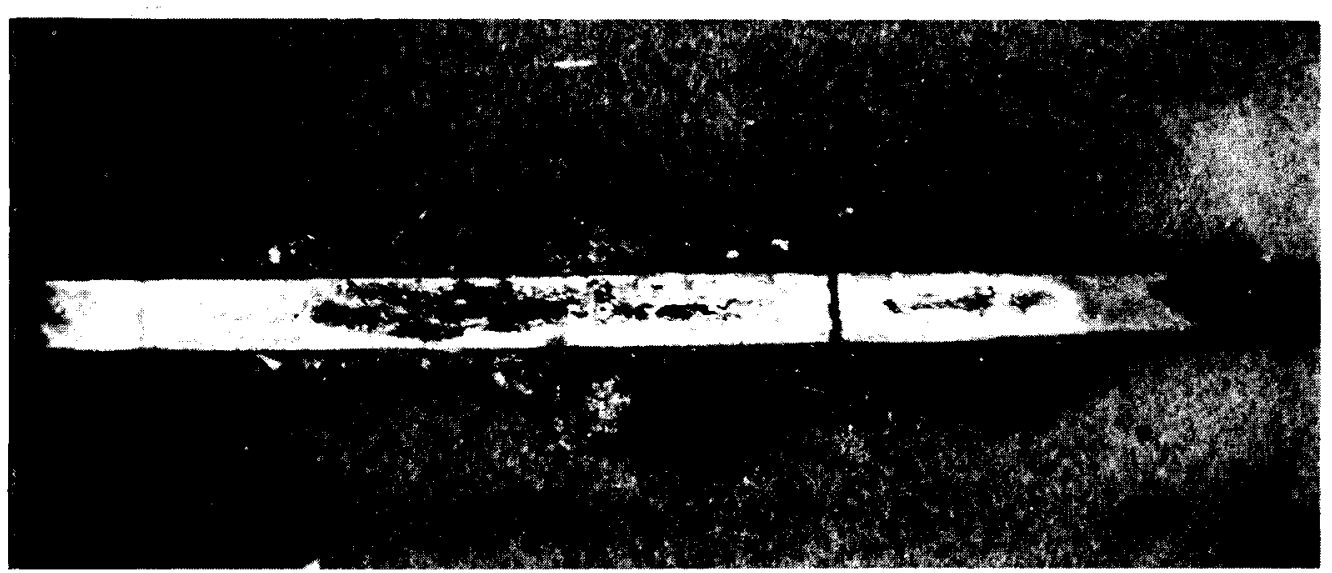

Figure 11. Companion to Previous Test Specimen

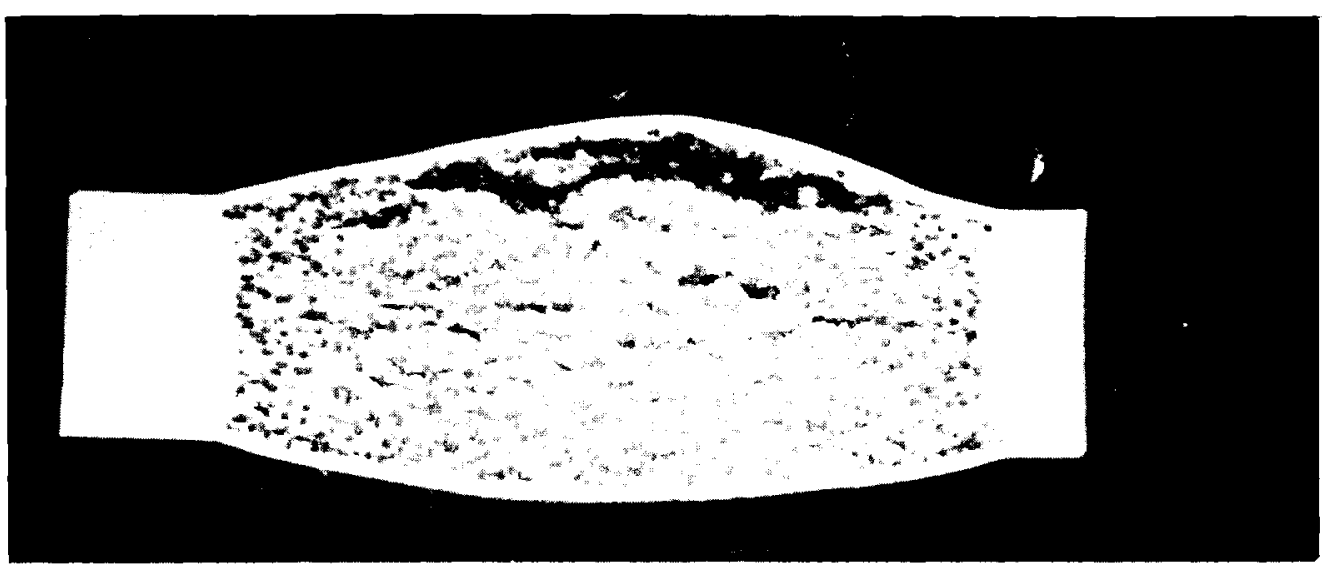

Figure 12. Fuel Plate Swelling 
Specimens 8 and 9 in the remaining capsule showed obvious swelling after a burnup of $33 \%$ of the $235 \mathrm{U}$ atoms. This behavior is illustrated in Figure 13 (LS-13903, R-14325) which shows specimen 9 in transverse section. Specimen 9 increased $6 \%$ in volume. A longitudinal section of specimen 8 , which increased $27 \%$ in thickness is shown in Figure 14 (LS-13901, R-14261). Temperature ranged from 950 to $1050^{\circ} \mathrm{F}$ during test; the average temperature was $1000^{\circ} \mathrm{F}$.

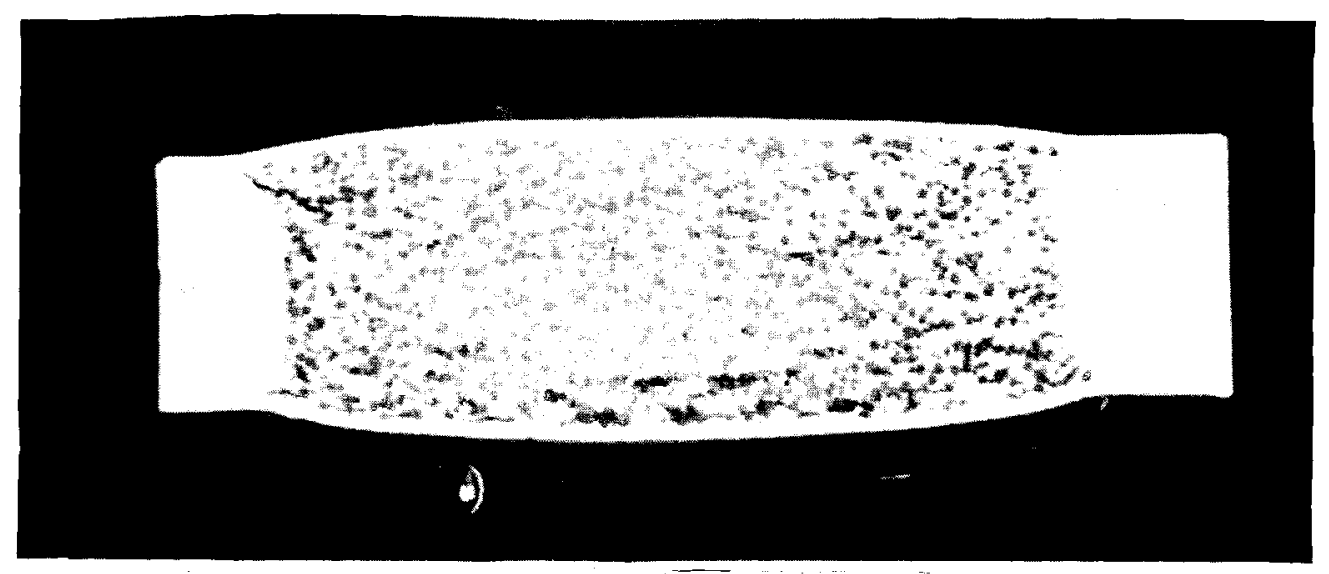

Figure 13. Fuel Plate Swelling After 33\% Burnup (Transverse)

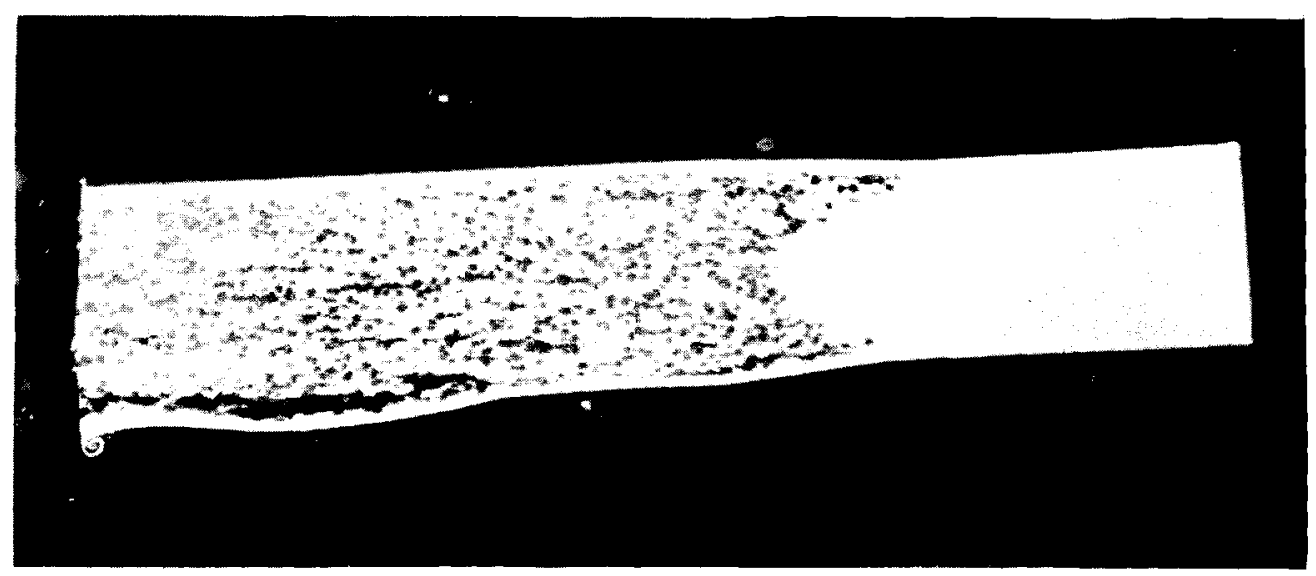

Figure 14. Fuel Plate Swelling After 33\% Burnup (Longitudinal)

These irradiation test results indicate that the temperatureburnup limitation of the improved $\mathrm{SS}-\mathrm{UO}_{2}$ cermet fuel for Fermi Core B was definitely exceeded. Moreover, it should be noted that the specimen size was relatively small, so one would expect better 
performance due to the restraint offered by the cladding and frame material. In addition, control of particle shape and distribution of fuel appeared to offer only marginal improvement in performance.

During the development of the Fermi Core B fuel element, several advances were made in the development of nondestructive testing techniques to ensure compliance with specifications and asmanufactured product reliability. Among the developments were:

- An ultransonic technique that is capable of detecting corecladding interface nonbond areas $1 / 8$ in. diam or larger,

- An eddy-current technique capable of measuring the fuel plate thickness to within an accuracy of $\pm 1 \mathrm{mil}$,

- A radiographic technique to detect $\mathrm{UO}_{2}$ concentration inhomogeneities in excess of the specified $\pm 5 \%$ tolerance,

- A spacing measuring device, based on recently improved eddy-current theory, that measures coolant-channel gaps between plates to $\pm 0.5 \mathrm{mil}$, and

- Methods for characterization of $\mathrm{UO}_{2}$ powder so that particles of the desired shape and integrity can be procured from the vendors with a high degree of confidence that the material is of high quality.

An analysis of pertinent irradiation data on the performance of $\mathrm{UO}_{2}-\mathrm{SS}$ dispersions has been compiled at ORNL. These data are plotted in Figure 15 (ORNL-LR-DWG-75068R3). It should be noted, however, that the temperature points plotted in this figure are based on estimated values since only limited data are available on actual surface temperature measurements during irradiation. The curve indicates that to achieve a burnup of $2 \times 10^{21}$ fissions/cc the maximum surface temperature of the fuel should be less than $800^{\circ} \mathrm{F}$. At a surface temperature of $1200^{\circ} \mathrm{F}$, the maximum burnup that can be achieved before uncontrolled swelling occurs is about $0.8 \times 10^{21}$ fissions/cc.

Ideally, a dispersion fuel should consist of components that are in equilibrium with one another as well as with their environment at all times. Chemical stability of the components, for instance, must be maintained in fabrication and during the lifetime of the fuel in the reactor. Plutonia and urania exhibit notable differences with regard to phase stability. Uranium dioxide is the oxide of lowest oxide content in the uranium-oxygen system whereas $\mathrm{PuO}_{2}$ is the highest oxide in the plutonium-oxygen system. Silicon, a tramp constituent in SS, will reduce $\mathrm{PuO}_{2}$.

\section{SUMMARY AND CONCLUSION}

Finally, I would like to state that in my considered opinion, the $\mathrm{PuO}_{2}$-SS dispersion fuel is a questionable fuel concept for FFTF operation at $1200^{\circ} \mathrm{F}$. At best its development to provide adequate service will be an expensive and uncertain proposition. Radiation-induced swelling 
of $\mathrm{PuO}_{2}$ and severe thermal stress under condition of high power density are likely to lead to premature failure of the potentially embrittled SS matrix and cladding. It is true that certain measures can be taken to improve performance at $1200^{\circ} \mathrm{F}$, such as built-in void space to accommodate fission gas and reduce swelling. Unfortunately, no one has come up with a practical way of achieving this objective despite the fact that its effect on performance has been suggested for some time. Other parameters that potentially influence or upgrade performance are: 1) higher strength matrix and cladding material, 2) lower concentration of the fissile phase, and 3) lower temperature. Yet when all these factors are considered, the $\mathrm{PuO}_{2}-\mathrm{SS}$ cermet fuel concept appears marginal for application in FFTF at temperatures of approximately $1200^{\circ} \mathrm{F}$.

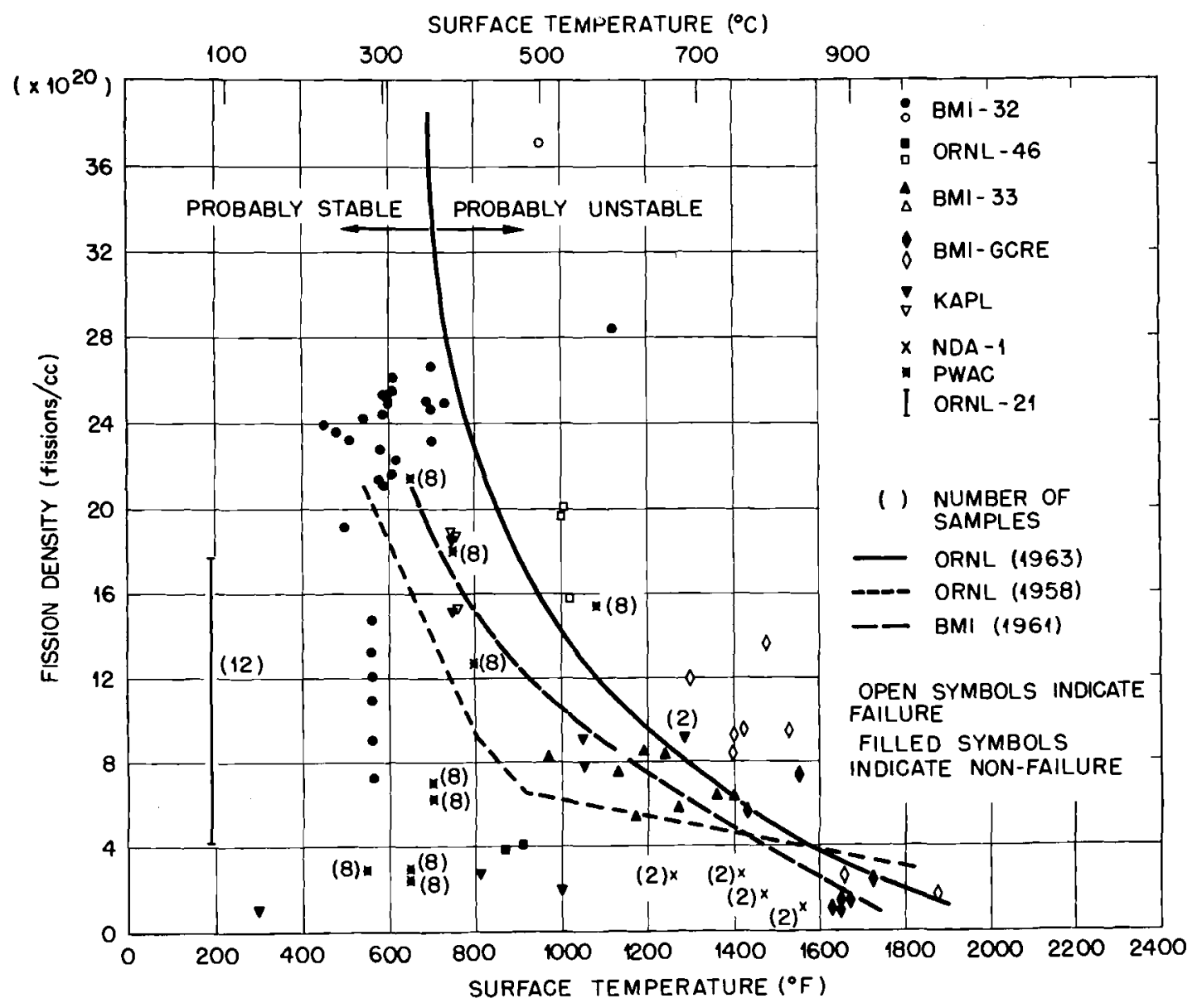

Figure 15. Irradiation Stability of $\mathrm{UO}_{2}$-Stainless Steel Dispersions 

CLADDING AND DISPERSION FUEL DEVELOPMENT AT ORNL

D. A. Douglas, Jr.

Oak Ridge Nationa1 Laboratory

Oak Ridge, Tennessee

\section{METALLURGICAL AND CERAMIC ASPECTS}

My purpose today is to touch 1 ightly on two aspects of the problem under discussion: one has to do with our analysis of the expected performance of the fuel element concepts as proposed in the BNWL Report CC-175, and the other has to do with our estimates of the cost of fabricating such fuel elements.

Although we completed a detailed analysis of each fuel element proposed, I will discuss performance of only one of these concepts; because, as a result of the formal and informal discussions at this meeting, it becomes quite evident that the one about which the most controversy rages is the cermet, and the consensus appears to be that the bulk oxide will meet performance requirements. Our analysis concurs with this view. There is another reason for considering the cermet, in that it gives me a chance to talk about irradiation damage of structural metals; certainly, this would be more of a problem in cermet fuel than in the clad bulk oxide concept.

I will begin by considering what we know about cermets and their performance. Since it is one of the oldest forms of fuel elements, there are years of actual operating experience. A comprehensive analyses of data from experimental and operating experience has produced several analytical mode1s. Jim Weir, at out Laboratory, and investigators at other sites have published mathematical treatments that predict fairly well the performance for any given configuration and various volume percent fuel loadings. The validity of these models depends on certain assumptions. In order to handle the analysis, one of these assumptions is that there is an ideal dispersion of spherical particles. As we know from discussions at this meeting, this is a perfectly achievable sort of circumstance. I am glad that peterson (and later Cunningham) showed the curve that has been generated over the years to predict the stable and unstable conditions for the cermet fuel. From these data, one notes that at $1000^{\circ} \mathrm{F}$, a 1 imiting curve is reached at about $12 \times 10^{20} \mathrm{fis}$ sions/cm 3 ; whereas, at $1200^{\circ} \mathrm{F}$, this 1 imit is reached about $10 \times 10^{20}$ fission/ $/ \mathrm{cm}^{3}$. Now let's ask ourselves a question 


\section{2}

"Since the data are based on thermal irradiations, how well are we able to apply this information to the fast test reactors?"

First, one must realize that there is a great deal more gas produced in a fast flux situation. In a stainless matrix fue 1 , for example the one that was projected in the CC-175 report, one assumes an exposure of somewhere around 200 full power days. This means that something like $3 \times 10^{20}$ atoms $/ \mathrm{cm}^{3}$ of gas will be generated. Of this total amount, $40 \%$ is hydrogen, some $5 \%$ is helium, and $55 \%$ is fission gas. These values correspond to about 300 to $350 \mathrm{ppm}$ of helium and approximately $7000 \mathrm{ppm}$ of hydrogen in the cermet. What effect will these copious quantities of gas have on the performance of the cermet? We don't see why the fission gas should behave any differently in this case. We know how to predict what the behavior will be in a thermal reactor. If the temperature of the ceramic is low enough, the majority of the gas will stay in the oxide particle. At higher temperatures the gas will diffuse to the interfaces between the oxide and the metal. In both cases the result is amenable to analysis. The very large generation of hydrogen is a phenomenon not encountered in our thermal reactor experience. Based on known diffusion rates of hydrogen in stainless steel, we calculate that the gas will leave the metal matrix about as fast as it is bred. We know of no deleterious reactions between hydrogen and stainless under the proposed conditions so I don't think the presence of hydrogen constitutes a problem. I would like to qualify this conclusion in that this applies only to stainless steel. Obviously, if the metal matrix is changed, one would have to re-examine the situation.

Now let us consider the problem of helium. Under thermal irradiation we know that the helium is born principally through an $(n, \alpha)$ reaction with boron -10 . It is probable that most of the boron is concentrated in the grain boundaries and that the presence of helium in the grain boundary leads to a reduced strain for fracture. Our laboratory, as well as others, has been working to try to find some way to alleviate this problem for a thermal reactor. In the fast reactor this situation is a little bit different; you can breed helium from all of the elements, with nitrogen and nickel being the two most prominent offenders. Therefore, we believe the gas will be distributed more generally throughout the structure. The problem will then be to prevent it from concentrating in one location, particularly the grain boundaries.

Since helium appears to be the culprit that affects the hightemperature ductility of metals, let's look at what is available to us in the way of data and decide how serious a problem we have to tackle.

The relationship between helium content and ductility as determined by irradiations in thermal reactors is shown on Slide 1 . The ductility of this stainless steel decreases with increasing helium content, the largest effect being noted at the highest deformation temperature. With respect to the importance of temperature, the 
amount of helium required to cause an initial decrease in ductility is lower as one increases the deformation temperature.

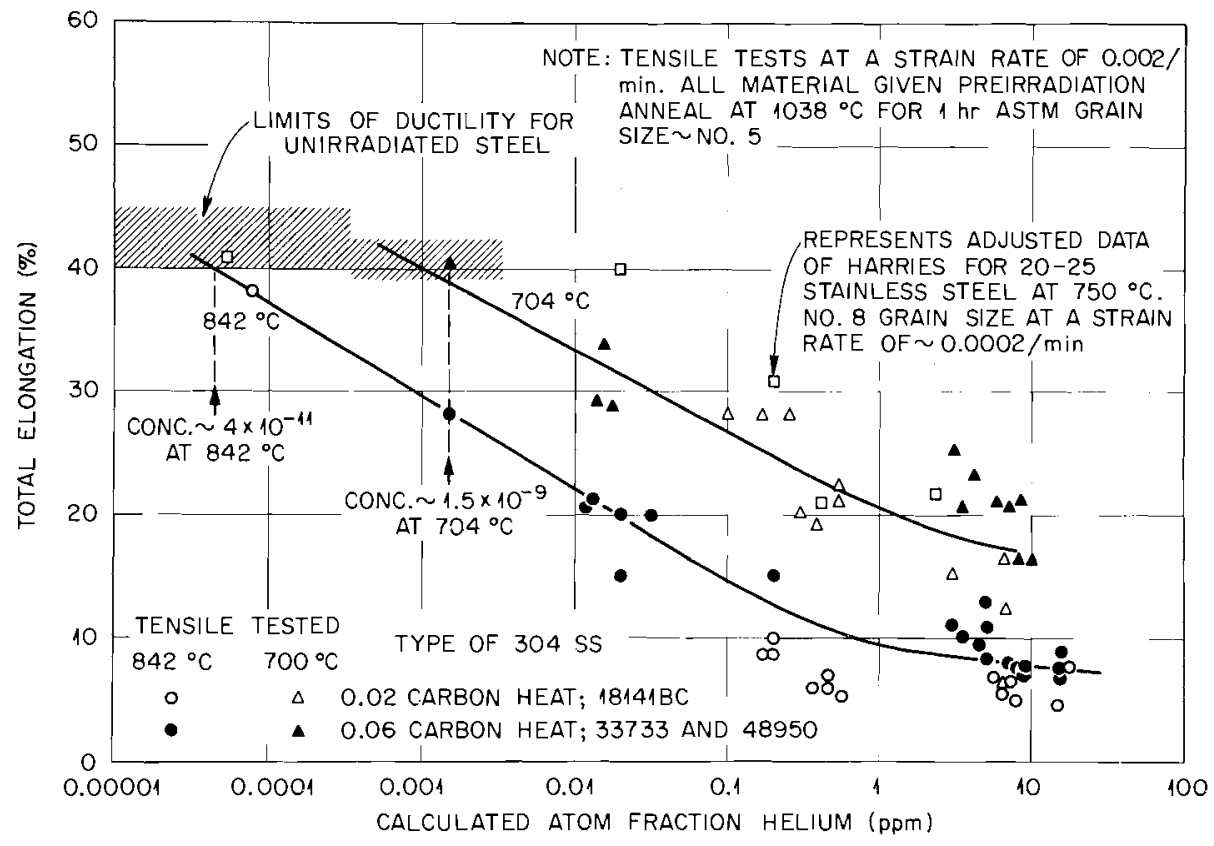

$\underline{\text { SLIDE } 1}$ Ductility of Irradiated Type 304 Stainless Stee 1

The embrittlement in a fast reactor can be approximated from these data, with certain assumptions about the helium distribution for which no data presently exists. A fast reactor operating at a plant factor of $80 \%$ for one year at a neutron flux of 1016 neutrons $/ \mathrm{cm}^{2}-\mathrm{sec}$ will produce slightly more than $100 \mathrm{ppm}$ helium. Thus, if one projected the curves linearly the tensile ductility would be 1 ess than $10 \%$ at $700^{\circ} \mathrm{C}$ with perhaps no ductility at all at $842^{\circ} \mathrm{C}$. Under creep conditions at stresses lower than the yield stress, the ductility values are lower than those found in short-time tensile tests. The data also suggest the possibility that there is a change in the slope of the curve at concentrations above $1 \mathrm{ppm}$ and thus the strain for fracture at $100 \mathrm{ppm}$ may not be as low as the above pessimistic assumption would predict.

I have suggested that the difference in distribution of helium under fast flux exposure might produce different results than presented on Slide 1 . Very recently the influence of fast reactor irradiation on the mechanical properties of austenitic type $316 \mathrm{~L}$ and a precipitation hardened 17-4 PH stainless steel were obtained through the combined efforts of Oak Ridge National Laboratory and Los Alamos Scientific Laboratory. The data for jrradiations in LAMPRE to a total neutron dose of $2 \times 10^{21}$ neutrons $/ \mathrm{cm}^{2}$ are given on Slide 2 . The numbers in brackets represent test data for the unirradiated control samples. No significant changes are observed until a test temperature of $700^{\circ} \mathrm{C}$ is reached. Both type 
316L and 17-4 PH suffer marked reductions in the total elongation at fracture. At higher temperatures the type $316 \mathrm{~L}$ continues to lose in both uniform strain and total elongation. The behavior of the type $316 \mathrm{~L}$ stainless steel is in general agreement with the prediction from the thermal-reactor-irradiation data for type 304 stainless steel given in the previous figure. Although the ductility of the type $316 \mathrm{~L}$ at 700 and $800^{\circ} \mathrm{C}$ is certainly adequate for most applications, the dose level of $2 \times 10^{21}$ neutrons/cm ${ }^{2}$ represents only two days of operation at a neutron flux of 1016 neutrons $/ \mathrm{cm}^{2}$ sec. Thus, the embrittlement of the austenitic stainless steels for a year or more of operation in a fast reactor could be quite severe even for operating temperatures of 600 and $700^{\circ} \mathrm{C}$. However, the conformance between the thermal irradiation data and the 1 imited data from fast neutron exposure gives some hope that the types of solutions being developed for the structural metals in thermal reactors will also work under fast reactor conditions. Metallurgists at Oak Ridge National Laboratory have shown that the embrittlement observed after irradiation in thermal test reactors is due to an effect of helium on the grain boundary fracture process. We have also shown that this effect may be reduced by modifying the composition and metallurgy of these steels. These methods are believed to be successful because the quantity of helium per unit length of the grain boundary is reduced and, in most cases, the stress necessary to nucleate and propagate a boundary crack is increased.

\begin{tabular}{|c|c|c|c|c|c|c|}
\hline \multirow{3}{*}{$\begin{array}{l}\text { Deformation } \\
\text { Temperature } \\
\left({ }^{\circ} \mathrm{C}\right)\end{array}$} & \multicolumn{6}{|c|}{ Irradiated Strength and Ductility (Unirradiated Values in Parenthesis) } \\
\hline & \multicolumn{2}{|c|}{$\begin{array}{c}\text { Yield Strength } \\
\text { (psi) }\end{array}$} & \multicolumn{2}{|c|}{$\begin{array}{c}\text { True Uniform Strain } \\
(\%)\end{array}$} & \multicolumn{2}{|c|}{$\begin{array}{c}\text { Total Elongation } \\
(\%)\end{array}$} \\
\hline & $316 \mathrm{~L}$ & 17-4 PH & $316 \mathrm{~L}$ & $17-4 \mathrm{PH}$ & $316 \mathrm{~L}$ & $17-4 \mathrm{PH}$ \\
\hline \multicolumn{7}{|c|}{$\times 10^{3}$} \\
\hline 25 & $34.2(33.1)$ & $148.0(162.0)$ & $51.7(51.0)$ & $7.8(6.9)$ & $75.6(72.2)$ & $12.6(10.2)$ \\
\hline 400 & $19.6(19.0)$ & $113.2(158.0)$ & $33.1(33.5)$ & $5.7(4.4)$ & $43.7(43.6)$ & $9.9 \quad(6.3)$ \\
\hline 500 & $20.5(17.8)$ & $89.5(109.8)$ & $34.0(33.5)$ & $3.1(2.7)$ & $44.4(44.8)$ & $8.0 \quad(8.2)$ \\
\hline 600 & $19.7(19.8)$ & $51.3 \quad(70.5)$ & $29.3(29.7)$ & $4.2(3.1)$ & $38.8(40.8)$ & $18.1(18.8)$ \\
\hline 700 & $18.6(15.6)$ & $33.5 \quad(23.1)$ & $17.7(18.3)$ & $(4.1)$ & $27.0(42.7)$ & $16.2(33.4)$ \\
\hline 800 & $15.0(13.2)$ & $15.2(15.9)$ & $4.5(14.0)$ & 4.8 & $7.3(42.3)$ & $37.8(44.0)$ \\
\hline 900 & $10.8(11.3)$ & 10.3 & $1.8(14.6)$ & $3.9(3.4)$ & $3.7(59.3)$ & $18.3(16.3)$ \\
\hline
\end{tabular}

arradiated specimens were exposed to a total neutron exposure of $2 \times 10^{21}$ neutrons $/ \mathrm{cm}^{2}$ at approximately $525^{\circ} \mathrm{C} \pm 25^{\circ} \mathrm{C}$ in LAMPRE by LASL and tensile tested by ORNL.

SLIDE 2 Comparison of Fast-Reactor Irradiation Effects on an Austenitic Stainless Steel and Age-Hardening Steel ${ }^{\text {a }}$

The influence of grain size on the ductility of stainless steel is illustrated in slide 3. Decreasing the grain size greatly enhances the ductility both for short-time tensile tests and in postirradiation creep tests. 
SLIDE 3 Influence of Grain Size on Irradiation Embrittlement

\begin{tabular}{|c|c|c|c|c|c|c|}
\hline \multirow[b]{3}{*}{ ASTM } & \multirow[b]{3}{*}{ Grain } & \multirow[b]{3}{*}{ Size } & \multicolumn{4}{|c|}{ Ductility $\left(\frac{0}{0}\right)^{*}$} \\
\hline & & & \multicolumn{2}{|c|}{ At $704^{\circ} \mathrm{C}$} & \multicolumn{2}{|c|}{ At $842^{\circ} \mathrm{C}$} \\
\hline & & & $\begin{array}{l}\overline{\text { Pre- }} \\
\text { irrad. }\end{array}$ & $\begin{array}{l}\text { Post- } \\
\text { irrad. }\end{array}$ & $\begin{array}{l}\text { Pre- } \\
\text { irrad. }\end{array}$ & $\begin{array}{l}\text { Post- } \\
\text { irrad. }\end{array}$ \\
\hline & 3 & & 30 & 19 & 23 & 8 \\
\hline & 5 & & 39 & 23 & 36 & 11 \\
\hline & - & 10 & 58 & 42 & 47 & 16 \\
\hline
\end{tabular}

Grain boundary precipitates and, hence, carbon are important also. The effect of carbon content with respect to irradiation temperature is shown in Slide 4. At temperatures such as $900^{\circ} \mathrm{C}$ where all the carbon is in solid solution, the the ductilities of both the low- and high-carbon steels are equal. But at the low temperatures, grain boundary carbides of the proper morphology may retard the nucleation and growth of grain boundary cracks. This is illustrated further by the data in S1ide 5 .

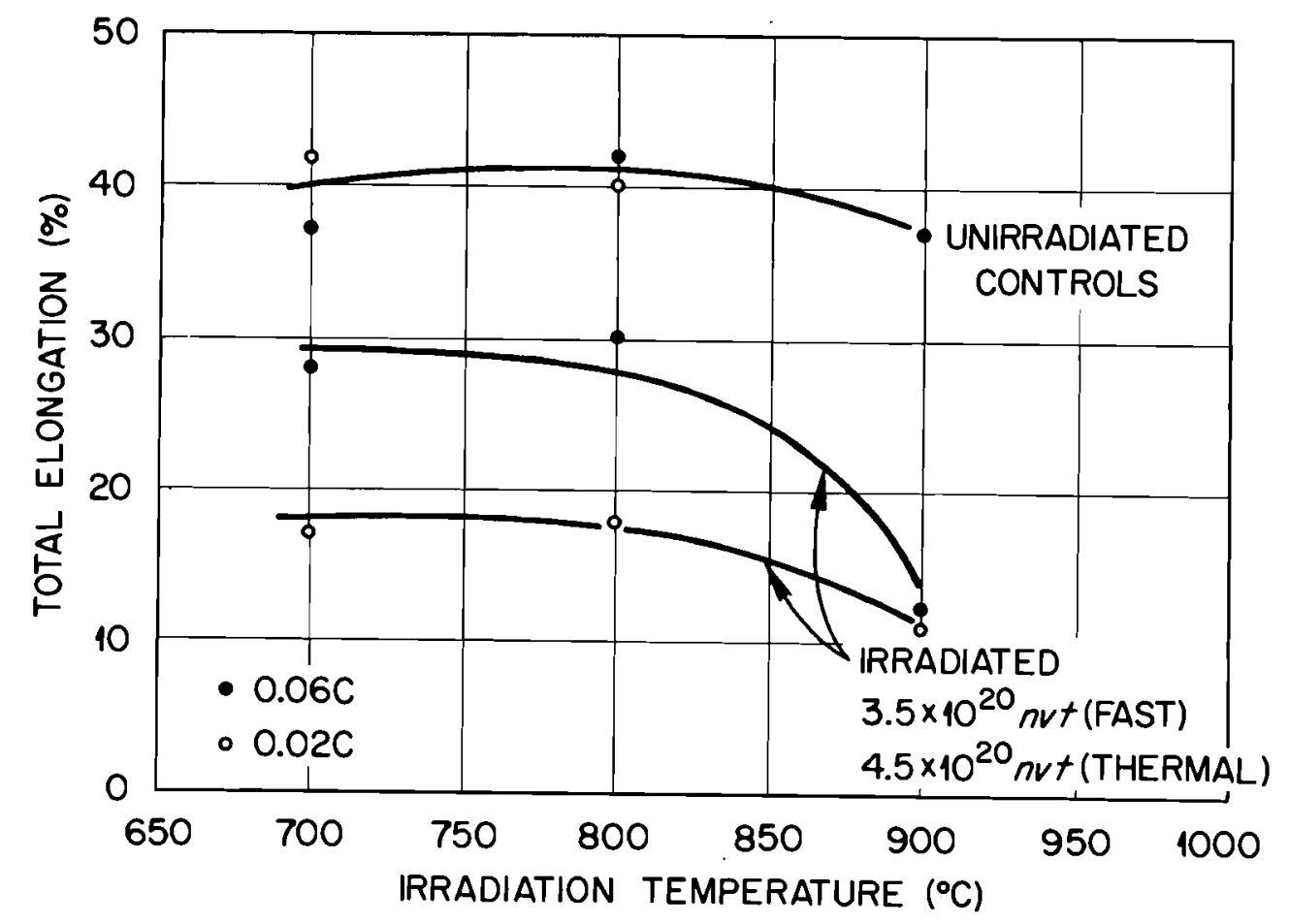

SLIDE 4 Ductility of Type 304 Stainless Steel at $650^{\circ} \mathrm{C}$ on Tensile Testing at $0.2 \%$ per min. 
Type 304 Stainless Steel

Deformation Elongation

Temp. $\left({ }^{\circ} \mathrm{C}\right)$

(\%)

704

20

704

30

842

8

842

14

14

\section{SLIDE 5 Effect of Aging}

One of the most remarkable results is a stainless steel containing titanium carbide precipitates distributed in such a way as to trap the helium at these precipitates before it reaches the grain boundaries. For example, helium bubbles can be found in the grain boundaries of irradiated type 304 stainless stee1 (S1ide 6); whereas, no bubbles are found in the grain boundary of the titanium stainless steel irradiated to a neutron dose of 2 x 1020 neutrons $/ \mathrm{cm}^{2}$.
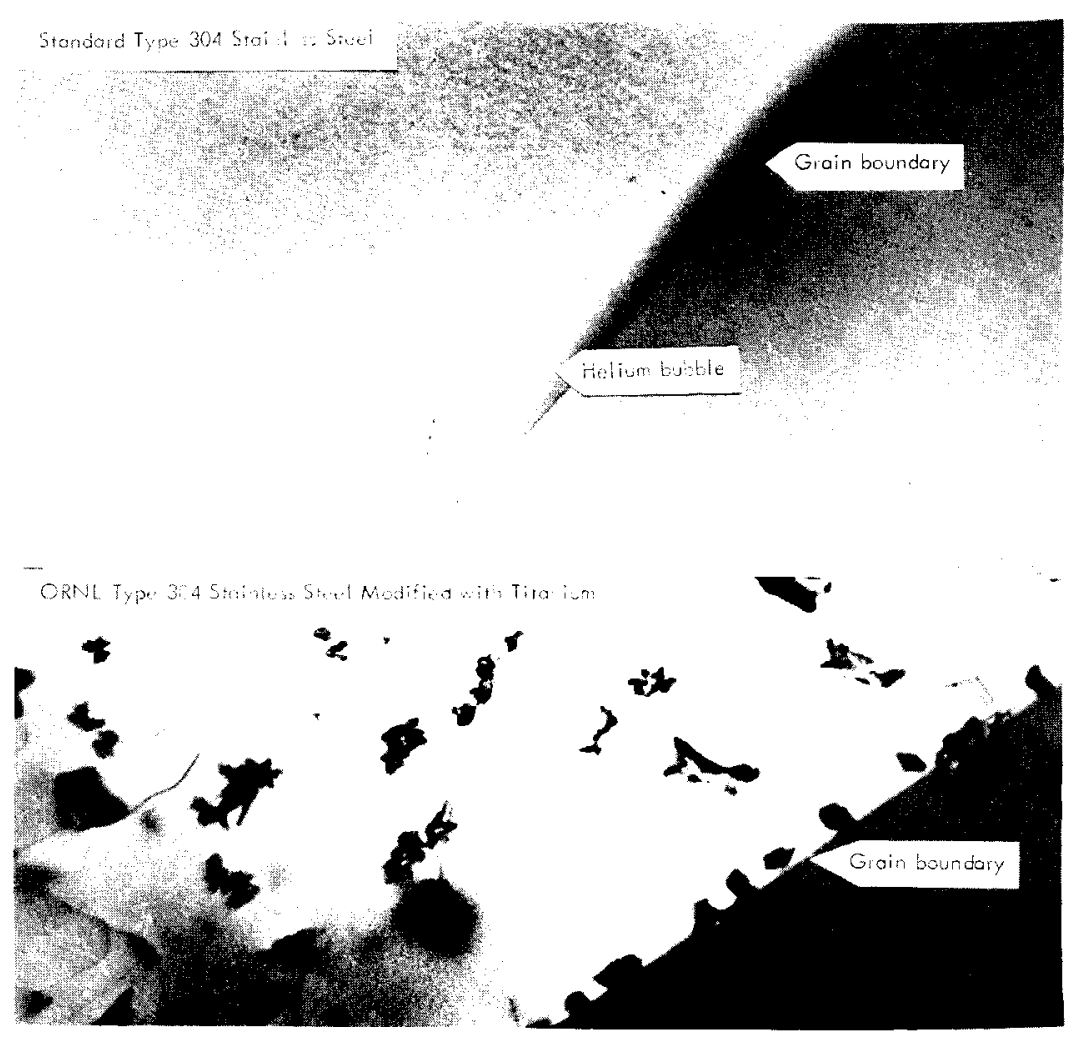

SLIDE 6 Comparison of Grain Boundaries in Irradiated Stain1ess Stee1

A comparison of the properties of this steel with standard stainless steel is given in Slide 7. The behavior of titaniumbearing stainless steel alloys in a fast reactor spectrum is 
17.7

unknown, but perhaps speculation is in order. The proposed mechanism for the behavior of this alloy in a thermal reactor can be successful in a fast spectrum. It is possible that the observed behavior of the 17-4 PH steel is due to the same mechanism and, thus, the degree of embrittlement of the titaniumbearing steel in fast reactors may be far less than that observed for the standard solid solution austenitic alloys.

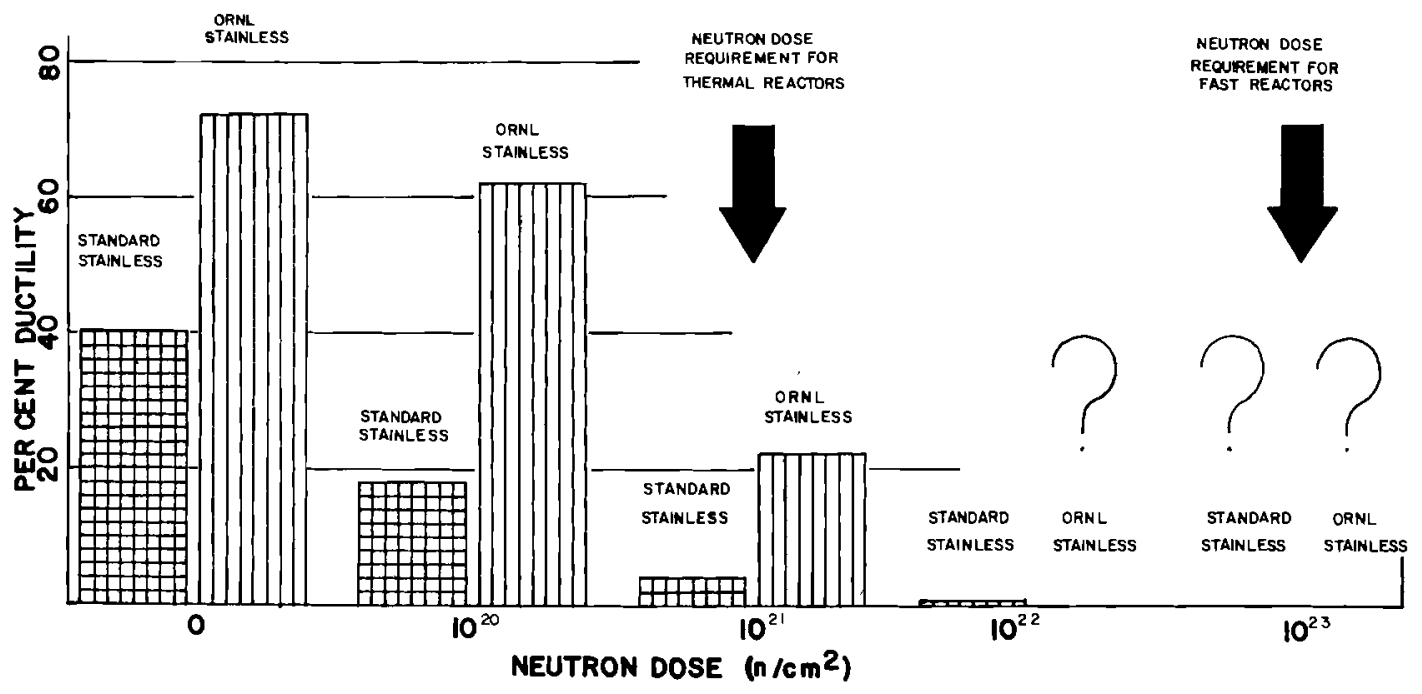

SLIDE 7 Irradiation Damage of Stainless Steel Alloys at Elevated Temperature

Summary On Cermet Fuels

I would like to summarize, at this point, our discussion on cermet fuels. It is my opinion that if we have a $1000^{\circ} \mathrm{F}$ surface temperature, there is a very high probability that the cermet fuel will meet and probably exceed the design objectives that are set forward for it in the present report. If the surface temperature is raised to $1200^{\circ} \mathrm{F}$, I become much less confident in its ability. Irradiation-induced embrittlement of the metal matrix appears to be a solvable problem by any one of several techniques so that this phenomenon will probably not be the controlling factor in limiting a cermet's performance.

None of this discussion should be inferred to mean that I have anything against the metal clad-bulk oxide fuel element; I think it is a good concept. I don't really care which type is selected, but I think that the final selection should be based on all of the facts.

\section{FABRICATION COSTS}

I'd like to dwel1 for a few minutes now on the subject of fabrication costs. I am impressed as a result of the firstday meeting with the magnitude of the problem of trying to optimize fuel cycle costs against so many variables. I think 


\section{8}

that the work that has been done so far is quite creditable, and the attempt to optimize the fuel element and reactor design simultaneously to achieve the minimum fuel cycle cost is a very refreshing approach and one that has been needed.

Cost analyses are very difficult to explain and even more difficult to defend. They must be appraised within the context of a certain set of assumptions. The most important variables in any cost analyses are the ground rules under which the study is conducted. So it is worthwhile discussing how our study was carried out. We approached this problem in a similar manner to the economic studies we have done over the past five or six years. I used the same people and the same computer codes as in previous studies; so all of our built-in prejudices were held constant. In consultation with our fabrication people in ceramics and in our metal forming groups, we laid out appropriate process flow sheets including all of the inspection and assembly requirements; we estimated the cost of the capital plant and the capital equipment that would be needed for each step in the process. Then, we amortized these capital charges at $22 \%$ as an annual cost. Following this, we looked at the man-hours for each individual step and applied an overhead factor. A11 of this information was used as input to our computer code, which then performed the calculations as a function of certain variables. On the basis of our ground rules, one would expect to obtain higher costs than Don deHallas would estimate as an in-house fabrication cost. Our cost estimate would also be higher than some commercial vendors might propose because they may already have a plant and equipment that are capable of plutonium production. We used the fuel elements as described in CC-175, which means that the bulk oxide pin had a diameter of 0.168 in. and the cermet had a diameter of 0.225 in. Under normal circumstances, the pin diameter has a greater influence on fabrication costs than any other single factor. Unfortunately, this case is much more complex, and we might wallow in semantic arguments all afternoon if we do not carefully define our units of measure. It is conventional in costing thermal power reactors to use dollars per kilogram of uranium. In this case comparing a mixed oxide ceramic with a pure $\mathrm{PuO}_{2}$ cermet, we didn't think it was quite fair to base it on dollars per gram fissile (S1ide 8).

Since I have been here and thought about it a little, I realize that this isn't fair either; because, ultimately, the Atomic Energy Commission and the rest of us are going to have to pay a cost per year or per core, and so the thing of most significance is the cost per pin. When we look at it from this basis and select a production rate of $10 \mathrm{~kg} / \mathrm{day}$, our analysis indicates a cermet pin cost of $\$ 150$ compared to $\$ 125$, if it is a bulk oxide. However, contrary to what this curve shows, there are only $13 \mathrm{~g}$ or so of plutonium in the bulk oxide, whereas there are $32 \mathrm{~g}$ in the cermet pin. So the use of a unit of dollars per gram fissile favors the cermet; whereas, the dollars per pin would favor the oxide. Finally, the fact emerges that we need a specific reactor design in order to tell 
how many pins there are of either type. There is one important consideration I want to draw to your attention; the slope of this cost curve as a function of production rates. To make this point, it really doesn't matter if we compare costs as grams fissile or dollars per pin. If we select a production

SLIDE 8 Comparative Cost Estimates for Fabricating Cermet and Oxide FFTF Fue 1 Elements (Glove Box Fabrication)

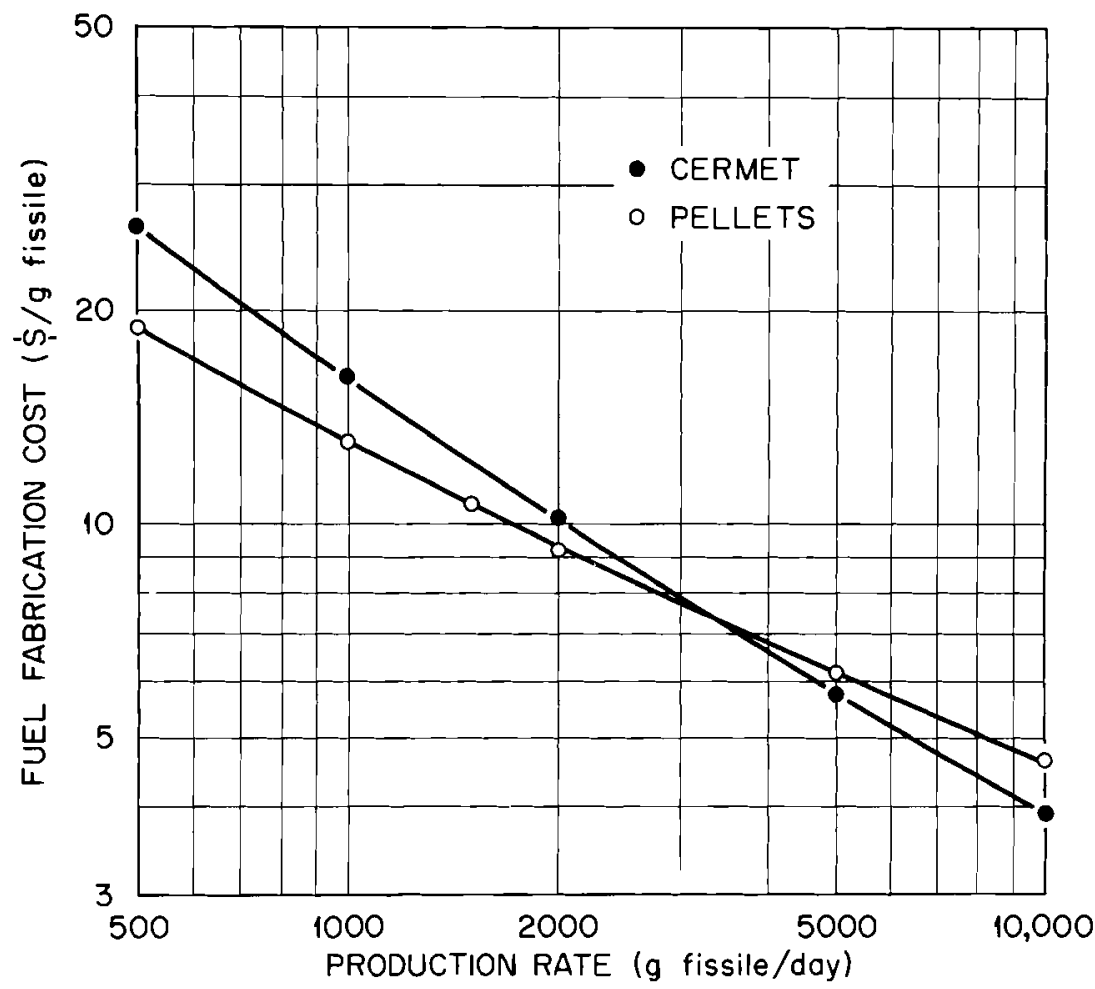

rate of $10 \mathrm{~kg} / \mathrm{day}, 310$ pins must be produced per day in the case of the cermet. This predicts a cost of $\$ 150$ per pin. If the operation is at a production rate of $2 \mathrm{~kg} / \mathrm{day}$, the cost rises to $\$ 300$ per pin. So I think that this factor should be an important aspect of future cost analyses and future estimates of what the core will cost for various reactors. We have to ask ourselves: "At what production rate are we going to make the pins?" Finally, (S1ide 9), let's consider the question of costs relative to conditions of fabrication.

There was some discussion about the possibility of using reprocessed fuels. We have looked at the costs of contact, semiremote, and complete remote fabrication relative to our Thorium Utilization Program; so we made a similar analysis for this case as we11. We estimate about a $7 \%$ penalty in changing from glove box fabrication to semiremote. Completely remote fabrication would result in a $20 \%$ cost penalty.

To summarize, no economic evaluation of these two concepts can be made until an optimized design for each type of fuel element is developed. The rate at which such elements will be fabricated is a potent economic factor. In general, this factor would favor the bulk oxide element since fabrication equipment is already in use to produce pins for irradiation tests and for critical experiments. 
17.10

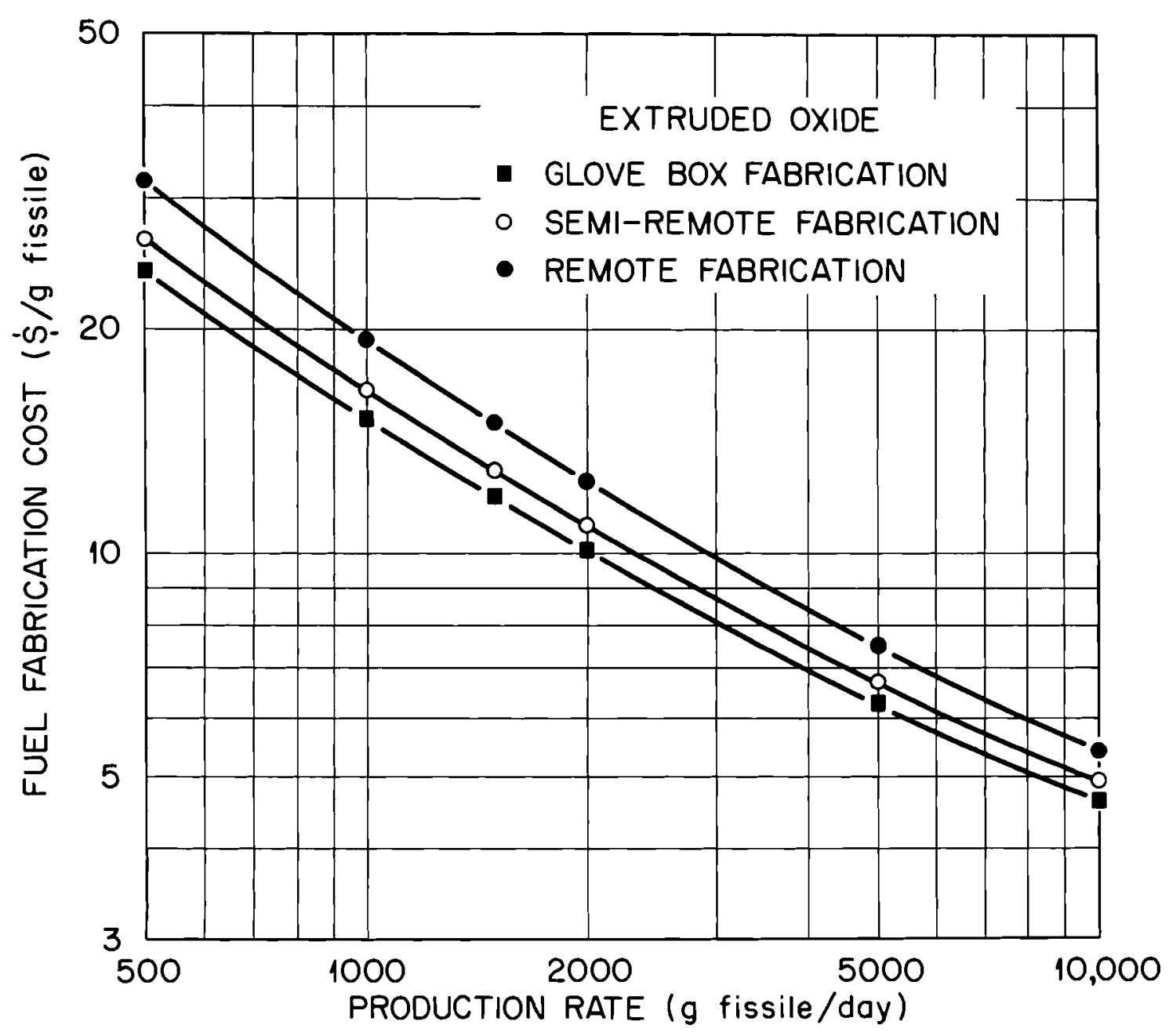

SLIDE 9 Comparative Cost Estimates for Fabricating Oxide

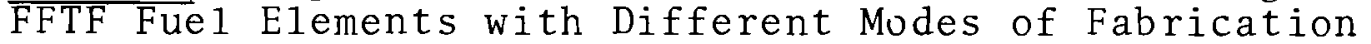


D17.1

CLADDING AND DISPERSION FUEL DEVELOPMENT AT ORNL

J. E. Cunningham, J.W. U11man, D. A. Douglas

Oak Ridge National Laboratories

Oak Ridge, Tennessee

\section{DISCUSSION}

MR. ANDERSON: The other day we heard on loss of ductility that you don't plan on failure of the irradiated sample and I agree, but I still think it is going to happen. The analysis that you had was the third top quarter and this would add a dollar. It is a rather modest reactivity position, and $I$ agree that the calculations Hofmann showed are good. However, with a temperature coefficient you are likely to have a dollar insertion, which means a raise of about $400^{\circ} \mathrm{F}$ in fuel temperature and stainless steel temperature. This takes the temperature up into the range where you do worry about this loss of ductility. It seems that it is something that should be considered. In other words, if you do get an insertion like that, you have zero ductility in your fuel.

MR. HENNIG: Instant zero ductility?

MR. LUCAS (Battelle): I think this is a real problem.

A VOICE: I don't think so at all.

MR. PETER HOFMANN: The fuel coefficient is about a half a cent a degree, so that would be a $200^{\circ}$ rise if there is no other feedback.

MR. ANDERSON: That is Centigrade.

MR. HOFMANN: Yes.

MR. ANDERSON: I said Fahrenheit.

A VOICE: We expect to take some action to reverse the insertion of reactivity.

MR. ANDERSON: It depends on the time scale, whether you can take action or not.

MR. HENNIG: Very roughly, the conditions would be nominal, at an average, taking the temperature of maybe $500^{\circ} \mathrm{C}$ base, ramped up to something under 700 and scrammed.

MR. CLAUDSON (Battelle): It is true that if the fuel type temperature reaches $700^{\circ} \mathrm{C}$, we are in a very dangerous condition, and I will talk about this in a few minutes by showing some slides. I think the work that Don Douglas put out at Oak Ridge would also show this. There is no doubt the ductility is going to be quite low, based upon thermal data that we have today. 


\section{D17.2}

MR. DOUGLAS: Again, the slides that I showed did not show ductility as a problem at $700^{\circ}$. It was at 800 .

MR。CLAUDSON: Let us say, it is worse at 800 than it is at 700. I think we are in fair agreement.

MR。DOUGLAS: Remember there are several sets of data. There are a great many things that affect ductility in your irradiated condition, grain size being one along with carbon content and the type of stainless stee1. I think it is very difficult at this time to hypothesize on a particular subject and say that you have a serious problem or you don't have a serious problem. When you pick the type of stainless steel and the fuel that you want, then you postulate a certain set of conditions, assess it, and see if there is a problem. I don't think we are going to get any place by hypothesizing on a hypothetical type of material.

MR, deHALAS: I would like to propose the following, which is probably even more complicated. It is really a design problem for the fuel. If you decide the fuel goes into a zero ductility stage, this is something that you have to design rapidly around, and you ought to be able to approach the problem.

MR. ANDERSON: That is what happened. You get internal pressures.

MR. deHALAS: This is true, but it doesn't have a simple answer.

MR. HECK: The temperature numbers that were quoted here were average temperatures. I am wondering if maximum temperatures might not be a little more important; a1so, the swing of the peak temperature is a lot bigger than the average temperature. This might affect the answer, whether you are going to have a gross loss of ductility locally or not.

MR, CUNNINGHAM: This is an engineering question.

MR. DOUGLAS: The answer, of course, is yes.

MR. MERCKX (Battelle): One of the things that you keep saying is that there is going to be a strain, but these are rising from thermal beads and when you even go up to fairly high temperatures, you are talking fractions of a percent. Normally, when you say you have lost ductility, that means small fractions of a percent from your thermal with a one shot. Even if you did something with a $1 \%$ strain, you have no ductility. Yet, for a thermal problem, this would be plenty of strain to stop you from breaking apart.

MR. DOUGLAS: We11, I should point out that we must have spent all of three or four man-months looking at this problem and analyzing the fuel performance for the Fast Test Reactor. Battelle-Northwest, by a crude estimate, has spent 3000 or 4000 man-months; so I think you should weigh our opinion at about $1 \mathrm{~g}$ versus $1 \mathrm{~kg}$ on their side. But I would be surprised if it turned out to be the only thermal stress problem. If you look 
D17.3

at the considerable amount of fission gas that has been generated, then you postulate a temperature increase, and then put the question to yourself; wouldn't you expect there would be a pressure generated from the copious quantities of helium and fission gases present?

A VOICE: At this time we would need short time strains or else you have got a ductility, a wave type problem of how fast this stuff is going to swell up or blow up.

MR. DOUGLAS: I didn't think the question was how it would fail, but does it fail.

MR. ROAKE: One of the things I think we haven't done is that you talk this way about the oxides or any other material. Because we have a test reactor, and failure should occur in some of the loops, we should have fuel elements or have the cores designed to go under certain incidents. Part of the economics in picking the core would have to be that these fuel elements under very transient conditions will live it out, and you cannot design that on the bas is you designed the breeders. You are going to have to have these incidences, and then you will be able to see how the fuel behaves. Really nothing has been presented on this problem. Maybe all the people have different type fuel elements. If I can't use your reactor, I am not very much interested in it. I might have just such a problem. Now with this type of transient thrown on your reactor, what would happen with our design problems, and how do you pick a fuel element with these type transients occurring? This could happen in an oxide. If you get an overpower transient, will the pressure blow the clad apart? There is not a word addressed to this. You talk about the oxide in a core which is stable and there isn't a failure. A failure doesn't have to occur in the material when you get the transient conditions happening from the failure in the test loop, where you didn't have any control. So, when we are addressing fuel design problems, the selection of the fuel, whether it will ride out all the things that will happen in test loops, will take a lot more study, and this hasn't been done.

MR. KIRKBRIDE: One further comment about this embrittlement problem is taking a rough look at the loops that are going to be built--I guess out of stainless steel in the FFTF, operating under (let us say) roughly, $1016 \mathrm{n} / \mathrm{cm}=\mathrm{sec}$ and with a $75 \%$ duty cycle, you will get something like $2 \times 10^{23}$ nvt per year. And it looks to me like there may be a need for plug-in loops that can be changed every six months or so. One further point is about the loops. I understand they are designed to take $1200^{\circ}$ sodium, and actually even higher, maybe $1400^{\circ}$. This puts us right in the range where the ductility is decreasing very rapidly. I think if you look at some of the data out of the EBR-II (this data plus maybe some of the reference data), it may look like we are passing through zero ductility before we get to 1023 nvt. 


\section{D17.4}

MR. SHOUDY: Getting back to the discussion of a minute ago, I wonder if Battelle-Northwest looked at the spert tests that were run in 1960 or 1961 on cermet fuel?

MR. deBOISBLANC: As I recall, that was unirradiated material, and it blew apart at 19,000 MW.

MR. SHOUDY: It was quite a transient but real ductile material, and it blew apart.

MR. deBOISBLANC: That was an internal pressure problem. I am from Idaho.

MR. SHOUDY: I don't know the details.

MR. deHALAS: I just want to comment that we certainly recognize that there is a problem here and the testing program has called for transient testing, so I don't think there is any answer, but it is an unknown problem.

A VOICE: I just wanted to mention briefly the state of the cladding in EBR-II. It has been referred to a couple of times. This is 304 stainless. It is operating about 500 to $550^{\circ} \mathrm{C}$. We have been doing some burst testing on this material after it is removed from spent fuel, and this material has dropped considerably in ductility. In the unirradiated condition, it showed about 13: elongation when it burst, for example, at $500^{\circ} \mathrm{C}$. After the exposure of about $1.3 \times 1022 \mathrm{nvt}$, the uniform elongation dropped to less than $1 \%$. It is still a ductile failure but there is remarkable change in the behaviour of this material. Now, just before I left, I looked at some data gotten last week; it has been burst at $700^{\circ} \mathrm{C}$, and here, to our surprise, there was an enhancement of the ductility. We are up around now 4 or $5 \%$ elongation for failure, so it is possible (in contrast to the remark you just made), that maybe the loops running at a higher temperature will have a more ductile situation.

A VOICE: What irradiation do you use?

A VOICE: They are running from 500 to $550^{\circ} \mathrm{C}$. That is the sodium type: We don't think we saw much in the way of recoil damage; there is still a sodium gap.

MR. deBOISBLANCE: How much nvt accumulated, do you know?

MR. CUNNINGHAM: About $1.3 \times 10^{22}$. It is a good hard spectrum.

A VOICE: Jack, I find it difficult to follow the basis of your design analysis when you merely had time to put that one curve on the board. I wonder what either you or the PNL people have done with respect to the evaluation. What criteria do you use? Do you use full gas release or no gas release? How much delay time before 


\section{D17.5}

you reach the yield point you are taking for $5,10,20$, or $50 \%$ porosity? What strength are you taking? How much ductility do you want? How much local strength is there on a particle when the cladding expands...? There are a lot of analytical tools or criteria tools or criteria that may have been brought to bear, but you can't see it (at least) from your presentation today. How did you evaluate performance capability, just solely from the curve you show? I happen to agree with your reservations.

MR, CUNNINGHAM: Essentially that is right. We looked to see what data we had obtained in the past. We looked for what capability (that we thought was in the system) that essentially could be near term and put to practice, and made allowances for this. We didn't go into a real detailed analysis at all.

MR, C. E. WEBER (Atomics Internationa1): How would you evaluate the improvement in going from 5\% porosity to 20 , if you can get it, or at 30? It isn't the more the merrier, if the gas is being released from the oxide.

MR. CUNNINGHAM: Are you familiar with Wier's treatment of this analysis, where he changed the particle size and the particle density and the amount of built in void space and this sort of thing? There is a report on this.

MR. WEBER: That isn't what $I$ am aware of, it is an elastic analysis.

MR。 CUNNINGHAM: It is an elastic analysis. Subsequent people in England and other people in the industry have refined this analysis, and this kind of information is available. We did not go through it in detail. We looked at the information we had. We took some educated guesses from our background and experience, and the trends are fairly obvious. I don't see us making a big jump in improvement in cermet fuels. I just don't think it is in the cards.

MR. WEBER: I am looking for the analysis then that shows how you are going to make them work, since you have got analyses that you conclude they won't work. This is alsc my question to Battelle, and I would like to see it answered.

MR, CUNNINGHAM: The whole basis of our examination is when we got this letter in on the fuels evaluation; it said the fast flux test facility would use cermet fuel at $1200^{\circ}$ outlet temperature. However, we looked at our curves, and we knew that we were in trouble,

A VOICE: I thought I still saw that number in the last report, $812^{\circ}$. 
MR。KITTEL: It sounds to me like you are talking about the peak outlet temperature, the closed loops.

MR, HENNIG: When we finish this, I will be offering you all copies of the design analysis basis of the fuel cycle cost analysis in this little bound package up here. There are 50 copies, about enough to go around.

MR. DOUGLAS: I would like to correct an impression that Jack left with you. It sounds like he and I haven't talked together, and it is true. We have not talked together for ten days. He was there when I was gone, and vice versa. In point of fact (the basis on which I made my talk), we have done a real detailed analys is of the fuel concept in BNWL-CC-175. A detailed analysis of this sort takes several weeks to do and covers many pages. I don't see any point in going through this whole complete analysis. What it ended up in indicating was that at the lower limit, as I tried to imply, we agree substantially with what Battelle-Northwest says in their document, that either concept will work. I am cautious about extending it up to a higher range. If you are interested in seeing the detailed calculations, I can send you a copy. I don't have them with me.

MR. WILLIS (A.I.): You have a photomicograph there in the top part of the picture. You show bubbles but no structure; and in the bottom part of the picture, you show structure but no bubbles. The indication is that titanium will solve a lot of our problems. I would like to know why the titanium is going to solve our problems in steel.

MR. DOUGLAS: Your analysis of what that photograph is supposed to show is correct. We have found these little bubbles, which (in our analysis) means it is helium there in grain boundaries. We don't want to promote the titanium addition as being something magic. I think any dispersed particle distributed through the matrix would have the same effect. We happened to use titanium, 17-7. T-8 will do the same thing if you treat it correctly, or it could be any number of other things. So please don't get the impression and say only titanium will solve this problem. This is one way of going about solving it, and it apparently works. There are other ways as well.

MR. WILLIS: Do you have any reason why it works?

MR. DOUGLAS: Yes, it works because it tends to give you a surface for the helium to collect on, before it has a chance to grow in size to the point that your surface tension produces a large bubble. It also indicates that the reason for titanium for a thermal reactor was that we wanted to see if we could get boron out of the grain boundary and distribute it in small concentration throughout the matrix. For this purpose you can't use just any dispersoid. 


\section{D17.7}

You have to use titanium or some other compound, and we have looked very hard, honestly, I think, to find helium bubbles in the grain boundaries on titanium treated material. We haven't found it. On the basis of this inference, we have come to the conclusion that we have gathered most of the boron out of the grain boundaries and captured it in the dispersoids. But when you talk about a fast reactor where your helium will be borne in many locations, I think that any kind of dispersoid will help to alleviate this ductility problem.

MR. WILLIS: We11, without really requesting an answer here, I would just like to make the point that boron is in the boundary You will find from the recoil; it will probably leave the boundary, and it doesn't look as though the partial density or the precipitate density or the dislocation density is high enough to selectively trap all the helium. In other words (getting back to the boundaries), even if it originated there, you have to have pretty high density to capture it. This is why I asked the question.

MR. deBOISBLANC: I would like to make a comment on this question of the service conditions for the reactor fue 1 in the light of experimental failure. I think that, firstly, it is not even considered an adequate reactor design to take the contemplated experimental failure in fuel without any damage to the fue 1 . I don't think one should equate that, however, with taking the maximum credible reactivity, which is a safety coefficient in this fue 1 slumping accident in the experiment. It is essentially chosen as a maximum credible accident that is occurring in an experimental sample. You have this problem. The power distribution (as has been demonstrated) is wel1 determined by the leakage characteristics of the core. Therefore, you postulate that you have experiment $A$, and experiments $B$ and $C$ (a11 experiments) running at limiting conditions, or many of them running at limiting conditions. If you had an excursion from one experiment, it would be probable that the experiments in the other two would fail. And when you realize what this means, this would be a very costly failure because the cost of the experi-. mental samples in terms of all the invested dollars (piled on the samples before you get into the reactor), is much more than the cost of the core. So when you say you are going to run the sample to its failure, you are going to talk about leakers and inductive type failures and things which do happen in the reactor, but not the catastrophic failures. I would like to say also that you are going to be hard pressed to produce a fuel slumping accident in an experimental sample deliberately. And you couldn't tolerate it, you would just ruin every experiment in the core. This would cost millions of dollars. I think this is the point. I think this is the question of what experimentalists want to be able to do; perhaps they need some clarification. They do not want to do their maximum credible reactivity efficient: Do you agree on that? 
D17.8

MR. CUNNINGHAM: I think that is true.

MR. R. E. MACHEREY: (Argonne): All the data we have on this business of swelling versus temperature has been on cracked plates. Is that what you show?

MR。CUNNINGHAM: Essentially, I think there are one or two exceptions to that, but basically what you say is true.

MR. MACHEREY: About 50\% of this stainless steel in this assembly is in the clad. Now, in the flat plate geometry, it is difficult for me to understand how this could contribute anything to the restraint of this swelling. If you change the geometry to circular, then you can get a contribution from this clad. Now would you care to venture how much it is going to push the curve up there by doing this, or how much temperature you get out of it?

MR. CUNNINGHAM: What you say is true, it is going to go up. You can see what effect the restraint had down in the end, with the closures down there, the way the swelling tapered off. But I don't know how much it is going to help.

MR, DOUGLAS: I might add, the first six or seven dispersions (when I worked with GE), which were failed, were very muchly carbides. The only thing that was wrong was that we did a tenth and eleventh, and we found out we no longer could correlate them. A1l you have to do is take something like a very dense dispersion and make a core eccentric in the circular plate, externally. You will find out you can get rid of this quite quickly. But I have looked at more dispersions than I care to admit. If we could get a correlation on them, from anything other than plate, then we could go from one geometry to another, I think I would frankly be surprised, due to the various conditions under which we operate, and how you operate. I just wish we had stopped at six.

MR. DOUGLAS: Then I would like to reply to Bob's question that has to do with a report the British published that treats the subject of flat plate geometry versus pin geometry. The results from that do show a much higher burnup for pin geometry than for the flat plate geometry. I happen to have a copy of it over in the room if you would like to see it. I mean you can make some calculations and say how much improvement this means. But they haven't run their pins to failure yet, as I recall. Do you recall, Don?

MR. deHALAS: I can't recall either. I think one would suspect that the cylinders would hold better than the plates, but we did look at the plates very carefully. My recollection is there were about a dozen points on that curve that were cylinders. You can't tell them apart from the plate on the mass figure correlation. 
Now Cliff asked the question a little bit ago that really hasn't been answered: How do we think we are going to get off the curve? We11, there are two parts to that. One is, if we do settle on a temperature under 1,000 on the curve; and the second is that we haven't completed to date a more detailed analysis using a modified correlation, for example. But the point does come out of it that it appears that the route to extend the 1 ine of a cermet probably goes to lower density particles. This is a new realm of problems which shows in the application.

MR。CUNNINGHAM: Don, I think that is the big hope myself, but the way we were all asked to comment on this as sort of an existing technique, something they are doing in the last year or so. Everybody has known this for 10 years, and nobody has come up with a solution.

MR. deBOISBLANC: I would like to read a note from the Battelle studies, the Battelle 400 Report. This refers to the fact that $2 \%$ volume increase in the matrix will result in rupture of the cladding. So the point made that the stress does get taken up by the hoop stress in the cladding is certainly true. But on the other hand, the deformation to failure is very small, and the volume changes that you are showing there are much larger than $2 \%$.

MR。 deHALAS: That is the bulk fue 1.

MR. deBOISBLANC: You are answering the solution to the problem, but for a given system $2 \%$ volume of change in the matrix in the cylindrical geometry will produce failure. More than $2 \%$ is tolerable. You can take several mils without failure. So it is a tradeoff。

A VOICE: I have seen zirconium go up something like $20 \%$ without failing. It all depends on how you run the test. I, myself, would like to push the curve that you have there upwards so that we can get the higher temperatures for the fuels. I would love to know how to do it. But basically, everybody sort of addresses themselves to the problem of matrix strength. But you go down to the region of around the particle, of the crack initiation and propogation, this potentially could be amore severe problem than the limitations on strength.

MR. CUNNINGHAM: I think it depends very much on the temperatures seen in the operation.

A VOICE: This is a real problem, but you aren't talking about correlation, a nice smooth curve. I would like to push it up, maybe reducing the $\mathrm{UO}_{2}$ volume. Putting porosity in is the way to do it. But I think the other question as to why it fails, why it doesn't fail in the cladding, but fails in the matrix (it has been doing this since 1954) is the thing we ought to put our attention to. 
MR。CUNNINGHAM: Some main things (we are pretty certain) are going to help us, and then there are a lot of subtleties and mechanisms we have got to look into. We have been looking at this; the $\mathrm{AEC}$ at large has been looking at it; and everyone concerned has been looking at it. We have gone a long way to make progress, but we may be running out of the thing, except for the two that I think will. add most is clad restraint--developing the clad thickness, and builtin void space.

A VOICE: But you are going right back to technology; right now we are worried about cladding and brittleness due to reactor irradiation. The reason we originally invented dispersions was to get an undamaged region around the particle, which supposedly is going to get brittle anyway. This is cladding effect. It is a contradiction.

MR. CUNNINGHAM: We11, we think we have got the thermal problems sort of iced in Oak Ridge on the cladding and matrix material. We don't have it in hand at all on past reactors.

MR. SHAPIRO: When you are talking about void space, are you talking about void space in the fuel itself or in the matrix?

MR. CUNNINGHAM: The on $1 y$ way I would ever talk is in the fue 1. In the matrix you defeat the whole purpose of the element, then you lose your thermal conductivity, if you don't have really a true dispersion fuel.

MR. SHAPIRO: No, I don't think that is necessarily so. In another application it was necessary for us to take fuel, and this happened to be coated particles, which was compacted to say, something in the neighborhood of $90 \%$ theoretical density. It was necessary for us to take this thing above the melting point; and the point was that in so doing, if you built in porosity within the matrix material, you were able to accommodate the almost instantaneous swe11ing that occurred under these conditions and keep the part intact.

MR. DOUGLAS: It depends on your heat flux, though. When you have got a severe heat flux problem you consider the fast reactor.

MR. SHAPIRO: The point is, what I am saying is that $10 \%$ or so in the porosity of the matrix material, you might be able to accomplish and still have a good thermal path through the matrix material.

MR. deBOISBLANC: There is a substantial imbalance between the central temperatures of the particle and the surface temperature of the plates. Until that is accommodated to more engineering adjustment, you are not limited by porosity in the plates. For example, the heat could be reduced by a factor of 10 , and you would still not get greatly increased surface temperatures. But I agree, however, that this is an incautious way to proceed with our problem. The proper way to do is to search for a stronger matrix or to search for a strong particle. 


\section{D17.11}

One that intrinsically does a better job of retention of fission products. If you can't find that, then you must go to a stronger matrix. All these things must be built in in carefully controlled voids, and the proper place to put them there is probably around the core. As a result of this, you are led to the logical conclusion that the proper thing to do is to put the voids around the particle...

Now, fabrication feasibility data has been established for that concept, but the irradiation data has not been established.

MR. SHAPIRO: The point that I was merely trying to make was that in other applications, which I think tolerated conditions even considerably more severe than I think are contemplated here, one would be able to do this by building in accommodations, not only within the fuel necessarily but also within the matrix material. I don't think that that particular route should be exactly overlooked.

MR. CUNNINGHAM: In your case, did you have a ceramic matrix or a metal?

MR. SHAPIRO: As a matter of fact, we had both. I don't think in this particular case, it makes any difference. The point that I was trying to make was that if you had almost instantaneous swel1ing by virtue of the fission of the fuel.

MR. CUNNINGHAM: You are deciding a pressure dampening kind of thing, is that correct?

MR. SHAPIRO: This, in effect, takes care of the kinds of things that one has worried about occurring within the reactor itself. I think if you have a lot of time, things can adjust themselves. It is when things occur rapidly, that you start worrying.

MR. CUNNINGHAM: We11, I don't think we have really investigated the whole area very thoroughly.

A VOICE: Since it is getting late in the afternoon, I will make one observation, which maybe helps the cermet barrier. It seems to me that of all the experimental failure (that I know of), with the possible exception of the very most recent Oak Ridge work with their uniform spherical material, all the data prior to that is extremely noisy. This is in the sense that the reproducibility. of the material and of the experimental conditions over the same standards is characteristic of the early work on the oxide fuel systems, highly unreversible. That is to say that the curve may be more favorable if you get well controlled conditions. But then the question is (if given the analysis, which seems to be really reasonably water tight), of how the plates deformed and failed, what happens if you postulate the irradiation damage corresponding to roughly 20 to 40 points higher, plus the thermal tests which 
D17. 12

have been done? How does that curve move with 20 times more fast neutron damage?

MR. CUNNINGHAM: Well, you would have an increased embrittlement on the stainless steel and you go down, which is one way of looking at it, and another way--I see Don is shaking his head there.

MR. deBOISBLANC: It might actually get better.

MR. DOUGLAS: I tried to show this in some of those curves relating to the amount of helium generated, which after all is the agent that is going to embrittle stainless steel. It is not the fission gas, not the hydrogen; and if you recall on a slide that showed, there is apparently a knee in the curve somewhere between one and ten parts per million of helium. It looks as if (and this is purely projection), the effect of annealing is to resaturate itself at about $700^{\circ}$. This was on 304 stainless steel, which happens to be one of the poorer acting...embrittlement points of the stainless; and we still had something around $10 \%$ ductility. I don't think we should try to magnify the size of the problem, and I don't think that the problem necessarily is a bad one. I don't think we should be gloomy about it. It certainly deserves a thorough testing. I am quite optimistic concerning the effect of fast irradiation damage at the higher exposures. 
18.1

\title{
MATERIALS CONSIDERATION
}

\author{
T. T. Claudson
}

Pacific Northwest Laboratory

Richland, Washington

Operated by Battelle Memorial Institute

\section{INTRODUCTION}

I would like to mention that all the materials considerations we have made to date have been based upon the FFTF interim design basis reports (the BNWL-CC-400 and BNWL-CC-175 reports). As far as

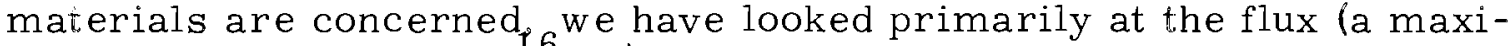
mum in excess of $1 \times 10^{16} \mathrm{nv}$ ), and at coolants which are of nominal purity levels and are typical in fast breeder reactor systems. Coolants in the closed loops are excepted because we will have to deal with failed fuel elements, fission products, and high oxygen and carbon contents. Temperature ranges, which have already been discussed, have been ranging from 700 to $1000^{\circ} \mathrm{F}$ as in the original conceptual design. Subsequently, we are looking, in some detail, at temperatures to $1200^{\circ} \mathrm{F}$ in the reactor. Maximum temperatures of the closed loops were $1400^{\circ} \mathrm{F}$. This, coupled with the expected environmental conditions in the closed loops, will place the most astringent conditions on materials of any application in the reactors.

Basically the materials have been selected on the basis of known technology, strength, stability, corrosion, mass transfer, and irradiation resistance as well as on the recommendations of known authorities in the field. The excellent ground work accomplished by Argonne in FARET analysis and design was also useful. Cost and availability were also included.

On the basis of these criteria listed in the interim reports, 304 stainless steel was chosen as the primary construction material for the reactor. Fuel cladding would also be in 304 stainless steel. There are components in the reactor that contact Sodium and, therefore cannot be 304 stainless. In fact, nickel base alloys must be used for components in the control rod mechanisms, springs, and other similar applications. In addition, Inconel 600 has been selected as shielding material. There are also several hard facing applications where we'll have to face the stainless with alloys such as Stellite $6 \mathrm{~B}$, or the Colmonoy alloys. These materials also have to be examined. Whatever we choose, and once we have the reactor going, I think it is obvious that we're going to ask' from these materials more than has been asked from them in the past in terms of flux and environmental exposure. For instance, EBR-2 is in the $10^{15}$ range, and in FFTF, we are prophesying $1 \times 10^{16}$ in terms of flux. Similarly, the operating temperatures will be higher; coolant impurities will be in the range I have mentioned already; and close loop applications will be raised,possibly,by the order of magnitude. 


\section{TEST TEMPERATURES AND ALLOYS}

Briefly, w ith the time we have left, I'd like to discuss materials in primarily three areas: what we've done in the past; what we know in the present; and what we hope to know in the future. The corrosion mass transfer studies for nuclear environments and stainless steel have been continuing since before the days of the Sea Wolf. I think we have estabprimarily that austenitic stainless steels are going to be fairly good in temperatures from 1000 to $1200^{\circ} \mathrm{F}$ in relatively pure sodium. While this is an asset, we have also introduced problems in self-welding, fretting, corrosion, galling, etc., where these materials are under contact and under pressure. Particularly important, we have shown a somewhat proven basis for mass transfer, which is leading to relationships for design criteria regarding flow, the delta $T$ in the system, and relative volumes of sodium and materials in the liquid metal system. I must emphasize, how ever, that the actual data at the elevated temperature conditions has not been obtained, especially in temperatures avove $1200^{\circ}$ F. Certainly, this is an area that must be looked at here.

In years past data regarding the irradiation effects on structural materials were required for low temperature radiations and low test temperature. These showed essentially increased strength and reduced ductilities that were, in many cases, recoverable by post irradiation annealing. Since the late $1950^{\circ} \mathrm{s}$, higher temperatures irradiations and test data have been in demand and several pertinent facts have been dis covered. High temperature properties of materials irradiated at low temperatures were not fully recovered by postirradiation annealing. The high temperature properties of materials irradiated at elevated temperatures were also not substantially recovered. The intermediate temperature range, the 300 to $500^{\circ} \mathrm{C}$ properties for irradiation temperatures in excess of $300^{\circ}$, were not fully recovered. All of these data have shown (as Don Douglas pointed out) that we have even more drastic reductions in ductility and strength as the radiation and test temperatures are increased.

One additional area, I would like to mention, is the long term metallurgical stabilities of elevated temperature materials. We know that the stability is influenced greatly by the chemical composition. This is shown from examples of molybdenum and phosphorus. They are ferride formers, which lend the material to form other phases, including sigma phases. We know that carbon precipitates are influenced by a balance between carbon and carbide formers, such as tantalum, tungsten, titanium, and chromium. All of these can lead to common problems such as sensitization and sigma phase formation which results in an imbrittlement of the material. We know very little about the weldments in various environments, including the irradiation environment. Typical austenitic stainless steel welding practice includes the use of 308 welding rod, which will throw 5 to $10 \%$ delta ferrite into the structure and which will subsequently transform into sigma phase. I think, all of these possibilities must be examined in detail.

If anything has been gained over the past few years, it's the multitude of mechanical test data for the most common structural materials, their subsequent use in devising code cases, and implementing these into design codes. 
Slide 1 shows the maximum allowable stress values for 304 SS according to the ASME boiler code, and compares it to the standard $100 \% 0.2 \%$ yield strength material. A gradual decrease in the allowable strength down to about $1000^{\circ} \mathrm{F}$ is shown for 304 and $304 \mathrm{H}$ grades. From $1000^{\circ}$ down to about $1400^{\circ} \mathrm{F}$, there is a rather rapid drop. I think the sacrifice in material strength beyond $1000^{\circ} \mathrm{F}$ is obvious. Translated into design criteria, a substantial increase in cost must result. It is not yet clear that 304 SS can be used at $1200^{\circ} \mathrm{F}$.

Slide 2 shows data, which I think may be more pertinent at these temperatures, and that is the allowable stress required to cause a creep of $1 \%$ in 10, 000 hours. Plotted here (Slide 2) is stainless steel 304 and 316 , and similar data for two nickel base alloys, Incoloy 800 and Inconel 600 , are shown for comparison. This slide shows similar results to the previous slide. On a log scale at about $1000^{\circ} \mathrm{F}$, a value of $20,000 \mathrm{psi}$ is shown. At $1200^{\circ} \mathrm{F}$, the stress is reduced by an order of 4 or about 5000 psi. As shown, 316 has an obvious advantage in creep strength.
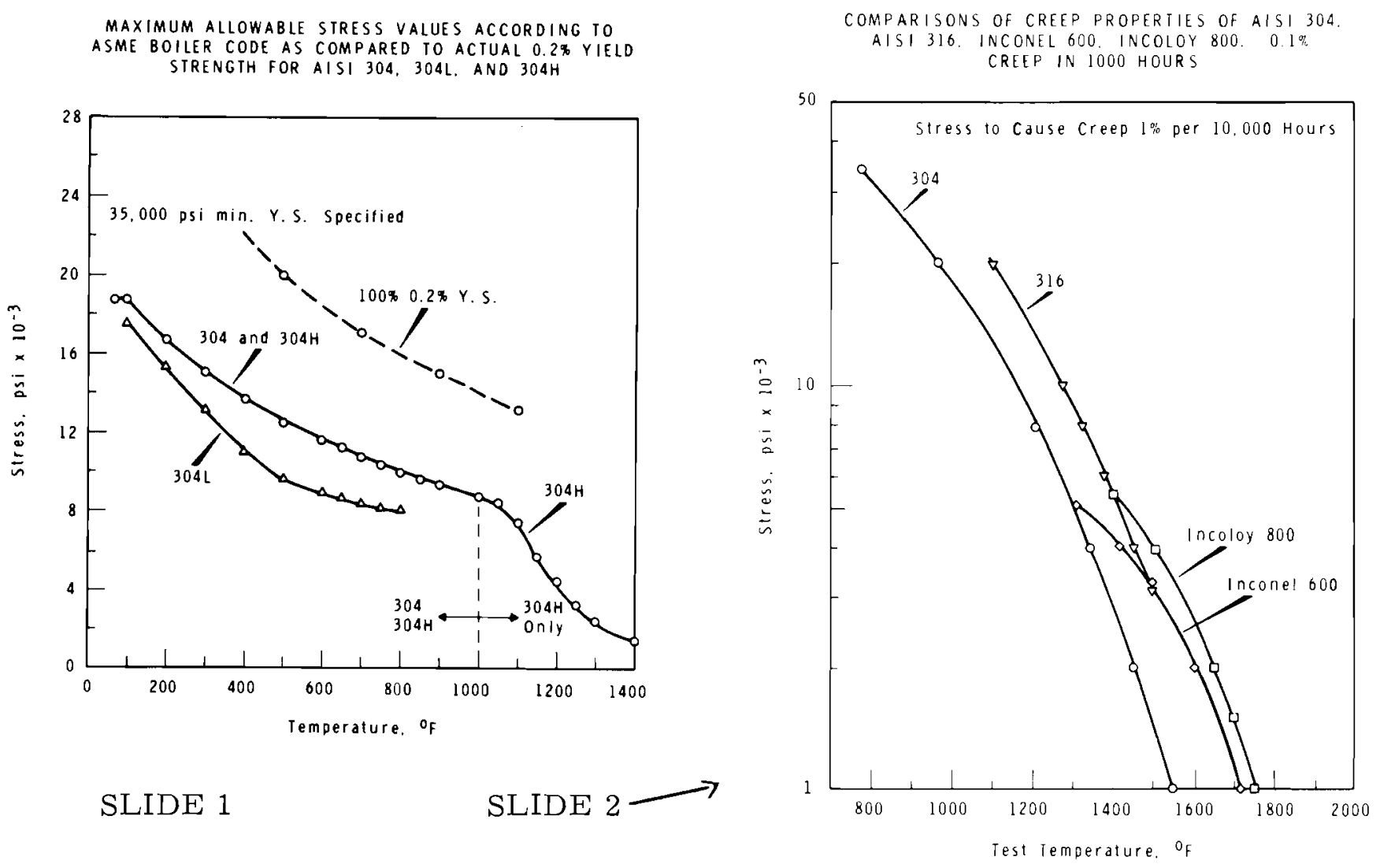

Presently, corrosion and mass transfer programs are planned for the FFTF project. These efforts will help to obtain mass transfer and corrosion data under FFTF operating conditions in both the primary cooling systems and the close loop systems. Radiation effects have been mentioned several times throughout the meeting. Don Douglas from Oak Ridge has already mentioned the presently sponsored AEC program. Progress is being made through this program which had its 
last general meeting not long ago in Cincinnati. Effort is being made there to make use of the existing thermal data for fast reactor systems and also to redirect some of the efforts in this program towards fast reactor application. In addition, there are commercial companies such as General Electric and Westinghouse that have substantial programs directed towards structural materials and fuel cladding problems.

These slides, I have prepared, somewhat complement those shown by ORNL. Data on Slide 3 have been obtained at Battelle Northwest and show the yield stress of $316 \mathrm{~L}$ as a function of test temperature. The radiation exposure was 1 x $10^{20}$ nvt, and the irradiation temperature was $290^{\circ} \mathrm{C}$. Test temperatures to about $850^{\circ} \mathrm{C}$ have been completed. We do get a drop in strength with test temperature in both the unirradiated and the irradiated. At lower test temperature an increase in strength is found due to the irradiation. This is both for the annealed and the cold work material.

Slide 4 shows the data for the ductility of $316 \mathrm{~L}$ in terms of total elongations versus test temperature. After $600^{\circ} \mathrm{C}$ we began to see a substantial drop in ductility, and at $750^{\circ} \mathrm{C}$ there's very little ductility left in the material.

Slide 5 is the elongation data on specimens of 304 and 348 irradiated under the same conditions as the $316 \mathrm{~L}$. Specimens were irradiated to exposures from $1 \times 10^{20}$ to $1 \times 10^{21}$ nvt. The ductility decreases rapidly at $700^{\circ} \mathrm{C}$, and recent data at $800^{\circ} \mathrm{C}$ shows the ductility is still dropping.

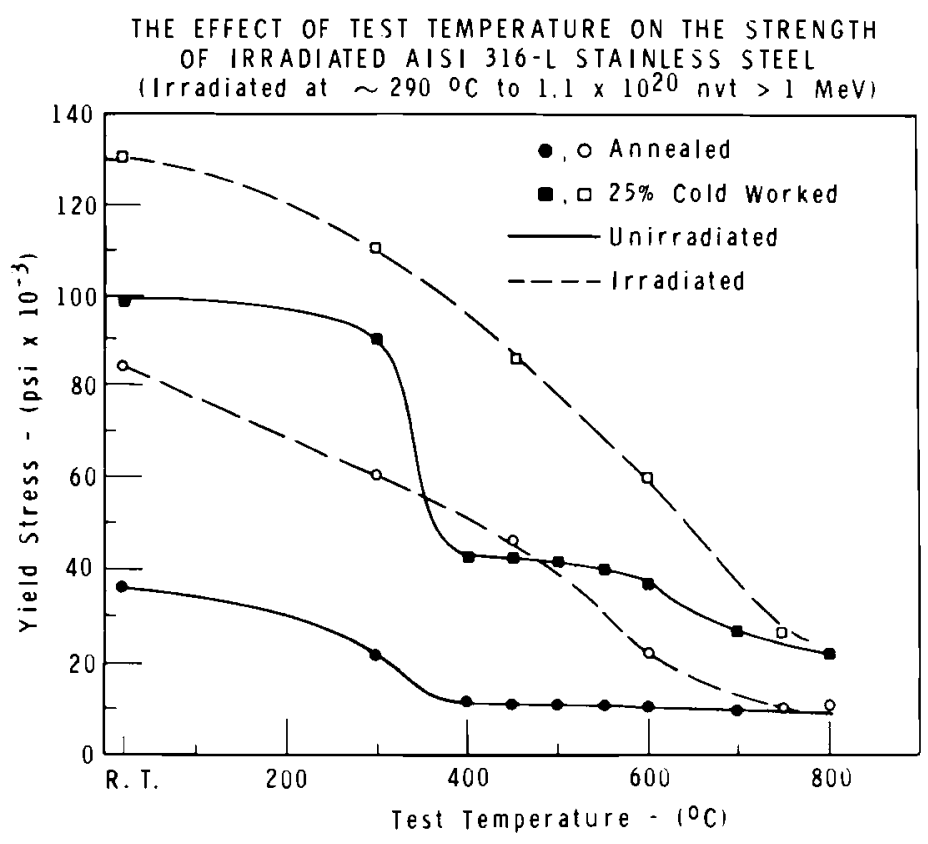

SLIDE 3 


\section{5}

SLIDE 4

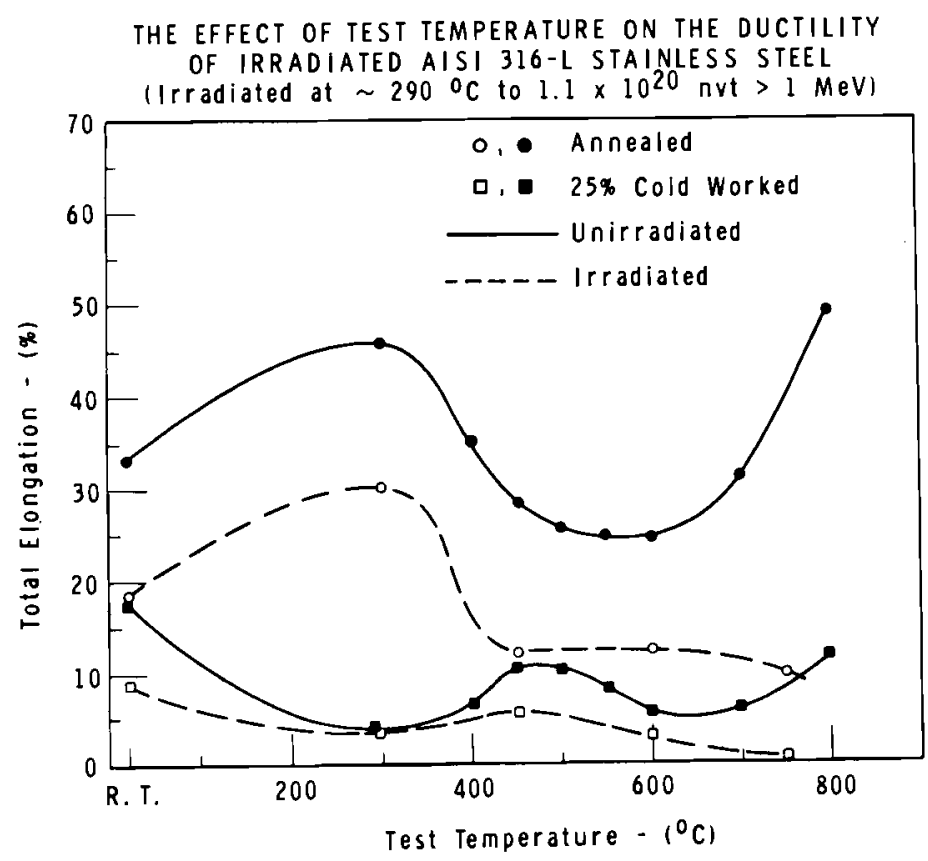

IHE EFFEC OF TEST TEMPERATURE ON THE OUCTILITY OF TRRADIATCD AIST

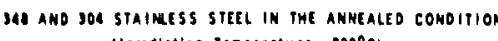
Clerecletion Tempereture $\sim 290^{\circ} \mathrm{Cl}$

SLIDE 5

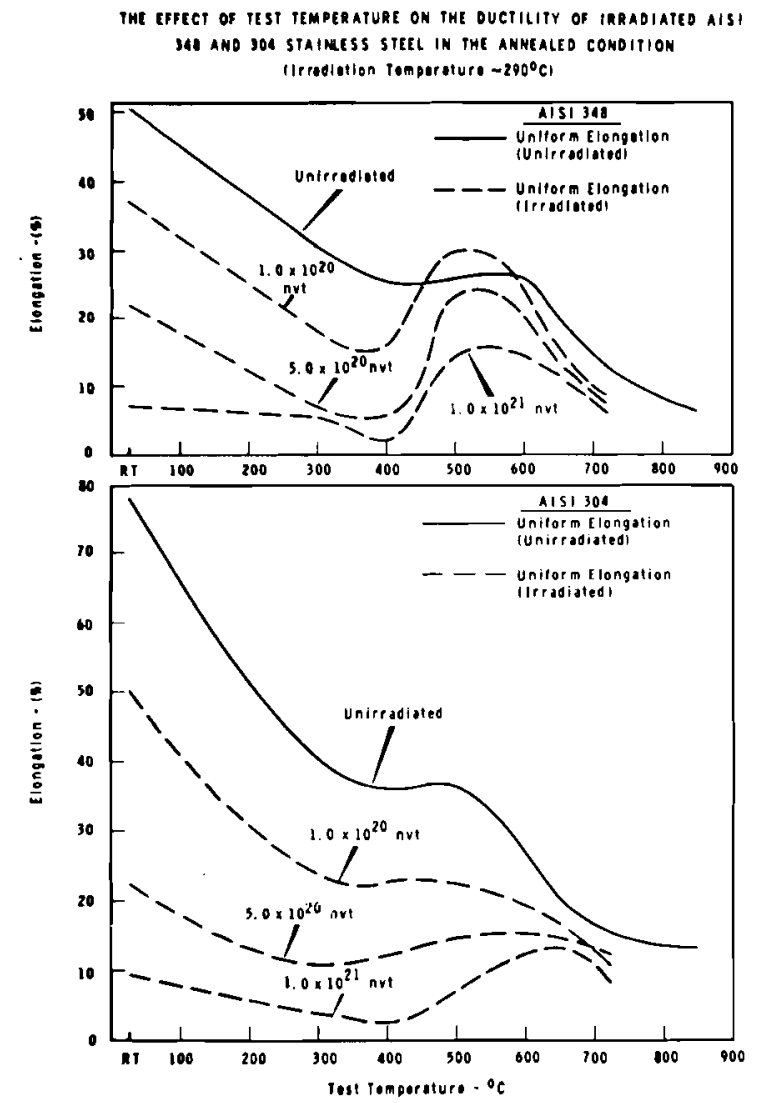


In-reactor creep properties of $304 \mathrm{SS}$ data is shown in Slide 6. We are performing these tests in a rig that measures the creep rate of material while under the influence of neutrons. We plotted the creep rate versus the reciprocal temperature for stress levels of $10,000,20,000,30,000$, and $40,000 \mathrm{psi}$ at temperatures from $350^{\circ} \mathrm{C}$ to $750^{\circ} \mathrm{C}$. The solid figures are the out-of-reactor controls, and the open figures are the irradiated controls. We found very little difference in the creep rate of $304 \mathrm{SS}$, in-reactor or out-of-reactor.

This sounds very optimistic until we look at the next slide (Slide 7) where we have plotted the Larson-Miller parameter, and indication of the log of the time-to-rupture versus the stress. The solid dots in Slide 7 are the out-of-reactor controls; the open dots are the in-reactor measured data. You can see that there is a shift to the left, which indicates a marked reduction in the time-to-rupture. If you take these data and calculate the time-to-rupture, you would find a difference of from 400 hours out-of-reactor to 80 hours in-reactor, or roughly a factor of 5 decrease in time-to-rupture.

ARRHENIUS PLOT OF IN-REACTOR AND EX-REACTOR CREEP RATES OF ANNEALED 304 STAINLESS STEEL

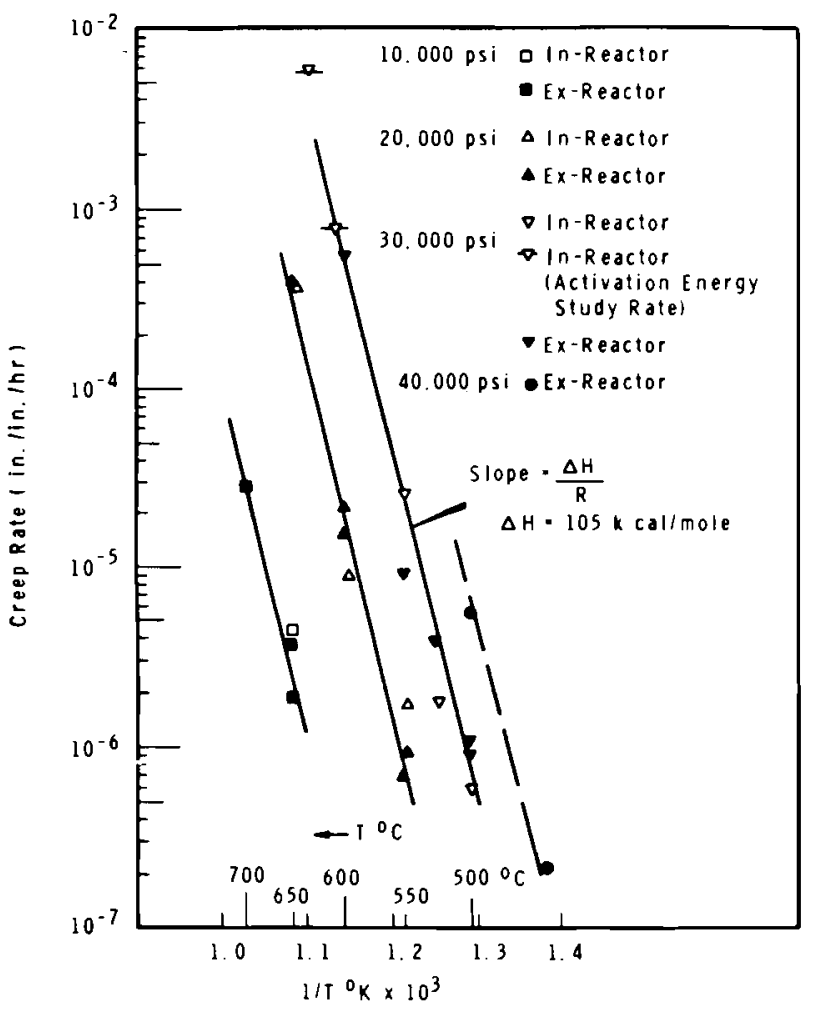

SLIDE 6
THE EFFECT OF IRRADIAIION ON THE CREEP-RUPTURE PROPERTIES OF ANNEALED 304 STAINLESS SIEEL

Stress vs the Larson-Miller Parameter $T$ in ${ }^{\circ} K$, tr in Hours

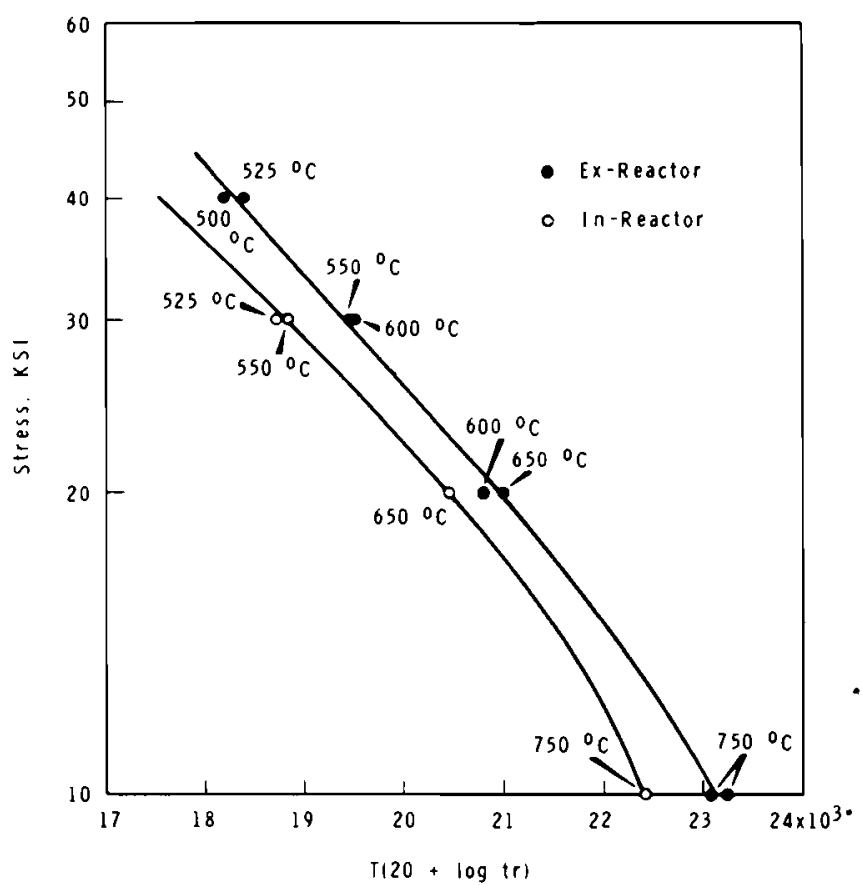

SLIDE 7 
In summary, I would like to show a slide (Slide 8) shown before, which points out some of the remarks made earlier. This is a rather complicated slide, so bear with me. On the far right we have the FFTF operating conditions where temperatures are operating at $1400^{\circ} \mathrm{F}$.

Exposures are shown where various reactor components would be seen in 30 days, one year, and 3 years in FFTF; which is way out into range of 7 to $8 \times 10^{23}$ nvt. Other data show that which has been collected to date. Data showing the irradiation temperature of $140^{\circ} \mathrm{F}$ is of interest because it is the only data on stainless steel that has gone out to exposure exceeding $10^{22}$ nvt. This work was done by Fred Shober at Battelle Columbus. He has tested at room temperatures and at about $600^{\circ} \mathrm{F}$. Shober has recently taken specimens from the same irradiation and run tests at $750^{\circ} \mathrm{C}$. The material was essentially perfectly elastic and shows very little plastic deformation before failure. Other data show irradiations at $550^{\circ} \mathrm{F}$, tested at various temperatures from 840 to $1200^{\circ} \mathrm{F}$, and a decrease in ductility.

\section{DAMAGED MECHANISMS}

We've talked about damaged mechanisms, and I think most of our attention this afternoon has been directed toward the gas transfor- mation and resulting damage that occurs in both thermal and fast reactors.

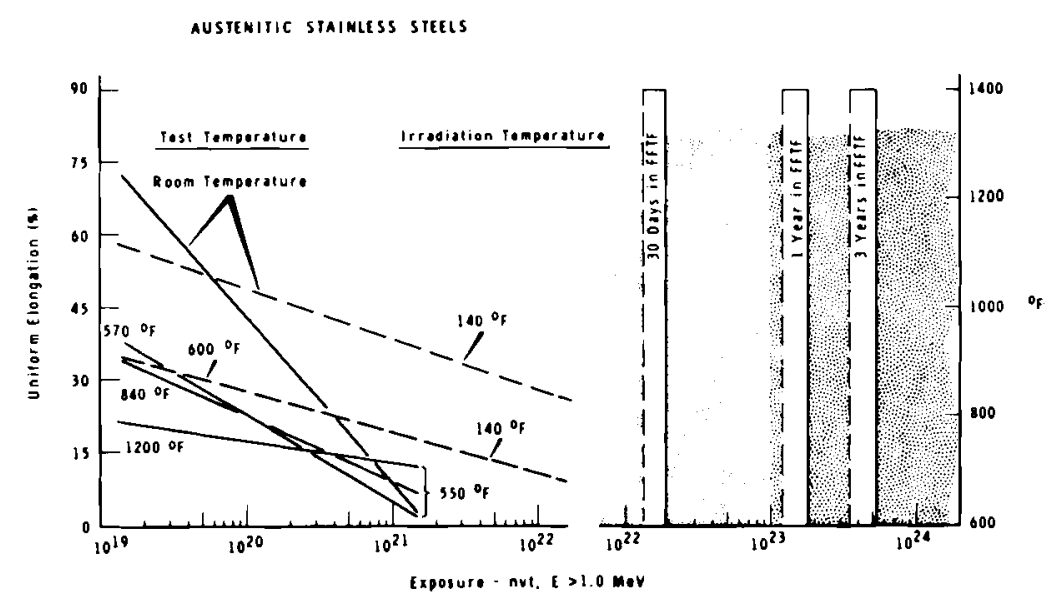


A schematic drawing (Slide 9) illustrates the various types of damage in terms of anticipated thermal and fast reactor damage. One is the grain boundary embrittlement problem due to the $\mathrm{B}^{10}$ burnup; another would be strictly a displacement phenomena talked about from the beginning. In addition, there are $(n, x)$ reactions occurring from the fast component of the flux primarily on the nitrogen iron, chromium and nickel components in the matrix. This would be primarily a homogeneous distribtution and could act as matrix strengthening agent through the formation of the interstitual helium.

Since we were worried about rates of damage in helium distribution in the material itself, we ran a few calculations showing the variations and how the helium is formed in the material (Slide 10). We plotted days in-reactor versus helium in parts per million from the $(n, \alpha)$ reactions on iron, nickel, chromium, and nitrogen in 304 stainless steel with about $14 \mathrm{ppm}$ boron in it. This data is for the ATR spectrum which burns out the $B$ quite rapidly. Since it is burned out, we have a constant concentration across the time scale. The total parts per million due to the $(n, x)$ reactions increases with time and the total curve is given. As a guide we used a $100,000 \mathrm{MWd} /$ ton exposure on a fuel pin plane in a facility. The total comes to something on the order of 18 or $20 \mathrm{ppm}$.

If we put a convertor around a fuel pin and try to shield out the thermal component, we might get a distribution as shown on Slide 11 where the $\mathrm{B}^{10}$ is still burned out quite rapidly. We have, however, a more rapid rate of the $(n, x)$ reactions on the iron, nickel, chromium, and nitrogen, and we get 100,000 MWd burn up in about a year corresponding to about $28 \mathrm{ppm}$.

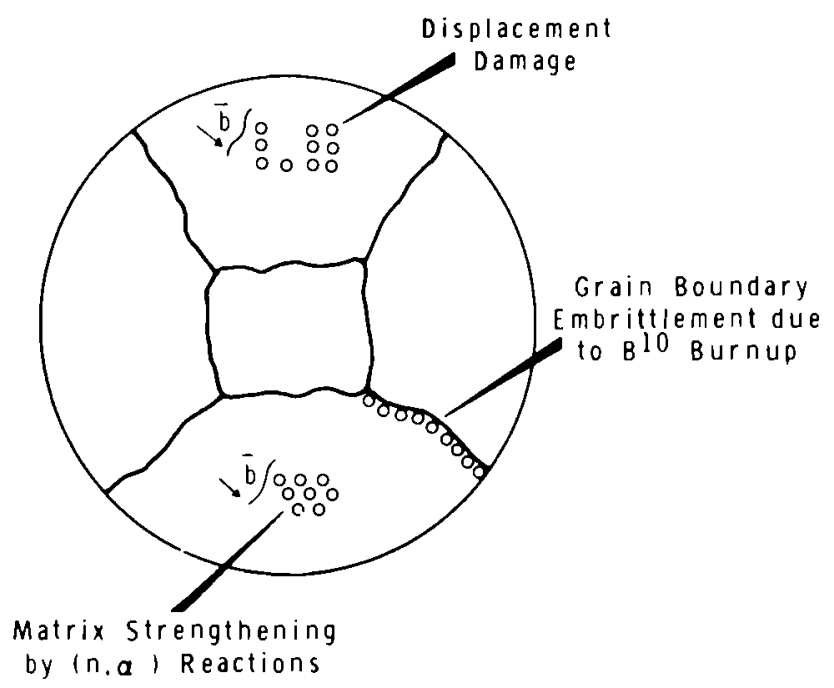


18.9

SLIDE 10

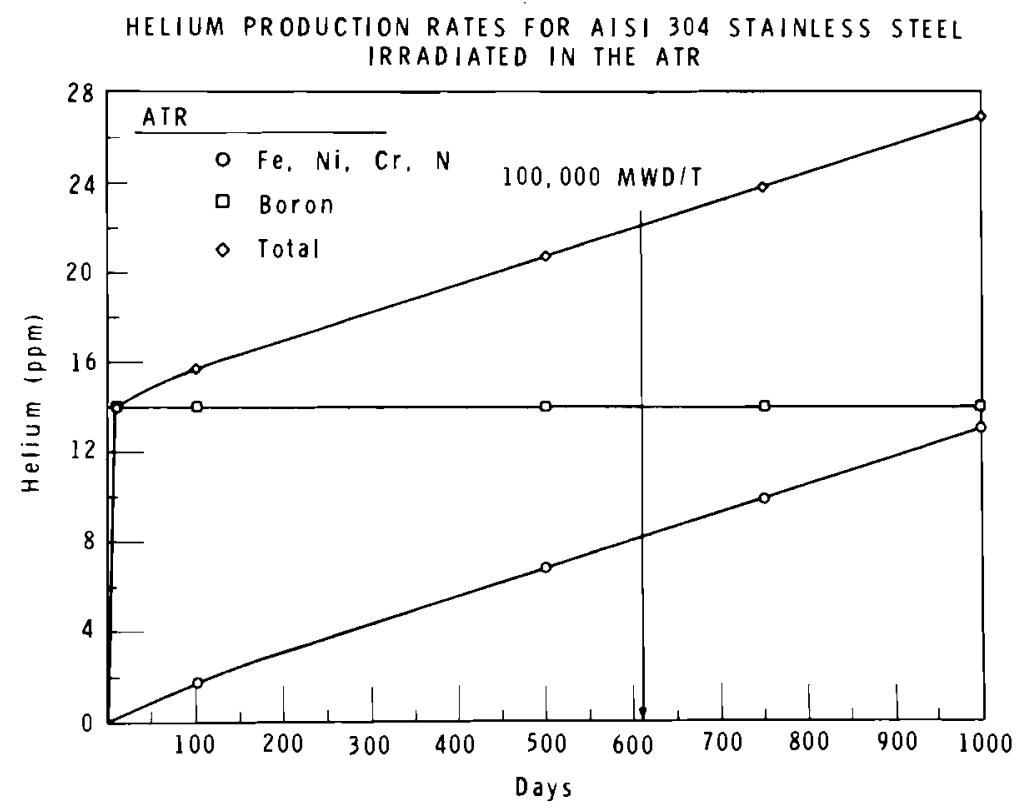

SLIDE 11

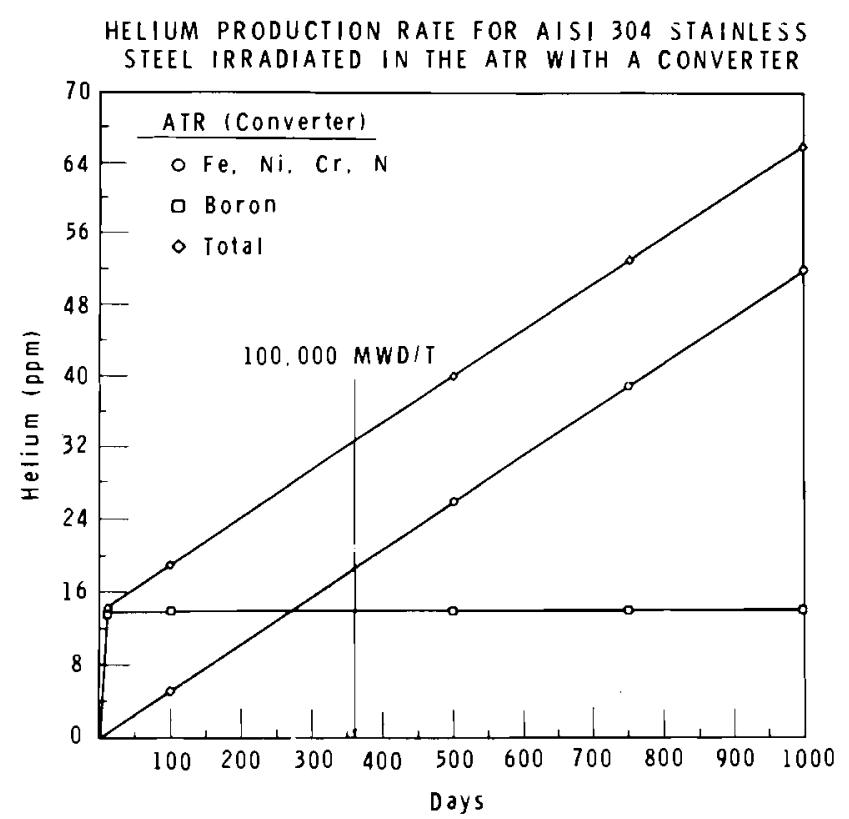


Slide 12 shows (FFTF) where there is very little thermal component, the $\mathrm{B}^{10}$ is never quite burned out even after 1000 days or the 100,000 MWd/ton exposure. In this case, in about 100 days we have something on the order of 6 ppm helium product.

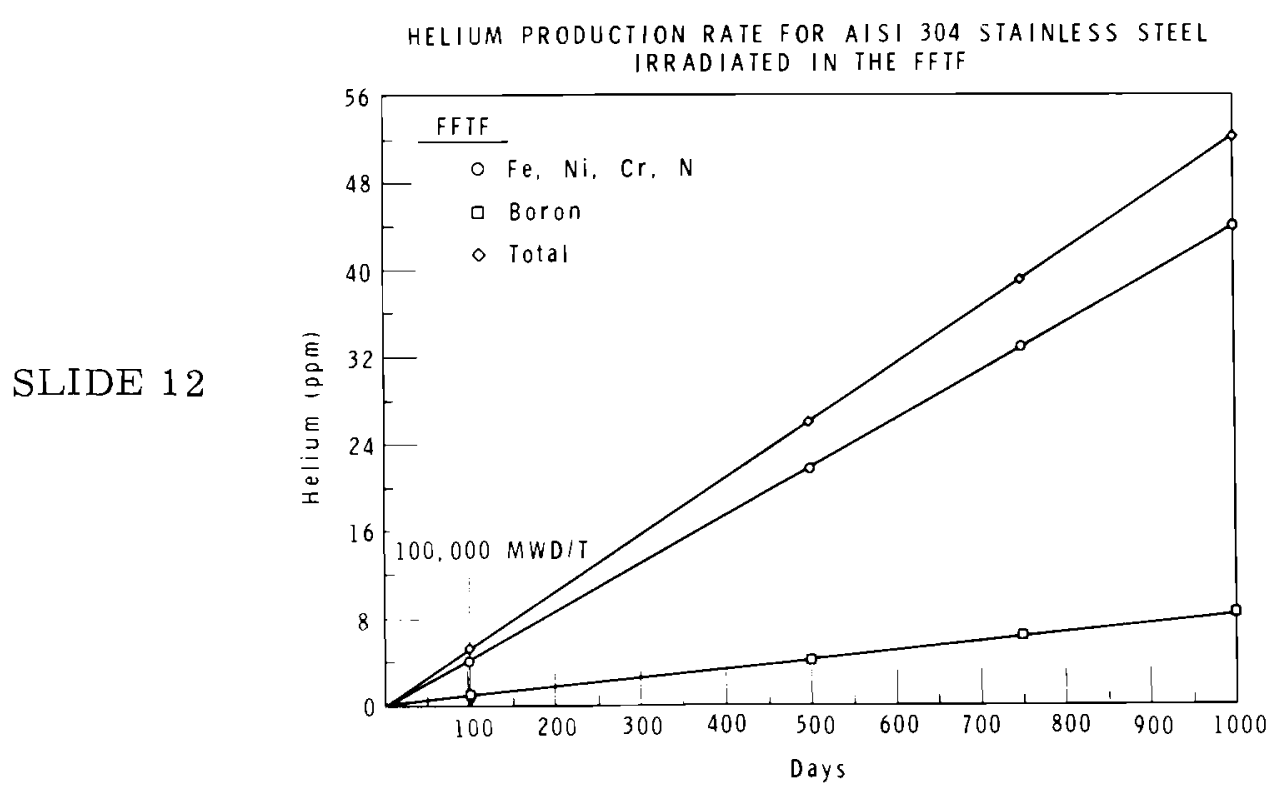

Slide 13 shows the difference in irradiation tests in a thermal reactor to simulate fast reactor testing. In an unshielded case we would have thermal neutrons coming in and primarily fast coming out, a fairly hard spectrum at the clad. Putting a cadmium shield around the pin, you would get primarily fast flux and 3 or 4 orders of magnitude thermal flux less than you had started with before. Again, fast flux would be coming out at the clad. In a fast spectrum we would have both fast flux going in and out of the cladding.

Relating this to Slide 14 then, we would have various distributions across a specimen or a fuel clad. If we had a grain boundary and a FOBR, we would have little increases in helium concentration at the grain boundary due to the boron ten burnup which had segregated at the grain boundary. In a thermal reactor such as the ATR, these would be quite significant with a constant $(n, \alpha)$ distribution.

I feel that worthwhile work is being done by Oak Ridge and other laboratories (including BNWL), in investigating improved fabrication methods for producing heats of steel which will alleviate some of the problems of irradiation damage. This work is being applied not only to stainless steel but to nickel base alloys and pressure vessel steels also. One thing we should not forget is the damage displacement portion of any effects that we might see in mechanical properties. 
HELIUM PRODUCTION RATE FOR AISI 304 STAINLESS STEEL IN FAST AND THERMAL SPECTRA

SLIDE 13

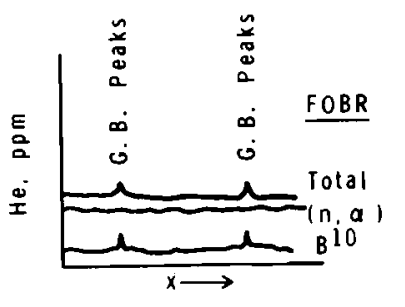

PFM HELIUM GENERATED TO

REACH GOAL EXPOSURE FOR FFTF TUBES

$\frac{\text { Reactor }}{\text { AIR }} \frac{B 10}{15.7} \quad \frac{\mid \mathrm{n}, a)}{2.6} \quad \frac{\text { Iotal }}{23.3}$

$\begin{array}{llll}\text { GETR } & 15.7 & 13 & 28.7\end{array}$

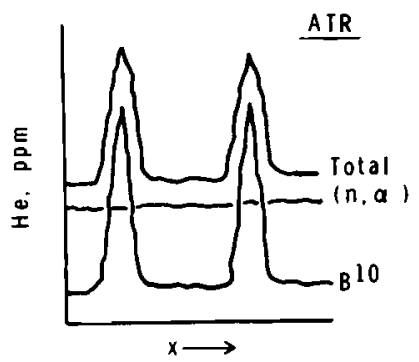

$\begin{array}{llll}\text { FOBR } & 1.6 & 4.3 & 5.9\end{array}$

$\begin{array}{llll}\text { FFIF } & 2 & 8 & 10\end{array}$

EFFECT OF NEUTRON SPECTRUM UPON FUEL CLAD DAMAGE MECHANISM

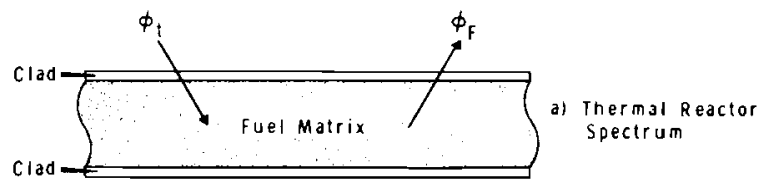

SLIDE 14
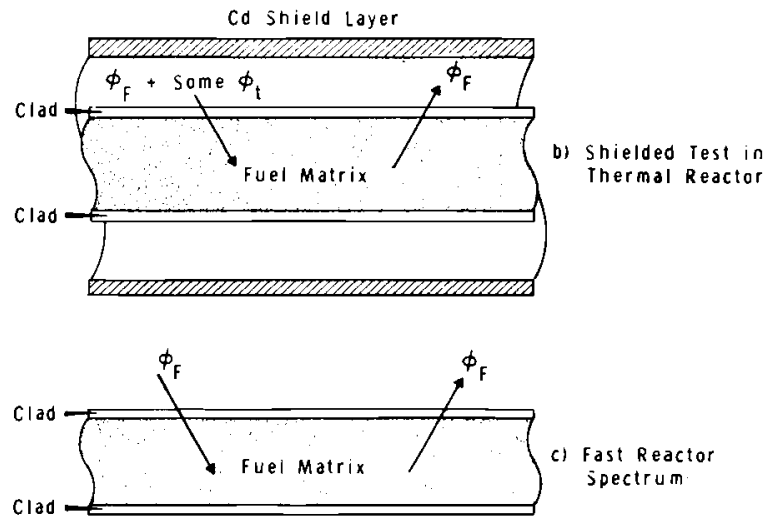
Having not paid too much attention to this type of damage in structural materials in thermal reactors, we've been lucky that this type of damage has not been catastrophic. I do not feel that this same statement can be made for fast reactor systems and especially when we consider the flux rates such as those in the FTR. An example is a very simple calculation that shows that each atom in the matrix of the material in the FTR will be displaced at least once a day. These rates are significantly higher than what we've ever seen before, but there is certainly a much greater chance of disorder reactions in the alloy than ever seen before in the thermal reactor spectrum.

\section{SUMMARY}

The future work we envision for this project right now is dealing with the corrosion mass transfer studies. This is where I think corrosion rates at the elevated temperatures are going to have to be determined. This will include nickel base and refractory metal systems because of the closed loop criteria. Also, we will have to look at corrosive effects in thin sections of fuel cladding on the mechanical property and phase stability of the alloys considered for use. We fully intend to continue irradiations in thermal reactors, including the ATR, which will be available to us in another few months. We are already using EBR-2 at our laboratory and Fermi reactor should be available to us in the near future. I think efforts to correlate material damage in thermal reactors to fast reactor spectrums is a necessity

We are quite interested in fuel clad and fuel interaction studies. They will have to be carried out under conditions where we have the proper rate of fuel burnup to fuel clad damage to get a realistic evaluation of just how serious our fuel problem is in terms of clad failure. I've already mentioned fretting, galling, and wearing studies which certainly will be required to get a feel for what is happening down at the grid plates between the fuel elements, and inside of the process tubes. One of the most important things to look at is, as I mentioned before, the effect of chemistry, fabrication processes, and environmental effects on the metallurgical stability and mechanical properties of the clad instruction materials. I think the idea of using titanium or other carbide formers is one approach to reduce irradiation damage. All these efforts, I feel must be directed toward the development of specifications, quality control, and nondestructive testing procedures to obtain the optimum heats of materials for use in the construction of reactor and fabrication components. 
D18.1

MATERIALS CONSIDERATIONS

T. T. C1audson

Pacific Northwest Laboratory

Operated by Batte1le Memorial Institute

Richland, Washington

\section{DISCUSSION}

MR. KITTEL: I have a question. What was the nvt? What flux were those done in and how appropriate is this to the to the $10^{16}$ flux that would exist in the FFTF?

MR. CLAUDSON: We11, Howard, I get this question every time I present these data, and I think I am going to quit presenting them. But these tests were conducted in a Hanford Production reactor, and such information as you request is classified. Someday, you come out with your little green badge on, and we can talk about this. I think it is fairly obvious, though, that the spectrum is certainly the same as the FFTF produces. I think that is safe to say. I am sorry, but that is all I can say.

MR. KELMAN (Argonne): I don't know why the 304, 316 creep strength keeps coming back, but the latest data seems to show the emperatures on the order of $1200^{\circ} \mathrm{F}$. They cross over, if anything. I don't know what data, you are showing. data.

MR. CLAUDSON: We11, these data are on the old ASME code now.

MR. KELMAN: These have been obsolete for 10 or 12 years

MR. CLAUDSON: They may be. The ASME new code values do indeed show that there is a cross over between 304 stainless and 316 at about at about 1200 to $1400^{\circ} \mathrm{F}$. Those data, (as I know) have not come out yet.

MR. KELMAN: They are out.

MR. CLAUDSON: They have come out?

MR. KELMAN: Yes. It seems to me that 316 got into this picture of socium corrosion and other programs early in the game, and it is very hard now to ever get 304 into the picture. Each time we repeat the old data, we continue the same old problem. Maybe once and for all, everyone could shift gears and decide that at this temperature (especially because 304 is considered more stabe material thermally), it is at least equivalent of 316 . 
D18.2

MR. CLAUDSON: In fact, as I mentioned, we have already selected 304 for the temperature ranges that I talked about (at $1200^{\circ} \mathrm{F}$ mixed outlet temperature). This means your fue 1 clad is up another 50 , or 200 degrees possibly, on the other side. This is $1400{ }^{\circ} \mathrm{F}$ for stainless steel. This is getting up to where I think people are going to have to do some serious thinking before using it.

MR. HANTHORN: Most of your irradiation data is radiation of 1 ow temperatures with the actual high temperature tests. Would you care to conjecture as to the effect of elevated temperatures?

MR. CLAUDSON: We have irradiated specimens (and I think Oak Ridge has too) up to about $1200^{\circ} \mathrm{F}$ or so. Our radiations have gone up to $750{ }^{\circ} \mathrm{C}$ (whatever that is in Fahrenheit), and we have tested up through 850 with it. These data show a marked reduction, still about the same level about that length of exposure, on the order of 5 or $6 \%$. And this is really dependent, I think, on each material variation, the processing etc., because you can irradiate several of these materials under the same conditions, and get quite a wide scatter range.

MR. KELMAN: You mentioned displacement as being a worse problem than we have experienced before in thermal conditions. I wonder, at the temperature you are talking about, if you don't expect to have these displacements annealed out? This is quite possible. I haven't done the study myself. It seems very likely to me that (with metal instability or any other property of material) once you start displacing one atom for the same atom every day, something is going to give somewhere. And certainly, just intuitively speaking, I feel that we are on as safe ground (as we are) in thermal conditions. The probability of annealing and so forth is very true. Meley gave a very excellent talk last week on this subject, and I can say that his model today shows annealing of the damage etc. To solve the question about the $10^{16}$ flux and displacing everything once a day, I would say that he has to work out a new model.

MR. CLAUDSON: I think the general position is that there will be more residual damage at the same temperatures at higher and higher fluxes because of the more complex clusters formed which are more difficult to anneal out. It is hard to put a quantitative value on it; qualitatively, it appears to be in that direction.

MR. deHALAS: I believe there have been studies indicating that this does happen.

MR. KITTEL: I think we do have data that bears this out. Some of the early irradiations were done in EBR-I which is, of 
D 18.3

course, a hard spectrum too. These show that the changes in the mechanical properties occurring in stainless steel radiated in that reactor could be almost entirely anneled out at $500{ }^{\circ} \mathrm{C}$. Yet in EBR-II, which has a factor of (perhaps) 10 to 100 greater, there is considerable embrittlement from displacements, but is still in after the irradiation of $550^{\circ} \mathrm{C}$.

MR. CLAUDSON: I showed this at the last one of these meetings here at Hanford, Howard, that we do see evidence of displacement damage in some of our elevated temperature tests. It is pretty difficult; there are so many things going on at the same time.

MR. KITTEL: We11, it does seem that the higher the flux, the more this annealing thing comes to the front.

MR. HENNING: Thank you a11. We have one last item on our agenda, but before we get to that, I want to tell you that I have up here on the table the remains of 50 copies of what is entitled The Analytical Mode1 for the FFTF Fuel Cycle Cost Studies. It also includes a core design analysis. Now many of you will have different specific interests in this. There were questions raised on fabrication costs; our model will show the constants that we used. Other questions are how we computed the temperatures and the bases for predicting the a allowable burnup; these are all cited in the paper. I think it is easy to follow. The important point I want to make about the whole thing is this: I think you can say we will stand behind the method completely; the physical constants and the correlations used are the subject of many discussions right here; and we are fully aware that these are not in any sense accurate and precise, but they are all indicative. 


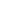


OXIDE AND CERMET FABRICATION

AT PACIFIC NORTHWEST LABORATORY

W. E. Roake

Pacific Northwest Laboratory

Richland, Washington

Operated by Battelle Memorial Institute

\section{INTRODUCTION}

Pacific Northwest Laboratory is engaged in a plutonium fuels development program which began in 1956. The program is broadly based, covering many aspects of fuel technology. Its objective is to develop and demonstrate the necessary technology for safe and economical fabrication of plutonium fuels. In addition to the Test Reactors in Idaho, our principal tool has been and continues to be the PRTR. More recently, we have joined other laboratories in providing plutonium-based fuel for experiments in the EBWR and the Saxton reactor. These development efforts have been supported strongly by basic research programs funded by Fuels and Materials Branch, Division of Reactor Development and Technology. Although fuel development work has been principally focused on thermal reactor systems, most of the technology developed is applicable to fast reactor systems.

Program efforts have been highly successful, thanks to the early planning and guidance of a lot of people including Ivor Thomas and Ersel Evans, both of whom have moved on to other pastures. Some of the more important contributions include:

- Development and demonstration of packed-particle fuels

- Ceramic fuel fabrication by vibrational compaction and by hot-or cold-swaging

- Plutonium alloy fuel fabrication by extrusion and rolling

- Determination of basic properties of fuel materials, particularly at high temperatures

- Advances in fuel element designs, materials and fabrication techniques

- Establishment of world-wide fuel material and information exchange

- Extensive fabrication cost studies based on experience

- Establishment at PNL of a broad plutonium fuel development capability. 


\section{DISCUSSION}

We have maintained a close working relationship between fabrication, research and support functions. Had the draftsman drawn two more lines in S1ide 1 , it would show this relationship as a tetragon, which has a stable structure no matter which face it rests upon.

SLIDE 1

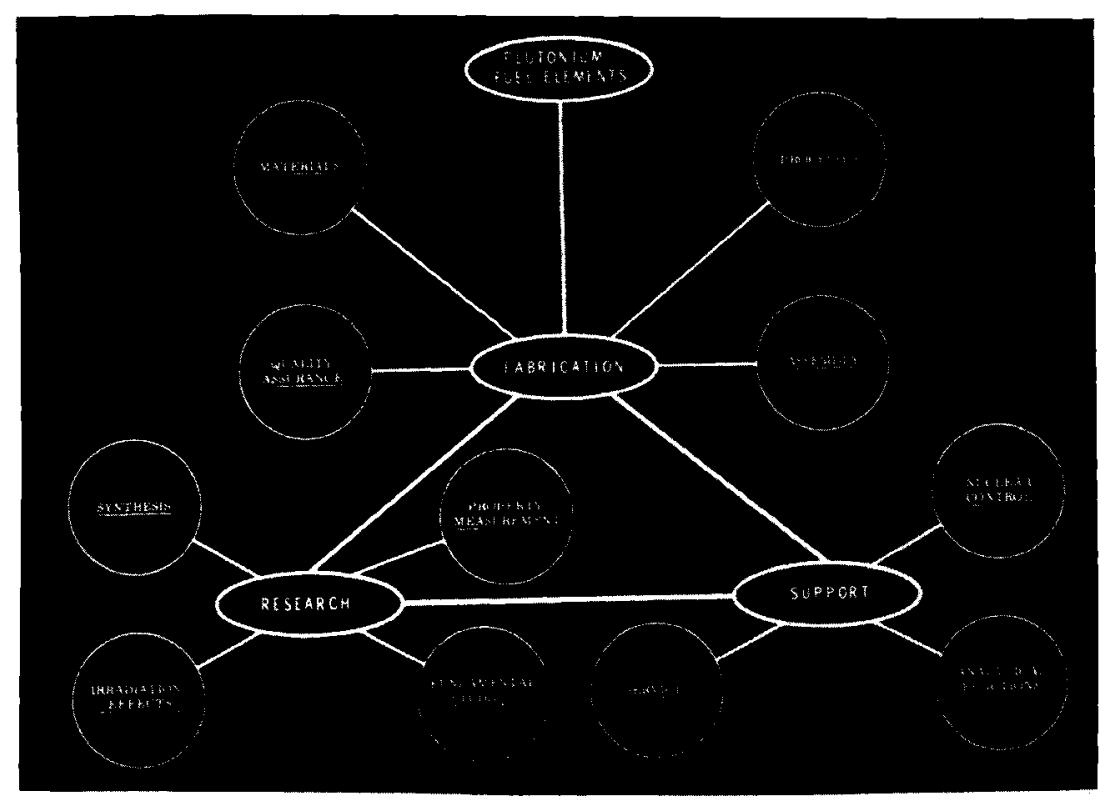

This is the Plutonium Fuels Laboratory (Slide 2). I show you this slide for two reasons: first, to indicate that our weather is not always overcast, and second, so that you will know when to get off the bus available immediately after this talk.

SLIDE 2

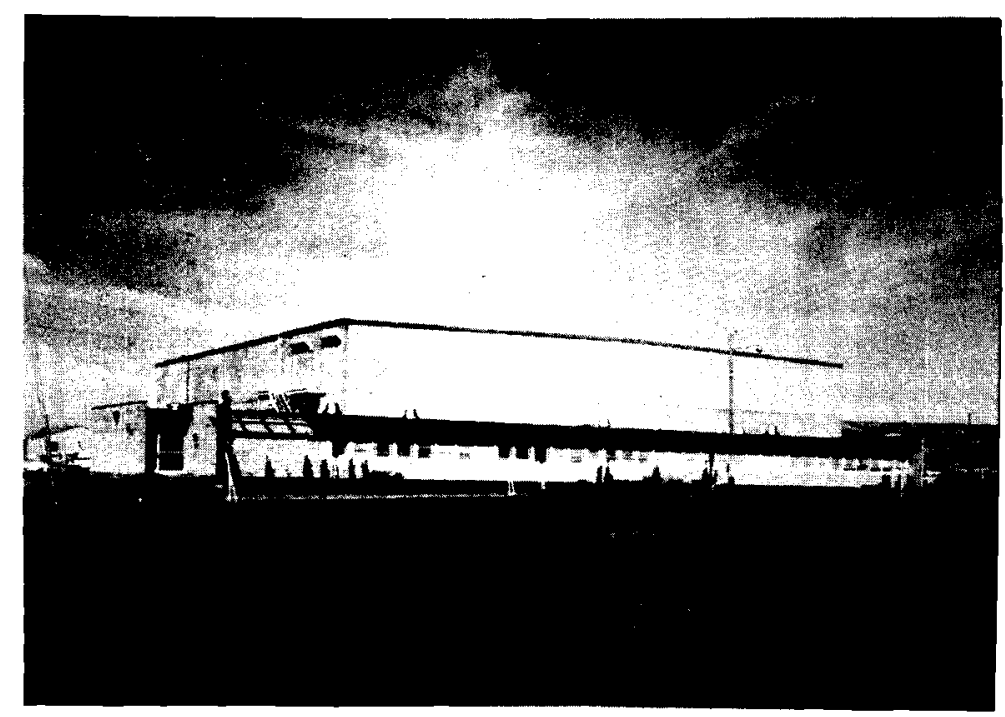


Process development capabilities are extensive. S1ide 3 shows some of the various types of test elements that have been made. Mass varies by six orders of magnitude.

SLIDE 3

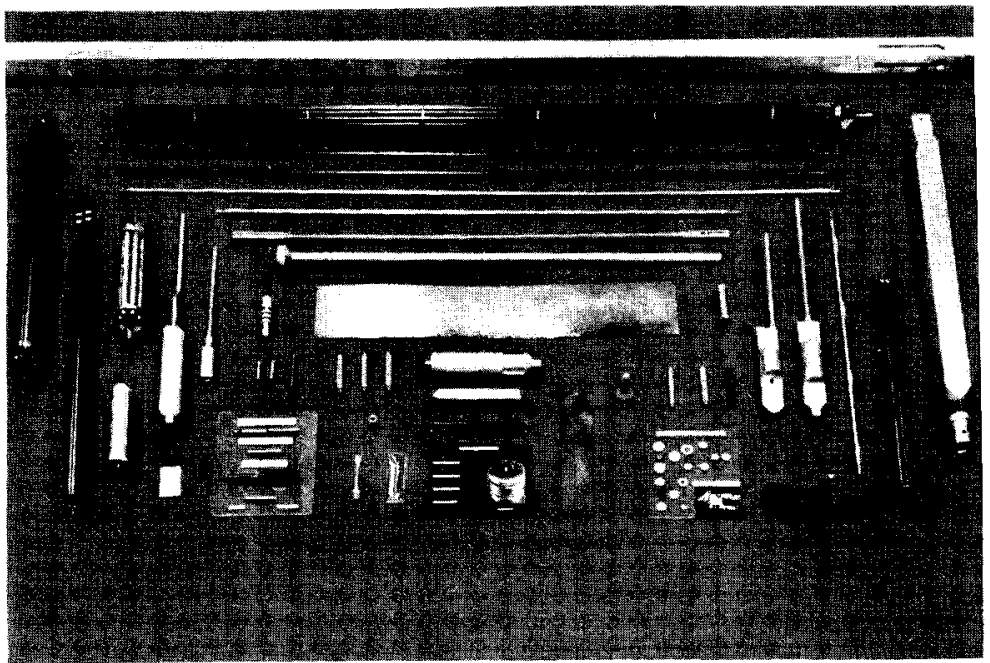

I am going to show you an old slide (S1ide 4). It is one that many of you have seen before, but one which illustrates the point that the irradiation program in PRTR has reflected continued changes in fuel element design and fabrication processes. Each color depicts such a change, evaluated on a statistically significant basis.

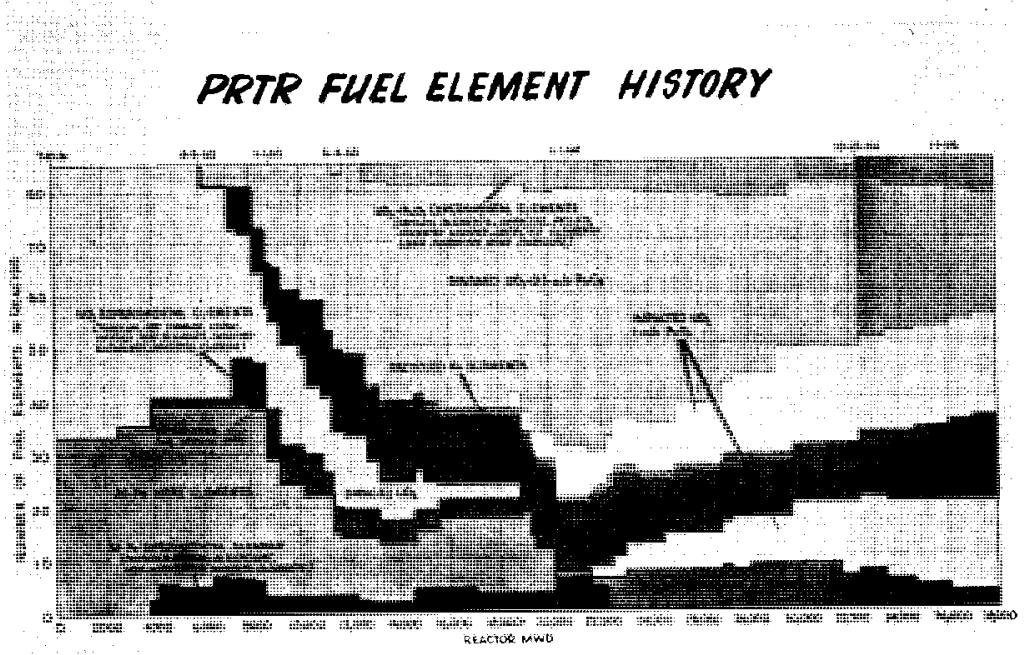


Three of the major design variations tested in PRTR are shown in Slide 5. Two of these are obviously much simpler to make from packed fuel particles. The 19 rod cluster can be made equal1y well and at about the same cost by either the pellet or packed particle route. We have specialized more on packed particle fuels because of the intensive and competent effort on pelleting at other laboratories.

SLIDE 5
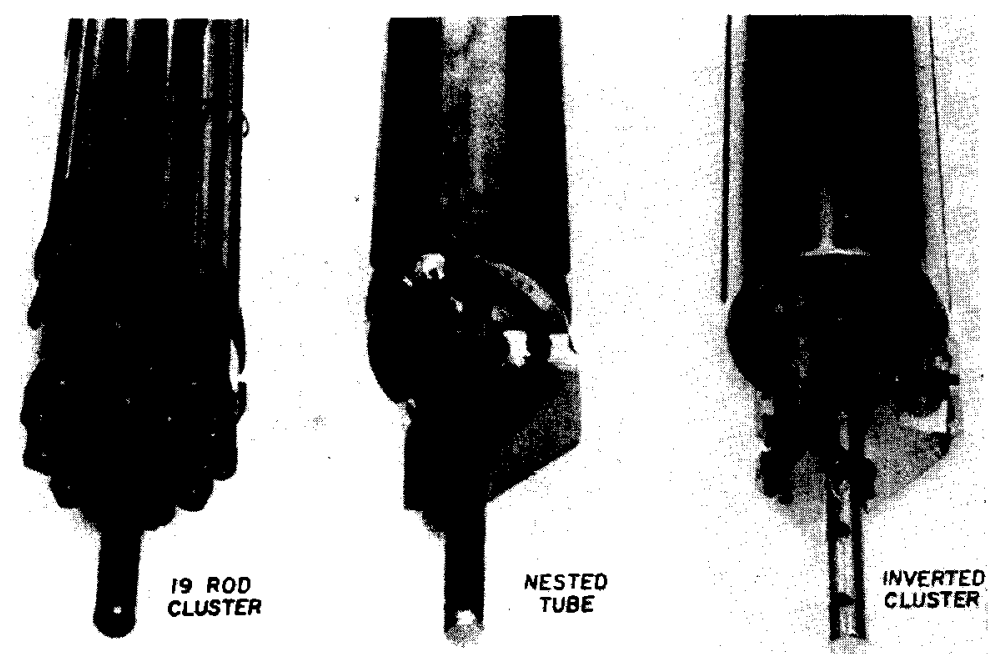

\section{PRTR FUEL ELEMENT CONFIGURATIONS}

From early studies on elements made from prefused $\mathrm{UO}_{2}$, we have progressed through several methods of loading mixtures of plutonium and uranium oxides into fuel elements. The most recently studied method for preparing high density particles comprise of dispersions of $\mathrm{PuO}_{2}$ in $\mathrm{UO}_{2}$ is high energy-rate impaction. I believe Walt Hampson intends a demonstration during the laboratory tour.

Impaction has several advantages. High densities are achieved without high temperatures, and the ratio of plutonium to uranium atoms in the product is that of the raw powder. Plutonium spatial distribution is fixed and depends on the degree of powder blending. Slide 6 outlines the process flow pattern.

Slide 7 shows Carl Burgess about to blow the whistle on a $151 \mathrm{~b}$ can of mixed oxides for PRTR.

A lot of words have been said about the need, or lack thereof, for atomically dispersed plutonium for safe fast reactor mixed oxide fuels. I don't want to get into that argument here. I will show you how high resolution autoradiographs of coprecipitated and sintered mixed oxide compares with those of compacted, mechanically mixed powders (Slide 8). 


\section{MIXED OXIDE PREPARATION}

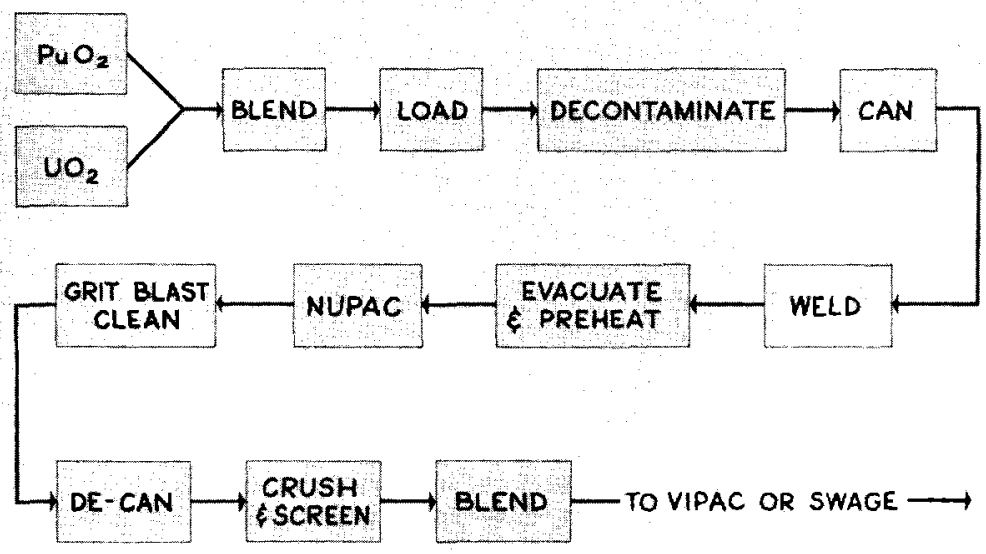

SLIDE 6

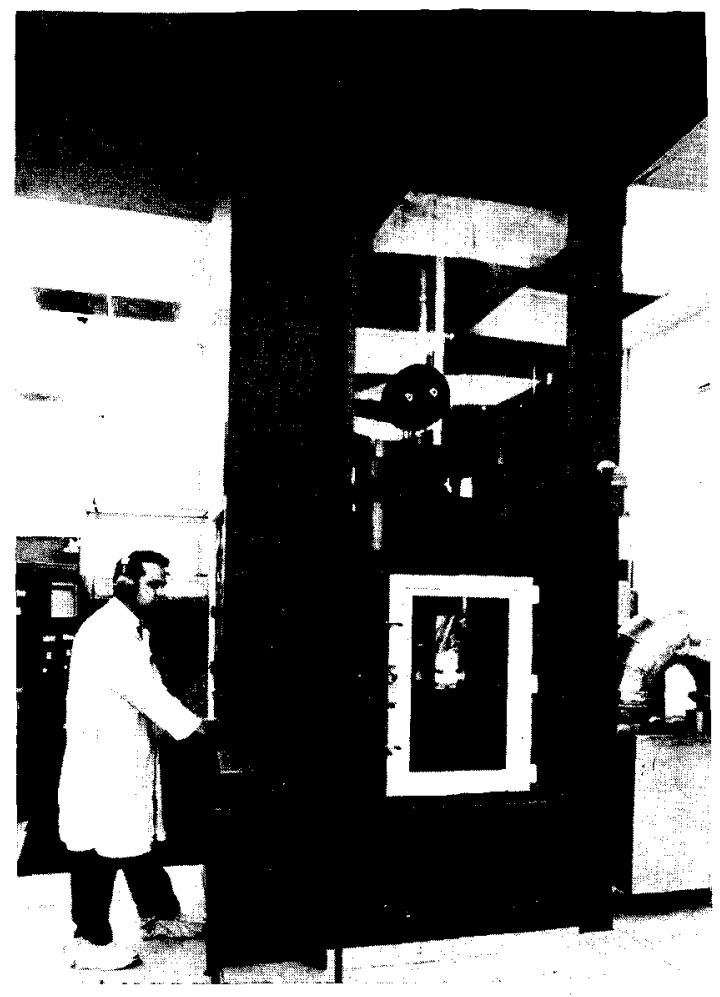

SLIDE 7
MECHANIGALLY MIXED-IMPACTED $\mathrm{UO}_{2}-20 \mathrm{~W} / \mathrm{O} \mathrm{PUO}_{2}$ FAST REACTOR FUEL STUDIES COPRECIPITATED \& SINTERED $30 x$ $500 x$

BALL-MILLED \& IMPACTED MILLING TIME (HOLRS)

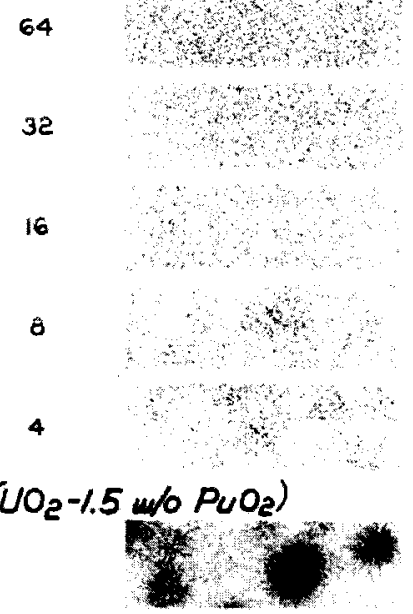

BLENDED $\left(\mathrm{UO}_{2}-1.5 \mathrm{wO}_{\mathrm{O}} \mathrm{PUO}_{2}\right)$ 
Russ Peterson calculated thermal time constants for transfer of heat from discrete $\mathrm{PuO}_{2}$ particles to surrounding $\mathrm{U}^{2} 38$ (S1ide 9). Impacted mixtures seem reasonably safe.

SLIDE 9

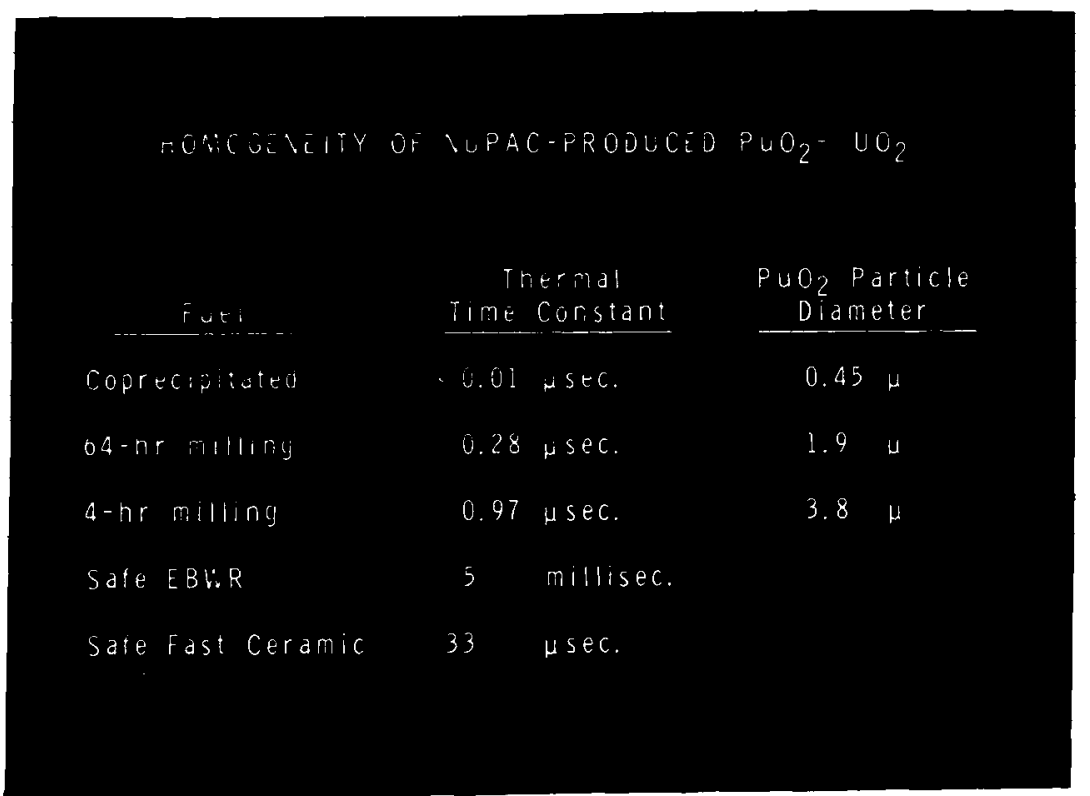

Stainless steel-PuO 2 cermet development studies were started during FY-1965. So far this has been a low level effort aimed only at providing quick and dirty answers needed to provide some respectability to our FFTF Conceptual Design studies. Efforts were focused on methods for fabricating cermets and on the irradiation behavior of these cermets. Three methods were screened:

- Coextrusion

- High energy rate impaction

- Cold pressing.

Coextrusion was found to be a relatively simple method for producing integrally clad and capped small diameter cermet pins. It is also useful for nonclad pins for sodium bonding. Impacted cermets were produced almost completely free of $\mathrm{PuO}_{2}$ particle damage or stringering and approached $100 \%$ of theoretical density. Cold pressing was used to produce cermets with bulk densities of 80 to $85 \%$ of theoretical. Stainless steel clad, stainless steel- $\mathrm{PuO}_{2}$ cermets with densities of 95 to $98 \%$ of theoretical, were routinely produced by coextrusion. For the most part, the high density $\mathrm{PuO}_{2}$ particles in these cermets were undamaged and unstringered. Preliminary studies were performed by using low density fuel particles $\left(\mathrm{UO}_{2}\right)$ to determine if the extrusion process can produce a cermet having a high density matrix and a low density fuel particle. This combination is considered desirable for accommodating the buildup of fission products during irradiations. These studies indicated 
that extruded cermets can be made which contain low density (80-85\% theoretical density) fuel particles. Some densification of the fuel particles occurs during extrusion--75\% dense particles end up 80-85\% dense. We've worked with low density particles made by powder agglomeration and sintering and by crushing low density pellets; the results are about the same.

Particle shape was studied as a process variable. Extruded cermets containing angular particles and spherical or rounded particles were made. The tendency for particle fracture and stringering was almost completely eliminated when rounded or spherical fuel particles were used (S1ides 10-11).

SLIDE 10

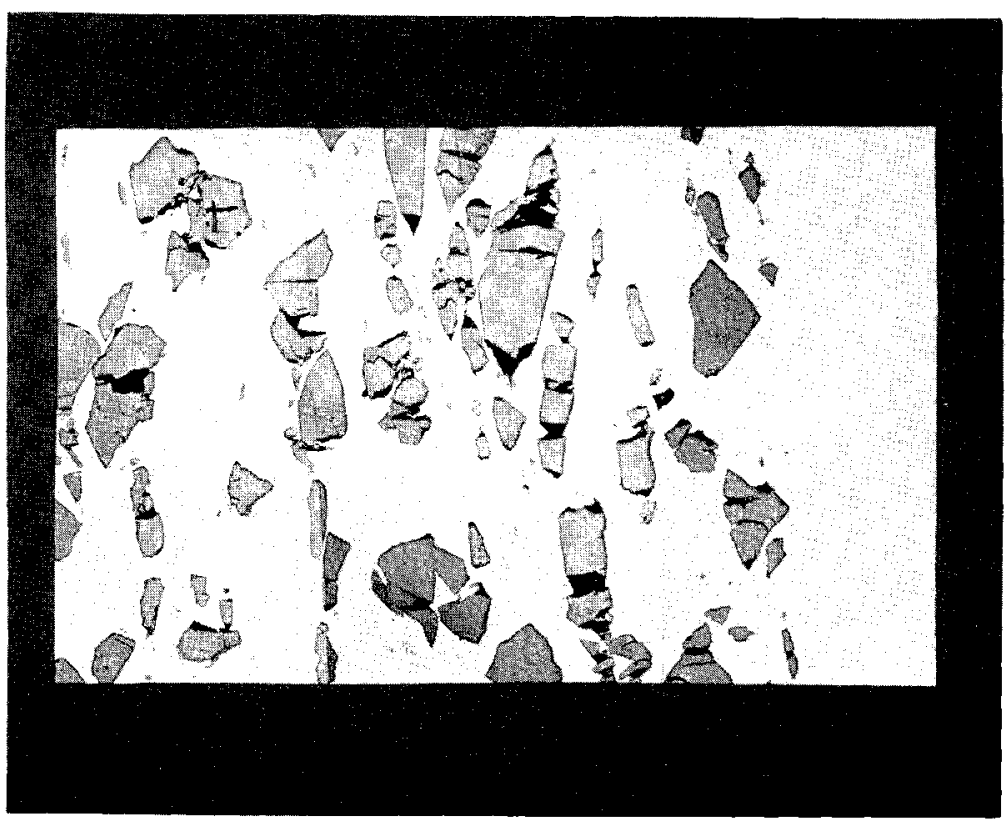

SLIDE 11

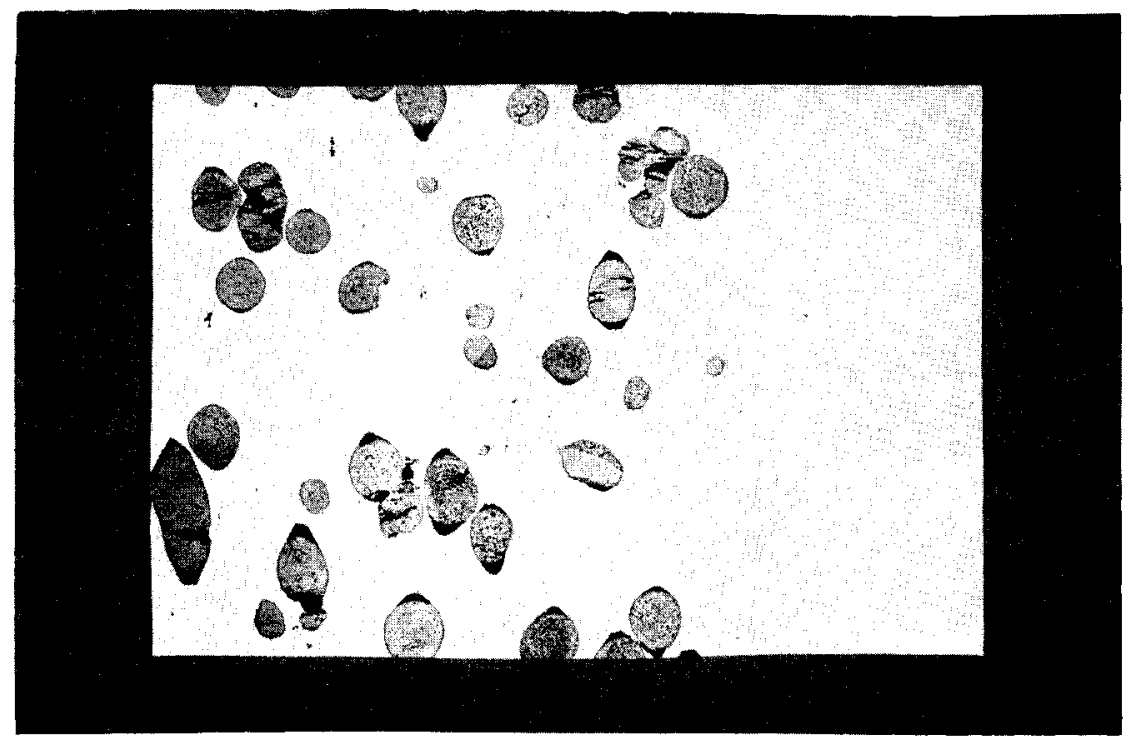


A method of "jet milling" was developed to produce rounded fuel particles from angular $\mathrm{UO}_{2}$ or $\mathrm{PuO}_{2}$ particles. Depending upon the fuel material, milling times between 30 and 120 minutes yielded particles sufficiently rounded for cermet use. The rounded particles retain the physical and chemical properties of the feed material (SIide 12).

Some of our experimental cermets have been irradiated. High and low density stainless steel-20 vol\% $\mathrm{PuO}_{2}$ cermet pellets made by impaction or by cold pressing were irradiated in MTR to $2.3 \%$ and to $6 \%$ burnup of the plutonium atoms. Peak rod power was $46 \mathrm{~kW} / \mathrm{ft}$. These were $1 / 2$ in. diam pellets clad in $304 \mathrm{SS}$ and double clad in aluminum.

The high density cermet operated with a maximum centerline temperature of approximately $1000^{\circ} \mathrm{C}$. A few small cracks were present in the central portion of the specimen; otherwise, the cermet appeared to be in excellent condition. A slight amount of sintering of the $\mathrm{PuO}_{2}$ occurred in the hotter part of the specimen. No evidence of any reaction between the stainless steel and the $\mathrm{PuO}_{2}$ was observed, nor was there any evidence of suboxide formation in the $\mathrm{PuO}_{2}$. You can examine the micrographs during the tour.

Central melting occurred in the low density cermet and caused a central void. Central temperature is estimated to have been over $1400^{\circ} \mathrm{C}$. Surrounding the central void was a region comprised of a dense pore-free matrix with agglomerated $\mathrm{PuO}_{2}$. Extensive sintering of the $304 \mathrm{~L}$ stainless steel matrix as well as the $\mathrm{PuO}_{2}$ particles had occurred. A change in appearance of the $\mathrm{PuO}_{2}$ gives evidence of a reaction between the $\mathrm{PuO}_{2}$ and some constituent in the stainless steel, possibly silicon. There appeared to be no degradation of the stainless steel matrix outside the melt region. In the hotter regions of the specimen, the $\mathrm{PuO}_{2}$ structure is indicative of suboxide. Fission gas release was $35 \%$.

Eight cermet fuel pins were irradiated in the ETR P-7 Loops to about 4 to $5 \%$ burnup of the heavy atoms (S1ide 13). These pins operated at a calculated peak rod ower of $14 \mathrm{~kW} / \mathrm{ft}$, with a surface temperature approximately $400^{\circ} \mathrm{F}$. Four contained cold pressed, low density cermet pellets ( $80 \% \mathrm{TD})$. The other four contained high density (95 to $97 \% \mathrm{TD})$ cermet cores coextruded with stainless steel cladding and integral end plugs. The fuel pins are $12 \mathrm{in.}$ long and $0.25 \mathrm{in.OD}$, with a core diameter of $018 \mathrm{in.}$ Core compositions include stainless steel-20 vol\% $\mathrm{PuO}_{2}$, stainless stee1-20 vol\% beta $\mathrm{Pu}_{2} \mathrm{O}_{3}$, and stainless stee1-30 vol\% $\mathrm{U}^{23} \mathrm{O}_{2}$. Postirradiation measurements revealed no significant dimensional changes in those pins.

Postirradiation examination of the low density cermet pins showed essentially the same results as did the MTR tests already mentioned. 


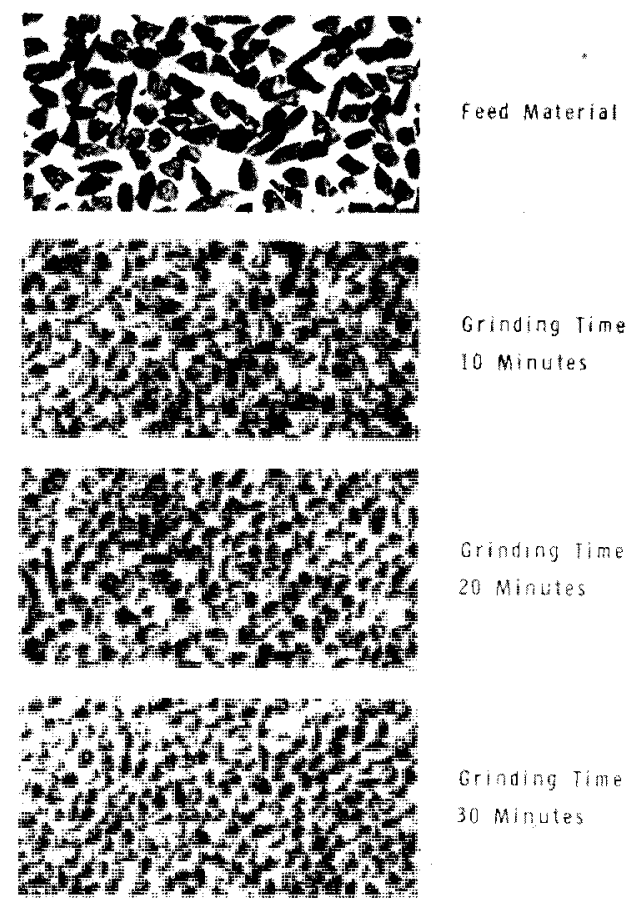

AIR GROUND UO, PARTICLES $(20 \times)$ $(-60+80$ Mesh)

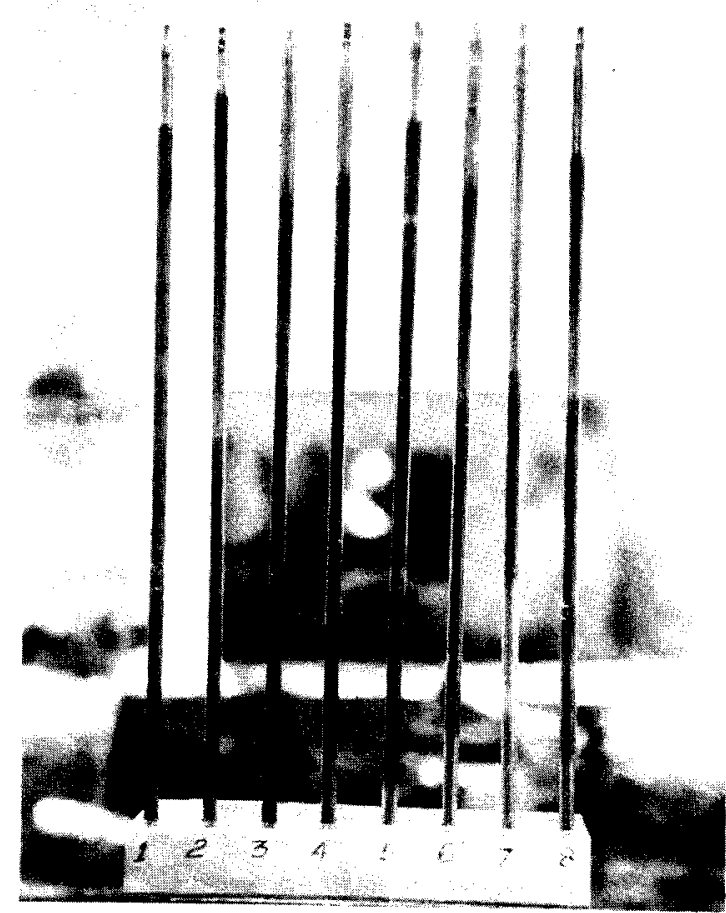

IRRADIATED CERMET FUEL PINS

3athles Steel 20 we $\mathrm{PuO}_{2}$ and stamless steel 30 wo 402 . Hign and low density Cermet fuel pins liratided at L. Kwin to 5 \% B. U. of the peav Alor. Max Cermet Tent. $800-1400$ oc

The four pins of extruded cermets were in excellent condition after irradiation. The centerline temperature of these pins was estimated at $1000^{\circ} \mathrm{C}$. There was no evidence of matrix degradation. The metallurgical bond between the cermet core and the stainless steel clad was retained throughout the irradiation. A slight reaction between the $\mathrm{PuO}_{2}$ and the stainless steel occurred, possibly silicon extraction. The $\mathrm{PuO}_{2}$ particles did undergo some sintering; however, no evidence of suboxides was detected. No reaction occurred between the $\mathrm{UQ}_{2}$ and stainless steel in the fuel pins containing $30 \mathrm{vol} \% \mathrm{U}^{235} \mathrm{O}_{2}$ cermet cores.

Extruded, high density ( $97 \% \mathrm{TD})$ cermet pins have been fabricated for irradiation in the EBR-II. The core compositions include stainless stee1-20 vo1\% $\mathrm{PuO}_{2}$, stainless stee1-30 vol\% $\mathrm{PuO}_{2}$, and stainless stee $1-20$ vol\% UO 2 . These pins are 0.3 in. in diameter by 18 in. long. They were made with high density ( $95 \% \mathrm{TD})$ fuel particles rounded by the "jet milling" process. They are in the hands of ANL for encapsulation and are scheduled to be changed into EBR-II during the next couple of months. They are to be irradiated to $\sim 2 \%$ and $5 \%$ burnup of the heavy atoms at a maximum centerline temperature between 600 and $680^{\circ} \mathrm{C}$. The expected maximum rod power will be $9.5 \mathrm{~kW} / \mathrm{ft}$. 
Other extruded $\mathrm{PuO}_{2}$-SS pins using low density particles are planned for irradiation tests in the near future.

We have also supplied Howard Kittel with $5 \mathrm{~kg}$ of ballmilled and impacted mixed oxide, which he has made into both Vipac and pelleted pins for EBR-II. Two vibrationally compacted pins are in the reactor now.

$\underline{\text { SUMMARY }}$

In summary, the PNL plutonium fuel development program has principally emphasized packed particle mixed oxides, with recent excursions into $\mathrm{PuO}_{2}$-stainless steel cermets. Approximately 10 tons of plutonium-based fuel involving more than $350 \mathrm{~kg}$ of plutonium have been involved in process development and fuel evaluation studies. 
20.1

OXIDE FUEL, STATUS AND STRETCH CAPABILITIES

\author{
E. L. Zebroski, W. E. Baily, S. A. Rabin, and J. H. Field \\ General Electric Company \\ San Jose, California
}

\title{
INTRODUCTION
}

We will cover some highlights of our oxide fuel program, having some bearing on the potential use of oxide as driver core for FFTF. The work covers experiments with pins ranging from $100 \mathrm{mil}$ diam to $220 \mathrm{mil}$ diam; mixed oxide with 20 to $35 \% \mathrm{PuO}_{2}$ content; and specific powers ranging from under one thousand to over three thousand $\mathrm{W} / \mathrm{g}$ of fissile, or plutonium content (some of them are spiked with $\mathrm{U}^{235}$ ). We will not really try to explicitly cover the stretch capabilities since the stretch capabilities seem to be in the same category as those being explored for conventional oxide fuels. This mainly entails running oxide in the molten condition. We have run mixed oxide fuel as high as $45 \mathrm{~kW} / \mathrm{ft}$, but I'm not sure that there is not as yet any reasonable design basis for operating routinely therein a reactor core. We will stay with the experiments that mainly pertain to the 15 to $20 \mathrm{~kW} / \mathrm{ft}$ range of operation. Also, we will not cover fabrication processes in any depth except to mention that in the experimental program described, we have both high and low density pellets ranging from $98 \%$ matrix density down to $80 \%$. We have both pellets and powder; vipac powder fuel at around $85 \%$ density; both preformed bushings and bushings formed in the first hour (or so) of irradiation.

The presentation will be in three parts. Mr. William Baily, manager of our ceramic fuel development for fast reactor fuel, will cover properties affecting design, especially the stoichiometry-dependent properties (including the $\int \mathrm{Kd} \theta$ work) which has recently had some very interesting and favorable results. We will mainly cover work that has been done since the April meeting in Denver last year, at which time a fairly good summary of the program was presented. The recent work is on the $\int \mathrm{Kd} \theta$ and gap conductance, on fuel-clad interactions, and on sodiumfuel compatibility. Mr. Stan Rabin, formerly involved in cermet fuel development at Oakridge and now leads our fast flux irradiation work, will describe the work on fuel redistribution, particularly on melting, plus the status of the current and scheduled fast flux test irradiations. Finally, Mr. John Field, who heads our core design analysis work, will discuss safety criteria in transient testing, and damage mechanisms in oxide fuel. If there is time, I'll conclude with a discussion of vented fuel. 
20.2

\title{
OUT-OF-PILE PROPERTIES AND CHARACTERISTICS
}

\author{
W.. E. Baily
}

We will start out very quickly and review some of the results that describe what mixed oxides are and some of the physical properties measured out of the pile. Slide 1 sets the stage for saying what mixed oxides are and shows the Vegards relationship across the phase diagram. This shows the linear relationship in which we find no presence of second phases across the phase diagram provided we keep stoichiometric compositions. Slide 2 is the phase diagram that we have measured for the mixed oxide fuel. I believe the most important thing to note is that we cannot see the maximum that has previously been reported at around 10 to $20 \mathrm{wt} \%$ plutonia. As a matter of fact, we see a very simple, standard, binary phase diagram with complete solid solubility across this system. The narrow solidus-liquidus range is similar to some of the other $\mathrm{UO}_{2}$-oxide phase diagrams previously reported. This work was done with thermar rest techniques, and we used about $10 \mathrm{~g}$ samples. Both the solidus and liquidus were measured by thermal arrest change in the curve slope. The lines noted are curves computed by ideal solution theory. You can see they fit very closely to the experimental data.

SLIDE 1. Lattice Parameters for the $\mathrm{UO}_{2}-\mathrm{PuO}_{2}$ System.

SLIDE 2.
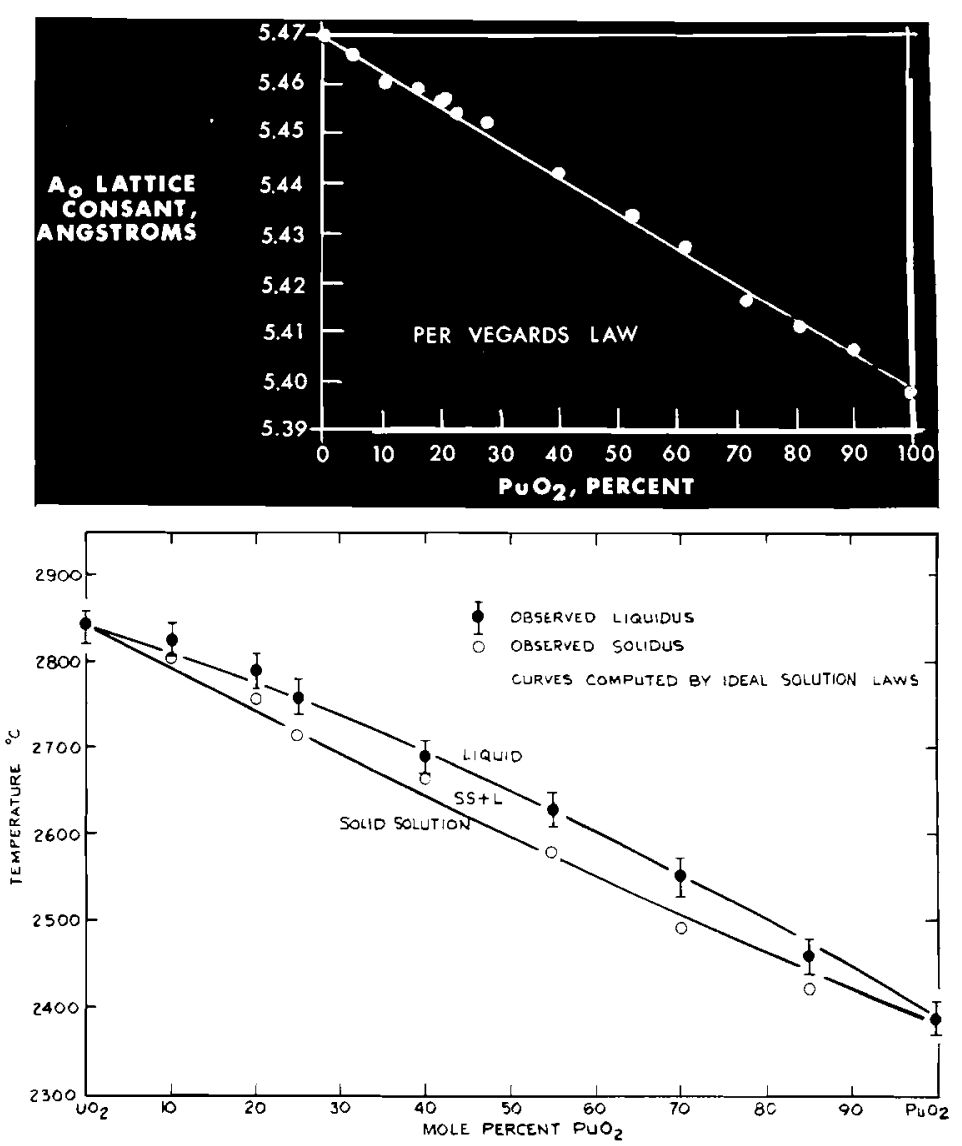

SOLID-LIQUID PHASE DIAGRAM FOR THE UO2-PUO, SYSTEM 
Slide 3 shows you the microstructures in coprecipitated mixed oxides; we see what we call striations within the grains themselves. I won't say we always see them, but we almost always see them in the coprecipitated mixed oxides at $20 \mathrm{wt} \%$ plutonia, independent of stoichiometry from about 1.97 to 2.01. We have not been able to identify the causes for these striations. We suspect, however, that they are formed during the coprecipitation process and may represent some step-change in composition. We do find that they are annealed out very quickly at temperatures of (say) $1400^{\circ} \mathrm{C}$ for approximately 10 hours (in-pile). In Slide 4, we can see the experimental data on the effect of stoichiometry on the melting point. We find decreasing melting point as a function of increasing oxygen content. The temperatures shown are a little different from the ones noted on the phase diagram, simply because this work was measured with the filament technique. Since that time we have measured the melting point change as functions of stoichiometry with thermal arrest techniques, and have found approximately an 80 to $85^{\circ} \mathrm{C}$ imcrease in the melting point going from 2.00 to 1.97. (I'll talk in a minute about the effect of this and how our in-pile tests show confirming information.)

SLIDE 3. Mic rostructure, Coprecipitated $\mathrm{UO}_{2}-\mathrm{PuO}_{2}$.

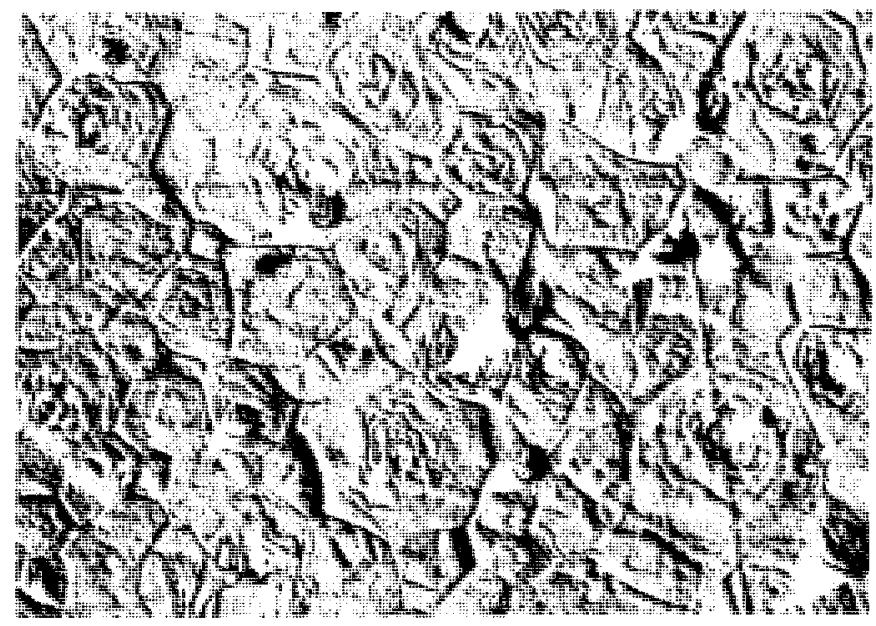

SLIDE 4.

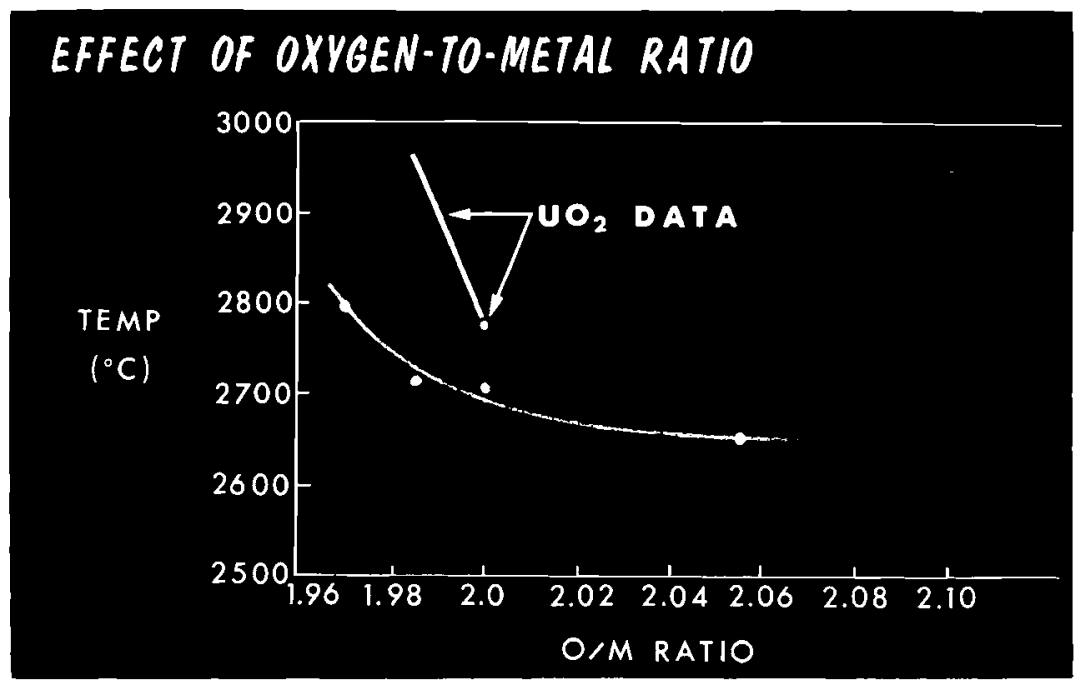


Slide 5 shows the relationship we have found for the lattice parameter change as a function of stoichiometry, and we find a linear decrease in the cell size as the function of stoichiometry from 1.97 to 2.01 . We make these different stoichiometries by simply controlling the atmosphere within the sentering furnace. By using a very dry atmosphere, we can make stoichiometries as low as 1.95. The 1.95 is not shown on Slide 5 because we do not have X-ray data completed on it. We do not find presence of second phases, particularly sesquioxide, as might be expected in hypostoichemetric fuel, (which is a little surprising). To date, we do occasionally find trace phases of $\mathrm{Pu}_{2} \mathrm{O}_{3}$ in the $\mathrm{X}$-ray work, but we find no rhyme nor reason to its presence. We occasionally see it independent of stoichiometry.

We measured the in-pile thermal conductivity of mixed oxides and $\mathrm{UO}_{2}$ (Slide 6) simultaneously, by using both tungsten-rhenium thermocouples within the fuel and a gas bulb thermometer at the end of the fuel (using gas law equations to calculate center temperature) and we came up with this general relationship. Now this is a difficult experiment at best, but the results do show a lower (low) temperature conductivity function for the mixed oxides compared to $\mathrm{UO}_{2}$. This doesn't work necessarily to our advantage, but it isn't too much of a burden because, in general, our fuel temperatures are about $1000^{\circ} \mathrm{C}$.and higher. In this range, the fact that you have a low temperature conductivity function of the mixed oxide does not appear to be significant.

SLIDE 5.

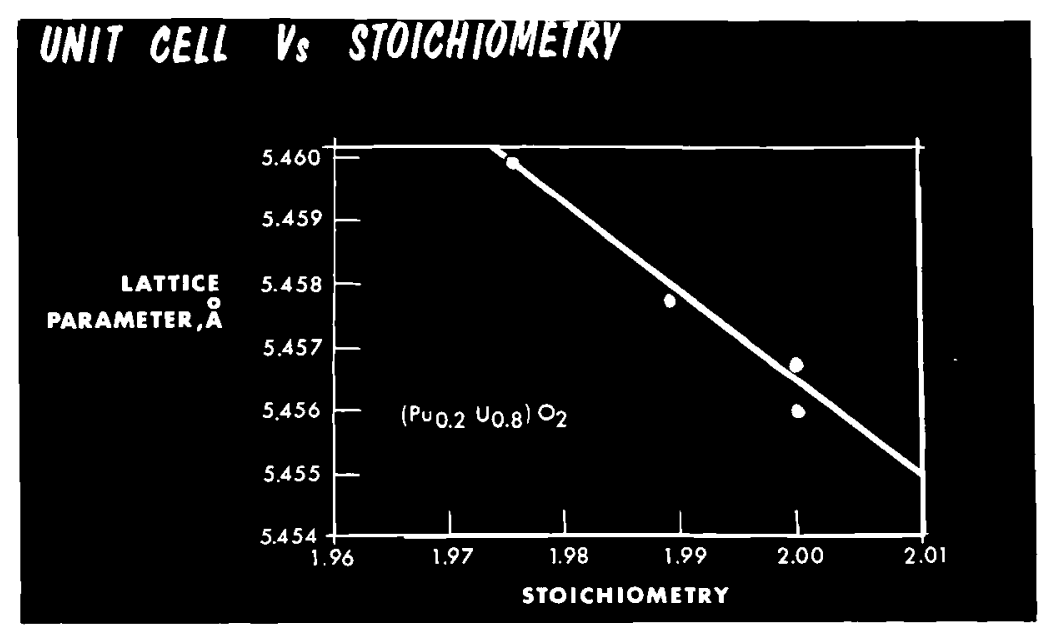

\section{IN-PILE TIERMAL CONDUCTIVITY}

Thermal Conductivity, $\mathrm{K}$, watts $/ \mathrm{cm}-{ }^{\circ} \mathrm{C}$

SLIDE 6.

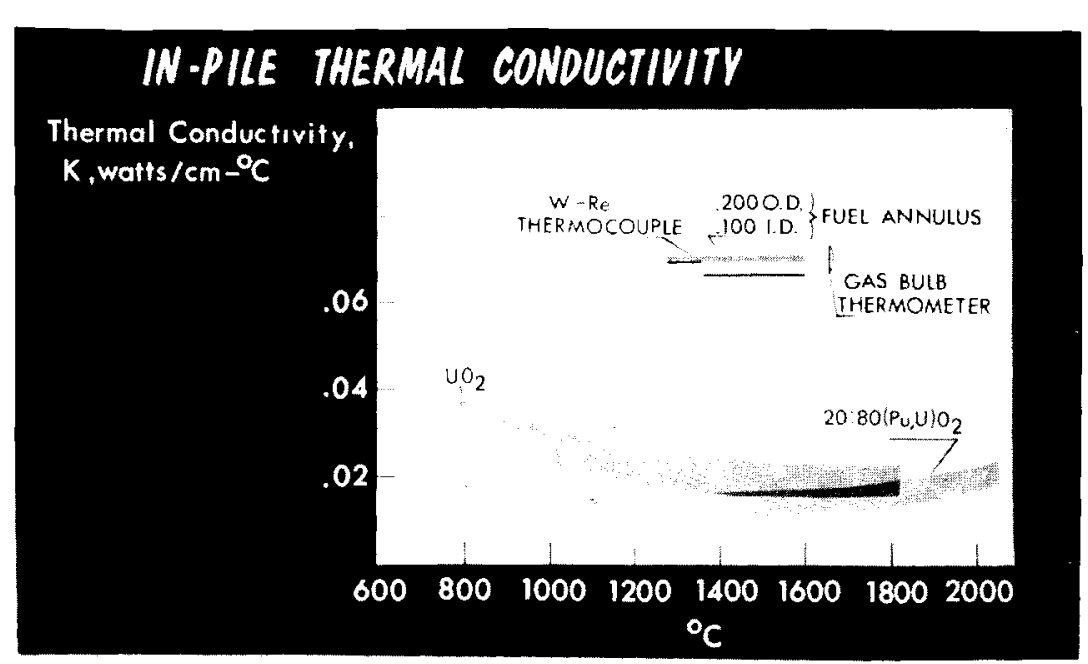


Slide 7 shows our summation of all of the thermal conductivity data to date. This includes both the in-pile results and the out-of-pile results, which were reported by Hetzler ahout two years ago; these measurements were made with a hot rod rig. Also, we have superimposed on this data smeared thermal conductivity measurements that we have made, which run from a surface temperature of approximately $1000^{\circ} \mathrm{C}$ to the melting point. Here is a complete listing of all the data; there are 9 data points shown here. We have doubt about the lowest data point, but what we are showing is an integral to melting of approximately 81 to $82 \mathrm{~W} / \mathrm{cm}$ with the tolerance factor not noted. Going from a temperature of about $1000^{\circ} \mathrm{C}$, we show a value approximately of $55 \mathrm{~W} / \mathrm{cm}$ to melting. We have taken the liberty to extrapolate the low temperature conductivity data on a linear basis rather than bringing it up. In first work with this curve we increase it by using the Oak Ridge out-of-pile $\mathrm{UO}_{2}$ thermal conductivity data, but we decided that wasn't necessarily correct since we suspect the low temperature conductivity of mixed oxide is lower than $\mathrm{UO}_{2}$. So we've taken the equation of this curve, and extrapolated it back to zero, and that is why our total integral is lower than that of $\mathrm{UO}_{2}$, which if it were shown, would be going up to here. But the value that one is concerned about is $T_{s}$ to $T_{c}$, and you only use zero to center temperatures for microstructure calculations.

\section{SLIDE 7.}

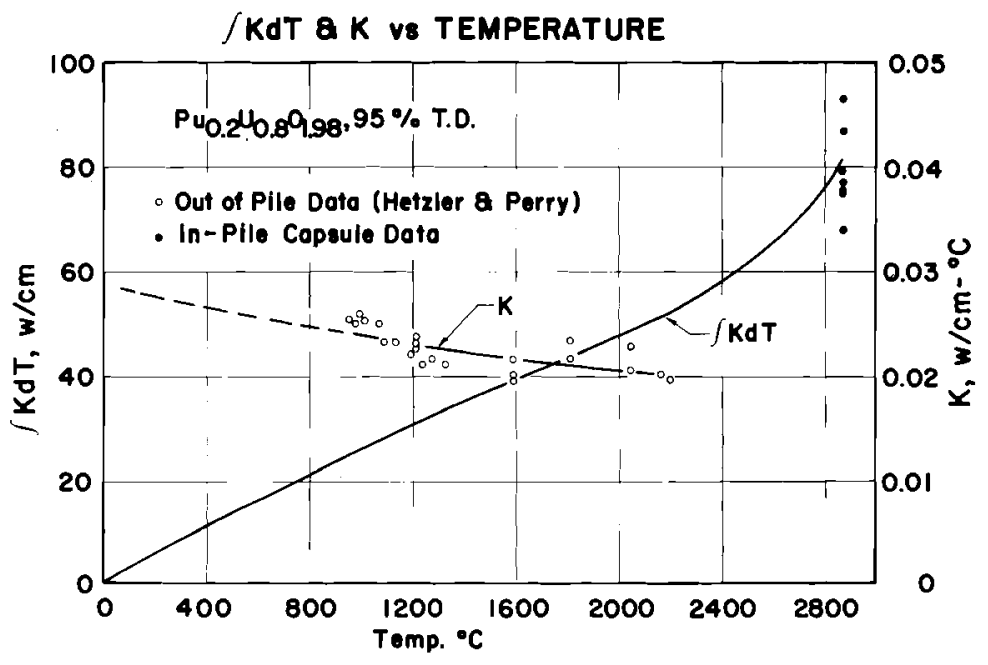

Slide 8 shows hypostoichiometric mixed oxide and stoichiometric mixed oxide. The message in Slide 8 is that we found significantly more melting in the stoichiometric pin, and these pins ran at $46 \mathrm{~kW} / \mathrm{ft}$. The molten boundary is shown in the stoichiometric and hypostoichiometric pins. We found significantly more melting in the stoichiometric pin at equal power levels. This is an alpha autoradiograph, and we see a plutonium segregation line at the molten boundary; we see this in all fuel pins that operate with molten fuel. 
SLIDE 8. Melting in Stoichiometric and Hypostoichiometric Mixed Oxide.

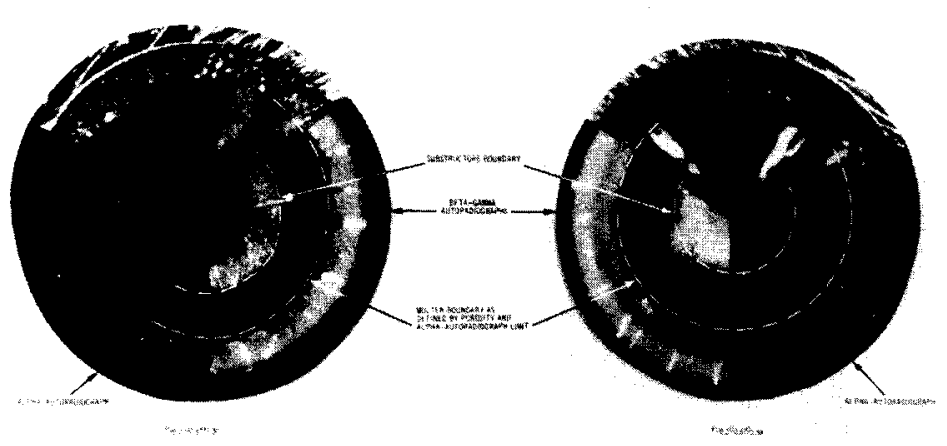

Slide 9 shows what we describe as the molten boundary. We see a substructure area and a porosity limit. When we first obtained data from pins such as these with high powers (we've run approximately eleven to date), we felt that the substructure was the molten boundary. We have since run pins, though, that exhibit both substructure and no substructure with essentially the same diametrical expansion of the cladding (due to the volume change on melting). We have now gone back and chosen the porosity limit as the molten boundary, and it fits with the volume change of approximately $10 \%$.

Slide 10 shows alpha segregation or gradient that we see in the molten fuel. Correlating it with the gamma activity regions, we find that almost all of the characteristic microstructure changes within the fuel have corresponding change in either beta, gamma, or alpha radiography rings. We've yet to develop a good understanding of this; however, we are doing core sampling now to appreciate just what this tells us.

In designing our fuel, we have this problem of void deployment, which we heard a little about yesterday. We need a certain void fraction within our fuel for transient melting case, as well as fuel swelling. We have the opportunity to deploy this void in:

- The radial gap

- Within annular pellets

- Low density pellets

- Powder fuel.

We haven't made a choice on this; our reference design uses the radial gap as the means for deploying the void.

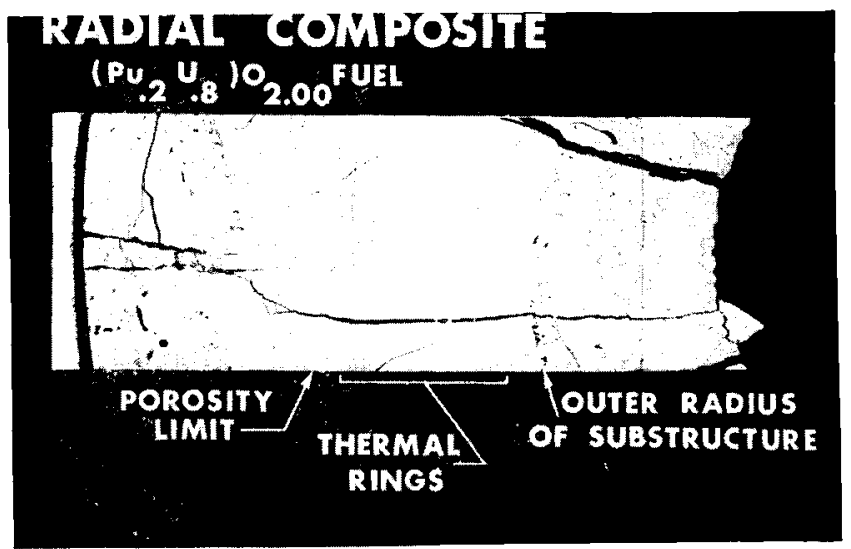

SLIDE 9.

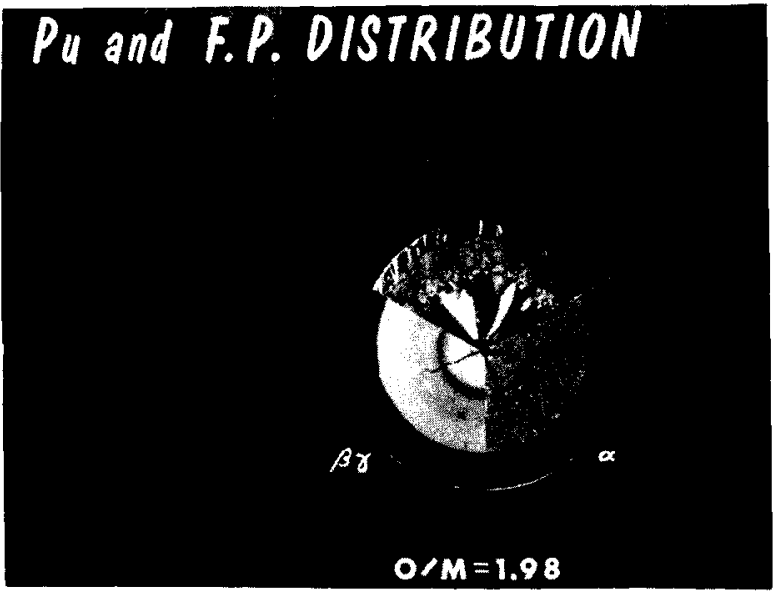

SLIDE 10. 
These are three pins that ran at $20 \mathrm{~kW} / \mathrm{ft}$ (Slides 11-13); they ran at the same time and they saw identical thermal history. The most important thing is to appreciate the structure we see as in Slide 11. This is the grain growth radius with the $2 \mathrm{mil}$ gap. If we go to the $4 \mathrm{mil}$ gap, as shown in Slide 12, we see a slightly larger radius of grain growth, at the same power level. In Slide 13, we see a $10 \mathrm{mil}$ gap; we find that this is the molten boundary of this pin. We see that the gap size very definitely controls the center temperature initially. We had a tungsten-rhenium thermocouple in a fourth pin to measure the center temperature, which showed that the effect of the gap on conductivity decreased very quickly in the irradiation period of the capsule. However, the microstructure reflects the hottest $t \mathrm{t}$ temperature that we see.

SLIDE 11. 2 Mil Diametral Gap Specimen-20 kW/ft.

SLIDE 12. 4 Mil Diametral Gap Specimen-20 kW/ft.

SLIDE 13. 10 Mil Diametral Gap Specimen-20 kW/ft.
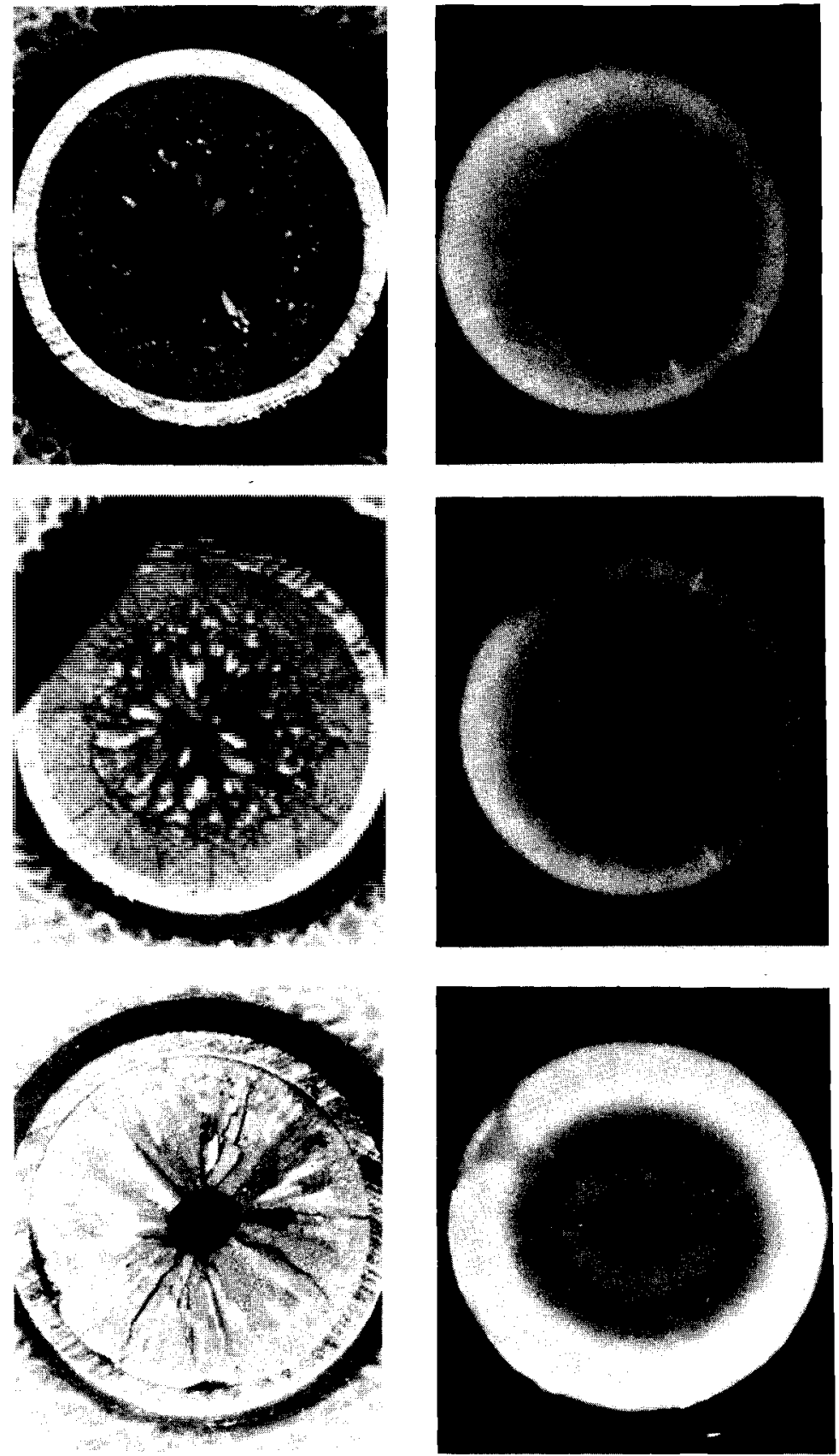
20.8

In Slide 14, which shows the gap conductivity values, we find that the value of 2000 for $h$ appears very nice for an interference fit. This is just a slight interference fit, and these are $h$ values calculated for both the $4 \mathrm{mil}$ and $10 \mathrm{mil}$ gap. You can see that the different $\mathrm{h}$ values result in approximately $800^{\circ} \mathrm{C}$ difference in the fuel temperature. We did find that the gap size changed. The 2 mil gap size was the same before and after the irradiation; the $4 \mathrm{mil}$ gap size dropped down to 2 mils; the 10 mil gap had dropped down to 5 mils; and all this is in a 42 hour period (with two complete thermal cycles).

Slide 15 shows profilometer results. We think the profilometer is the most valuable tool known for establishing the effect of mechanical fuel-cladding interaction. I've listed the diameter changes for the $4 \mathrm{mil}$ gap specimen as well as for the $10 \mathrm{mil}$ gap. I should have had the $2 \mathrm{mil}$ gap in here, which is essentially the same as for the $4 \mathrm{mil}$ gap. You notice these are rather small changes, and we see little bumps at the pellet interfaces. However, we do not see the large bumps, for example in the SEFOR and BWR work, where there are 3 mil ridges. In those cases, interference fits produce a very large ridge (We do not see this.). On the $10 \mathrm{mil}$ gap, we see an increase, and this is actually ovality that we are looking at. These are the 0 and $90^{\circ}$ traces, and is an ovality factor. This fuel pin did melt; fuel did flow downward; and it did have two thermal cycles. On the second thermal cycle, it moved downward. So we have some sort of a bending of the element caused by the melting condition within the fuel (or caused by the fuel melting).

We had a choice of using annular fuel pellets, and we had a thermal flux test with this fuel (Slide 16). The central void in the rod was generally the same along the length. We do not see any disadvantage as was recorded in the LSBR data on the use of annular fuel. We do see, interestingly enough, a little change (indentations) at the internal pellet interfaces. Some of the rods show sort of a slight wave function at the pellet interface. We see no evidence that annular fuel is unstable, and these tests ran to 7000 MWd/ton and for about 600 hours.

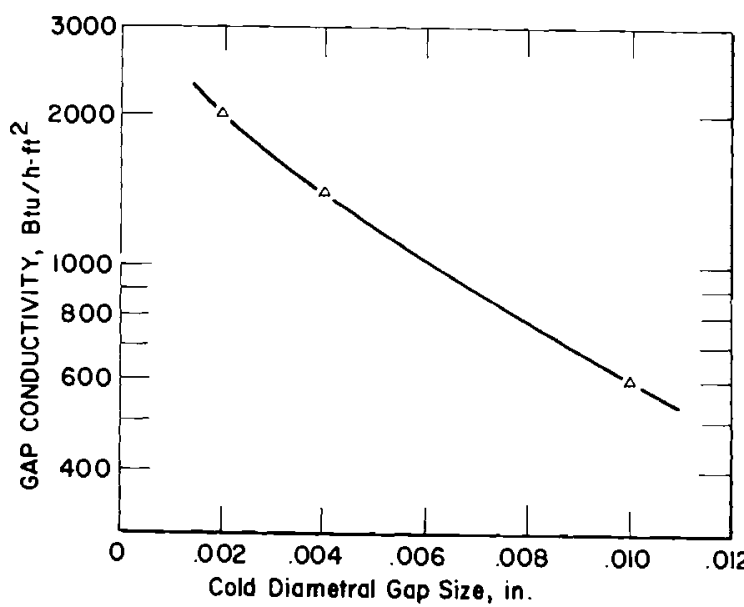

SLIDE 14. Conductivity vs Gap Size, Argon Atm.

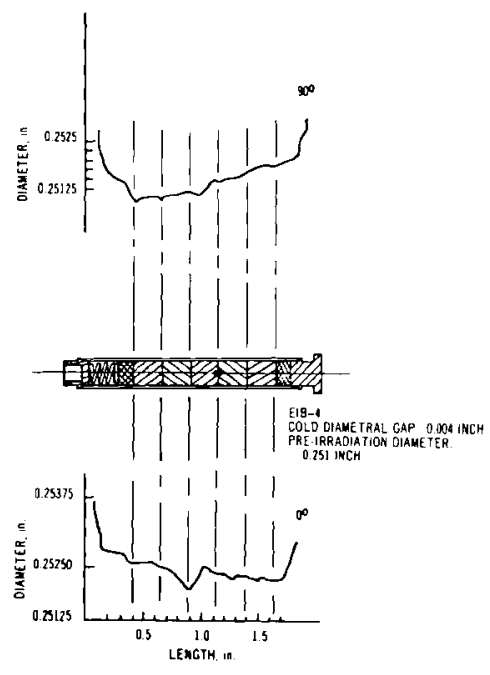

Figure 5-4. Cladding Protsle of Pin E1B-4

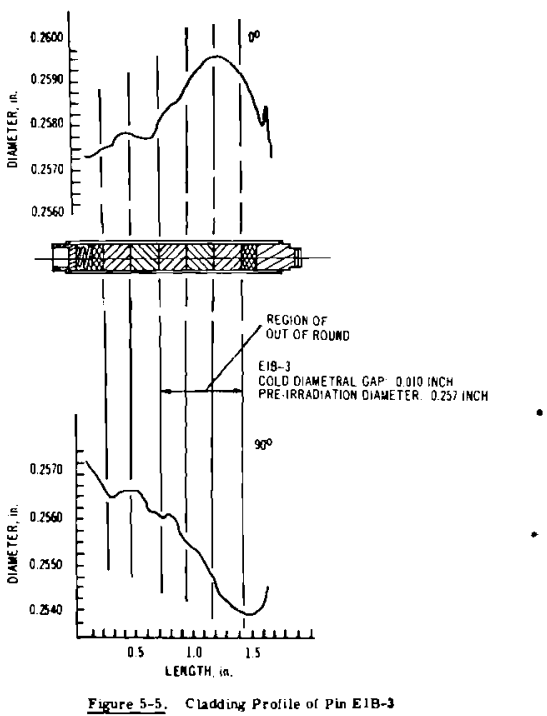

Figure 5-5. Cladding Profile of Pin E18-3 
This pin (shown in Slide 17) ran at peak power of $20 \mathrm{~kW} / \mathrm{ft}$ and a burnup of $70,000 \mathrm{MWd} / \mathrm{ton}$. This is the cold end of that fuel rod. The average burnup in this average section was $40,000 \mathrm{MWd} / \mathrm{ton}$. The interesting thing is the gradual change in the porosity limit, and this continues essentially all the way up the fuel rod until we get up to the peak power sections. Now, most of us would say, "Ah, that looks like a plate fuel with high burnup." In fact we have a lot of fuel at the other end of the rod that looks just like this. In other words, we don't see the porosity in the high burnup fuel that runs at high temperatures. We feel that it is very important that fuel swelling models, which may actually work for water cooled reactors where you have a fuel surface temperature of $400^{\circ} \mathrm{C}$, are not necessarily applicable to sodium cooled tests whe re the fuel surface temperature (in this case for example) is closer to a $1000^{\circ} \mathrm{C}$. The fact that we don't have considerable high burnup swelling data on mixed oxides is discouraging, but perhaps the fact that we don't have may even be encouraging.

SLIDE 16.

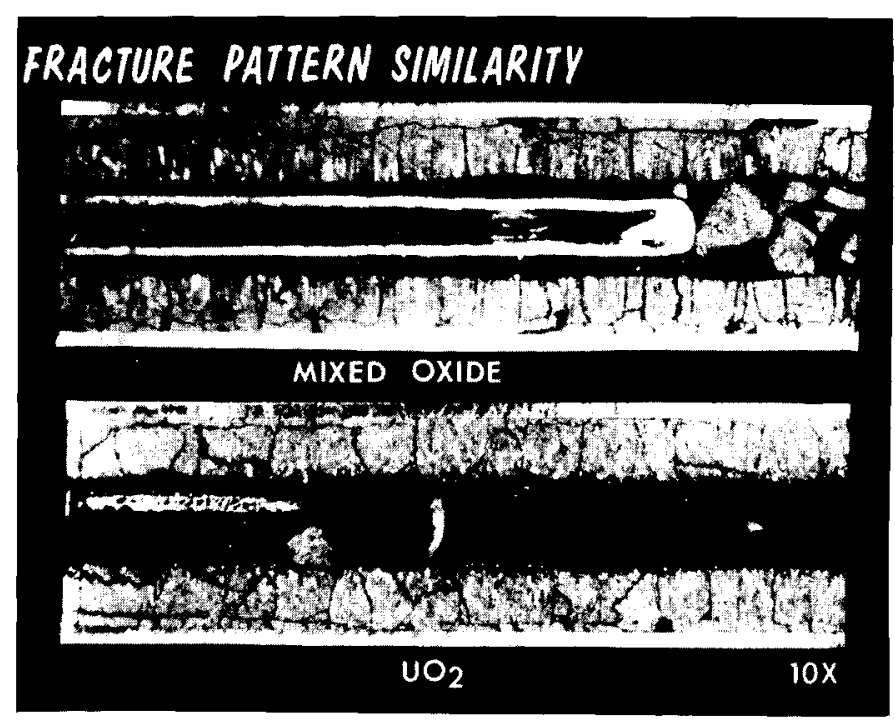

SLIDE 17. Porosity of High Burnup, High Temperature Fuel.

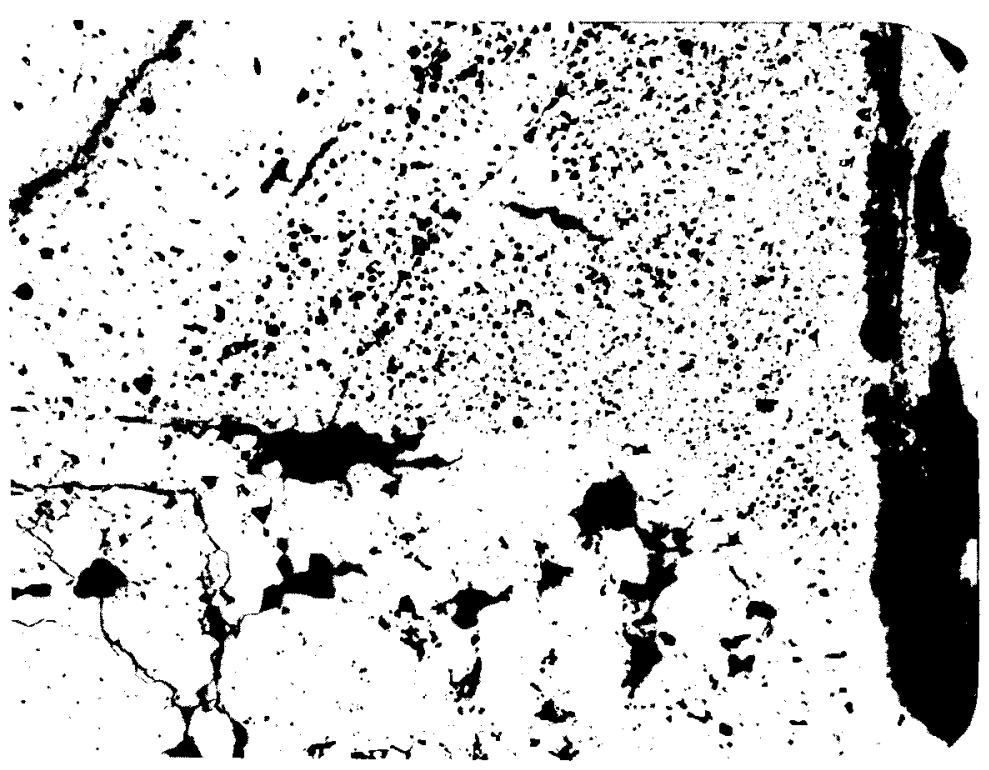


We have data on 65, $000 \mathrm{MWd} /$ ton, and John Field might cover some of this information on one of the pins that was transient tested after a high burn-up. We found no diametrical expansion of this fuel with $92 \%$ smear density after 65,000 MWd/ton, and the center void was essentially intact. I don't think I can say unequivocally that the fuel will swell into the center void, but the center void was certainly there; it could have swollen into the center void had it wanted to.

\section{Sodium Compatibility}

Slide 18 shows some $\mathrm{UO}_{2}$ specimen test results from our dynamic sodium loops. These were run to establish sodium compatibility with $\mathrm{UO}_{2}$. We find that stoichiometric $\mathrm{UO}_{2}$ exhibits excellent compatibility with dynamic sodium. We found, however, that hyperstoichiometric $\mathrm{UO}_{2}$ in static tests clearly falls apart in a matter of two or three minutes.

\begin{tabular}{|c|c|c|c|c|c|c|}
\hline & & 2 PELL & $\begin{array}{l}\text { - SODIUM } \\
\text { (flow }\end{array}$ & $\begin{array}{l}\text { COMPATIBIL } \\
\text { te }=16.5 \mathrm{ft} /\end{array}$ & $\begin{array}{l}\text { _ITY TESTS } \\
(\mathrm{sec})\end{array}$ & $\mathrm{T} 1050^{\circ} \mathrm{F}^{\star}$ \\
\hline & $\begin{array}{c}\text { TEST } \\
\text { PERIOD (his) }\end{array}$ & $\begin{array}{l}\text { O/U } \\
\text { RATIO }\end{array}$ & $\begin{array}{l}\text { DENSITY } \\
\text { gm/c.c. }\end{array}$ & $\begin{array}{c}\text { INITIAL } \\
\text { WEIGHT }(\mathrm{gm})\end{array}$ & $\begin{array}{c}\text { FINAL } \\
\text { WEIGHT (gn) }\end{array}$ & REMARKS \\
\hline & 118 & 2.01 & 10.77 & $38.61^{* *}$ & 38.64 & Slight surface reaction \\
\hline TDF 18 & 118 & 2.01 & 10.33 & $39.05^{* *}$ & 39.07 & Slight surface reaction \\
\hline LIDE 18. & 263 & 2.01 & 10.44 & $38.99 * *$ & 39.00 & Slight surface reaction \\
\hline & 27 & 2.10 & 10.14 & $1.60^{* * * *}$ & - & Decomposed \\
\hline & 168 & 2.18 & 10.49 & $1.80^{* * *}$ & - & Decomposed \\
\hline & 168 & 2.18 & 10.61 & $1.72^{* * *}$ & - & Decomposed \\
\hline & $\begin{array}{l}\text { OXIDE CONTEN } \\
\text { ANNULAR PELL } \\
\text { CYLINDRICAL }\end{array}$ & $\begin{array}{l}\text { OF SODI } \\
\text { T I inch } \\
\text { LLET } 0.2\end{array}$ & $\begin{array}{l}\text { ARIEO FRO } \\
\cdot 0.6 \text { inch } \\
\text { Ch OD } 0.2\end{array}$ & $\begin{array}{l}10 \text { to } 190 \mathrm{ppm} \text {. } \\
0 \cdot 0.5 \text { inch LC } \\
5 \text { inch LONG. }\end{array}$ & ONG. & \\
\hline
\end{tabular}

This is a mixed oxide pin (Slide 19). It was not too high a temperature $\left(750^{\circ} \mathrm{C}\right)$, but we do find that both stoichiometric and hypostoichiometric mixed oxides exhibit excellent corrosion resistance in sodium. We also find that the hyperstoichiometric mixed oxide completely disintegrates in sodium, and that the oxygen level of the sodium (which is initially 30 to $50 \mathrm{ppm}$ ) increases to about $350 \mathrm{ppm}$ with the hyperstoichiometric specimens. You will find that density has a deleterious effect on the compatibility, and in lower density material, which exhibits poor mechanical integrity, the pellet is liable to fracture and pulverize much more quickly than at a high density. However, as far as chemical attack goes, we found no difference as a function of density. 


\section{(PU..2 $\left.U_{0.8}\right)_{1.97}$ EXPOSED TO No AT $750^{\circ} \mathrm{C}-150 \mathrm{hrs}$}

SLIDE 19.

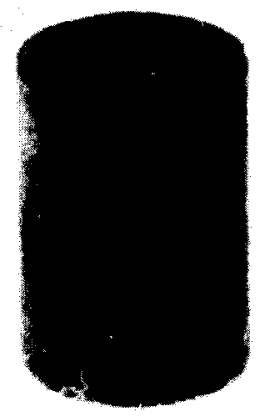

Slide 20 relates to one of the points that was raised yesterday; namely, the fact of sodium logging, which is some work that was completed about two years ago. The first sample was a control sample. This next specimen had a core of sodium placed within the center of the fuel rod; with an intact cladding there was no way for the sodium to get out of the fuel. The capsule was irradiated at a high power level. Sodium was expelled and pushed out to the peripheral region, but did not burst the cladding. We had another specimen with a hole at the center in the side of the cladding, where the sodium was permitted to cycle in and out of the fuel rod during the test. It was cycled (I believe) a hundred times and, in all three cases, there was no diametral change in the fuel.

\section{SODIUM LOGGING, PUO2-UO2 SERIES}

0.015 INCH CLAD

FCR PROGRAM

SLIDE 20.
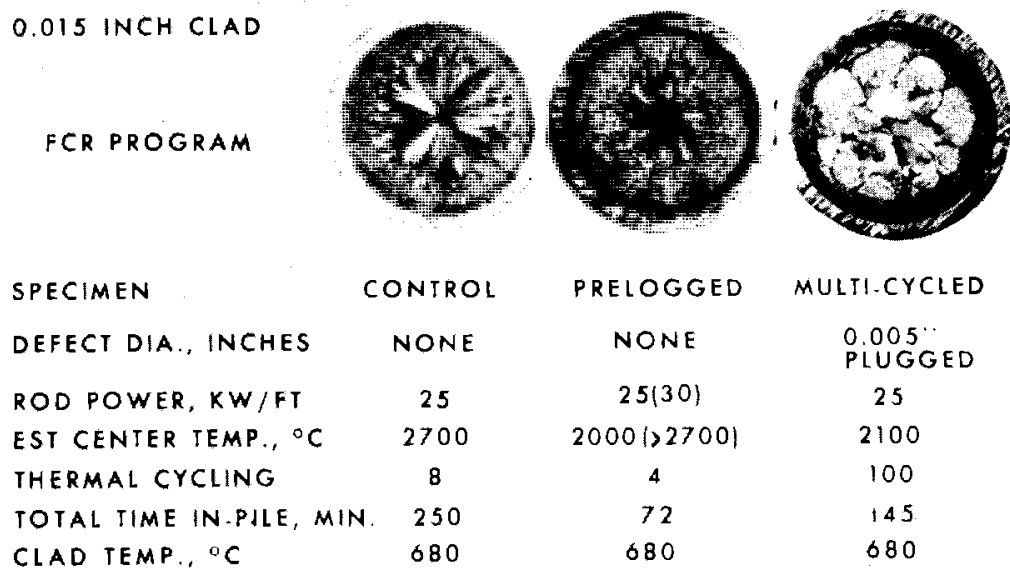

ONIROL
NONE
25
2700
8
250
680

PRELOGGED

MULTI.CYCLED NONE

$$
25(30)
$$$$
20001227001
$$$$
0.005
$$$$
\text { PLUGGED }
$$$$
25
$$$$
2100
$$$$
100
$$$$
145
$$ 
FUEL MOVEMENT

S. A. Rabin

First, let me summarize our results on the subject of fuel movement. In general, we avaluate fuel movement in terms of the two phenomena of fuel-clad interactions and reactivity changes (Slide 1). For fuel-clad interactions, our initial design accommodates differential fuel cladding expansion. What we would be concerned with would be any fuel movement that would alter the initial deployment and change this expansion accommodation; and, if this occurs, what remedial action could be taken (such as modifying the method of void deployment or mechanical restraint). In the area of reactivity changes, we are concerned with any movement of fuel that is rapid and toward the reactor mid plane. Areas of concern here are subassembly bowing, plutonium-uranium separation, thermal expansion of the fue 1 column, and bulk axial redistribution.

SLIDE 1

\section{EXPERIMENTAL OBSERVATIONS}

ON

MIXED OXIDE FUEL MOVEMENT RELATIVE TO

(1) FUEL-CLADDING INTERACTION

(2) REACTIVITY CHANGES

In Slide 2 we see a summary of our experimental results. The differential thermal expansion can be accommodated under representative conditions. Radial movement occurs, but it introduces no fuel-clad interactions. High burnup swelling tests in both thermal and fast spectra are in progress, and at high burnup, this should be an area of concern. Molten expansion in an overpowered condition can be accommodated provided sufficient void volume is available; we have observed this both in steady state and transient tests (which John Field will discuss subsequently). For molten expansion, it is possible to calculate how much initial smear density is necessary to accommodate the approximately $10 \%$ expansion on melting. The $90 \%$ theoretically dense smear density could accommodate $75 \%$ of fuel in the molten state. 
20.13

SLIDE 2. SUMMARY OF EXPERIMENTAL WORK ON MIXED OXIDE FUEL MOVEMENT

1. Differential fuel-cladding thermal expansion can be accommodated under representative FCR conditions.

2. Radial movement occurs as in $\mathrm{UO}_{2}$ but introduces no fuelcladding interactions.

3. High burnup swelling tests in thermal and fast fluxes are in progress.

4. No evidence of significant axial redistribution exists in mixed oxide operated below center melting.

5. Both upward and downward movement of fuel are noted as a result of transient melting.

6. Molten expansion in an overpower condition can be accommodated in short fuel segments provided sufficient void volume is available.

Slide 3 shows a summary of specimens where we have evaluated the extent of axial redistribution, both below and above melting. What we split the fuel pin into axial nodes (in this case 3 nodes), and evaluate any density changes. You'll observe that we observe no axial redistribution below melting. Above melting, we do observe a densification upon solidification of the fuel. Relative to separative effects between the plutonium and the uranium above melting, there is some plutonium segregation, but the effect on the Doppler is quite small, approximately $4 \%$ reduction of the calculated shut down reactivity. This was described in greater detail by Phil Novak and his associates in a paper presented at the fast reactor meeting in Chicago in October.

In conclusion about this work on fuel movement, we have (for the present) selected fuel design limits that preclude axial fuel movement due to melting; that is to say, all fuel keep low incipient melting in the steady state, and no fuel rises above the liquidus during an overpower transient.

Thermal Flux Experiments

Before moving on to the Fast Flux irradiation program, I'd like to summarize the present program we have in our thermal flux irradiations. The first category test results in pile thermal conductivity, (smear conductivity at low burnup) were described by Bill Baily. We also have high burnup smear conductivity experiments. Of two pins both going to $100,000 \mathrm{MWd} / \mathrm{ton}$, one will be examined, and the other will be rabbit tested. Low burnup, direct thermal conductivity tests have been completed. These were instrumented capsules containing thermo-couples and also pressure transducers by which we use the central void as a gas bulb thermometer. Following these are high burnup, direct conductivity capsules using the same technique. 
20.14

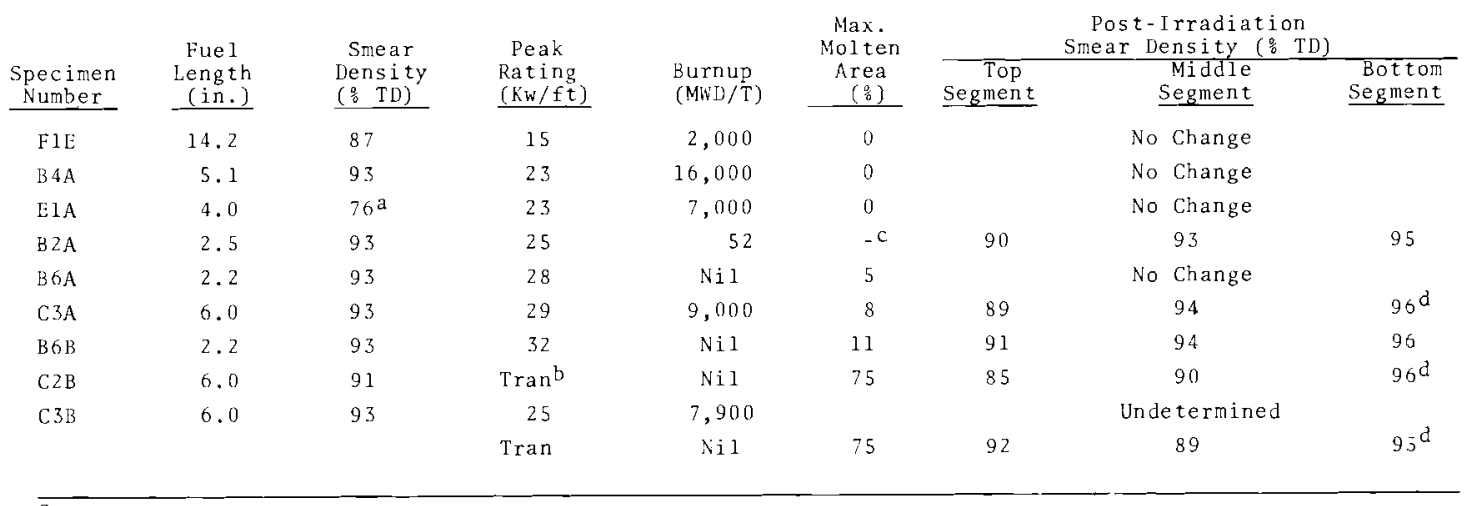

\footnotetext{
Annular pellets (95\% TD)

${ }^{b_{T r a n}}=$ Transient irradiated

$\mathrm{C}_{\text {Metallographic preparation may have missed coring }}$

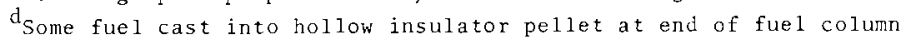

SLIDE 3. Axial Distribution of Irradiated Mixed Oxide Fue1

In the area of fuel swelling, we have four capsules in pile going to high burnup, and these are given interim examinations by gamma scanning; they have been examined thus far at 20,000 MWd/ton with no fuel swelling evident. We also have planned high fuel swelling experiments where we will be looking at various degrees of restraint. Finally, there are high burnup tests where we will be looking at a variety of parameters; these will be instrumented capsules run in V-rafts so you can control where the peak power occurs.

Fast Flux Fue 1 Irradiations

I will briefly summarize our Fast Flux program. Most of these irradiations are being performed in EBR-II. The Group 1 capsules have been irradiated for a short time and examined (I will discuss these results momentarily). The Group 2 and 4 are medium and high power scoping studies going to high burnup 50,000 to $100,000 \mathrm{MWd} / \mathrm{ton}$. The 21 (Group 2) capsules are in pile and have been for some time in EBR-II. The Group 0 and 6 are low burnup swelling tests to evaluate the effects of retained fission products; the Group 0 capsules are in pile; and the group 6 is going in very shortly. We have planned some bare pin irradiations, probably to be conducted in Fermi. The Group 5 represents high cladding temperature capsules. Currently, we can get only about $1150^{\circ} \mathrm{F}$ peak cladding temperature; we would like to get up to around $1300^{\circ} \mathrm{F}$. This will be a design to accomplish this. Finally, in our current program, a series of capsules would be preirradiated EBR-II and then transient tested in TREAT. 
I will now descirbe the results of our first short term irradiations in EBR-II. Slide 4 shows the parametric input: pellets versus vibratory compacted stoichiometry, density, diametrical and axial gap, and cladding material. Coprecipitated powder was used for all of the fuel.

The flip chart shows the operating conditions. The main points here were a short term irradiation, $1800 \mathrm{MWd} / \mathrm{ton}$, linear power 14 to $16 \mathrm{~kW} / \mathrm{ft}$ and cladding temperature peak of about $1150^{\circ} \mathrm{F}$. The irradiation was conducted in Row 4 of EBR-II. This next chart summarizes the results of this test. First of a11, the cladding integrity was maintained. There was no cladding distortion; that is, no diametrical increases. Fuel morphology was similar to that found in a thermal spectrum. No significant axial fuel redistribution occurred, and this is shown in Slide 5, which shows a gamma scan of the low density pellet fuel after irradiation. This profile is very smooth and comparable to the flux profile.

This shows transverse sections from 3 of the pins; all of the information as to power, density, and stoichiometry is given on Slide 6. As you can see, the central void size and region of columnar grain growth vary as functions of these parameters.

GROUP I FAST FLUX

$\mathrm{PuO}_{2}-\mathrm{UO}_{2}$ FUEL DESIGN PARAMETERS

Fuel

\begin{tabular}{lcccccc} 
Fuel Pin & $\begin{array}{c}\text { Fuel } \\
\text { Process }\end{array}$ & $\begin{array}{c}\text { Stoich. } \\
(\text { O/M) }\end{array}$ & $\begin{array}{c}\text { Density } \\
(\% \text { T.D.) }\end{array}$ & $\begin{array}{c}\text { Dio. Gop } \\
\text { ( in ) }\end{array}$ & $\begin{array}{c}\text { Axial Gop } \\
\text { ( in ) }\end{array}$ & $\begin{array}{c}\text { Clodding } \\
\text { Mot'l }\end{array}$ \\
\hline F-I-A & Pellets & 2.00 & 93 & 0.0040 & 0.48 & 347 SS \\
F-I-B & Pellets & 2.00 & 95 & 0.0025 & 0.48 & 347 SS \\
F-I-C & Pellets & 2.03 & 94 & 0.0022 & $<0.002$ & Incoloy 800 \\
F-I-D & Pellets & 1.97 & 93 & 0.0030 & 0.48 & 316 SS \\
F-I-E & Pellets & 2.00 & $\mathbf{8 7}$ & 0.0030 & 0.48 & 347 SS \\
F-I-F & Vipock & 2.00 & $\mathbf{8 5}$ & N.A. & 0.31 & Incoloy 800
\end{tabular}

Co-precipitoted 20 wt $\% \mathrm{PuO}_{2}-80$ wt $\% \mathrm{UO}_{2}$ Clodding dimensions (ref.) $0.250 "$ O.D. $\times 0.015^{\prime \prime}$ wall 


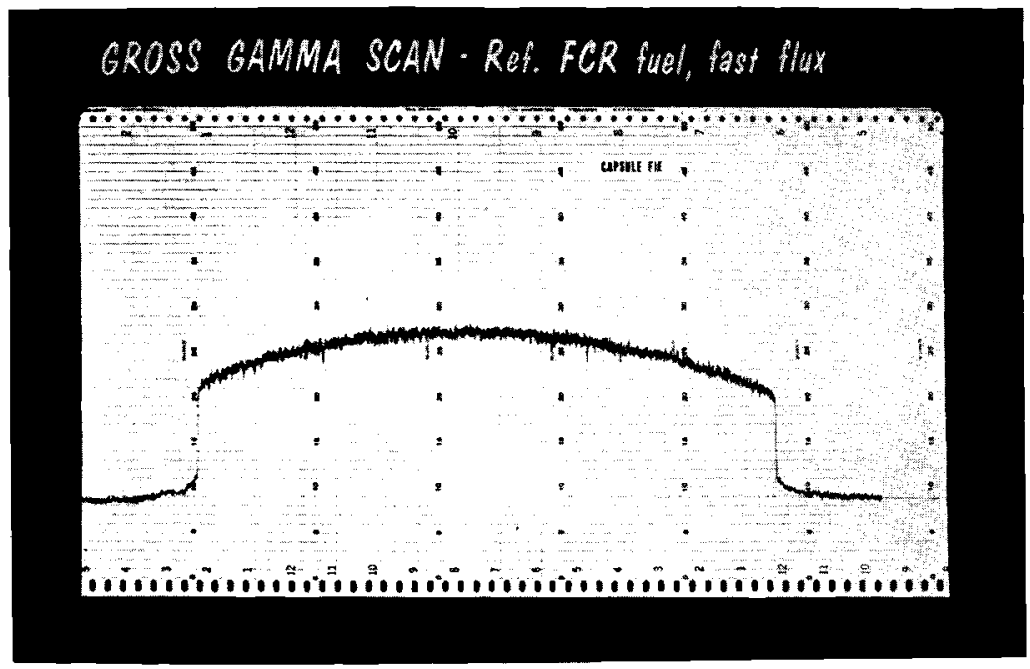

$\underline{\operatorname{SLIDE} \quad 6}$

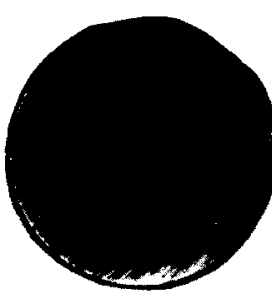

Rel. Power 1.000

\% T. D.

$O / M$
94 2.03

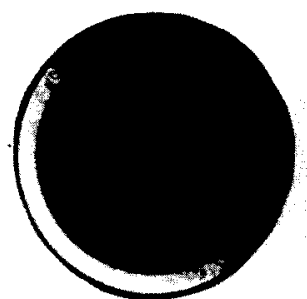

0.950

93

1.97

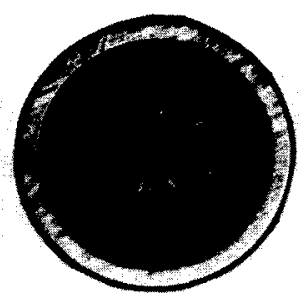

0.865

87

2.00

Slide 7 shows a diametral composite of one of the fuel pins, and as you can see from the microstructure; the morphology is similar to that which we find in a thermal spectrum. There is a result here where I have shown some functional relations in evaluating the data that we were able to establish between these parameters. The central void size appears to depend most on the density and diametral gap. The extent of the columnar grain growth depends on stoichiometry, and fission gas release is most dependent on density. This is shown dramatically, where in evaluating grain growth and central void formation, we used an integral to normalize the power. In fission gas release for high 
density specimens, it was about $5.7 \%$; for low density specimens, it was up around 24 or $26 \%$. I might point out that in a high burnup specimen, where you have designed a plenum to accommodate the gas, you like the gas to get up to the plenum. F1F is the vipac specimen. It exhibits the highest gas release and the lowest integral to columnar grain growth, but interestingly, not the lowest to central void formation.

SLIDE 7

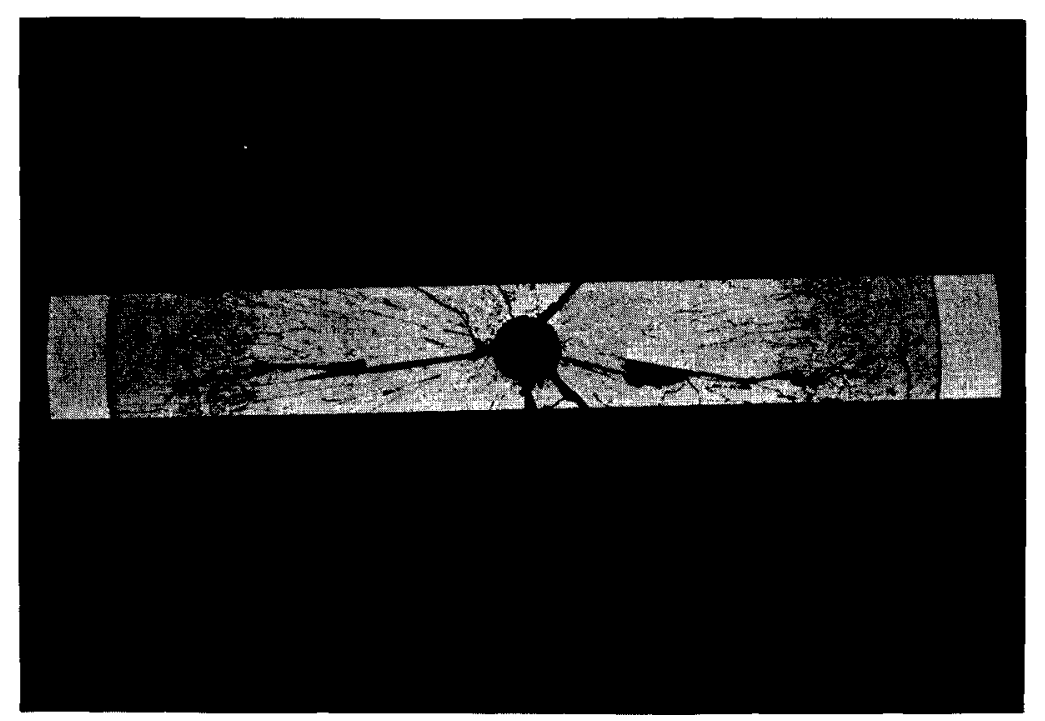

For our conclusions on these short time tests, the fuel element performance was as predicted, and successful proof testing of the capsule concept (the standard capsule used in EBR-II) was demonstrated. We have proceeded with high burnup tests in EBR-II, which are now in progress. 


\title{
TRANSIENT PERFORMANCE
}

\author{
J. H. Field
}

In arriving at a fuel design of any fast reactor (FFTF included), attainment of an optimum balance between safety and economics is a prime consideration. This consideration of safety has led us to an analytical and experimental investigation of what I like to call certain nonstandard operating conditions. This separates them from steady state operations. I want to emphasize that we don't plan to run fuel under these conditions, certainly not in large commercial reactors, and we wouldn't plan to run the FFTF driver fuel under these conditions.

My purpose this morning is to briefly review some of the results of the tests involving one of these off standard conditions--that of fuel performance under transient overpower conditions. We have done other nonstandard investigations such as the sodium logging example (that Bill Baily mentioned), and the very high power steady-state fuel relocation work (which Stan mentioned). To help put this experimental transient work in proper perspective, we might first consider those areas in our safety criteria that relate directly to these experiments. For example, one of these criteria, which was listed in Dr. Cohen's recent paper at the October Argonne meeting, was as follows:

"The integrated negative Doppler reactivity from operating power level to the point of fuel rupture is required to be large compared to credible outside sources of rapid reactivity insertion."

This criterion is aimed at limiting the effect of rapid reactivity insertions, and it sets, for example, the maximum control rod reactivity. However, to give relevance to such a criterion, it becomes necessary to experimentally determine this point of fuel rupture. In doing this, it must be done not only on new fuel, but fuel at various burnups, up to the maximum burnups that we would plan to reach in our reactors. Another relevant safety criterion as far as our experimental transient work is as follows:

"The performance of mechanical devices used to ultimately shut down the reactor shall be sufficiently rapid to avoid any damage, except minor localized fuel damage, following any credible reactivity addition."

This sort of a criterion sets requirements for the inherent negative feedbacks as well as requirements for mechanisms and instruments, etc. Here it is necessary to define this small local damage factor (I like to call it the point of incipient damage), where we begin to deform the cladding. 


\subsection{9}

We need to define this in terms of fuel temperatures, and this must be done experimentally. This is not to say it can't be done analytically, and we do it analytically first; but before we believe it, we do it experimentally.

Now the type of transient experiments that we have run to date have been basically aimed at a simulation of accidents involving reactivity insertions up to the range of about $\$ 2 / \mathrm{sec}$. This is the range that we would consider credible. I have a couple of slides that will help to establish this comparison between what we calculate for such a transient at about $\$ 2 / \mathrm{sec}$, and what we have actually been able to achieve in our transient experiments in the TREAT Reactor in Idaho.

Slide 1 shows the clad and coolant temperatures as calculated with our FORE code for an insertion of about $\$ 2 / \mathrm{sec}$. The effect of the large Doppler coefficient (high leakage core with a degraded spectrum) is quite evident in increasing the time before either boiling or burnout occurs, which is very important to us.

SLIDE 1

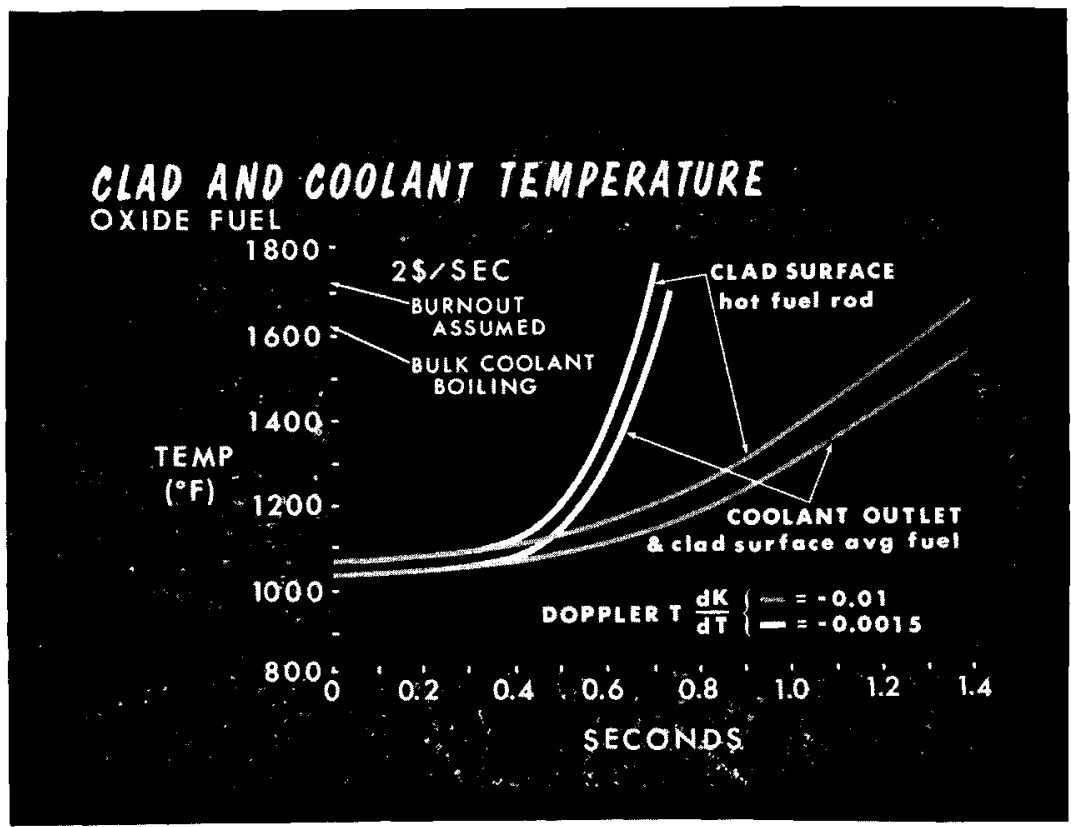

Slide 2 is a companion to this one and shows the peak fue 1 temperatures we would expect from the same sort of $\$ 2 / \mathrm{sec}$ insertion. Incidentally, there is no scram assumed here, so you would expect these temperatures actually to be limited before they climb off the top end of the chart. We are concerned with the time required to reach the assumed failure limit, the temperature range of interest being from the melting point up to about $6500^{\circ} \mathrm{F}$. I' 11 mention the $6500^{\circ} \mathrm{F}$ again a little later, but this is the area where we might expect failure (depending on the burnup of the fuel). However, the time to reach such a temperature is approaching about one second, and this is a relatively long time compared to what we used to hypothesize in-ramp insertions of $\$ 100$ or $\$ 200 / \mathrm{sec}$. This relatively long time is a result of the strong Doppler and 


\subsection{0}

the fact that we have a low conductivity fuel system, thus retaining much of the heat in the fuel itself. This relatively slow fuel temperature rise enables us to make an adequate experimental simulation using the TREAT Reactor.

SLIDE 2

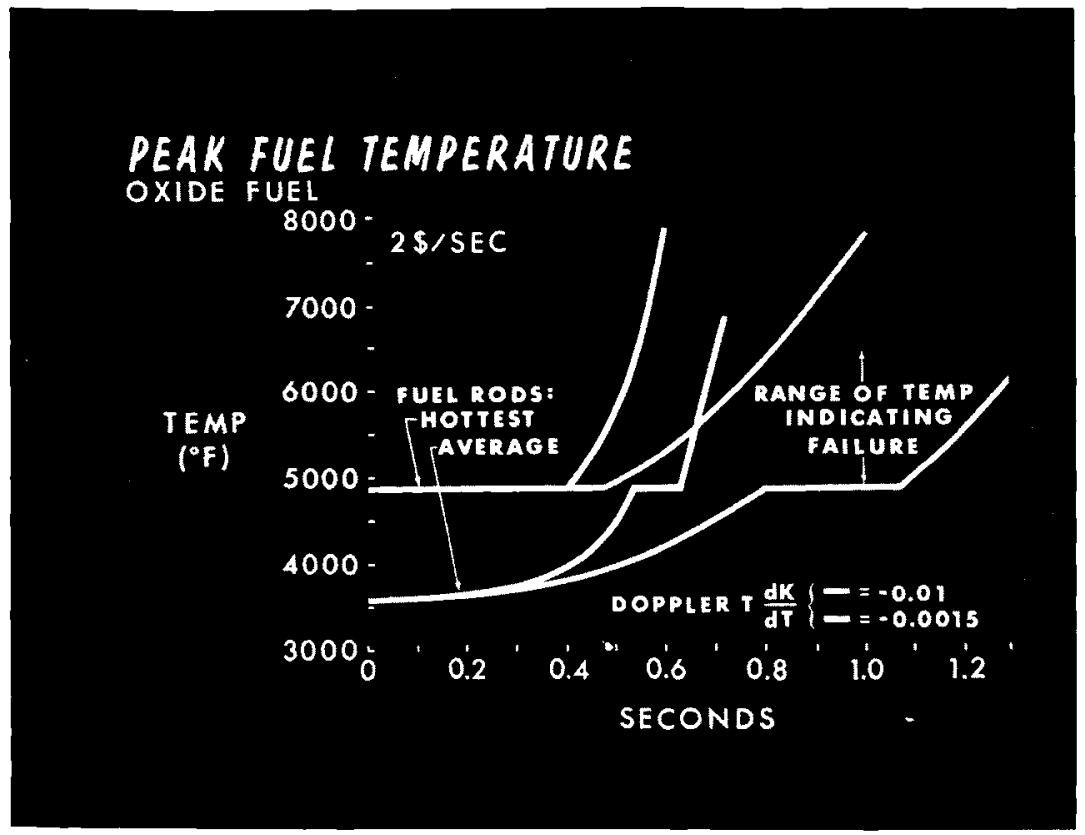

Slide 3 illustrates a typical transient run earlier in the program. The fuel temperature trace is a calculated one; the rest of the traces were actually measured in the TREAT Reactor. We did, in fact, have theremocouples in some of our early tests in TREAT and we were able to follow at least a portion of the fuel temperature rise until failure of the thermocouple. Therefore, we feel fairly confident that this

SLIDE 3500 MW-Sec Transient Maximum Temperature Versus Time

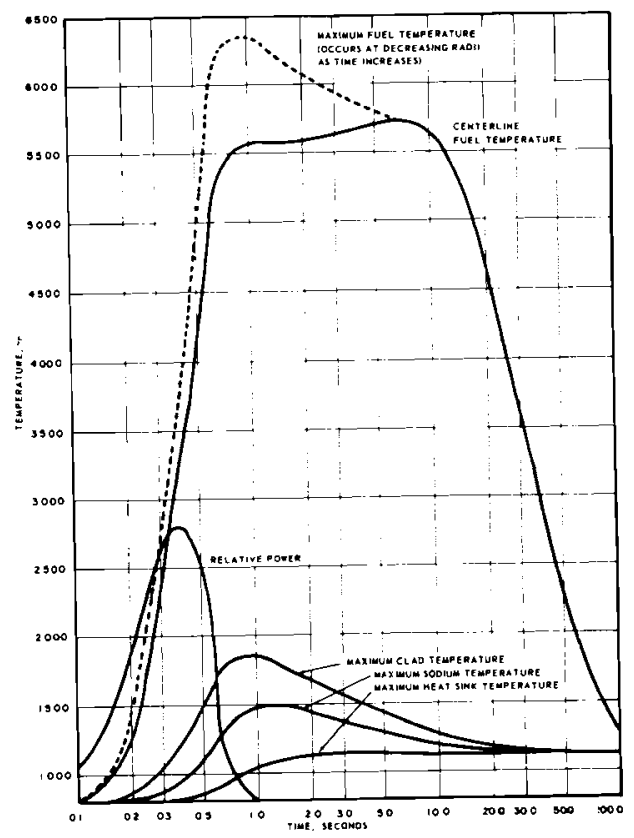


20.21

is the sort of temperature response we get in the fuel. You can see again that this temperature (peak temperature in the range of $\left.6500^{\circ} \mathrm{F}\right)$ is reached in something like 1 sec or possibly a little slower than that; so as far as the temperature rise is concerned, it is comparative with what we calculate for the $\$ 2 / \mathrm{sec}$ insertion.

With that rather lengthy introduction I'd like now briefly to review some of our experimental results to date. In general, our attack has been to try to identify incipient damage and final failure mechanisms, first with unirradiated fuel (as a matter of fact with $\mathrm{UO}_{2}$ fuel), then with unirradiated mixed oxide, next with low burnup fuels, and finally with high burnup fuels under transient conditions.

S1ide 4 is an example of a single transient (the temperature traces were seen previously). The estimated peak fuel temperature was $6500^{\circ} \mathrm{F}$, approximately $80 \%$ of the volume molten in the fue 1 pin at its peak operating condition. The molten fuel extended to within 10 or $20 \mathrm{mils}$ of the cladding. This happens to be a 1 in. diam fuel rod on which we started our very early tests. There was approximately a $20 \mathrm{mil}$ cladding deformation, uniform along the length of the fuel pin as a result of the mechanical interaction with the clad, at this extremely high temperature. The interesting void

\section{POLISHED SECTION OF I-D}

SLIDE 4

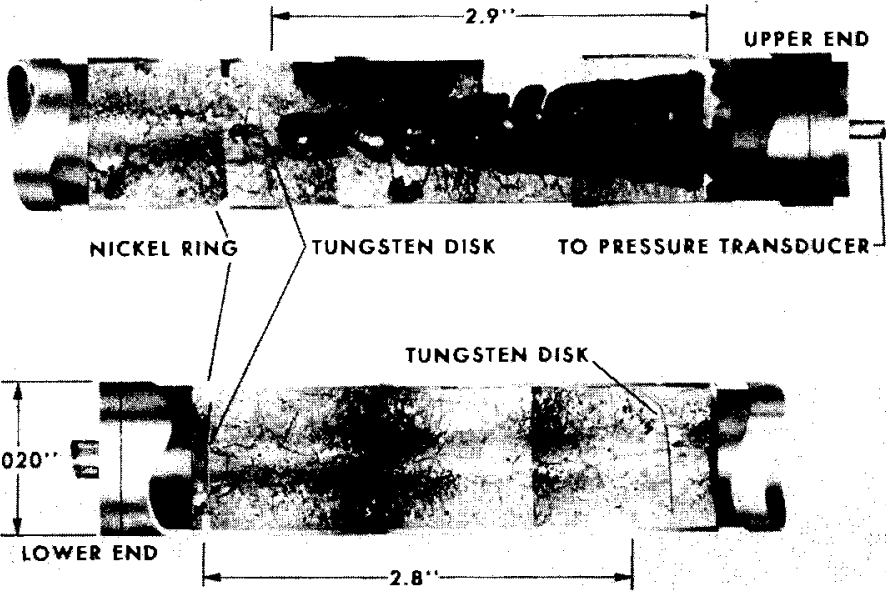

structure, at the top of the pin resembles what you would expect to see in a shrinkage void in a casting. The length of the fue 1 column actually increased (measured down to the first spacer); note the shelf for a segmentation scheme. The fuel column height increased slightly and the void formed on cooling much like in a normal casting process.

Slide 5 (cross sections) compares with a transient that we calculated would go just beyond the melting point. The error here, plus or minus, is probably 300 to $400^{\circ} \mathrm{F}$; so absence of molten material may well be explained by that. We have a case where we ran more than one transient, and there seems to be some difference in performance. This points out one fact, which we now believe, that the first transient (at least to melting point, and a little above) actually doesn't cause the problem. It's the second transient that causes the problem, and we have seen this again in our SEFOR tests 


\subsection{2}

where we have run as few as two transients iniating them from a steady state operating condition. Apparently after the first transient, the fuel densifies locally. After the second transient, there simply isn't room for the differential radial expansion, and you begin to treat the cladding in this manner.

\section{POLISHED TRANSVERSE SECTIONS}

\section{SLIDE 5}
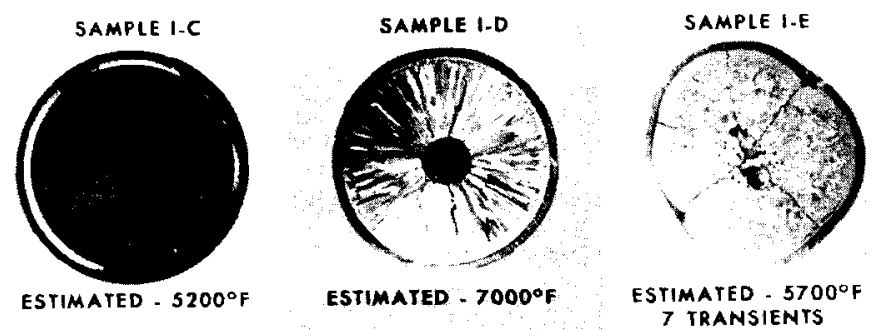

I think we should emphasize that in a power reactor, and hopefully in the FFTF, you would have only one transient. The second one we hope to avoid (or at least replace the fuel).

Following this early work with $\mathrm{UO}_{2}$, we went on to perform experiments with zero burnup mixed oxide to establish the failure limit. The most severe of these tests reached an estimated fuel temperature of $7700^{\circ} \mathrm{F}$ (I'm sorry I don't have a picture of it). Essentially, what we found was that the failure mechanism was that of molten fuel burning its way through the cladding. This established clad melting rather than fuel vapor pressure as the ultimate failure mechanism in zero burnup fuel.

Final1y, we turn our attention to preirradiated tests with mixed oxide fuel, and we have established a comparison between low burnup transient irradiated fue 1 and zero burnup transient irradiated fuel. Slide 6 shows two specimens: the $\mathrm{C}_{2} \mathrm{~B}$ specimen was cold fuel; whereas, the $\mathrm{C}_{3} \mathrm{~B}$ specimen approached a burnup of about $8000 \mathrm{MWd} / \mathrm{ton}$.

SUMMARY OF TRANSIENT IRRADIATION OF FUEL SAMPLES

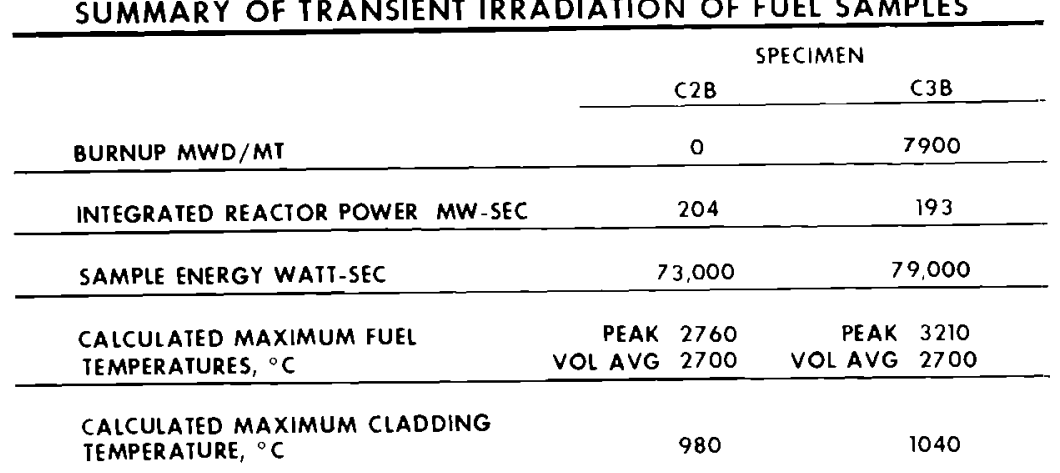

Slide 7 summarizes the results of the test. The specimen in the middle, is a control specimen which was run to the same 
20.23

burnup but was not trans-irradiated: It was examined after the steady state irradiation to help establish the condition of this fuel pin before the transient; so, you have a transient with no burnup, a steady state irradiation, and a steady state irradiation with transient. You'11 note that we did get cladding deformation

$\underline{\text { SLIDE } 7}$

\begin{tabular}{|c|c|c|c|}
\hline \multicolumn{4}{|c|}{$\begin{array}{l}\text { SUMMARY OF POST-IRRADIATION } \\
\text { EXAMINATION OF FUEL SAMPLES }\end{array}$} \\
\hline \multirow[b]{2}{*}{ POST-IRRADIATION EXAMINATION } & \multicolumn{3}{|c|}{ SPECIMEN } \\
\hline & $\mathrm{C} 2 \mathrm{~B}$ & C3A & С $3 B$ \\
\hline CLADDING DEFORMATION & No & NO & YES \\
\hline PIN BOWING & YES & YES & YES \\
\hline PELLET CRACKING & YES & YES & YES \\
\hline GRAIN GROWTH & YES & YES & YES \\
\hline CENTRAL VOID FORMATION & YES & YES & YES \\
\hline FUEL MELTING & YES & No & YES \\
\hline FUEL RELOCATION & $\begin{array}{l}\text { DOWNWARD } \\
\text { ONIY }\end{array}$ & NO & $\begin{array}{l}\text { UPWARDS AND } \\
\text { DOWNWARDS }\end{array}$ \\
\hline CLADDING MELTING & NO & No & NO \\
\hline
\end{tabular}

in $C_{3} B$ but not in $C_{2} B$; keep in mind that the only difference was an amount of fission gas, very small in relationship to $100,000 \mathrm{MWd} / \mathrm{ton}$. We do believe that it was this fission gas that caused the cladding deformation. Another difference is in fuel relocation; in the case of $\mathrm{C}_{2} \mathrm{~B}$ we found fuel relocation downward on $1 \mathrm{y}$. In $\mathrm{C}_{3} \mathrm{~B}$ we found fue 1 being relocated both upward and downward in the fuel specimen. The cladding deformation in $\mathrm{C}_{3} \mathrm{~B}$ was about $3 \mathrm{mils}$, and (as I said) it is attributed to the unreleased fission gases suddenly released in the transient.

The longitudinal cross section from $\mathrm{C}_{2} \mathrm{~B}$ is shown in Slide 8 . You can see an apparent movement of fuel, from the upper end of the pin which has apparently solidified at the point near the center line. Stan pointed out in one of his slides, the density of this lower region is somewhat higher than it is toward the middle of the fuel column. However, it is questionable whether this much fue 1 relocation would be enough to cause a significant reactivity insertion, and (as a matter of fact), the net movement of the fuel toward the center of the core may be zero.

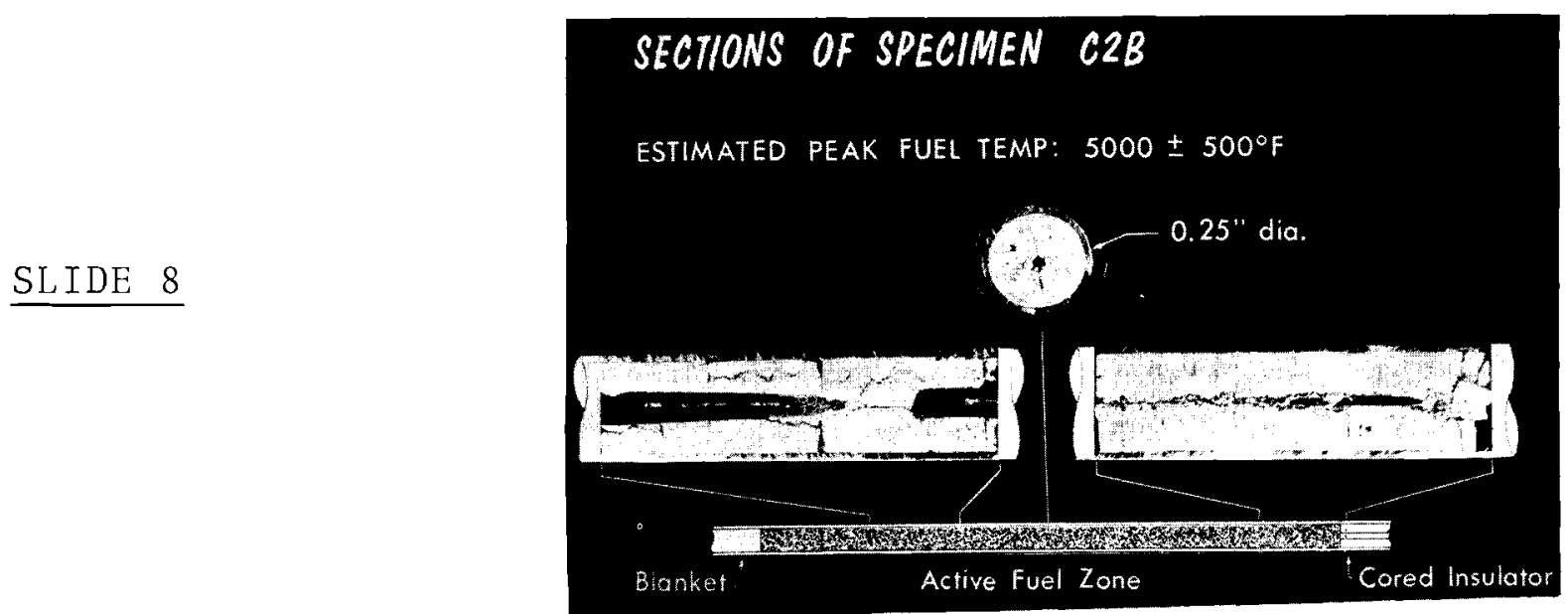


The specimen $\left(\mathrm{C}_{3} \mathrm{~B}\right)$ which saw low burnup followed by transient irradiation is shown in Slide 9. The difference between the two can be seen from the upper section of the fuel rod, where we don't see the conical void that we observed in $C_{2} B$ (nor even a large, circular void). There is a small shrinkage cavity, and there is fuel above that, so it is a very dense structure in this area.

\section{SPECIMEN C3B FOLLOWING TRANSIENT IRRADIATION}

SLIDE 9

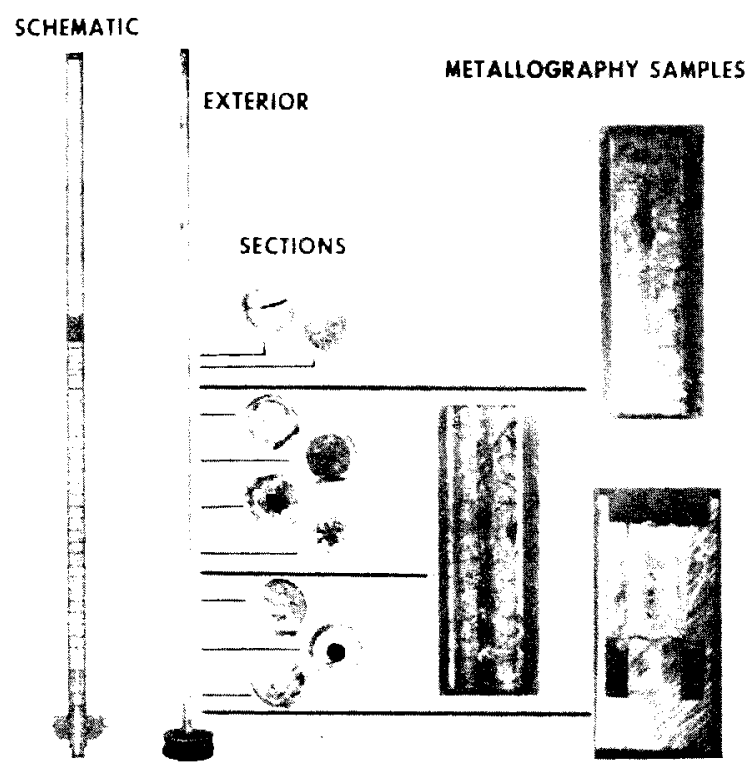

Down toward the center of the fuel pin, you see a continuous central void; down at the bottom, you see again a very dense structure with no void. The indication here is that fue 1 was expelled in both directions by an expanding bubble of fission gas. This expanding bubble of fission gas, we believe, was the unreleased fission gas. This was fission gas, which was unreleased during the steady state, but, was suddenly released during the transient, causing fuel to be pushed in both directions away from the core center line (the theoretical core center line). Now, I am told by physicists that this movement away from the core center is a highly desirable situation. Apparently, the fuel does not fall back again; essentially, it freezes at the top of the fuel pin.

In S1ide 10 is an analytical model, (in the process of development) to try to explain this sudden release of the fission gas observed in previous tests. I might mention that the previous tests utilized a pressure transducer. We observed a considerable pressure pulse, so there is some empirical indication that there was a large release of fission gas during the transient. In the thermal flux environment in which the preirradiation of this pin took place, the gas generation in this model has been assumed to follow the flux depression line (S1ide 10). If you superimpose upon this, the steady state temperature profile where it was run (as shown) you can 
make some assumptions about the amount of gas release. We have assumed $100 \%$ gas release above about $3300 \% \mathrm{~F}, 50 \%$ gas release be tween $2900^{\circ}$ and $3300^{\circ} \mathrm{F}$, and $4 \%$ gas release in the zone from $2900^{\circ} \mathrm{F}$ out to the edge of the fuel. This results in a total gas release

SLIDE 10

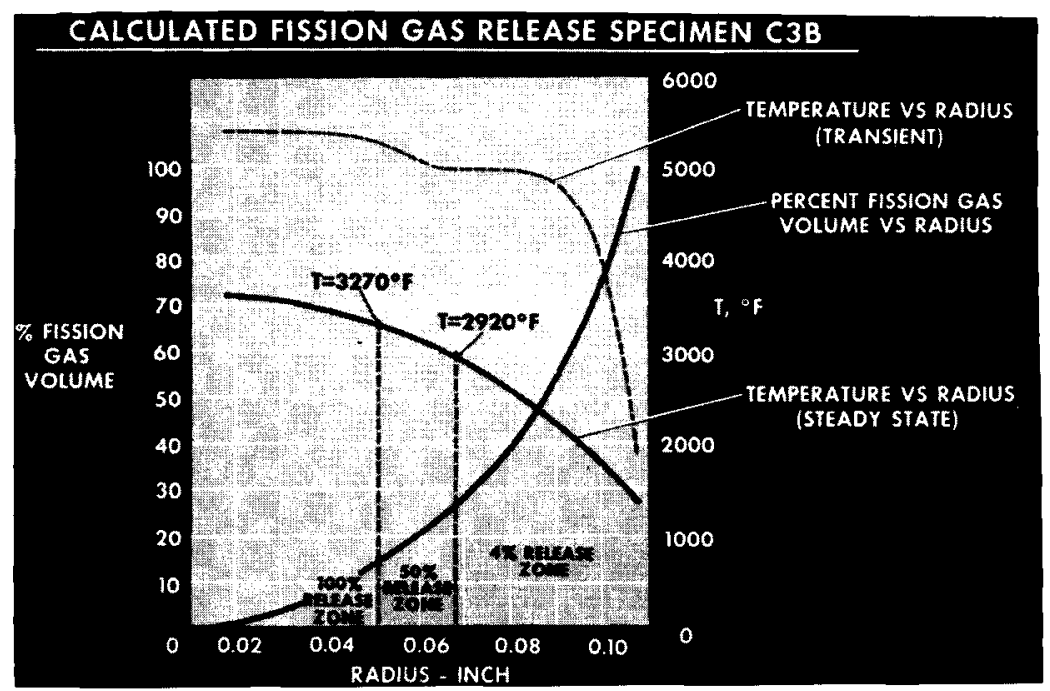

of about $29 \%$, which is consistent with measurements made on the control sample. Now, if we assume that in the transient, additional gas is released from only that fuel which becomes molten, (and this is strictly an assumption), the maximum temperature profile shown would result in an additional $38 \%$ gas release. We performed pressure calculations based on this total release and got surprisingly good correlation for the $3 \mathrm{mil}$ cladding deformation by using a simple hoop stress calculation. At this point we stopped calculating. I think it is obviously partly fortuitous, but at least, it indicates that we have a meaningful model here and we will continue to work on it.

We subsequently ran a high burnup specimen in a transient designed to melt fuel only in this $100 \%$ gas release region; in other words, avoiding any extra release of gas based on a theoretical mode1. As hoped, this transient caused no cladding damage, despite the fact that there was $11 \%$ molton fuel volume during the test.

We will soon be running another high burnup specimen. By high burnup, I mean 65,000 MWd/ton. We are going to expose this to a larger transient than the previous sample, designing a transient to try to release just enough gas in the $50 \%$ release zone to cause about $1 \%$ cladding deformation. If we come anywhere near that, then I think we will confirm that this model is a reasonable one, and we can proceed with more analytical work based on these assumptions.

Before leaving this exercise in geometry, I should mention the application of this model to a fast flux environment (keep in mind that we are doing this with a thermal flux steady state irradiation). Using this model in a fast flux environment indicates that the transient performance of the fuel may be significantly better since less gas is generated per fission event; plus you have a flat power profile and temperature profile which will result in less unreleased gas just prior to the transient to protect the cladding. We are proceeding to verify this point with a number of specimens that will be preirradiated in EBR-II (as Stan mentioned), and we may try to use 


\section{VENTED FUEL}

\section{E. L. Zebroski}

I would like to add a comment on the transient tests. It may not be obvious on these temperature traces that many of these tests are severe in a different way than the "50\% overpower" capability at steady state, which is an assumed criterion for the driver fuel reference design. These tests run from five hundred to several thousand times full-power peak instantaneous power during transient. On this bases, $50 \%$ overpower operation at a steady state corresponds to a transient of some tens of thousands of times full power in the peak. This may be an unrealistically large allowance for overpower. It would correspond to extremely large reactivity ramps, which should be designed out of the reactor.

Coming now to the vented fuel, the obvious advantage of vented fuel is not necessarily the most important. The obvious advantage is to lower the hoop stress on the cladding; however, the fission gas may itself be a small contribution to the total stress picture on the cladding. Allowing for that gain, you can reduce the amount of clad material and make a small gain in breeding ratio, giving a small economic incentive on the order of a few hundredths of a mil per kilowatt hour in fuel cycle costs. Possibly, important consequences of vented fuel may seem unexpected; that is, you may have a cleaner reactor system with vented fue 1 than without vented fuel. This is based on the assumption that with experience, you will always run fuel by actual experience on how far you can go before too much of the fuel fails and releases excessive amounts of fission products. There is a good chance that for a given burnup level there will be fewer gross failures with vented fuel than with nonvented fuel. The actual radioactivity, with which the reactor operator would have to contend, may well be smaller with vented fuel than with nonvented fuel.

Finally, the vented fuel offers a safety bonus in that it avoids a sudden release of fission gas, with possible gas blanketing of adjacent pins. This may also be a bonus reducing the amount of fuel that would fall if you should have a small accidental transient. The results of our transient tests show the fuel can experience a high power transient well into gross central melting with no immediate damage. However, the fuel then may not be in a condition to take a second transient. Therefore, it would be necessary to examine and unload the portion of the fuel which went beyond melting in such a transient. With properly vented fuel, the threshhold for incipient damage may be increased appreciably. (We should note that the occurrence of such transients is not assumed to be normal operation of the reactor. Hopefully, this is the sort of thing that might happen once in a lifetime to a reactor, so we are going in the direction of the reactor safety considerations rather than normal operating conditions). 
The status of the vented fuel testing is that there is only one test fully completed and examined so far; and that went uneventfully to $15,000 \mathrm{MWd} / \mathrm{ton}$.

Two additional tests have been operated, and one of these is in the process of being examined. These are long pin tests that use the capsules having circulating sodium. There are about four more long-pin tests under construction, most of which we hope to run this year.

In terms of design, there are two kinds of vents being considered. One is the "diving bell," which makes no attempt to control the delay time of the fission gasses. It gives an effective delay time of about 5 days, and that has certain consequences to the amount of radioactivity in the system. The other approach is to introduce a delay time in the gas release path. It appears practical to get delay times on the order of 1,000 hours. In this case the activity of the system is markedly reduced. In the first case, there is significant contribution to the activity level of the coolant. In periods of the order of two years, the buildup of cesium-134, reaches a level comparable with sodium-22. With delay times of $-10^{3}$ hours or better, you get essentially no contribution to the liquid coolant radioactivity. However, you do get a contribution to the cover gas radioactivity, mainly the 10 year krypton-85 ( $10^{4} \mathrm{Ci}$ at equilibrium). You do have to consider the cost of some additional gas-handling system for vented fuel. However, if you as sume that normal fuel 1 ife would be failure-rate limited, it is necessary to have a gas handling system even without postulating vented fuel.

The vented fuel tests (contrary to some expectations) may turn out to be relatively clean tests. For example, we have looked briefly at the possibility of doing a long pin vented fuel test in SRE. We find we would have to encapsulate the vent, not to protect the reactor coolant, but to protect the test. It would not be possible to measure the activity released from the vent if it were exposed to open reactor coolant.

Slide 1 summarizes the different vent concepts, the simple "diving bell", the closed plenum, and the high pressure concept (which no one is seriously considering).

Slide 2 is a specific capsule for which extensive examination has already been completed; the current ones running are long pins, with circulating sodium. This one used stagnant sodium. There are two regions of cover gas space; fission gas was found in both of these regions.

Slide 3 gives you the cesium buildup in the coolant if there is no effort to control the delay time; this gets to 
quite a high level. After one to two years, it becomes equal to the sodium-22 level in the reactor and then goes on to nearly a factor of five (higher than the sodium-22 if there is no separation or clean-up effects).

SCHEMATIC - FISSION GAS

ACCOMMODATION CONCEPTS

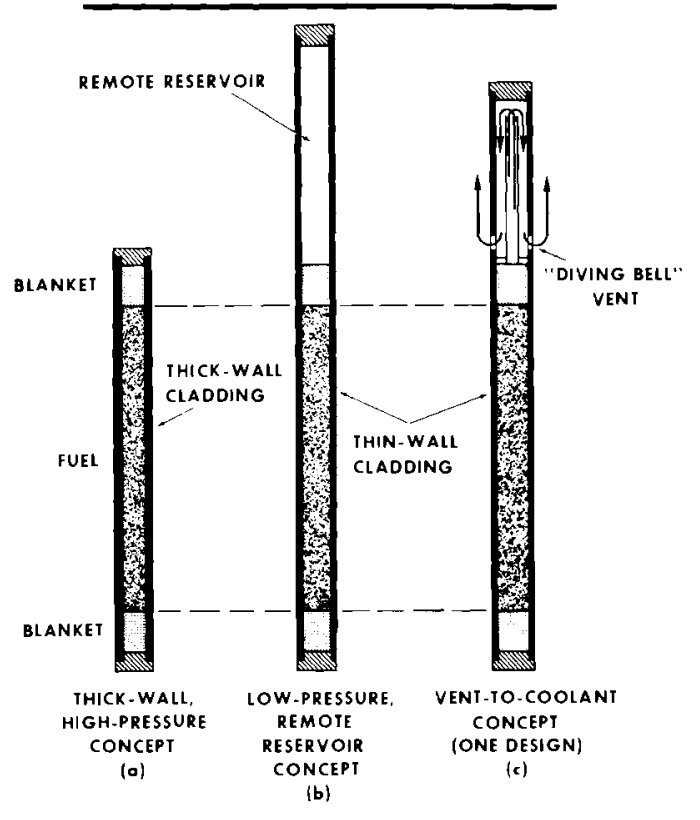

SLIDE 1 .

CESIUM-133 BUILDUP

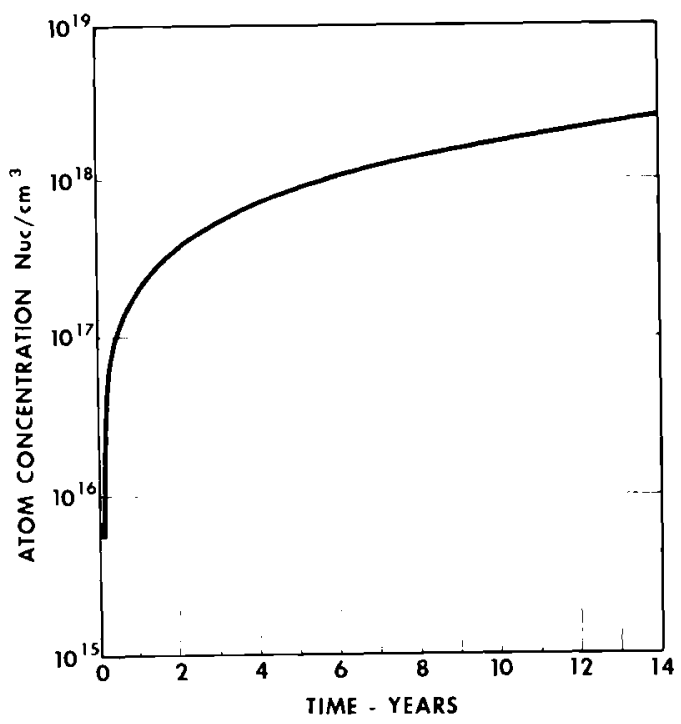

VENTED FUEL CAPSULE SCHEMATIC

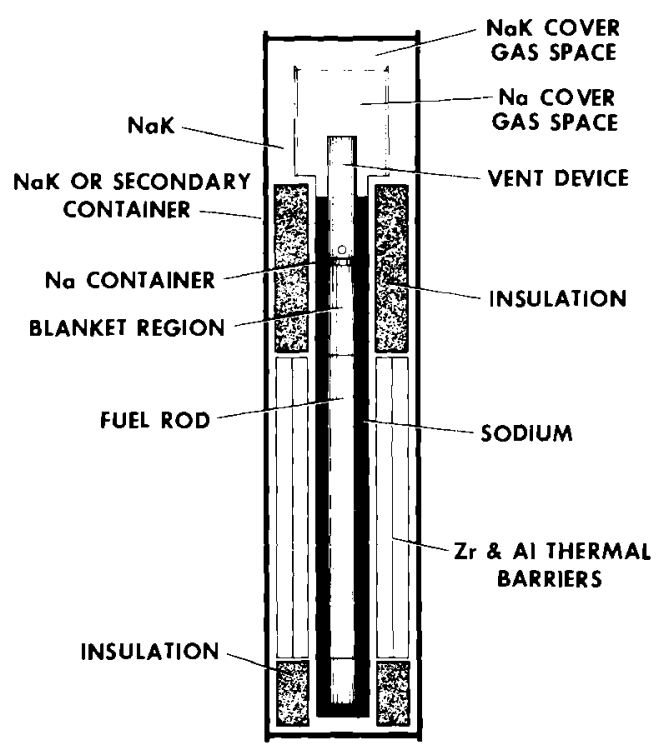

SLIDE 2.
EQUILIBRIUM COVER GAS ACTIVITY (CURIES) FOR VARIOUS HOLD-UP TIMES" FOR VENTED-TO-COOLANT

\begin{tabular}{|c|c|c|c|c|c|c|c|c|c|c|}
\hline ISOTOPE &, $1 / 2$ & o & $10 \mathrm{~m}$ & $1 \mathrm{~h}$ & $5 \mathrm{~h}$ & $15 \mathrm{~h}$ & $1 d$ & $5 d$ & $20 \mathrm{~d}$ & $60 \mathrm{~d}$ \\
\hline Kt-89 & $3.2 \mathrm{~m}$ & $3.54 \times 10^{7}$ & $4.06 \times 10^{6}$ & $3.54 \times 10^{1}$ & & & & & & \\
\hline$x_{e} .137$ & $3.8 \mathrm{~m}$ & $4.52 \times 10^{7}$ & $7.18 \times 10^{5}$ & $6.8 \times 10^{2}$ & & & & & & \\
\hline$x_{0}-135 m$ & $15 \mathrm{~m}$ & $1.38 \times 10^{7}$ & $8.84 \times 10^{6}$ & $9.6 \times 10^{2}$ & $1.38 \times 10^{-1}$ & & & & & \\
\hline$x_{*}-138$ & $17 \mathrm{~m}$ & $4.20 \times 10^{7}$ & $2.82 \times 10^{7}$ & $3.77 \times 10^{6}$ & $2.1 \times 10^{2}$ & & & & & \\
\hline$K_{r-87}$ & $1.3 \mathrm{~h}$ & $2.08 \times 10^{7}$ & $1.91 \times 10^{7}$ & $1.22 \times 10^{7}$ & $1.04 \times 10^{6}$ & $7.3 \times 10^{3}$ & $5.72 \times 10^{1}$ & & & \\
\hline$k_{1}-83 m$ & $1.86 \mathrm{~h}$ & $3.70 \times 10^{6}$ & $3.45 \times 10^{6}$ & $2.56 \times 10^{6}$ & $5.55 \times 10^{4}$ & $1.45 \times 10^{4}$ & $1.37 \times 10^{2}$ & & & \\
\hline$x_{r}-28$ & $2.8 \mathrm{~h}$ & $2.84 \times 10^{7}$ & $2.73 \times 10^{7}$ & $2.22 \times 10^{7}$ & $8.5 \times 10^{6}$ & $7.1 \times 10^{5}$ & $7.4 \times 10^{4}$ & & & \\
\hline$k_{r}-b_{s m}$ & $4.4 \mathrm{~m}$ & $1.15 \times 10^{7}$ & $1.12 \times 10^{7}$ & $9.8 \times 10^{6}$ & $3.2 \times 10^{5}$ & $1.06 \times 10^{6}$ & $2.63 \times 10^{5}$ & & & \\
\hline$x_{e-135}$ & $9.2 \mathrm{~h}$ & $4.90 \times 10^{7}$ & $4.75 \times 10^{7}$ & $4.52 \times 10^{7}$ & $3.34 \times 10^{7}$ & $1.45 \times 10^{7}$ & $8.04 \times 10^{6}$ & $5.23 \times 10^{3}$ & & \\
\hline$x_{e} .133 \mathrm{~m}$ & $23 \mathrm{~d}$ & $1.30 \times 10^{6}$ & $1.30 \times 10^{6}$ & $1.28 \times 10^{6}$ & $1.22 \times 10^{6}$ & $1.08 \times 10^{6}$ & $9.62 \times 10^{5}$ & $2.86 \times 10^{5}$ & $3.25 \times 10^{3}$ & \\
\hline$x_{e} \cdot 133$ & $5.3 \mathrm{~d}$ & $5.32 \times 10^{7}$ & $5.32 \times 10^{7}$ & $5.32 \times 10^{7}$ & $3.15 \times 10^{7}$ & $4.9 \times 10^{7}$ & $4.66 \times 10^{7}$ & $2.76 \times 10^{7}$ & $3.72 \times 10^{6}$ & $2.12 \times 10^{4}$ \\
\hline$x_{e}-131 m$ & $\begin{array}{ll}12 & d\end{array}$ & $1.54 \times 10^{5}$ & $1.54 \times 10^{5}$ & $1.54 \times 10^{5}$ & $1.54 \times 10^{5}$ & $1.48 \times 10^{5}$ & $1.45 \times 10^{5}$ & $1.15 \times 10^{5}$ & $5.4 \times 10^{4}$ & $9.8 \times 10^{3}$ \\
\hline$K_{r-\theta S}$ & $10.6 \mathrm{r}$ & $75 \times 10^{2}$ & $7.5 \times 10^{2}$ & $7.5 \times 10^{2}$ & $7.5 \times 10^{2}$ & $7.5 \times 10^{2}$ & $7.5 \times 10^{2}$ & $7.5 \times 10^{2}$ & $7.5 \times 10^{2}$ & $7.5 \times 10^{2}$ \\
\hline TOTAL & & $276 \times 10^{8}$ & $2.11 \times 10^{8}$ & $1.51 \times 10^{8}$ & $1.01 \times 10^{8}$ & $0.07 \times 107$ & $5.6 \times 10^{7}$ & $2.0 \times 10^{7}$ & $3.70 \times 10^{6}$ & $2.67 \times 10^{4}$ \\
\hline
\end{tabular}

- ASSUME 35\% RELEASE FRACTION FROM IHE FUEL

SLIDE 3. 
Slide 4 summarizes the equilibrium activity if reasonable delay times are obtained. This corresponds to the total core and a generous assumption of the release fraction of fission gas from the fuel, $35 \%$ overall gas release fraction. With delay times in the range of 20 to 60 days, then krypton- 85 becomes a predominant long-lived activity because the two long-lived xenons are on 1 y 12 days and 5 days, respectively. From the standpoint of the gas handling system, there is practical1y pure krypton-85. This is a problem that you would have to face anyhow since processing the fuel also releases this gas. So going to vented fuel doesn't change the total contamination to the world.

\begin{tabular}{|c|c|c|}
\hline \multicolumn{3}{|c|}{$\begin{array}{l}\text { MEASURED FISSION PRODUCTS } \\
\text { RELEASED TO COOLANT } \\
\end{array}$} \\
\hline NUCLIDES & HALF LIFE & RELEASE FRACTION \\
\hline \multicolumn{3}{|l|}{ GASES: } \\
\hline$X e-134$ & $\infty$ & 0.46 \\
\hline$K_{r-85}$ & $10.6 y$ & 0.44 \\
\hline$x_{e-131 m}$ & $12 d$ & 0.3 \\
\hline$x_{e-133}$ & $5.3 \mathrm{~d}$ & 0.27 \\
\hline$x e-133 m$ & $2.3 \mathrm{~d}$ & 0.05 \\
\hline$X_{e}-135$ & $9.2 \mathrm{~h}$ & $<0.0003$ \\
\hline
\end{tabular}

SOLIDS:

$\begin{array}{lccl}\text { Sr-89 } & 51 \text { d } & 7 \times 10^{-7} \\ \text { Sr-90 } & 28 \text { y } & 7 \times 10^{-8} \\ \text { Y-91 } & 58 \text { d } & 4 \times 10^{-8} \\ \text { Zr-95 } & 65 \text { d } & 5 \times 10^{-8} \\ \text { Ru-103 } & 39.7 \text { d } & 4 \times 10.10 \\ \text { I-131 } & 8 \text { d } & 1 \times 10.8 \\ \text { Cs-137 } & 29 \text { y } & 1 \times 10.6 \\ \text { Ba-140 } & 12.8 d & 6 \times 10^{-10} \\ \text { C }-144 & 285 \text { d } & 4 \times 10^{-7}\end{array}$

SLIDE 5.

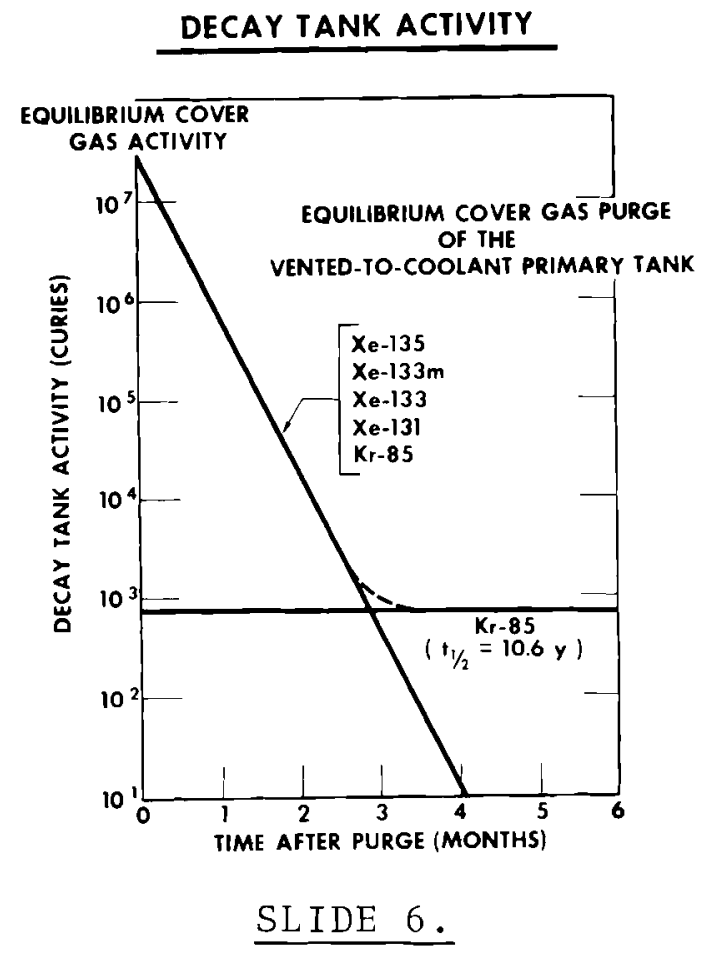

Slide 5 shows the measured fission products released to the coolant in the previously pictured vented fuel capsule. The average release fraction was about $44 \%$. The solids that were "released" are not necessarily real, since these are right down in the background level of detectability of these activities. The release tapered off within the first few hundred mils in the blanket region above the fuel pin (there was a $1-13 / 16$ in. blanket region above the fuel pin).

Slide 6 shows what you get in the decay tank of the system. This indicates that you're going to have to have some sort of a gas handling system in any event for either vented or nonvented fuel, because it is assumed that some fuel will fail and release gas. If you have a decay tank and if you have a two to three month hold up time, you virtually release krypton -85 . 
Slide 7 illustrates that if you do not control the delay time and if you must take the ambient (roughly five day) delay time that you get with the open vent, then the cesium-134, which is formed by neutron capture from cesium-133, becomes a dominant activity in the case of vented fuel.

SLIDE 7.

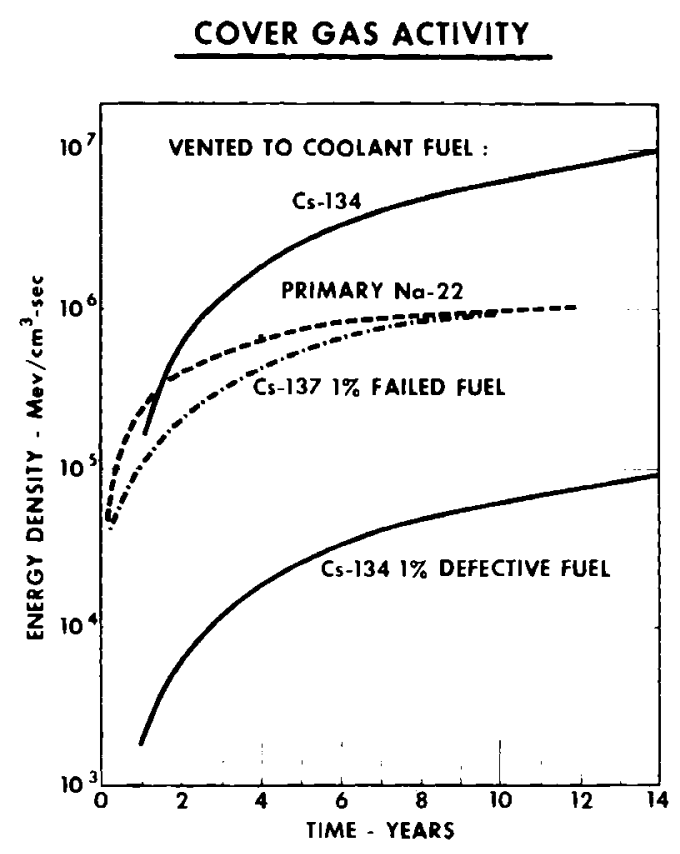


OXIDE FUEL, STATUS AND STRETCH CAPABILITIES

E.L.Zebroski, W. E. Bailey

S. A, Rabin, and $j, H$, Field

\section{DISCUSSION}

MR。R。E。 PETERSON (Pacific Northwest Laboratory):

Regarding the selection of overpower factors, I was curious. You mentioned only one extreme condition. Our studies also included essentially incipient melting as a normal operating state. I was wondering, between the two extremes we looked at, do you have any feeling where the normal operating conditions should lie?

MR. ZEBROSKI: That is a function of the speed of the scram system. Your scram system, we have assumed pessimistically, would require $400 \mathrm{msec}$ to insert a scram rod. If there is an incentive, you can speed that up considerably. If you take the kinds of transients John Field was showing and chop them off at 400 msec essentially nothing has happened to the fuel except you would have to write a report on the reason for the scram. That is very safe for the two-dollars-a-second kind of accidents. If you want to postulate a much more serious accident, approaching 10 or 20 dollars a second, you can still be in a low fuel damage situation. But then you would like to chop it off with a faster scram system. I haven't really answered your question, but obviously it is a function of the design of the control system and the size of the Doppler coefficient.

MR. PETERSON: I have one more question along this same 1ine. That is, what sort of fuel criteria should be adopted here? In your estimation, should this be the same as adopted for a large fast breeder, or more conservative?

MR. ZEBROSKI: Obviously you are in a more exposed situation because of the variety of fuel loadings possible. You would have to defend the proposition that a greater variety of accidents is also possible. So I think you would want to put in somewhat more margin.

MR。BURNHAM (Pacific Northwest Laboratory): Are you proposing, Ed, that the FFTF driver fuel include BeO or not?

MR. ZEBROSKI: Right now our best estimate is that the uncertainty of the Doppler coefficient calculation for a large reactor concept is on the order of $\pm 40 \%$. For a $\mathrm{PuO}_{2}$ cermet the uncertainty might be much greater than that. The value may remain smaller, but the uncertainty, on a percentage basis 
is greater. If the plutonium Doppler is very sma11, then for the cermet the choice of spectrum is not a safety question, but a question of what you want to simulate in your test environment. You would not have a firm answer to the Doppler question for $\mathrm{U}^{238}$ until the SEFOR transient tests are run. These will give a measured integrated Doppler so that you won't have to allow for such a big calculational uncertainty.

MR. BURNHAM: Let me say, if we were to specify a fuel today, would we have to specify BeO?

MR。 ZEBROSKI: You would probably want to keep that option both for safety and to simulate the spectrum of a large dilate core. The feeling of our own group is that the $\mathrm{BeO}$ is a conservative allowance. There is a good chance that you won't need it (or not very much of it) in a large reactor. You mainly want to preserve the option of adjusting the Doppler coefficient to whatever value your safety analysis tells you you must have.

MR deBOISBLANC (Phillips): What range of temperatures did you cover in studying the vent release rate?

MR. ZEBROSKI: Most of them have been run at the nominal peak power condition of large core design which is 20 to 24 $\mathrm{kW} / \mathrm{ft}$, and central temperatures near $2600^{\circ} \mathrm{C}$.

MR。deBOISBLANC? The other question related to postulating a holdup time up to say 60 days. I am now thinking of the FFTF. If the delay time is zero then the benefits of venting in the sense of the lower internal pressures are realized; if on the other hand, the delay time is infinite, there is no advantage from the fuel. So the question is, in the FFTF type of condition, with burnup in relatively short periods of time, would it be advisable to have a delay time of, say, 60 days? Obviously not.

MR. ZEBROSKI: For oxide fue1, burnup times would stil1 be several years. You can get the thousand-hour delay time with a plenum pressure of $100 \mathrm{psi}$, which is still negligible compared with 1500 psi you would have if you didn't vent.

MR. WEBER (Atomics Internationa1): How far have you proceeded along the lines of testing to the design burnup levels of oxide elements representative of the cooler region, as well as the higher? I ask this because of this uncertainty on the elasticity of oxides at lower temperature, and at what burnup levels did you change the stress level on the pins?

MR. ZEBROSKI: We11, that was covered by the chart that Mr. Stan Rabin showed. There are one or two bundles running out about Row 7 in EBR-II which are addressed just to that question. 
MR. WEBER: You don't have a lot of data now?

MR. ZEBROSKI: We don't have a lot of data now. However, there is one very interesting set of experiments going on right now, and we have already had the first couple of points. This is a swelling experiment as a function of central temperatures where the capsules are designed like a dilatometer. The problem of the big scatter in the swelling data is that it is an extremely difficult measurement to make with any degree of precision. We hope to have avoided this by a design of a gun-barrel type of experiments with very thick aluminum cans around the stainless steel, and the means of reading out the deformation of the fuel column. We can read it every $25,000 \mathrm{MWd} / \mathrm{t}$ on or so, and then put it back in the reactor. We have the first four of these right now, and the first four readings tend to show that the swelling is just barely above the experimental noise level, $35 \mathrm{mils} \pm 25 \mathrm{mils}$ reading at this point. These experiments are being run at a series of different peak temperatures. This should give a precise measure of swelling as a function of the peak temperature.

MR. de HALAS: Ed, a couple of days ago when I gave a brief summary of our reasoning for a choice of fuel, I indicated that the thing we were most scared about was the oxide; the fact it looked like it needed some reasonable porosity in there to accomodate swelling, and that we didn't know what was needed in terms of a program actually to demonstrate this porosity was going to stay where you wanted it. Al Shoudy expressed a similar doubt the other day, which I think kind of best expressed it. What do you do to prove, for example, to the ACRS that you have a safe system? I mean it is like any reactor pressure vesse1; I don't know how you would get a final answer on it. I wondered what your comments were. Is this going to be an easy or hard part of the problem?

MR. ZEBROSKI: The question of fuel positioning is clearly important, and has some encouraging answers which we can discuss. However, the charge from Bob Hennig just a minute ago suggested that we put such questions that require extended discussion over to the afternoon panel. 


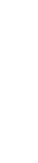

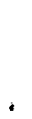




\section{STATUS OF STAINLESS STEEL-UO ${ }_{2}$ CERMETS \\ D. L. Keller}

Battelle Memorial Institute

Columbus, Ohio

INTRODUCTION

First, I would like to comment on some of the discussions pertinent to cermet fuels.

- I agree with Ken Merkx who summed up discussions on $\mathrm{UO}_{2}$ porosity collecting at the fuel particle interface and 2 forming a heat transfer barrier as being a ghost problem. Experimental results have shown (to my knowledge) no evidence of this happening, so we shouldn't concern ourselves too much with this problem.

- I disagree with the suggestion that high gas release, resulting from high fuel particle temperatures, may not be undesirable. Every effort should be made to retain the fission gas within the $\mathrm{UO}_{2}$, either as a fine precipitate or as isolated gas bubbles. Release of gas from the $\mathrm{UO}_{2}$ can be minimized by keeping the particles as cold as possible. If, for example, we release all the gas from the FTR fuel containing $20 \%$ porosity, we must contend with about 9,000 psi internal gas pressure.

- The reference to English cermets showing superior irradiation stability because of improved structure is incorrect.

- The use of metallic coatings on fuel particles to insure thermal bonding is unnecessary. Using coatings to improve homogeneity will not likely result in improved radiation stability, and the suggestion that coatings can be provided at $10 \%$ additional cost over the cost of shaping the particle is not reasonable. However, I do believe that coatings must be an important part of any substantial improvements in cermet performance.

- Reduction of $\mathrm{PuO}_{2}$ to a lower oxide or free plutonium in hydrogen at $1200^{2}{ }^{\circ} \mathrm{C}$, which is the maximum temperature for sintering stainless $\mathrm{PuO}_{2}$ mixtures, will not likely occur.

- Cermet pins will not have increased burnup capability over plates. Cermet pins will provide more restraint, and thus have a lower growth rate, but they will probably fail at a lower volume change since the cladding will be strained more severely.

- Cermet technology has not (in my opinion) shown any significant improvement in burnup capability during the past five years. Another way of saying this is that the performance of 
these fuels is quite predictable and insensitive to most recent inovations, particularly, improvements in homogeneity and particle shape.

Now, I hope I can bring out most of these points during the course of the presentation I have prepared for this meeting.

My talk will be made in three parts: A discussion of the growth characteristics of dispersion fuels that occurs prior to failure, a review of the failure limits of stainless stee 1 dispersion fuels with emphasis on data obtained during the past three years; and, finally, a brief discussion of the stretch capability of dispersion fuels.

\section{GROWTH OF DISPERSION FUELS}

One method of evaluating the growth characteristics of disper sion fuels is to measure the per cent density change in an entire specimen, converting this to a volume change in the UO fuel particles, and calculating the growth rate of the UO, particles in terms of a unit burnup. It is useful then to compare the UO particle growth rate in dispersion fuels to the growth of bulk fo as measured by Bleiberg and shown in Slide 1. In this manner, the relative restraint by the stainless steel matrix and clad, or the effect of porosity on the growth of the $\mathrm{UO}_{2}$ particles in dispersion fuels can be evaluated.

In S1ide 2, the percent growth of UO fuel particles is plotted versus the total burnup in the fuel particles for a number of similar dispersion fuels. Results obtained at 700,1050 , and $1300^{\circ} \mathrm{F}$ are identified.

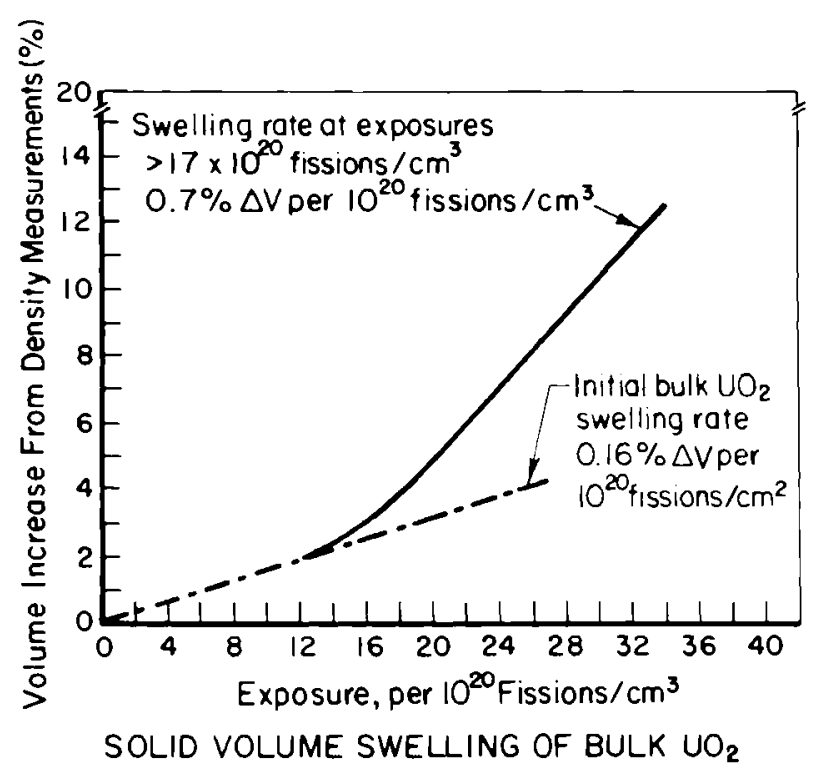

$\underline{\text { SLIDE } 1}$

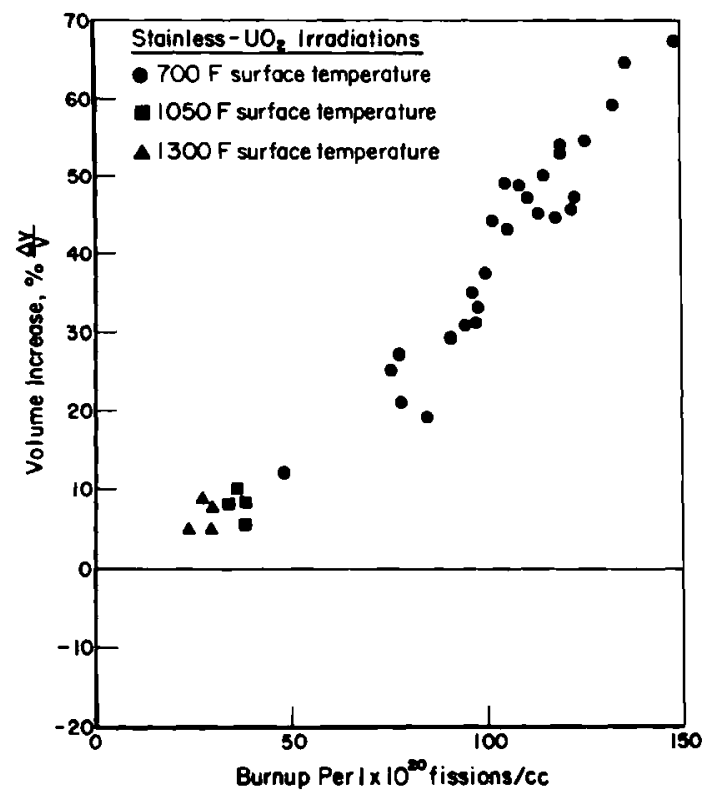

SLIDE 2 
In Slide 3 , the results are interpreted to show an initial growth rate of $0.22 \Delta \mathrm{V} / 10^{20} \mathrm{f} / \mathrm{cm}_{3}^{3}$ followed by a second and higher rate of $0.57 \Delta \mathrm{V} / 10^{20} \mathrm{f} / \mathrm{cm}^{3}$. The change in rate occurred at a burnup of about $65 \times 10^{20} \mathrm{f} / \mathrm{cm}^{3}$, suggesting the pores filled at this point after which a final growth rate is established that is characteristic of dense $\mathrm{UO}_{2}$. Extrapolating the final growth rate back to zero burnup indicates an initial $\mathrm{UO}_{2}$ porosity of $25 \%$.

Using the same data points, a temperature dependency (related to the restraining force of stainless steel) of $\mathrm{UO}_{2}$ growth is shown in Slide $4 a$. It is assumed in this case that the lower burnup-higher temperature data would extrapolate back to the same point at zero burnup. The author prefers this interpretation.

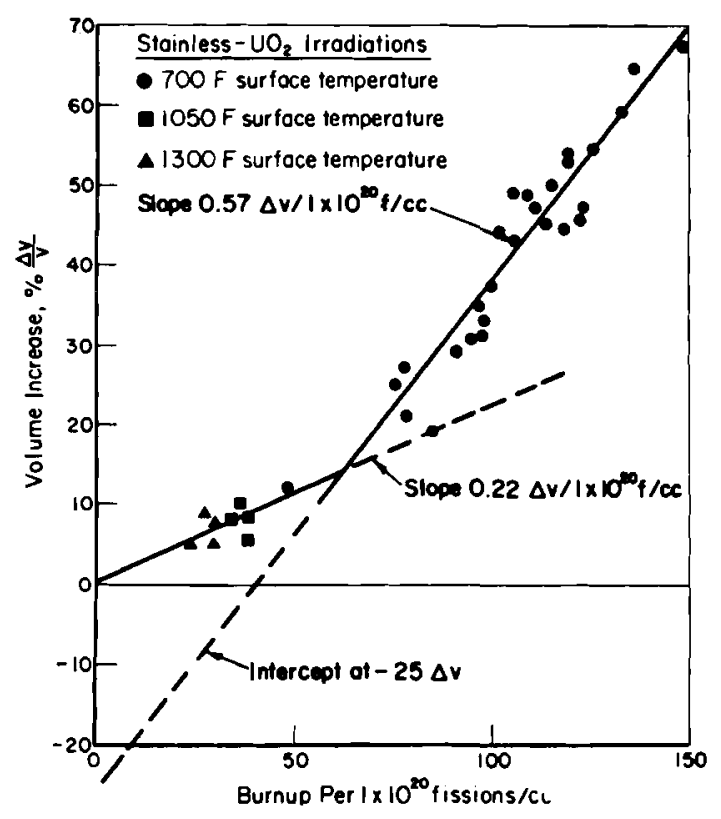

SLIDE 3

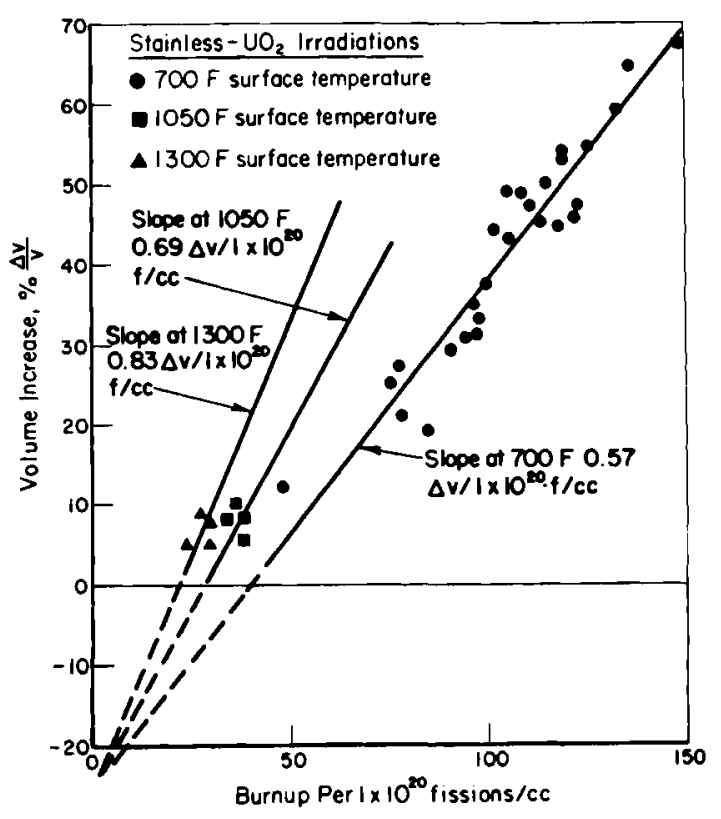

SLIDE $4 \mathrm{a}$

The initial porosity of the oxide fuel particles in all cases was approximately $5 \%$; however, their growth seems more characteris tic of particles containing $25 \%$ porosity. Quantitative metallographic pore counting of irradiated specimens has shown that the volume of residual porosity in the $\mathrm{UO}_{2}$ usually exceeds the total volume of oxide swelling. This is most reasonably explained by the diffusion of porosity from the stainless matrix to the $\mathrm{UO}_{2}$ fuel particles. Here, it agglomerates along with the fission gas without causing an external change in specimen dimensions. These observations point toward a conclusion that trace porosity in the stainless matrix accounts for much of the observed porosity in irradiated $\mathrm{UO}_{2}$ particles and, in some manner, is responsible for the initially dense oxide particles behaving like they contain significantly larger amounts of porosity. 
In SIide 4b, the swelling or growth rate of dispersion fuels prior to failure is plotted against temperature. It is predicted from this curve that the FFTF driver fuel will expand at a rate of 0.6 to $0.7 \Delta V$ per I x $1020 \mathrm{f} / \mathrm{cm}^{3}$ at a temperature of $900^{\circ} \mathrm{F}$.

SLIDE 4 b

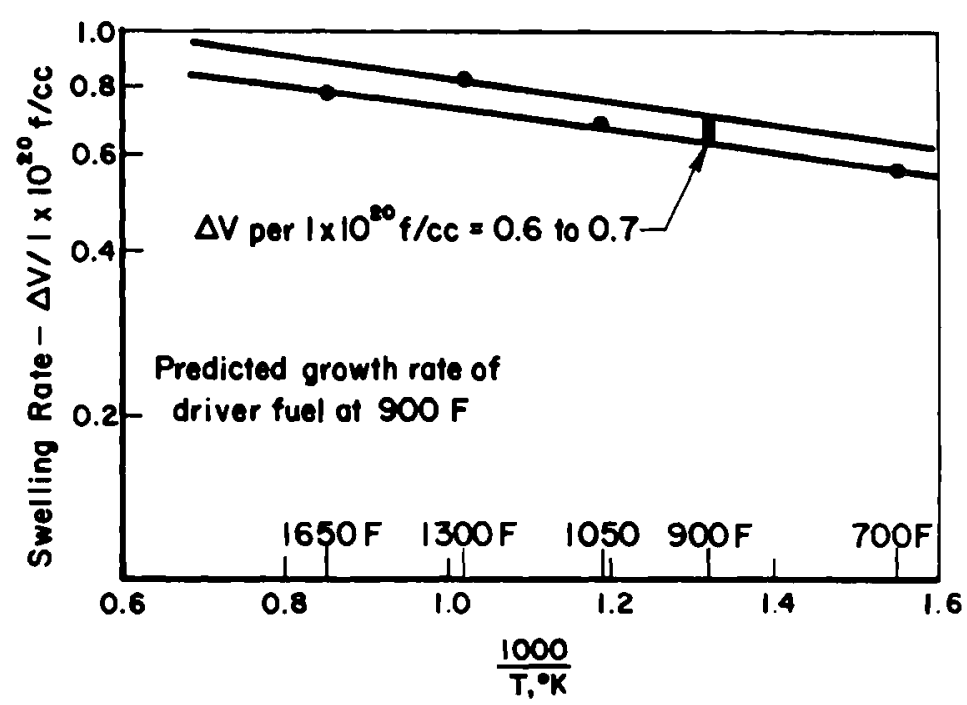

FAILURE OF DISPERSION FUELS

As the growth of the oxide particles continues and as fission gases excape the $\mathrm{UO}_{2}$ to react against the restraining matrix, the stainless steel is eventually strained beyond its ductility limit and failure occurs. The curve relating the combination of burnup and temperature at which this happens in stainless stee1-UO $\mathrm{J}_{2}$ is persions is shown in Siide 5. The hashed region, drawn by Keller of BMI in 1961, shows a zone between those conditions which cause failure and those which do not. The solid line is a curve drawn by Thurber of ORNL in 1963 and shows a sharper transition between failure and nonfailure and is based on some additional data. It is clear, however, that no significant changes occurred in the performance of dispersion fuels during that period.

More recent data on dispersion fuels is somewhat sparse but quite significant.

Stainless steel-UO $\mathrm{UO}_{2}$ elements, being developed for the $\mathrm{A}^{2} \mathrm{~F}^{2}$, were irradiated at $500^{\circ} \mathrm{F}$ then heated at $100^{\circ} \mathrm{F}$ intervals until blisters appeared.(1) As shown in Slide 6, failure did occur where expected.

Information provided by Vic Pearson, ANL and Martin Garbor, Philips Petroleum Company. 
21.5

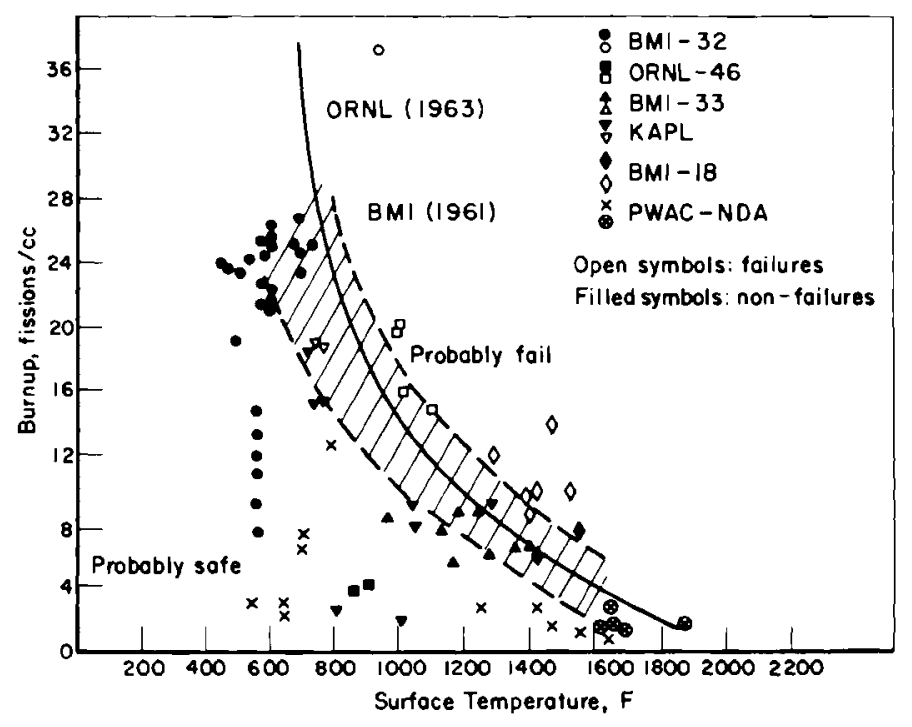

IRRADIATION STABILITY OF UO $\mathrm{U}_{2}$-STAINLESS DISPERSIONS

\section{SLIDE 5}

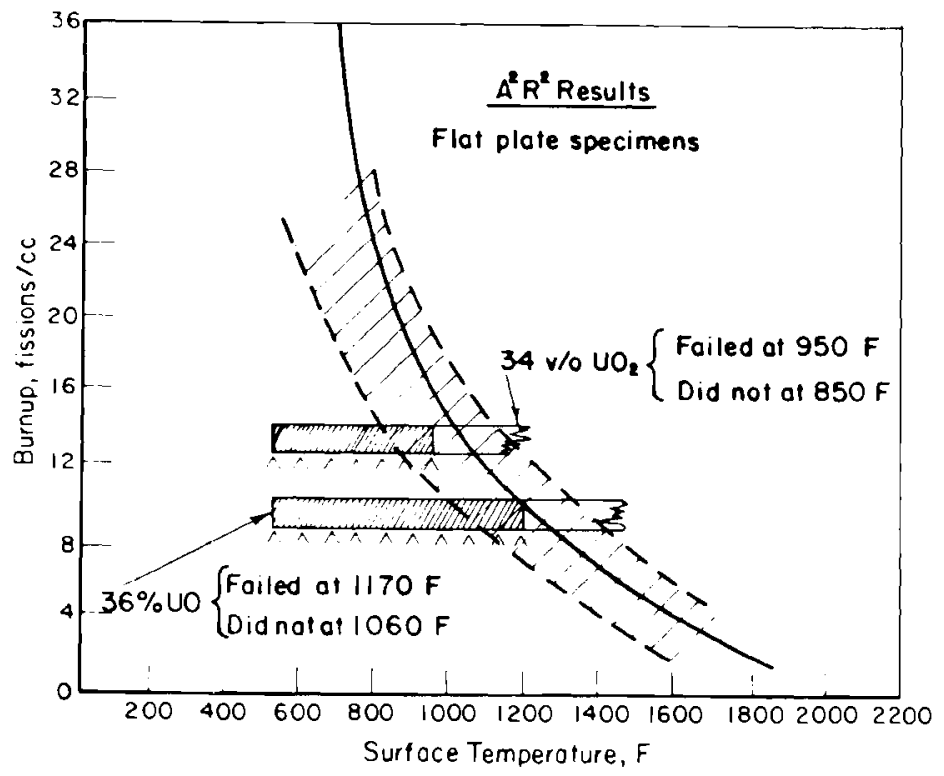

SLIDE 6 
Nichrome V-UO2 elements, irradiated at surface temperatures between 1400 and $1600^{\circ} \mathrm{F}$ and possessing a more ideal distribution of fuel particles than is obtained by conventional fabrication processes, nonetheless, failed (S1ide 7) in accordance with the established curves. (2)

\section{$\underline{\text { SLIDE } 7}$}

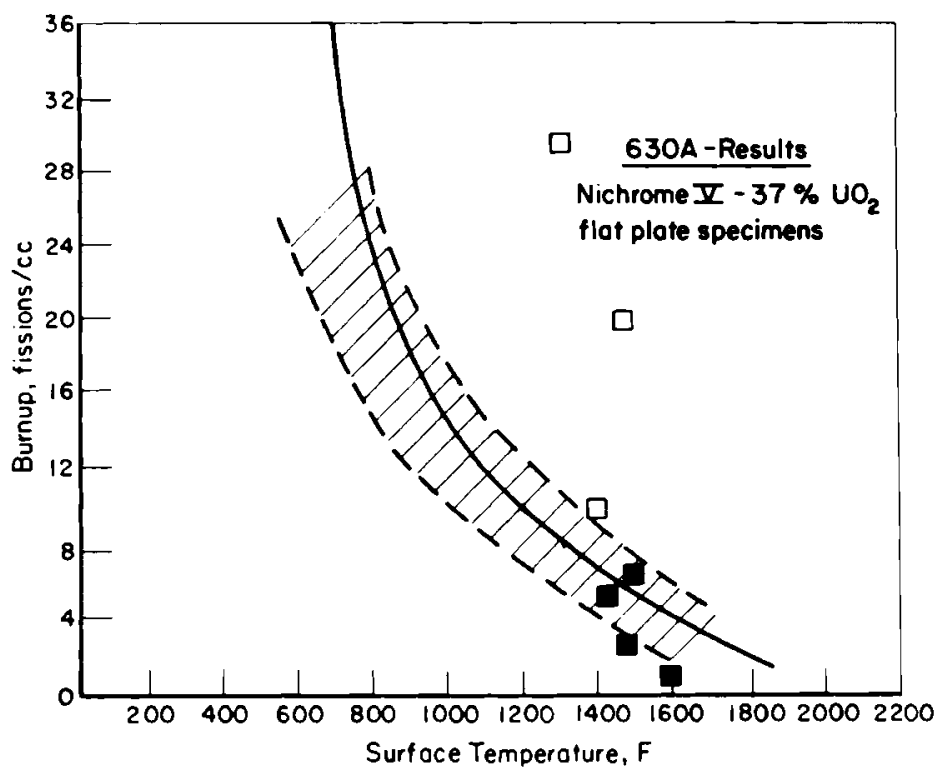

Harwel1 data, Siide $8-11,(3)$ is often referred to as showing superior performance of dispersion fuels when a near ideal distribution of $\mathrm{UO}_{2}$ particles is achieved. The data instead demonstrates that if unusualiy poor structures are obtained, whether in plates or pins, the irradiation performance is markediy reduced. The "improved" or "ideal" structures, although near perfect in distribution of fuel particles, have so far performed in line with expected behavior.

(2) Information provided by R. Robertshaw of GE-NMPO. 


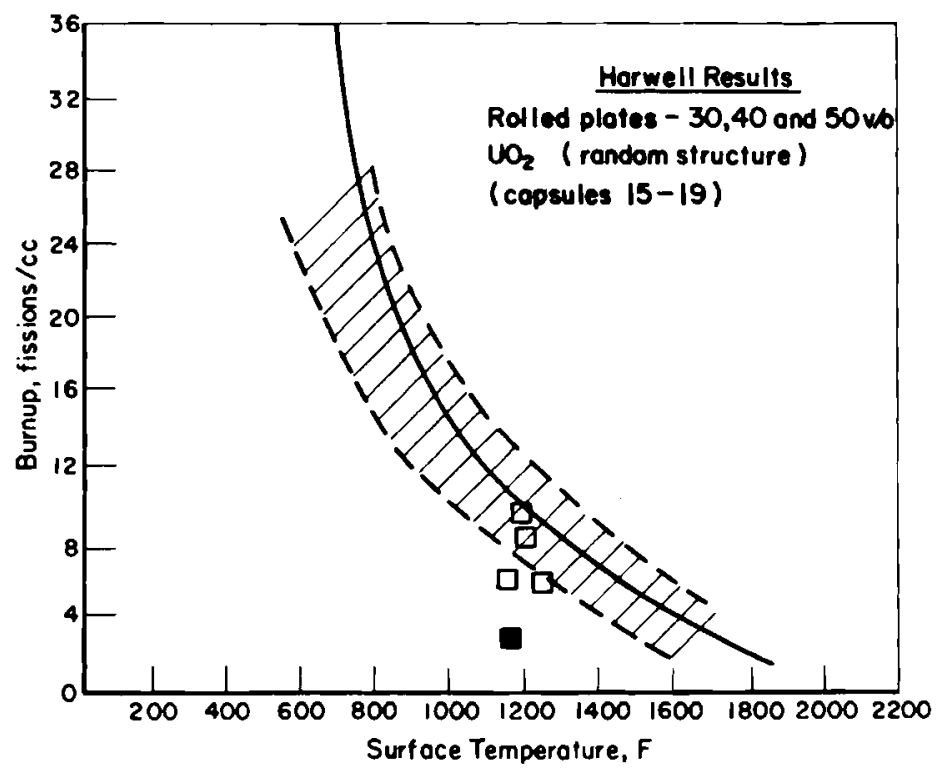

\section{SLIDE 8}

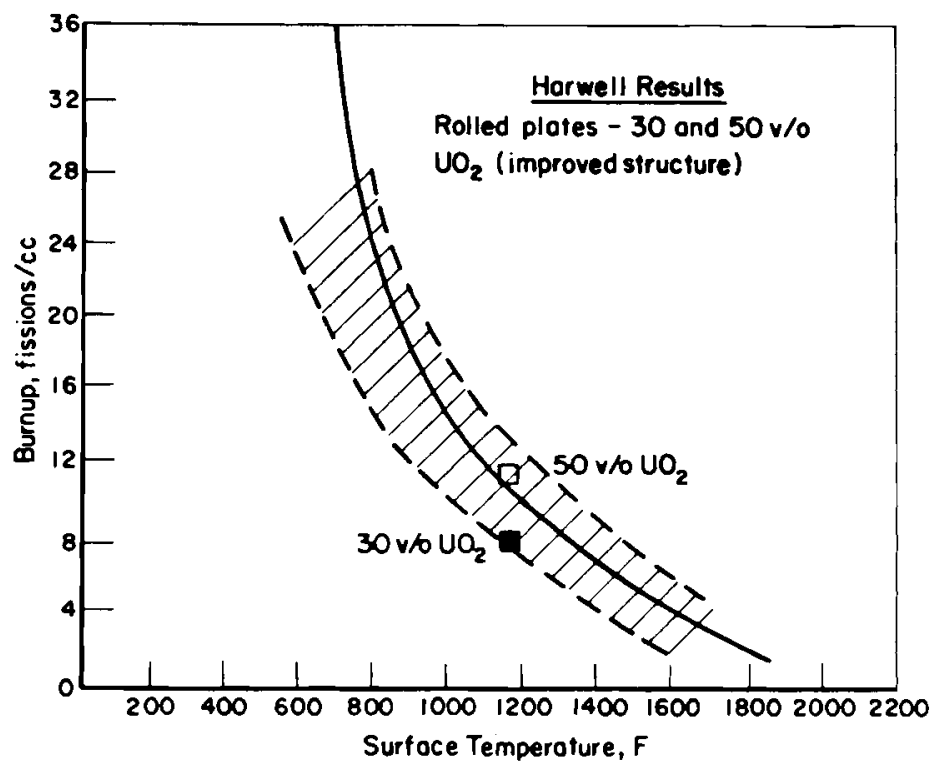


21.8

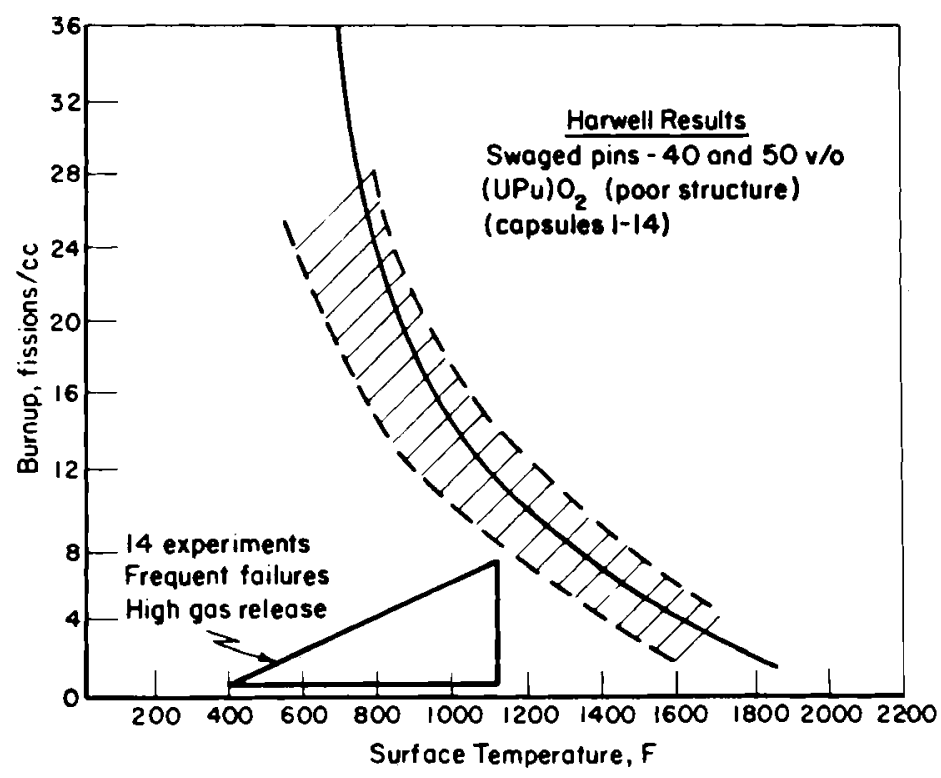

SLIDE 10

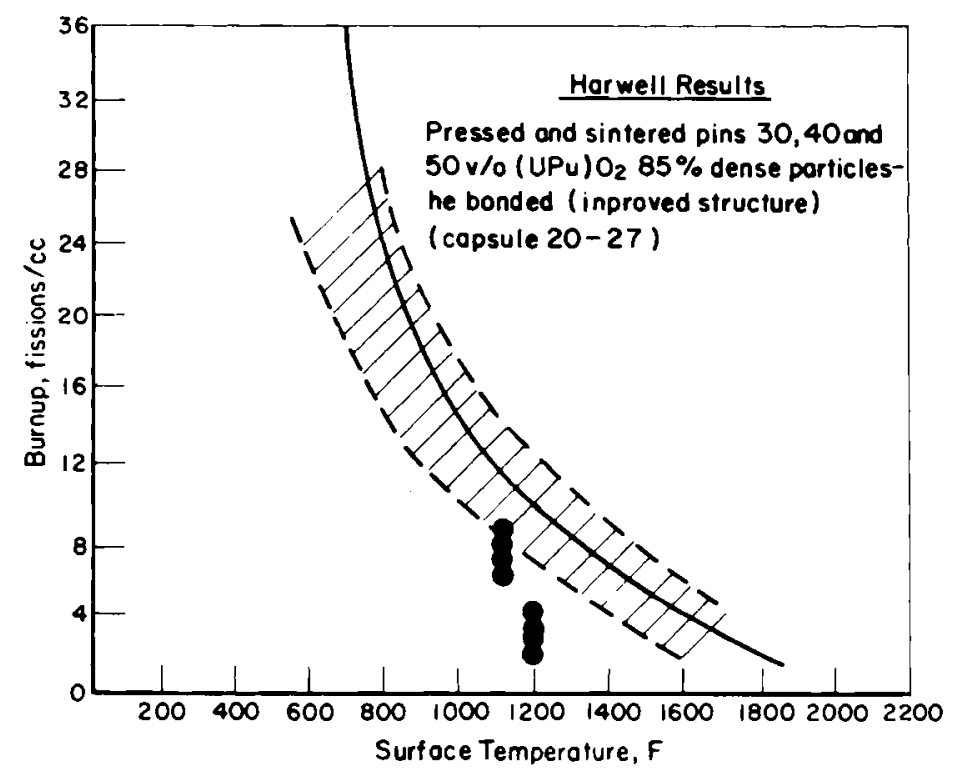

SLIDE 11 
In Slide 12, the design fuel life for the SM-1, SM-1A, and PM- $2 \mathrm{~A}$ reactors is shown. These cores contain flat plate dispersion elements and each has operated to $100 \%$ of design. The performance of the tubular dispersion elements in the PM-1 and PM-3A cores is depicted in S1ides 13-14, respectively. In each case, and activity release was detected at about $12 \times 10^{20} \mathrm{f} / \mathrm{cm}^{3}$. Operation has continued to nearly design life in the PM-l core with no further activity release.

\section{SLIDE 12}

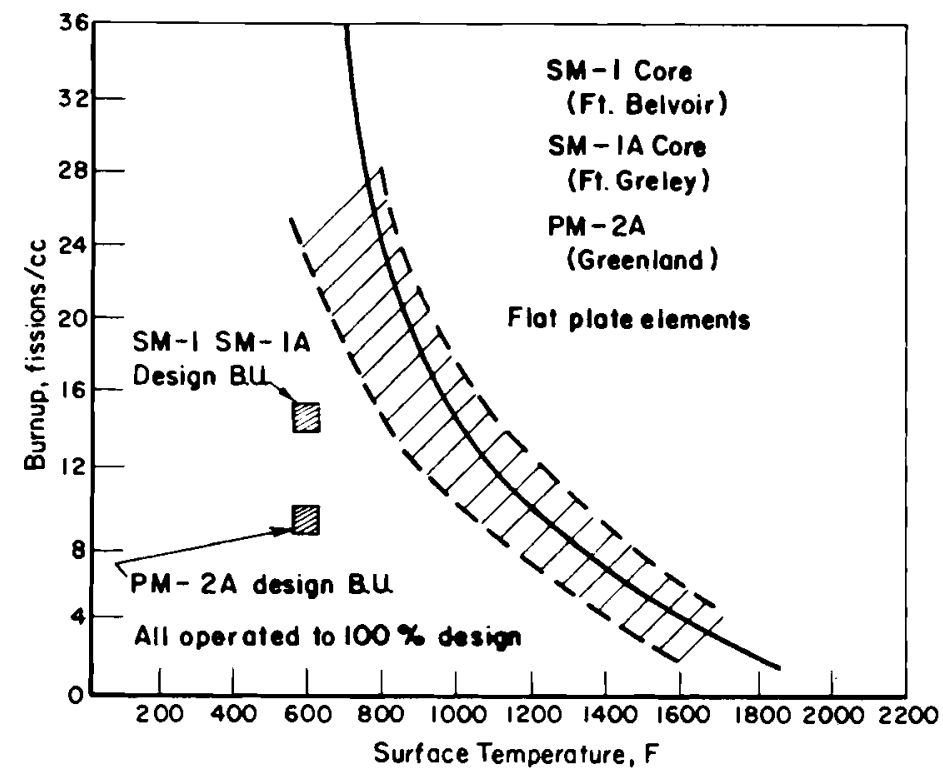

SLIDE 13

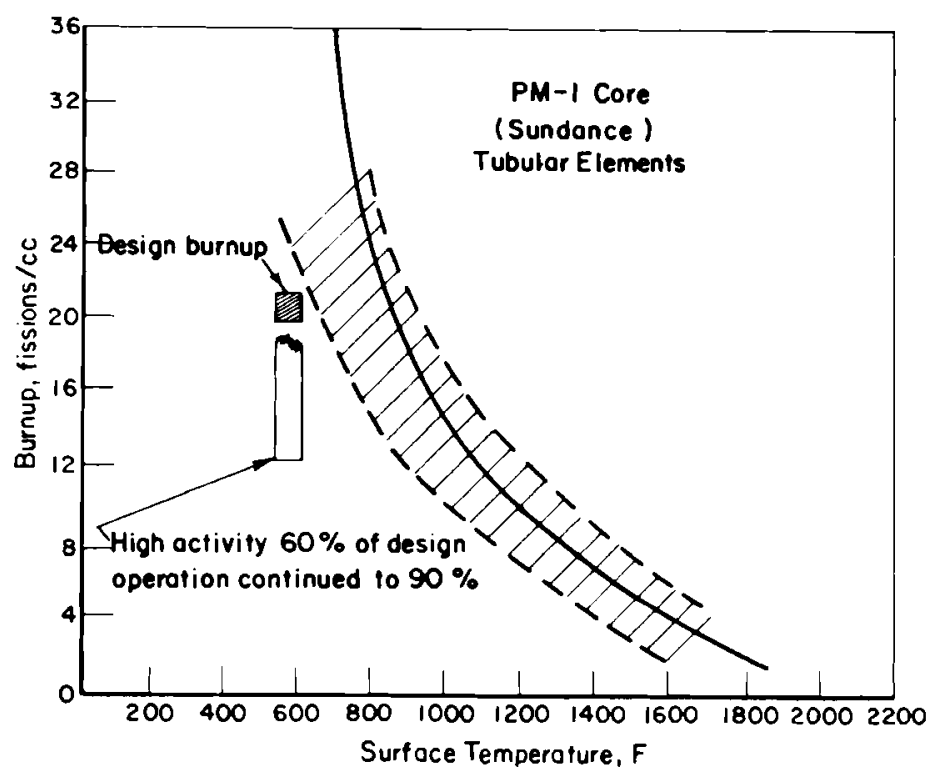

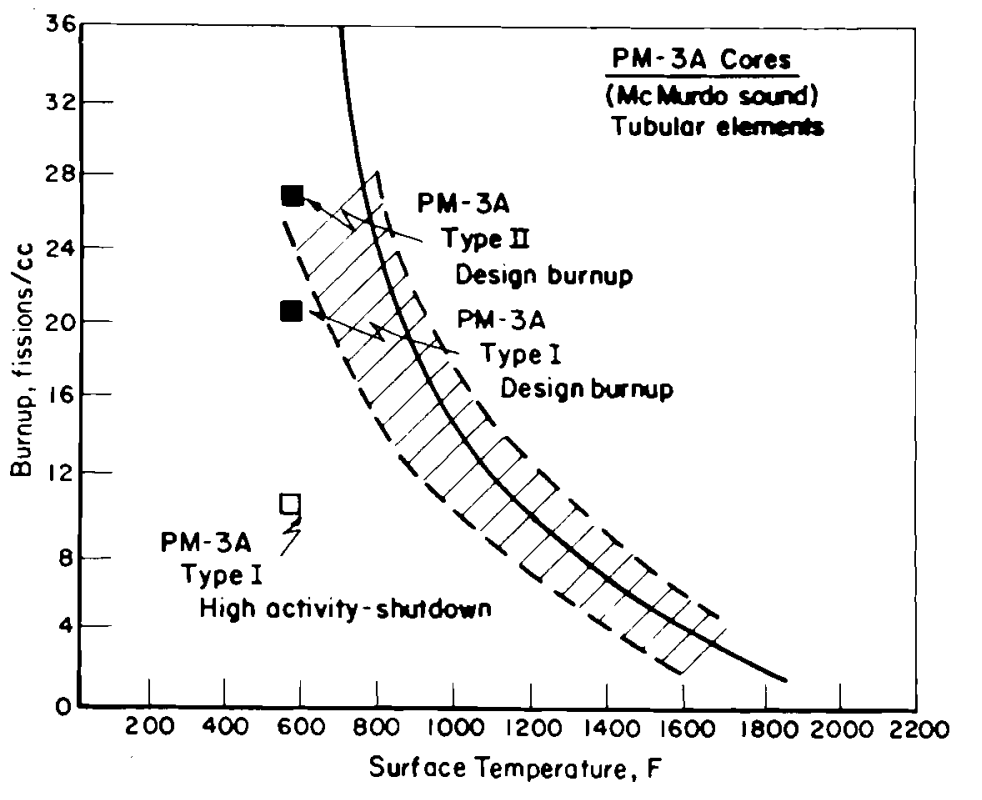

SLIDE 14 
It is concluded, therefore, that the failure curves constructed by Keller (1961) and Thurber (1963) still provide an accurate prediction of the performance of stainless steel-UO 2 dispersion fuels. The required burnup for the FFTR driver fuel is shown in Slide 15 in reference to these curves.

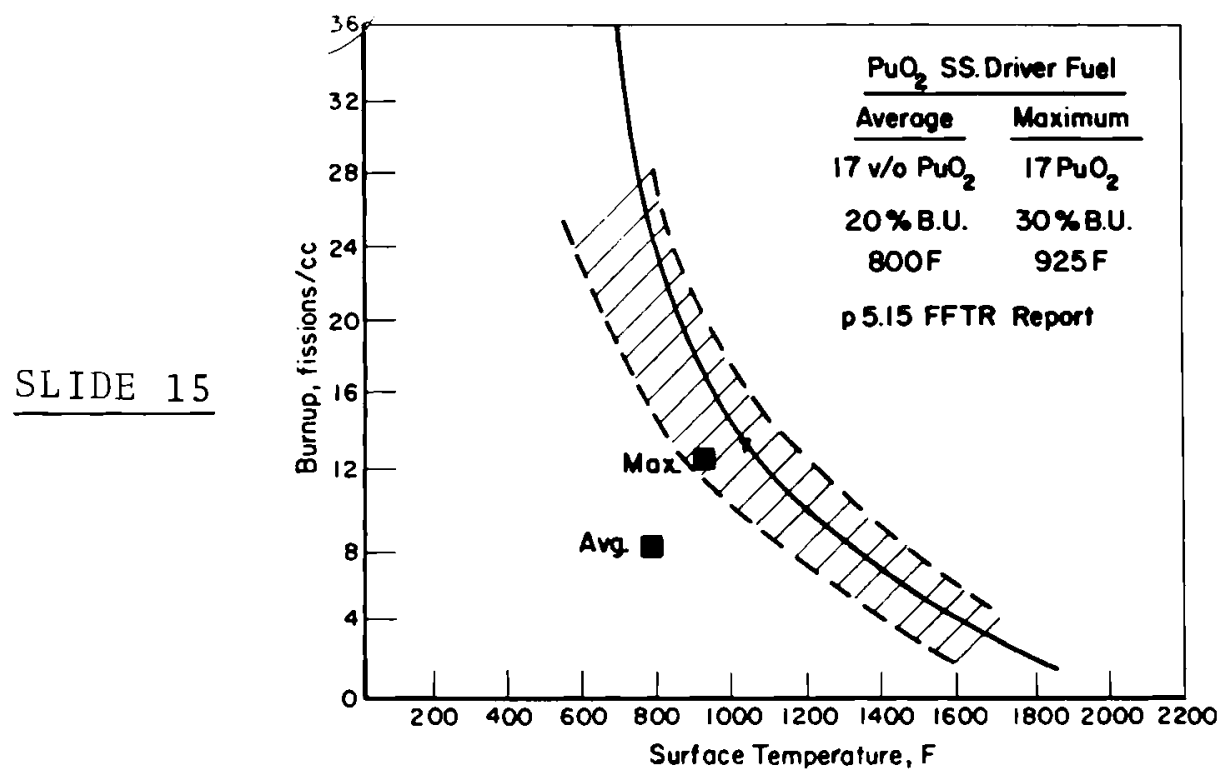

\section{STRETCH CAPABILITY OF DISPERSION FUELS}

With regards to the stretch capability of dispersion fuels, I offer the following comments:

- It is unlikely that any further improvement in radiation capability will be achieved by simply learning to fabricate a more perfect or ordered arrangement of $\mathrm{UO}_{2}$ fuel particles in a steel matrix.

- It seems certain, however, that if fuel structures comparable to those readily achieved in today's technology are not obtained, then radiation stability will fall off sharply.

- It seems only reasonable that the use of porous fuel particles will improve the performance of dispersion fuels; however, there is no experimental support of this as yet.

- High fuel loadings do not decrease the burnup capability of dispersion fuels. These are shown in some of the Harwell results and in slide 16.

- Therefore, I would propose that significant increases in the burnup capability of dispersion fuels may be achieved by: 


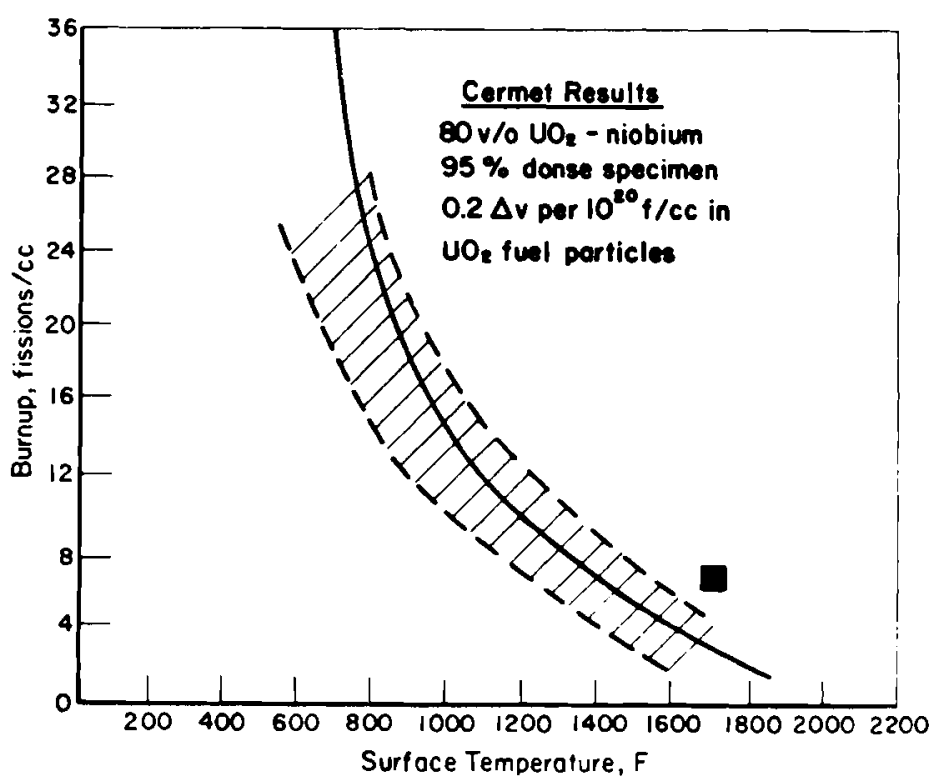

(a) Decreasing the amount of metal matrix to that which will provide corrosion resistance in the event of a clad defect, good thermal conductivity, and reliable expansion characteristics. This should be about 40 to 50 vol\% stainless steel.

(b) Incorporating into the remaining volume high strength $\mathrm{UO}_{2}$ fuel particles containing about 15 to $20 \%$ porosity.

(c) Coating these fuel particles with a high strength metallic coating such as tungsten, which will serve as a diffusion barrier between fuel and matrix.

(d) Developing a technique for fabricating this type element that does not damage the coated fuel particle nor lose continuity of the stainless steel matrix.

(e) Considering the use of high density fuel particles such as UN that will permit the use of thicker coating at similar volume loadings and particle sizes. 


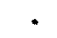


D 21.1

\author{
STATUS OF STAINLESS STEEL-UO 2 CERMETS \\ D. L. Keller
}

\title{
DISCUSSION
}

MR. ZEBROSKI: First, have you tried to make any estimate of how this prediction curve might be shifted if you allowed a 20 to 50 times greater fast flux component for the matrix, and is this true of all the tests you just demonstrated in the curve?

MR. KELLER: No.

MR. ZEBROSKI: Well, I am sure no one has that data, but maybe you can do some speculation on trends. And secondly, you made a great leap forward here, I should point out, in the assumption that $\mathrm{PuO}_{2}$ is similar to $\mathrm{UO}_{2}$. One thing we can say, when you only go to $20 \%$ solid solutions, the re are substantial and significant differences in diffusional properties, at the rate they tend to diffuse out, in the conductivity. That is, the temperature pattern and the melting point which is much lower and chemical reactivity which is much higher, for $\mathrm{PuO}_{2}$ relative to similar $\mathrm{UO}_{2}$ elements would lead me to suggest that at least some factor of safety be allowed for the fact that you are predicting that $\mathrm{PuO}_{2}$ be similar to $\mathrm{UO}_{2}$ results.

MR. KELLER: The original comment was that we might be faced with a problem of $\mathrm{PuO}_{2}$ being reduced to a lower oxide in the presence of stainless steel. I am not making any assumption, just giving you the results of the experiment-it was not reduced in hydrogen to a lower oxide.

MR. SHAPIRO: I won't comment on the question as to the desirability of coating particles if a cermet is used. However, I think it would be desirable, with regard to the cost of coating and particles generally. In view of the fact that you made the comment you felt that the ten-and I said 10 to $20 \%$, would be the additional cost, of where the coating was. The cost of making particles in the first place on a large scale from highly enriched uranium oxide, approximately $70 \%$ of the total cost constitutes the cost of losses with flow charges, discharges, insurance, etc. $70 \%$. Therefore, even if you were to double the cost of the fabrication of the particles themselves, the most you could. add under those circumstances would be $20 \%$ of the total cost. That 
is, if you add to. If you subtract, you are only talking in terms of $15 \%$. If you were simply to double the cost of fabrication, then the fact of the matter is the cost of the coating is not $50 \%$ of the total cost of the actual fabrication. Is that clear?

MR. KELLER: It is clear but let me tell you what I meant. The implication, from what I heard-the base cost was not the cost of fabricating the particle, it was cost of shaping the particle.

MR. SHAPIRO: The question is, what is the cost of a particle coated and uncoated, and I am saying, the cost of a particle coated, is not a lot more than the cost of a particle uncoated, because when you have to start to make a particle in the first place, you have to assume all the costs associated with making a particle. I think that has to be borne in mind in connection with any estimates made.

MR. KELLER: If you are saying something different there, I will withdraw it. There is one other statement, I think you made. Are you inferring that it makes no difference whether it is nickel coating or carbide coating or tungsten coating? Is this true of all coatings?

MR. SHAPIRO: I believe I made the statement it would be dependent on the nature of the coating and the specifications but that it would probably run somewhere between 10 and $20 \%$ additional, depending on the nature of coating and the specification, and I am assuming roughly $50 \%$ and I am talking about large scale manufacture.

MR. SHAPIRO: (Interposing) I suggest the experiences we have had in literally thousands of kilograms of material would be much more valid than the experience you have had with a few grams.

MR. KELLER: I stick by my statement.

MR. deHALAS: I would just like to make some comments. I actually don't disagree with anything. In particular, an important point Don says there are no data on the effect of really low porosity particles on cermet behavior. This perhaps is a hope, if you like, because we don't have any points to check against that occurred. I would like to just sketch something on the blackboard for a minute. It is a point of great confusion to me. I am sure it isn't to Don, but he didn't really cover it too much. All I want to show here is what the swelling behavior really is, and I rather suspect that some of you people are confused on this also. If you plot this swelling-first it is burnup, and then you get the curve that takes off more steeply. The thing that wasn't really apparent to me, until you stop to think about it a little bit, was the talk about closing porosity and this taking off. I have made an assumption mentally that the difference was that we were getting mainly into solid fission products swelling versus gas fission products swelling. So that after the compressible gasses kind of close, or the pores kind of close up, due to the constraint, that you were getting into an uncorrectable type swelling. This is not the case at all. This swelling is almost entirely fission gas swelling. A third or less of it is solid swelling. 
The point then comes that you have a hold in your stainless steel matrix with a particle in it and this is swelling and exerting a force on the matrix. The force is mainly the force of the fission gasses, not the solid fission products in this particle. Now it is obvious to see if I put a hold like that in the matrix and have a little pea rattling around in it, this thing is going to swell at a pretty high burnup. This would be my $1 \%$ dense particle or something in there. So there is something to gain by going up in porosity or going down in density in the particles. There are practical limitations to this but it is going to gain you something. The question then gets into the two questions Ed had, and that is that it simply becomes a problem of restraint. That is, this man gets into the matrix problem properties, and how these are effected by the radiation. One of the questions with these cermets is what happens under this fast irradiation because here you have got the matrix much more highly irradiated, if you like, than all the data that formed the curve. If it is just a question of strength, it won't hurt anything. If it was a question of relative creep rates, the swelling rate of the particles will hurt something. The other is $\mathrm{PuO}_{2}$ versus $\mathrm{UO}_{2}$. The $\mathrm{PuO}_{2}$ swelling rate is not necessarily or even suspected to be the same as the straight $\mathrm{UO}_{2}$ swelling rate, so you are dealing with some different set of curves there.

MR. WEBER: I think you did a good job of putting that curve on. There are data, in my opinion, that show a delay with increasing porosity. You don't really get a sharp break in curve, but putting in porosity does infer getting to a point where you start to go up. The slope of the upward curve is quite obviously dependent upon the strength of the material. And you have already covered a major problem which is: When you start yielding, what is the ductility of the material? It is probably more important than the strength, and I would like to agree completely with Don, that it is certainly true the primary thing going on is the gas release, not a little bit of void which is decreasing in the initial phases of this due to solid fission products. They are important. You can't ignore them. I would be concerned about getting more release from plutonium oxide sooner than you would get from $\mathrm{UO}_{2}$.

MR. KELLER: I didn't want to give the impression only porosity would help. When I looked at the SM-2 data, it shows a very distinct behavior. It seems like you could go up to $40 \times 10^{20}$ fissions/cc in fuel without any swelling. I don't know that it is the porosity that is providing the delay in swelling. I am not at all certain some other effect isn't completely masking this effect out. But I don't want to give the impression it isn't porosity. It's just not clear in my mind what it is. 
MR. deBOISBLANC: Do you feel you can understand systematically why the high volume loading seems to be behaving better because it seems to be in the opposite direction as to what you presented as your convincing argument?

MR. KELLER: I really didn't come to this conclusion until two weeks ago and it is hard to explain.

MR. deBOISBLANC: In order to be consistent, you must provide your first explanation of $\Delta V / V$ times the fraction of volume.

MR. KELLER: I hadn't thought of that.

MR. deBOISBLANC: You assumed that you could explain the observable matrix swelling by a linear combination of the swelling in the matrix, which was small. You weighted down the particle expansion by the fraction of the particle and got the correlation with the volume fraction change in the matrix. Now if you double the percent of volume in your particles then for the same nvt, you would expect to see twice the expansion. However, I think when you do this you should go to another parameter, not the fissions per $\mathrm{cm}^{3}$ but the fissions per $\mathrm{cm}^{3}$ for a unit of volume of particle. That is another factor that in the cther direction it would indicate at least they ought to be the same.

MR. deHALAS: I would just comment on this volume percent sensitivity. I don't think it is too logical if you consider it is function of restraint, and if you have got a look at the particle, if you like - in one case it has a lot of stainless steel on it ... so you are still providing a relatively equal amount of restraint. As a matter of fact, the straight $\mathrm{UO}_{2}$ element will somewhat swell at the same rate as a cermet element, if you take account of the volume percent $\mathrm{UO}_{2}$ in it. It is either restrained by the matrix or the $\mathrm{UO}_{2}$. The one point I forgot to bring up concerning restraining volume, there is ${ }^{2}$ one distinct advantage to low volume percent; it is easier to fabricate.

MR. KELLER: No question about that.

MR. SHAPIRO: It would seem to me that the possible explanation of the difference in behavior, depending upon the population of the particles, is that if you look at the nature of the failure which occurs, these are sort of striations within the element as a result of apparent stringering of the material. And in effect what happens is that each particle and its tails tend to form crack starters. If you increase the population of the particles, such that you have these things terminate in effect against another particle, it would seem to me therefore that the behavior on this would be a little bit better under those circumstances than it would be if you had an ability for the thing to tear all the way up, without having a point of termination.

MR. RABIN: I won't try to educate you, Don, I will just ask you a question. You mentioned no correlation in the thermal cycling data that you could find. In FFTF, in the maximum condition we are talking 
about a Delta-T, which $I$ think is conservatively greater than we are accustomed to. Do you have any feeling how this might effect the behavior?

MR. KELLER: Well certainly--The assumption is that the cladding must fail by some kind of a strain. If you have got other stresses that add to or supplement, I can't help but believe that something is going to happen earlier. But nonetheless I think Shoudy described the Fermi core B radiation and they are indeed plotted on that curve, and they are awfully high Delta-T radiation, and they don't change that curve one bit.

MR. BABIN: What were the Delta T's there?

MR. KELLER: About 285.

MR. SHOUDY: About 280 .

MR. KELLER: 280. I think we were expecting premature failure and it didn't happen.

MR. ZEBROSKI: I wonder if Don or someone could comment. Actually failure is not the criteria. Remember this hot channel effect creates a small amount of swelling. I wonder if anybody can comment on what is realistic criteria as to what swelling tolerance is.

MR. deBOISBLANC: I will comment on that. It is a prime problem actually. The thermal hydraulic analysis of a test reactor, or any other reactor for that matter, must consider the hot channel factors. Some of them are imaginary and some of them are real. The one you just referred to is real. The deviations in the channel which statistically are expreienced in the fabrication essentially set the spread and the statistical allowance that you must make for this factor. Now when you have a systematic effect added to this, this offsets the statistical spread of your values and must be treated, not as a hot spot channel factor, but a systematic error. So it doesn't enter into the statistical picture. For example, in the ETR fuel the specifications for deviation are 10 mills. That is the production specification. With the probability that you would exceed 10 mills by 10 mills would be one part in a thousand. Now if you had a 2 mill or a 3 mill systematic spread, this simply removes your. limit from one part in a thousand to roughly one part in a hundred. 


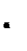




\title{
ALLOY FUEL AND CARBIDE DEVELOPMENT
}

\author{
J. H. Kittel and R. E. Macherey
}

Argonne National Laboratory

Argonne, Illinois

\section{INTRODUCTION}

As was mentioned a day or two ago at this meeting, essentially all the fast reactors that have been operated to date have used metallic fuel; but as is well known, when plutonium alloys were pushed to higher temperatures, the swelling problem appeared. Also, at about the same time, $\mathrm{UO}_{2}$ was found to be performing well in water-cooled reactors. However, in many of these thermal reactor applications, the thermal rating of the fuel was rather low. Nevertheless, there developed a wholesale switch to the selection of ceramic fuels, particularly oxide, for the next generation of fast reactors. In the last year or two, there have been two developments that have caused people (both in the U. S. and abroad) to reappraise some of these decisions.

First, to the surprise of many people, ceramic fuels have also been shown to have a swelling problem; we have heard of some of these problems in the last day or two. The results have been particularly disturbing in connection with some of the recently reported irradiations for the LSBR reactor. These results have not yet been described in detail, but some of the high lights have appeared in the yellow pages of Nucleonics and elsewhere.

The second development is that metal fuels have made some rather remarkable advances recently in showing that the swe11ing problem can be overcome in these fuels. In contrast, at the present time, I think it is fair to say that less is known on how to cope with the swelling problem in ceramic fuels, and in particular, to what extent jacket restraint can be used. It is the recent results on metallic fuels that I would like to discuss this morning.

Swelling in metal fuels can be attacked in different ways. The two methods that have received the greatest attention are: first, the use of fine particles as a second phase dispersion; and secondly, the use of cladding restraint. Considering first the use of second phase particles, the principle here is to attempt to use very small particles in a very fine dispersion to act as trapping sites for bubbles of fission gas. If the bubbles can be kept very small, then surface tension forces are effective in preventing further growth, and they can store 
relatively large amounts of fission gas at high pressures. A11 other things being equal, fuel with smal1 bubbles will show less swelling than fuel with larger bubbles. This approach has been followed in this country primarily by people at Atomics International; the group at Harwell has also been active in this area.

At Argonne, we found that this mechanism did indeed seem to operate with thorium-uranium fuels; Slide 1 shows an example of this. Here is a thorium $10 \%$ uranium alloy, irradiated to $4 \%$ burnup. Note that the second phase particles of uranium (shown here) in many instances have a pore attached to them. There are numerous similar examples scattered throughout the structure of this specimen. The number of pores attached to particles is generally proportional to the burnup the specimen received.

S1ide 2 shows a comparison of the behavior of these alloys as represented by Curve 6 in comparison to a number of other uranium base alloys. Here the swelling has been plotted as a function of irradiation temperature.

Now, I don't think we are prepared to say that the reason the throium-uranium alloys appear to be so good relative to other alloys is entirely due to the second phase particles. There are other considerations, including the higher melting point of the thorium alloys; nevertheless, it does seem quite likely that the small particles are acting as they had been predicted to do. Looking at the other extreme, Curve 1 is the alloy which really, at one time, interested us the most. This is a plutonium alloy, of the type that one would like to use as recycled fuel in a fast breeder reactor.

We see here that the fissium alloy was the worst of the group. The use of second phase particles is not feasible in this material because the operating temperature is so close to the melting temperature that any second phase which could be distributed in the material would quite likely be dissolved during operation. Therefore, we sent to the second approach for controlling swelling that makes use of clad restraint.

This particular approach is ideal for fast reactor applications since the fuel is normally in the form of smal1 diameter rods. The tubing which is used as cladding is also of relatively sma11 diameter so that it is capable of containing relatively high pressures without unduly large wall thicknesses. The type of specimen that was used in the studies of clad restraint is shown in Slide 3 .

These specimens have been irradiated in instrumented capsules in the CP5 reactor. They are irradiated in the center of the fuel elements. These are annular fuel elements, so that the fast flux on the capsule is enhanced. The spectrum is much harder than we find, for example, in MTR. The specimen is, in effect, a scaled-down EBR-II fuel element. The diameter and 


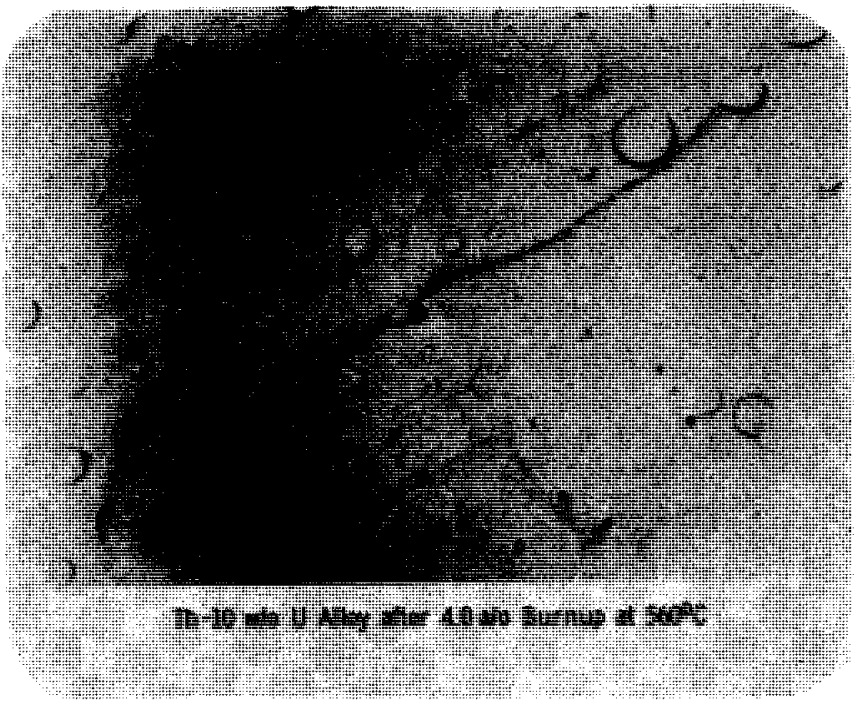

SLIDE 1

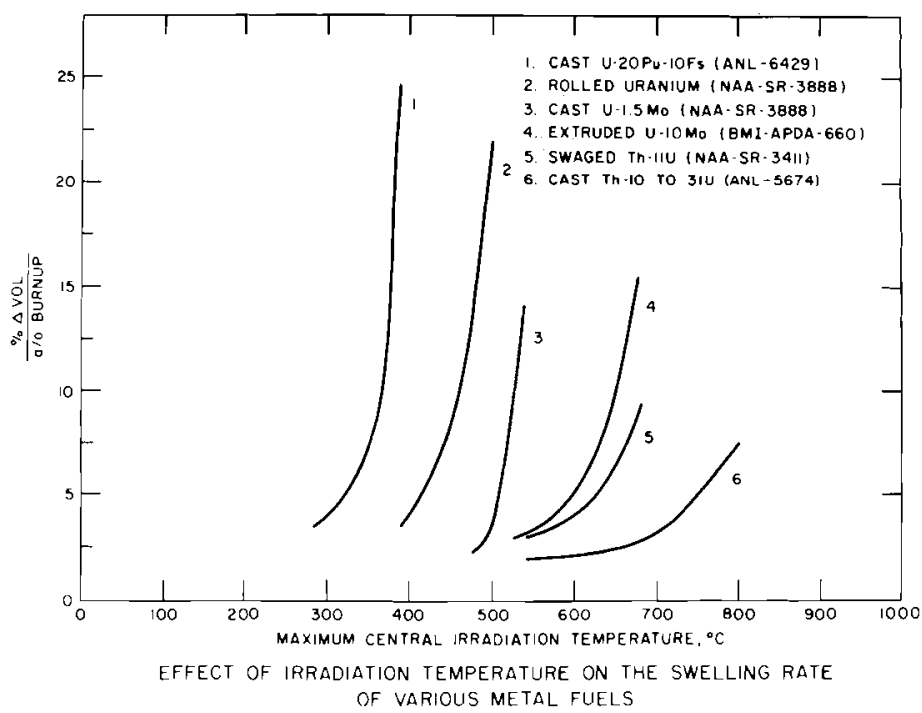

OF VARIOUS METAL FUELS

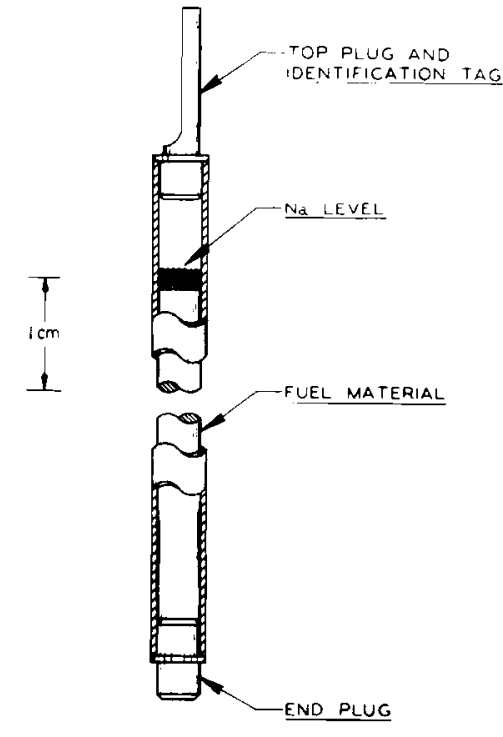

SLIDE 3 
other radial dimensions are kept the same as the reference element; that is, the outer diameter is 0.176 in., the clad thickness is $9 \mathrm{mil}$, there is a $6 \mathrm{mil}$ thick sodium annulus between the fuel and the jacket, and the fuel diameter is 0.144 in. To make up a specimen of reasonable length, we scaled down the length of the EBR-II element by a factor of 7. The void space and the depth of the sodium cover were scaled down in the same proportion.

The principle variations in the experiments have been clad thickness, the amount of void space left above the sodium, and the composition of the cladding. Because this was a fissium alloy at the time these experiments started, the fuel alloy was not compatible with stainless steel, and almost all of the jackets that were investigated initially were made of refactory metals.

This series of experiments led to the demonstration a few months ago of a group of specimens that achieved burnups of $8.7 \%$. These are shown in slide 4 .

SLIDE 4

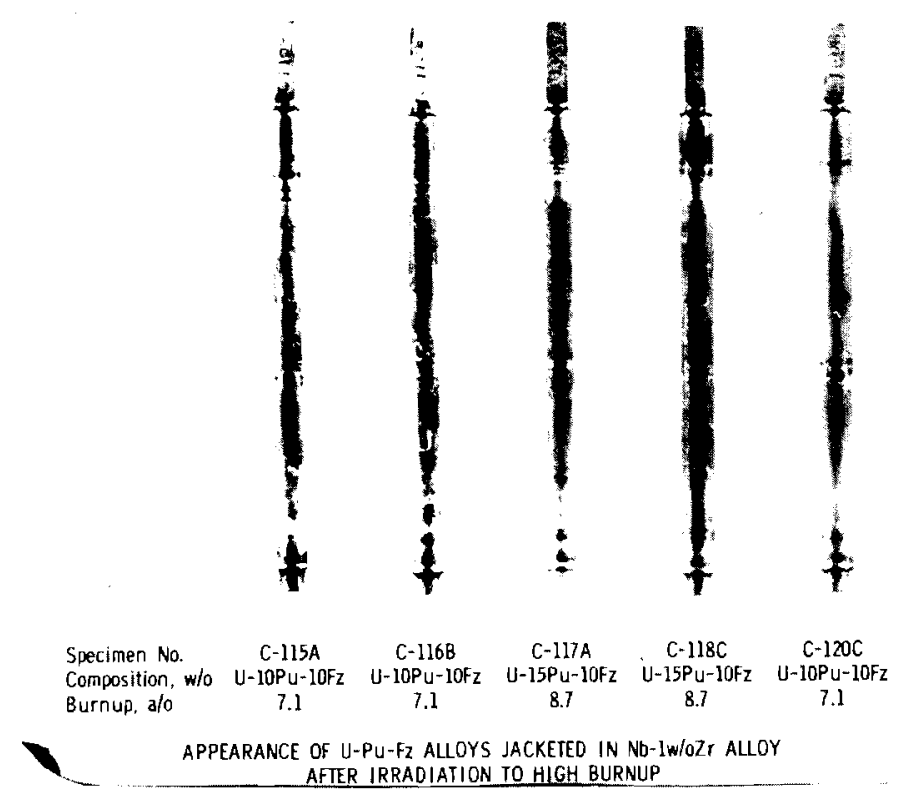

$10^{21}$ The $8.7 \%$ burnup corresponds to a fission density of $2.8 \mathrm{x}$ $10^{21}$ fissions per $\mathrm{cm}^{3}$. None of these specimens showed any measurable changes in dimensions. The cladding thickness varied from $15 \mathrm{mils}$ to 20 and $25 \mathrm{mils}$ in one. These fission densities are among the highest, certainly, that have been achieved in any plutonium fuel, ceramic or metaliic. I will have more to say later on high-burnup plutonium fuel irradiations.

More recent work has been in the direction of looking at the effects of void space within the cladding and in looking at the higher melting fuel alloys. If the fuel is assumed to have negligible strength, the effect of void space is readily calculated by using the ordinary gas 1 aws. 
Slide 5 shows the type of achievable burnup curves that develop where the fuel is assumed to have negligible strength parameters. The $Z$ shown along the top curve is an indication of the percent axial growth of the fuel and as indicated, this represents isotropic swelling. If, for example, fuel with a diameter of 0.144 in. is irradiated in a jacket with an ID of $4.2 \mathrm{~mm}$, it would expand in diameter $14.2 \%$ before it is in contact with the jacket. Similarly, if $14.2 \%$ axial growth is permitted, then, theoretically, the achievable burnup should be on the order of $8 \%$ before the maximum allowable jacket stress is exceeded. These calculations are only a first approach. There are many refinements that are not included, such as strain in the jacket which would act as a relaxation mechanism to lower clad stresses. With these types of calculations as a guide, a number of experiments are in progress to determine the effect of void space within the jacket. If metallic fuel is irradiated with effective or smeared densities below about $80 \%$, our data and data from Dounreay indicate that the fission gas voids within the specimen become interconnected, and the fission gas then escapes from the material.

Similar data were also obtained in a series of postirradiation annealing experiments (shown in Slide 6). Here a

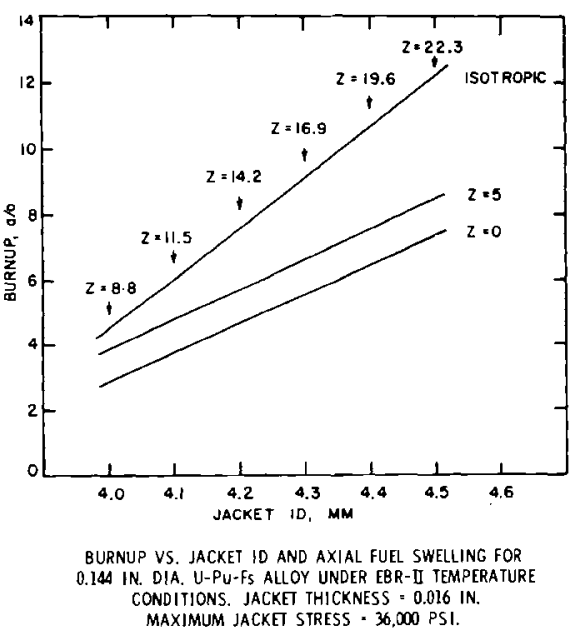

SLIDE 5

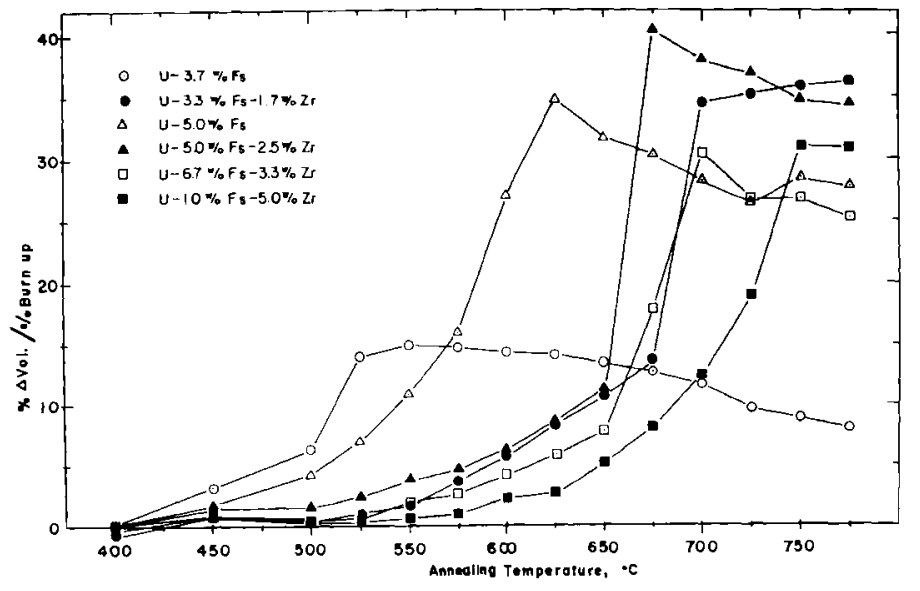

Figure, Comporison of the Swelling Rates of U-Fs \& U-Fs-Zr Allays

SLIDE 6

series of uranium-fissium alloys were annealed after having been irradiated to burnups of several percent. At various intervals, they were removed from the annealing furnace and the densities measured. All of them showed that, depending on the alloy content, above a characteristic temperature no 
further volume change occurred, or in some cases a contraction was observed. Later experiments that were done where gas release was measured simultaneously showed that as the fuel began to swe11, fission gas was also released. These experiments correlated very well with the work done in-reactor. It appears, therefore, that this type of behavior opens up many possibilities. If the fission gas does leave the fuel as freely as it appears, then the gas can either be stored in a plenum above the fuel or outside the core, or the element could be vented. Some experiments are already in progress to test this. Venting a metallic fuel is particularly attractive because the fuel is quite compatible with the coolant, and diving bells or other arrangements to keep the coolant out of the jacket are not required.

Using some of these newer ideas, we have a number of prototype elements under irradiation in CP5 and EBR-II. These elements include fissium alloys, although these are being phased out in favor of the uranium-plutonium-zirconium and titanium alloys. In CP5 at the present time, there are 9 prototype elements that have reached burnups of $2 \times 10^{21}$ fissions per $\mathrm{cm}^{3}$ at maximum cladding temperatures of 530 to $580{ }^{\circ} \mathrm{C}$. The atom burnups which correspond to these fission densities are from 5 to $5.7 \%$. The diameters are a 11 near 0.2 in., and the cladding thickness is $16 \mathrm{mils}$. Based on neutron radiography, which we use frequently to inspect the specimens within the capsules, the uranium-plutonium-zirconium alloy, which is compatible with stainless steel, appears especially stable under irradiation, even though this is the specimen that is operating at the highest clad temperature and that has gone to the highest burnup. The power density in this particular specimen is $2.7 \mathrm{~kW} / \mathrm{cm}^{3}$, and it is being operated with the 1 inear heat rating of $13 \mathrm{~kW} / \mathrm{ft}$. In EBR-II at the present time there are $18 \mathrm{full}$ length uranium-plutonium-zirconium alloy rods under irradiation. The fuel length is 14 in., the same as the driver fuel. Two. of these rods are clad in vanadium-20 titanium alloy, and the balance are all in iron- or nickel-base alloys including 304 , 316, and Haste110y $x$. The maximum cladding temperature in the EBR-II experiments ranges up to $650{ }^{\circ} \mathrm{C}$, and the maximum burnup to date is 1.1 at. $\%$ or $4 \times 10^{20}$ fissions per $\mathrm{cm}^{3}$. These elements are al1 designed to go to burnups in the range of 5 to $10 \%$. Two full-length rods of the uranium-plutonium-titanium alloy are also under irradiation under similar temperature and burnup conditions, jacketed in vanadium-20 titanium alloy. Also, we are now designing experiments that will take the zirconium alloy and, perhaps, the titanium alloy as well to higher linear heat ratings we 11 beyond $20 \mathrm{~kW} / \mathrm{ft}$.

I would like to mention the experience with EBR-II driver fue1. EBR-II, of course, is operating on metal fuel, and the coolant temperatures are significantly higher than those mentioned yesterday as being acceptable for FFTF. The inlet temperature in EBR-II is $700^{\circ} \mathrm{F}$; at the present time it is 
operating with an outlet temperature of $832^{\circ} \mathrm{F}$. This is at $45 \mathrm{MW}$ or $3 / 4$ full power. When it goes to full power, the outlet temperature will approach $900^{\circ} \mathrm{F}$. Based on what we have learned in the last year or two, we tend to regard the Mark I driver fuel as a rather primitive design. The element was designed about 10 years ago, not by metallurgists I might add, and it suffers from several design deficiencies. The clad thickness is only 9 mils. We have found it difficult to buy hundreds of thousands of feet of this type of tubing with high quality in such a thin wall thickness. But most seriously, the plenum above the sodium is not large enough to accommodate the bond sodium as it is displaced by fuel swelling. Nevertheless, these elements are of interest because they furnish information on the behavior of actual fast reactor fuel elements under fast reactor operating conditions.

Slide 7 shows the reasoning that has lead to our specification of a maximum allowable burnup of $1.2 \%$ in the Mark I fuel. The data shown here are volume measurements made on the fuel after the cladding has been removed. As you can see, the curve has begun to slope upwards after approximately $1 / 2 \%$ burnup. At about 1.2\% burnup, the fuel swelling has reached something on the order of $8 \%$. With this amount of swelling, the clad stress has built up to a level that could give us some concern, since, statistical1y out of the thousands of elements in the reactor, we may be in a region where occasional fuel clad failure might be expected.

SLIDE 7

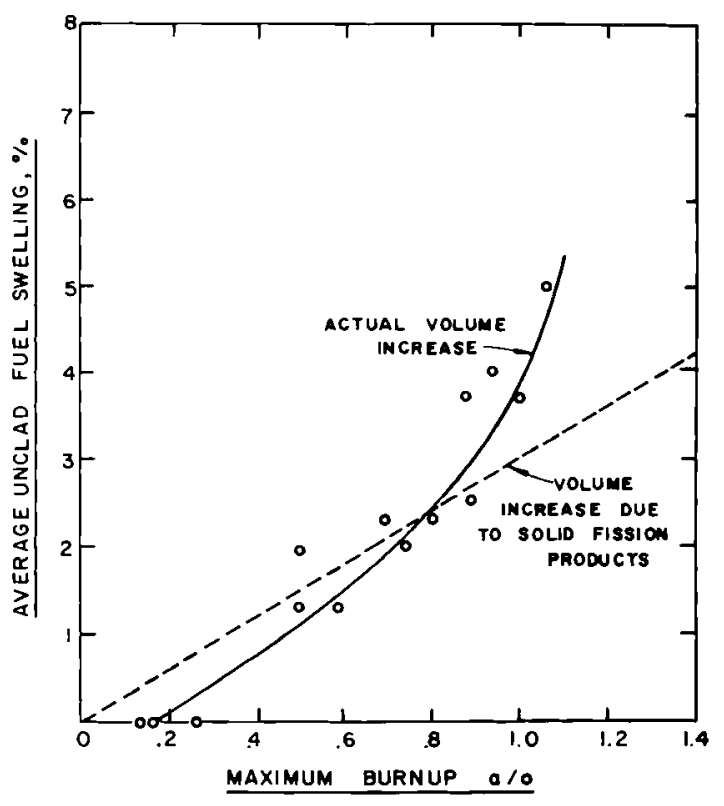

SWELLING OF UNCLAD EBR-II MARK I FUEL RODS (BASED ON SODIUM LEVEU 
Although many thousands of rods have been put through the reactor, we have not yet encountered a clad failure. On the order of 2500 of these rods have reached the maximum burnup of $1.2 \%$ without any evidence of jacket failure. Late last year we began making rods designated as Mark IA which have a modest design change. The fuel was shortened in length slightly to increase the size of the plenum over the fuel so that all of the bond sodium could be accommodated above the fuel. We calculate that, with the same degree of conservatism, this should increase the allowable fuel burnup to about $1.8 \%$. The effective fuel density is still maintained at $86 \%$, and the cladding thickness is still $9 \mathrm{mils}$. Designs are also being made of Mark II and III elements which will permit much higher burnups, and therefore allow longer fuel cycles in keeping with EBR-II's role as a fast test reactor.

A typical Mark II design is an element with the outer diameter raised to 0.22 in., the cladding thickness increased to $15 \mathrm{mils}$, the effective density $78 \%$, and the number of pins per subassemblies decreased from 91 to 61. We calculate that this element should readily go to 5 at.\% burnup, and in some instances, possibly to 10 at. $\%$ burnup.

It is of interest to compare the behavior of metal fuels under irradiation with work that has been published on other fuels. I've made a tabulation on the board based on a literature survey (which we're presently engaged in) of high burnup results on plutonium fuels for fast reactors. The work is listed in slide 8 and shows only U. S. data; we're in the process now of gathering what the Europeans have published.

These are specimens that have been irradiated under conditions where the cladding has not failed and where they have gone to burnups that are at least $1 \times 10^{21}$ fissions/cm ${ }^{3}$. The first column depicts the oxide specimens which were irradiated and reported by GE in about 1961. In the second column are the carbide specimens described yesterday by UNC. The third column is for a group of mixed carbide specimens irradiated by Argonne. The next column includes the uranium-plutonium-fissium alloy specimens; five of these were shown in the slides. In the last column are the high burnup irradiations of the uranium-plutoniumtitanium and uranium-plutonium-zirconium alloys.

Comparison of High-Burmup Irradiations on Fast Breeder Reactor Uranium-Plutonium Fuels

\begin{tabular}{|c|c|c|c|c|c|c|c|}
\hline \multirow[b]{2}{*}{ Reporting Site } & \multicolumn{2}{|c|}{ OXIDE } & \multicolumn{3}{|c|}{ CARBIDE } & \multicolumn{2}{|c|}{ AIIOY } \\
\hline & GE-APED & DERE & UNC & ANL & $\mathrm{AERE}$ & ANL & ANI \\
\hline No. of Unfailed Specimens & 9 & 3 & 4 & 4 & 14 & 12 & 2 \\
\hline Fuel Composition, w/o & $\mathrm{UO}-\mathrm{POPuO}^{3}$ & $\mathrm{UO}_{2}-\mathrm{ISPO}_{2}$ & UC-2OPuC & UC-2OPuC & vC-15PuC & $\mathrm{U}-10,15 \mathrm{Pu}-10 \mathrm{Fz}$ & $\mathrm{U}-1.5 \mathrm{Pu}-10 \mathrm{~T} i, \mathrm{zr}$ \\
\hline Cladding Material & $347 \mathrm{SS}$ & $316 \mathrm{~L} \mathrm{SS}$ & Niobium & $304 \mathrm{SS}, \mathrm{Nb}-12 \mathrm{r}$ & Nim. $80,316 \mathrm{~L}$ SS & $\mathrm{Nb}-1 \mathrm{Zr}, \mathrm{V}-2 \mathrm{OT} 1$ & v-20Ti \\
\hline Cladding wt. / Fuel wt. & $0.36-0.68$ & $0.27-0.34$ & $0.73-0.79$ & $0.25-0.27$ & (a) & $0.18-0.45$ & 0.21 \\
\hline Avg. Cled Outer Surf. Temp., ${ }^{\circ} \mathrm{C}$ & $350-465$ & $(a)$ & $460-640$ & $525-600$ & (a) & $430-545$ & $520-540$ \\
\hline Burnup, $10^{21}$ fissions/cc & $1.0-2.1$ & $1.1-1.2$ & $1.0-3.8$ & $1.3-1.8$ & $1.1-3.8$ & $1.5-2.8$ & $1.2-1.3$ \\
\hline
\end{tabular}

(a) Information not available. 
The cladding thickness is of particular interest for all of these specimens. We have no apologies to make for having achieved high burnup in so-called "gun barrel" type of experiments. I think this is indicated most clearly in the numbers that show the ratio of clad weight to fuel weight. For the metallic fuels, these are on the low side. The only lower values are those that Argonne has used for carbide irradiations. The highest fission densities have been achieved in the carbide specimens (as described yesterday). In the case of metal fuels, fission densities were achieved second only to the carbide but in cladding of much lower thickness. The surface temperatures of all the specimens are comparable and are within the range of the FFTF requirements.

\section{METAL FUELS SUMMARY}

Summing up on metal fuels, we believe that because of the high achievable burnup which has been demonstrated in these fuels under conditions applying to the FFTF and because of the low fuel cycle costs of metal fuels, they deserve consideration as a primary candidate for the FFTF, rather than merely as a backup.

Our original goal with mixed carbide irradiations was to reach burnups on the order of 5 to $10 \%$, but more recently we are thinking in terms of burnups on the order of $15 \%$. We assumed at the start of this work several years ago that achieving high burnup in ceramic fuels would require low density fuel, unless the "gun barrel" type of approach were to be used. I think that the correctness of this assumption has been borne out by our experiments as well as by experiments from other sites where in some cases dense ceramic fuels have encountered difficulties. We used the AI data on swelling of uranium carbide as a basis for estimating the amount of void space that would be required. It appeared that fuels with effective densities on the order of 80 to $85 \%$ would meet our requirements. Vibratory compaction is an ideal way to obtain fuels with these orders of densities. This technique also appealed to us because of its adaptability to remote operation and because it can be used to fabricate tube-and-shell type fuel elements.

Our first irradiation specimens were, therefore, made by vibratory compaction. We used two different cladding materials, Type $304 \mathrm{SS}$ and niobium-1\% zirconium. We also used two different fuels, UC $20 \% \mathrm{PuC}$ as a mechanical mixture, and a few specimens of pure PuC were put in for comparison. The carbon content in the PuC fraction was considerably varied on both sides of stoichiometry to determine the effect of carbon content. We used a range from $3.4 \%$ to $6.4 \%$ carbon. The UC fraction was stoichiometric monocarbide; it was unenriched because these irradiations were being done in a thermal reactor.

The burnups in the first group of specimens ranged up to $6.8 \%$ of all metal atoms. This corresponds to a fission density 


\subsection{0}

of $1.8 \times 10^{21}$ fissions $/ \mathrm{cm}^{3}$, and these are the specimens 1 isted in Slide 8. The clad surface temperatures in these experiments ranged from 400 to $675^{\circ} \mathrm{C}$. Slide 9 shows the data for $304 \mathrm{SS}$ clad specimens. I would discount the temperature calculations as being anything other than relative, because we really don't know the conductivity of this fuel. The main thing of interest is the diameter change. Here the specimen that had $4.7 \%$ change in diameter is also the material that is hypostoichiometric. The hyperstoichiometric specimen (immediately above it) only went to a burnup that was slightly less. Nevertheless, there is a considerable difference in the amount of swelling which it experienced.

Slide 10 shows the same data for the niobium-zirconium clad mixed carbide. Here, there were no significant dimensional changes in any of the specimens; the indicated changes in diameter and volume are essentially within the error of measurement.

Slide 11 shows the data on the PuC. Five specimens were irradiated; the second specimen on the 1 ist was a specimen we were using in CP5 as a flux monitor. We inadvertently moved it into a high flux position and melted the cladding on it. The third specimen also showed a clad failure of a much less drastic type. This clad failure appeared to be due to the pressure of contained fission gas within the cladding. You will notice that this is a hypostoichiometric specimen, and the appearance of the fracture suggests that it is of a ductile type.

Slide 12 shows a tabulation of fission gas release. There is nothing surprising here. The rate of gas release, of course, was determined by the stoichiometry of the PuC since this was a source of essentially all fissions. As one would expect, the hypostoichiometric material released considerably more gas than the hyper. We were somewhat surprised at the low apparent release of the two specimens having carbon contents near $6.2 \%$, even though they went to rather high burnups. There is some uncertainty about these two numbers, but we do know they are very low.

Two weeks ago at Harwe11, I learned that similar results had been obtained there by Brian Bradbury. His explanation was that the original porosity in his powdered specimens had become converted to spherical closed pores during irradiation. In our metallography we see a similar type of behavior.

IRRADIATION OF VIPAC UC-ZOPUC IN 304 SS

SLIDE 9

\begin{tabular}{|c|c|c|c|c|c|}
\hline $\begin{array}{c}\text { Burnup, } \\
\% \%\end{array}$ & 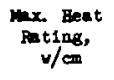 & $\begin{array}{l}\text { Mex. Center } \\
\text { Temperature, } \\
\text { "C }\end{array}$ & $\underset{\substack{\text { Carbon, } \\
\text { /o }}}{ }$ & $\max _{\Delta \mathrm{DL}}$ & $q \Delta v$ \\
\hline 1.35 & 200 & 390 & 6.42 & 0.3 & 0.6 \\
\hline 1.40 & 200 & 820 & 3.40 & 0.5 & 0.2 \\
\hline 5.8 & 180 & 915 & 0.22 & 2.8 & 3.5 \\
\hline 6.8 & 205 & 1040 & 4.24 & 4.7 & 7.3 \\
\hline
\end{tabular}




\subsection{1}

IRRADIATIOX OP VIPAC UC-2OPUC IN

SLIDE 10

\begin{tabular}{|c|c|c|c|c|c|}
\hline $\begin{array}{l}\text { Dumup, } \\
1 / 0\end{array}$ & $\begin{array}{l}\text { Mx. Heat } \\
\text { Rating, } \\
\text { v/cen }\end{array}$ & $\begin{array}{l}\text { Max, Center } \\
\text { Teapereture, } \\
\text { "c }\end{array}$ & $\begin{array}{c}\text { Carbon, } \\
w / 1,\end{array}$ & $4 \Delta \mathrm{DAO}$ & $\$ \Delta v$ \\
\hline 1.21 & 185 & 750 & 6.42 & 0.5 & 0.3 \\
\hline 1.57 & 260 & 945 & 3.40 & 0.3 & 0.5 \\
\hline 6.8 & 195 & 1000 & 6.22 & 0.2 & 0.4 \\
\hline 6.8 & 200 & 1025 & 4.24 & 0.4 & 0.5 \\
\hline
\end{tabular}

SLIDE 11

\begin{tabular}{|c|c|c|c|c|c|}
\hline $\begin{array}{l}\text { Jecket } \\
\text { Waterial }\end{array}$ & $\begin{array}{c}\text { Bumup, } \\
\text { a } / \mathrm{c}\end{array}$ & $\begin{array}{l}\text { Max. Heat } \\
\text { Rating, } \\
\text { w/cm }\end{array}$ & $\begin{array}{l}\text { Max. center } \\
\text { Temperature, } \\
\text { 'C }\end{array}$ & $\begin{array}{c}\text { Carbon, } \\
w / 0\end{array}$ & 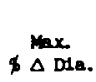 \\
\hline 304 BS & 1.95 & 310 & 1095 & 4.24 & 1.0 \\
\hline 304 SS & 5.43 & 505 & 1610 & 6.22 & $(0.8)$ \\
\hline $304 \mathrm{sS}$ & 6.0 & 210 & 1080 & 4.24 & 14.3 \\
\hline$M b-12 x$ & 2.18 & 335 & 1255 & 3.40 & 0.5 \\
\hline$a-12 x$ & 5.3 & 170 & 870 & 4.24 & 0.1 \\
\hline
\end{tabular}

SLIDE 12

\begin{tabular}{|c|c|c|c|c|}
\hline $\begin{array}{c}\text { carbon, } \\
\text { v/o }\end{array}$ & $\begin{array}{l}\text { Max. Hest } \\
\text { Rating, } \\
\text { w/cm }\end{array}$ & $\begin{array}{l}\text { Max. Center } \\
\text { Temperature, } \\
\text { 'c }\end{array}$ & $\begin{array}{c}\text { Burnup, } \\
\mathbf{a} / \mathrm{o}\end{array}$ & $\begin{array}{c}\text { Cas } \\
\text { Helease, } \\
\text { of Theor. }\end{array}$ \\
\hline 3.40 & 220 & 820 & 1.40 & 25.7 \\
\hline 3.40 & 260 & 945 & 1.57 & 21.5 \\
\hline 3.40 & 335 & 1255 & 2.18 & 45.9 \\
\hline 4.24 & 170 & 870 & $5.3^{(1)}$ & 31 \\
\hline 4.24 & 200 & 1025 & $5 . \hat{8}^{(1)}$ & 20 \\
\hline 4.24 & 205 & 1040 & $6.8^{(1)}$ & 40 \\
\hline 4.24 & 310 & 1095 & 1.95 & 35.2 \\
\hline 6.22 & 180 & 915 & $5.8^{(1)}$ & $<1$ \\
\hline 6.22 & 195 & 1000 & $6.8^{(1)}$ & $<1$ \\
\hline 6.42 & 185 & 750 & 1.21 & 5.7 \\
\hline 6.42 & 200 & 890 & 1.35 & 8.8 \\
\hline
\end{tabular}

(1) Bstimated 


\subsection{2}

The four vibratory compacted specimens with the highest burnups are shown on Slide 13. As I mentioned, the two niobiumzirconium specimens showed negligible dimensional changes.

SLIDE 13

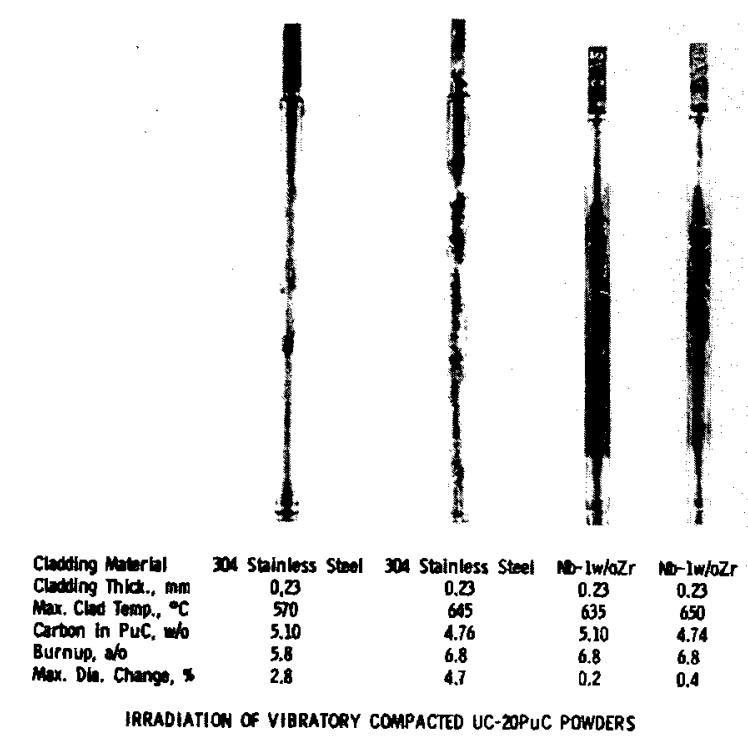

S1ide 14 shows the two PuC specimens that went to high burnup. Again, the niobium-zirconium specimen came through in good shape. Shown here is the clad failure that developed in the stainless steel. The thing that really interests us is the remarkable amount of plastic deformation shown by the stainless stee1. Although this specimen was under irradiation for about $2-1 / 2$ years and it picked up an nvt of about $3 \times 10^{21}$ neutrons/ $\mathrm{cm}^{2}$, the cladding still showed highly ductile behavior.

SLIDE 14. Irradiation of Vibratory Compacted PuC Powder

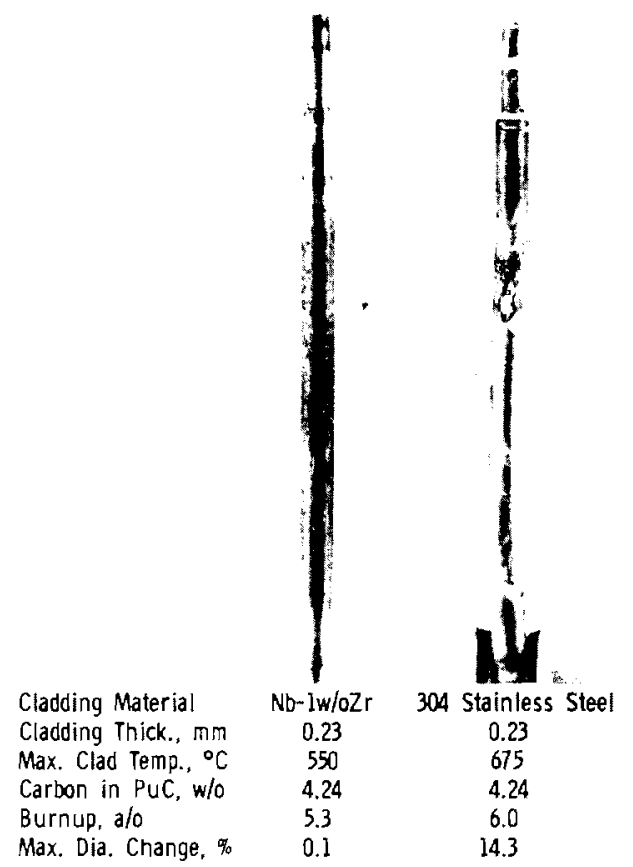


We are just now doing the metallography on the mixed carbide specimens.

The mixed carbide with UC as the large particles are shown on Slide 15. The PuC is distributed as an intermediate sized particle and is shown as gray material. This specimen went to a burnup of about $1.6 \%$, and very little sintering is evident. In fact, we had considerable difficulty in keeping the sample in the mount during polishing. This particular specimen had $3.4 \%$ carbon in the PuC fraction.

Slide 16 shows one of the PuC particles that has gone to a burnup of $8 \%$ since all of the fissions have taken place in this phase. A little bit of sintering has developed around the outside.

Here the material shown has gone to $6.8 \%$ burnup (S1ide 17). The PuC has swelled into almost all of the available void space around the UC particles.

Here (Slide 18) is the same material at a higher magnification so that you can see a white phase which has formed around the grains in the UC.

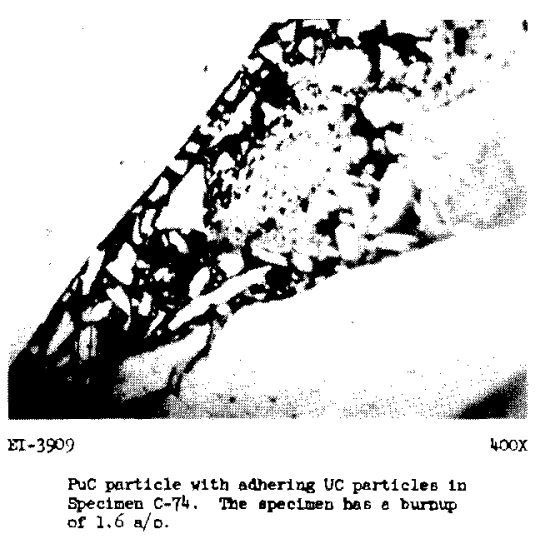

SLIDE 15

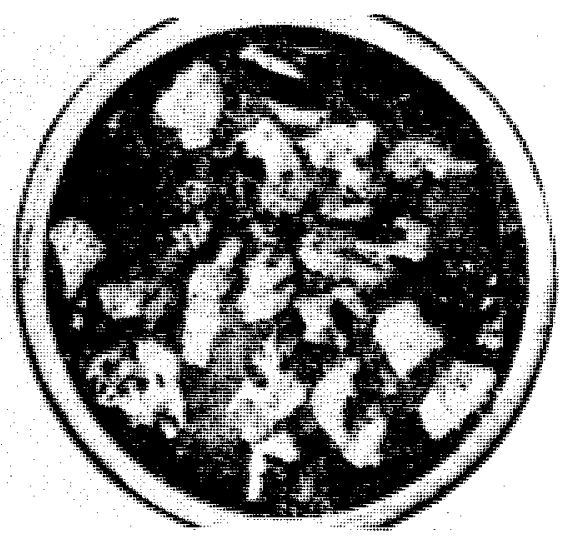

SLIDE 17

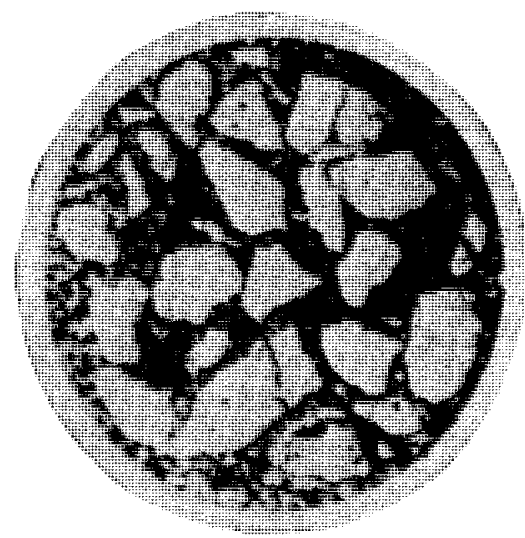

SLIDE 16

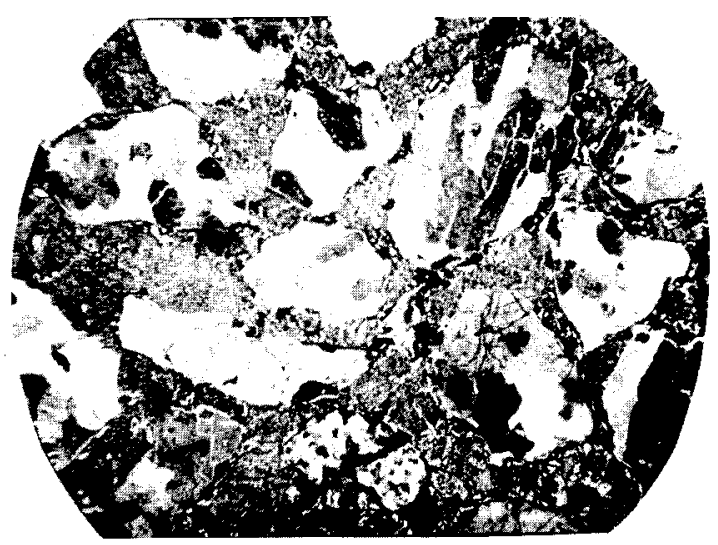

SLIDE 18 


\subsection{4}

Slide 19 shows this at higher magnification. We believe that the white phase is sesquicarbide, and may have been formed from carbon released from the PuC during fission. This particular specimen was hyperstoichiometric material. Notice the large amount of porosity in the PuC fraction. Slide 20 shows another area at high magnification. One can see the spherical pores that have developed in the PuC.

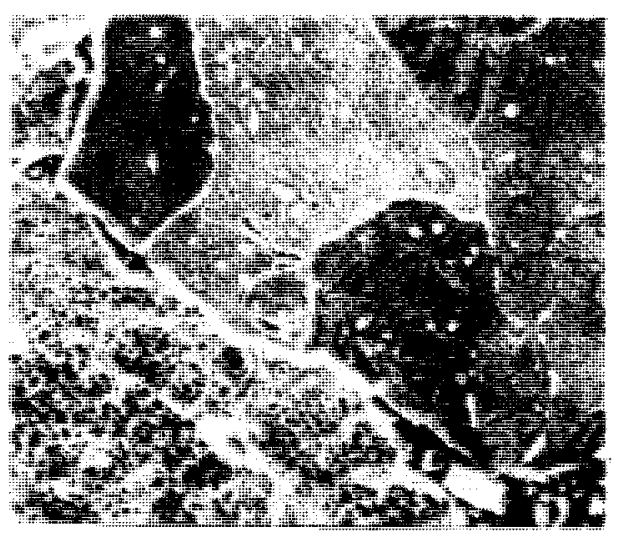

SLIDE 19

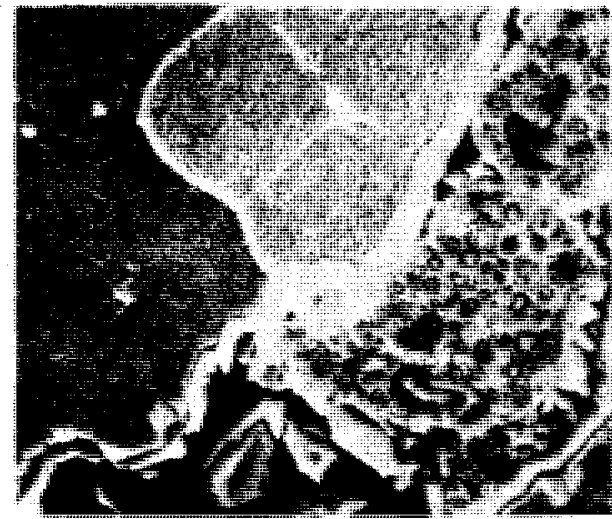

SLIDE 20

\section{CARBIDE SUMMARY}

Concluding the discussion of the carbide, we are continuing the irradiation of mixed carbide in MTR capsules, and in both EBR-II and Fermi. The work in EBR-II is on full-length rods; eleven of these rods are in the reactor now; and they are running with power densities of $2.5 \mathrm{~kW} / \mathrm{cm}^{3}$, and heat ratings up to $25 \mathrm{~kW} / \mathrm{ft}$. The cladding materials presently include $304 \mathrm{SS}$ Hastelloy $X$, and niobium $1 \%$ zirconium; Inconel 625 will be added at a later date. The maximum burnup to date is $1.4 \%$. our opinion is that mixed carbide would very easily satisfy the burnup and temperature requirements of the FFTF; particularly, if the low density modification is used. The lack of manufacturing capability, which was discussed at length yesterday, is admittedly a problem. However, we tend to feel that it is somewhat unrealistic to consider only the types of fuel where large manufacturing capabilities exist. If such a restrictive philosophy had been followed with the MTR, the reactor would have been fueled with unalloyed uranium. Based on our discussions with industrial colleagues, we are assured that if a large demand develops for mixed carbide, then the required production capability would appear. 
PART II. R. E. Macherey (ANL)

\section{URANIUM-PLUTONIUM ALLOYS}

The uranium-plutonium alloys have properties that make them attractive as fuels for fast power breeder reactors. Some of these properties are directly applicable to the FFTF. The attractive properties are high density, good thermal conductivity, large reliable thermal expansion, and no reaction with sodium. In addition, these materials are quite easily prepared; they are amenable to pyrorefining, and they can be fabricated and remotely refabricated. Uranium-plutonium alloys, however, particularly the fizzium variety, do have disadvantages such as relatively low melting temperature, incompatibility with otherwise attractive jacket materials, and a very low radiation swelling temperature. In spite of these latter properties, irradiations have been taken to 8.7 atom percent burnup, in a U-15wt $\%$ Pu-10wt $\% \mathrm{Fz}$ al1oy jacketed in Nb-1wt $\mathrm{Zr}$ tubing. This fuel depends upon its jacket to restrain swelling.

The current effort at ANL on metal1ic fuels is aimed at overcoming the disadvantages of U-Pu-Fz alloy fuel while still maintaining the ability to pyrorefine and not degrading their attractive features. The first objective of this development was to raise the rather low melting temperature for better performance and reliability of the fuel; and the second objective was the elimination of phase transformations in the operating temperature range of the fuel. The first step in the development was the removal of the fizzium elements, which in itself raised the melting temperature, followed by alloying with those elements that are known to raise the melting temperature. Molybdenum, niobium, scandium, titanium, yttrium and zirconium were among those explored. Through study of available information and some experimental work, it was soon evident that titanium and zirconium are the most potent in raising the melting temperature of uraniumplutonium alloys.

Rather marked increase in the solidus temperature where fuel me1ting begins with modest additions of either titanium or zirconium is apparent in Slide 1. Alloys of interest do not degrade density very much. The effects of plutonium additions on these zirconium and titanium alloy fuels are also shown in slide 1 along with solidus isotherms.

In the chart shown in S1ide 2, it is evident that about 10wt: $\mathrm{r}$ or $\mathrm{Ti}$ increases the solidus temperature close to or even above the melting temperature of uranium fuel itself; and even more dramatically, these alloys increase melting temperature some $300^{\circ}$ above fizzium alloys. These alloys also have good density. The 10wt:zr alloy and the 6wt $\%$ Ti alloy have a density of about 16, equivalent to the fizzium a11oys . 


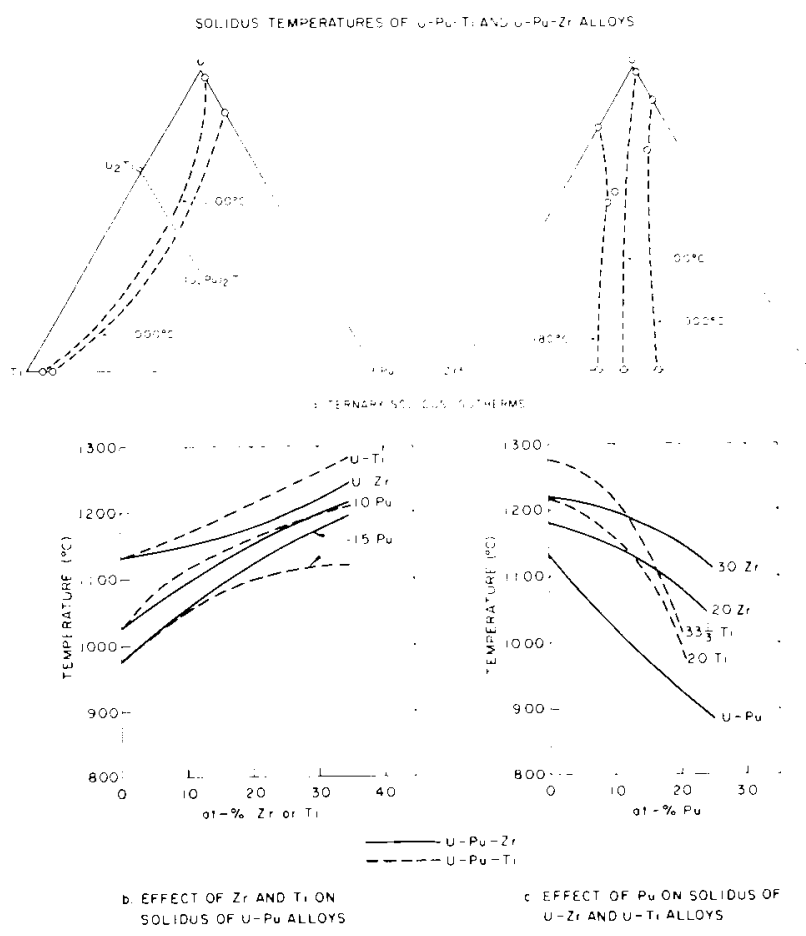

SLIDE 1

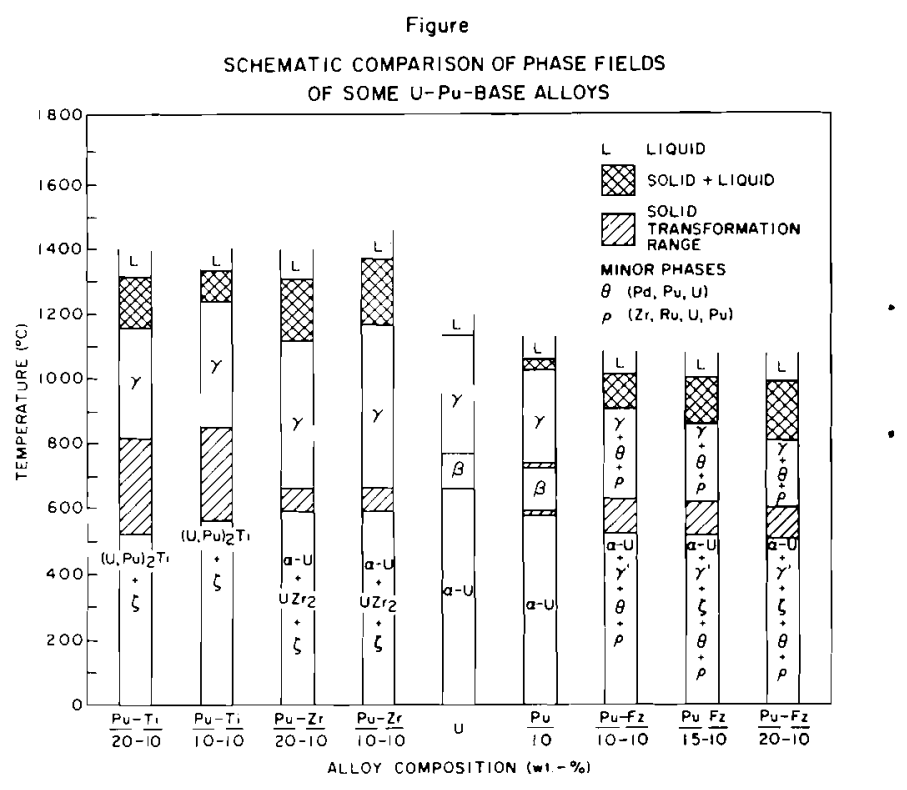

SLIDE 2

Thermal conductivity, although somewhat below the fizzium alloy, is high and increases with temperature, as may be seen in Slide 3. Thermal cycling of U-Pu-Zr and U-Pu-Ti alloys between $350^{\circ} \mathrm{C}$ and $830^{\circ} \mathrm{C}$ does not cause appreciable dimensional change in 100 cycles. The objective of raising melting temperature of U-Pu alloys has been fulfilled by this development.

Phase transformations within the operating temperature range of alloy fuels are not considered favorable. In the uraniumtitanium system, there exists the compound $\mathrm{U}_{2} \mathrm{Ti}$ that has no transformations up to $900^{\circ} \mathrm{C}$ where it transforms into a gamma uranium phase. It was hoped that plutonium could be substituted for uranium in this compound. Plutonium substitutions in this compound lowers the transformation temperature to such an extent that the U-Pu-Ti compound is of little interest. In addition, the necessary high alloy reduces the density to about 14.5. This work has been discontinued.

The most exciting feature of this alloy development has been the discovery of compatibility of certain uranium-plutoniumzirconium alloys with stainless steel (Type 304). The additions of zirconium to this fuel have two effects: first, the temperature at which molten products occur between jacket and fuel to temperature is raised well above reactor operating temperature; second, the diffusion of fuel into the jacket is reduced to a very low rate. 


\subsection{7}

In the graph shown in Slide 4 , the vast superiority of the 10 and $14 \mathrm{wt} \% \mathrm{Zr}$ alloys in raising the temperature at which molten products form between the fue 1 and the $\mathrm{jacket}$ is apparent. The reaction temperature has been raised to over $800^{\circ} \mathrm{C}$, which gives a substantial margin in operating temperature. In comparison, the fizzium alloys form molten products at about $650^{\circ} \mathrm{C}$. Reducing the zirconium content, even to a $6 \%$ level, makes it better or equivalent to the uranium alloy.

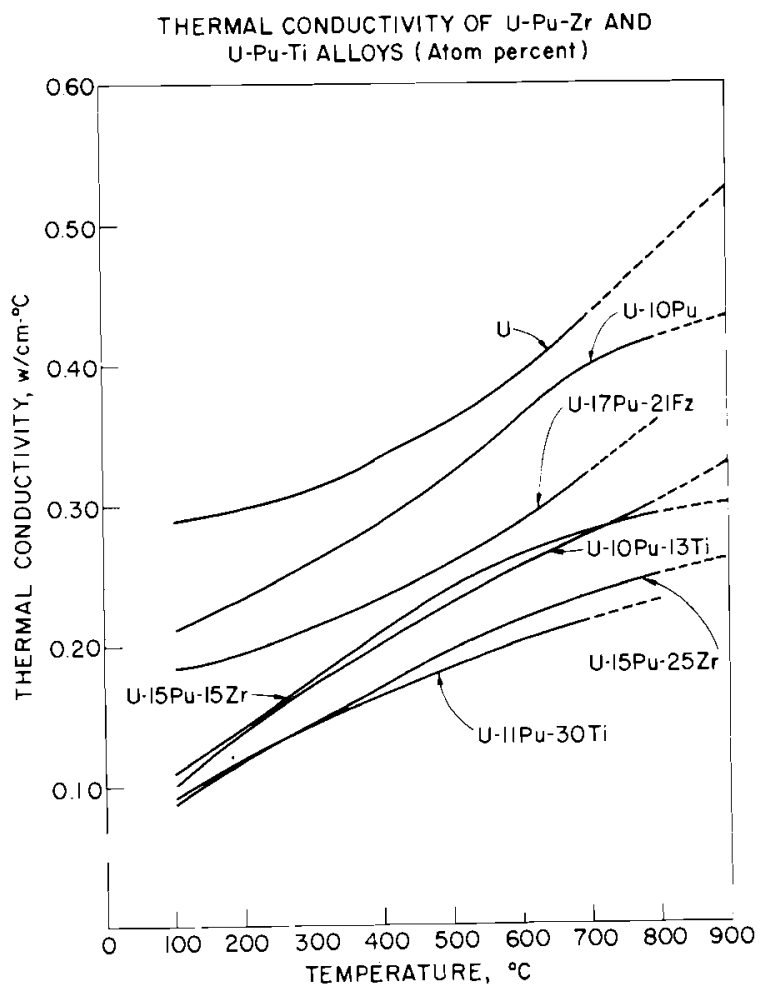

SLIDE 3

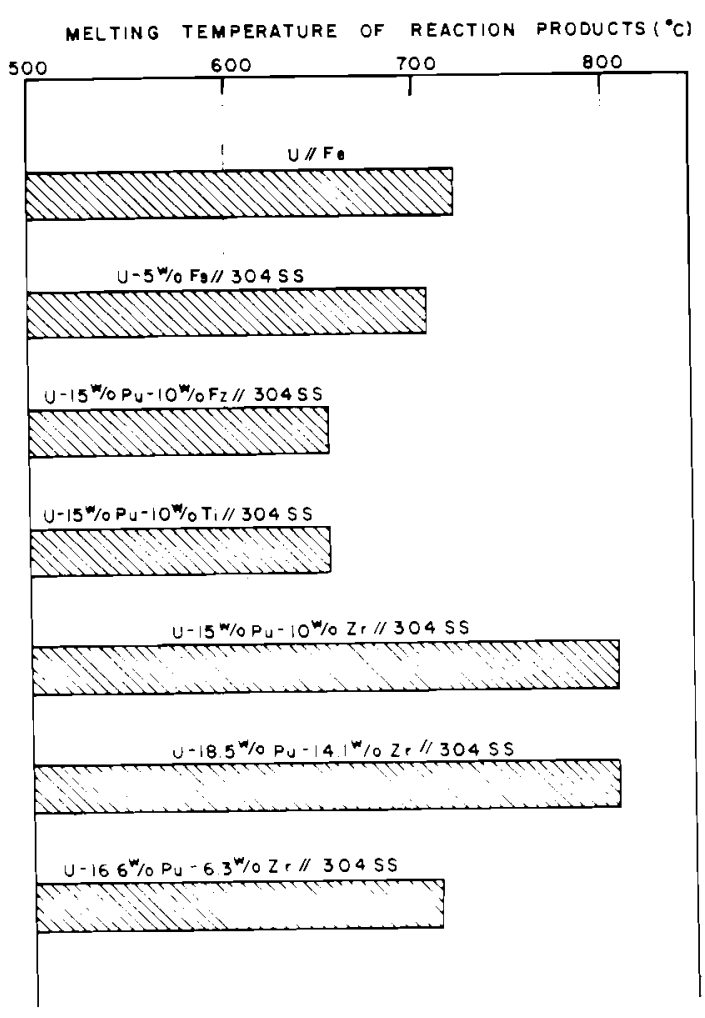

SLIDE 4

Photomicrographs of diffusion couples are shown in Slide 5.. The very low penetration rate of U-15wt\% $\mathrm{Pu}-14 \mathrm{wt}: \mathrm{Zr}$ alloy into Type 304 SS even at $800^{\circ} \mathrm{C}$ is apparent. U-Pu-Fz and U-Pu-Ti alloys form molten phases with stainless steel at this temperature. 

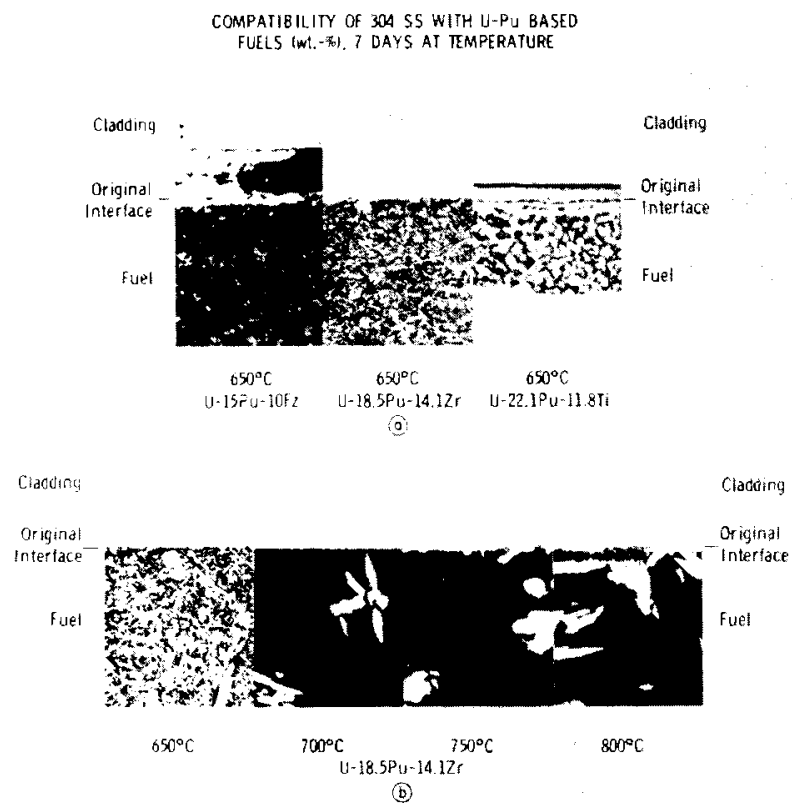

Since irradiation performance of these metallic fuels depends upon restraint of irradiation swelling by the jackets employed, higher strength alloys are sought for use with them. Some nickel base alloys and higher nickel iron alloys have the higher strength sought. Compatibility studies have been made to determine the effect of nickel content upon interdiffusion. Haste11oy-X, Incoloy and Type 304 SS are compared in S1ide 6 . Photomicrographs are presented in Slide 7 showing diffusion couples of these materials with U-Pu-Zr alloys. Compatibility decreased with increasing nickel content. Hastelloy-X, 50\% Ni, is poorer than Incoloy, $30 \% \mathrm{Ni}$, which in turn is poorer than Type $304 \mathrm{SS}, 18 \% \mathrm{Ni}$. Incoloy appears useable but Hastelloy-X is probably not. Alloys having intermediate nickel contents have also been explored. The N 55 alloy and 16-15-6 alloy show slightly more reaction with U-Pu-Zr alloy than does Type 304 stainless.

TABLE $\vee$

PENETRATION OF U-PU-Zr FUEL INTO 304 SS, HASTELLOY-X AND INCOLOY-800 CLADDING

\begin{tabular}{|c|c|c|c|c|c|c|c|c|c|c|}
\hline \multirow{3}{*}{ CLADOING } & \multirow{3}{*}{$\begin{array}{l}\text { TEMP. } \\
\left({ }^{\circ} \mathrm{C}\right)\end{array}$} & \multicolumn{9}{|c|}{ PENETRATION $\left(\mathrm{cm} \times 10^{4}\right)$} \\
\hline & & \multicolumn{3}{|c|}{$U-16.6 w / 0 P u-6.3 w / 0 Z r$} & \multicolumn{3}{|c|}{$u-15 w / 0 \mathrm{Pu}-10 w / 0 \mathrm{Zr}$} & \multicolumn{3}{|c|}{$U=18.5 \mathrm{w} / \mathrm{oPu}-14.1 \mathrm{w} / \mathrm{o} \mathrm{Zr}$} \\
\hline & & 7 DAYS & 17 DAYS & 42 DAYS & 7 DAYS & 17 DAYS & 42 DAYS & 7 DAYS & 17 DAYS & 42 DAYS \\
\hline 304 SS & $\begin{array}{l}650 \\
700 \\
750 \\
800 \\
850\end{array}$ & MELTED & 8 & & $\vdots$ & 2 & & $\begin{array}{c}<1 \\
1 \\
2 \\
9 \\
\text { MELTEO }\end{array}$ & 2 & $\begin{array}{l}6 \\
17\end{array}$ \\
\hline HASTELLOY-X & $\begin{array}{l}700 \\
750 \\
800\end{array}$ & MELTED & 50 & & MELTED̈ & 10 & 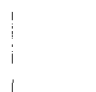 & $\begin{array}{l}\vdots \\
\text { MELTED }\end{array}$ & & 12 \\
\hline INCOLOY -800 & $\begin{array}{l}750 \\
800\end{array}$ & & & & $\begin{array}{c}16 \\
\text { MELTED }\end{array}$ & & 38 & & & \\
\hline
\end{tabular}

WELTING OF THE REACTION PRODUCTS AND ACCELERATED PENETRATION OCCURRED.

SLIDE 6 


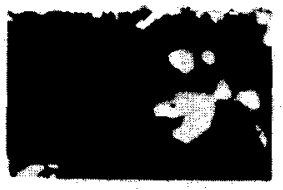

304. ss

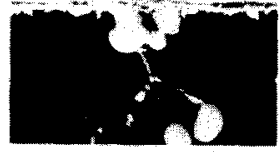

Nis

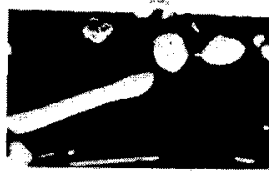

$26-150$

SLIDE 7

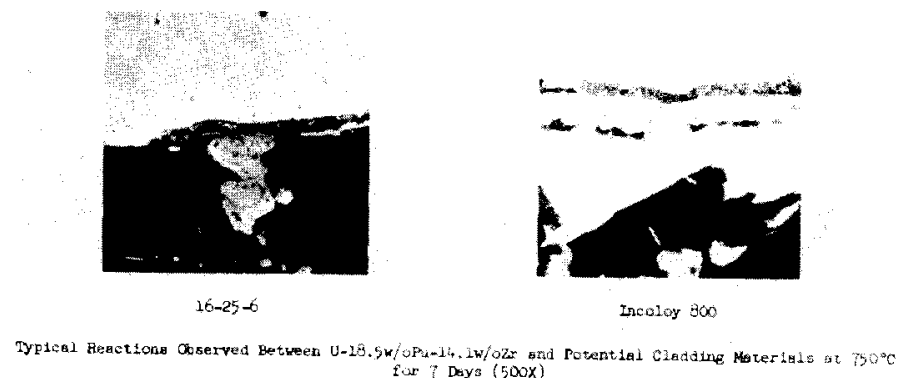

Assuming a parabolic rate law, penetration of less than $10 \mu$ is predicted for U-Pu-Zr alloy into Type 304 SS at $650^{\circ} \mathrm{C}$. This indicated compatibility is certainly satisfactory.

Uranium-plutonium-zirconium and other alloys can now be factored into a pyrorefining cycle by using a salt separation process which removes nearly all fission products. Restrictions on alloy imposed by the melt-refining process can be eliminated. The U-Pu-Zr alloy system, even though melting temperatures are much higher than the fizzium alloy, can be injection cast and hot refabricated by well developed processes proven on fizzium alloys.

Prior to the development of the U-Pu-Zr alloy fuel, stainless steel and nickel base alloys could not be used with U-Pu alloy fuels, primarily because of low interaction temperature. The choice of jacket materials was restricted to refractory alloys. Vanadium alloys, because of their good strength, compatibility with U-Pu alloys, and attractive nuclear properties, are the prime candidates. A V-20wt $\mathrm{Ti}$ has all the necessary attributes for a jacket material except for verification of corrosion resistance to sodium containing moderate oxygen contamination. The compatibility of V-20wt\% Ti with U-Pu-Ti and U-Pu-Fz alloy fuel is excellent in the temperature ranges of interest. Penetration of various jacket materials, projected in mils per year by U-Pu-Fz and U-Pu-Ti alloys, is shown in Slide 8 . The substantial superiority of vanadium alloys and niobium alloys (another considered material) is apparent. 
CALCULATED PENETRATIONS FOR ONE YEAR (mils)

SLIDE 8

\begin{tabular}{|l|c|c|c|c|c|}
\hline \multirow{2}{*}{ Combination } & \multicolumn{5}{|c|}{ Temperature ${ }^{\circ} \mathrm{C}$} \\
\cline { 2 - 6 } & 550 & 600 & 650 & 700 & 750 \\
\hline $\mathrm{Cr} / \mathrm{U}-10 \mathrm{Pu}-10 \mathrm{Fz}$ & & & & $* 1.3$ & \\
Hast X/U-10 Pu-10 Fz & 1.0 & 2.0 & 4.2 & & \\
Hast X/U-15 Pu-10 Fz & & & 7.3 & & \\
$\mathrm{Mo} / \mathrm{U}-10 \mathrm{Pu}-10 \mathrm{Fz}$ & & 0.5 & 0.7 & 0.9 & \\
$\mathrm{M} / \mathrm{U}-15 \mathrm{Pu}-10 \mathrm{Fz}$ & & 0.6 & $* 1.0$ & $* 1.4$ & \\
$\mathrm{Nb} / \mathrm{U}-10 \mathrm{Pu}-10 \mathrm{Fz}$ & 0.8 & 1.8 & 27.3 & & \\
$\mathrm{Nb} / \mathrm{U}-20 \mathrm{Pu}-10 \mathrm{Fz}$ & 1.1 & 3.0 & 52.0 & & \\
$\mathrm{Nb}-1 \mathrm{Zr} / \mathrm{U}-10 \mathrm{Pu}-10 \mathrm{Fz}$ & 0.9 & 1.4 & 23.6 & & \\
$304 \mathrm{SS} / \mathrm{U}-10 \mathrm{Pu}-10 \mathrm{Fz}$ & 0.6 & 1.0 & 2.3 & & \\
$304 \mathrm{SS} / \mathrm{U}-15 \mathrm{Pu}-10 \mathrm{Fz}$ & & & 4.1 & & \\
$\mathrm{~V}-10 \mathrm{Ti} / \mathrm{U}-10 \mathrm{Pu}-10 \mathrm{Fz}$ & 0.2 & 0.3 & 0.8 & & \\
V-20 Ti/U-10 Pu-10 Fz & 0.1 & 0.3 & 0.6 & 1.2 & $* 2.9$ \\
V-20 Ti/U-15 Pu-10 Fz & & $* 0.3$ & 0.6 & 2.0 & 3.8 \\
\hline
\end{tabular}

*Calculated from only one data point assuming an $x^{2}=k t$ law.

Corrosion resistance of vanadium alloys to reactor grade sodium has not yet been established. It is known that no vanadium alloy has been developed which is useable in sodium containing 50 ppm oxygen; however, it is known that $\mathrm{V}-20 \mathrm{wt} \% \mathrm{Ti}$ alloys have very low corrosion rates in high purity, gettered sodium. The maximum acceptable oxygen level appears to lie somewhere between these two levels. The current development includes the establishment of acceptable levels. Alloy development has been extended beyond the $\mathrm{V}-\mathrm{Ti}$ series with promising results. $\mathrm{V}-\mathrm{Ti}-\mathrm{Cr}$ alloys have been found superior to the binary $V-T i$ alloy on a comparative basis in screening tests; and a $\mathrm{V}-\mathrm{Cr}-\mathrm{Al}$ alloy has shown superiority over both these alloys. The corrosion rates for a V-20wt:Cr-10wt:Al alloy were an order of magnitude lower than $\mathrm{V}-20 \mathrm{wt} \mathrm{Ti}$ a $110 y$ in sodium contaminated to $50 \mathrm{ppm}$ oxygen. These vanadium alloys hold considerable promise, particularly since oxygen levels in operating reactors sodium is relatively low. Vanadium alloy will continue to be part of the metallic fuel development as backup for stainless steel, and as advanced alloys for better performance.

\section{URANIUM-PLUTONIUM CARBIDE FUEL}

Uranium-plutonium-carbide fuel development at ANL currently emphasizes irradiation, compatibility, and fabrication, with some physical property determination and synthesis. Fuel loadings around $80 \%$ effective density have been selected as the most promising for developing high burnup; fabrication and irradiation work is in this range. Helium bonding (contact) has been selected as the main method of heat transfer, although sodium bonding is considered. Fabrication emphasizes vibratory compaction although 


\subsection{1}

pellet processes are included where void space is incorporated in annulus between fuel and jacket, in a central hole, or in porosity in low sintered density. Compatibility between fuel and jacket, of course, is of major concern with compatibility between fuel and sodium of secondary interest.

Initial compatibility work is being done on stoichiometric, single phase (U,Pu)C specimens pressed and sintered. Characteristic structure of this material is shown in Slide 9.

SLIDE 9

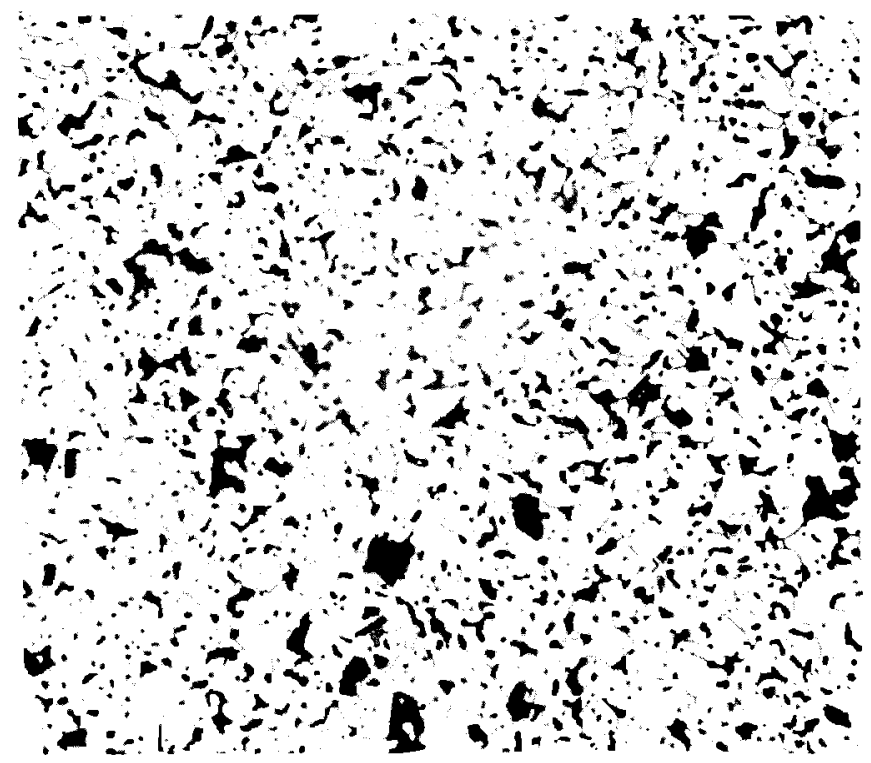

Its carbon content is $4.75 \mathrm{wt} \%$, oxygen is $900 \mathrm{ppm}$, nitrogen is $300 \mathrm{ppm}$ and its density is $86 \%$ of theoretical. Capsules for compatibility studies were prepared by polishing and pressing couples in molybdenum frames and encapsulation. The initial experiments were conducted on the candidate jacket materials 1 isted in S1ide 10.

EXTENT OF HIGH TEMPERATURE REACTIONS OF (U0.8PU0.2 $\mathrm{KC}$ WITH POTENTIAL CLADDING MATERIALS FOR 7 days $(168$ hours)

\begin{tabular}{|c|c|c|c|}
\hline Material & $\begin{array}{c}\text { Temperature } \\
\left({ }^{\circ} \mathrm{C}\right)\end{array}$ & $\begin{array}{l}\text { Reaction } \\
\text { Zone Width } \\
\text { (L) }\end{array}$ & Remarks \\
\hline Type 304 SS & $\begin{array}{r}950 \\
1100\end{array}$ & $\begin{array}{r}3 \\
130\end{array}$ & Heavy grain boundary penetration. \\
\hline Hastelloy-X & $\begin{array}{r}950 \\
1100\end{array}$ & $\begin{array}{c}45 \\
\text { Melted }\end{array}$ & Reaction zone in direction of fuel. \\
\hline $\begin{array}{l}\text { Vanadium- } \\
20 \text { w/o Titanium }\end{array}$ & $\begin{array}{r}950 \\
1100\end{array}$ & $\begin{array}{l}160(320) \\
750(>1300)\end{array}$ & $\begin{array}{l}\text { Nonuniform continuous band, poorly defined } \\
\text { due to reaction along stringers. }\end{array}$ \\
\hline Vanadium & $\begin{array}{r}950 \\
1100\end{array}$ & $\begin{array}{l}\text { Nil } \\
365\end{array}$ & $\begin{array}{l}\text { Irregular second phase growth along grain } \\
\text { boundaries. }\end{array}$ \\
\hline Niobium & $\begin{array}{r}950 \\
1100\end{array}$ & $\begin{array}{l}2 \\
8\end{array}$ & Uniform continuous band. \\
\hline Molybdenum & 1100 & 6 & Uniform continuous band. \\
\hline Tantalum & 1100 & 4 & Uniform continuous band. \\
\hline Chromium & 1100 & Nil & \\
\hline Tungsten & 1100 & Nil & \\
\hline
\end{tabular}

values in parentheses indicate extent of reaction along stringers. 
Penetration rates and interdiffusion band widths are also given. The high temperature $\left(1100^{\circ} \mathrm{C}\right)$ were employed to accelerate the test. Stainless steel does very well, showing good compatibility. Hastelloy-X and (U,Pu)C interdiffuse somewhat more. Vanadiumtitanium alloy interacts quite badly with this fuel, although unalloyed vanadium is quite good. Niobium, molybdenum, tantalum show little interdiffusion with chromium and tungsten experiencing the least reaction of those listed.

Photomicrographs of some diffusion couples are shown in Slide 11. The extensive penetration into vanadium-titanium alloy is apparent. Even at high temperature, stainless steel shows little general penetration although some intergranular penetration has occurred.

\section{SLIDE 11}

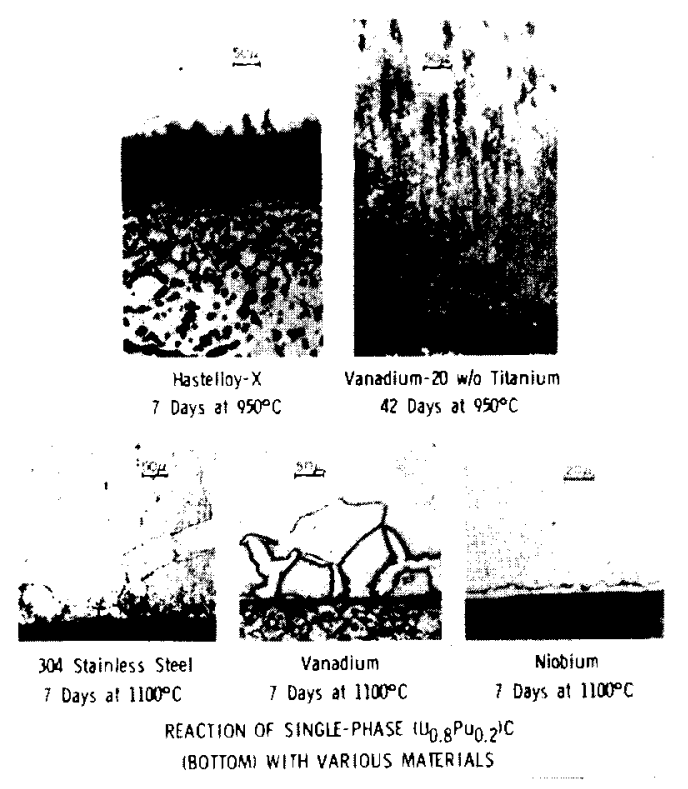

Compatibility work was extended to include hyperstoichiometric carbide for comparison with the previous work on stoichiometric material. Interactions with both materials are presented in Slide 12. Interaction with stainless steel by the hyperstoichiometric (U,Pu)C is greater than that developed by the stoichiometric carbide at $800^{\circ} \mathrm{C}$, Slide $12 \mathrm{a}$. 


\subsection{3}

EXTENT OF $800^{\circ} \mathrm{C}$ REACTIONS OF STOICHIOMETRIC AND HYPERSTOICHIOMETRIC URANIUM-PLUTONIUM CARBIDES WITH POTENTIAL CLADDING MATERIALS FOR 42 days (1008 hours)

\begin{tabular}{|c|c|c|c|c|}
\hline \multirow[t]{2}{*}{ Material } & \multicolumn{2}{|c|}{$\begin{array}{l}\text { Stoichiometric Carbide } \\
(4.83 \text { Equivalent w/o } \mathrm{C}): \\
\left(\cup_{0.8} \mathrm{Pu} \mathrm{u}_{0.2} \mathrm{l} \mathrm{C}\right. \\
\text { Reaction Zone Width }(\mu)\end{array}$} & \multicolumn{2}{|c|}{$\begin{array}{l}\text { Hyperstoichiometric Carbide } \\
(5.25 \text { Equivalent w/o C): } \\
(\mathrm{U}, \mathrm{Pu}) \mathrm{C}+(\mathrm{U}, \mathrm{Pu})_{2} \mathrm{C}_{3} \\
\text { Reaction Zone Width }(\mu)\end{array}$} \\
\hline & Average & Maximum & Average & Maximum \\
\hline Type 304 SS & Nil & Nil & 24 & 29 \\
\hline Hastelloy-X & 8 & 10 & 8 & 11 \\
\hline $16-15-6$ & 28 & 34 & 35 & 38 \\
\hline $16-25-6$ & 26 & 30 & 32 & 36 \\
\hline $\mathrm{N} 155$ & 15 & 37 & 19 & 43 \\
\hline
\end{tabular}

"Equivalent $w / 0$ Carbon $=w / 0 \mathrm{C}+$ w/o 0 times $0.75+w / 0 \mathrm{~N}$ times 0.857 .

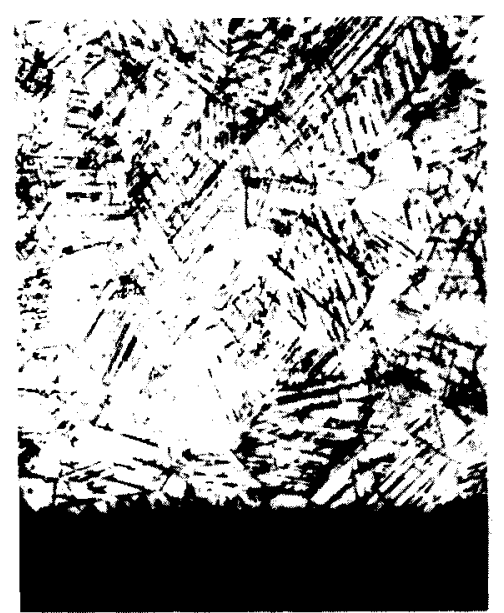

Stoichiometric $(4.83 \mathrm{w} / \mathrm{O} \mathrm{C})$

URANIUM-PLUTONIUM CARBIDE VS TYPE 304 STAINLESS STEEL $800^{\circ} \mathrm{C}-42$ days

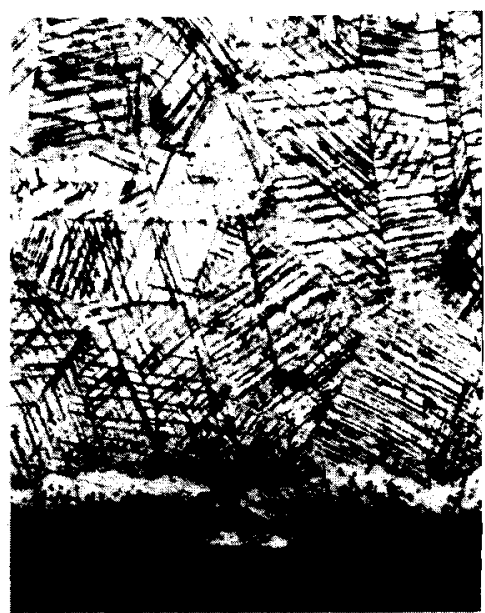

Hyperstoichiometric $(5.25 \mathrm{w} / 0 \mathrm{C})$ URANIUM-PLUTONIUM CARBIDE VS TYPE 304 STAINLESS STEEL $800^{\circ} \mathrm{C}-42$ days 
The other materials do not show much effect of carbon content. Hastelloy-X does show some reaction with the fuel at $800^{\circ} \mathrm{C}$ as shown in Slide 13 .

As mentioned previously, fabrication development is centered around vibratory compaction. This process is attractive because of its simplicity and adaptability to remote operation; also the process lends itself well to the density range of interest for good irradiation performance. The method being developed by Ayer at ANL consists of infiltration of a packed bed by a sequence of loading operations. Relatively large particles are loaded first to form a porous bed into which fine particles are vibrated.

Simple equipment shown in Slide 14 is used in this work. The basic equipment is a 60 cycle vibrator equipped with amplitude control, accelerometer, and other monitoring devices. A very necessary piece of equipment is the rattle cage positioned around the loading tube. This cage impacts random vibration, including a horizontal mode which is thought to break up bridging of particles. Particles are fed into the jacket tubes from the plastic bottle screwed into the loading funnel at the top.

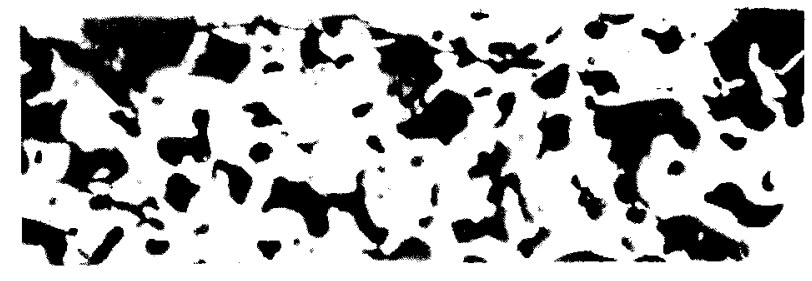

$\left(\mathrm{U}_{0.8}\right.$ PU0.2) C VS HASTELLOY $-X$ $800^{\circ} \mathrm{C}-42$ days

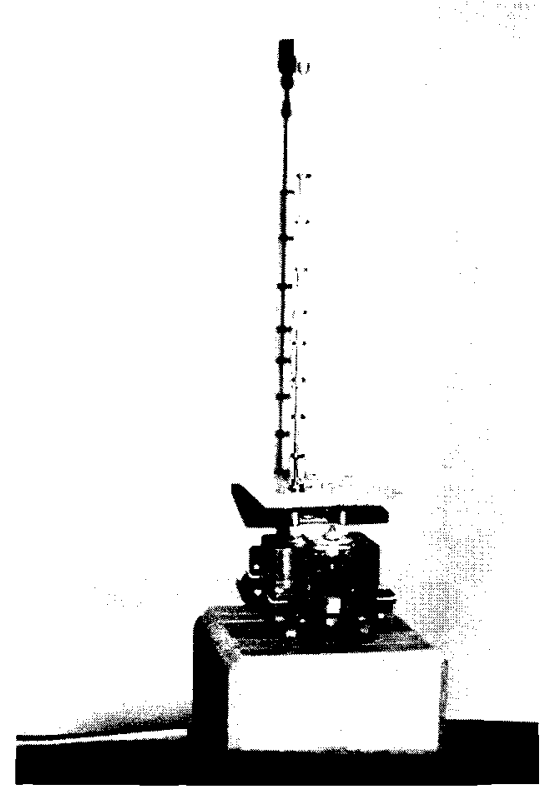

SLIDE 14

A three component system is used for loading in which the large particle fraction is about one-fifth of the jacket inside diameter. The second fraction is about one-half the size of the larger. The third fraction is very fine for infiltration. The large particles, $1.2 \mathrm{~mm}$, are used in loading $6.5 \mathrm{~mm}$ ID tubing. Using these particles about $59 \%$ packing efficiency can be obtained. Addition of a second fraction about one-half the above size will yield a packing efficiency of $66 \%$. Higher densities (above $90 \%$ ) can be developed by the addition 
of a third very fine fraction. In practice, the two larger fractions are blended together before loading into the jacket tubing then loaded to develop a fixed bed into which the fine particles are loaded. This method has potential for producing uniform loadings. The proportions employed by weight are $62 \%$ of the large fraction, $18 \%$ of the intermediate, and $20 \%$ of the fine fraction.

The initial work on uranium carbide-plutonium carbide was done using crushed, angular, high density particles. A packing efficiency of $86 \%$ was realized in small diameter tubing about 12 in. long.

Radiographs of a loaded rod are shown in Slide 15. 


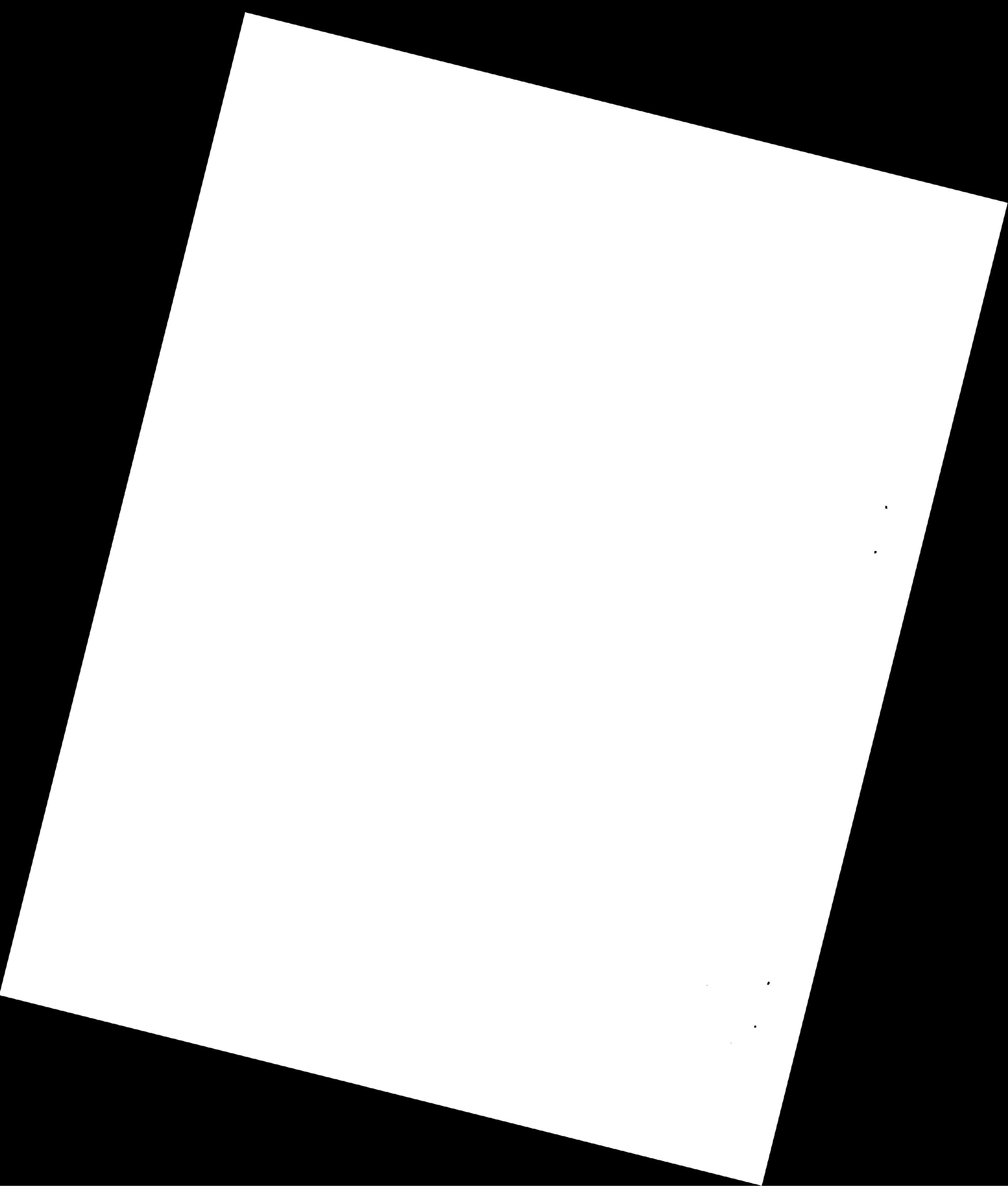


APPLICATION OF SODIUM THERMALLY BONDED CARBIDE FUEL ELEMENTS IN FFTF

\author{
C. E. Weber \\ Atomics International \\ Canoga Park, California
}

\title{
INTRODUCTION
}

I plan to discuss one specific type of carbide based fuel element; namely a sodium thermally bonded, slightly hypostoichiometric to stoichometric carbide, stainless steel canned. This type is not to be confused with other carbide types such as (a) a contact bonded, sintered or cast hypostoichiometric carbide with no annular allowance for fuel expansion (at high burnup levels the cladding must be ductile to accommodate fuel growth) and (b) compacted powders of UC-PuC. This type of fuel is highly developmental at present and may have no advantages over mixed oxides. To set the stage of my review, one may classify fuel elements for FFTF into two classes:

- COLD OPERATION-C1ass A-Fuel operating below the temperature for fission gas release and fission gas bubble induced swelling.

- HOT OPERATION-Class B-Fuel, at least a part of which operates above the temperature where gas release occurs and concurrent fuel swelling may occur. Plenum space for the gas and solid fission product expansion is required if cladding or matrix strain is to be avoided with Class A operation.

During the meeting, the advantages to FFTF of maximum use of existing technology, minimum development time and funds, nuclear safety (which I do not feel fully qualified to discuss), a stretch capability for FFTF, and a desirable applicability of FFTF type fuel in Fast Breeder Reactors have been empasized. The sodium thermally bonded, carbide fueled, stainless steel canned element must be seriously considered for FFTF application. A conventional canning material, stainless steel, can be used. The stress on the jacket will be low since (in fuel elements sized for FFTF) gas release and gas bubble induced swelling will not occur and space can be provided in the sodium filled annulus to accommodate fuel growth due to solid fission product accumulation. This type of fuel element will run cold in FFTF. A11 of the fuel can be run safely below the temperature for breakaway swelling and gas release. Under these conditions, the carbides act like the stable thorium and zirconium base metal fuels. These metal fuels have been taken to very high burnup levels with little or no volumetric expansion than due to the 
solid fission products as long as the fuel operated below the critical swelling temperature (which in itself only decreases slowly with increasing fuel burnup). The thickness of the sodium annulus can be sized to accommodate the volumetric growth at the design burnup level.

\section{CARBIDE WITH SODIUM BONDING}

In the series of examples developed ind this review, I have used a growth rate of $3 \%$ per 10,000 Mtu which is conservative, based on values of $2 \%$ (or slightly less) in good agreement with the theoretical value and with experimental values to exposures of 30,000 MWd/Mtu. However, a value of $3 \%$ provides a safety factor. The gas release from high density carbides is negligible, we 11 less than $1 \%$ unless the central temperature is pushed up to where gas diffusion and concomitant fuel swelling occurs. Operating below the swelling temperature, a small plenum chamber at the top of the fuel element will provide for the small amount of gas released and the sodium thermal expansion. The hoop stress in the stainless steel can be kept very low which is a desirable feature considering the minimum ductility of stainless steel under irradiation.

Another consideration is that of the compatability of the fuel with stainless steel. Hyperstoichiometric carbides will carburize the stainless steel and will strengthen and embrittle the can. A slight amount of excess carbon and some carburization can be tolerated; however, stoichiometric or hypostoichiometric carbides do not carburize stainless steel. No reaction occurs until the stainless steel-carbide temperature reaches the eutectic temperatures (e.g. Cr-UC eutectic,) which are very high at approximate 1 y $1800{ }^{\circ} \mathrm{C}$.

A second source of incompatability is reaction between second phase grain boundary metal (U in highly hypostoichiometric UC). This does not occur unless intimate contact is established, and does not occur at a rapid rate until this eutectic temperature is reached ( 1340 o F). At lower temperatures, on $1 y$ local reaction occurs, with the rate 1 imited by the rate of diffusion of the metal along the grain boundaries. Our experience has shown that there is an adequate broad composition range in the $U_{1-x}$ to $U C$ system such that this carbide can be operated without cladding carburization and without local reaction of uranium to cladding temperatures of $1300{ }^{\circ} \mathrm{F}$. By reducing the amount of excess metal, the compatibility limits can be improved.

A final parameter is the "critical temperature" for swelling in UC and UC-PuC fuels operating at FFTF conditions. Consider the percent increase in volume per $10^{4} \mathrm{MWd} / \mathrm{Mtu}$ as a function of fuel centerline temperature. Conservatively, slightly hypostoichiometric UC can be operated to $30,000 \mathrm{Mwd} / \mathrm{Mtu}$ at a centerline temperature of $1900^{\circ} \mathrm{F}$. Granted, hyperstoichiometric 
carbide as a fuel material alone is more resistant to swelling than "hypo" due to free (second phase) uranium in $\mathrm{UC}_{1-\mathrm{x}}$. However, limiting this discussion to the "hypo" system which is "cladding compatible", we have good central fuel temperature data based on thermocouple measurement in the center of the fuel during irradiation, and there is no uncertainty with respect to contact resistance between the fuel and the cladding. The best single experiment ran at a fuel central temperature of $1800^{\circ}$ plus a burnup of $30,000 \mathrm{MWd} / \mathrm{Mtu}$, and a cladding inner surface temperature of $1150^{\circ} \mathrm{F}$. For the purpose of rating carbides for FFTF running to somewhat higher burnup at 40,000 Mwd/Mtu, I have arbitrarily downgraded the maximum permissible centerline fuel temperature to 1700 of to allow for the increase in burnup. This reduction assumes this class of fuel behaves like a metal, e.g. the thorium and zirconium base alloys, and is consistant with the observed volumetric stability of carbides to very high burnup at lower central temperatures. Further conservatism is gained, as mentioned previously, through the choice of $3 \% \Delta \mathrm{V}$ per $10,000 \mathrm{MWd} / \mathrm{Mtu}$.

I will not spend time on the microstructure of hypostoichiometric UC after irradiation to $30,000 \mathrm{MWd} / \mathrm{Mtu}$ due to my reduced time for review. Slide 1 shows the center, middle and edge of UC (4.6 wt: C) irradiated slightly below the critical temperature for swelling and gas release.

Finally, what is the capability of stoichiometric to slightly substoichiometric carbides sized for FFTF based on the critical temperature for swelling and the volumetric expansion rates just developed? We have not explored all permutations of fuel rod size, fuel composition, etc. As shown in the table. we have chosen a fuel rod of 0.226 OD, a $0.008 \mathrm{in.}$ SS wall thickness, and a fuel OD of $0.202 \mathrm{in}$. with sodium in the annulus. This compares with the 0.226 in. OD cermet element at $27.7 \% \mathrm{PuO}_{2}$ and 29 metal atom percent burnup and $27 \%$ porosity. For this case, the burnup is $14.3 \times 10^{20}$ fission $/ \mathrm{cm}^{3}$, the rod 1 inear power is $16.3 \mathrm{~kW} / \mathrm{ft}$, and the coolant outlet temperature is $800 \mathrm{OF}$. The cladding inner temperature is about $888^{\circ} \mathrm{F}$, and the fuel central temperature is $1277 \mathrm{oF}$.

TABLE I. FFTF CARBIDE FUELS

(Fuel $\overline{O D}=0.202$ in., $\operatorname{Rod} O D=0.226$ in.)

\begin{tabular}{|c|c|c|c|c|c|c|c|c|}
\hline \multirow{2}{*}{$\begin{array}{c}\text { Design Parameters } \\
\text { Composition vol\% } \\
\text { Puo }\end{array}$} & $\begin{array}{c}\mathrm{FFTF} \\
\mathrm{PuO}_{2}-\mathrm{SS}\end{array}$ & \multicolumn{2}{|c|}{$U C_{1-x}$} & \multicolumn{2}{|c|}{$(\mathrm{UPu}) \mathrm{C}$} & \multicolumn{3}{|c|}{$(\mathrm{ZrPu}) \mathrm{C}$} \\
\hline & 27.7 & - & - & - & & - & & \\
\hline $\mathrm{PuC}^{2}$ & - & $73-27$ & - & $\overline{19} .4$ & & $\overline{16} .4$ & & \\
\hline UC & - & - & 一 & 75.6 & & - & & \\
\hline $\operatorname{2rC}$ & - & - & - & - & & 78.6 & & \\
\hline $\begin{array}{l}\text { Fission/cc } \times 10^{20} \\
\text { Metal atom\% }\end{array}$ & 14.3 & $\begin{array}{r}15.5 \\
4.5\end{array}$ & $\begin{array}{r}15.5 \\
4.5\end{array}$ & $\frac{15.5}{5}$ & & 15.5 & & \\
\hline Rod Power (Kw/ft) & 16.3 & 16.3 & 16.3 & 16.3 & 29.5 & 16.3 & 16.3 & 28 \\
\hline Coolant outlet (oF) & 800 & 800 & 1200 & 1200 & 800 & 800 & 1200 & 1200 \\
\hline $\operatorname{Max} . \operatorname{clad}\left({ }^{\circ} \mathrm{F}\right)$ & 888 & 888 & 1288 & 1288 & 959 & 888 & 1288 & 1359 \\
\hline Fuel $\$(O F)$ & 1277 & 1222 & 1677 & 1700 & 1700 & 1263 & 1663 & 2000 \\
\hline Max. allowable & - & 1700 & 1700 & 1700 & 1700 & 2000 & 2000 & 2000 \\
\hline
\end{tabular}




\section{4}

First consider UC and today's technology. This fuel, sized for FFTF, will run cold, and a carbon content as 10 as 4.4 wt carbon could be used at a $800^{\circ} \mathrm{F}$ coolant outlet temperature. It should be noted that 0.2 in. OD rods of UC have been cast at A.I., although it is cheaper and easier to use 0.25 in. material. Also a 0.008 in. cladding thickness has been used in these examples, and this would have to be increased for coolant outlet temperatures above $800 \mathrm{~F}$.

The first case shows $25 \%$ enriched UC, operating at $800{ }^{\circ} \mathrm{F}$ outlet temperature, $16.3 \mathrm{KW} / \mathrm{ft}$ to a burnup of $15.5 \mathrm{x} 10^{2}$ fissions $/ \mathrm{cm}^{3}$, and a central temperature of $1222^{\circ} \mathrm{F}$. If the coolant outlet temperature is raised to $1200^{\circ} \mathrm{F}$, the maximum central temperature is $1677^{\circ} \mathrm{F}$ limit. In this second case the allowable spread in carbon content would probably be decreased, e.g. 4.6 to 4.8 wt $\%$

The third case is stoichiometric to slightly substoichiometric UC-PuC. Process development is required, particularly fabrication rate scale up. Either cast UC-PuC or higb density $(<95 \%)$ closed porosity sintered carbides are desired. This avoids a prolonged discussion ranging into estimates of the behavior of low density, open porosity carbides under irradiation. The examples have been sized for 95\% density. In the case of UC-19.4\% PuC, the central temperature is again very low, $1279{ }^{\circ} \mathrm{F}$ at a $800{ }^{\circ} \mathrm{F}$ outlet temperature. It is an acceptable $1700^{\circ} \mathrm{F}$ at a 1200 oF sodium outlet temperature. Varying the power rating, if the outlet temperature is fixed at $800 \mathrm{oF}$, then the rod linear power can be raised from $16.3 \mathrm{KW} / \mathrm{ft}$ to 29.5 $\mathrm{KW} / \mathrm{ft}$ before the $1700{ }^{\circ} \mathrm{F}$ central temperature limit is reached. It should be noted that the $1700^{\circ} \mathrm{F}$ limit for UC-PuC has been set the same as UC, because the absolute melting points are close to the same for UC and UC-20\% PuC. Other parameters can also be changed to optimize the use of UC-PuC, e.g. rod diameter, cladding thickness, etc. It is clear that with some process development and restriction of the composition so close to stoichiometric then a UC-PuC, sodium bonded element can be designed to operate below the swelling and gas release temperature, with no cladding ductility required.

The stretch capability for carbide systems is outstanding provided a $\mathrm{ZrC}-16.4$ vo1\% PuC carbide can be fabricated. In this case on a homologous temperature scale, the critical temperature for swelling of $\mathrm{ZrC}-\mathrm{PuC}$ (solid solution) can be conservatively set at $2000^{\circ} \mathrm{F}$. For an $800^{\circ} \mathrm{F}$ outlet temperature, the central temperature is $1263{ }^{\circ} \mathrm{F}$ at $16.3 \mathrm{KW} / \mathrm{ft}$. It goes to 1563 of at a 1200 of outlet temperature. The linear power can be increased to $28 \mathrm{KW} / \mathrm{ft}$ at $1200^{\circ} \mathrm{F}$ outlet sodium before the 2000 of central temperature limit is reached. With a ZrC-PuC system, the neutron spectrum is harder than with UC-Puc; the rods could be bigger, etc. 
To summarize carbides as fuel for FFTF, with sodium bonding they will run very cold at an $800^{\circ} \mathrm{F}$ outlet temperature and can be run at $1200^{\circ} \mathrm{F}$ sodium outlet temperature. This type of element can be made. In larger diameters, we have processes tons of UC, making fuel elements for HNPF and SRE. The use of sodium bond presents no fabrication and handiing problem. It appears to me that for FFTF, if emphas is is placed on maximum use of existing technology, minimum development time and funds, and freedom from plastic deformation of embrittled stainless steel cladding; sodium bonded fuels must be seriously considered. May I recommend that an overall system analysis and fuel cycle cost analysis be carried out by the FFTF project along the 1 ines now underway for the $\mathrm{PuO}_{2}{ }^{-}$ cermet and $\mathrm{UO}_{2}-\mathrm{PuO}$ element.

In the reduced time available, I will not be able to review our fast reactor cladding work in detail. We have a wel1 integrated program on cladding, which started last october, addressed to the general question of the environmental behavior of thin section cladding material in reactor quality sodium. The major part of our program will be to look at creep, stress rupture and fatigue in sodium, not under irradiation, and determine the effect of normal impurities. Tests will be run over a wide temperature range and for a long period of time. A number of carididate alloys will be procured, and tubing will be made available to other sites as needed so that a single heat of material can be studied for comparison purposes. We are working with 304,316 , Incoloy 800, and Incone 1625 . We will initiate tests later on vanadium base alloys and possibly niobium base alloys (here we get into the question of physics). We will be looking at the effect of cyclic and steady heat flux on stress rupture and creep behavior using high flux heaters that will go up to about 1,500,000 BTU/hour/ft ${ }^{2}$ per square foot. We want to tie down the specific effect of the mass transfer disturbed surface layer on stainless steels by comparing stress rupture behavior in a stagnant and flowing sodium $\Delta$ ' system. We will be doing some testing in SRE, where we have an excellent overall environment. The major thing missing is high fast neutron flux. Tests are also planned for FERMI to look at stress structure and creep after irradiation in sodium.

The embrittling effect of helium will be studied in detail to determine amount of helium, its motion, interaction with second phases, and change in mechanical properties in alloys. We will complement the current irradiation programs by the use of tritium decay to helium in situ and by cyclotron injection. This will give data on the faster, easier, and cheaper relative behavior than with reactor irradiation alone. 


\section{6}

Moreover, FFTF and FBR helium levels cannot be reached in existing fast reactors in any reasonable period of time. We will also be using a fixed constant quality sodium, developing and improving some of the analytical techniques for sodium. We are planning to interact with other sites, comparing not just analytical

techniques but also sampling and handling practices. We have hopes that we can apply a solid source mass spectrometor

to the analysis of sodium under such conditions that it will not be contaminated by the atmosphere. 
D23. 1

\section{CARBIDE FUELS AND CLADDING DEVELOPMENT AT AI}

C. E. Weber

\section{DISCUSSION}

MR. CHERNOCK: Cliff, I just want to raise a few questions with respect to the model you have thrown out for the use of the mixed carbide. Using as a basis the uranium carbide data, I would like to see if we can maybe project as to what the plutonium effects are going to be. For example, I will grant you that you can operate hypo-carbide at temperatures up to $1700^{\circ} \mathrm{F}$. It is hypo-uranium carbide. I question very, very strongly whether the behavior that you see in uranium carbide can be in any way, shape, or form applied to uranium plutonium carbide when the melting point is so low. The statement that we can straddle the fence and stay around 4.75 to 4.85 , I think, buys you two problems, one of which is quite difficult to lick. I would suggest that anything below the stoichimetric will give you the melting swelling problem in plutonium, and anything above stoichimetric is going to give you the carbon transport problem. I don't think that we can really talk in terms of degrees in stoichimetric. I think if you go below stoichimetric even to the smallest extent, you have shown this very, very vividly to have a metal swelling problem. If you are above stoichimetric to the smallest extent, as has been shown, you have the carbon transport problem. Now with respect to the latter item, there is one piece of work at AI that I am wondering why we haven't used more frequently in the literature, and why more people are not aware of it. I would like to make more people aware of it. There has been some work performed at AI on carbon transport with hyper-stoichimetric carbide, around $6.3 \%$ carbide, in which a simple heat treatment converted the di-carbide to a mono-carbide structure. The carbon transport went from 1. $4 \%$ down to . $04 \%$. I haven't seen any follow up data on this and I am wondering if you might be in a position to comment on this also.

MR. WEBER: I don't think I can really comment on this. I think the problem was that we might slow it down, but we couldn't guarantee without more work that you could eliminate transport by going to mono-carbide. Let me answer your second comment. You can by going on the hyper side if you don't go too far. You don't have to stay at 4.80 to 20 and use that as a guideline. I don't think you can go as far down on the hypo side as you can with the UC, but how far you can go down I think depends on the amount of metal there and what the metal is. I think our UC case experience shows the more free metal the worse you are. A lot of the irradiations, the best data supporting both, are around $4.65 \%$ carbon. I will quarrel a little bit, I don't know the data of the relationship so I can't quarrel too much, but I think you can go at least to lower temperatures somewhat below stoichimetric and I have not tried to claim that we have solved the problem of making a stoichimetric mixed carbons. 
D23. 2

A VOICE: In your design basis, what happens if you are going to 3 or $4 \%$ burnup and you get a transient of that temperature? Is that a catastrophic swelling failure? Second, have you been able to put PUC solid solution with your carbide?

MR. WEBER: I haven't tried it.

A VOICE: I think we have a little bit and it has been unsuccessful.

MR. WEBER: On your first question, though, I could extend the discussion by asking you what happens to cermet. But in the case of carbide, if you go fast enough you are going to start getting swelling. Start pushing sodium around inside the can and you go too far, as I have, and you will open up the can. In detailed analysis you might find it is better or worse. I think you have a $20 \%$ power transient between particles based on data that people have seen. I don't think that is the reason for dominating the cermet material.

MR. KAZI: In this same connection, I would say that one of the major reasons why our fuel designs tend to go more towards a hot fission carbide rather than the low temperature carbide is the safety policy. In a way we feel that we have to accommodate the swelling and anticipate fission gas leaks, rather than keeping it there and taking the consequences during a transient. I am wondering whether this should not be particularly important in a test reactor where maybe transients are more likely. All I want to say is that if we depart from the materials or the physics point of view, I feel that it is easier to live with fission gas leaks rather than keeping the stuff in the fuel.

MR. HECK: I would like to comment here on something you said earlier in the meeting. You remarked that one of the reasons for not choosing one fuel is that there are seven or eight possibilities and you don't know which one is best. It seems to me that what has come out in the meeting is that three or four people at least have proposed carbide within variations and each one of them would work. So, in effect, maybe this is an advantage rather than a drawback. It looks like there are three or four possibilities, any one of which would be superior to several of the other fuels.

MR. WEBER: My only comment on that is that I think we ought to look harder at it and I think we ought to look at specific fuel elements besides and not too much in general ... without looking at the fuel cladding and materials and so on. We have had a little bit of generalization at this meeting.

MR. deBOISBLANC: This brings up an extraordinarily good time to mention something. You notice a number of the specimens on which one person made his case. Also if you want to go into it a little more deeply, if you look at the generation of these failure curves in cermet, and count the number of specimens, you get another number. Now if you calculate the number of pins in this machine, at 214 per element, you get roughly 14, 000 pins for 60 elements. Now if you argue that you are going to operate this thing for a cycle with a probability of failure in the order of one part in a thousand, you recognize that you are talking about an extraordinary low failure problem built in a pin. Now from a statistical point of view, you would attack a set day like 


\section{D23. 3}

this and wonder what the problem is. If there were a failure that could prevail here in any one of these tests, wouldn ${ }^{p} t$ you have observed it in 90 or 100 tests? There isn't statistical confidence attached to this, and that factor in this irradiation testing has been given far too little concern, because it answers questions like the one you raised. The se subordinate clauses are the things that make a difference. Yes, here is a system that will work, but it takes some development on this and some development on that. All of us are in the same boat. There is not enough data on any one of these systems to guarantee that 15,000 pins in a reactor can operate without a $50 \%$ chance that in one cycle you will have one failure.

MR. WEBER: We have at least had the experience that you have the same old failure below the sodium line and your fission gas pushes the sodium out. But as it gets hotter it will contact the clad and stop. You certainly can never say it caused the cladding to fail under all conditions.

MR. deBOISBLANC: The thing of concern is not the probability of failure but the probability of non-failure.

MR. YEVICK: If you get right down to the statistics of 13, 000 elements in the reactor and you want to make sure you get less than one failure per reactor load, it is very easy to just check the fourth reactor load. Then you have the confidence, at $99.9 \%$ confidence, that you have already built the reactor four times.

MR. deBOISBLANC: Precisely. You must do roughly 60, 000 tests. 


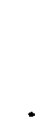


$\operatorname{SD} 24.1$

SUMMARY DISCUSSION

FFTF DRIVER FUEL MEETING

E. R. Astley (BNW)

INTRODUCTORY REMARKS

MR. HENNIG: Gentlemen, welcome back. The summary group will give us their presentations now, and $\mathrm{Mr}$. Astley will

lead off with some remarks.

MR. ASTLEY: I would like to make a few general remarks thanking all of you for your very fine presentations, for sticking to the subject, and for bringing out a lot of data very pertinent to the FFTF. It is obvious that much work has gone into the presentations, and as far as I am concerned, this has resulted in a very successful meeting.

We have chosen not to call this a panel, incidentally. It has more than just the name involved. We are not interested at this time in a small group of people standing up in front trying to make a pretense or even an effort at saying they have been able to absorb everything they have heard in the last three days and summarize it for the group. So rather than that, what we hope to do is at least have each individual, in his own field, briefly summarize what he considered to be important and worth retaining.

Upon completion of the summary presentations I hope we can then bring the audience into this session as an audience panel discussion to the extent that time permits.

Now I must confess that I anticipated that we would all sit here for three days and then take 15 minutes to summarize al1 of our thoughts. It is a bit of an imposition on this group, so you will have to bear with that. I persuaded Al Amorosi to attempt to guess what was going to be said in the three days and to come prepared to put a few graphs and curves up which he thought might pertain to the meeting, and then to use the presentations of the past three days to organize these data into a 30 minute summary as he saw it. I think this is going to be worthwhile because Al has had the advantage over many of you in that for over a year he has been assisting us, and looking at many different things. Therefore, he has quite a bit of background that some of the rest of you do not have. Now I restricted A1 to 30 minutes, because we do have to keep on schedule and because some of you are taking an early plane. 
First, if I may I would like to take just a very few minutes and make a very general observation concerning the meeting. Being facetious, I think that as I understood what was said, each of the groups that are now involved and working hard on a particular fuel feel this fuel should receive consideration as FFTF driver fuel. I think we can say then that the group decided that cermets, mixed oxides, carbides, and metal alloys, as well as the molten plutonium fuels should be considered in the selection of driver fuel.

At some time or other during the meeting I believe I heard these following factors brought out that are important considering the over-all choice. These are the fabrication cost of the core, the reprocessing costs, the cost of developing the fuel, and the application of your fuel choice to the LMFBR program. These are to be considered from the standpoint of dollars invested, as well as the technical manpower tied up, and of course the know-how that could be transferred to this program. In other words, what is the state of the art of the fuel, and this depends on when you might get the FFTF on line, and its availability there after. The special nuclear safety requirements of the FFTF driver fuel choice is a significant consideration as we 11 as the influence that driver fuel choice has on capital cost of the FFTF.

I think at one point or another all of these items were brought out by the various speakers at the meeting, and they are all very pertinent. I just want to assure you that one of the requirements in writing that the Commission has placed upon Battelle-Northwest is that we evaluate all these factors and make recommendations to them in a visible manner on paper.

At this particular time our group has been concentrating, however, on two fuels, the cermets and the mixed oxides. I believe after this meeting I am convinced that we need to expand our scope in this area. I think we can summarize the fact that the goals of the FFTF are a high degree of safety, and a high degree of reliability. These two conditions mean, in my opinion, that we need a series of operations of the first and second driver core. We should not forget this, in light of some of the recommendations that were made. The full range of operating conditions that we hope to achieve should come in the following cores as the fuels are proved. I believe you can make a generalization that all fast fuels are expensive at this particular time, probably in the range of three to five thousand dollars per kilogram. We are talking of perhaps around $600 \mathrm{kilo-}$ grams a year, and thus a cost of one to three million dollars a year in the optimized system that is finally chosen.

The factors that I have mentioned have to be considered on the whole; costs are not the only factor. Many of the other factors were brought up during the course of this meeting. 
$\operatorname{SD} 25.1$

SUMMARY DISCUSSION

R. Avery (ANL)

REMARKS

MR. HENNIG: I will next ask Bob Avery to speak.

MR. AVERY: Not too much was really said about physics during the meeting. I think it is fair to say that there are no basic, major uncertainities on physics. I would like to make it understood that I am not implying that physics considerations are not important, but I don't believe there are any major uncertainities. I believe we understand quite well what the effects of flux, power, power densities, size (etc.) are. Also, in the detailed designs, there will be certain details surely, dealing with control and details of loop designs that (perhaps) might be quite sticky problems and will require detailed physics studies. But I do not believe these are major uncertainties that would have a definite bearing on the ultimate choice of system. Perhaps, the only excention (and I do not reallv feel that) might relate to the Dopplereffect. But I don't think even the uncertainty of that number would effect an ultimate choice of system.

I will not repeat any of our major considerations on physics. I think what was said generally is agreed to, and I think, probably well understood.

In the area of safety, this obviously is a much more difficult question. Perhaps to clarify the thinking, certainly in my own mind and perhaps in others, I think one wants to consider different aspects to the safety. There is one where we are given the change in the physical state of the system, how the fue 1 is moved, how the temperature is changed, what is the associated change in reactivity; and this is one of the areas, perhaps, that physicists are more concerned with. Here again, I do not believe that there are any major uncertainties. I think you will be able to calculate, and, if not, make critical measurements on what these changes would be. Given the change in reactivity, one can then calculate power histories by rather straightforward means. As the means are straightforward, one has to look at detailed questions like the value of the dollar, and the delayed neutron fraction that would enter into it. There are some types of accidents that would be crucial items; there are others where it would be not so important.

So, going from the physical state to the reactivity and from the reactivity to the power histories is straightforward. The main item is what this means in terms of state:how does the stuff 
move, how does the temperature change. I think it is obvious that there are many questions and much work to be done. Clearly this is the function of all the previous history of the system (how it was built). Much was said here, and I think that we are all aware that much more has been done on all the systems in this area. It is, perhaps, a falr statement that probably there is less doubt in the area of the cermet than there is in the others, but certainly the cermet has many troubles itself. I believe, also, it is fairly clear that there was much uncertainty, when we were talking about safety, as to what the design criteria would have to be, particularly with the test loops. Here, I am talking not about general principles of design criteria for all fast reactors, which I think would be great, but rather of design criteria that the FFTF people will have to decide on.

For example, there are very specific decisions: one could say that the melt down reactivity associated with loops would be less than a dollar; or one could be limited to less than the amount of integrated, inherent reactivity (he will have) until some failure point. There are, obviously, many such decisions that one has to make on this. More often than not, they are specifically spelled out very early in the game.

I would like to take a few minutes on what the major advantages and disadvantages of the various possible fuels are as I heard them; obviously there is a fair amount of subjectivity in such considerations. With the cermet, I think one could say the main advantages are the high flux. This is high flux per unit power density. Probably, it has a good power density in terms of the other systems. It probably has a reliable expansion coefficient as an inherent feedback coefficient even after burn up, but others will be more expert in that area than $I$.

The disadvantages, (as I see them) and at the moment I am talking about the plutomium fuel, is the small delayed neutron fraction. The fact that there is no shut down mechanism after the melting has occurred, and that its inherent only to that point, I considered quite an important point.

Next, I will go on to the ceramic oxides and carbide. I think their advantages are that there is an inherent negative feedback in the Doppler effects, It may be even greater up to the point than, let's say, the cermet if one artificial degrades the spectrum; but even without it, one has a value, and as a further advantage, this is a shut-down mechanism which would continue even after the melting point. The main disadvantages would be the effect of a lower power density for the oxide; but, perhaps not so for the carbide. The expansion is probably unreliable; in the case of both oxlde and carbide, it gives a lower value for the flux as a function of power density. There is also the question of possible movement of fuel, which I must say again, is a subjective reaction. Perhaps, it was somewhat over emphasized during the meeting. Again, I think one wants to be clear whether one means in terms of lack of fixing precisely 
$\operatorname{SD} 25.3$

where the fuel is, whether one is worried about getting into trouble, or whether it is something which would not get one out of trouble through expansion. I think that getting one into trouble through movement of the fuel (somehow or another) may be not a real problem.

The main advantages of metal fuel elements are again relatively high power densities. It suffers from the lack of having an inherent negative feedback coefficient. I believe that in the present context, it could only get an adequate result if we artificially moderated it and if it would have a very good negative Doppler effect. Its expansion is unreliable if one wants to have very high burnups. Also, it has other disadvantages in the low flux values.

Let us proceed to two other alternates, $\mathrm{U}^{235}$ versus plutonium. I think the advantage with uranium, obviously, is a high beta value, which is a quite important value. There may be other straight economic values. The main disadvantage, of course, is the reduced flux.

The berillium oxides are moderating materials. The main advantage is that BeO enhances the Doppler effect and probably fully makes up for whatever we do to the power density (which it leads to) by giving a higher reaction rate. To me, it seems like a desirable thing to seriously consider. I have no knowledge or opinion in terms of the metallurgical or the material aspects of it.

Generally, my conclusion, then, is in terms of required $R$ \& $D$, insofar as the things that I have been talking about. But of course, it seems to me the main question relates to determining how things move and what is the physical state of the system as various power histories occur. 


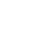

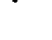


$\operatorname{SD} 26.1$

SUMMARY DISCUSSION

A. Amorosi (APDA)

\section{REMARKS}

It is always a difficult task to make a choice on the design of an advanced system; the Fast Flux Text Facility is no exception. To assist BNW in making this decision, the author will discuss general requirements in the following:

Nuclear Characteristics

Thermal Performance of Fuel

Economics

Safety

For each of the above-mentioned areas, the general effect of various factors in the design will first be reviewed, and then the properties, which specific fuels have, will be indicated. Lastly, the performance of the major fuels being considered will be summarized.

There are, of course, a great many factors that must be considered before the detailed design of a particular fuel element is frozen. However, in order to attain a perspective view, only a broad-brush treatment will be given. To concentrate on large first order effects, many important factors will not be covered that were covered during the meeting. Such factors are as follows:

- Effect of power rating on overall costs. An arbitrary figure of $\$ 80 / \mathrm{kW}$ is used for the incremental cost of the plant.

- Structural materials will not be covered, even though these are important in the design of the reactor.

- Reactivity adjustment is not considered to be a large first order effect and, therefore, will not be covered.

- The maximum accident is certainly important, but the fact that the major fuel systems being considered for FFTF would appear to be acceptable in this regard, for Fermi is evidence that this is not a major issue.

In addition, certain assumptions have been made to minimize the variables which have to be taken into account. These are as follows:

- Driver core characteristics will dominate for malfunctions which do not arise from the test fuel. It may be necessary to do such things as 
(a) Pressure the closed loops to prevent transient boiling

(b) Insulate the closed loops to avoid problems associated with high temperature rise in test loops

(c) Limit power density (kW/liter) in lattice position test pins to be comparable to the driver core.

- Little penalty is imposed by operating the firver core at about 600 to $900^{\circ} \mathrm{F}$, since insulated closed loops can be operated at their own conditions, and some flexibility is possible to operate open loops to $1100^{\circ} \mathrm{F}$.

Many requirements have to be met by the friver fuel. Some of the key assumptions made for this presentation are as follows :

- The cross sectional area assumed devoted to loops is $1 \mathrm{ft}^{2}$ evenly distributed across the core. This is equal to three 6-in. holes can be provided in the blanket area.

- It is assumed that two core types should be investigated. The first is the type which will be used for, say, the first three loads of FFTF. This core can run to a lower flux, say, $0.5 \times 1016$, and has as its main objective reliability and minimum development cost. The second type of core is really the reference driver, which should be able to give a peak flux of at least 1016 , and preferably $1.35 \times 10^{16}$. The facilities for building the first core type should be adaptable for making the reference cores if transition time and costs are significant.

From a nuclear standpoint, the major interest is the effect of fuel on power and size requirements. This and a number of other nuclear questions were discussed in considerable depth by Messrs. Hofmann, Heck, Avery and Cohn. The relationship between power, fuel and bolume for a fixed flux is given in Slide 1. The liquid-metal-fueled concept of LASL is i included. Although this reactor has plutonium fuel the critical mass requirements correspond with those of $U^{2} 35$ because of the high cross section of the tantalum clad presently required to contain molten plutonium fuel. Examination of Slide 1 indicates that the preferred fissile material is plutonium. Fuels other than $\mathrm{U}^{235}$ and plutonium have been reviewed, and their properties are given in Table IV, but there is no overwhelming reason to oyerride the fact that the major choice is between $\mathrm{U}^{235}$ and $\mathrm{Pu}^{239}$. There is a distinct edge in favor of $\mathrm{Pu}^{239}$, aside from a safety consideration to be discussed later. 


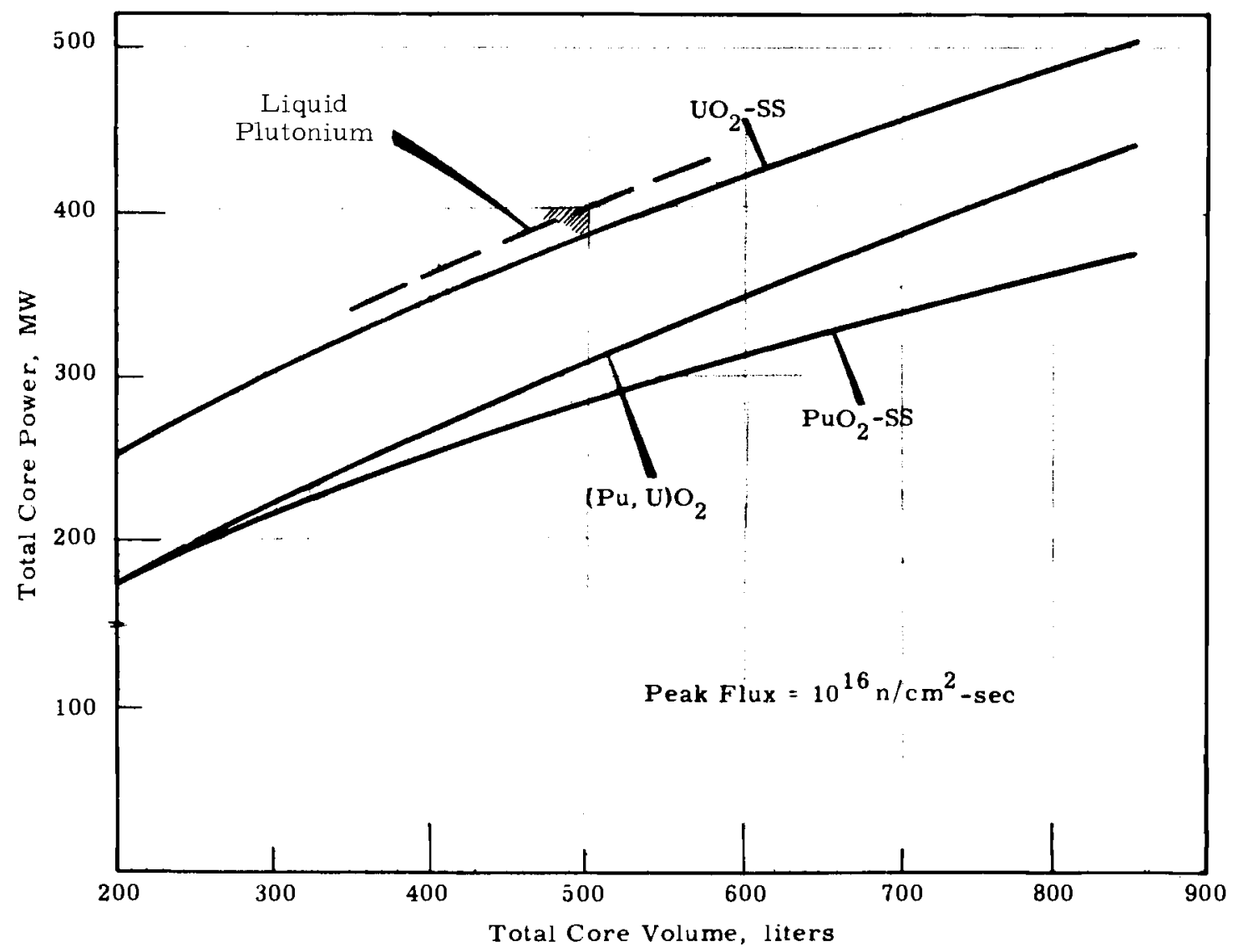

SLIDE 1. Effect of Fue1 Type on Total Reactor Power as a Function of Core Volume (From APDA Report MD65-625)

A major problem for high flux, minimum power cores is to transfer heat to the coolant; the specific power, which can be achieved being related to conductivity, and the $\Delta T$ which can be taken through the fuel. In comparing fuels on the thermal performance basis, the $\int k d \theta$ is commonly used. Slide 2 shows the core power which can be achieved for different $\int k d \Theta$ for pin sizes $3 / 16$ and $1 / 4$ in. 


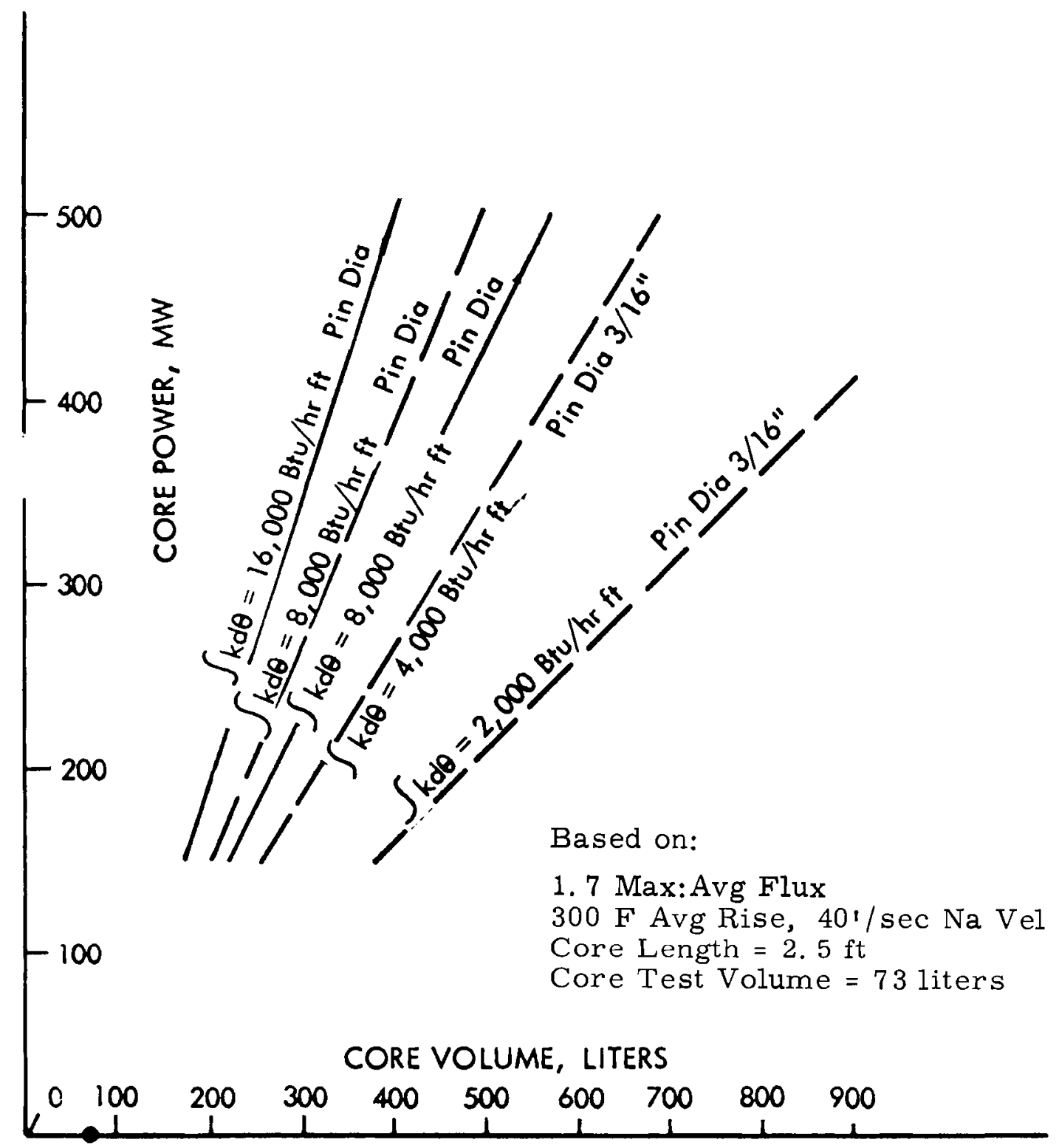

SLIDE 2. Effect of Fuel Specific Power, ${ }^{\mathrm{I}} \mathrm{kd} \theta$, Achievable on Core Power

If the heat transfer performance and the nuclear data are superimposed, the core power can be obtained for any ${ }^{\prime} \mathrm{kd} \Theta$ for the fuels of interest. These are given in Slides 3, 4, and 5. They cover two pin sizes, $3 / 16$ and $1 / 4$ in., and two fluxes of interest, $10^{16}$ and $1.5 \times 10^{16}$.

The performance of the LASL molten plutonium fuel has been added to Slide 4. This performance shows that if the pin diameter is double and the heat flux quadrupled, the design point remains unchanged. 


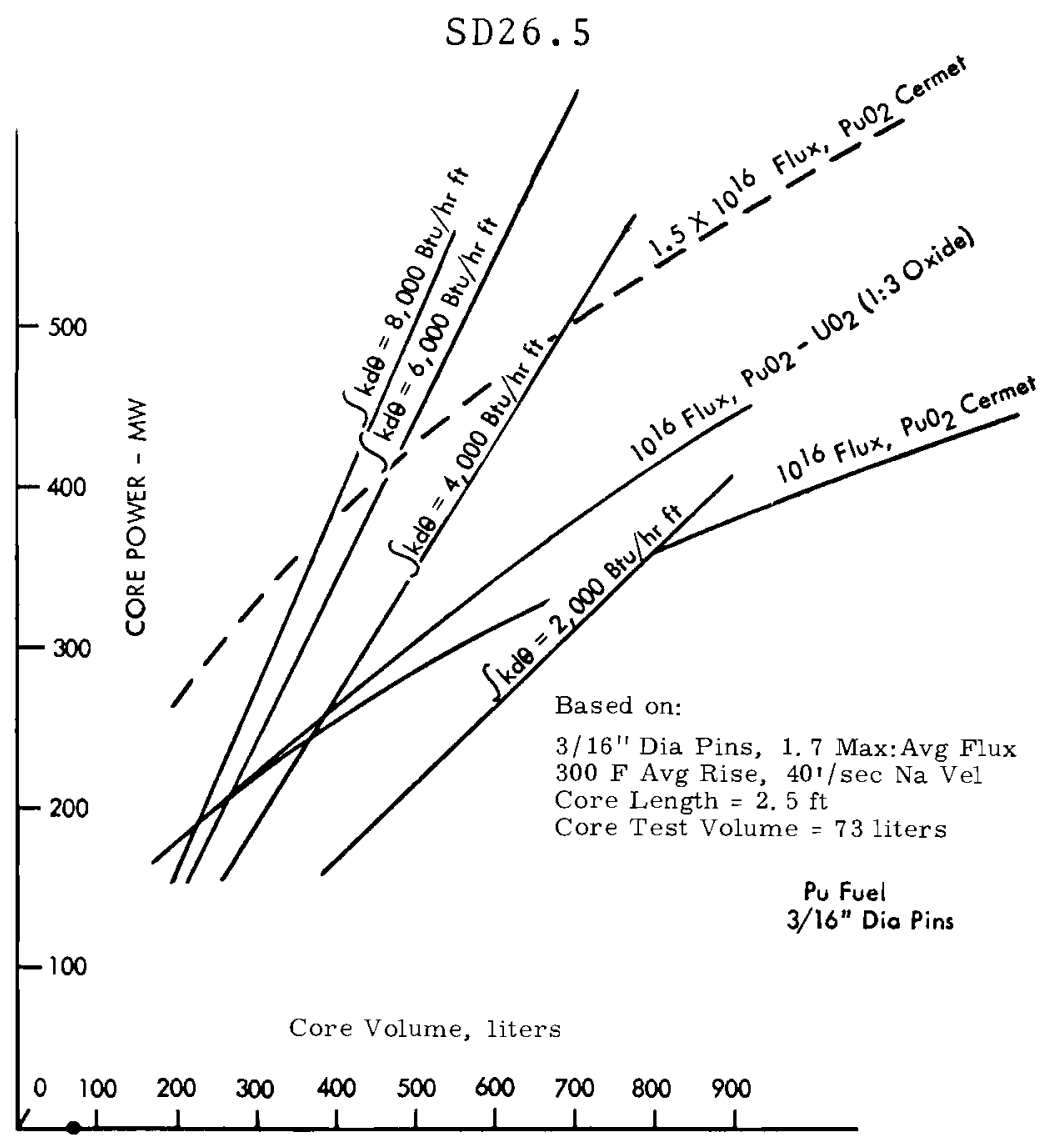

SLIDE 3. Effect of Fuel Specific Power, ${ }^{j} \mathrm{kd} \theta$, on Core Power Required ( $1.5 \times 10^{16}$ Flux)

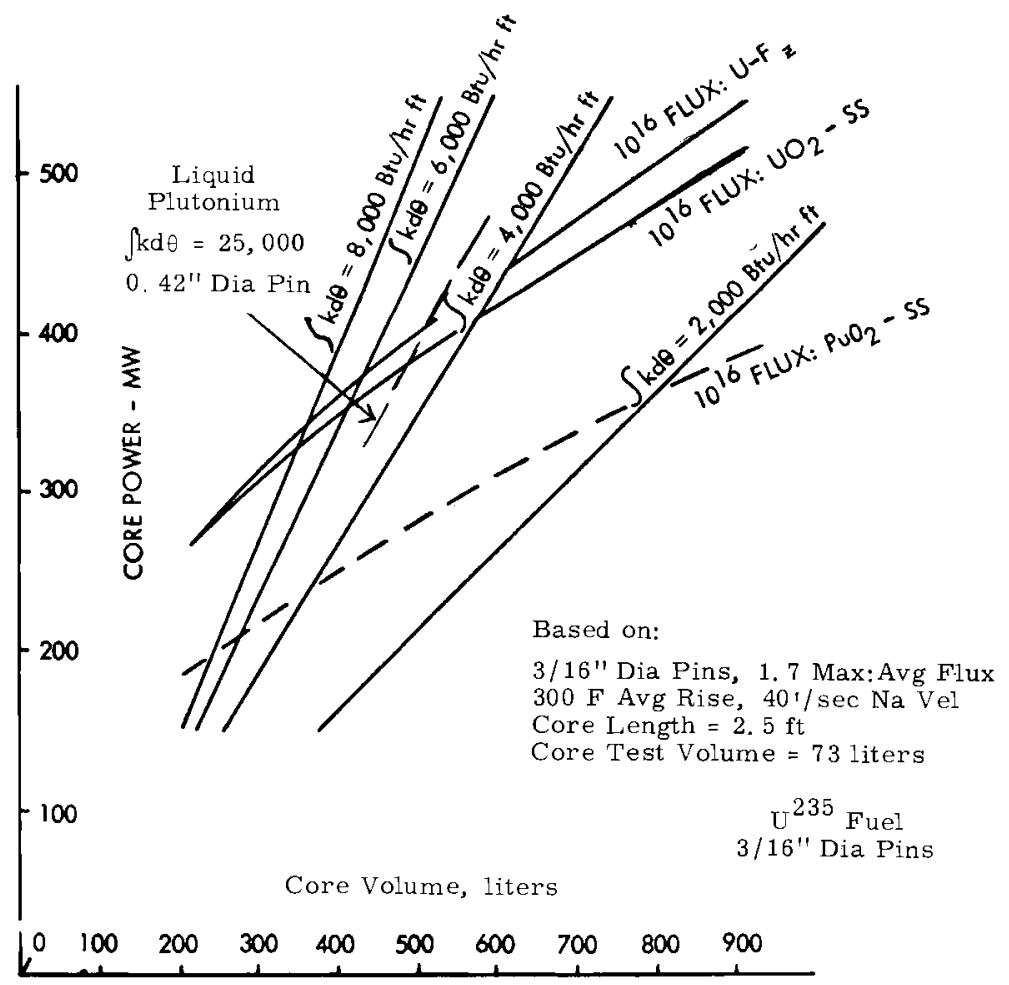

SLIDE 4. Effect of Fuel Specific Power, ${ }^{j} k d \theta$, on Core Power Required (1016 Flux) 


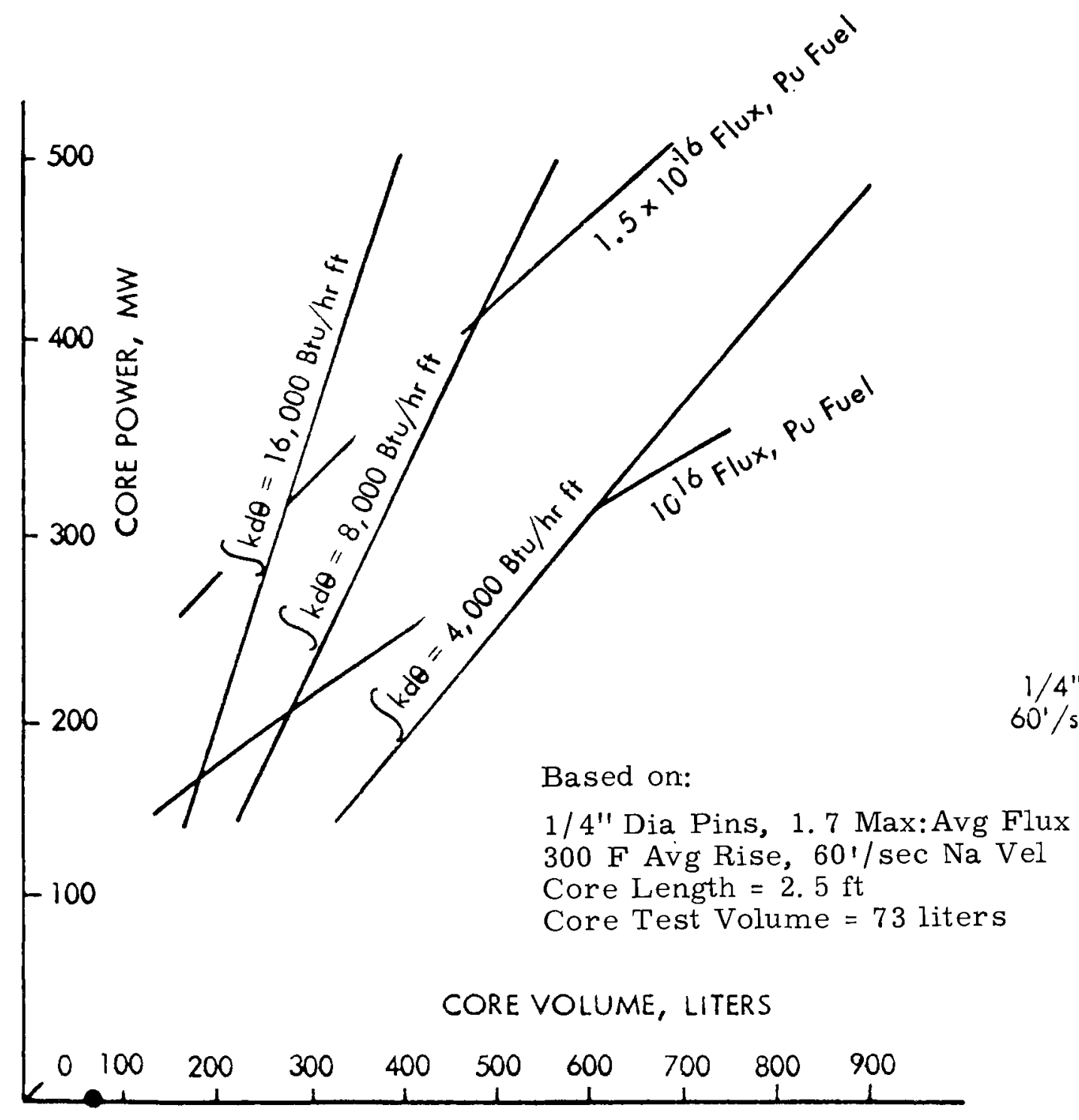

SLIDE 5. Effect of Fuel Specific Power, skde, on Core Power Required ( $1 / 4$ in. Dia Pins)

As a check on the general validity of the information presented, Table II of APDA document MD-65-625 is included as Table I. For those studies, the allowable heat flux was taken as $1.1 \mathrm{~kW} / \mathrm{in}$. for the cermet, 1 for the oxide, and 1.2 for the metalic fuel. This resulted in small pin sizes indicated in Table I. 
$\operatorname{SD} 26.7$

TABLE I. Results of Fue1 Study

\begin{tabular}{|c|c|c|c|c|c|c|c|c|}
\hline & \multicolumn{4}{|c|}{ Sma11 Loops } & \multicolumn{4}{|c|}{ Large Loops } \\
\hline & \multirow{2}{*}{$\begin{array}{l}\text { Cermet } \\
\mathrm{PuO}_{2}-\mathrm{SS} \\
\end{array}$} & \multicolumn{2}{|l|}{ Oxide } & \multirow{2}{*}{$\frac{\text { Metal }}{\mathrm{U}-\mathrm{Fz}}$} & \multirow{2}{*}{$\begin{array}{l}\text { Cermet } \\
\frac{\mathrm{PuO}_{2}-\mathrm{SS}}{}\end{array}$} & \multicolumn{2}{|l|}{ Oxide } & \multirow{2}{*}{$\frac{\text { Metal }}{U-F z}$} \\
\hline & & $\begin{array}{ll}\mathrm{Hi} & \mathrm{PuO}_{2} \\
\end{array}$ & $\overline{3: 1}$ & & & $\begin{array}{ll}\mathrm{Hi} & \mathrm{PuO}_{2} \\
\end{array}$ & $3: 1$ & \\
\hline Core Volume, Liters & 332 & 246 & 650 & 310 & 478 & 372 & 905 & 425 \\
\hline Core Diameter, Inches & $27^{\prime \prime}$ & $23^{\prime \prime}$ & $37.5^{\prime \prime}$ & $26^{\prime \prime}$ & $32^{\prime \prime}$ & $28.4^{\prime \prime}$ & $44^{\prime \prime}$ & $30^{\prime \prime}$ \\
\hline Critical Mass, kg & 248 & 239 & 427 & 413 & 310 & 281 & 543 & 491 \\
\hline Power, Mw & 229 & 202 & 348 & 309 & 273 & 258 & 438 & 361 \\
\hline Pin Size, oD, Inches & .170 & .125 & .197 & .125 & .171 & .125 & .197 & .125 \\
\hline$\rho \mathrm{kd} \theta, \mathrm{Btu} / \mathrm{hr}-\mathrm{ft}$ & & & & & 3,700 & 3,300 &, 300 & 4,000 \\
\hline
\end{tabular}

It should be noted that the larger the ${ }^{\mathrm{J}} \mathrm{kd} \theta$ (which can be used as a design), the smaller the power needs to be for a given performance. The ultimate reference driver fuel has a high value of $\mathrm{J} k d \theta$. Incidentally, this also has a strong implication for central station plants in that it means a small core size can be chosen with a better corresponding sodium void coefficient, with larger pins (which are easier to make), and with a larger pitch simplifying the fuel support structure. The smaller core, of course, also simplifies the reactor vessel design; particularly, as the power rating of the plant increases.

Table II lists the heat transfer properties of various representative fuels. The table gives the approximate melting point, the design maximum fuel temperature, and the average conductivity as well as the ${ }^{\top} \mathrm{kd} \theta$ to melting for the various fuel systems. It also gives a potential design ' $k d \theta$. This design is based upon the assumption that the sodium temperature at the point of maximum fuel temperature is $1000^{\circ} \mathrm{F}$. It assumes arbitrarily $1000^{\circ} \mathrm{F} \Delta \mathrm{T}$ across the gap of a gas-bonded pin. The maximum temperature for which the different fuels can be normally operated varies. It is common practice with an oxide system to allow this temperature to get close to the melting point. As a result, a slight degree of central void formation is accepted. Central hole formation can take place at sintering tmeperatures of 3000 to $3500{ }^{\circ} \mathrm{F}$ while the melting point is $-5000^{\circ} \mathrm{F}$. 
SD26.8

TABLE II. Heat Transfer Properties of Fuels

\begin{tabular}{|c|c|c|c|c|c|c|c|}
\hline Fuel Systems & $\begin{array}{l}\text { Melting } \\
\text { Temp, F }\end{array}$ & $\begin{array}{l}\text { Design } \\
\text { Max. Fue 1 } \\
\text { Temp, F } \\
\end{array}$ & 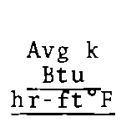 & $\begin{array}{l}\quad \int k d \theta \\
\text { Btu/hr-ft } \\
\text { To Melting } \\
\end{array}$ & $\begin{array}{l}\int k \sin \theta \\
\text { Watts/cm } \\
\text { To Melting } \\
\end{array}$ & \multicolumn{2}{|c|}{$\begin{array}{l}\text { Potential } \\
\text { Design } \\
\quad / \mathrm{kd \theta} \\
\text { Watts/cm } \\
\end{array}$} \\
\hline$\frac{\text { Metal Alloys }}{\text { U-Fs }}$ & 2100 & 1700 & 18 & 18,000 & 170 & $\begin{array}{r}110 \\
60\end{array}$ & $\begin{array}{l}\text { (potential) } \\
\text { (present } \\
\text { practice) }\end{array}$ \\
\hline $\begin{array}{l}\text { U-Mo } \\
\text { U-Th }\end{array}$ & $\begin{array}{l}2100 \\
2000\end{array}$ & $\begin{array}{l}1700 \\
1600\end{array}$ & $\begin{array}{l}15 \\
25\end{array}$ & $\begin{array}{l}15,000 \\
22,500\end{array}$ & $\begin{array}{l}145 \\
215\end{array}$ & & \\
\hline $\begin{array}{l}\mathrm{U}-\mathrm{Pu}-\mathrm{Zr} \\
\text { Molten } \mathrm{Pu}\end{array}$ & 2100 & 1700 & 11 & $-11,000$ & 106 & $\begin{array}{r}63 \\
240\end{array}$ & \\
\hline$\frac{\text { Dispersions }}{\mathrm{UO}_{2}-\mathrm{SS}}$ & 2600 & 2000 & 8 & 12,000 & 115 & $\begin{array}{l}70 \\
40\end{array}$ & $\begin{array}{l}\text { (potential) } \\
\text { (present } \\
\text { practice) }\end{array}$ \\
\hline $\begin{array}{l}\mathrm{UO}_{2}-\mathrm{Ni} \\
\mathrm{UC}-\mathrm{Graphite} \\
\mathrm{PuN}-\mathrm{Mo} \\
\mathrm{PuO}_{2}-\mathrm{SS}\end{array}$ & $\begin{array}{r}-4100 \\
2600\end{array}$ & $\begin{array}{l}3500 \\
2000\end{array}$ & $\begin{array}{r}59 \\
8\end{array}$ & $\begin{array}{r}180,000 \\
12,000\end{array}$ & $\begin{array}{r}1700 \\
115\end{array}$ & 70 & \\
\hline $\mathrm{PuO}_{2}-\mathrm{W}$ & 4100 & 3500 & 80 & 240,000 & 2300 & & \\
\hline $\begin{array}{cl}\text { Ceramic Compounds } \\
\mathrm{UO}_{2} & \mathrm{p}=0.7 \mathrm{TD} \\
& \mathrm{p}=0.8 \mathrm{TD} \\
& \mathrm{p}=0.9 \mathrm{TD} \\
\mathrm{PuO}_{2} & \mathrm{p}=0.9 \mathrm{TD}\end{array}$ & $\begin{array}{l}5000 \\
5000 \\
5000 \\
4100\end{array}$ & $\begin{array}{l}4400 \\
4400 \\
4400 \\
3500\end{array}$ & & $\begin{array}{l}2100 \\
2600 \\
3700\end{array}$ & $\begin{array}{l}20 \\
25 \\
35\end{array}$ & & \\
\hline $\begin{array}{l}25 \% \mathrm{PuO}_{2}, 75 \% \mathrm{UO}_{2} ; \mathrm{p}=0.9 \\
25 \% \mathrm{PuO}_{2}, 60 \% \mathrm{UO}_{2}, 15 \% \text { Mo } \\
\mathrm{p}=0.9\end{array}$ & $\begin{array}{l}4800 \\
4700\end{array}$ & $\begin{array}{l}4200 \\
4100\end{array}$ & $\begin{array}{l}-1.2 \\
-3.0\end{array}$ & $\begin{array}{l}3400 \\
8100\end{array}$ & $\begin{array}{l}33 \\
78\end{array}$ & 33 & $\begin{array}{l}\text { (present } \\
\text { practice) }\end{array}$ \\
\hline $\begin{array}{l}\text { UC } \\
\text { PuC-UC }\end{array}$ & $\begin{array}{r}4700 \\
-4500\end{array}$ & $\begin{array}{l}3800 \\
1700\end{array}$ & $\begin{array}{r}7 \\
-7\end{array}$ & $\begin{array}{l}25,000 \\
24,000\end{array}$ & $\begin{array}{l}240 \\
230\end{array}$ & $\begin{array}{r}-190 \\
35\end{array}$ & $\begin{array}{l}\text { (potential) } \\
\text { (present } \\
\text { practice) }\end{array}$ \\
\hline $\begin{array}{l}1102^{-00} 2 \\
\text { PuN-UN }\end{array}$ & $\begin{array}{l}5800 \\
5140\end{array}$ & & & .6000 & 58 & & \\
\hline
\end{tabular}

Notes:

1. $\quad s k d \theta$ to melting is based on:

a. For metal, dispersion and carbide fuels, the $\Delta \mathrm{T}=$ melting temperature $-1100^{\circ} \mathrm{F}$, where sodium temperature is assumed to be $900^{\circ} \mathrm{F}$ and $200^{\circ} \mathrm{F}$ is taken for sodium, clad and bond $\Delta T$ ' 's.

b. For ceramic compounds which are not sodium-bonded, the $\Delta \mathrm{T}=$ melting temperature $-2000{ }^{\circ} \mathrm{F}$, where the sodium temperature is assumed to be $1000^{\circ} \mathrm{F}$ and $1000{ }^{\circ} \mathrm{F}$ is taken for sodium, $c l a d$ and bond $\Delta T^{\prime} s$.

2. Potential design is based on design maximum temperatures of:

a. $400{ }^{\circ} \mathrm{F}$ less than melting point for metal fue1s

c. Conductivity of the irradiated oxide and carbide as $2 / 3$ of unirradiated values.

3. Present equitable design practice is based on:

a. $1700{ }^{\circ} \mathrm{F}$ maximum temperature per weber.

b. Conductivity of .02 Watts/cm per GE presentation.

c. Design $\Delta \mathrm{T}$ for metal system of $600^{\circ} \mathrm{F}$ per discussions with ANL.

Based upon today's technology and the data presented at this meeting, it would appear that a reasonable ${ }^{J} \mathrm{kd} \theta$ of an oxide pin is $33 \mathrm{~W} / \mathrm{cm}$. It is noted that where reduced density oxide fue 1 is taken, the design ${ }^{3} \mathrm{kd} \theta$ must be lower, perhaps as low as 15 . Conversely, if one were to try to design to achieve a high value, it is conceivable that a fuel element with perhaps molybdenum added to increase its conductivity could be used. For such a system, it would appear probably to be able to design for a ${ }^{5} \mathrm{k} \theta$ 
as high as 65 to $70 \mathrm{~W} / \mathrm{cm}$. These, of course, are not high specific powers for a reactor where high power density is important. The PuC-UC ceramic fuel is attractive because of the higher conductivity of this fuel. It is some six times higher than the mixed oxide if the irradiation effects on conductivity are ignored.

We have been presented with problems of the design of the carbide system; on one hand with carburization problems if hyperstoichiometric form is used, and adverse swelling if the hypostoichiometric form is used. Weber pointed out that for a conservative design, he would recommend that the hypostoichiometric form be used, and that the design temperature be 1 imited to approximately $1700^{\circ} \mathrm{F}$. This would give a ${ }^{\prime} k d \theta$ of about 35 , assuming $2 / 3$ of nonirradiated conductivity properties. There may be other ceramic compounds which could give a high thermal performance, and it may readily be that the carbide itself could be operated we 11 above $1800^{\circ} \mathrm{F}$ if it were proved that the canning material is strong enough to restrain growth, or if an alloying additive could improve its properties.

It can be seen from the table that metal alloys have a better conductivity and (by and large) can be run at ' $k d \theta^{\prime} s$ in the range of $100 \mathrm{~W} / \mathrm{cm}$. In order to achieve this type of heat transfer, it may be necessary to accept some cracking of the fuel. This would seem to be acceptable if sodiumbonded elements are used. It would appear that the first core for FFTF could be operated with a metallic fuel at a power of about $60 \mathrm{~W} / \mathrm{cm}$ and that for the reference design it should be possible to run at $100 \mathrm{~W} / \mathrm{cm}$. For the dispersion system, a limit of about $400^{\circ} \mathrm{F} \Delta \mathrm{T}$ has been chosen where the clad is metallurgically-bonded to the fuel because it is felt that a combination of thermal stress and growth might crack the clad at a higher temperature difference. It would appear acceptable to design a cermet with the sodium bond and then to allow a larger $\Delta \mathrm{T}$ through the fuel. Also, it would appear acceptable to design a stainless steel cermet for $80 \mathrm{~W} / \mathrm{cm}$, whether it be a $\mathrm{UO}_{2}$ or $\mathrm{PuO}_{2}$ dispersion.

It is rather interesting to look for fuel systems giving a very large ${ }^{J} \mathrm{kd} \theta$ as the ultimate driver core. There are, of course, several materials with high conductivity. Dispersions could be made with nickel, molybdenum or tungsten as the matrix material; indeed, some rather astounding thermal performance could be envisioned.

At this time it would appear that quite a penalty must be paid if a gas gap is accepted between the fuel and the clad because of the poor conductivity of this bond. There also may be a large penalty paid if a metallurgical bond is used because a large $\Delta \mathrm{T}$ cannot be accepted without rupturing the clad. This could result in pin warpage and blockage of the coolant passage. A sodium-bonded fuel seems to be preferred. One potential 
$\operatorname{SD} 26.10$

problem with a sodium-bonded element is that as the fuel swells, it will touch the clad and any fission gases which are released below the contact ring may cause a gas pocket which could result in melting of the fuel.

It is recommended that the fuel element be vented and that the venting be radial. Very little porosity need be provided because the hole spacing equal to approximately $1 / 4$ diameter of the pin would seem to be acceptable, and the hole size could be several mils in diameter. The purpose of the clad is, therefore, to restrain growth and not to contain fission products. If one were to feel uncomfortable about a large release of fission products to the system, coated particles could be used with cermet fuel. Very little attention had been given to this type of element, and the procedure to achieve the sodium bond must be given careful consideration.

Quite a bit of information has been presented in regard to the relative cost of fabrication of different types of fuel. On one fuel system, Fermi Core $A$, we have a fairly accurate cost of approximately $\$ 100 / \mathrm{pin}$, or about $\$ 2,000,000 /$ core. These pins are U-10 Mo metallurgically bonded to a zirconium clad. If such a pin could give $2 \%$ burnup, then the total fuel cycle cost per year would be of the order of $\$ 6,000,000$.

As presented in the meeting, it is expected that EBR-II fue 1 elements should give $2 \%$ burnup. Since these fuel elements should give $2 \%$ burnup. Since these fuel elements are considerably cheaper to fabricate than Fermi, it could be expected that such a fuel element would have an operating cost of the order of $\$ 4,500,000 / y e a r$. The plutonium oxide cermet pin is expected to give a burnup of somewhat less than the EBR-II element (as indicated in Table II), and its operating cost could be expected to be somewhat higher--perhaps $\$ 6,000,000$. Its lower megawatt rating minimizes the discrepancy in the cost. An oxide core because of its much lower ${ }^{\prime} k d \theta$ requires many more pins than the other fuel systems, and its annual operating cost is expected to be slightly above $\$ 6,000,000$. This data is presented in Table III.

It is concluded that there is not a great deferential in the operating cost between the various fuel systems being considered for the first core. The difference between the various fuel systems is expected to be much smaller than the accuracy of the absolute numbers. It would, therefore, appear that the choice of the first core should be primarily related to its performance reliability and its capability of developing into a more promising system. 


\section{TABLE III.}

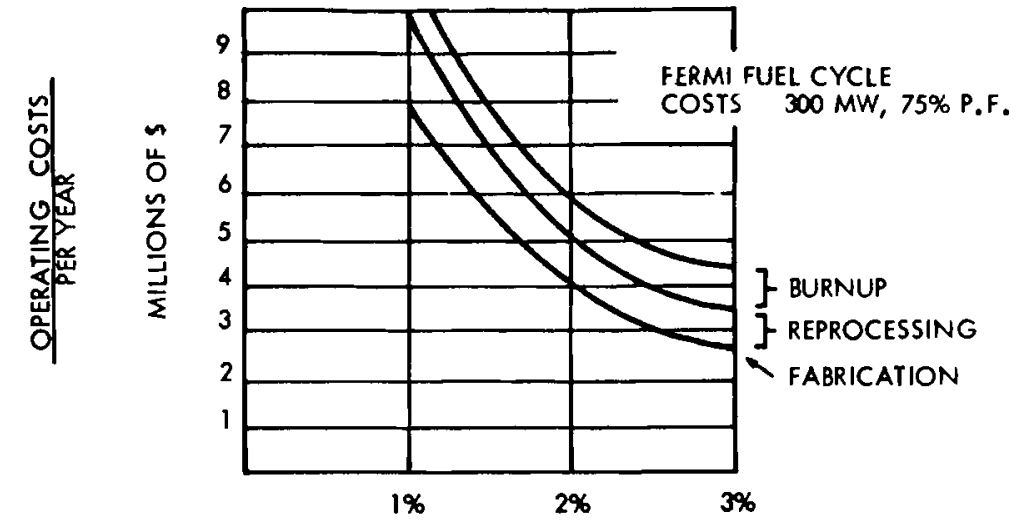

AVG BURN-UP

ESTIMATED RELATIVE FUEL COSTS*

\begin{tabular}{|l|c|c|c|c|c|c|c|}
\hline \multicolumn{1}{|c|}{ FUEL } & $\int \mathrm{kd \theta}$ & $\mathrm{MW}$ & $\begin{array}{l}\text { TOTAL } \\
\text { NUMBER } \\
\text { OF PINS }\end{array}$ & $\begin{array}{l}\text { FAB } \\
\text { Cost } \\
\text { Per } \\
\text { Pin }\end{array}$ & $\begin{array}{l}\text { EST } \\
\text { Cost } \\
\text { Per } \\
\text { Core }\end{array}$ & $\begin{array}{c}\text { Bumup } \\
\text { Fissions } \\
\text { cc meat }\end{array}$ & $\begin{array}{c}\text { Approx } \\
\text { Cores } \\
\text { Per } \\
\text { Year }\end{array}$ \\
\hline $\begin{array}{l}\text { FERMI (.156" Dia) } \\
\mathrm{U}^{235}\end{array}$ & 6,000 & 375 & 11,600 & $\$ 100$ & $\$ 1.2 \times 10^{6}$ & $2 \%=10^{21}$ & $\begin{array}{l}\text { at } 2 \% \\
=2 \text { cores } \\
2.1\end{array}$ \\
$\begin{array}{l}\mathrm{Pu}^{239} \\
\text { (like cermet + Na bonded) } \\
\begin{array}{l}\text { Pu239 } \\
\text { (like oxide) }\end{array}\end{array}$ & 6,000 & 210 & 6,500 & $\$ 200$ & $\$ 1.3 \times 10^{6}$ & $\sim 0.7 \times 10^{21}$ & 2.0 \\
\hline
\end{tabular}

- All pins 3/16" Dio

A great deal had been said about safety requirements and, indeed, a great deal of work will be required before a hazards report is developed. There are, however, two major areas of safety that have an impact on the choice of fuel. These are the delayed neutron fraction and the total $\Delta K$ from operation to boiling of sodium. The delayed neutron fraction is the major index to the number of test pins which can be safely melted in a transient short of damage to the driver core. The second is related to the capability of the driver core to accommodate a loss of power accident without sodium boiling when the safety system fails to scram (This was discussed by Nims). The characteristics of the various fuels from the standpoint of safety are given in Table IV. 
TABLE IV. Nuclear and Safety Characteristics

1. Nuclear Data-0.75 of flux above $0.1 \mathrm{MeV}$

\begin{tabular}{|c|c|c|c|c|c|c|}
\hline & $\beta$ & $\sigma_{\mathrm{f}}$ & $\sigma_{c}$ & $\begin{array}{l}\text { Fission } \\
\text { Threshold, } \\
\text { MeV } \\
\end{array}$ & $\eta$ & $\left(\frac{\text { Power }}{\phi}\right)$ where $\left(\frac{\mathrm{P}}{\phi}\right)_{\mathrm{Pu}}=1$ \\
\hline $\mathrm{U}^{235}$ & 0.0065 & 1.74 & 0.42 & & 2.0 & 1.7 \\
\hline $\mathrm{U}^{233}$ & 0.0027 & 2.45 & 0.35 & & 2.2 & \\
\hline $\mathrm{Pu}^{239}$ & 0.0021 & 1.90 & 0.39 & & 2.46 & 1.0 \\
\hline $\mathrm{Pu}^{240}$ & 0.0026 & 0.46 & 0.41 & 0.2 & & \\
\hline $\mathrm{U}^{238}$ & 0.0157 & 0.062 & 0.24 & 1.2 & & \\
\hline Thorium & 0.022 & 0.014 & 0.40 & 1.25 & & \\
\hline
\end{tabular}

2. Core Safety Characteristics

$\begin{array}{lcccc}\beta & \begin{array}{l}\Delta \mathrm{k} \text { to } \\ \text { Sodium } \\ \text { Boiling* }\end{array} & \begin{array}{l}\text { Doppler } \\ \text { Coef } \\ \Delta \mathrm{k} /{ }^{\circ} \mathrm{C}\end{array} & \begin{array}{l}\text { Fuel } \\ \text { Expansion } \\ \text { Coef, } \\ \Delta \mathrm{k} /{ }^{\circ} \mathrm{C}\end{array} \\ 0.0072 & 0.6 & 2 \times 10^{-6} & 10 \times 10^{-6} \\ 0.0065 & 0.6 & -\cdots & 10 \times 10^{-6} \\ 0.0021 & 0.6 & -\cdots & 10 \times 10^{-6}\end{array}$

Mixed Oxide, $\frac{\mathrm{Pu}}{\mathrm{U}^{238}}=\frac{1}{3}$

0.0033

0.3

$3 \times 10^{-6}$

Thorium Metal, $\frac{\mathrm{U}^{233}}{\mathrm{Th}}=\frac{1}{3}$

$0.0031>0.6$

? $\quad 10 \times 10^{-6}$

Cermet, $\mathrm{PuO}_{2}-\mathrm{Th}$

$0.0025>0.6$

? $\quad 10 \times 10^{-6}$

Molten Plutonium

$0.0021 \sim 1.7$

$60 \times 10^{-6}$

*No credit for sodium coefficient or for structural but credit for Doppler and fuel expansion. 
SD26.13

The reason why the delayed neutron fraction is the key to the number of test pins that can be melted short of damage to the driver core was well brought out by the Germans in their paper presented at the safety conference held last fall at ANL. A fast reactor was examined with various reactivity coefficients. The time to fuel melting as related to the ramp rate of reactivity insertion was evaluated. The results are given in siide 6. It can be seen that for ramp rates greater than $\$ 4 / \mathrm{sec}$, there is not a great contribution from the Doppler effect within the magnitude which can be produced in the FFTF reactor. At lower than this ramp rate, it is expected that both the Doppler coefficient and the fuel expansion should be helpful (even though it doesn't show up in the German work). The curve indicates that any pin melting circumstance can be handled as long as it has a ramp rate less than $\$ 4 / \mathrm{sec}$ if the control rods can be counted on to operate within 0.3 second. As Hofmann and Nims pointed out, it is reasonable to expect that the rate of test fuel movement will be less than about $3 \mathrm{ft} / \mathrm{sec}$ and that, for the equivalent of the movement of $1 / 100$ of the core, the ramp rate even with plutonium fuel will be under $\$ 4 / \mathrm{sec}$. It is further observed that if the $\Delta \mathrm{k}$ inserted is less than $\$ 1$ and less than $\Delta k$ to boiling, no serious damage results regardless of the ramp rate.

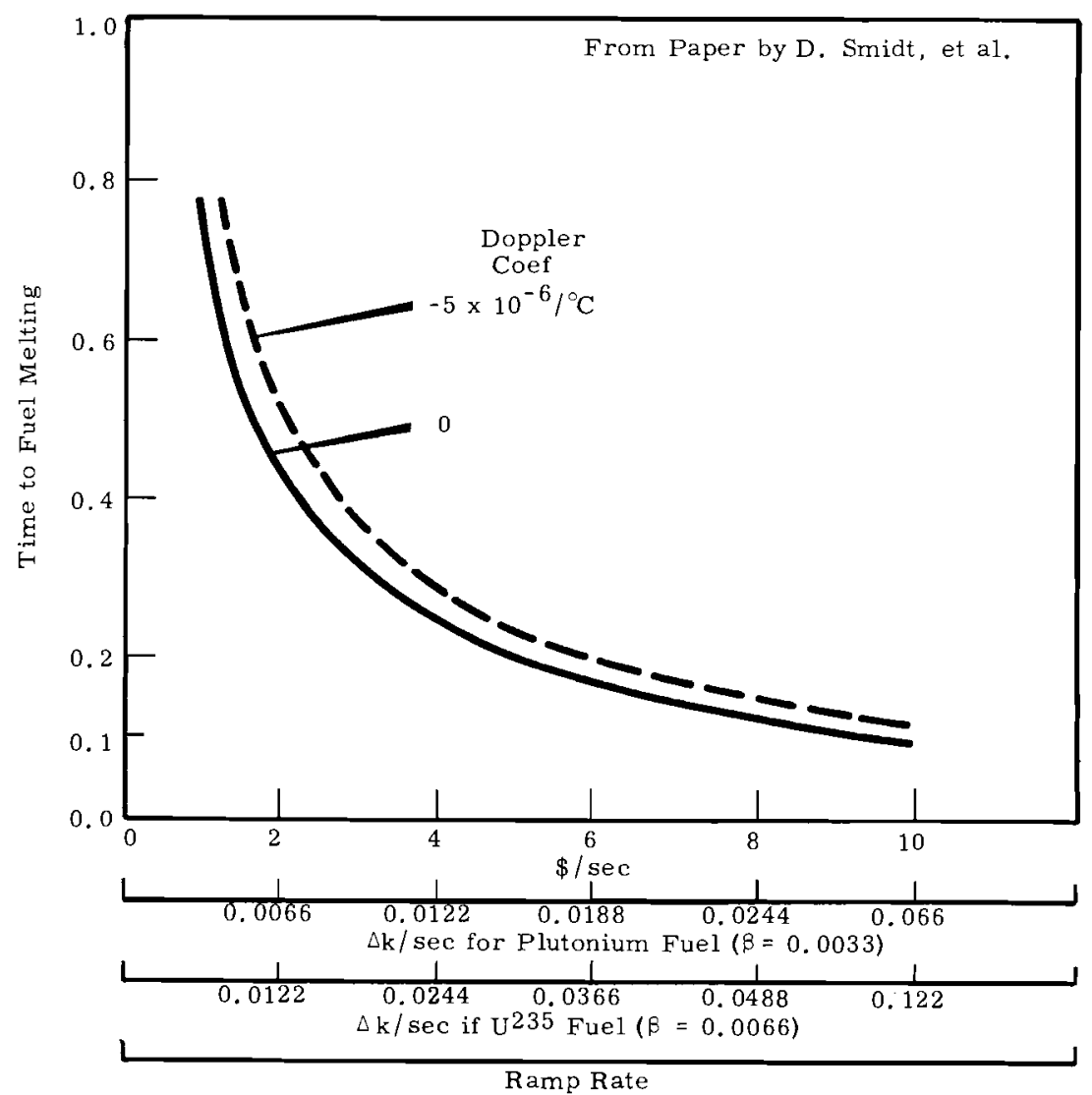

SLIDE 6. Effect of Doppler Coef and Ramp Rate on Time to Fue 1 $\overline{\text { Melting }}$ 
$\operatorname{SD} 26.14$

The question of the rate at which reactivity might be introduced under the condition of melting of the test pin has been given some consideration by both Nims and Hofmann. Considering both gravitational effects and the results of the Huebotter experiments (1) as we 11 as the real geometry that would exist in a test element and the nature of the fuel, it is difficult to see that the fuel could move at even an average speed of $3 \mathrm{ft} / \mathrm{sec}$ toward the center of the reactor. A refinement of the physical situation will probably indicate that these rates are conservative, and the 1 ikelihood of stopping at the center of the reactor is small.

Table $V$ gives data on power transient accommodation capability of various pin type fuel systems. Shown is the heat capability of the fuel and the time constant for heat to be transferred from the fuel elements to the sodium. The time to melting of a fue 1 element, if it is operated at $100 \%$ power with no heat transfer to the coolant, is also given. Al1 systems are expected to have minor differences in time to melting ranging in time constants between 0.5 and 2 seconds. This time difference between the various fuels is not significant when it is recognized that the power increase will be exponential. The heat transfer constant is such that for certain systems such as the metaliic ones, there is a good chance that some credit for the sodium temperature coefficient can be taken for the probable type of accident that is expected to be under $\$ 1.00 / \mathrm{sec}$. This credit is not considered to be great enough to take into account on a broad evaluation of the type that is being made here.

Table VI shows a rough estimate of the number of test pins that can be melted for the various types of driver fuels and specific power of the test pins.

It is to be noted that there is an effect of critical mass of the driver core. It is also to be noted that if all cores were made the same critical mass, the relative pins which could be handled at $1 \mathrm{~kW} / \mathrm{in}$. are

$\begin{array}{ll}\text { - } \mathrm{PuO}_{2}-\mathrm{SS} & 160 \\ \text { - } \mathrm{UO}_{2} \text {-SS } & 320 \\ \text { - } \mathrm{Mixed} \text { Oxide } & 175 \\ \text { - } \mathrm{U} 235 & 340\end{array}$

(1) APDA-155 "Study of Fast Reactor Me1tdown Accidents Using Simulant Materials" 
SD 26. 15

TABLE V. Data on Power Transient Capability of Various Pin Type Fuel Systems

\begin{tabular}{|c|c|c|c|c|c|c|c|}
\hline & $\frac{\rho \mathrm{C}_{\mathrm{p}}}{\mathrm{Btu} / \mathrm{ft}^{3}-{ }^{\circ} \mathrm{F}}$ & $\begin{array}{l}\int \mathrm{kd} \theta \\
\frac{\mathrm{Btu}}{\mathrm{hr}-\mathrm{ft}}\end{array}$ & $\begin{array}{r}\Delta \mathrm{T} \\
{ }^{\circ} \mathrm{F} \\
\end{array}$ & $\begin{array}{r}\mathrm{k}\left(1000^{\circ} \mathrm{F}\right) \\
\mathrm{Btu} / \mathrm{hr}-\mathrm{ft}-{ }^{\circ} \mathrm{F}\end{array}$ & $\begin{array}{l}\text { to Melting } \\
\frac{\rho \mathrm{C}_{\mathrm{p}} \Delta \mathrm{T}}{\mathrm{k}} \\
\text { sec }\end{array}$ & $\begin{array}{l}\text { of Fuel } \\
\frac{\mathrm{r}^{2} \mathrm{C}_{\mathrm{p}} \rho}{4 \mathrm{k}} \\
\mathrm{sec}\end{array}$ & T Melting \\
\hline $\begin{array}{l}\text { Uranium Alloy } \\
\text { Metal }\end{array}$ & 40 & 6,000 & 700 & 21 & 1.0 & 0.22 & 2,070 \\
\hline SS Cermet & 65 & 6,000 & 650 & 8 & 1.6 & 0.8 & 2,500 \\
\hline Ni Cermet & 70 & 12,000 & 700 & 25 & 0.9 & 0.3 & 2,400 \\
\hline Oxide & 35 & 3,000 & 800 & 1.85 & 2.0 & 2.0 & 4,400 \\
\hline Carbide & 40 & $\begin{array}{r}12,000 \\
6,000\end{array}$ & 700 & $\begin{array}{r}10 \\
5\end{array}$ & $\begin{array}{l}0.5 \\
1.0\end{array}$ & $\begin{array}{l}0.4 \\
0.8\end{array}$ & 3,000 \\
\hline
\end{tabular}

Data based on 3/16 in. diameter of fuel.

Where:

$$
\begin{aligned}
\frac{\rho \mathrm{C}_{\mathrm{p}} \Delta \mathrm{T}}{\mathrm{k}} & =\text { Time to melting of fuel at } 100 \% \text { power and no heat transfer } \\
\frac{\mathrm{r}^{2} \mathrm{C}_{\mathrm{p}} \rho}{4 \mathrm{k}} & =\text { Time constant for pin element fuel heat transfer } \\
\Delta \mathrm{T} & =\begin{array}{l}
\text { Temperature difference between melting temperature and } \\
\text { operating temperature of fuel }
\end{array} \\
\mathrm{p} & =\text { Heat generation per unit volume of fuel at hot spot } \\
& =\frac{4 \int_{\mathrm{kd} \theta}}{\mathrm{r}^{2}} \\
\mathrm{r} & =\text { Radius of fuel }
\end{aligned}
$$

For reference:

$$
\begin{aligned}
& \text { Average neutron lifetime } \cong 0.05 \mathrm{sec} \text { for } \beta=0.033 \\
& \cong 0.1 \mathrm{sec} \text { for } \beta=0.066 \\
& \text { Fuel rod expansion response } \cong 10^{-3} \mathrm{sec} \\
& \text { (Approx. } \ell \div \text { speed sound in fuel) } \\
& \text { after heating } \\
& \text { Doppler }=\text { Instantaneous } \\
& \text { takes place } \\
& \text { Prompt neutron lifetime } \cong 10^{-7} \mathrm{sec}
\end{aligned}
$$


TABLE VI. Rough Estimate of Relative Number of Test Pins Which Can be Melted Short of Melting Driver Fuel

\begin{tabular}{|c|c|c|c|}
\hline & \multirow{3}{*}{$\begin{array}{l}\beta=0.0021 \\
\text { critical mass }=200\end{array}$} & \multicolumn{2}{|c|}{$\begin{array}{l}\text { Test Pins Which Can be Melted, } \\
\begin{array}{|l}\text { Specific Power of Test Pin } \\
\end{array}\end{array}$} \\
\hline & & $1 \mathrm{~kW} / \mathrm{in}$. & $5 \mathrm{~kW} / \mathrm{in}$. \\
\hline $\mathrm{PuO}_{2}-\mathrm{SS}$ & & 80 & 16 \\
\hline $\mathrm{UO}_{2}-\mathrm{SS}$ & $\begin{array}{l}B=0.0065 \\
\text { critical mass }=400\end{array}$ & 330 & 66 \\
\hline Mixed Oxide & $\begin{array}{l}\beta=0.0033 \\
\text { critical mass }=300\end{array}$ & 130 & 26 \\
\hline $\mathrm{U}^{235}$ Metal & $\begin{array}{l}\beta=0.0072 \\
\text { critical mass }=450\end{array}$ & 380 & 76 \\
\hline
\end{tabular}

Based on:

1. $10^{16}$ maximum flux for driver core

2. Test pins at average core position

3. Accident is of the order of $\$ 2 / \mathrm{sec}$ or less

4. Core critical masses corresponding to Slides 3 and 4

5. $\int \mathrm{kd} \theta$ corresponding to Table III

6. Metal and cermet systems are assumed to have fuel expansion coefficient of $10 \times 10^{-6} /{ }^{\circ} \mathrm{C}$ and oxide a Doppler of $3 \times 10^{-6} /{ }^{\circ} \mathrm{C}$.

The general conclusion drawn from the evaluation of safety is that when a detailed evaluation is made as to what happens when the test pin melts, it will be found rather unlikely that large positive reactivity insertions will take placu. Furthermore, as long as a control system is chosen that can insert reactivity within $0.3 \mathrm{sec}$, the likelihood of damage to the driver core will be small. Lacking present evidence of this position, preference from a safety standpoint should be given to $U^{235}$ fue $1 \mathrm{~s}$ and to fuel elements that can provide fuel expansion such as the metallic or cermet type. 
GENERAL CONCLUSIONS

A summary is given in Table VII.

$\underline{\text { Nuclear }}$

- There is about a 1.7 advantage in using $\mathrm{Pu}^{239}$ fuel in place of $\mathrm{U}^{235}$ fuel in power requirements.

- Larger reactors produce a larger flux per megawatt.

Thermal Performance

- Choose high ${ }^{j} k d \theta$ fuel for the ultimate reference. This probably means ruling out both gas-bonded and metallurgically-bonded elements. If a high ${ }^{\mathrm{I}} \mathrm{kd} \theta$ developed, there should be no need to design the plant for greater than 250 to $300 \mathrm{MW}$ for $1.35 \times 10^{16} \mathrm{flux}$.

- suggest consideration of (a) allowing large $\Delta \mathrm{T}$ of fue 1 and radial fracture of fuel where necessary, and (b) designing to prevent melt-through of the clad by radial venting. A $3 / 16$ in. diam pin is acceptable for metal, cermet and carbide pins which are sodium-bonded.

- As planned by BNW, the primary system should be designed for vented fuel.

- Design plant for ultimate use with plutonium fuel. If the plant is operated initially with U235 fuel, do not run plant at a power above the plutonium rating even if some sacrifice is made in the achievable initial flux. For initial fuel testing, $0.5 \times 10^{16}$ flux could be adequate.

Economics

- There seems to be very little difference in the fuel operating cost per year for the major fuels being considered for initial operation.

- Since the fuel element can be made large in diameter, real economies can be achieved if high ${ }^{\prime} \mathrm{kde}$ fuel is used.

- Burnups now achievable of about $10^{21}$ fissions/ $\mathrm{cm}^{3}$ give reasonable enough economics for initial operation. The penalty in development cost and/or performance to design for much greater burnup seems unwarranted.

- Establish general nature of the ultimate reference fuel before choosing the initial fuel and the costs of making the transition. 
$\operatorname{SD} 26.18$

\section{Safety}

- Two driver core fuel characteristics appear most important from a standpoint of prevention of driver core damage; they are fuel expansion and the delayed neutron fraction of the fuel. The latter is the major governing factor in the number of test pins that can be melted short of damage to the driver core. Fuel expansion affects both the test pins, which can be allowed to me1t, and plays a large role in the loss of power accident. If tricks are not relied on to provide the $\Delta \mathrm{k}$ to handle the power loss accident, then a fuel element should be chosen that has a reliable fuel expansion (such as the metal or cermet).

- Design the safety system to start introducing reactivity in $0.3 \mathrm{sec}$.

- A11 fuels being considered for the initial operation seem to have no "go, no-go" problem in regard to the maximum accident. However, the FFTF plant should be designed for the largest maximum accident possible without incurring undue cost. Future drivers may have maximum hypothetical accidents greater than now presumed. Furthermore, bolder fue 1 tests than now envisioned may be run.

FURTHER EVALUATIONS REQUIRED

The following further evaluations should be made before a choice of fuel is made for the initial loading:

- Recheck primary use of facility.

(a) Are the initial tests going to be primarily those associated with trying to understand fuel behavior as proposed by D.R. deBoisblanc?

(b) What types of power densities are envisioned (1ike $5 \mathrm{~kW} / \mathrm{in}$.$) ?$

(c) On how many of the initial tests will fue 1 be run to failure?

- Establish overpower conditions for which the driver core should be designed.

- Establish design of the possible fuels for both $0.5 \times 10^{16}$ and $10^{16}$ flux, since FFTF can be a bootstrap operation from the standpoint of its own fuel development.

- Low burnup designs are acceptable for first fuel cores.

- The AEC should establish the relative importance they attach to the choice of the initial fuel because this might assist in the development of near-term central station fuel. 
TABLE VII. Summary of Characteristics of FFTF Driver Fuels

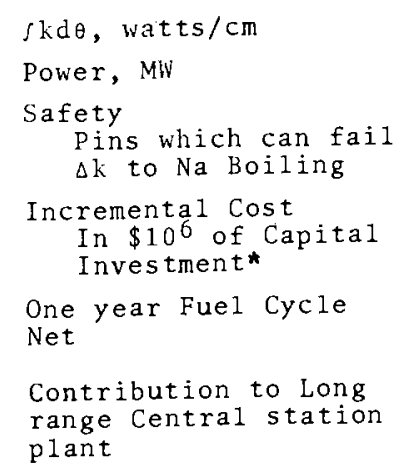

\begin{tabular}{|c|c|c|c|c|c|c|c|c|}
\hline \multicolumn{3}{|c|}{ METAL } & \multicolumn{2}{|c|}{ CERMET } & \multicolumn{2}{|l|}{ OXIDE } & \multicolumn{2}{|c|}{ CARB I DE } \\
\hline $\begin{array}{l}\text { Present } \\
\text { Practice }\end{array}$ & Potential & Potential & $\begin{array}{l}\text { Present } \\
\text { Practice }\end{array}$ & Potential & & & & \\
\hline $\begin{array}{c}\text { U-Fs } \\
\text { Na-Bonded }\end{array}$ & $\begin{array}{c}\mathrm{Pu}-\mathrm{U}-\mathrm{Zr} \\
\text { Na-Bonded } \\
\text { Vented }\end{array}$ & Liquid $\mathrm{Pu}$ & $\mathrm{PuO}_{2}-\mathrm{SS}$ & PuX - W & $\mathrm{PuO}_{2}-\mathrm{UO}_{2}$ & $\begin{array}{l}\mathrm{PuO}_{2}, \\
\mathrm{UO}_{2}, \\
\mathrm{Mo}^{2}\end{array}$ & $\begin{array}{l}\text { PuC, } \\
\text { UC }\end{array}$ & $\begin{array}{l}\text { PuC, } \\
\text { UC, } \\
\text { Cr }\end{array}$ \\
\hline 60.0 & 100.0 & 250.0 & 60.0 & 500.0 & 33.0 & 60.0 & 35.0 & 200.0 \\
\hline 375.0 & & & 210.0 & & 340.0 & & 320.0 & \\
\hline 380.0 & & & 80.0 & & 130.0 & & 120.0 & \\
\hline 0.6 & 0.6 & 1.7 & 0.6 & 0.6 & 0.3 & 0.3 & 0.3 & 0.3 \\
\hline 13.0 & & & & & 10.4 & & 8.8 & \\
\hline$(-) \frac{1.4}{11.6}$ & & & 0 & & $\frac{0.3}{10.7}$ & & $-\frac{0.4}{9.2}$ & \\
\hline & Has potenti & & $\begin{array}{l}\text { Higl dipe } \\
\text { type has }\end{array}$ & $\begin{array}{l}\text { ersion } \\
\text { potential }\end{array}$ & $\begin{array}{r}\text { May b } \\
\text { for g } \\
\text { steam } \\
\text { rea }\end{array}$ & $\begin{array}{l}\text { good } \\
\text { s or } \\
\text { cooled } \\
\text { tors }\end{array}$ & Has & potential \\
\hline
\end{tabular}

* Based on $\$ 80 / \mathrm{kW}$ 
- Check the assumption that no credit is given to ceramic fuel for fuel expansion. This is a severe penalty; however, if the assumption is to be changed. Show proof of why. Also, confirm fact that, for a sodium-bonded metal pin, can take credit for expansion.

- Review stress criteria for clad such as the interaction of material with clad and the creep or stress rupture. criteria.

- Establish criteria for allowable stress in metallurgicallybonded metal fuel. What is the nature of failure, and how serious is it?

- Establish the nature of failure of the bottle-type (nonvented) closures 1 ike that used for oxide fuel and for sodium-bonded metallic fuel. What is a safe design criterion under transient conditions? Can large gas pressure be tolerated at a11? What is the power to which the oxide-type element can be taken before failure; what is the mechanism of failure?

- Establish the importance of the delayed neutron fraction on the choice of the driver fuel. What are the limits of rates of fue 1 movement and degree of movement which might be normally expected? TREAT tests to determine rates and magnitudes should be run.

- How are the transients for various ramp rates affected by $\beta$; by Doppler; by fuel expansion? Confirm the belief of relative insignificance of the Doppler for $3: 1 \mathrm{U}^{238}$ and the plutonium fuel on the test fuel meltdown accident.

- If Doppler contribution is smalla (as is expected), then there is little reason to use $\mathrm{U}^{238}$ or thorium in the fuel.

- Check the scram rate that will be relied on to prevent damage by the fuel movement.

- Establish a schedule for the decision on the initial driver fue 1 .

- The clad for all fuels needs more evaluation: for instance, establish why eutectic formation at about $1350^{\circ} \mathrm{F}$ was considered a problem with EBR-II type fuel, and it is no longer considered a problem with Pu-U-Zr fuel. 
$\operatorname{SD} 27.1$

SUMMARY DISCUSSION

E. L. Zebroski (GE)

$\underline{\text { REMARKS }}$

It seems clear that the FFTF driver fuel has advocates for either oxide or metal or carbide or cermet. The selection cannot intelligently be made on any single factor, but will have to be based on an exhaustive, weighted balance of all of the relevant factors. Unfortunately, I don't think any of us would attempt to say that he has yet made an exhaustive, weighted balance of all of the factors. However, we should be able to agree that the amount of development and testing required to get some assured, useful level performance should be one of the major factors.

From that view point, because he put it so smoothly, I found myself almost going along with Howard Kittel on how equivalent the experience is on carbide, oxide and metal. But thinking it over, I cannot really agree that there is nearly as much experience on metal or on carbide fuel. There is really a great deal more experience on oxide fuel than on metal fuel, for conditions of interest to large fast reactors.

While the "box score" basis is not the only important factor, we should keep the record straight about the large number of fast reactors that are going to operate on oxide fuel. Looking at the reactors committed or actually under construction right now, there are $2030 \mathrm{MW}$ thermal of fast reactors with oxide fuels against less than 200 with metal fuels. About 10 to 1. So Howard, I just cannot agree with you on that.

MR. KITTEL: No, I said, "that have been operated."

MR. ZEBROSKI: We11, even there I think you have to be a little generous to metal in order to discount the Dounreay data on three 77 pin bundles of oxide. If you want to make the comparison, they have it there on a large number of pins now. That is a large fraction of the experience (not even counting SIR A and $B$ and $B R-5$, all using oxides).

The question of fuel movement has been given a great deal of weight. I wish I could agree with Al Amorosi's position that you cannot give safety much weight. I would not want to live within 50 miles of the "zero-zero" coefficient reactor which he put on the board there. I wonder if the ACRS would let you build it at all. You could possibly build that in Russia on their ground rules, but--

MR. AMOROSI: No, I was talking about the Germans. 
MR. ZEBROSKI: I don't believe they propose to build the "zero-zero" coefficient type either.

MR. AMOROSI: They have not had very much background。

MR. ZEBROSKI: Possibly the main reason for still considering the cermet is to get the assurance of the fuel location (the point raised earlier today by Dr. Don de Halas). The problem of axial fuel expansion or relocation is a problem which is central to the accuracy of the Doppler coefficient measurements which SEFOR is scheduled to do. There, the calculations show that if you have a one-segment oxide fuel rod, then the possible $\Delta \mathrm{k}$ for the axial expansion is about equal to the Doppler effect. If you divide the fuel into two segments, then the axial expansion drops to about $50 \%$ of the Doppler effect. If you segment the fuel to three segments, it drops to $20 \%$ of the Doppler effect. If you segment it into an infinite number of segments, it is still about $20 \%$ of the Doppler effect. If the main advantage of cermet is to get assurance of where the center of gravity of fuel is, you can match this with a few segments rather than pay the price for "infinite segmentation" as with cermet.

Rather than try to make exhaustive comments on a lot of the points which Al Amorosi has raised, I would like to try a more general perspective. One real danger is that we make comparisons of the choice of fuel on what we might call "scientism"--in which we give a great deal of weight to a few things which are easy to calculate and to make trade-offs with. But we tend to ignore the more crucial and slippery issues--which affect feasibility, timeliness, and overall costs and objectives--that are more qualitative and hard to calculate。In making a judgment of what the crucial issues are, we have to start out with some assumptions and some interpretations of the criteria。

Let me suggest one generalization of the criteria as I read them: first, that the FFTF has a reasonable objective of putting in as much test fuel as possible, as soon as possible, so as to increase the U.S. fast reactor test capabilities. Secondly, I think that the further the driver fuel and the reactor design is from the known technology, the more likely that problems will occur which have to do with bailing out the test reactor, and then the value of the testing capability can for some time become small or even negative. Thirdly, maximizing the research and development required to obtain a special-purpose driver fuel is not a worthy objective of the LMFBR Program.

I think we can all agree that some of the "scientism" considerations should be iittle regarded. The appeal has long been gone on novelty for its own sake even in RED; novelty is no longer synonymous with virtue in nuclear design. 
$\operatorname{SD} 27.3$

I am concerned that ultra-high flux target may be in the category of novelty.

May I digress again before I get to the specific points. For the early 1970's, in addition to the FFTF there are now at least six large and four small fast reactors committed (all with oxide fuels initially - - except one which may have carbide also). Most of these reactors are in Europe. Let us hope that the FFTF will truly contribute in a timely fashion to U.S. technical and industrial capability on fast reactors. This reactor must not be an exercise in schedule delays and budget escalation, which have been almost universal on projects of this kind. It may hurt some in the audience here when I read the record of three to six year delays and more (over the initial schedule) of EBR-II, ETR, ATR and Fermi, not to mention EGCR. All of these were much easier reactors to design and build than FFTF as it is now specified. If this program follows the route of diversion of scarce test facilities to objectives which are not relevant to fast-spectrum power reactors (skewed cores, cermet fuel, giant handling machines, super-temperature loops, etc.) you will have the unpleasant satisfaction of first reading the results of most of the experiments which you are interested in doing in the foreign literature. You could see U.S. industry having to buy its technical results and having to license its fast power reactor technology from overseas. To bring it closer to home, there would be fewer jobs and at lower levels in the U.S. power reactor industry and thus also in the supporting laboratories.

I think that the driver fuel choice should go the same route as for most reactors to date. You take a balanced judgment of what you can do with the greatest certainty and the least possible compounding of development. This looks to be an oxide core with an initial flux target from about $0.7 \mathrm{x}$ $10^{16}$ to $1 \times 10^{16}$.

It appears possible at a firmly calculable cost to make the design adaptable for subsequent cores at higher power density (with either oxide or carbide). Limited segmentation of the fuel can limit axial effects.

Finally, it seems to me that cermet is worthy of being only a very low-level back-up. It seems to me that cermet could turn out to be the best choice only on the assumption of failure of most of the fast reactor development efforts in the U.S., and the rest of the world as well.

Now on the blackboard we have a chart along the line of decision theory. The coding numerically represents things which are difficult to make quantitative, this is a numerical way of expressing a priori judgments or an opinion. Everyone can make his own judgments on these factors, but one of the useful inputs on such judgments is what other experienced people think about it. 


\section{$\operatorname{SD} 27.4$}

This chart compares the choice of different driver fuels in terms of the expected value of the result. On the first line, the assurance of sufficient development and test by 1969 or 1970 (which is about the earliest you have to freeze on a fuel design for fabrication of the first core) we can rate oxide at about 10. This will drop down somewhat for carbides and metals, and still lower for cermet (at the target performance level needed to justify an advantage over ceramic fuels)。

Assuming that your commitment did not allow a serious compromise with the performance target set for cermet (see the second item in the table) the benefits to the United States' fast breeder program of cermet development is a negative value. This judgment comes about as follows: If cermet is to be seriously tested, critically short central test space in EBR-II will have to be diverted to something having no relevance to any power reactor program, and which even then cannot be fully and realistically tested in EBR-II regardless of priority.

On the third item, the long-term benefit to advanced fast breeder reactors, it seems to me that carbide, and possibly nitride, are the only legitimate additional candidates now visible for high power density operation. It seems to me that metal fuel, even granting that burnup capabilities can be raised, does have the defect of limited temperatures. It faces a ceiling on allowable temperature versus swelling rates. You would have to take a fairly sizable increase in your ultimate power cost target if you accepted that temperature ceiling as a limit for your technology. Both metal and cermet suffer to some extent from this defect of temperature limitation.

On the fourth item, the cost-effectiveness of the FFTF tests, I will observe again that cermet again rates poorly. This is because you must devote most of the reactor core to the driver fuel function which gives you no useful data. Whereas, if you put in either oxide or carbide, you can learn something useful to power reactor fuel. You could do this with metal also, but EBR-II is already capable of doing it on a large scale.

In the right hand column, you see weighting factors giving the relative importance to some of these things. This is by no means exhaustive, there are many other considerations. But the "assurance of development" and "near-term benefits" items should have important weight, while questions like calculated near-term fuel cost are much less relevant. Near-term fuel cost differences are trivial (relative to the extra development cost of a singlepurpose fuel) and probably not projectible with meaningful accuracy since the specifications are not known. Similarly, the issue of ultra-high peak flux for accelerated testing is almost not directly relevant, or are incomplete. A reasonable figure of merit (used in Item 4) is proportional to ( $f 1 u x$ ) times (operating factor) times (useable test volume of the core). High flux alone is not a bargain to the extent that a high target value of peak flux tends to force lower values of the other two factors. 
$\operatorname{SD} 27.5$

An ultra-high flux target also forces a generally more complex and trouble-prone design which sharply increases development costs and the probability of delays. This leads to Item 5 , which is the "timeliness" factor or "present worth" factor. The exponential term (see table, footnote 4) describes the observation that the technical and strategic value of a multipurpose test reactor decays somewhat with time. In a competitive world, the prime objectives on which an FFTF can have useful impact must very largely be realized in the period before 1980. If we choose speculative (and in some cases untestable) performance targets for the fuel and reactor design, it will inevitably take several years longer to accomplish the pre-operational and postoperational R\&D - - and we may still end up with the need to compromise performance to more modest levels.

TABLE I

A SUGGESTED RATING PROCEDURE FOR DRIVER FUEL CANDIDATES EVALUATTION

\begin{tabular}{|c|c|c|c|c|c|}
\hline Factor/Candidate & Carbide & Cermet & Oxide & Meta1 & $\begin{array}{l}\text { Relative } \\
\text { Rating } \\
\text { of Factor }\end{array}$ \\
\hline $\begin{array}{l}\text { 1. Assured development } \\
\text { of target performance } \\
\text { by } 1969-70 \text { for } \sim \$ 10^{7}\end{array}$ & 6 & 3 & 10 & 6 & 10 \\
\hline $\begin{array}{l}\text { 2. Near-term benefit to } \\
\text { LMFBR capabilities } \\
1970-75\end{array}$ & 5 & $-3^{(1)}$ & 10 & 5 & 10 \\
\hline $\begin{array}{l}\text { 3. Long-term benefit } \\
1976-80\end{array}$ & 10 & $3^{(2)}$ & 6 & $5^{(2)}$ & 5 \\
\hline $\begin{array}{l}\text { 4. Cost-effectiveness of } \\
\text { FFTF test programs ( } 3 \text { ) }\end{array}$ & 8 & 1 & 10 & 3 & 10 \\
\hline $\begin{array}{l}\text { 5robability of } \\
\text { avoiding unscheduled } \\
\text { delays of } \geq 2 \text { years }(4)\end{array}$ & 5 & 2 & 9 & 4 & 20 \\
\hline Overa11 Ratings & 6 & 1 & 9 & 4.5 & \\
\hline
\end{tabular}

Notes:

(1) Because of diversion of scarce facilities and skills to work of little direct value to the LMFBR power reactor program

(2) Because of top temperature limitations

(3) Reflecting (a) the product of ( $f 1 u x$ ) x (operating factor) $x$ (test volume useful to main objectives of LMFBR program) and (b) the greater cost and time required for loop testing versus open-core testing.

(4) This factor could also be expressed by crediting each candidate with the "timeliness value" for example: $10 e^{-.5}(t-1972)$ reflecting the decay in usefulness of delayed test capabilities. 
SD28. 1

\title{
SUMMARY DISCUSSION
}

\author{
W. E. Ray (Westinghouse)
}

\section{REMARKS}

I find that all of rny points have been covered by panelists who have preceded me. I would like to mention, however, that Dr. Zebroski raises a strong point: there is, in our estimation at Westinghouse, considerable incentive to investigate several fuel systems for the FFTF, provided that all might have eventual commercial utility. Early development of commercial fast reactors is the paramount consideration.

We feel that the commercial program for such power reactor development will reach full scale by about 1972 and that there will be two prototypes on line by 1975. Therefore, it is mandatory that FFTF driver fuel development information not only support the test facility needs, but also provide data for commercial reactor design.

I raise these points for the sole reason that, from our point of view, the FFTF driver fuel decisions will have some effect on power reactor fuel developement. One rather obvious reason for this (as Dr. Amorosi pointed out) is that there will be no time for a three- or four-year "debugging" period involving low use factors for the FFTF facilities. We feel that the best approach to assuring maximum FFTF usefulness lies in careful selection of the fuel element design.

Materials selections should be based on the conservation of resources of which money is perhaps the least important. (The engineering design effort is going to cost something in the order of 8 to 20 million dollars.) The most important expenditure involved is that of the nation's fast neutron supply. We feel that a sensible approach to these expenditures of fast neutrons, time, and money is to utilize them more efficiently by placing additional emphasis on fuels having potential commercial merit. The importance of this point is established since it has been raised several times during the past several days and by a number of types of organizations.

I believe that the carbide fuel system, which obviously we espouse, has considerable potential as a commercial fuel. Its technical merits have already been well covered, so there is no reason to reiterate them at this time. We recommend that carbide fuels be included in the FFTF development program scope.

There is a further point which may be of some interest and which has been only indirectly cited during the past three days. The FFTF driver fuel is going to receive an enormous amount of irradiation testing. New test programs which might be conducted in the FFTF after it is completed cannot supply the total need for commercial for fuel developement. Test 
assemblies, by their very nature, are atypical, not typical, of commercially manufactured products. The samples they contain are individually produced under conditions of abnormally close process and quality control and, therefore, do not reflect statistical variations inherent in mass-produced elements. The significance of these statistical data must be established by commercial organizations assessing the financial risks which must be assumed in marketing a large FBR. During the course of fuel load manufacture for all FFTF, such data will be generated. The FFTF driver fuel will, after all, accumulate a considerable number of design confirmation exposures. These data can be applied, even though it be to some extent indirectly, to the statistical assessment of a given commercial fuel system.

I think there is, and will be, enormous financial involvement in this country's fast breeder industry. This financially motivated interest can greatly influence the rate at which the FFTF program proceeds. If the program is well-planned and answers the long-term commercial need, financial interest can supply considerable stimulus. If only the short-term need is served, the eventual result will eventially be the direct opposite.

A result of this three-day conference has been a healthy difference of technical opinion as to which fuel should be developed for a given application. During the last sessions in particular, we have heard many systems mentioned. Previous speakers today have (even at this late point in the symposium) brought up entirely new candidate materials. We feel that this is as it should be, since all of the systems do have merit. However, very soon a reference fuel and a backup fuel system must be selected and carried aggressively toward the final goal. I think this will be done.

Again, let me refer to Dr. Zebroski's presentation. I see no reason why I shouldn't assess the situation with regard to the status of various driver fuel candidates as he has summarized on the blackboard. I believe it is in keeping with information concerning both reference fuel systems for FFTF in docum entation given us over the last month. It certainly corresponds both with the remarks by speakers during earlier sessions and those presented at this time. 
SD29.1

SUMMARY DISCUSSION

W. P. Chernock

Combustion Fngineering, Inc. Windsor, Connecticut

$\underline{\text { REMARKS }}$

MR. CHERNOCK: To come up here at this stage of the symposium and say anything that is astounding and brand new, I think, would be astounding.

First of a11, I think that the most significant item that has been brought home in this session to me is the fact (and one that I came here with) that the design of a fast test reactor is going to be an entirely difficult task. To design this particular test reactor to essentially complete and fulfill all the requirements being imposed on it is even more of a task. However, in looking at the output that may be realized from this type of an effort., I believe that we would all be highly remiss if we didn't take advantage of the fact that there will be between 7000 and 10,000 fue 1 rods built per year in support of this activity. The amount of Fuel element technology in the area of fabrication, quality control, and (hopefully) in the area of fuel element performance, should not be overlooked.

Now I have had mixed emotions on the kind of comments I should make. I must admit that right after Don deHalas made his short presentation on Tuesday, I felt very strongly that he had hit the mark, and that the cermet fuel would actually cut the mustard. I think that his basic approach to it will work. The real question I raise is that after we spend the $x$-millions of dollars developing that particular fuel system, all that we basically end up with is a particular fuel system that has (as far as I can see) absolutely no future significance to the fast reactor program other than as test fuel. And as I stated earlier, I think we might be a little remiss in not taking advantage of the large amount of fabrication and technology that will be developed during the fabrication of a large number of fuel rods per year.

I tinink I have made some comments in the session concerning the feelings that I have had about a fuel selection. I don't think that these have changed abruptly. My basic comment is that 
I think there are two fuels in the running: the mixed oxide and the mixed carbide. I do not at the present time submit to the argument that because there happens to be today possibly more fuel rods being irradiated that contain $\mathrm{UO}_{2} / \mathrm{PuO}_{2}$, that this should govern the future of the fast reactor program for the next 5 to 10 years. I believe wholeheartedly that we should take a very good look at the various candidate fuels and come up with the prime selection of that fuel best meeting the needs of this country's fast reactor program. I feel very confident, based on what I heard at this session, that that particular fuel can be made to work as a fast test reactor fuel in FFTF. I think the advantage to be gained from this approach to the program as a whole is immeasurable.

I think I would like to leave my comments at that point with one major exception; that is, I don't think anybody previously has touched on one area, and I (being a metallurgist of course) might be a little more concerned with this area than other people. However, I think that the problem of cladding materials and structural components is something that may be a little more overbearing and a little more limiting than the fuel problems. Note that I separate fuel from fuel element. I have much reservation concerning the ability of structural materials (as we know them today) to perform under the very severe flux requirements which would be imposed on them in this test reactor. 
SD50.1

SUMMARY DISCUSSION

D. R. deHalas (BNW)

REMARKS

MR. HENNIG: Our final speaker is Don deHalas of PNWL.

MR. deHALAS: My first remark really ought to be made by the Commission, but the subject has been touched upon so much here I can't ignore it. The subject keeps arising as to the commitment of the full fast neutron resources on this program. With what simple arithmetic that I can make on the subject, I feel that the amount of effort going in on this program can be no more than $10 \%$ of the Commission efforts on fuels and materials in the next 10 years. It doesn't seem to make any gross perturbation on the overall program. Furthermore, when you come to the problem of deciding which fuel would be best for the fast breeder program, if you want to use this as a criteria, one certainly can take arguments with the chart that Ed Zebroski put on the board there. For example, along the first line ( $I$ think if I want to put some of my personal numbers on there), I would write about 7-10 as the number as a chance for success. In other words, I think putting an effort on it, you could bring about any one of those fuels from a materials standpoint. The line I would like to quarrel with most, however, and I think this is subject to a lot of different opinion, is this third line; that is, the long term benefit to the fast breeder program. And this is why it gets so difficult to design what is good for everything, and make a secondary objective. I feel strongly that you will have to have a fast doubling time reactor. This would, then, rate carbide at 8 , cermet at 0 , oxide at 0 , and metal at about 10. I might be generous enough to put oxide at 1. I don't feel that oxide is going to make it in the long run.

The main thing I would like to summarize is that, as far as the reactor is concerned and getting the thing on the line, we are going to have to pick a fuel that is fairly well developed (as the point we brought up earlier), so that you can take maximum benefit of the existing technology. This, unfortunately, does drop some interesting looking long term things like the liquid metal. It probably leaves, at best, four systems: the oxide, the cermet, the alloy, and the carbide system. Although I think there are some doubts about how far along the source development is for the mixed carbide material, this is something that is still questionable. 
From a material standpoint, I think that all of these systems 10ok relative1y good. I think presentations have been made here by people who firmly believe what they are saying with good reason--that the carbide, the oxide, the alloy and the cermet probably could be made to meet FFTF fuel objectives from a material standpoint. The caution, of course, is that none of these are demonstrated fuels. None of them have ever been irradiated to the extent in fast reactors that we are talking about.

Now what we really get down to is that materials are certainly not the entire basis for the choice of a fue 1 in a reactor. I firmly believe that the choice of fue 1 will have to be strongly dictated by the other reactor considerations talked about here (the cost, the safety, and whathave-you), in spite of the fact that people brush over them as not being important. I think that they may be the overriding factors. 
SD 31.1

SUMMARY DISCUSSION

\section{FINAL GENERAL REMARKS}

MR. ASTLEY: Thank you, Don. I would like to indulge the group again; however, I believe I really did cut Al Amorosi off short. A1, if you wouldn't mind coming up, I would appreciate your taking another few minutes to complete your remarks as you saw it.

MR. AMOROSI: That reminds me that I said I was amazed at how much I felt that all the information and data presented seemed to be fairly consistent from one speaker to another.

I said also that I wasn't sure that we (being from different backgrounds) would come to the same conclusions, even though we agreed with the data, but I didn't think we were going to be as far apart as we are.

FFTF is being built for a purpose. I do want to digress (as others have) about central stations. Central station fast reactors and breeders have a long way to go. We have made numbers on the relative economics of the capital plant versus the pressurized water reactor, and one for one, the size of the heat exchangers, the surfaces, and the thickness of pipe. We can show on first principles that the fast reactor is cheaper in capital cost than water reactors. It is certainly far from that today. On first principals, we can show the same for fuel economics, too.

I would like to talk not about extrapolating oxides and carbides, but about the systems 1ike Kirkbride talks about. Here we are struggling with rather small power densities, and looking at our systems from the standpoint of their thermal performance. About all you can say about the oxides and carbides are that the visionaries who talk about the nitrides and some of the others are right. Those systems have a lot of potential. I don't want to overlook the potential of the ceramic system, nor the potential of the metal systems. You can't overlook the middle ground either, which is cermet. There is a good chance that you might be able to put $30 \%$ matrix material with high density, and you can argue about what other properties are.

What I am telling you is that there is that middle ground between the two that might give you very high power densities and very low inventory charges. I don't think you sacrifice very much on breeding, although we've got a long ways to go to get there. We must get down to the individual particle to see how it effects the behavior, and to see how the matrix behaves. I can visualize an element where I can make the gas 
go where I want. We don't have these answers today. Is there anybody who can say we are advancing the long range aspects of the fast reactor by pushing the oxide? We borrowed from water technology before; I wish we hadn't .

Now we have a facility that we can count to give us the long range fuel. I plead that we don't, in the process of using this facility, block out its capability by predetermining what it should do. So may I at least go back to the rating, which Ed had up there, and where he gave positive credit for the oxide? All I will say is if we are predetermining by that process, let's give it a negative value. The purpose of FFTF is, primarily (in my opinion), to do basic things to learn how these fuels behave; and after we learn, I am convinced that the dreamers won't have dreamed big enough.

I won't bother you with my check-off list of detailed recommendations of thermal stress and plates; instead, I will hand it to you. I realize we must do these things before we can make a choice of fuels. I only want to conclude the meeting as I started, and to say again I have enjoyed it and I have learned a great deal here. I think, indeed, it was a very worthwhile meeting and tough to change a lot of thoughts.

MR. ASTLEY: Thank you very much, A1.

There are others in the group who have not had the opportunity to express their opinions; the time is still early enough to open up the forum to others who feel they can still add something to what we spent three days working on .

MR. PEUCHL: One thing that surprised me, we haven't heard much about water reactor technology, and the lessons that we've learned from this as being applicable to FFTF. One thing I am thinking about is that test reactors and water technology consistently have shown that unless you have higher power density than the reactor being built, the test reactors aren't utilized. If we built an FFTF that is going to be (essentially) a first generation prototype with closed loops, I think we're dead before the next generation. We don't need the FFTF for the first generation prototype because, as Ed pointed out, these will be built anyhow. We need to go on from there to develop the next generation. Therefore, we shouldn't lose sight of the long range reason for the FFTF, and we shouldn't confuse the FFTF with the first generation prototype development. Let's divorce the two. Let's make sure that in designing it, we don't compromise to such an extent that we don't get the highest flux, the hottest spectrum, and the higher power densities.

MR. ASTLEY: I believe that is reflected well by the first set of criteria you put together for us.

If you recall at the opening of this meeting, I said this might well become the first of several meetings that we 


\section{$\operatorname{SD} 31.3$}

might hold on several different subjects. In this particular case we have invited a lot of you who have worked a long time in the field of driver fuel itself. I think that we could hold another meeting at a later date to discuss criteria, and we would hope to have others from your same companies specializing in that area.

Thank you very much again for coming, and we will attempt to write this all up and get it out to you. 
-

. 